


\section{NATIONAL INSTITUTE OF STANDARDS \& TECHNOLOGY \\ Research Information Center \\ Gaithersburg, MD 20899}



National Bureau of Standards U.S. Department of Commerce Boulder, CO 80303

November 1983 



\section{NBSIR 83-1695}

FITNESS-FOR-SERVICE CRITERIA FOR PIPELINE GIRTH WELD QUALITY
R. P. Reed
M. B. Kasen
H. I. McHenry
C. M. Fortunko
D. T. Read

Fracture and Deformation Division

National Measurement Laboratory

National Bureau of Standards

U.S. Department of Commerce

Boulder, CO 80303

Prepared for:

Materials Transportation Bureau

U.S. Department of Transportation

400-7th Street SW

Washington, DC 20590

and

Welding Research Council

United Engineering Center

345 E. 47th Street

New York, NY 10017

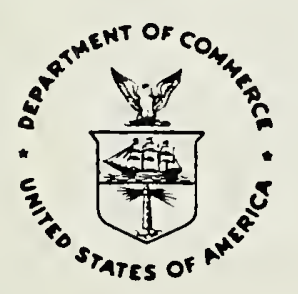

U.S. DEPARTMENT OF COMMERCE, Malcolm Baldrige, Secretary 



\section{CONTENTS}

Page

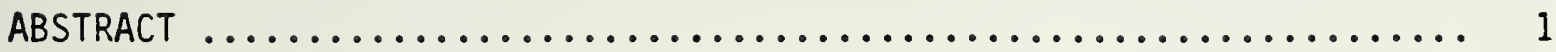

EXECUTIVE SUMMARY $\ldots \ldots \ldots \ldots \ldots \ldots \ldots \ldots \ldots \ldots \ldots \ldots \ldots \ldots \ldots \ldots \ldots \ldots$

1. INTRODUCTION $\ldots \ldots \ldots \ldots \ldots \ldots \ldots \ldots \ldots \ldots \ldots \ldots \ldots \ldots \ldots \ldots$

2. DEVELOPMENT OF ALLOWABLE FLAW-SIZE CURVES $\ldots \ldots \ldots \ldots \ldots \ldots \ldots$

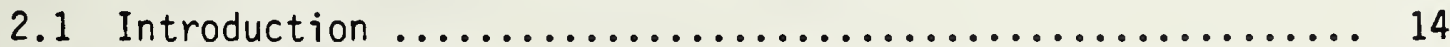

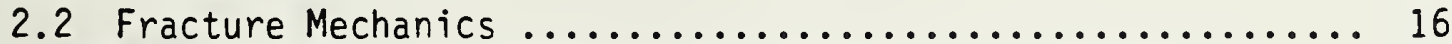

2.3 Model Evaluation Tests for Surface Cracks in Tension....... 38

2.4 Model Verification Tests on Large-Diameter Pipes ......... 71

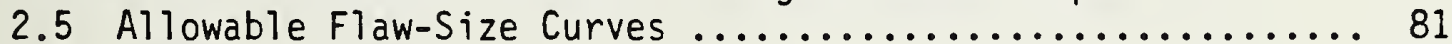

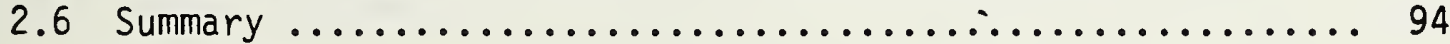

3. SHARP-FLAW DIMENSIONING $\ldots \ldots \ldots \ldots \ldots \ldots \ldots \ldots \ldots \ldots \ldots \ldots \ldots \ldots \ldots$

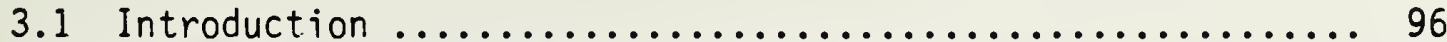

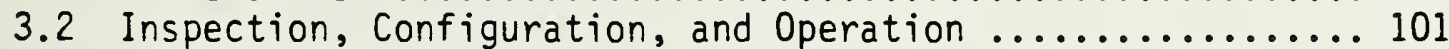

3.3 Theory of Horizontally Polarized Plate Wave Propagation.... 104

3.4 Theory of Scattering of Horizontally Polarized Shear Waves

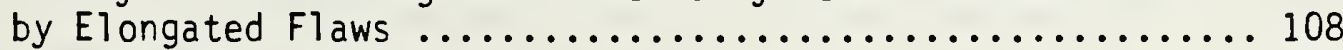

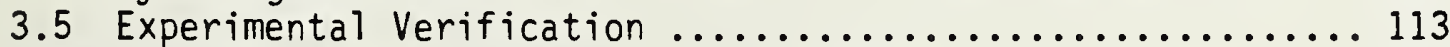

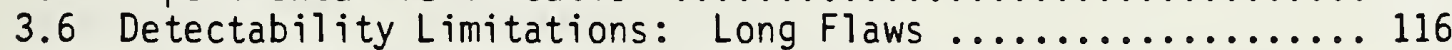

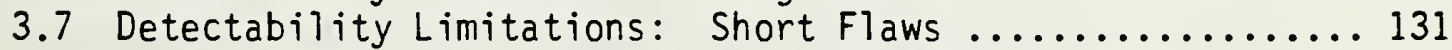

3.8 System Performance Limitations and Standards of

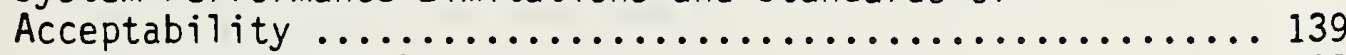

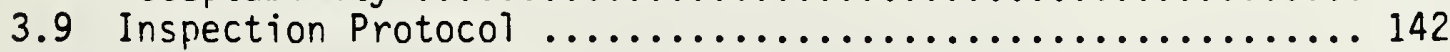

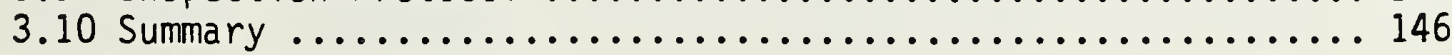

4. SIGNIFICANCE OF BLUNT FLAWS ..................... 148

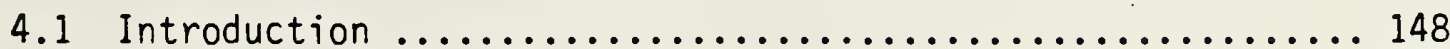

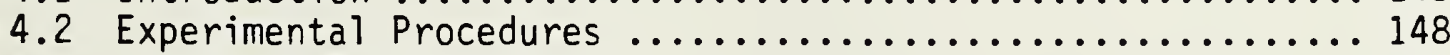

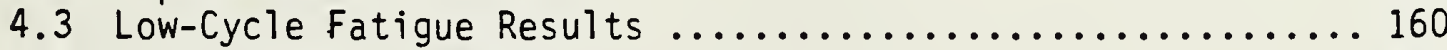

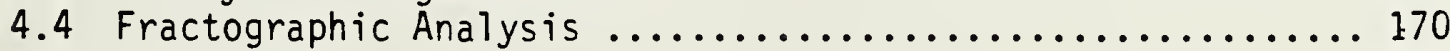

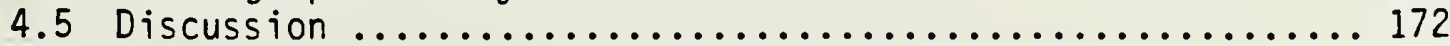

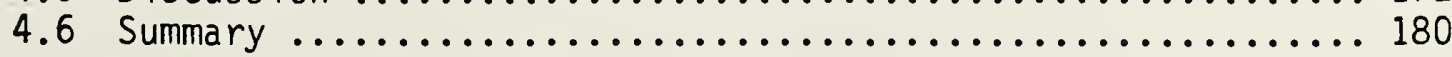

5. INTRINSIC LIMITATIONS ON DIMENSIONS OF BLUNT FLAWS ........ 182

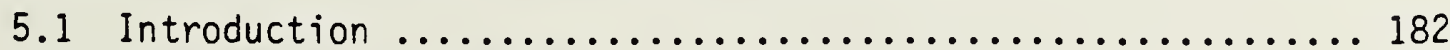

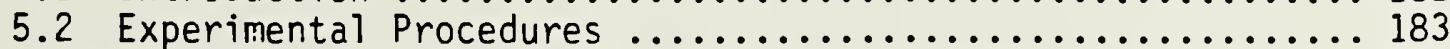

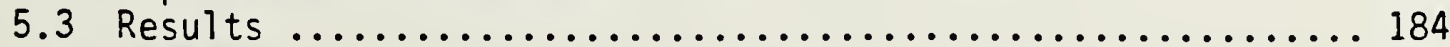

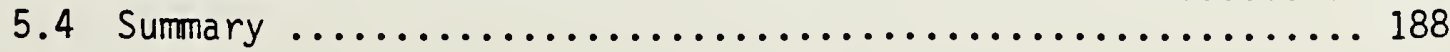



6. PRACTICAL LIMITATIONS ON BLUNT FLAW CONTENT USING

RADIOGRAPHIC INSPECTION............................. 189

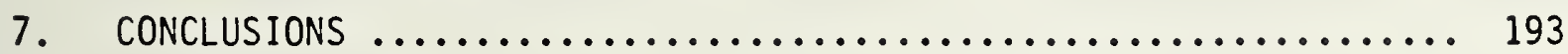

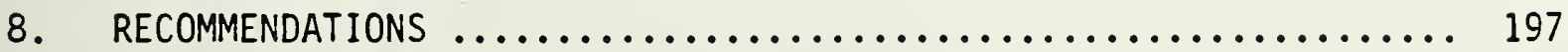

8.1 Implementation of Inspection Methods ............... 197

8.2 Implementation of Fitness-for-Service Criteria........... 202

8.3 Future Research.............................. 207

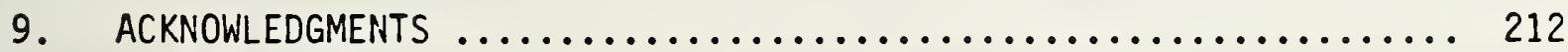

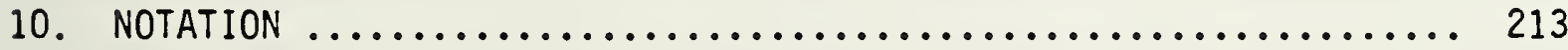

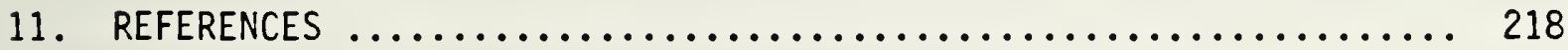

12. BIBLIOGRAPHY OF RELEVANT NBS PUBLICATIONS $\ldots \ldots \ldots \ldots \ldots \ldots \ldots . \ldots . \ldots$

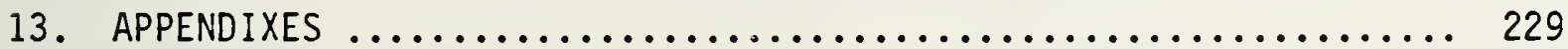

Appendix A - Details of Calculation of Allowable Flaw-Size Curves ................................. 229

Appendix B - General Research on Corrosion .............. 246

Appendix C - Radiographic Evaluation of the Significance of Weld Defects ............................ 267

Appendix D - Defect Characterization and Dimensioning of Cracks in Welds by the U1trasonic Diffraction Method ..... 278

Appendix E - Flaw Characterization ................... 300

Appendix F - Ultrasonic Examination of Girth Welds in 40-Inch, X-65 Line Pipe Specimens................... 326

Appendix G - J-Integral Analysis of Surface Cracks in Pipeline Steel Plates .............................. 363

Appendix H - Metallographic Analysis ................... 370 

FITNESS-FOR-SERVICE CRITERIA FOR PIPELINE GIRTH WELD QUALITY

R. P. Reed, M. B. Kasen, H. I. McHenry, C. M. Fortunko, and D. T. Read

\section{ABSTRACT}

Criteria have been developed for applying fitness-for-service analyses to flaws in the girth welds of the Alaska Natural Gas Transmission System pipeline. A critical crack-opening-displacement elastic-plastic fracture mechanics model was developed and experimentally verified. Procedures for constructing flaw acceptence criteria curves based on this model are provided. A significantly improved ultrasonic method for detecting and dimensioning significant weld flaws was developed and demonstrated on pipeline sections. The probability of crack initiation from blunt flaws was shown to be very low under severe low-cycle fatigue. Suggestions are offered for technical implementation of field inspection procedures and for practical implementation of the flaw acceptence criteria. 



\section{EXECUTIVE SUMMARY}

The utility of fitness-for-service criteria for judging the need to repair pipeline girth welds containing flaws exceeding that permitted under prevailing workmanship standards was demonstrated during construction of the Trans-Alaska Pipeline System (TAPS). Here, fracture mechanics principles provided a quantitative technical basis for decision making, eliminating the need for unnecessary repair of many welds while maintaining assurance of the structural integrity of the pipeline.

The National Bureau of Standards (NBS) provided the Department of Transportation (DOT) with the technical information for implementing the TAPS fitness-for-service approach. The DOT recognized that the general use of fitness-for-service criteria in general pipeline construction would be beneficial. In 1978 NBS was requested to initiate a research program leading to this objective. However, soon after the work began, the DOT requested that the NBS program concentrate on developing criteria that could be implemented during construction of the proposed Alaska Natural Gas Transportation System (ANGTS), which was then in the planning stage. It was the intention, then, to redirect the program to generalization of the ANGTS research and, indeed, a portion of the research was redirected in 1981. However, the present report emphasizes the specifics of the ANGTS line rather than the case of pipelines in general.

Fracture mechanics analysis was used in this research. Fracture mechanics permits mechanical understanding of the relationship between the maximum stress and strain imposed on a pipeline during its lifetime, the pipeline and weldment properties, and flaws of different sizes and configurations. This understanding required development and verification of a model relating these 

parameters to weldment performance. Although the basic principles of fracture mechanics are well understood and widely implemented in the aerospace industry, their application to pipeline fabrication was complicated by the relatively high ductility of the materials used in their construction. It was necessary to understand crack initiation and propagation in the elastic-plastic range instead of linear-elastic failure typical for materials and structures displaying lower ductility.

It was assumed that a pipeline would be subjected to predictable stresses, that it would be constructed from API line-pipe steel having known minimum properties, and that the pipe would be welded by a process producing predictable minimum weldment strength and toughness. This design and materials information was then fed into the theoretical model to develop a series of curves relating the combination of maximum flaw length and through-wall depth permissible for the maximum applied load (allowable flawsize curves). Practical use of such curves presupposes the ability to detect and measure girth-weld flaws, and methods to accomplish this had to be developed.

Research was directed into three areas:

1. Refinement and experimental verification of the fracture mechanics model linking flaw size (length and through-wall depth) to pipeline stresses and weldment strength and toughness. Analytical predictions were compared with experimental results obtained on surface-cracked tensile panels and large-diameter pipe tests. The critical crack-opening-displacement (COD) model (used in the TAPS evaluation) was modified to optimize agreement between analysis and experiment. Modifications included: superposition of the elastic and post-ligament-yield solutions for crack-tip-opening displacement, use of a strip-yield plastic-zone correction on both crack length and crack 

depth, and use of a curvature correction for pipes. In summary, a fracture analysis model that relates allowable flaw sizes to the applied stress level and the weldment strength and toughness was developed, verified experimentally, and used to calculate allowable flaw sizes for proposed operating conditions of the ANGTS pipeline.

Procedures for the use of elastic-plastic fracture mechanics for the derivation of allowable flaw-size curves are in the early stages of development. Further work is recommended to increase confidence in the analytical results.

2. Development of a practical method of determining the through-wall flaw dimension. The TAPS experience showed that estimation of dimensions from conventional radiographs was so inaccurate that very large safety factors were required. This resulted in fitness-for-service criteria that were far more restrictive than necessary. Initially, efforts were made to refine the densitometry method of assessing flaw through-wall dimensions. Although some improvement was realized, it became evident that this approach was intrinsically deficient in its ability to measure sharp flaws, such as lack of fusion or cracks, which are the most significant in a fitness-for-service analysis. A more promising approach was provided by ultrasonic methods, which are intrirsically sensitive to sharp flaws. Although ultrasonic inspection systems based on high-frequency piezoelectric transducers have long been used for flaw detection in welds, incorporation of even the most advanced automation and signal processing has thus far failed to produce systems capable of providing the required flaw measuring capability. Therefore, we tried a new approach -- the development of an ultrasonic inspection system optimized for pipeline use based on electromagnetic transducers (EMATs), which transmit and receive low-frequency shear/horizontal (SH) waves. Unlike conventional systems, this approach provides a signal amplitude that increases 
monotonically with through-wall flaw depth. The system can be gated to ignore flaws of noncritical dimensions and to trigger an alarm automatically when flaws of significant size are present. Coupling agents are not required between the transducer and pipe, and reliability is not affected by geometric factors such as the weld reinforcement. Its accuracy is comparatively insensitive to operator skill, and a permanent record is produced. The theory underlying flaw detection and sizing by this new method has been developed and validated by the inspection of welds containing a variety of artificial and real flaws at different depths and orientations. The necessary transducer arrays and associated signal processing equipment have been developed. Field trials and assessment of reliability are needed to confirm the usefulness of this system as an adjunct to the fitness-for-service approach to girth-weld quality assessment.

3. A convincing demonstration of the probably innocuous nature of blunt flaws, such as porosity, slag, and arc burns. We found that blunt flaws (porosity, slag) and arc burns do not decrease weldment strength and fatigue life. Subjecting pipeline welds containing large quantities of such flaws to severe low-cycle fatigue at $-2^{\circ} \mathrm{C}$ had little effect on crack initiation. However, should it be desired to include blunt flaws in a fracture mechanics analysis, metallographic studies of welds containing porosity and slag showed that their depth is limited to the depth of one weld pass, eliminating the necessity for through-wall dimensioning. In metallographic studies during the TAPS program, arc-burr, width was related to arc burn depth, thus providing an easy size characterization procedure for such flaws.

On the basis of these results, three technical options are suggested for implementing field inspection oriented toward fitness-for-service. 1) The use of ultrasonic methods for primary inspection combined with traditional radiography for assessing workmanship and for materials and procedure control 

appears feasible and is the most technically desirable. 2) Conventional radiography could be used as the primary inspection method, using a simplified ultrasonic system for measuring flaws. We believe that the EMAT system developed in this work appears to be the most promising inspection method for either of these two options; however, other systems of equivalent performance could be used should they become available. 3) In the event that ultrasonic inspection systems of demonstrated performance are not available at the time of construction of a given pipeline, radiography must be relied on exclusively. In this case, fitness-for-service criteria can still be used to assess blunt flaws. The demonstrated inherent limitations on the height of such flaws would very much simplify this effort by el iminating the need for complex flaw-measuring procedures. Even under this least desirable option, a substantial reduction in remedial welding can be anticipated since the majority of detectable flaws would be of blunt configuration.

We, suggest two ways in which the overall fitness-for-service approach might be implemented in pipeline construction: A generalized approach could prequalify designated sections of the line by establishing maximum allowable flaw sizes based on limits placed on weld-metal toughness, operating stresses, and other relevant variables. A specific-site approach might be used wherein the particular conditions existing for a given weldment are used to calculate safe flaw sizes.

We expect this report to contribute to a rational, technically defensible approach to assessing girth-weld quality, which will provide the fabricator with the maximum flexibility to accommodate his particular needs while maintaining pipeline safety. Additional research has been recommended to assist in generalization of the program's results. 
Specific support for this work was provided by the Materials Transportation Bureau, DOT, and by the Welding Research Council. The Northwest Pipeline Company provided materials and consultation. Consultation was also provided by Nova, an Alberta Corporation. 



\section{INTRODUCTION}

Extensive repair to girth welds on the Trans-Alaska Pipeline System (TAPS) resulted from the discovery that a large number of completed and buried welds failed to meet the Department of Transportation (DOT) standards ${ }^{1}$ related to girth welds of liquid pipelines, which incorporate the workmanship criteria of the 15 th edition of API Standard $1104 .^{2}$ The costs of this remedial welding were very high because pipe sections buried under permafrost and rivers at remote locations had to be excavated or looped.

At that time it was recognized that many of the flaws outside workmanship standards were unlikely to endanger the safety of the line and that repairs might do more damage to the integrity of the line and to the environment than if the flaws were left unrepaired. Unfortunately, there was no established technical basis for such a decision. Repairs were therefore begun.

At the same time, fracture mechanics studies were begun to establish a technical foundation for assessing flaw significance on a fitness-for-service basis as an alternative to workmanship criteria. The results of this work ${ }^{3,4}$ brought about federal acceptance of this approach. Consequently, the amount of TAPS repair welding was reduced and a substantial amount was saved in unnecessary repair. ${ }^{5}$ The DOT concluded in 1976 that "fracture mechanics analysis is acceptable as a basis for granting exemptions from existing standards in appropriate circumstances, if such analys is produces a convincing and conservative estimate of structural integrity." 6

It was anticipated that the need for long-distance transport of $0 i 1$ and gas would lead to additional use of fitness-for-service analysis in pipeline 

construction. The proposed Alaska Natural Gas Transportation System (ANGTS) was to operate at lower temperatures than the TAPS line and would be entirely buried, placing additional demands on system performance. Furthermore, since adoption of the API 1104 workmanship standards upon which the DOT standards are based, pipeline wall thickness and diameter have increased significantly, increasing the complexity and cost of weld repair. It was evident that much additional work was required before the fitness-for-service concept could be efficiently used to facilitate construction of a safe pipeline at minimum cost.

Research at NBS was initiated in 1978 to address the generic problems associated with possible amendment of the DOT liquid and gas pipeline federal regulations to include a fitness-for-service alternative. This included research on fracture mechanics, radiography, blunt flaws, and corrosion of pipelines. In 1979, the apparent imminent start of construction of the ANGTS gas pipeline led the DOT to request a change of focus of the NBS program to specifically address the operating conditions and materials anticipated for that line. This report, therefore, concentrates primarily on developing fitness-for-service criteria for the ANGTS line.

At the time of the TAPS experience, two fracture mechanics models were proposed $^{3,4}$ to assess the conservatism of the Draft British Standards methodology ${ }^{7}$ (later published officially by the British Standards Institution ${ }^{8}$ ), which was used in the Alyeska waiver request to DOT. One was based on elastic-plastic fracture mechanics principles [critical crack-tipopening displacement (CTOD) model]; the other considered only plastic instability of the uncracked ligament. Both of these newer models proved less conservative than the Draft British Standards. 

In this study we have focused on the extension of the critical COD model. Modifications relating to crack-tip physics and mechanics were developed and measurements were performed, leading to an experimentally verified elasticplastic fracture mechanics model. Consideration of crack growth from fatigue stresses, included in the TAPS assessment, has not been addressed here. Except near pump statons, cyclic stresses in the ANGTS line were not considered significant. Enhanced crack growth from environmental interactions, such as stress corrosion, also was not considered in the ANGTS case, because of this origin was not consdered likely.

There are serious deficiencies in the use of radiography as an inspection tool in a fitness-for-service analysis. The important flaw dimension affecting fracture is the through-wall depth of elongated, sharp flaws. But radiography provides direct information only on the projected dimensions of the flaw normal to the plane of the weldment. It is theoretically possible to relate the change in radiographic density caused by a flaw to the depth of the flaw; This approach was used in the TAPS analysis. ${ }^{3,4}$ However, it became evident that the combination of uncertainties in the conditions under which field radiographs are taken and processed, human bias, and the technical subjectivity of such determinations led to excessive conservatism in estimating flaw dimensions.

The majority of the repairs remove flaws of a blunt configuration--porosity, slag, and arc burns. This reflects, at least partly, the sensitivity of radiography to such flaws and its relative insensitivity to sharp flaws, such as lack of fusion or incomplete penetration. Yet, from a fitness-for-service approach, sharp flaws are more significant, since they produce the highest stress intensities and, consequently, have the highest probability of initiating fracture. A better inspection system for detecting and dimensioning flaws in girth welds was required, preferably one having optimal sensitivity to sharp flaws. 

The significance of blunt flaws in crack initiation was questioned. Results of a number of studies had suggested that the presence of such weld flaws is irrelevant to the performance of structures joined with tough weld metal, but the credibility and the boundary conditions of this assumption had not been examined for pipeline girth weids.

It was likely, we thought, that the through-wall depth of blunt flaws would intrinsically be restricted to the depth of one weld pass, allowing that dimension to serve as a maximum in a fracture mechanics analysis. Although such a limitation is frequently assumed, a thorough search of the literature did not reveal the required documentation. A study was therefore conducted on inherent dimensional limitations of blunt flaws with the purpose of confirming or refuting this assumption.

It is instructive to compare the results of this study with those of other pipeline fitness-for-service approaches that have been suggested:

1. The British Standard specifically related to pipeline welding, "Standard for the Field Welding of Carbon Steel Pipelines," 9 is currently under review. One proposal incorporating fracture mechanics analys is has been recently discussed by Carne and Harrison. 10 They proposed a three-tier approach. Tier one corresponds to workmanship standards similar to those now in existence, but incorporating minimum toughness requirements. Tier two consists of very conservative generalized fracture mechanics analysis, intended to be applied by field engineers not necessarily specialized in fracture mechanics. Tier three is applied by a specialist to a specific site and labeled engineering critical assessment (ECA). Inspection methodology was not discussed in this approach.

2. Glover and Coote ${ }^{11}$ pointed out that the British Standards Institution fracture mechanics guidelines are also being used for ECA of segments of the new large-diameter gas pipelines in Canada. Their assessment 

showed that radiographic inspection could not properly and consistently detect sharp flaws. They found that planar flaws not detected by radiography were consistently detected by high-frequency ultrasonic techniques, but that sizing was unreliable. They suggested that both radiography and ultrasonics methods be used, assuming that flaw depths are restricted to the thickness of one weld pass. The British Standards Institution Guidelines is therefore applied on the basis of flaw length and knowledge of the applied axial strain and weldment toughness. Note that this approach is limited to girth welds made by an automatic process.

3. The Japan Welding Engineering Society has recently published their first report, "Method of Assessment for Defects in Fusion-Welded Joints With Respect to Brittle Fracture."12 This report outlines procedures for using a fitness-for-service analysis in specific cases for establishment of allowable sharp flaw sizes in terms of local weldment strain and COD weld toughness. Inspection methodology is not addressed.

4. A major revision of the American Petroleum Institute 1104 Standard for Welding Pipelines and Related Facilities (issued in May, 1983) was discussed by Von Rosenberg and Royer. ${ }^{13}$ This generalized fitness-for-service approach requires calculation of maximum axial design stress and strain and minimum weldment toughness in terms of $\operatorname{COD}$ values of either $0.13 \mathrm{~mm}(0.005 \mathrm{in})$ or $0.25 \mathrm{~mm}(0.010 \mathrm{in})$. Fatigue crack growth is also considered. Inspection techniques are left to the individual companies. This revision appears as Appendix A, 16th Edition, API 1104 Standards. However, at the time of this report, the 16th Edition has not been incorporated into the Federal gas and liquid pipeline safety standards. 

These programs demonstrate international recognition of the need to supplement welding workmanship standards with fitness-for-service criteria for pipelines. Reduced pipeline construction time and costs through the elimination of unnecessary repairs are expected to accrue from this attention.

In summary, our research has focused on the development of a comprehensive model to calculate allowable flaw sizes and laboratory experiments to assess the validity of such models, the assessment of the significance of blunt flaws in girth weld structural integrity, the measurement of inherent blunt flaw size limitations, and the inspection methods for sharp flaws.

Our report first discusses the development and experimental verification of an elastic-plastic fracture mechanics model applicable to sharp flaws. We then address development of a new inspection technique that is optimized for the fitness-for-service approach. Research related to the significance of blunt flaws is then presented.

Finally, we recognize that nondestructive inspection has two roles in pipeline construction. One relates to quality control--the monitoring of welder and equipment performance and the quality of consumables used in the fabrication. The other is acceptance or rejection of a weld on the bas is of fitness-for-service. Although this report concentrates on the latter role, both roles must be considered in devising an effective inspection system. Possible inspection options for achieving these goals and for implementation of the fitness-for-service approach are therefore addressed in this report.

A series of Appendixes provide more detailed information on selected subjects addressed in the main body of this report. 



\section{DEVELOPMENT OF ALLOWABLE FLAW SIZE CURVES}

H. I. McHenry, Y. W. Cheng, R. deWit, J. G. Early, R. B. King, and D. T. Read

\subsection{Introduction}

Allowable flaw sizes for pipeline girth welds are set forth in Section 6 of API $1104^{2}$ on the basis of workmanship considerations; that is, flaw-size limits are based on quality levels that can reasonably be expected from a qualified welder using satisfactory materials, equipment, and procedures. In 1976, the U.S. Department of Transportation permitted the use of alternative weld quality standards to evaluate certain girth welds in the Trans-Alaska Pipeline System (TAPS). The alternative standards were in the form of allowable flaw size curves derived on the basis of a fracture mechanics analysis.

An allowable flaw-size curve is a plot of allowable flaw depth, a, as a function of flaw length, $\ell$, for a planar (crack-like) flaw as shown schematically in Fig. 1. Dimension a refers to the through-wall dimension of the flaw projected in the plane of the weld. A point on the curve represents the maximum flaw size ( $l$ vs. a) that can be tolerated without impairing the service performance of the pipeline under worst-case operating conditions. Flaw sizes that fall below the curve are acceptable because they will not impair performance, and flaw sizes that lie above the curve must be repaired because they are potentially detrimental.

The purpose of this part of the program was to develop allowable flawsize curves suitable for use in evaluating ANGTS pipeline girth welds. The specific objectives of the investigation were: 1) to develop a fracture mechanics analysis model to relate allowable flaw size to applied stress and 



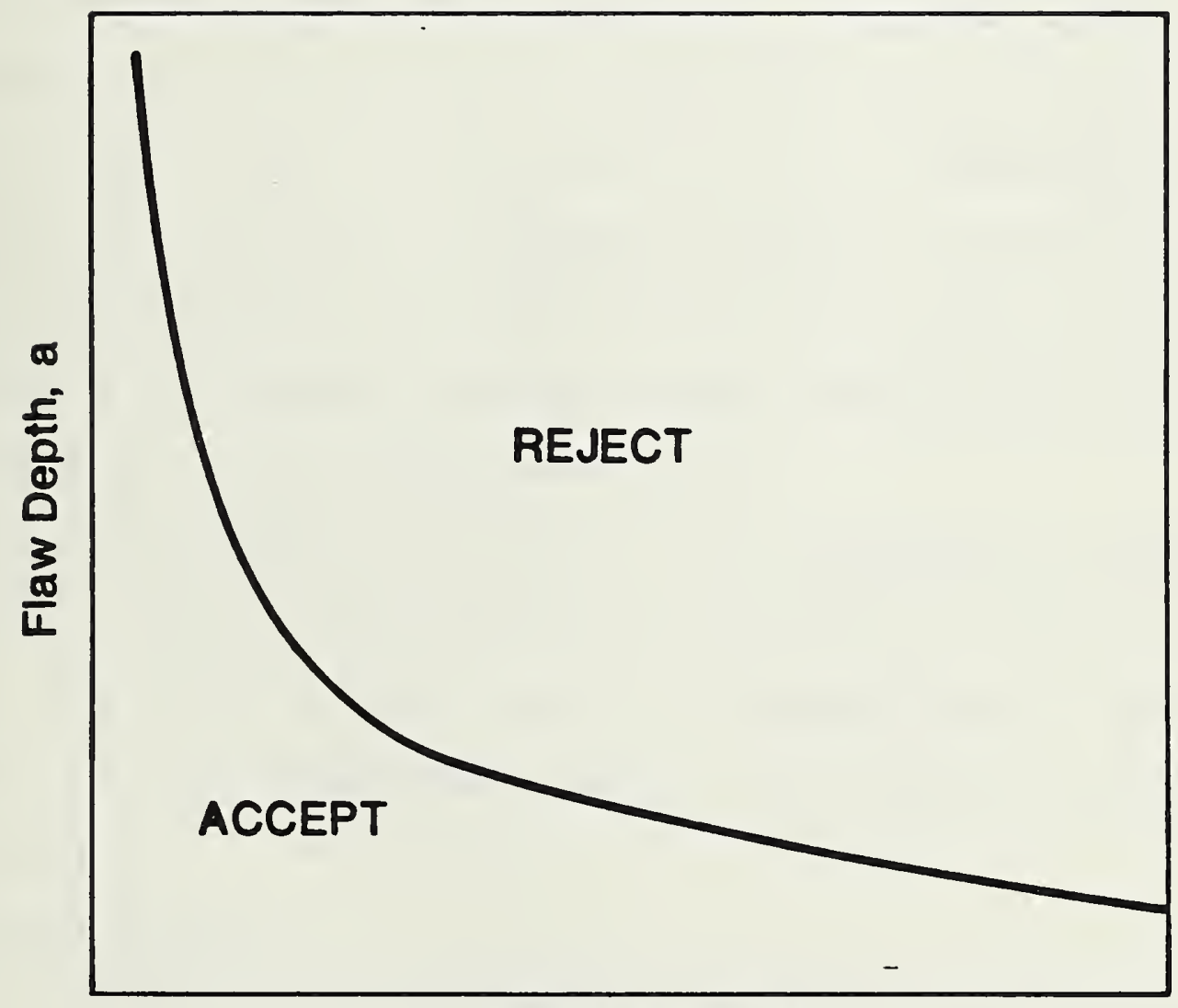

Flaw Length, $\ell$

Fig. 1 - Schematic of allowable flaw-size curve. 

weld toughness, 2) to verify the analytical model experimentally, and 3) to use the model to calculate allowable flaw-size curves for representative operating conditions and weld properties.

The achievement of the first two objectives required extensive research in analytical and experimental fracture mechanics. These results are summarized herein. Many of the results have been published previously, and these publications are cited where appropriate in this report. The achievement of the third objective is based on the results of the fracture mechanics studies. The allowable flaw-size curves for representative operating conditions and weld procedures are presented in Section 2.5, Allowable Flaw-Size Curves, along with the procedures used to calculate them. Section 2.5 is self-contained, and thus, readers primarily interested in the resulting curves can skip the preceding sections.

Fatigue and corrosion contributions to crack growth were not considered significant in the case of the ANGTS pipeline and are not included in this analysis. Preliminary corrosion research was conducted early in the program (Appendix B) for generalized pipeline analysis and fatigue was included on the TAPS analysis. 3,4

\subsection{Fracture Mechanics}

The principles of fracture mechanics provide the technical basis for the development of allowable flaw-size curves. Fracture mechanics is the study of the influence of loading, crack size, structural geometry, and material properties on the fracture resistance of structures containing cracks. There are two main ideas in fracture mechanics: First, fracture occurs when the driving force for fracture, a function of stress and flaw size, exceeds the resistance of the material to fracture, referred to as the fracture toughness. Second, fracture toughness is a geometry-independent material property; that 
is, a simple laboratory specimen and a large structure both fracture at the same critical value of driving force. The second idea is only directly applicable to linear elastic (brittle) fracture and to certain cases of fully plastic (ductile) fracture; however, it is a conservative assumption for elastic-plastic fracture if the notch constraint in the test specimen exceeds the notch constraint in the structure. Such is the case for pipeline girth welds when full-thickness single-edge-notch-bend specimens are used to measure fracture toughness.

Several fracture criteria are used to characterize the driving force for fracture and the fracture toughness. These include the stress intensity factor, $\mathrm{K}$, the $\mathrm{J}$-integral, $\mathrm{J}$, and the crack-tip-opening displacement, CTOD. Since $K$ is a linear elastic parameter, it was not suitable as a fracture criterion for ANGTS pipeline girthwelds, which must operate safely at stress levels near the yield strength. However, $K$ calculations were useful for determining the elastic component of $\mathrm{J}$ and CTOD, and thus, $\mathrm{K}$ calculations for surface flaws are discussed in this section. The main emphas is in this section is on the analytical work related to the development and experimental verification of elastic-plastic fracture mechanics models based on the CTOD concept. Research related to the $J$-integral concept is summarized in Appendix G.

\subsubsection{Linear Elastic Fracture Mechanics}

The stress intensity factor, $K$, for surface cracks has been the subject of numerous studies. Recently, the fracture committee of the Society for Experimental Stress Analysis compared several elastic numerical solutions for a surface flaw in a plate under remote tension and bending. ${ }^{14}$ The results of Newman and Raju ${ }^{15}$ compared favorably with the "best-estimate" results of the committee. The results of Newman and Raju are expressed in terms of numerical 

equations that facilitate computation, and thus, their $K$ solution has been used in the present study. The $K$ solution for semielliptical cracks in simple tension is:

$$
K=\sigma(\pi a / Q)^{\frac{1}{2}} F_{b}
$$

for $a / \ell \leq 0.5, a / t<1.0, \ell / W<0.5$, and $0 \leq \emptyset \leq \pi$;

where $\sigma=$ stress

$$
\begin{aligned}
& Q=\text { is the square of the complete elliptic integral of the second kind } \\
& F_{b}=\text { boundary correction factor, given below } \\
& a=\text { crack depth } \\
& \ell=\text { crack length } \\
& t=\text { specimen thickness } \\
& W=\text { specimen width } \\
& \emptyset=\text { anguiar position along crack front }
\end{aligned}
$$

The geometric variables $(a, \ell, t$, and $\emptyset$ ) are illustrated in Fig. 2 . In this study, the $K$ at maximum crack depth $\left(\varphi=90^{\circ}\right)$ was of interest.

The numerical expression for $Q$ developed by Rawe and used in Ref. 16 is:

$$
0=1+1.464(2 a / l)^{1.65}
$$

The boundary correction factor for tension is:

$$
F_{b}=\left[b_{1}+b_{2}(a / t)^{2}+b_{3}(a / t)^{4}\right] f_{w}
$$

where

$$
\begin{aligned}
& h_{1}=1.13-0.09(2 a / l) \\
& b_{2}=-0.54+0.89(0.2+2 a / l) \\
& b_{3}=0.5-1.0 /(0.65+2 a / l)+14(1.0-2 a / l)^{24}
\end{aligned}
$$

The finite width correction, $f_{w}$, of Nevman ${ }^{17}$ is used:

$$
f_{W}=\left[\sec (\pi l / 2 W)(a / t)^{\frac{1}{2}}\right]^{\frac{1}{2}}
$$





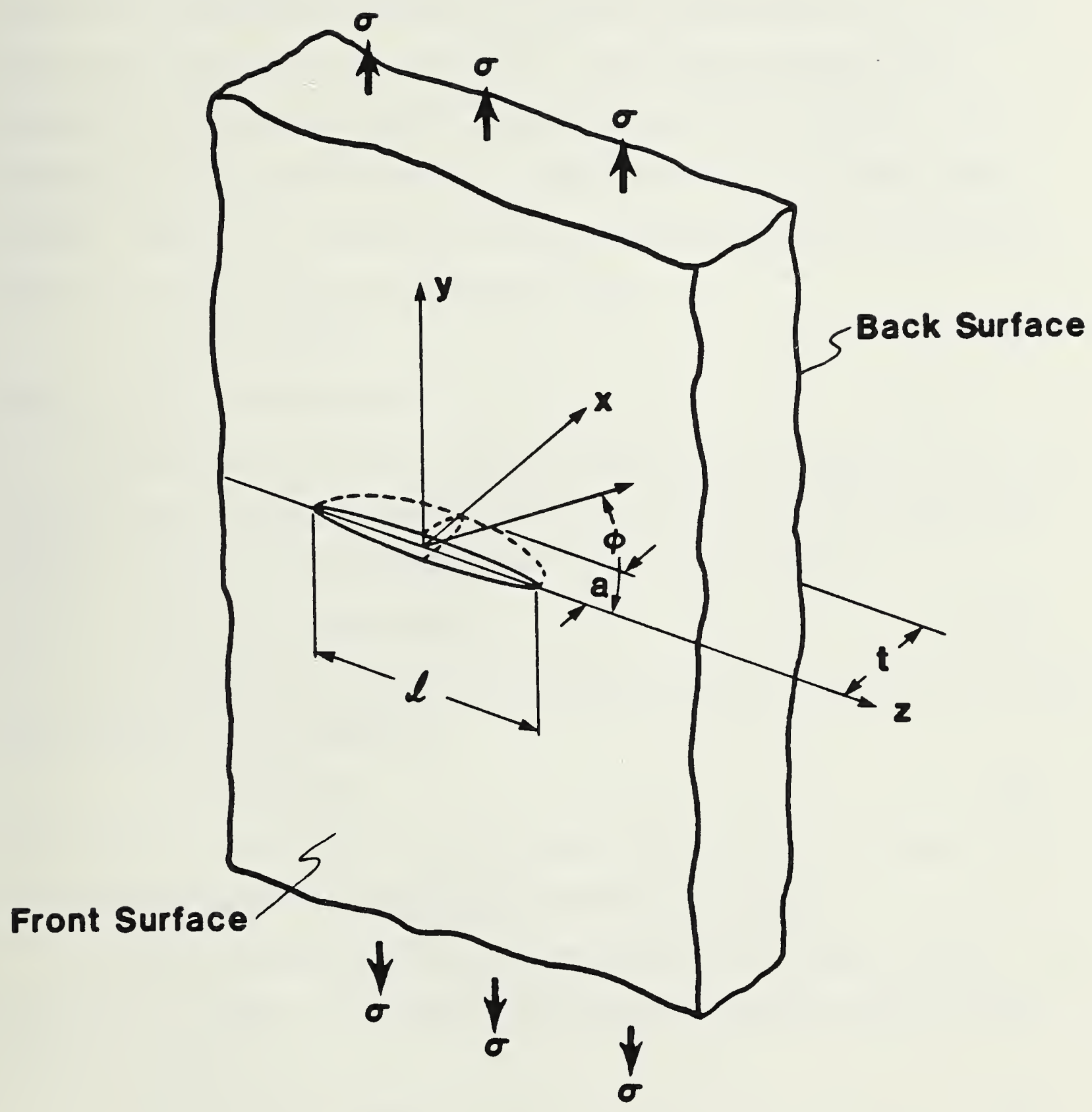

Fig. 2 - Geometry of surface flaw. 

Applicability of the linear elastic analysis has been extended to conditions approaching net-section yielding by correcting for the zone of plasticity that exists at the crack tip. The idea is that the plastic material at the crack tip strains without carrying the incremental load; therefore, in the elastic sense, the crack behaves as if it were slightly longer. The Irwin ${ }^{18}$ correction is made by adding the radius of the plastic zone, $r_{y}$, to the apparent crack length, where:

$$
r_{y}=\left(k / \sigma_{y}\right)^{2} / 2 \pi
$$

and $\sigma_{y}$ is the yield strength

Alternatively, the plastic zone has been modeled by Dugdale 19 as a thin strip of plastic material extending from the crack tip in the plane of the crack. The extent of the Dugdale plastic zone, $\rho$, is given by

$$
a /(a+\rho)=\cos \left(\pi \sigma / 2 \sigma_{y}\right)
$$

If the higher order terms of the series expansion of the cosine function are neglected, Eq. 9 reduces to

$$
\rho=\pi\left(k / \sigma_{y}\right)^{2} / 8
$$

The plastic-zone correction, $r_{y}$, is taken in this report to be equal to $p / 2$ and is approximately $23 \%$ greater than the Irwin correction.

A.s plasticity develops, the crack tip blunts. The extent of this blunting is the CTOD. The CTOD is calculated for the small-scale yielding case as follows:

$$
C T O D=K^{2}\left(1-v^{2}\right) / E^{-}
$$

where $E$ = Young's modulus

$$
\begin{aligned}
& \nu=\text { Poisson's ratio } \\
& \bar{\sigma}=\text { flow stress }=\frac{1}{2}\left(\sigma_{y}+\sigma_{u}\right) \\
& \sigma_{u}=\text { ultimate tensile strength } \\
& k=K \text { from Eq. } 1
\end{aligned}
$$





\subsubsection{Modified Critical-COD Model}

The CODs of a surface flaw in a flattensile panel that occur after the uncracked ligament has yielded have been modeled originally by Irwin $^{20}$ by assuming the surface flaw behaves as a through-thickness crack of the same length. The opening of the equivalent through-thickness crack is reduced by closure forces equal to the product of the flow stress times the area of the uncracked ligament.

In the TAPS evaluation, a model developed from Irwin's original concept, referred to as the critical-COD model, was used to calculate allowable flaw-size curves. ${ }^{4}$ However, there were no experimental data to evaluate the model. In this investigation, the analytical predictions have been compared with experimental results, and the critical-COD model has been modified to optimize agreement between analysis and experiment. Modifications include: superposition of the elastic and post-ligament-yield solutions for CTOD, use of a strip-yield plastic-zone correction on both crack length and crack depth, use of a finite-width correction for flat plates, and use of a curvature correction for pipes.

The critical-COD model treats the surface crack in a plate as a through-thickness center crack after the ligament has yielded. The COD at the middle of the center crack of length, $\ell$, in an infinite plate under a remote stress, $\sigma$, is given by

$$
C O D=2 \ell \sigma / E
$$

For a surface crack, the COD of Eq. 12 is reduced by the remaining ligament. The effect of ligament depth can be estimated by considering a closing force distributed over the crack-face area. Assuming the ligament is yielded, the total closing force, $F_{C}$, is

$$
F_{c}=\ell(t-a) \bar{\sigma}
$$



where $\bar{\sigma}$ is flow stress and is estimated as the average of the yield strength and the ultimate tensile strength, $t$ is the plate thickness, and $a$ is the crack depth. Distributing this closing force over the area et gives a closing stress, $\sigma_{c}$, on the equivalent through-thickness crack of

$$
\sigma_{c}=(1-a / t) \bar{\sigma}
$$

This closing force opposes the remote stress, $\sigma$, and the resultant opening of the surface crack is then

$$
C O D=2 \ell\left(\sigma-\sigma_{C}\right) / E
$$

To account for the crack opening due to the crack-tip plasticity, the effective crack length, which includes the plastic-zone size correction, $r_{y}$, is used in place of $\ell$. The resulting expression when Eq. 14 is substituted into Eq. 15 becomes

$$
\operatorname{COD}=[\sigma-(1-a / t) \bar{\sigma}] 2\left(e+2 r_{y}\right) / E
$$

where $r_{y}$ is a plastic-zone size correction with a finite width correction and is evaluated 21 as

$$
\sin (\pi \ell / 2 W) / \sin \left(\pi \ell_{p} / 2 W\right)=\cos \left[(\pi / 2)\left(\sigma^{\prime} / \bar{\sigma}^{\prime}\right)\right]
$$

where $\ell_{p}$ is the length of the crack measured to the ends of the plastic zone, defined as $\ell+4 r_{y}, \sigma^{\prime} / \bar{\sigma}^{\prime}=1-t / a(1-\sigma / \bar{\sigma})$, and $W$ is the plate width.

Using CTOD as the fracture criterion, the relationship among CTOD, $\sigma$, and flaw size is

$$
\text { CTOD }=\operatorname{CTOD}(\text { elastic })+\text { COD }
$$

where $\operatorname{CTOD(elastic)}$ is given in Eq. 11 and $\operatorname{COD}$ is given in Eq. 16. The value of $C O D$ is zero when $\sigma$ is less than ligament yield stress, given by $(1-a / t) \bar{\sigma}$.

Comparison of analytical predictions of the critical-COD model with experimental results discussed in Section 2.3 , was done in terms of CMOD, (i.e., the $\operatorname{COD}$ at $x=0$ in Fig. 2) where:

$$
\text { CMOD }=\operatorname{CMOD}(\text { elastic })+\text { COD }
$$



where $\operatorname{COD}$ is given in Eq. 16 and $\mathrm{CMOD}$ (elastic) is evaluated using the elasticity solution of Kobayashi ${ }^{22}$ for cracks with aspect ratios ( $\left.l / a\right)$ less than 10:

$$
\text { CMOD }=\frac{-8(1-v)}{G a l} C_{00}+\frac{4}{3 a^{2}} C_{20}+\frac{16}{3 l^{2}} C_{02}
$$

where $G=$ shear modulus and the coefficients $C_{00}, C_{20}$, and $C_{02}$ are governed by the geometry and loading conditions. The values for $C_{00}, C_{20}$, and $C_{02}$ are taken from Kobayashi. ${ }^{23}$ Solutions to Eq. 20 are shown in Fig. 3.

For long cracks $(\ell / a>10)$, the Kobayashi solution is not applicable and the elastic CMOD is calculated using King's ${ }^{21}$ simplified line-spring model (discussed in Section 2.2.3).

\subsubsection{Derivation of Yielded-Ligament Model from Line-Spring Model}

The line-spring model was developed by Rice and Levy ${ }^{24}$ for elastic surface cracks. Extension of the model to elastic-plastic surface cracks was speculated upon by Rice 25 and carried out by Parks. ${ }^{26}$ For the purposes of this program, the line-spring model provided an analytical framework for extending the critical-COD model to the case of surface cracks in pipes rather than flat plates.

The use of the line-spring model was suggested after the validity of the critical-COD model was confirmed for the case of surface cracks in tensile panels (see Section 2.3.2). Thus, the same physical assumptions used in the critical-COD model were introduced into the line-spring model, and the two models predict the same results for the flat-plate case. However, the linespring model made it possible to account for a number of complexities that exist for the case of long surface flaws in pipes, specifically, the elastic displacements of long cracks, the curvature effect, and plasticity development. On the other hand, these same physical assumptions resulted in considerable 



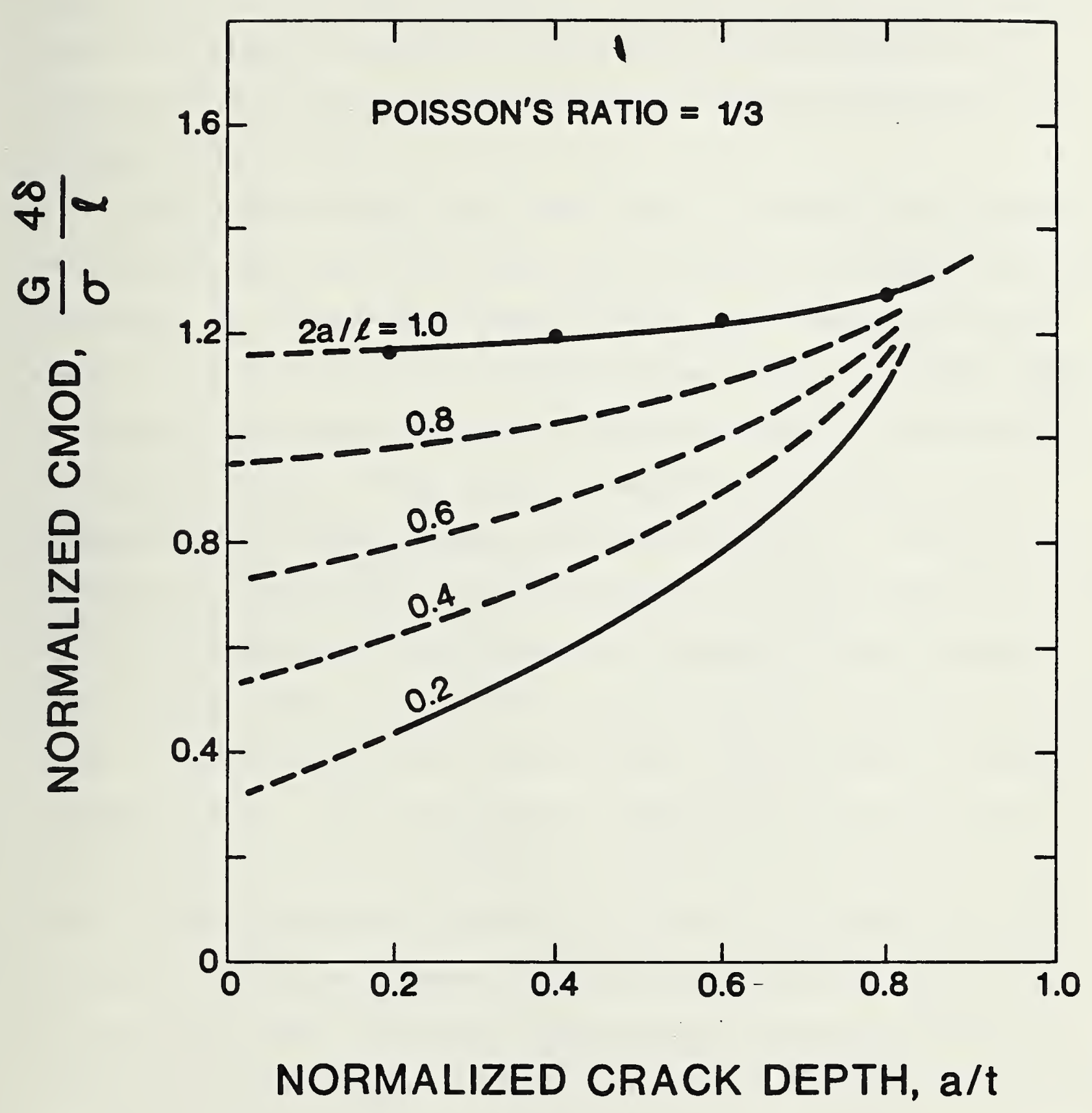

Fig. 3 - Theoretical solution for CMOD in a surface-flawed tension plate. $G=$ shear modulus, $\delta=$ CMOD, $\ell=$ crack length,$a=$ crack depth, and $t=$ plate thickness. 

simplification of the line-spring model. The derivation of the model for the case of flat plates is discussed in this section and the extension of the line-spring model to the case of surface cracks in pipes is discussed in the following section, 2.2.4.

In the line-spring model, the surface crack is treated as being equivalent to a through-crack of the same length as the surface crack. However, owing to the presence of the uncracked ligament, certain closing forces and bending moments act on the faces of the equivalent through-crack. The bending moments are caused by the eccentricity of the ligament with respect to the centerline of the plate thickness. These forces and moments can be related to the deformation of the ligament through compliance expressions. The place of the ligament can thus be thought of as being taken by "springs" connected from one face of the through-crack to the other. The compliance of these springs at a point along the crack is assumed to be equal to that of an edge crack in plane strain of the same depth as the surface crack at that point and in a plate of the same thickness. This is motivated by considering the limiting cases. If the crack were very long, it would begin to behave like an edge crack in a thick plate (thus deforming in plane strain) with all the applied load being carried by the uncracked ligament behind the crack. The other limit is that of a very deep crack, in which the ligament becomes increasingly thinner and eventually disappears entirely. In this case, the crack becomes a through-crack, and all the load is carried by the material on the sides of the crack. The actual situation is between these two extremes, and to analyze it approximately, we assume it is a mixture of the two limiting cases, so that the load is carried partially by the "edge crack" and partially by the "through-crack," as shown in Fig. 4. To determine the unknown force, $F(x)$, and moment, $M(x)$, that 



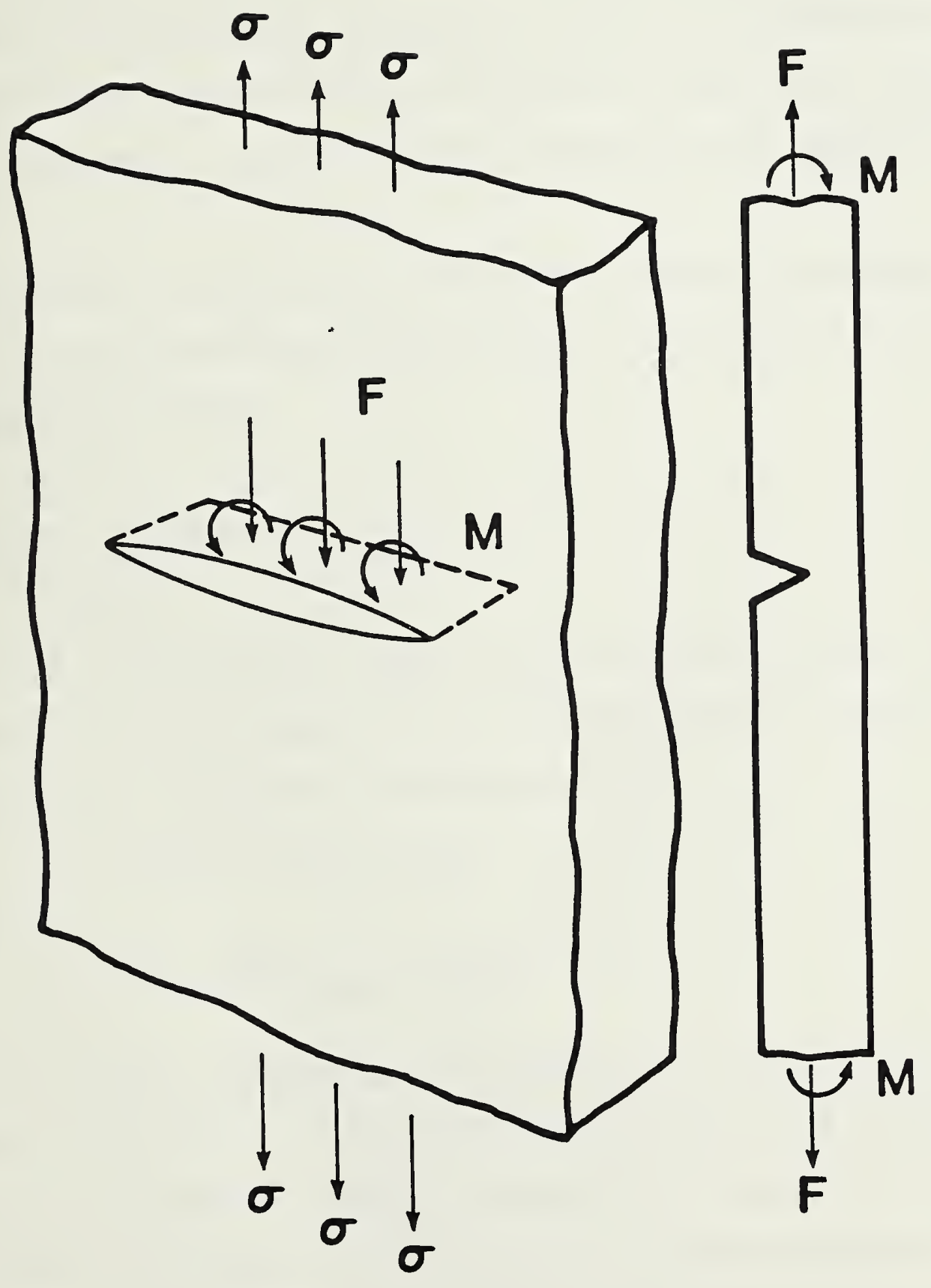

Fig. 4 - Line-spring model. 

act on the edge crack at a point $x$ along the crack and by equilibrium al so act on the through-crack faces, displacement compatibility is enforced; that is, since the "through-crack" and the "edge crack" coexist in the same plate, they must exhibit the same opening displacement of the crack and the same rotation of the crack faces. Once the force and moment are known, we can compute any parameter of interest, such as $\mathrm{J}$ or $\mathrm{COD}$, by focusing our attention on the edge crack.

To apply the line-spring model, expressions relating displacements and rotations to forces and moments must be set up. It is convenient to introduce the average tensile stress,

$$
\sigma_{0}=F / t
$$

and the bending stress,

$$
m=6 M / t^{2}
$$

where $t$ is the plate thickness, and $F$ and $M$ are the force per unit length and moment per unit length, respectively, on the edge crack. In terms of $\sigma_{0}$ and $\mathrm{m}$, the compliance expressions for the edge crack are

$$
\begin{aligned}
& \Delta_{c}=\frac{2(1-v) t}{E}\left(a_{11} \sigma_{0}+a_{12} m\right) \\
& \theta_{c}=\frac{12\left(1-v^{2}\right)}{E}\left(a_{12} \sigma_{0}+a_{22} m\right)
\end{aligned}
$$

where the compliance constants $a_{11}, a_{12}$, and $a_{22}$ are found by considering the edge crack loaded with $\sigma_{0}, m$ and with $\Delta_{c}$ and $\theta_{c}$ defined as the additional displacement and rotation of the ends caused by the presence of the crack. The procedure of Pice and Levy ${ }^{24}$ was used to calculate the compliance constants $\left(a_{i j}\right)$, and the results for the $a_{i j}$ as functions of crack depth are shown in Fig. 5 .

The mathematical complexities in the line-spring model arise in deriving the expressions relating displacement and rotation to $\sigma_{0}$ and $m$ in the throughcrack, because the force and moment at any position along the crack front. 



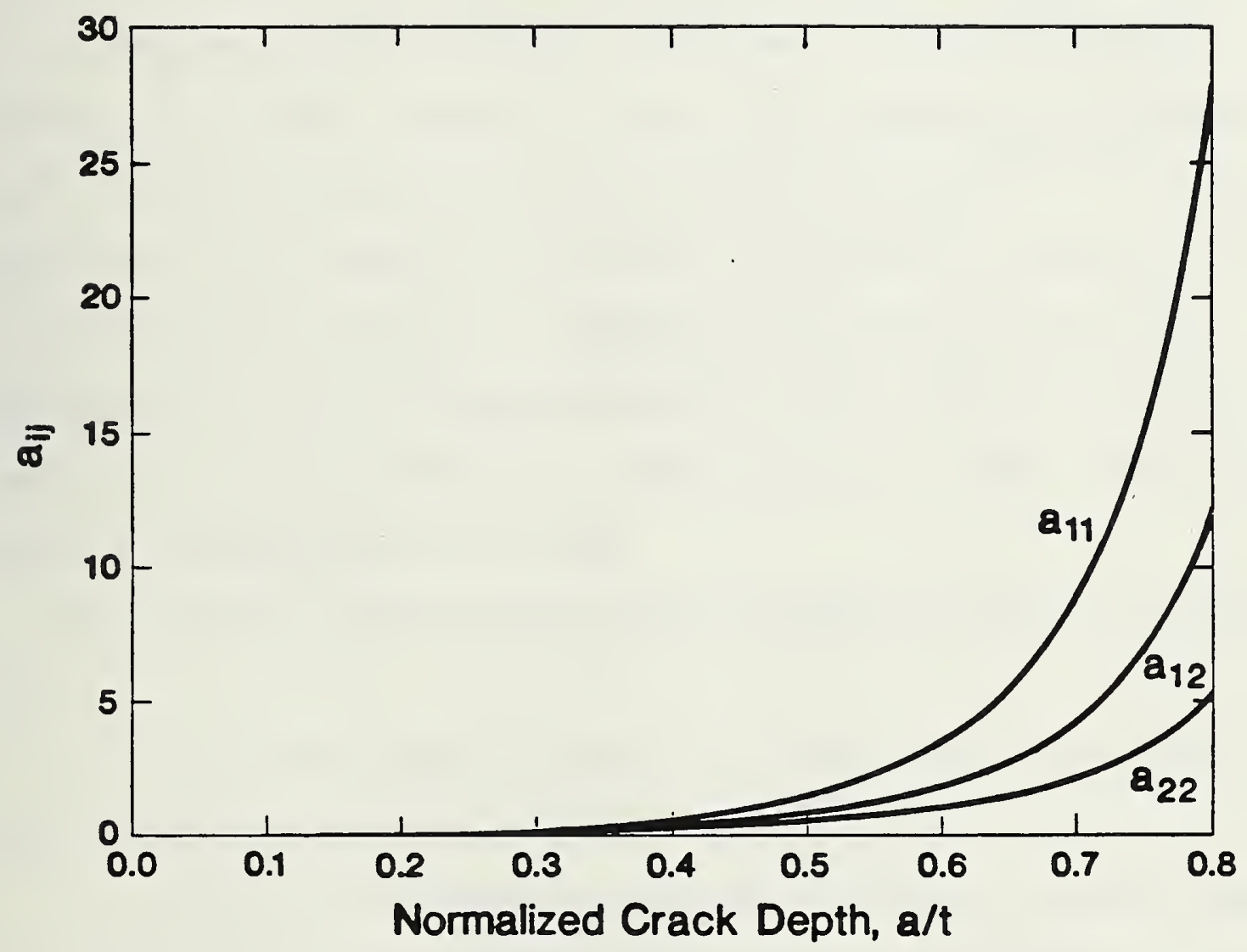

Fig. 5 - Compliance coefficients $a_{11}, a_{12}$, and $a_{22}$ as a function of crack depth. 

affect the displacement and rotation at any other point. Enforcing displacement compatibility results in a pair of coupled integral equations that must be solved numerically, 24,26 or the line spring may be inserted into a suitably modified finite-element program. 27,28 Extension of the model into the elastic-plastic region has been carried out by inverting Eqs. 23 and 24 and rewriting the result in an incremental form, thus relating $d \sigma_{0}$ and $d m$ to $d \Delta_{c}$ and $d \theta_{c}$ through a stiffness matrix. At sections in which yielding has occurred, the "tangent stiffness" is used. This procedure was proposed by Rice $^{25}$ and successfully carried out by Parks and Lockett. ${ }^{26,27,29}$ A further complication is the spread of plasticity from the sides of the crack. Rice 25 speculated on the possibility of handling this by using a strip-yield approach, but this has not been applied previously. If the line-spring is inserted into a finite element code, plasticity at the sides of the crack can readily be accounted for by the code.

The following simplifications reduce the model to a purely analytical one:

1. The surface crack is treated as existing in an infinite flat plate loaded in remote tension.

2. The actual crack front is replaced by a crack of constant depth, and displacement compatibility between ligament spring and through-crack is enforced only at the center of the crack. This reduces the coupled integral equations to a pair of linear algebraic equations, a simplification proposed in a slightly different form by Rice and Levy. ${ }^{24}$ The constant crack depth used in deriving the model presented here was the maximum depth of the actual crack, so that the correct depth in the actual crack at the point of most interest is matched by the model. 

3. The spring is elastic perfectly plastic; that is, it remains linear elastic until the force and moment in the spring reach a yield criterion.

4. Spread of plasticity from the sides of the through-crack is approximately accounted for by using an effective crack length of $\ell+2 r_{y}$, where $\ell$ is the crack length and the plastic-zone size correction, $r_{y}$, is obtained from a strip-yield analysis of a finite-width plate, the only manner in which finite width enters the model.

A simplified model results. The compliance of the ligament spring is still given by Eqs. 23 and 24 . For an infinite plate loaded in tension by $\sigma$, with the average tensile stress, $\sigma_{0}$, and bending stress, $m$, acting on the crack faces, the displacement and rotation at the center of the crack are

$$
\begin{aligned}
& \Delta_{C}=2 l / E\left(\sigma-\sigma_{0}\right) \\
& \theta_{C}=\frac{-4(1+v) \ell}{(3+v) E t} \mathrm{~m}
\end{aligned}
$$

Equation 26 is derived by integrating the solution for a point-moment applied on the crack faces. 24

Forcing equality between $\Delta_{c}$ in Eqs. 23 and 25 and $\theta_{c}$ in Eqs. 24 and 26 results in

$$
\begin{aligned}
& {\left[a_{11}+\ell / t\left(1-v^{2}\right)\right] \sigma_{0}+a_{12} m=\left[l / t\left(1-v^{2}\right)\right] \sigma} \\
& a_{12} \sigma_{0}+\left[a_{22}+\frac{\ell / t}{3(1-v)(3+v)}\right] m=0 \\
& \sigma=\alpha \sigma \\
& m=-B \sigma \\
& \alpha=\left\{l /\left[t\left(1-v^{2}\right)\right]\right\}\left(a_{22}+\frac{l / t}{3(1-v)(3+v)}\right) / S \\
& B=\left\{l /\left[t\left(1-v^{2}\right)\right]\right\} a_{12} / S \\
& S=\left\{a_{11}+\ell /\left[t\left(1-v^{2}\right)\right]\right\}\left[a_{22}+\frac{l / t}{3(1-v)(3+v)}\right]-a_{12}^{2}
\end{aligned}
$$



The spring remains elastic until satisfaction of a yield criterion is achieved in the edge crack. Various yield criteria were tried, including an upper bound slip-line solution presented by Rice, ${ }^{25}$ a lower bound static equilibrium condition that assumes yield occurs when a plastic hinge develops in the ligament, and a simple average stress condition. The average stress condition was chosen for simplicity and because its results agreed with the experimental data presented in Refs. 30 and 31 as well as any of the yield criteria tried. Yield is assumed to occur when the average tensile stress in the ligament, $\sigma_{0}$, reaches a closure stress, $\sigma_{c}$, shown in Eq. 14 , resulting in

$$
\sigma_{0}=\frac{t-a}{t} \bar{\sigma}
$$

From Eqs. 29 and 34 the value of applied stress at which ligament yield occurs, $\sigma_{L Y}$, is determined:

$$
\sigma_{L Y}=\frac{1}{\alpha} \frac{t-a}{t} \bar{\sigma} .
$$

Subsequently, $\sigma_{0}$ remains constant, given by Eq. 34 , and $m$ remains constant, given by Eq. 30 with $\sigma=\sigma_{L Y}$.

Equations 25 and 26 are still used for determining $\Delta_{c}$ and $\theta_{c}$, but after ligament yield, plasticity at the sides of the crack becomes important and the crack length, $\ell$, is replaced by the "effective" length, $\ell+2 r_{y}$. The expression for $r_{y}$ is

$$
r_{y}=\left(\ell_{p}-\ell\right) / 4
$$

and $\ell_{p}$ is evaluated from:

$$
\sin (\pi \ell p / 2 W)=\sin (\pi \ell / 2 W) /\left[\cos \frac{\pi}{2}\left(\frac{t}{a} \frac{\sigma}{\sigma}-\frac{t}{a}+1\right)\right]
$$

The plate will continue to carry additional load until the strip yield reaches the edge of the plate, which occurs when the applied stress reaches the net section yield stress

$$
\sigma_{N S Y}=\bar{\sigma}[1-\ell a /(W t)]
$$


. 
This results directly from equilibrium or from setting $\ell_{p}=W$ in Eq. 37 .

Equations 37 and 38 are valid for finite-width flat plates. If this model is applied to other situations, such as cracks in pipes, use of these equations would not be appropriate, and the strip-yield solution for an infinite plate might be more suitable, for which

$$
\ell_{p}=\ell / \cos \frac{\pi}{2} \frac{t}{a} \frac{\sigma}{\sigma}-\frac{t}{a}+1
$$

and the net-section yield stress becomes simply $\bar{\sigma}$.

The values of $\sigma_{0}$ and $m$ are now known for the entire range of applied stress. From these, both $C O D$ and the $J$-integral at the root of the crack can be computed. To calculate COD, it must be noted that $\Delta_{C}$ in Eq. 25 is the displacement at the centerline of the plate thickness. The CTOD is used as a fracture parameter, whereas the CMOD is more easily measured and is used for experimental verification of model predictions. From the geometry shown in Fig. 6,

$$
\begin{aligned}
& \text { CTOD }=\Delta_{C}+\theta_{C}(t-2 a) \\
& \text { CMOD }=\Delta_{C}+\theta_{C} t
\end{aligned}
$$

A.n alternative method for calculating CTOD prior to ligament yield is to use the relation between CTOD and $K$ (Eq. 11). The stress intensity factor, $K$, is calculated using the line-spring model, as discussed below. This method was preferable because accuracy of estimates of $K$ using the line-spring model can be checked using published elastic numerical results. ${ }^{15}$ After ligament yield the incremental value of CTOD was calculated using Eq. 40. This is justified because the assumption of crack faces opening as parallel lines is accurate after ligament yield, as has been confirmed experimentally using replication. studies. $^{31}$ The result is

$$
\text { CMOD }=(2 \ell \sigma / E)[1+2(1+\nu) B /(3+v)-\alpha]
$$





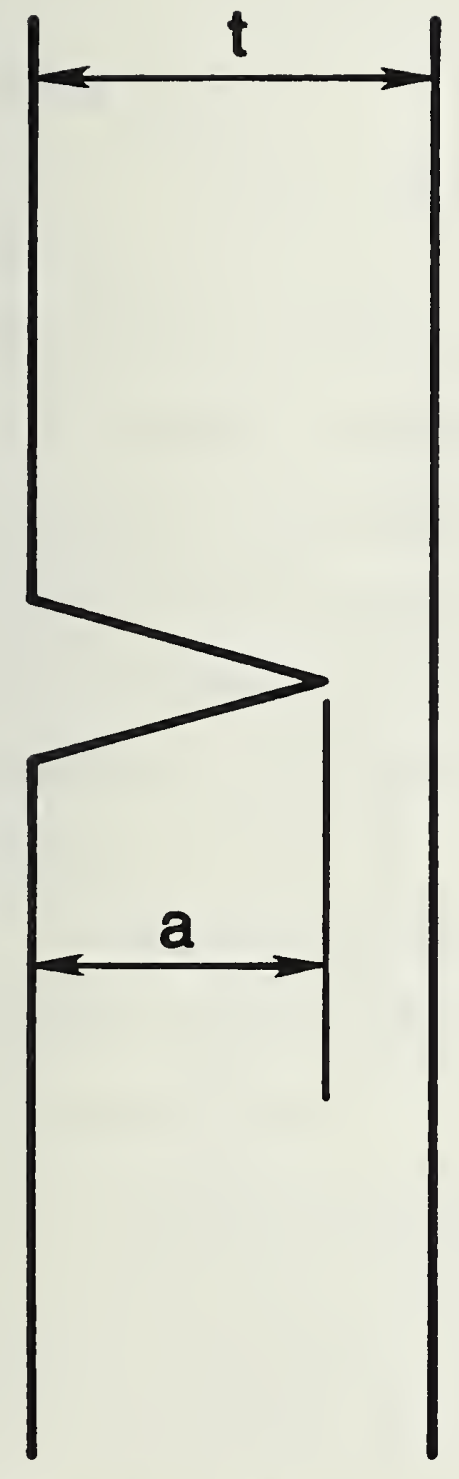

Actual Crack

(a)

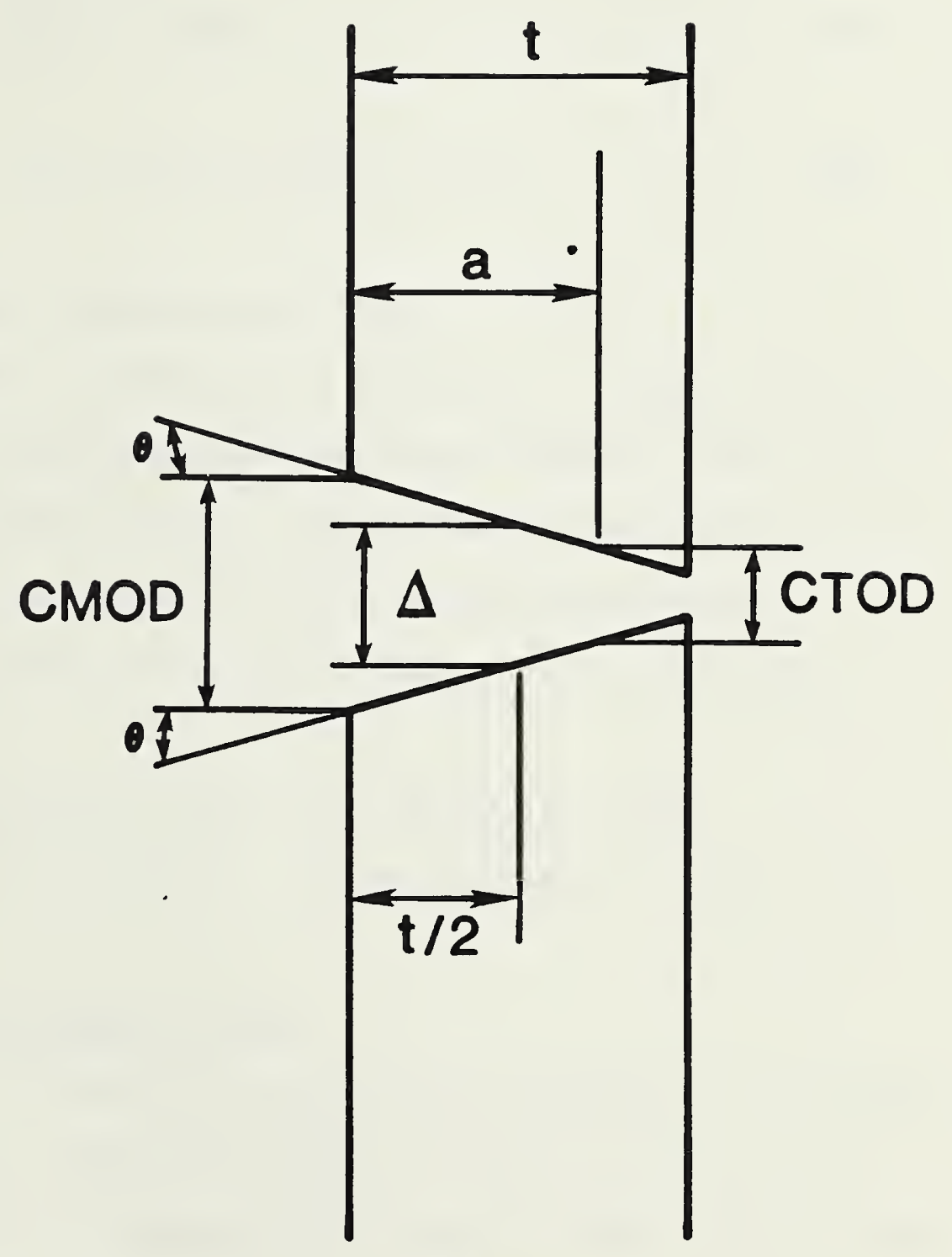

Model

(b)

Fig. 6 - Calculation of CMOD and CTOD. 
. 
for $\sigma \leq \sigma_{L Y}$

CMOD $=\left[2\left(l+2 r_{y}\right) / E\right] \sigma+\bar{\sigma}(t-a / t)\{[2(1+v) B] /(3+v) \alpha-1\}$

for $\sigma_{L Y}<\sigma<\sigma_{N S Y}$

$$
C T O D=K^{2}\left(1-\nu^{2}\right) / E \bar{\sigma} \equiv C T O D_{E}
$$

for $\sigma \leq \sigma_{L Y}$

$$
\text { CTOD }=\operatorname{CTOD}_{E}+\left[2\left(\ell+2 r_{y}\right) / E\right]\left[\sigma(1-a / t) \bar{\sigma}_{-}\right.
$$

for $\sigma_{L Y}<\sigma<\sigma_{N S Y}$

Both CMOD and CTOD increase without bound at $\sigma_{\text {NSY }}$.

The model presented is for flat plates in tension. To apply it to actual structures, the value of the nominal membrane stress component normal to the crack in the vicinity of the crack (i.e., the stress that would exist in that region if the crack were not present) is calculated and used as the applied tension, $\sigma$. The redistribution of stresses caused by the crack is thus ignored.

The model that has been described in this subsection is the yielded-ligament model for flat plates. Further development of this model is discussed below.

\subsubsection{Crack-Opening Displacements in Pipe}

The driving force for fracture in pipes differs from that in flat tensile panels in two ways: First, shells have greater stiffness than flat plates. Thus, the rotation of the crack faces, and the resulting CMOD, is less in pipe than in plates, particularity for long cracks. Second, the axial stresses in pipes are caused by bending or by bending plus tension, whereas, the flat plates were tested in tension. The bending stresses result in a stress gradient along the circumference. Therefore, as the plastic zone develops at the ends of a crack (located in the region of maximum tension), plasticity spreads into a decaying stress field. Thus, the stress for plastic collapse 

is greater for pipes in bending than for plates in tension. These factors were accounted for in the analyses of the large-diameter pipe tests, Section 2.4, and in the calculation of allowable flaw-size curves.

The higher stiffness of shells relative to flat plates against rotation is approximately accounted for in the simplified line-spring model. The line-spring model treats a surface crack as being equivalent to a throughcrack with closing forces and out-of-plane bending moments acting on its faces. The magnitude of these forces and moments is determined by enforcing compatibility of displacement and rotation between the through-crack faces and the ligament. The compliance of the ligament is determined by considering an edge crack of the same depth as the surface crack. The modification to the line-spring model, introduced to model long surface cracks in pipes more accurately, was to account for the effect of curvature on the relation between moment and rotation in a pipe. This relation enters the line-spring model when the compatibility calculation is made. For a flat plate, the relation between moment on the crack faces and out-of-plane rotation is

$$
\theta=-24(1+v) \ell M /\left[E t^{3}(3+v)\right]
$$

The compliance is seen to become very large, approaching infinity in the limit for long crack lengths. However, a circumferentially cracked pipe has significant rotational stiffness, even if the crack extends completely around the circumference. For long cracks, the effects of the ends of the crack on rotation at the crack center may be neglected, and the moment-rotation relation may be obtained by considering the axisymmetric problem of a cylindrical shell subjected to moments distributed around its end. The relation between moment and rotation is, then

$$
\theta_{C}=-(2 R)^{1}{ }^{2}\left[12\left(1-v^{2}\right)\right]^{3} 4 M /\left(E t^{5}{ }^{2}\right)
$$





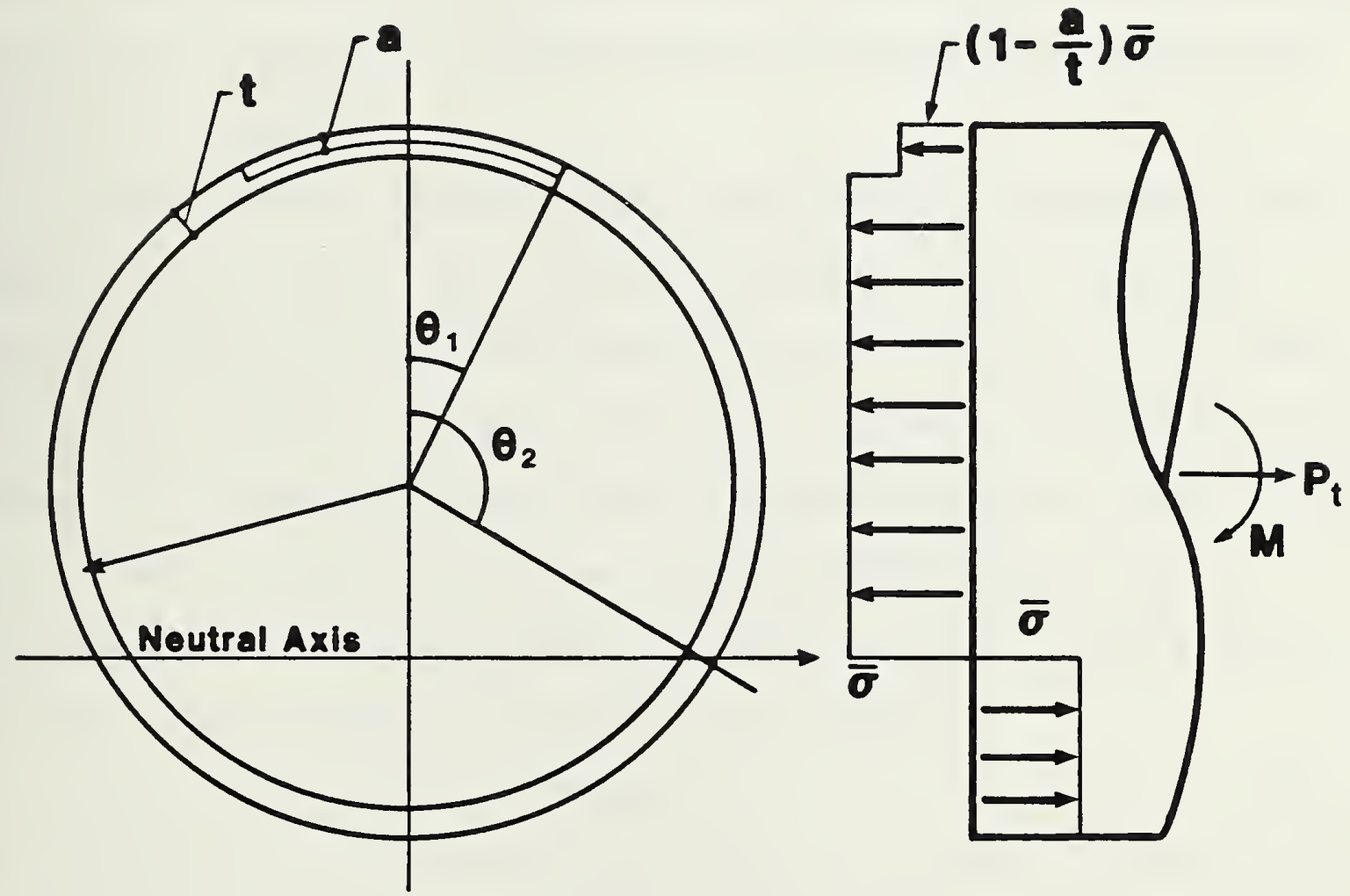

Fig. 7 - Schematic stress distribution of a pipe containing a circumferential crack under bending. 

The line spring calculations have been done in terms of bending stress $\mathrm{m}$ rather than moment $M$. these quantities are related according to (22).

$$
m=6 M / t^{2}
$$

Writing (47a) in terms of bending stress $m$, we have

$$
\theta_{c}=-\left\{(2 R)^{\frac{1}{2}}\left[12\left(1-v^{2}\right)\right]^{3} / 4 /\left(6 E t^{\frac{1}{2}}\right)\right\} m
$$

Equation 47 is derived using axis symmetric shell theory. The relation between $M$ and $\theta_{c}$ enters the line-spring model in the compliance calculation by replacing Eq. 26 with Eq. 47 and then obtaining curvature-corrected results for Eqs. 27 through 33.

In tensile panels, plastic collapse occurs when the net-section stress exceeds the flow stress. The collapse mechanism consists of localized deformation along $45^{\circ}$ slip lines emanating from the crack tips to the edges of the specimen. However, in a pipe subjected to bending or combined tension and bending, the collapse mechanism differs from that in a tensile panel. A lower bound estimate for the collapse load is obtained by assuming a plastic hinge develops at the cracked section, as shown in Fig. 7 . Force balance at the plastic hinge determines the neutral axis location:

$$
\theta_{2}=\left(\frac{1}{2}\right)\left[P_{t} /(2 \sigma t R)\right]+(a / t)\left(\theta_{1}+\pi\right)
$$

where the angles $\theta_{1}$ and $\theta_{2}$ are defined in Fig. 7, $P_{t}$ is the resultant of all applied loads, and $R$ is the pipe radius. Moment balance results in

$$
M=\left(2 \bar{\sigma} R^{2} t\right)\left(2 \sin \theta_{2}\right)-(a / t) \sin \theta_{1}
$$

where $M$ is the applied moment. For a given crack size, Eqs. 46 and 47 will give an interaction curve of values of $P_{t}$ and $M$ corresponding to plastic collapse.

The special case $P_{t}=0$ is of interest in the present study. For this case, the collapse moment is given by

$$
M_{c}=2 \sigma^{2} R^{2} t\left\{2 \cos \left[(a / t) \theta_{1}\right]-(a / t) \sin \theta_{1}\right\}
$$



which reduces to

$$
M_{c}=2 \sigma^{-} R^{2} t\left[2-(a / t) \theta_{1}\right]
$$

for small crack lengths. The moment at which flow first occurs in an uncracked pipe is given by

$$
M=\pi R^{2} t \bar{\sigma}
$$

Comparing Eqs. 52 and 51, for small crack lengths the collapse moment is approximately $27 \%$ higher than the moment corresponding to incipient yield. Thus, the pipe is capable of carrying significantly higher moments before the CMOD increases without bound than is predicted by the critical-COD model.

A modification to the critical-COD model has been derived that addresses this objection. The fully elastic contribution to CMOD is calculated as described above. The additional contribution to CMOD in the post-ligamentyield range, $C M O D_{L Y}$, is computed using a new expression. The expression for $\mathrm{CMOD}_{L Y}$ is deriving using an approach similar to that used by Heald, Spink, and Worthinton $^{32}$ in deriving strip-yield solutions for the COD of finite-width tensile panels. del!it and $S \operatorname{mith}^{33}$ followed this approach in deriving a model for a finite-width tensile panel containing a surface crack that gives analytical results for $C_{M O D}$ comparable to those obtained using the yielded-ligament model.

First the strip-yield expression for CMOD of a center crack in an infinite plate under tension is considered:

$$
\text { CMOD }=2 \bar{\sigma} \ell /(\pi E) \cdot \ln \{1+\sin (\pi \sigma / 2 \bar{\sigma})] /[1-\sin (\pi \sigma / 2 \bar{\sigma})]\}
$$

Equation 53 can be thought of as an expression that increases without bound as $\sigma$ approaches $\bar{\sigma}$, correctly reduces to the linear elasticity expression for CMOD at small stress levels, and interpolates in between these limits using the function in braces. The desired expression for $C M O D_{L Y}$ should have similar features with the following exceptions: 

1. The expression for $\mathrm{CMOD}_{\mathrm{LY}}$ shculd increase without bound as the plastic collapse moment is approached. When axial load is present, proporticnal loading must be assumed.

2. At small moment levels, the expression for $\mathrm{CMOD}_{\mathrm{LY}}$ should reduce to the expression for $\mathrm{CMOD}_{\mathrm{LY}}$ that results when plasticity at the sides of the crack is negligible.

An expression which meets the above criteria is

$$
\mathrm{CMOD}_{L Y}=\left[2 \ell \bar{\sigma} /\left(\pi^{2} E\right)\right][4-a \ell /(t R)-\pi(1-a / t)] \cdot \ln [(1+\sin \gamma) /(1-\sin \gamma)
$$

where $\gamma=(\pi / 2)\left(M-A_{0}\right) /\left(M_{c}-A_{0}\right), A_{0}=\pi R^{2} t(1-a / t) \bar{\sigma}$, and $M_{c}$ is evaluated

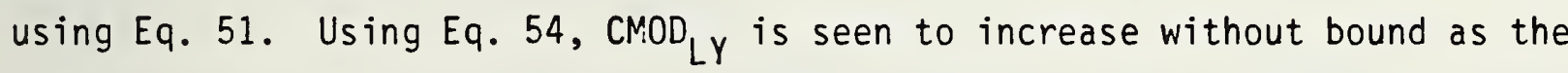
collapse moment is approached. For small moments, the pipe is elastic, the nominal membrane stress is

$$
\sigma=M / \pi R^{2} t
$$

and Eq. 54 reduces to Eq. 53. The logarithmic function interpolates between the two extremes. The model described here is called the pipe yielded-1igament model.

\subsection{Model Evaluation Tests for Surface Cracks in Tension}

The critical-COD and flat plate yielded-ligament models provide an analytical relationship among the COD, applied stress, flaw size, material properties, and specimen (or structural) geometry. Both models can be expressed in terms of the CMOD or CTOD. The experimental program was based on the assumption that verification of the model in terms of CMOD is sufficient to verify the models in terms of CTOD. This assumption is required because CMOD can be readily measured, whereas CTOD cannot.

In this section, the experimental evaluation of the critical-COD and flat plate yielded-ligament models is discussed for the case of surface cracks 

in tension. The CMOD's of surface cracks are measured as functions of stress and strain in tensile panels of API $5 L X-70$ steel plates and welded pipe segments. The experimental results are compared with analytical predictions.

\subsubsection{Experimental Procedures}

The test materials were API $5 L X-70$ pipeline steel in the form of 15.9-mmthick plate and pipe segments with transverse girth welds. The pipe segments were taken from $1220-\mathrm{mm}(48-i n)$ diameter, $15.9-\mathrm{mm}(0.625-i n)$ thick API $5 \mathrm{LX}-70$ pipe welded using representative field practices. Metallography and properties of the base plate and welds are described in Appendix $H$. Chemical composition is given in Table 1, along with compositions of materials considered elsewhere in this report.

Three series of specimens with different surface crack lengths and depths were tested: one series each for the base metal, the manual welds, and the automatic welds. The test matrix is shown in Table 2. The base-metal test specimens were tensile panels (Fig. 8) that were notched in three different ways: 1) a saw cut of $0.4-\mathrm{mm}(0.016-\mathrm{in})$ width, 2) a saw cut followed by fatigue sharpening, or 3) an electrical-discharge-machined (EDM) notch of 0.4-mm $(0.016-i n)$ width. The weld-metal test specimens were tensile panels taken from the $1220-\mathrm{mm}(48-i n)$ diameter pipe with the weld transverse to the tensile axis. The pipe curvature was retained in the specimens and the weld reinforcement was removed to obtain a uniform cross section. The surface notches, all prepared by EDM, were located on the concave side of the pipe at the root of the $V$-shaped weld.

Each of the specimens in Table 2 was loaded in tension at room temperature, and the CMOD was measured as a function of nominal stress, $\sigma$, and gage-length strain, $\varepsilon_{L}$. In the base-metal specimens, the $\varepsilon_{L}$ was taken as the average strain over a gage length of $305 \mathrm{~mm}(12-\mathrm{in})$, except for the $381-\mathrm{mm}$ (15-inch) 

Table 1 - Chemical Analyses in Weight Percent

\begin{tabular}{lccccccccccccc}
\hline & $C$ & $S t$ & $M n$ & $P$ & $S$ & A1 & Cu & Cr & Ni & Mo & V & Nb & $N$ \\
\hline $\begin{array}{l}\text { Base metal } \\
\text { (API 5LX-65) }\end{array}$ & 0.08 & 0.3 & 1.45 & 0.015 & 0.003 & 0.039 & 0.08 & 0.09 & 0.03 & 0.10 & 0.07 & 0.036 & 0.009 \\
$\begin{array}{l}\text { Base metal } \\
\text { (API 5LX-70) }\end{array}$ & 0.076 & 0.2 & 1.3 & & & & 0.12 & 0.14 & 0.13 & 0.33 & $<0.05$ & & \\
Manual weld & 0.12 & 0.17 & 0.67 & 0.005 & 0.010 & & & 0.13 & 1.6 & 0.031 & & & \\
Automatic weld & 0.11 & 0.43 & 1.3 & 0.011 & 0.005 & & & 0.073 & 0.92 & 0.053 & & \\
\hline
\end{tabular}



Table 2 - Test Matrix for the Experimental Evaluation of the Critical Cún Model and Flat Plite YieldedLigament Model

Specimen Specimen width, W, Crack Depth, a, Crack Length, \&, Number $\mathrm{mm}$ $\mathrm{mm}$ mm

\begin{tabular}{lrrr}
\hline P1 * & 102 & 6.65 & 40.9 \\
P2 & 102 & 9.07 & 43.5 \\
P3 & 102 & $5.64^{\S}$ & 31.2 \\
P4 & 102 & $6.88^{\S}$ & 48.3 \\
P5 & 76 & $10.54^{\S}$ & 31.2 \\
P6 & 76 & 4.57 & 13.5 \\
P7 & 76 & 3.56 & 31.8 \\
P8 & 76 & 5.08 & 14.7 \\
P9 & 381 & 3.81 & 114.3 \\
A1 & & & 29.7 \\
A2 & 76 & 8.13 & 47.5 \\
A3 & 102 & 5.72 & 17.3 \\
A4 & 76 & 2.49 & 11.8 \\
M1 & 76 & 4.57 & 29.2 \\
M2 & 76 & 8.13 & 47.5 \\
M3 & 102 & 5.84 & 11.8 \\
M4 & 76 & 4.32 & 15.0 \\
\hline
\end{tabular}

* P: base metal

+ A: automatic weld

* M: manual weld

5 specimen also used to evaluate the J-integral model

$1 \mathrm{~mm}=0.0394 \mathrm{in}$. 

Front

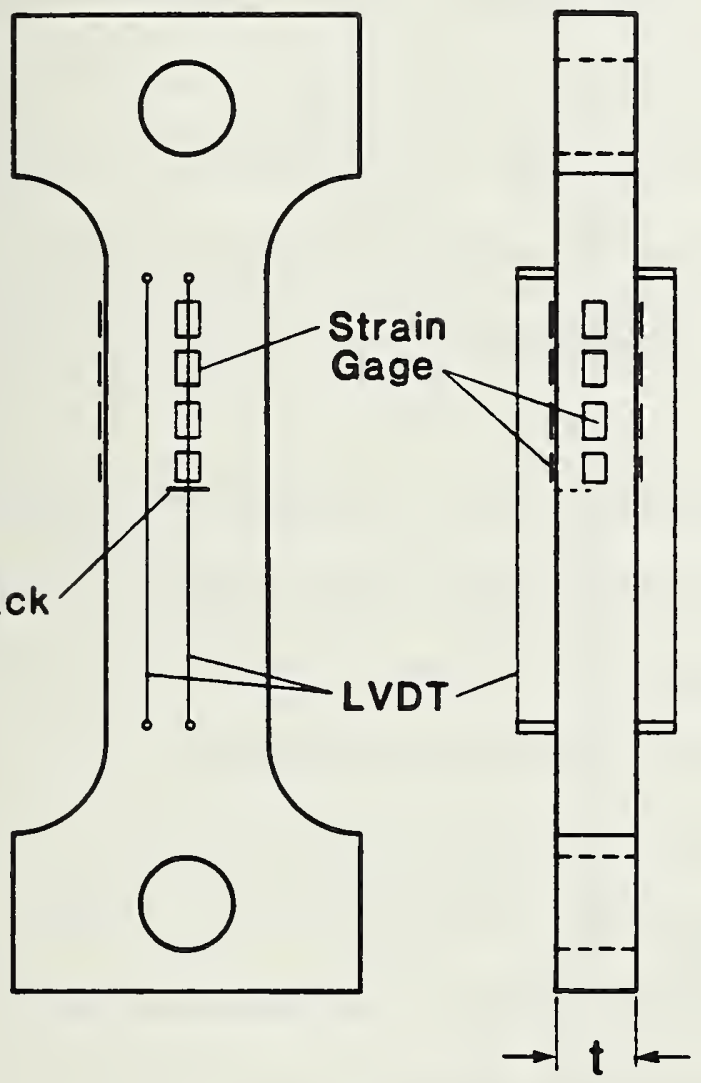

Back

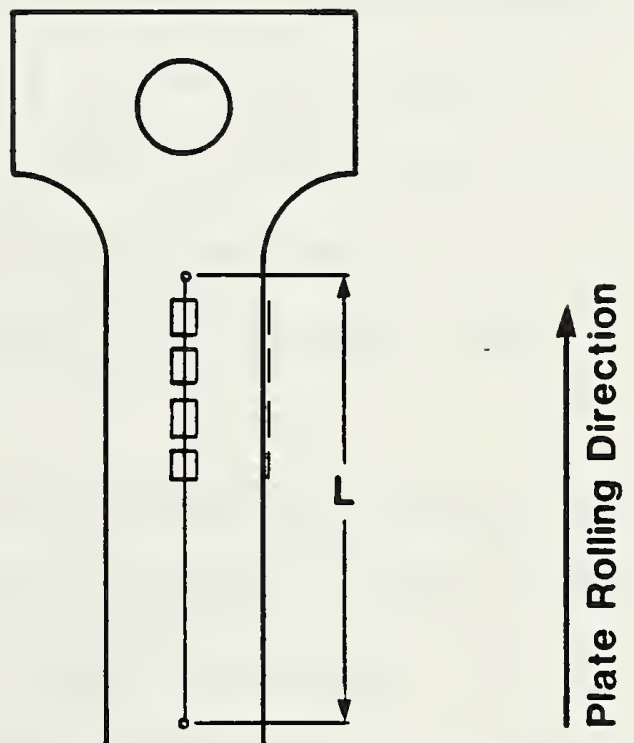

Fig. 8 - Test specimen configuration. 

wide specimen that had a gage length of $610 \mathrm{~mm}$ (24-inch). The specimens were pulled in tension under displacement control with a closed-loop servo-controlled hydraulic testing machine. The values of CMOD were measured with a clip-on displacement gage mounted directly onto the crack mouth with the clip-on teeth about $0.13 \mathrm{~mm}(0.005-i n)$ into the crack. The $\varepsilon_{L}$ was obtained from displacements measured over the chosen gage length with linear-variable-differential transducers (LVDTs). All signals from electrical resistance strain gages, LVDTs, the clip-on gage, and the load cell were acquired and stored by a multichannel minicomputer for on-line and post-test analysis. For observation of the deformation patterns near the cracks, the specimens were coated with photoelastic material and instrumented with electrical-resistance strain gages.

The CODs in specimen P5 were measured by a replication technique. The specimen was loaded under displacement control to a CMOD of $0.2 \mathrm{~mm}\left(0.0079-\mathrm{n}_{\mathrm{n}}\right)$. A liquid, silicone base, precision-impression material was mixed with a hardener and inserted into the crack. The replica material was allowed to harden at constant CMOD for about $15 \mathrm{~min}$. Subsequently, loading was continued until a CMOD of $0.5 \mathrm{~mm}(0.02-i n)$ was reached. The replica was removed and the replication procedure was repeated. The procedure was repeated at CMOD values of $0.9,1.2$, and $1.8 \mathrm{~mm}(0.035,0.047$, and $0.071-i n)$. Each of the replicas was cut along the minor axis of the semielliptical crack and mounted in phenyl salicylate. The CODs were measured at several distances ( $x$ values) from the crack mouth with a metallographic microscope. The precision of measurement was within $\pm 0.03 \mathrm{~mm}( \pm 0.0012$ in) under $100 \mathrm{X}$ magnification.

\subsubsection{Results and Discussion}

For the test matrix shown in Table 2, CMOD has been measured as a function of applied stress, $\sigma$, and of gage-length strain, $\varepsilon_{L}$. The experimental results 

of CMOD vs. $\sigma$ are compared with the analytical predictions of the analytical models discussed previously.

$$
\begin{aligned}
& \text { CMOD }=\text { CMOD (elastic), for } \sigma<[1-(a / t)] \bar{\sigma} \\
& \text { CMOD }=\left[2\left(e+2 r_{y}\right) / E\right][\sigma-(1-a / t) \bar{\sigma}]+\text { CMOD }
\end{aligned}
$$

for $\sigma \geq[1-(a / t)] \bar{\sigma}$

Equations 56 state the critical-COD model applied to prediction of crack mouth opening displacement. For short cracks, the critical-COD and flat plate yielded ligament models agree. They agree for all cracks after full ligament yielding.

Equation 56 a is evaluated using the elasticity solution of Kobayashi, ${ }^{23}$ (Eq. 20) for cracks with aspect ratios $(a / l)$ greater than 0.1 . For long cracks $(a / \ell<0.1)$, the Kobayashi solution is not applicable and the elastic CMOD is calculated using King's ${ }^{21}$ simplified version of the line-spring model (see Section 2.2.3). Also, for long cracks, the plastic zone development through the thickness before full ligament yielding becomes more important, and it is accounted for by adding a plastic-zone correction, $r_{y d}$, to the crack depth. The Dugdale plastic zone in a center-cracked finite-width panel is used. In using this solution for an edge crack in the line-spring model, bending is neglected; the underestimation of $r_{y d}$ is considered negligible for present purposes. The addition of the through-thickness plastic zone correction to the flat plate yielded-ligament model produces a model called the flat plate yielded-ligament model with through-thickness plasticity correction.

Equation $56 \mathrm{~b}$ is used from the onset of ligament yielding, $\sigma \geq[1-(a-t)] \bar{\sigma}$, unti] net-section yielding occurs. After net-section yielding, CMOD increases without bound at a constant applied stress and is shown as a vertical line in the CMOD-vs.-o curve, at $\sigma=\bar{\sigma}(1-a l / t W)$. The 



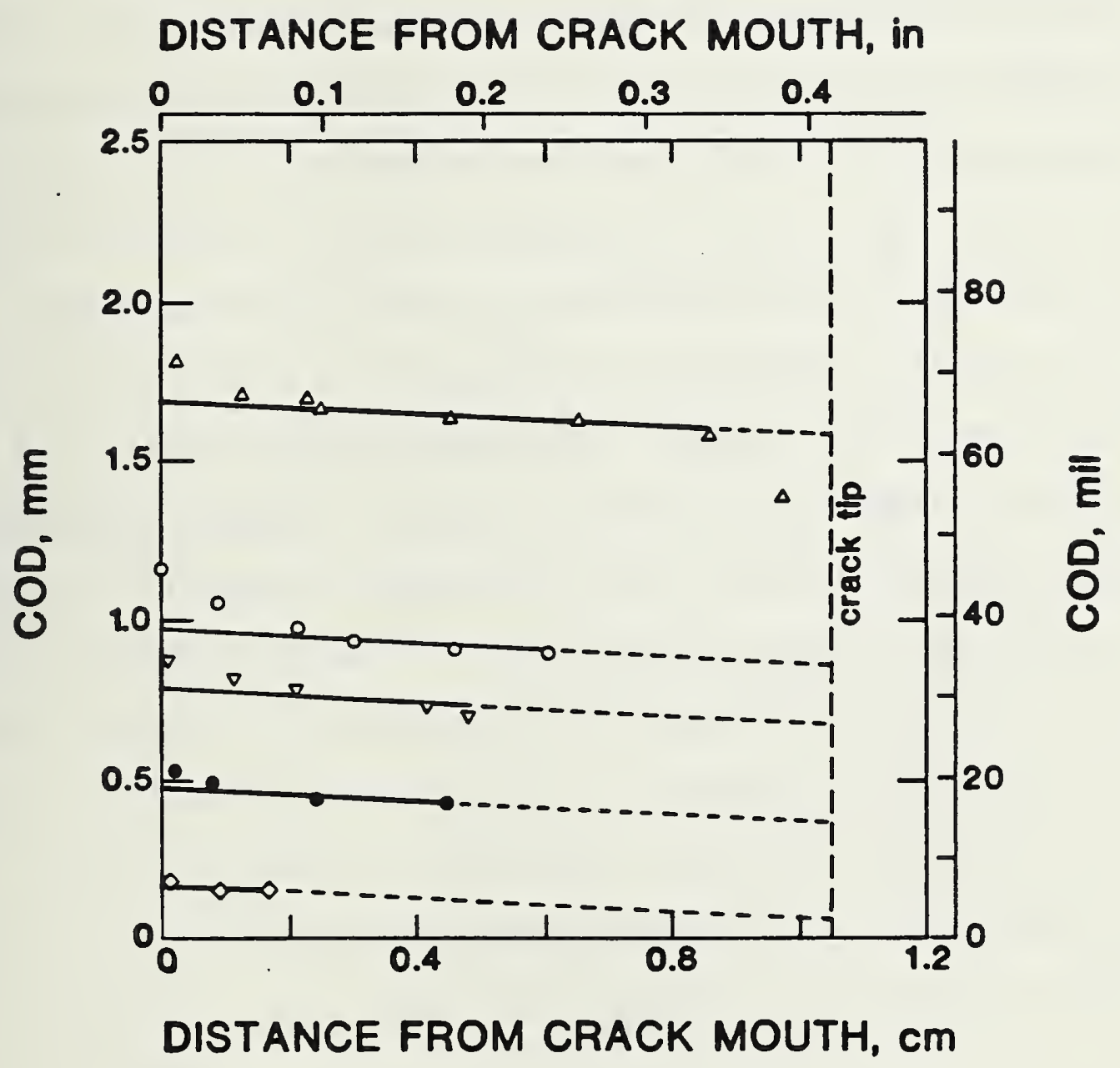

Fig. 9 - COD as a function of location along the crack face after ligament yielding. Measured with replication technique. Crack depth $=10.5 \mathrm{~mm}(0.413-i n)$; crack length $=31.2 \mathrm{~mm}(1.23-i n)$. 

important quantity in the application of fracture mechanics is CTOD and not CMOD, because CTOD is recognized as being related to crack initiation. With moderate length and deep surface cracks, it is assumed that the rotation of the crack faces owing to out-of-plane bending is negligible after the ligament has yielded and the increments of CTOD at the leading edge and CMOD are equal. Measurements of the crack profile are shown in Fig. 9. At low values of COD, the replicating material did not penetrate all the way to the crack tip. Thus, there are more data points at the higher values of COD than at the lower end. As shown in Fig. 9, the assumption of negligible rotation of the crack faces owing to out-of-plane bending after ligament yielding is reasonable.

Base-Metal Results. As shown in Table 2, nine base-metal tests were conducted. The results, summarized in Figs. 10 and 11, show good agreement between experiment and analysis. Note that the shape of the CMOD-vS-o curve for the long crack ( $l=114.3 \mathrm{~mm}, 4.5$-in) test differs from that of the shorter crack tests; nonlinearity due to crack-tip plasticity occurs at a lower stress, and there is a more gradual transition to net-section yielding. Addition of the Dugdale plastic-zone correction to the crack depth accounts for this behavior. Thus, the analysis appears to be valid for a broad range of crack sizes.

The chronological events associated with the CMOD-vs-o curves can be described as follows: Initial loading produces elastic specimen extension and the relationship between CMOD and stress is linear. As the stress increases, the plastic-zone size ahead of the crack tip enlarges. Eventually, the plasticity spreads to the back face of the specimen; this is termed ligament yielding. After ligament yielding, the plasticity contributing to the CTOD tends to occur in slip bands emanating from the crack tip at angles of $\pm 45^{\circ}$ from the plane of the crack, and the nonlinearity of CMOD-vs- $\sigma$ curves becomes 


STRESS, ksi

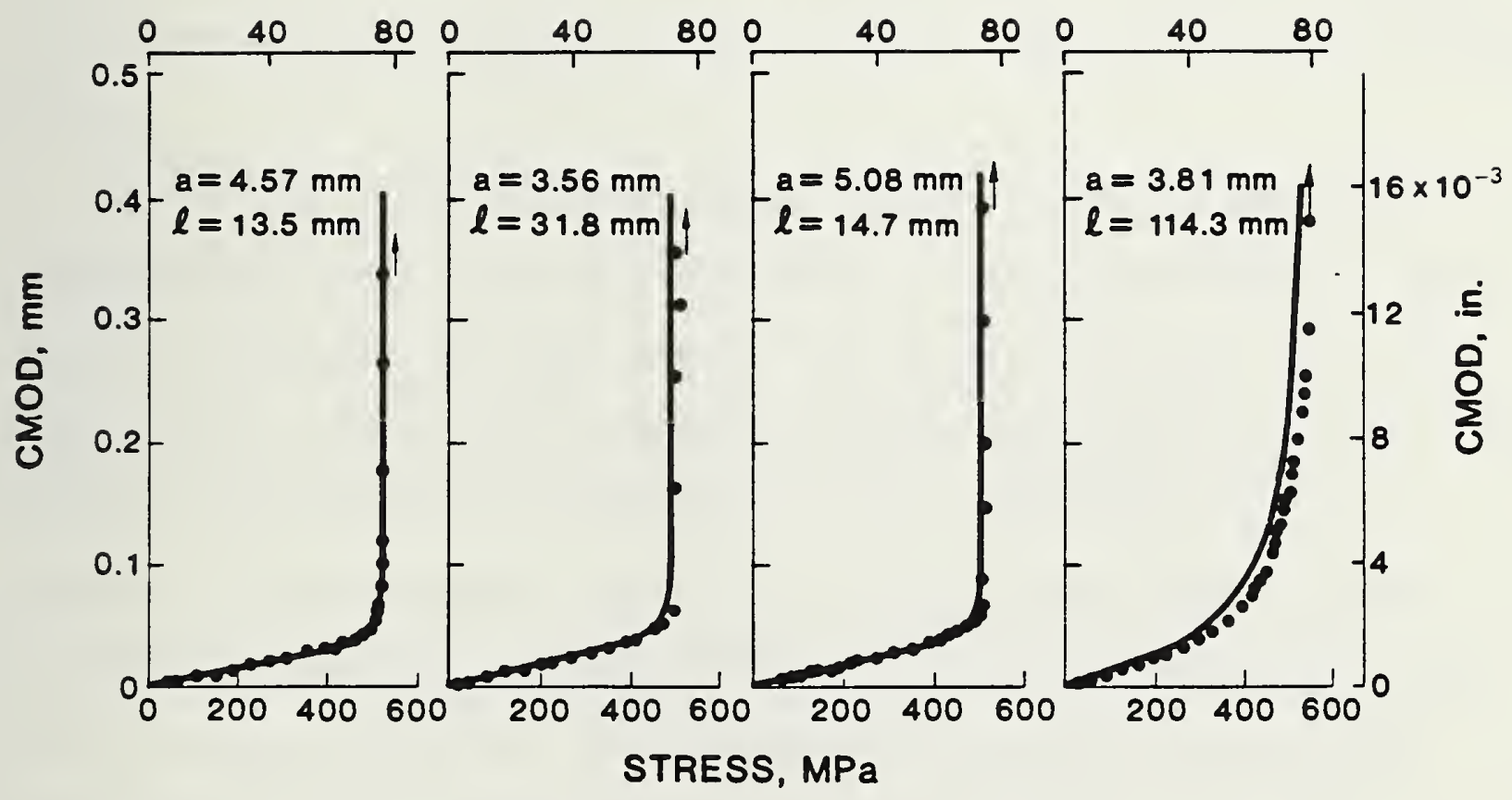

Fig. 11 - CMOD vs. nominal stress for base-metal specimens. Symbols are experimental data and solid lines are analytical predictions based on the modified critical-COD model. 

noticeable. Initially, the slip bands are between the crack tip and the back face. Finally, the net section yields along $\pm 45^{\circ}$ slip bands between the crack and the specimen edges, and the CMOD increases asymptotically to the net-section yielding stress. These phenomena were revealed by the brittle lacquer on the front and back surfaces of the specimen. On the front surface, Fig. 12, values of strain $3.8 \mathrm{~mm}$ above the center of the crack were more than two orders of magnitude lower than those of remote strain. The strain increased with distance from the crack plane and eventually reached the values of remote strain.

On the back surface of the specimen, the deformation phenomena were examined with brittle lacquer and electrical-resistance strain gages. As the stresses increased, the highest strains were observed at regions about $\pm 45^{\circ}$ from the plane of the crack emanating from the leading edge of the crack. The ligament yielding stress estimated from $\sigma=(1-a / t) \bar{\sigma}$ agreed well with readings from strain gages at the highest strain regions. After ligament yielding, the deformation pattern on the back of the specimen was an ellipse that surrounded the position of the crack until net-section yielding was approached. Then the pattern developed slip lines to the edge of the specimen. The strains at the region right behind the crack remained relatively low compared with the remote strains. This low-strain region could be seen necking down after extensive net-section yielding, and then the crack grew to the back surface. The strain patterns revealed by the brittle lacquer are shown in Fig. 13.

The results in terms of CMOD vs. $\varepsilon_{L}$ are shown in Figs. 14 and 15. Two distinct behaviors are observed. Seven of the specimens exhibited a nearly bilinear relationship between CMOD and $\varepsilon_{L}$, with the slope sharply increasing at strain levels slightly below the yield strain, as shown in Fig. 14. The 


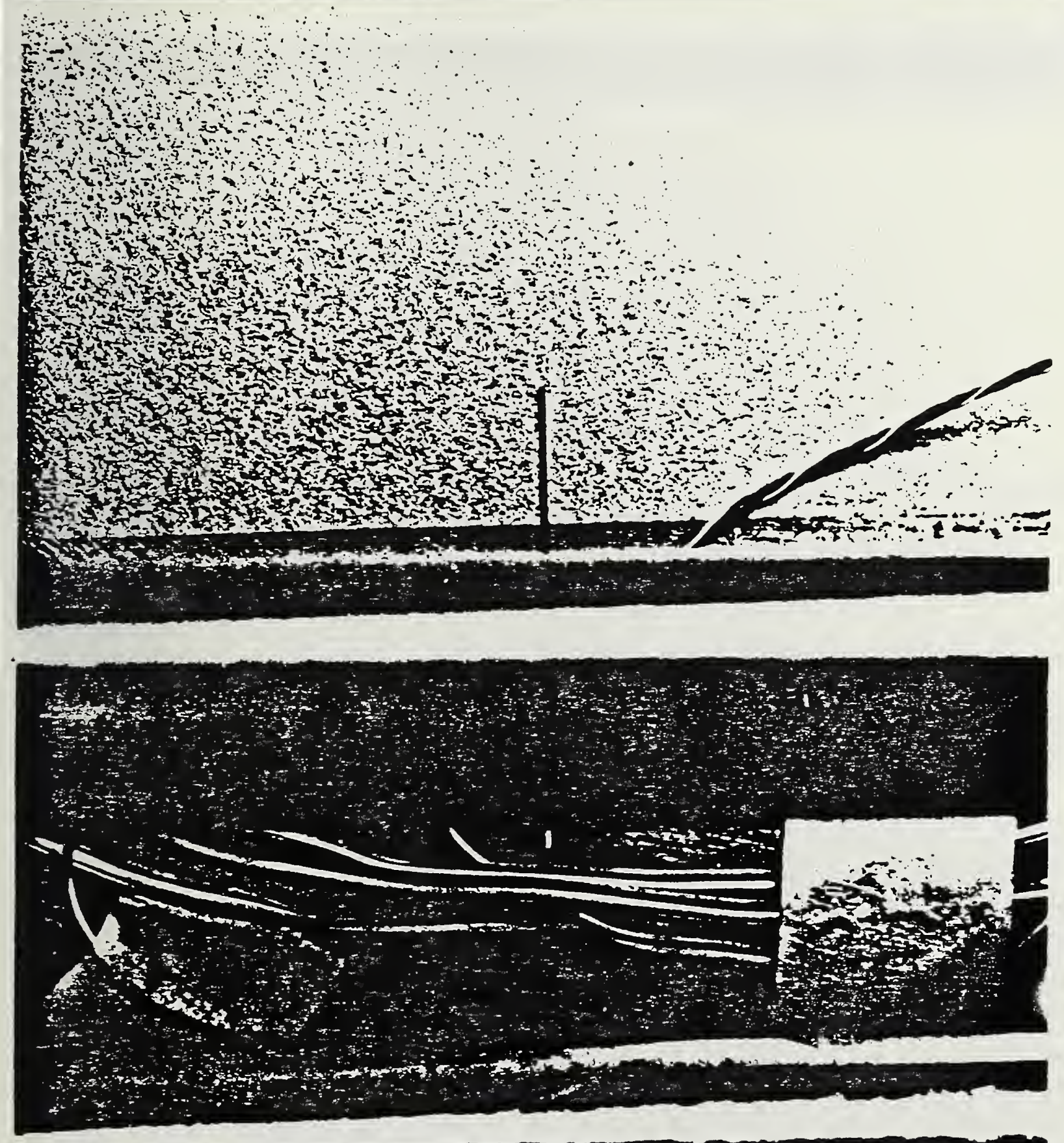

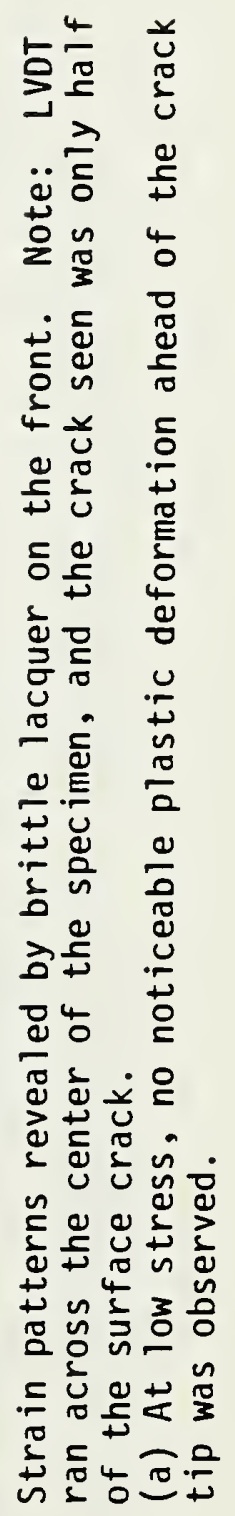

N

문 



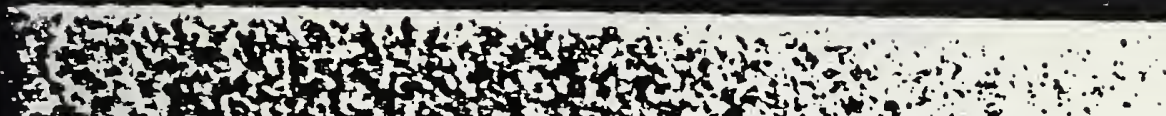

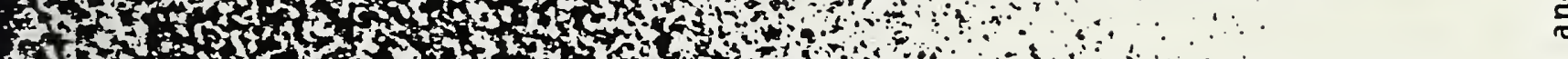

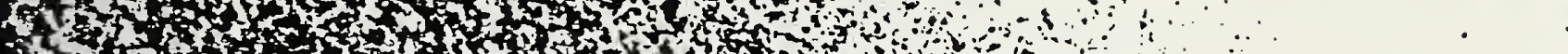

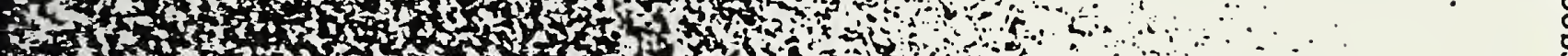

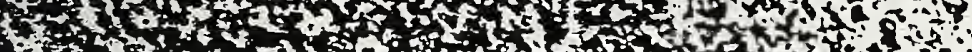

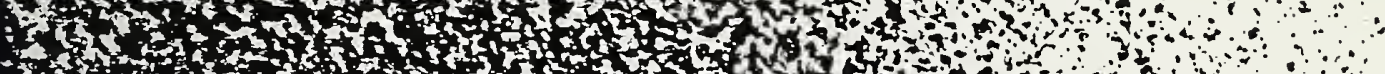

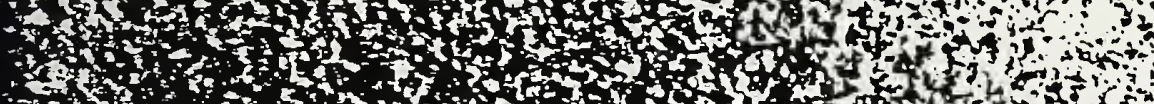

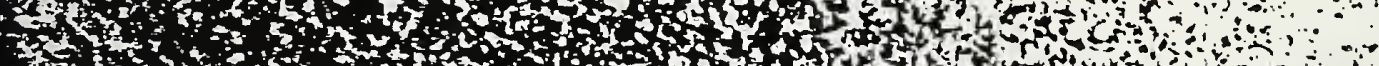

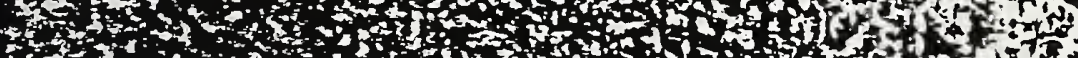

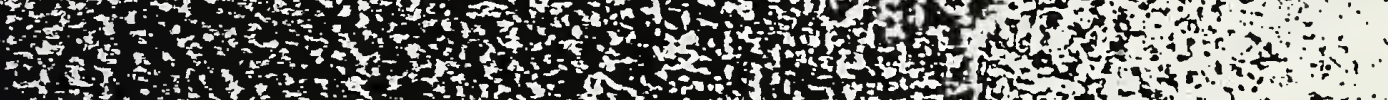

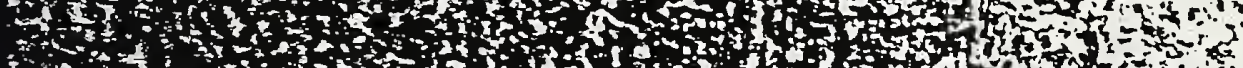

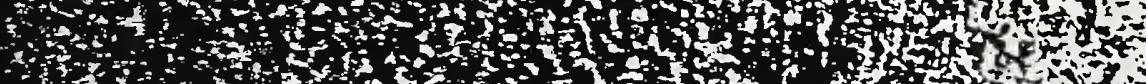

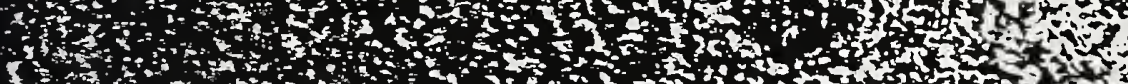

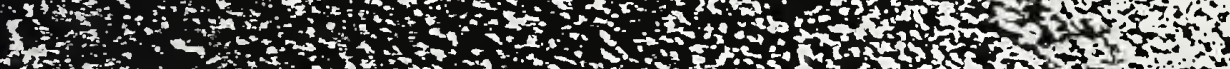

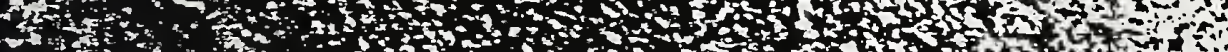

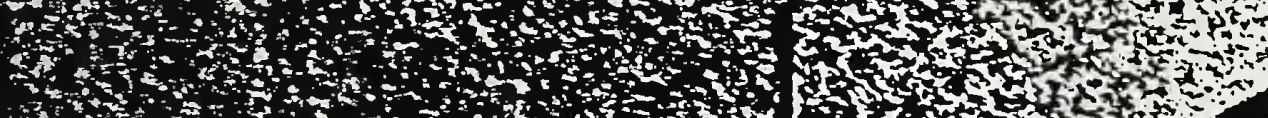

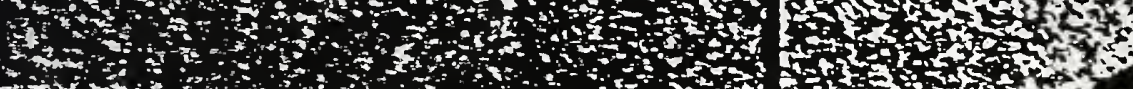

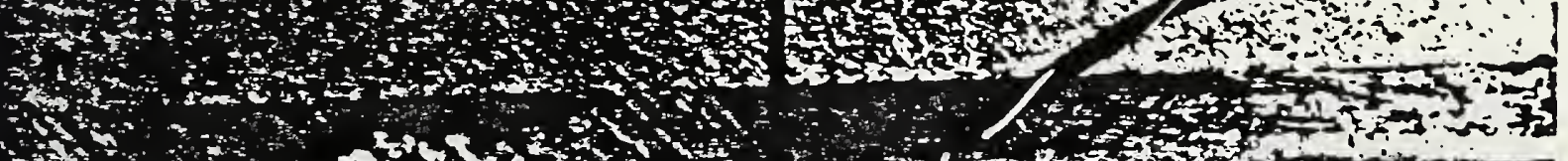

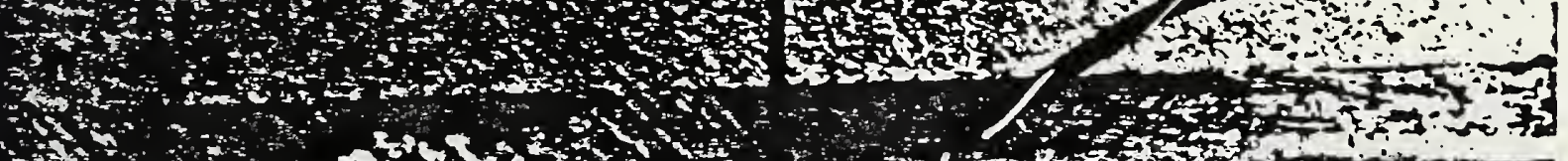

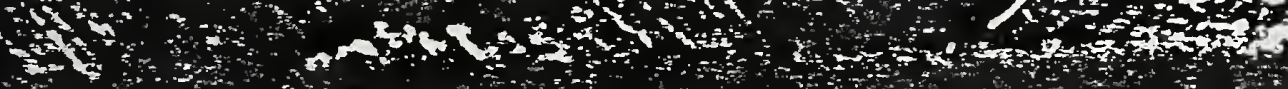





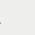




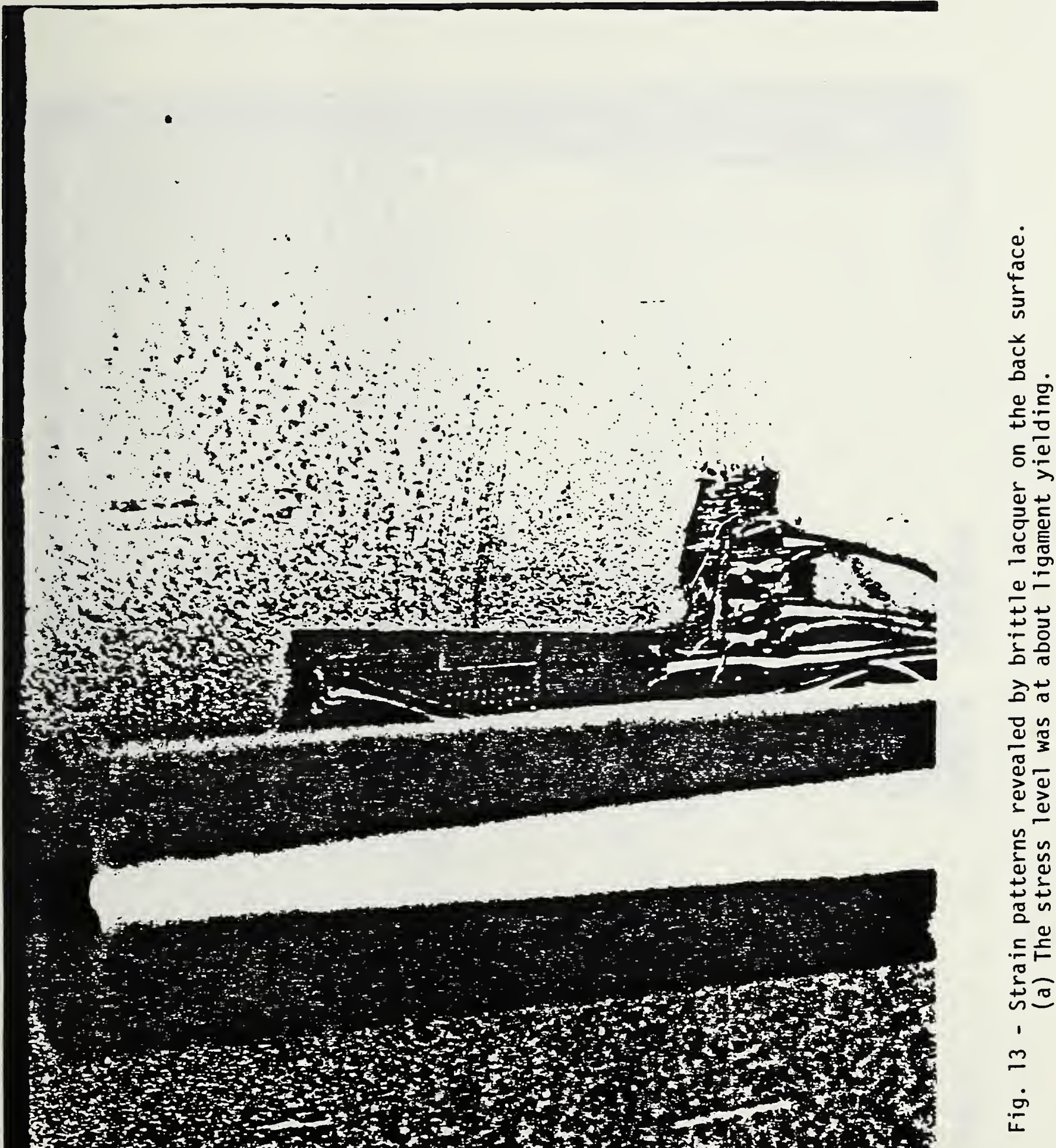





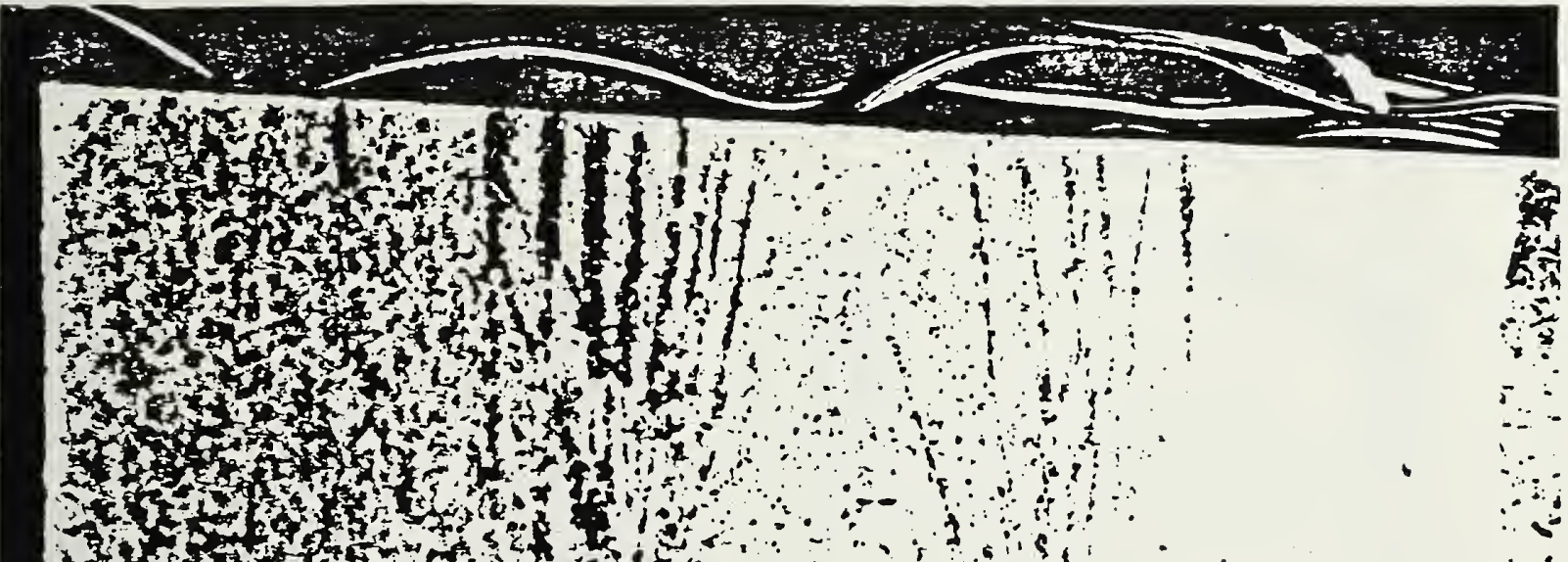

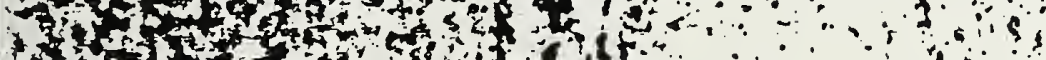

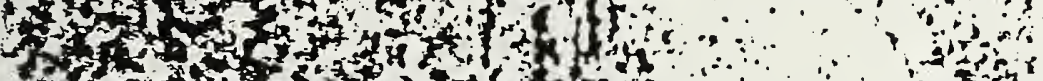

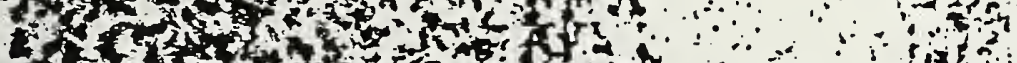

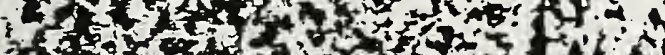

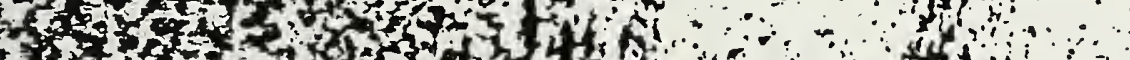

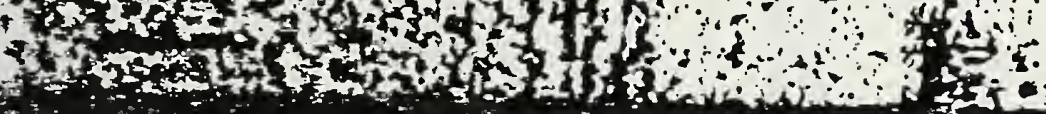

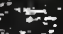

tran

-

:

. 


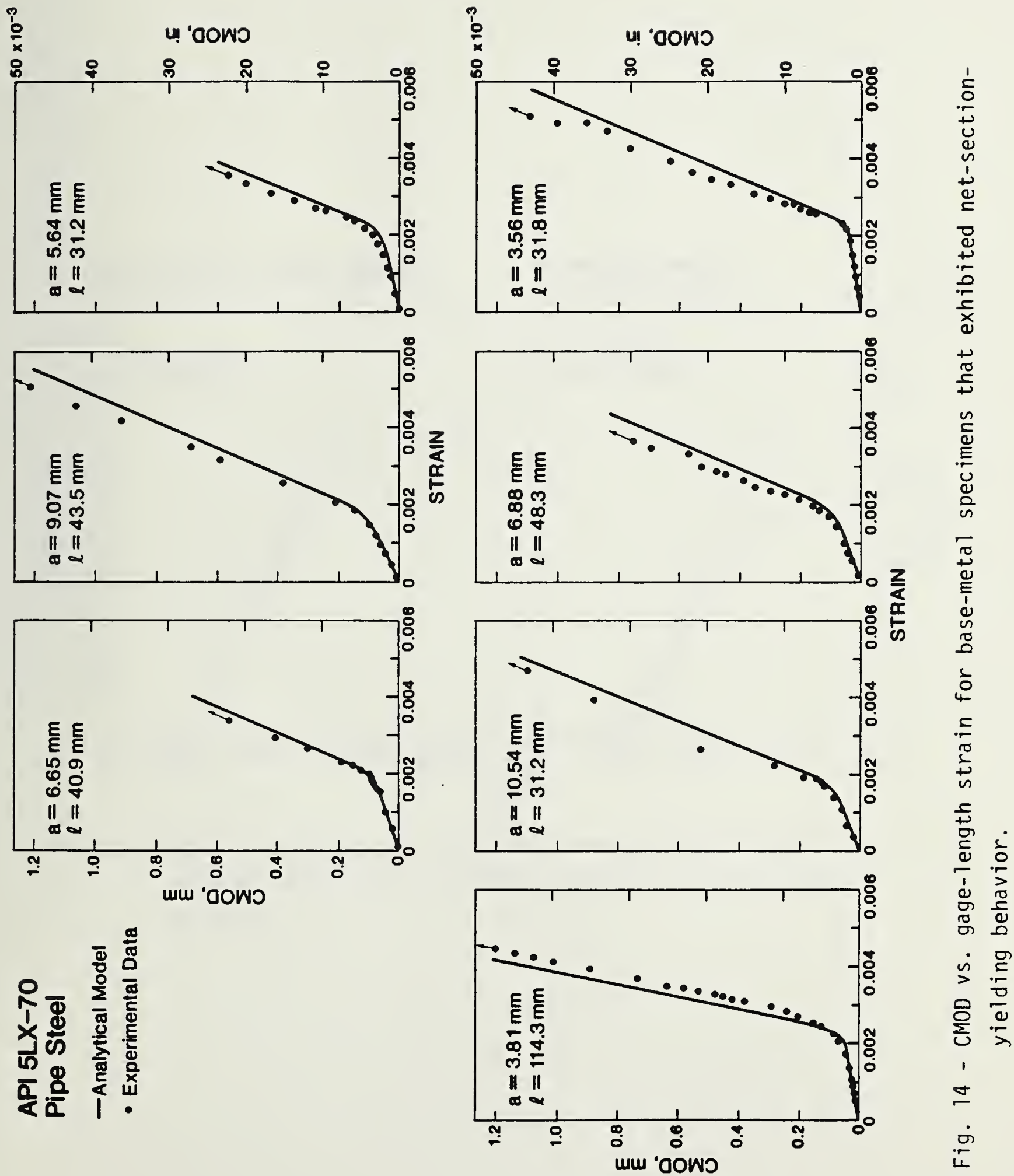



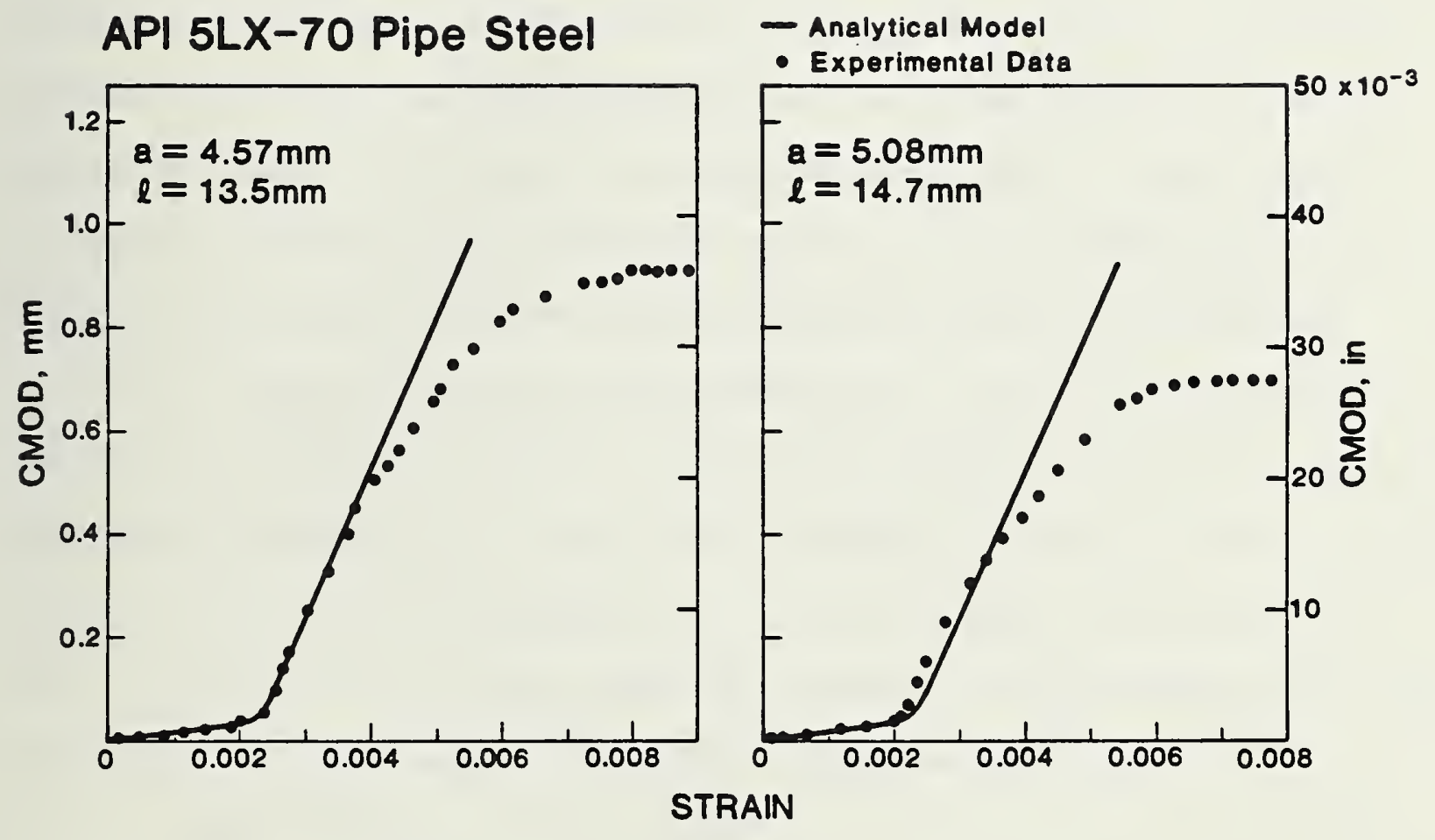

Fig. 15 - CMOD vs. gage-length strain for base-metal specimens that exhibited net-section yielding followed by gross-section yielding. 

bilinear behavior results when the strains that exceed yield only occur in the net section, that is, net-section yielding. Photoelastic observations and strain gage measurements indicate that, for net-section yielding, yielding is confined to slip bands, which extend at approximately $45^{\circ}$ angles from the crack tip to the specimen edges. As shown in Fig. 15, specimens 6 and 8 exhibited similar behavior at low strains, but at high strains the CMOD reached a plateau value, increasing only slightly with strain. The plateau develops when strain hardening elevates the flow strength in the slip bands and strains above yield occur in the gross section, that is, gross-section yielding. Once gross-section yielding occurs, the remote displacements are absorbed along the entire test section and CMOD increases slowly with $\varepsilon_{L}$.

The bilinear relationship between $C M O D$ and $\varepsilon_{L}$ can be modeled at stress levels below net-section yielding using Eq. 56, providing that the appropriate relationship between $\varepsilon_{L}$ and $\sigma$ has been established. At strains above net-section yielding, the slopes of the CMOD-vs. $-\varepsilon_{L}$ curves are equal to the gage length; this occurs because all of the remote displacement is transmitted to the crack tip through the slip bands. Thus, after net-section yielding,

$$
C M O D=C M O D_{N S Y}+L\left(\varepsilon_{L}-\varepsilon_{N S Y}\right)
$$

where $L$ is the gage length and the subscript NSY refers to the value of the quantity at the onset of net-section yielding.

A relationship between $\varepsilon_{L}$ and $\sigma$ is necessary to model CMOD vs. $\varepsilon_{L}$ at stresses below net-section yielding and to compute $C_{M O D}$ NSY and $\varepsilon_{\text {NSY }}$ for Eq. 57. The $\varepsilon_{L}$ is the sum of the elastic strain, $\sigma / E$, plus the increment of $\varepsilon_{L}$ due to the presence of the crack. The remote displacement, $\Delta^{\prime}$, in a center-cracked panel is given by

$$
\Delta^{\prime}=(2 \sigma l / E)[V(\ell / W)]
$$



where $V(\ell / W)$ is given by Tada, Paris, and Irwin. ${ }^{34}$ For a surface crack, Irwin's equivalent through-thickness crack concept ${ }^{18}$ can be used to modify Eq. 58; that is, $\sigma$ is reduced by the closing stress, $\sigma_{C}$, caused by the uncracked ligament,

$$
\sigma_{c}=\bar{\sigma}(1-a / t)
$$

To account for the crack-tip plasticity, the effective crack length, $\ell_{\text {eff }}$, which includes the plastic-zone size correction, $r_{y}$, is used in place of थ. The resultant expression becomes

$$
\varepsilon_{L}=\sigma / E+\left[2\left(\sigma-\sigma_{c}\right) / E\right]\left[e_{e f f} / L\right]\left[V\left(e_{e f f} / W\right)\right]
$$

Given Eqs. 56a, 56b, and 60, it is possible to calculate CMOD vs. $\varepsilon_{L}$ for the net-section yielding case. For stresses below and equal to net-section yield stress, Eq. 56 is used to calculate CMOD and $\sigma$ is converted to $\varepsilon_{L}$ using Eq. 60. For stresses abcve net-section yield stress, Eq. 57 is used. Comparison of the experimental and analytical results of CMOD vs. $\varepsilon_{L}$ shown in Figs. 14 and 15 indicates good agreement.

For specimens 6 and 8 , net-section yielding is followed by gross-section yielding at high strains. The transition from net-section yielding to grosssection yielding, and CMOD vs. $\varepsilon_{L}$ for gross-section yielding have not been modeled. In the present study, the transition occurred at a ratio of crack area to cross-sectional area of $5 \%$. The area ratio at which the transition occurs should be higher for materials with more strain hardening.

Weld Metal Results As shown in Table 2, four automatic-weld specimens and four manual-weld specimens were tested. As shown in Table 3, the yield and ultimate strengths of the welds were significantly different for both types of weld and for the base metal. These differences were far less in transverse-weld tensile tests on 76-mm (3-in) wide, full-thickness tensile panels with a $305-\mathrm{mm}(12-$ in) gage length. The stress-strain curves obtained 

using strain gages mounted in the center of the weld at midthickness on both edges are shown in Fig. 16. For the CMOD-vs.- $\sigma$ model, only the flow stress values are important: $596 \mathrm{MPa}\left(86.5 \times 10^{3} \mathrm{psi}\right)$ for the automatic weld and $557 \mathrm{MPa}\left(80.8 \times 10^{3} \mathrm{psi}\right)$ for the manual weld. However, for CMOD-vs. $-\varepsilon$ modeling using the two-tensile-bar model (discussed below), the shape of the stress-strain curve is also important.

The experimental results of CMOD vs. $\sigma$ for the automatic and manual welds are compared with the analytical predictions in Figs. 17 and 18. The general behavior trends observed in the experiment are reasonably modeled; however, the agreement between experiment and analys is is not as good as it was for the base-metal tests. The correlations are best at low stresses, but the CMOD is underestimated (specimens $A 2$ and $M 4$ are exceptions) for stresses between the stress required for 1 igament yielding and the stress required for net-section yielding. At still higher stresses, CMOD is overestimated. To model the observed behavior with Eq. 56b, it would be necessary to use a variable flow stress. This option is physically sensible, because the stress-strain curves for the welds exhibit more work hardening than the base-metal curves. The quality of the correlations is also influenced by an increase in experimental uncertainty for testing the welded specimens over that encountered in base-metal tests. The principal sources of error are: 1) bending caused by distortion, misalignment, and the curved test section, 2) the presence of residual stresses in the welded specimen, and 3) thickness variations caused by hand grinding the crown and root of the weld.

The experimental results of CMOD vs. $\varepsilon_{L}$ for the automatic and manual welds are shown in Figs. 19 and 20. The two characteristic trends observed in the base-metal tests, a bilinear curve for net-section yielding and a plateau when gross-section yielding occurs, are not apparent in the weld-metal 
Table 3 - Summary of Weld Metal Tensile Properties

\begin{tabular}{|c|c|c|c|c|c|}
\hline $\begin{array}{l}\text { Weld } \\
\text { Type }\end{array}$ & $\begin{array}{l}\text { Test } \\
\text { Temperature, } \\
{ }^{\circ} \mathrm{C}\end{array}$ & $\begin{array}{l}\text { Ultimate } \\
\text { Tensile } \\
\text { Strength, } \\
\text { MPa }\end{array}$ & $\begin{array}{c}\text { Yield } \\
\text { Strength } \\
0.2 \% \text { Offset, } \\
\mathrm{MPa}\end{array}$ & $\begin{array}{l}\text { Percent } \\
\text { Elonga- } \\
\text { tion in } \\
25 \mathrm{~mm}\end{array}$ & $\begin{array}{l}\text { Percent } \\
\text { Reduction } \\
\text { in Area }\end{array}$ \\
\hline Automatic & +24 & 549 & 491 & 26 & 69 \\
\hline Automatic & +24 & 820 & 735 & $15 *$ & $31 *$ \\
\hline \multirow[t]{2}{*}{ Automatic } & +24 & 800 & 715 & 21 & 66 \\
\hline & Average & 810 & 725 & 21 & 66 \\
\hline Manual & +24 & 560 & $470^{+}$ & 28 & 68 \\
\hline \multirow[t]{2}{*}{ Manual } & +24 & 550 & $460^{+}$ & 29 & 64 \\
\hline & Average & 555 & 465 & $28 \frac{1}{2}$ & 66 \\
\hline Automatic & -101 & 955 & 820 & $\neq$ & 61 \\
\hline \multirow[t]{2}{*}{ Automatic } & -101 & 910 & 790 & 27 & 63 \\
\hline & Average & 930 & 805 & 27 & 62 \\
\hline Manual & -101 & 665 & $58 C+$ & 30 & 65 \\
\hline \multirow[t]{2}{*}{ Manual } & -101 & 675 & $560+$ & $21 *$ & $30 \star$ \\
\hline & Average & 670 & 570 & 30 & 65 \\
\hline
\end{tabular}

*Slant fracture; other specimens exhibited cup-cone fracture.

tyield point observed; other specimens did not exhibit a yield point. $\neq$ Gage marks not visible on specimen after test.

psi $\times 10^{3}=\mathrm{MPa} / 6.894$ 


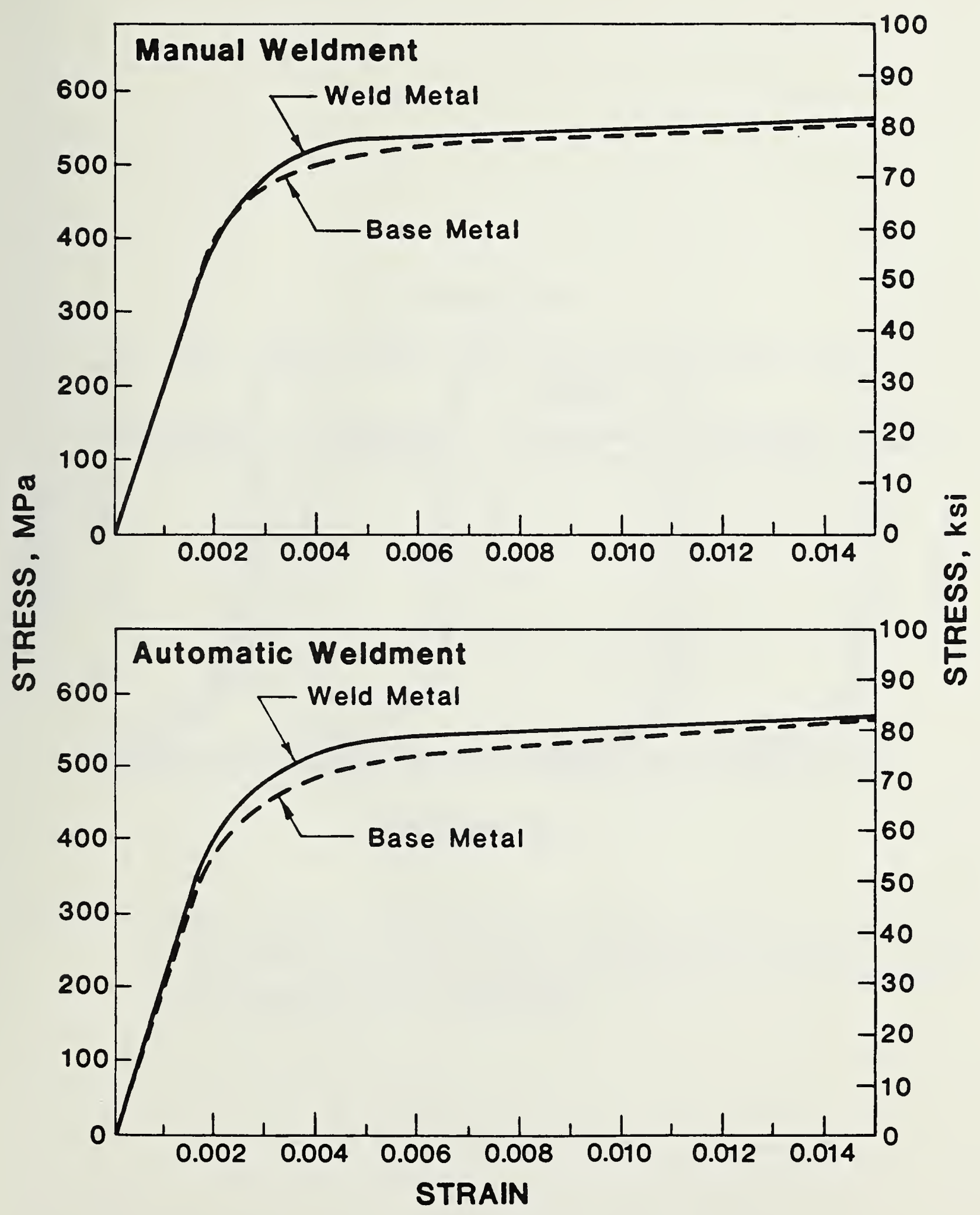

Fig. 16 - Stress-strain curves of automatic and manual weldments, as determined in transverse weld tensile tests. 



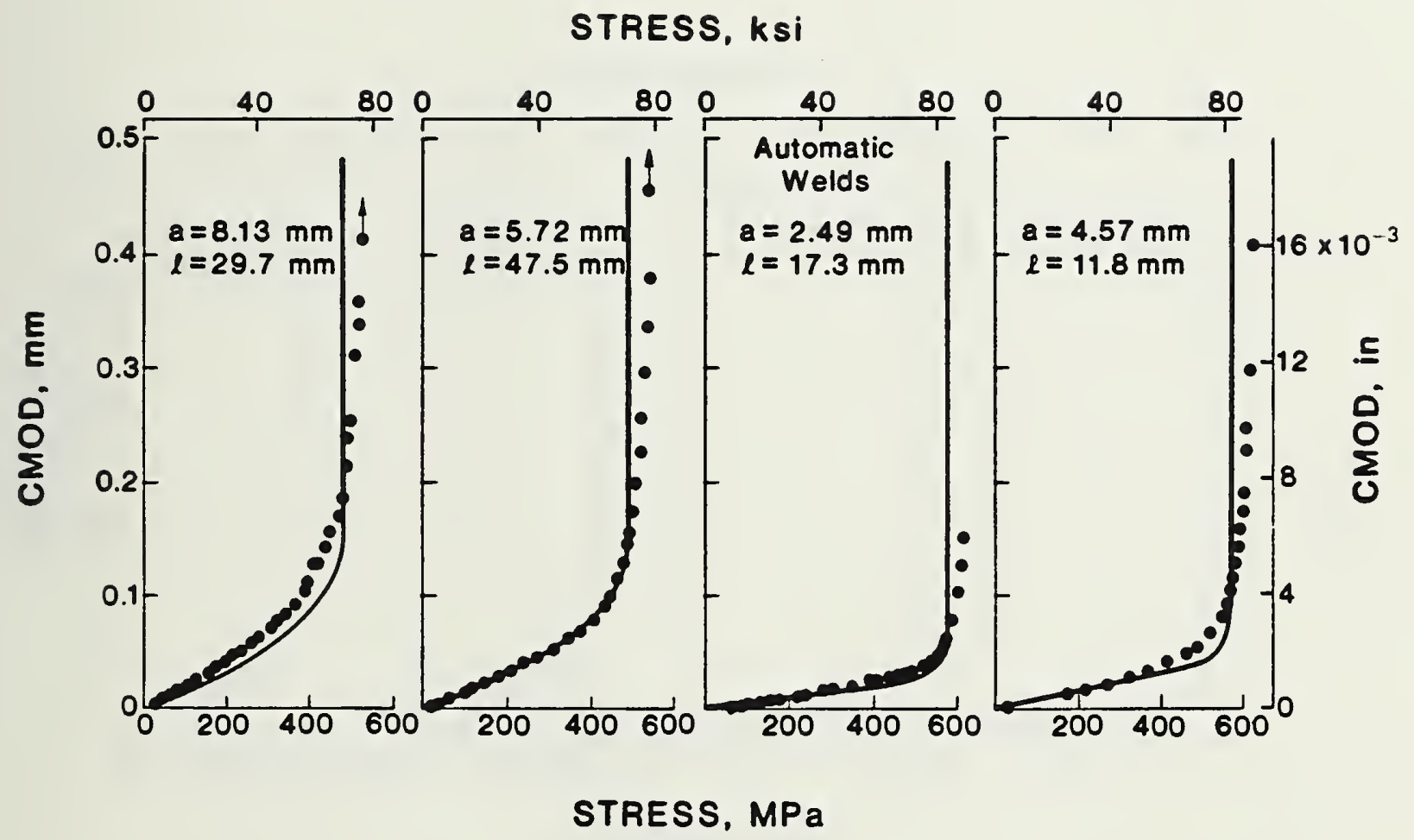

Fig. 17 - CMOD vs. nominal stress for automatic-weld specimens. Symbols are experimental data and solid lines are analytical predictions based on the modified critical-COD model. 

STRESS, ksi

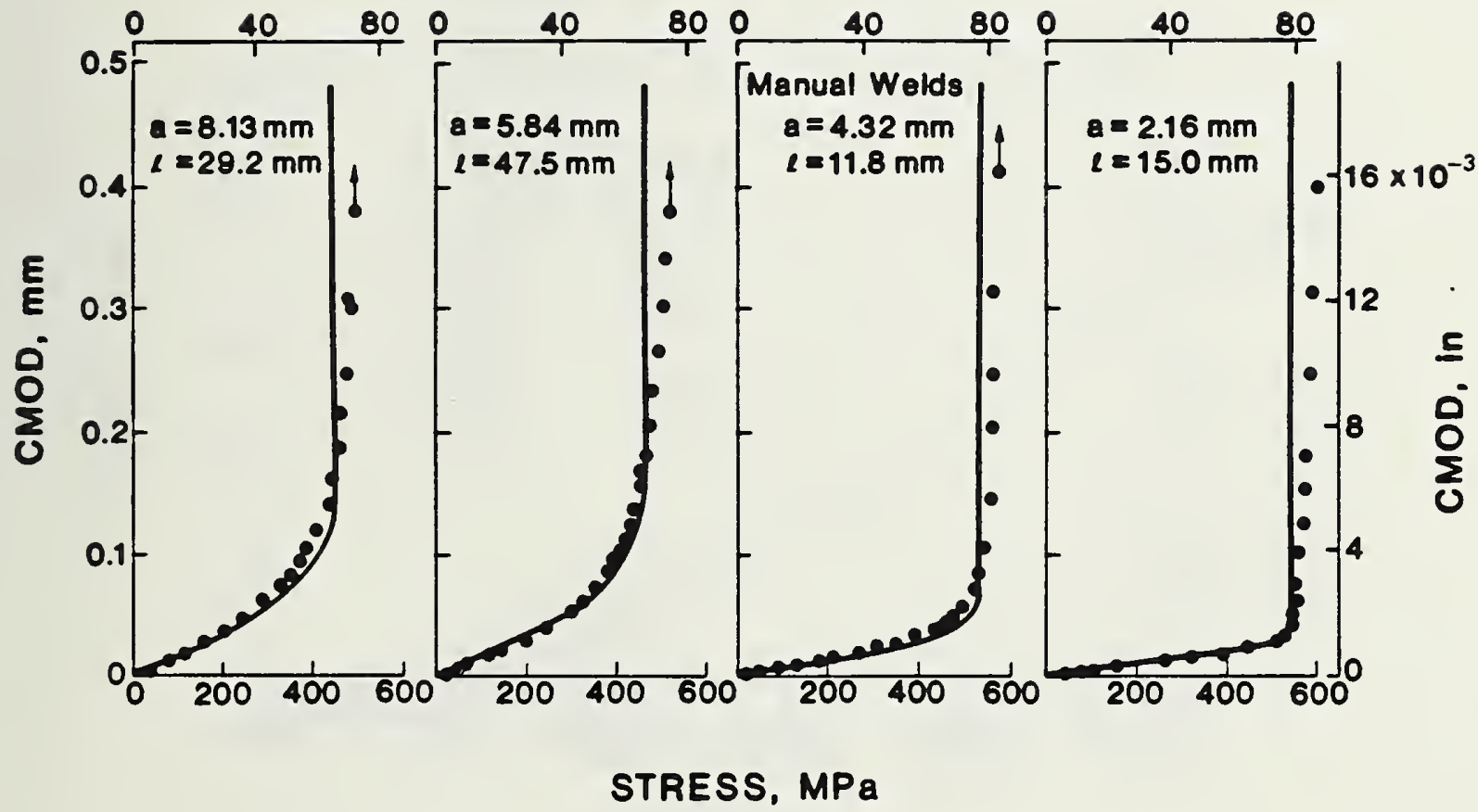

Fig. 18 - CMOD vs. nominal stress for manual-weld specimens. Symbols are experimental data, and solid lines are analytical prediction based on the modified critical-COD model. 



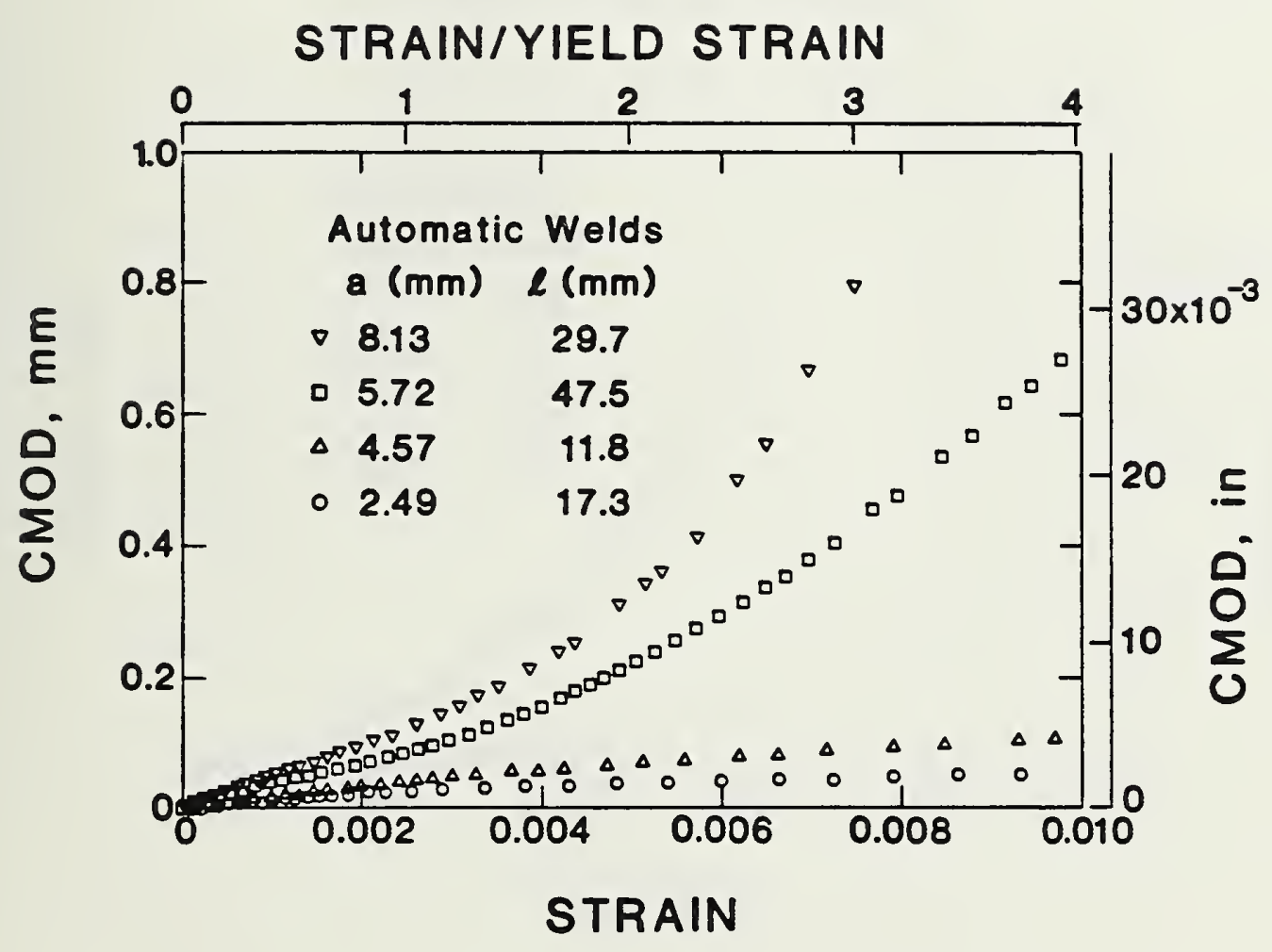

Fig. 19 - CMOD vs. gage-length strain for automatic-weld specimens. 



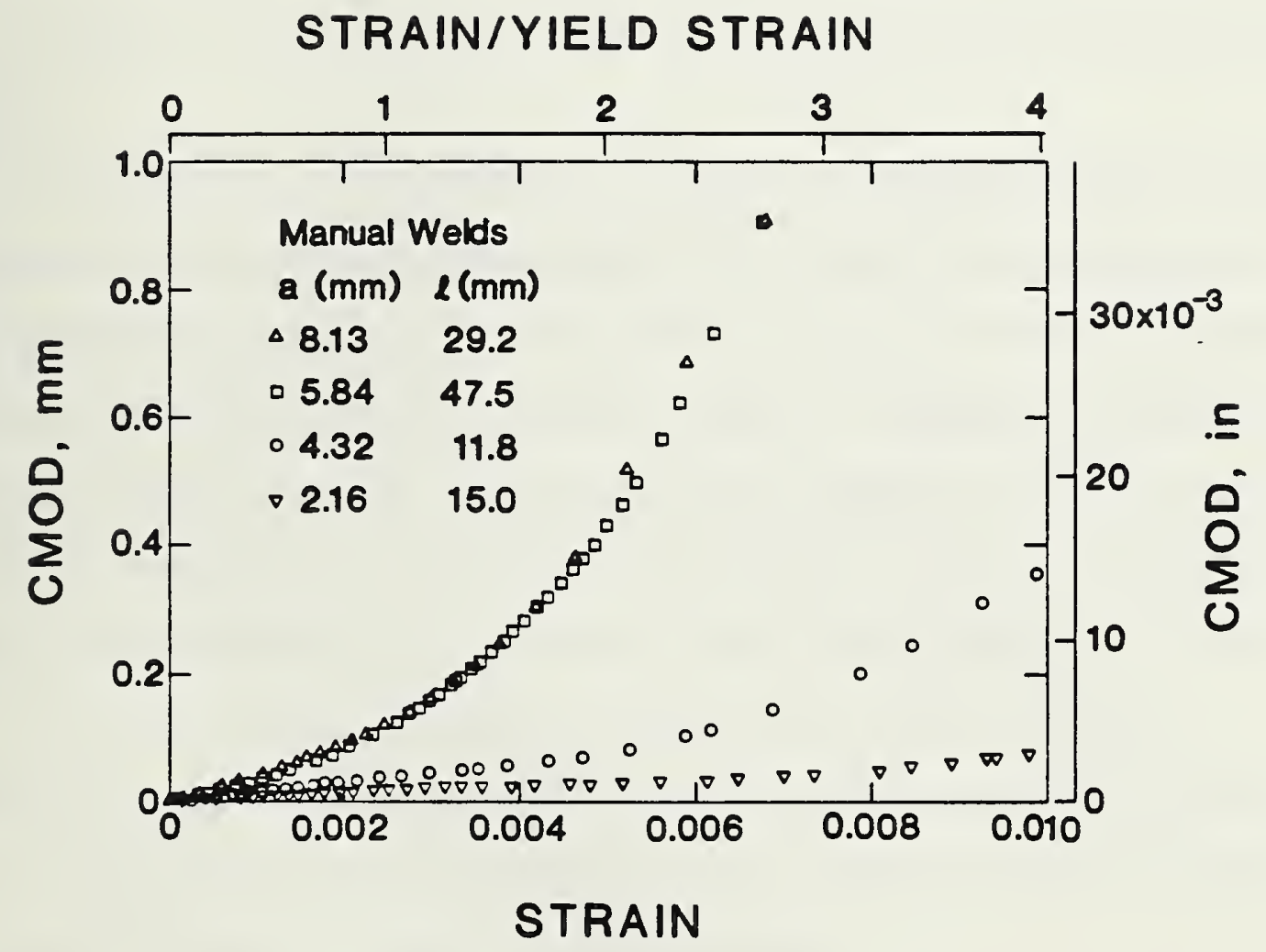

Fig. 20 - CMOD vs. gage length strain for manual-weld specimens. 

results; however, the net-section yielding and gross-section yielding designations are still appropriate. The CMOD-vs. $-\varepsilon_{L}$ curves for net-section yielding behavior in welded specimens are more parabolic than bilinear; the four specimens with the largest cracks ( $A 1, A 2, M 1, M 2)$ exhibited net-section yielding. The CMOD-vs. $-\varepsilon_{L}$ curves for gross-section yielding behavior in welded specimens show a very small increase in CMOD with $\varepsilon_{L}$; the four specimens with the smallest cracks ( $33, A 4, M 3, M 4)$ exhibited gross-section yielding.

The differences in the CMOD-vs. $-\varepsilon_{L}$ curves for the base metal and the welds can be explained by consideration of the higher yield strength and increased work-hardening rates in the weld. For small cracks, the base metal starts yielding while the weld is still elastic. Subsequent increases in stress applied to the weld depend on the work hardening in the base metal. Simply, the base metal stretches and the weld does not, and consequently CMOD increases slowly with strain. For the larger cracks (specimens Al, A2, M1, M2), $16 \%$ to $20 \%$ of the cross-sectional area of the weld is cracked. Consequently, the crack plane yields first and net-section yielding can occur. Even at the $20 \%$ crack-to-cross-sectional-area ratio, the weld work-hardens sufficiently to cause yielding in the base metal.

For the net-section yielding case, the apportionment of strain in the base metal and the weld can be modeled with the two-tensile-bar analogy shown in Fig. 21.

For a given value of remote imposed displacement, $\Delta^{\prime}=\varepsilon_{L} L$, at the ends of two tensile bars in series, the compatibility condition is

$$
\varepsilon_{1} L_{1}+\varepsilon_{2} L_{2}=\varepsilon_{L} L
$$

where $L$ is the gage length, $L_{2}$ is the width of the weld, and $L_{1}=L-L_{2}$ (see Fig. 21). The force equilibrium is 


$$
\left[\sigma_{1}\left(\varepsilon_{1}\right)\right] \cdot A_{1}=\left[\sigma_{2}\left(\varepsilon_{2}\right)\right] \cdot A_{2}
$$

where $\sigma_{1}\left(\varepsilon_{1}\right)$ and $\sigma_{2}\left(\varepsilon_{2}\right)$ are the stress-strain curves for materials 1 and 2 , and $A_{1}$ is the gross-section area and $A_{2}$ is the net-section area in the plane of the crack (see Fig. 21). Since the stress-strain curves are nonlinear, Eqs. 61 and 62 are solved numerically for $\varepsilon_{1}$ and $\varepsilon_{2}$. Assuming all the strain in the weld, $\varepsilon_{2}$, goes into the crack, then $C M O D=\varepsilon_{2} L_{2}$.

A comparison of the measured CMOD-vs. $-\varepsilon_{L}$ data with the values calculated using the two-tensile-bar analogy is shown in Fig. 22. The results indicate that strain partitioning due to the difference in the stress-strain curves of the base metal and the weld is a usable physical model of weldment behavior.

\subsubsection{Summary and Conclusions}

Crack-mouth-opening displacements (CMODs) of surface cracks have been measured as functions of stress and strain in tensile panels of API $5 L X-70$ steel plates and welded pipe segments. The conclusions drawn from the investigation are:

1. For CMOD vs. stress, the flat plate yielded-ligament model with through-thickness plasticity correction agrees well with the experimental results for the base metal and the welds. 



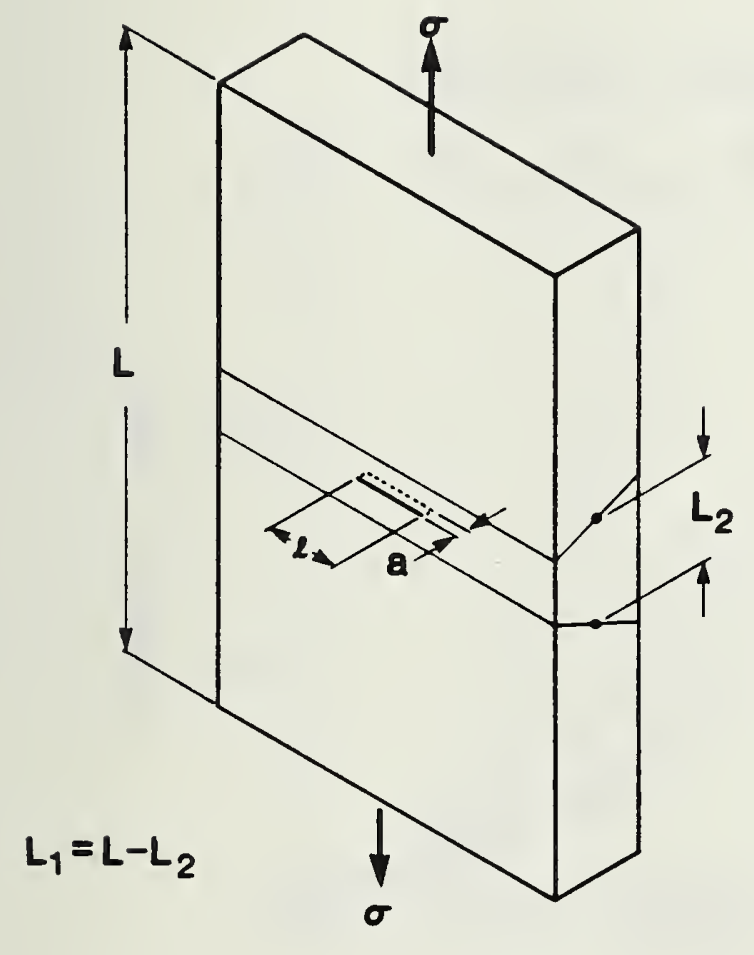

(a)

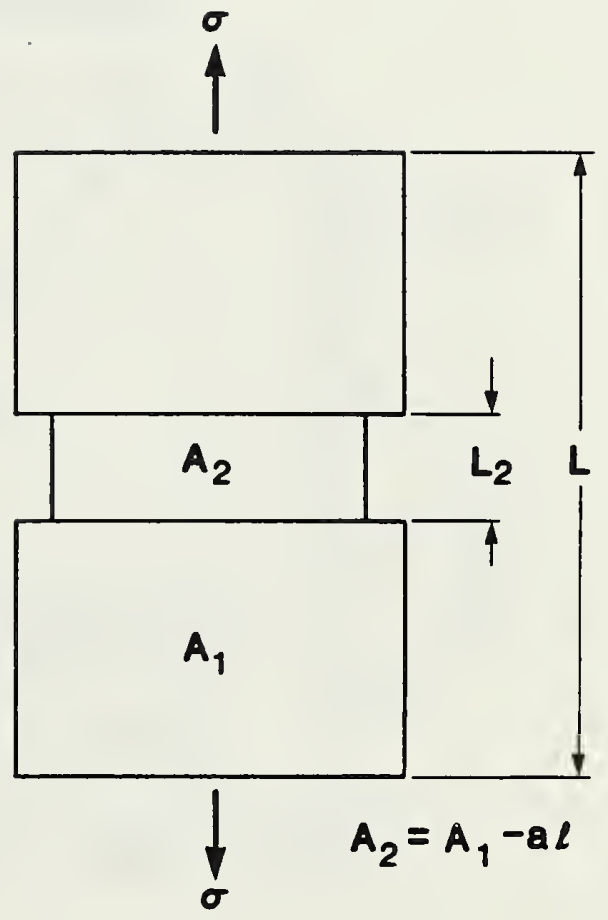

(b)

Fig. 21 - Two-tensile-bar model schematics: (a) specimen and (b) analogy. 



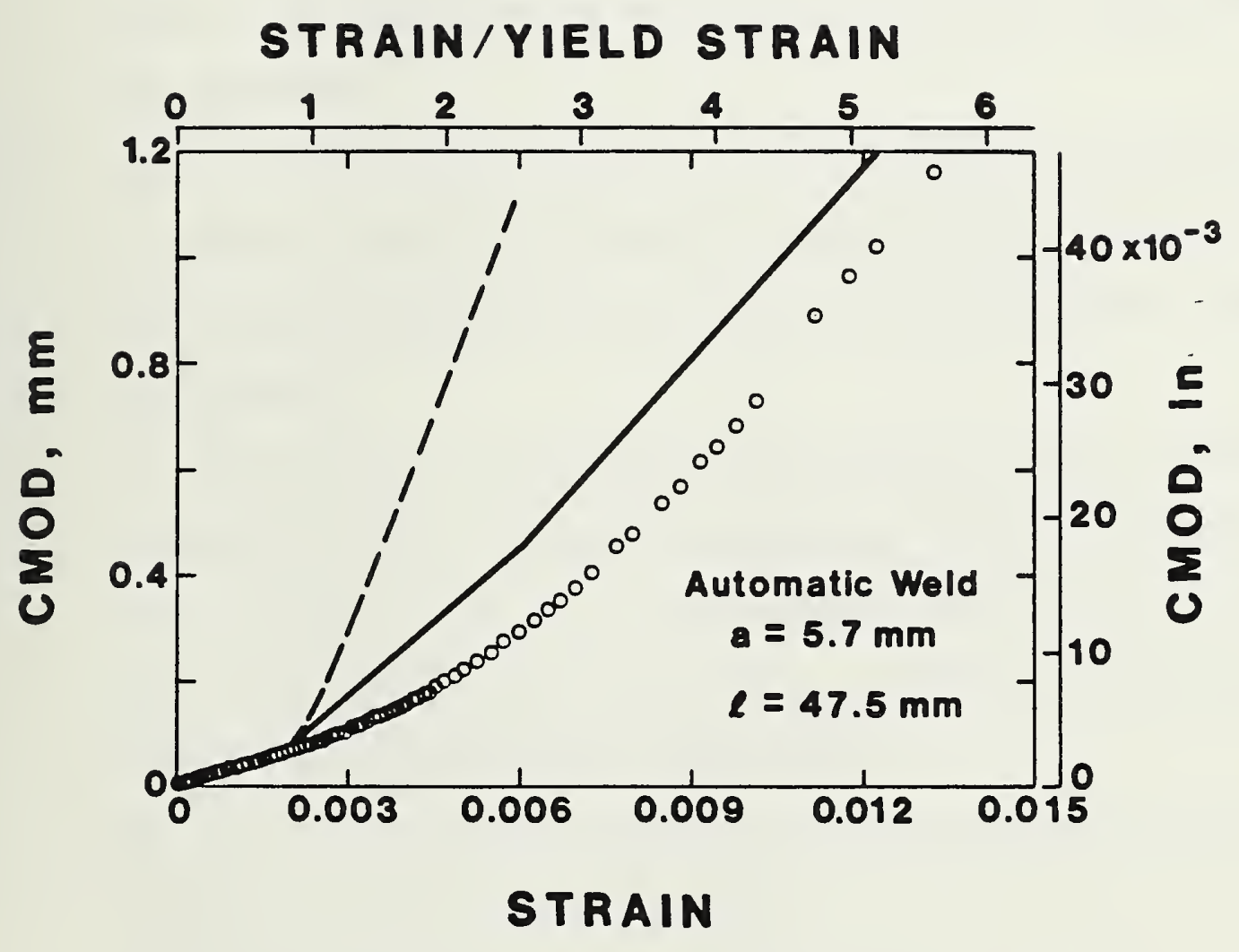

Fig. 22 - CMOD vs. gage-length strain for an automatic weld-metal specimen exhibiting net-section yielding. Symbols are experimental data; solid lines are prediction from twotensile-bar model; dashed lines are predictions from simple net-section yielding models. 

2. For strains above net-section yielding, the relationship between CMOD and strain is complex, depending upon the specimen type (plate or weld) and crack size.

a. For large cracks in base metal, all of the remote displacement goes into the crack tip through the slip bands extending from the crack tip to the plate edges at $45^{\circ}$, that is, net-section yielding. This behavior was modeled and predictions from the model agree well with the experiment.

b. For cracks with crack area less than $5 \%$ of the cross-sectional area, net-section yielding is followed by gross-section yielding in which the remote displacement is distributed evenly along the length of the specimen.

c. For large cracks in steel welds, net-section yielding is observed. The observed CMOD is lower than predicted by the model because the yield strength of the weld is higher than that of the base metal. A model, based on strain partitioning, accounts for the observed behavior and provides better predictions.

d. For small cracks in steel welds, gross-section yielding is observed throughout the strain range studied.

\subsection{Model Verification Tests on Large-Diameter Pipes}

An extensive series of full-scale experiments on pipes were conducted by the Welding Institute of Canada. ${ }^{35}$ The pipe was API $5 L X-70$ steel, $914 \mathrm{~mm}$ (36-in) in diameter and $11.5-\mathrm{mm}(0.45-$ in) thick. Pipes containing surface cracks were tested to failure. The surface cracks varied in length from $58 \mathrm{~mm}$ $(2.28 \mathrm{in})$ to $300 \mathrm{~mm}(11.8 \cdot \mathrm{in})$ and in depth from $3 \mathrm{~mm}$ (0.118 in) to $10.2 \mathrm{~mm}$ $(0.40 \cdot \mathrm{in})$. The tests included in this report are summarized in Table 4 . The tests were conducted at temperatures ranging from $-90^{\circ} \mathrm{C}$ to $20^{\circ} \mathrm{C}$. Twelve-meter-long pipe sections were loaded in bending by 20 hydraulic 

Table 4 - Test Matrix for Large-Scale Tests Conducted at the Welding Institute of Canada

\begin{tabular}{lcccc}
$\begin{array}{c}\text { Test } \\
\text { Numbert }\end{array}$ & $\begin{array}{c}\text { Crack } \\
\text { Length, mm }\end{array}$ & $\begin{array}{c}\text { Crack Depth, } \\
\mathrm{mm}\end{array}$ & $\begin{array}{c}\text { Wall } \\
\text { Thickness, mm }\end{array}$ & $\begin{array}{c}\text { Temperature, } \\
{ }^{\circ} \mathrm{C}\end{array}$ \\
\hline $2 B$ & 60.4 & 5.6 & 11.3 & 20 \\
4 & 63.5 & 5.9 & 11.2 & 20 \\
6 & 68.6 & 7.8 & 11.1 & -90 \\
7 & 61.0 & 5.4 & 11.5 & -90 \\
8 & 76.4 & 10.1 & 12.0 & -90 \\
9 & 81.7 & 8.8 & 11.6 & -50 \\
11 & 79.0 & 9.3 & 11.3 & -50 \\
13 & 59.6 & 6.2 & 10.8 & -50 \\
14 & 64.8 & 5.5 & 11.0 & -5 \\
15 & 60.4 & 5.5 & 11.2 & -5 \\
18 & 300.0 & 3.0 & 11.1 & 20 \\
19 & 300.0 & 3.0 & 11.1 & 20 \\
\hline
\end{tabular}

* Welding Institute of Canada identification numbers.

$1 \mathrm{~mm}-0.0394 \mathrm{in}$. 

jacks at 610-mm (24-in) spacing. A clip-on gage was used to measure CMOD, and the jack pressure was recorded. Strain gages were placed at various locations to determine the strain distribution in the pipes. In the low-temperature tests, the temperature distribution was measured with thermocouples. The surface cracks were located in the girth weld or in the base metal. Manual arc welding was used. The flow strength of the welds was approximately 590 $\mathrm{MPa}$, which slightly undermatched the base metal.

On the basis of beam theory, the maximum membrane stress induced at the cracked section was calculated to be

$$
\sigma=30.6 p
$$

where $p$ is the applied jack pressure. The bending moment at the crack section was

$$
M(\text { in } N \cdot m)=9.96 \times 10^{6} p
$$

Eqs. 63 and 64 were used in the present work to permit analytical computation of CMOD as a function of jack pressure.

\subsubsection{Results and Discussion}

Experimental results for CMOD as a function of jack pressure are shown in Figs. 23 through 27 . The tests on plain pipe, conducted at $20^{\circ} \mathrm{C}$, are plotted in Fig. 23. The remainder of the tests were on pipe sections containing girth welds. The tests in Fig. 24 were conducted at $-90^{\circ} \mathrm{C}$, the tests in Fig. 25 were conducted at approximately $-50^{\circ} \mathrm{C}$, and those in Fig. 26 at $-5^{\circ} \mathrm{C}$. The tests in Fig. 27 involved relatively long surface cracks and were conducted at $20^{\circ} \mathrm{C}$. The general form of the results is the same for each test: a linear relation between CMOD and $\mathrm{p}$ prevailed at low pressures, followed by a nonlinear region as yielding occurred in the vicinity of the crack, and finally a vertical asymptote as the pressure causing collapse at the cracked section was approached. 



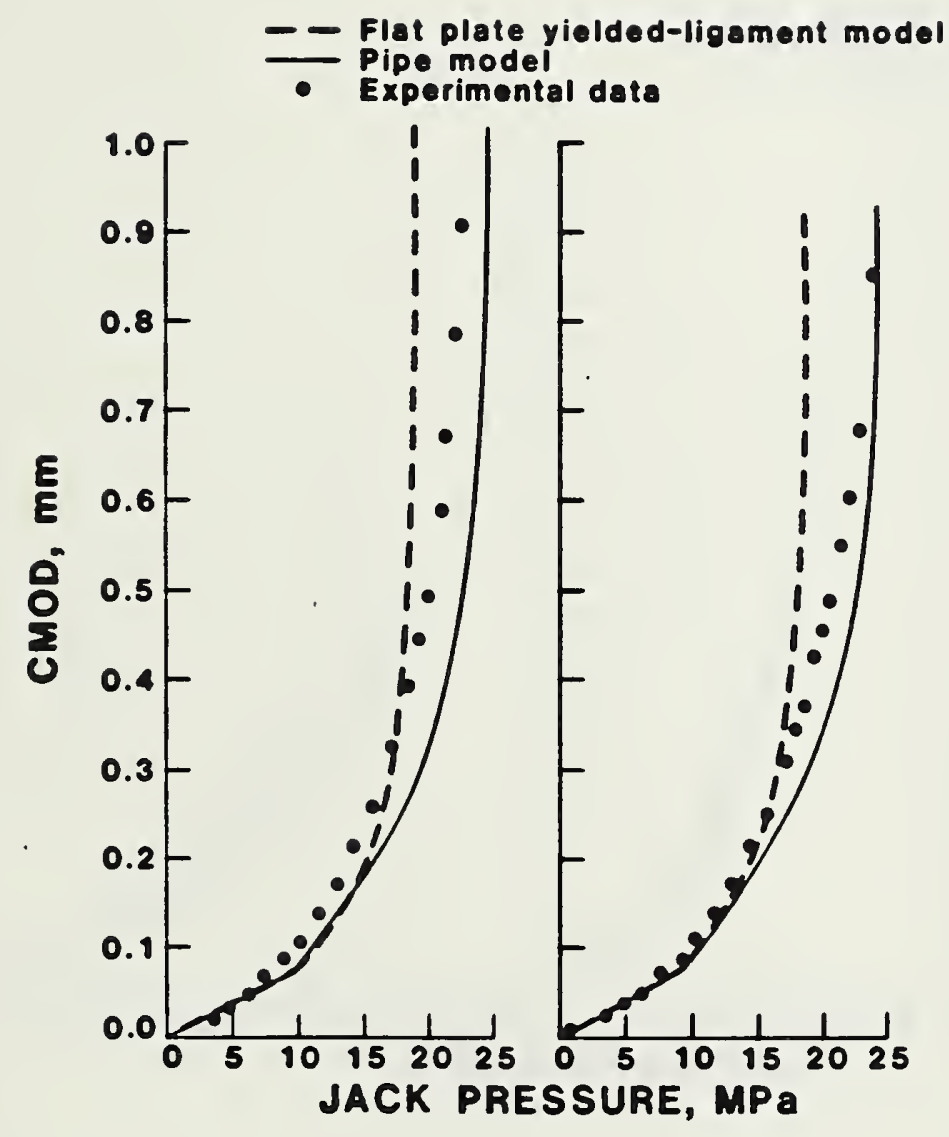

Fig. 23 - Comparison of model predictions and experimental results for CMOD vs. jack pressure. Tests were conducted at room temperature. 



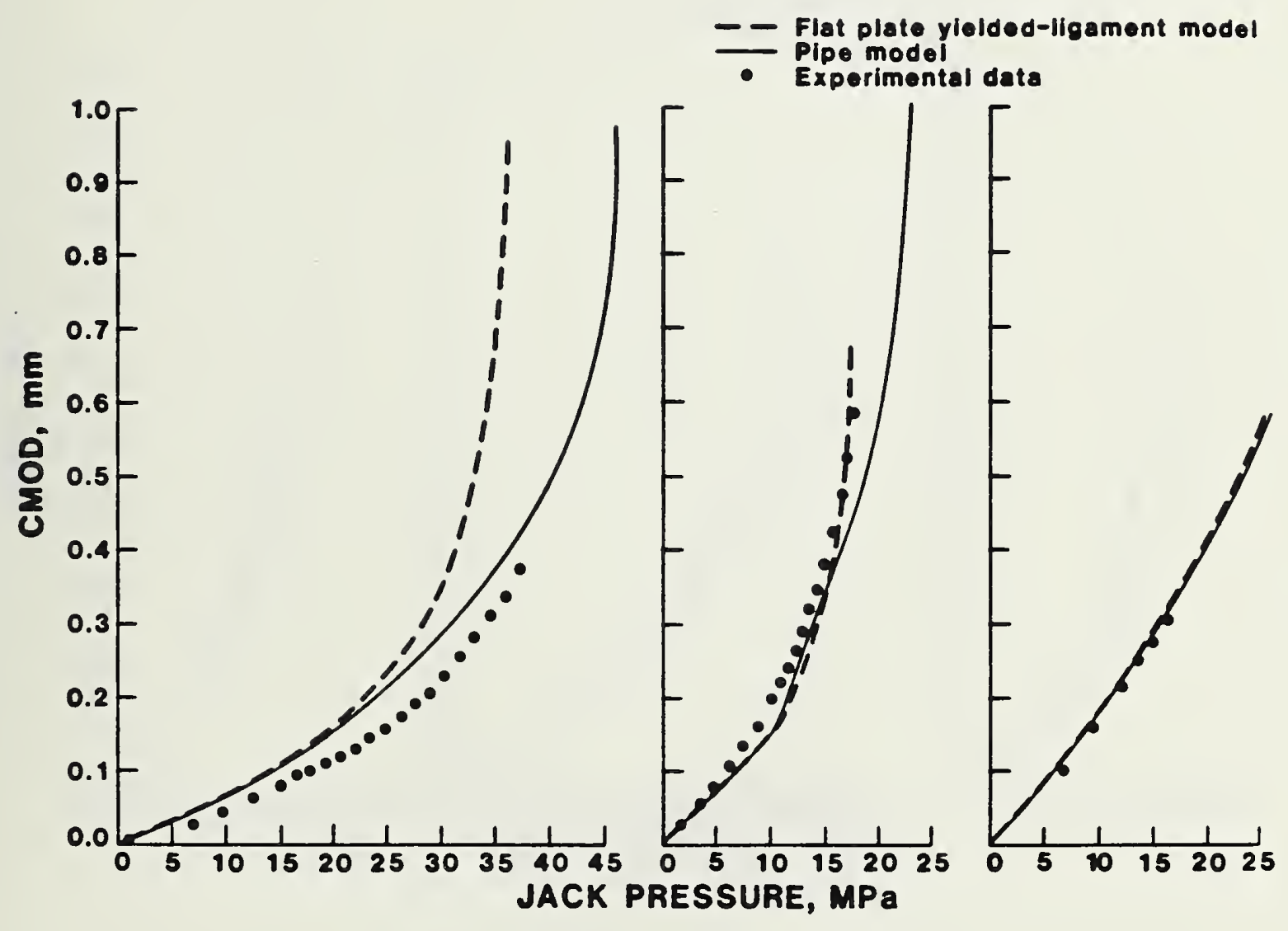

Fig. 24 - Comparison of model predictions and experimental results for CMOD vs. jack pressure. Tests were conducted at $-90^{\circ} \mathrm{C}$. 



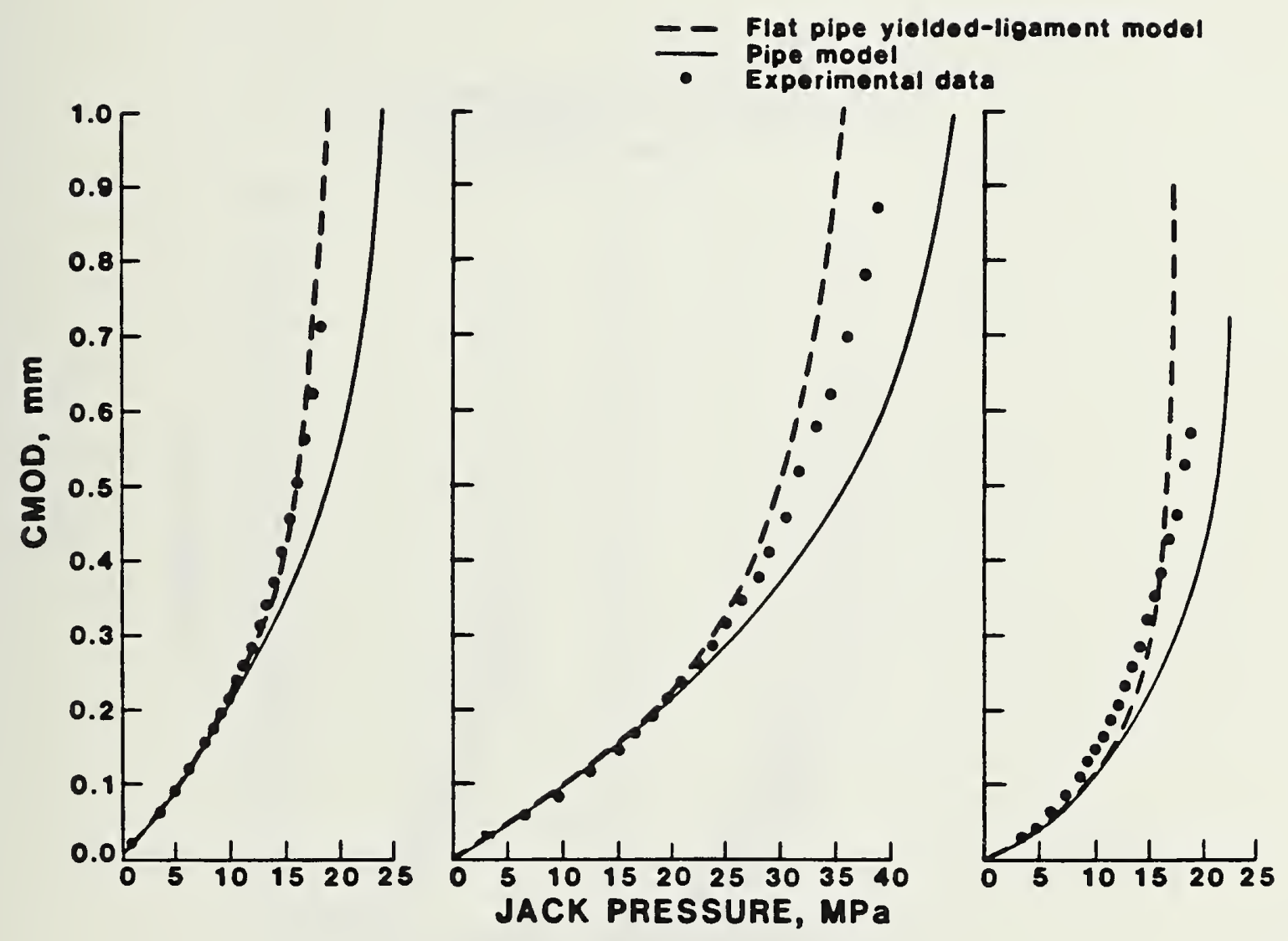

Fig. 25 - Comparison of model predictions and experimental results for CMOD vs. jack pressure. Tests were conducted at $-50^{\circ} \mathrm{C}$. 



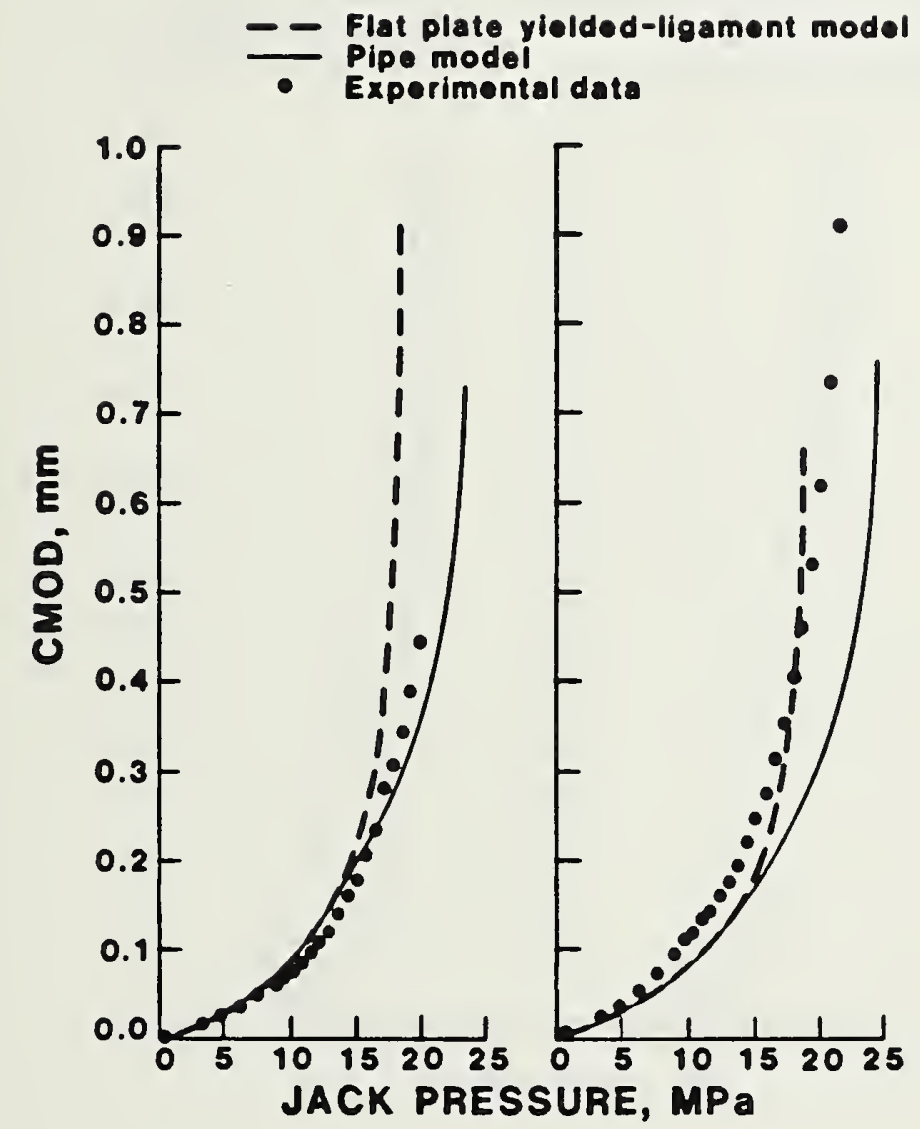

Fig. 26 - Comparison of model predictions and experimental results for CMOD vs. jack pressure. Tests were conducted at $-5^{\circ} \mathrm{C}$. 

- Exporimental data Flat plate yieldedligament model

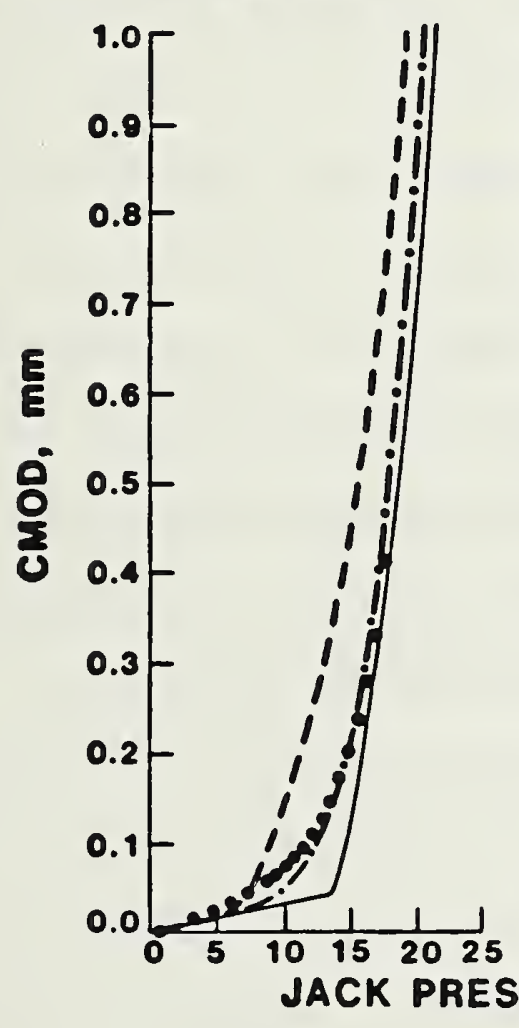

\section{Line-epring model with plestle zone}

\section{correction in depth direction:}

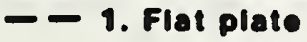

- - 2. With shell correction

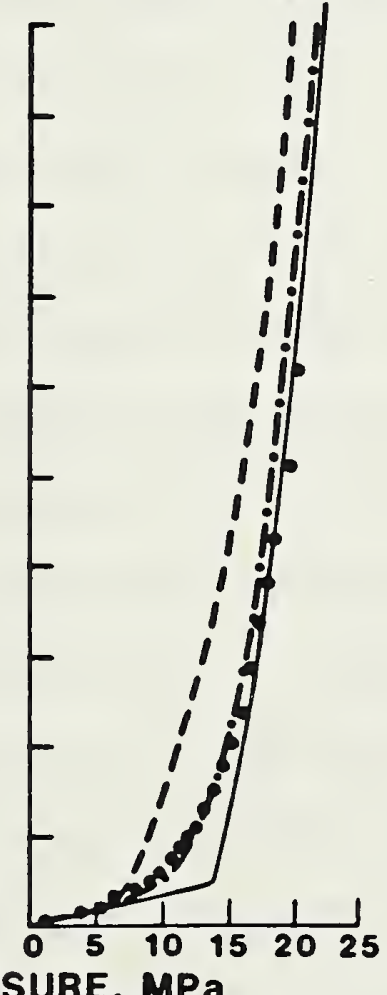

Fig. 27 - Comparison of model predictions and experimental results for CMOD vs. jack pressure. Tests were conducted at room temperature. 

Analytical predictions of CMOD as a function of jack pressure are compared with the experimental results described above. In the discussion, the original version of the yielded-ligament model, Section 2.2.3, will be referred to as the flat-plate yielded-ligament model. The modified version, which accounts for the plastic collapse mechanism of the pipe, discussed previously in Section 2.2.4, will be referred to as the pipe yielded-ligament model.

Typically the flat-plate yielded-ligament model agrees fairly well with observed values of CMOD until higher jack pressures, at which the model predictions are higher than the observed values. However, the discrepancy does not typically occur until higher values of CMOD have been reached. For example, if it is assumed that the highest CTOD fracture toughness value of pipeline weld metal that might be used in a fitness-for-service assessment is $0.25 \mathrm{~mm}(0.010-i n)$, then typical surface-flaw geometries such a toughness value (i.e., CTOD value) would be equivalent to a CMOD of approximately $0.28 \mathrm{~mm}(0.011-i n)$. The model predictions of CMOD agree favorably with the experimental results until values of CMOD higher than this are reached. Thus the flat-plate yielded-ligament model is, in general, sufficiently accurate over the range of interest. An exception was test 6, Fig. 24, in which the model predictions were conservative (by a maximum of approximately $40 \%$ ) at lower pressures. The reason for this discrepancy has not been explained.

The conservatism of the flat-plate yielded-ligament model at higher pressure values appears to be attributable to incorrect modeling of the plastic deformation in the pipe. The model predictions are vertically asymptotic at a value of pressure corresponding to the tensile net-section yield stress, $\sigma_{N S Y}$, given by Eq. 38. The model asymptote is typically to the left of the data asymptote. The modified version of the model, Eq. 54, called the pipe yielded-ligament model, assumes a more realistic plastic collapse 

mechanism and seems to agree more favorably with the experimental results at higher pressure levels. Exceptions are tests 7, 9, 13, and 15 in which the predictions of the flat-plate yielded-ligament model agree more closely. This observed behavior is curious because it is reasonable to expect the data to adhere more closely to the modified model. Two possible explanations are inelastic buckling and tearing. Buckling behavior was observed in several of the tests. Inelastic ovalization during buckling would significantly lower the plastic collapse pressure. Some ductile tearing was also reported in some of the tests. The model predictions based on the original crack length would, therefore, be too low.

In the two tests shown in Fig. 27 the crack length was relatively long $(300 \mathrm{~mm}, 11.8-i \mathrm{n})$. A straightforward application of the flat-plate yielded-ligament model to the calculation of CMOD in this case gives poor results. It is seen that the data become nonlinear much sooner than the model predicts. This was attributed to the importance of yielding through the ligament depth in this case, because the center of a long surface crack receives very little support from the material at the sides of the crack. Use of the flat-plate yielded-ligament model to compute the elastic component of CMOD and accounting for through-depth yielding via the plastic-zone size correction presented in Section 2.3 .2 resulted in extremely conservative curves. Upon examining the line-spring calculation more closely, it was found that the largest contribution to CMOD was from the rotation of the crack faces. The increased stiffness of a curved shell against rotation in comparison with the stiffness of a flat plate must, therefore, be accounted for. This was done in the manner described in Section 2.2.4, resulting in the pipe yielded-ligament model with through thickness plasticity correction. Prediction of this model are shown in Fig. 27 , which agree well with the experimental values of CMOD. 


\subsubsection{Summary}

On the basis of experimental results from full-scale tests of pipes in bending, the flat-plate yielded-ligament model was found to give predictions of crack driving force accurate to within experimental scatter or conservative by 1 ess than $20 \%$ over the range of practical interest in a fitness-for-service assessment. At driving force values above this range of practical interest, the model predictions become more conservative. This has been explained by introducing a modification to the model that more properly accounts for the plastic deformation in a pipe, the resulting model was called the pipe yielded-ligament model. Calculations of $\operatorname{COD}$ by the model in its original form are relatively inaccurate for long surface cracks. However, a modification to the model has been proposed that greatly increases its applicability to the long crack case. This final model was named the pipe yielded-ligament model with through-thickness plasticity correction. Because the model is relatively simple to apply, yet predicts crack driving forces that compare well with experimental checks, being accurate or conservative by less than $10 \%$ over the range of practical interest, it is felt to be a useful tool in determining the driving force for surface cracks under elastic-plastic deformation. This is an important ingredient in setting allowable flaw-size standards in pipeline girth welds and other structures.

\subsection{Allowable Flaw-Size Curves}

An allowable flaw-size curve is a plot of flaw depth as a function of flaw length, as shown schematically in Fig. 1. Each point on the curve represents a critical flaw size (length and depth) calculated using an appropriate fracture mechanics model and specified values for applied stress, residual stress, fracture toughness, tensile properties and pipe dimensions. The specified values are conservatively chosen to preclude the possibility of 
achieving critical conditions in service. In this section, the fracture mechanics model developed in this program for the calculation of allowable flaw size curves, the pipe yielded-ligament model with through thickness plasticity correction, is described. Guidelines for the selection of stress levels, pipe and girth-weld material properties, and pipe dimensions for use in the analysis are presented. Finally, a representative allowable flaw-size curve is given, and the influence of selected parameter variations on the position of the curve is shown.

\subsubsection{Fracture Mechanics Model}

The analytical model used to calculate allowable flaw-size curves is the pipe yielded-ligament model wih through thickness plasticity correction. The fracture criterion is the CTOD:

$$
\delta_{C}=C T O D_{E}+C T O D_{L Y}+C T O D_{P . S}
$$

where $\delta_{C}=$ fracture toughness in terms of CTOD

$\mathrm{CTOD}_{E}=$ elastic component of CTOD

$C T O D_{L Y}=1$ igament-yielding component of CTOD

$C T O D_{R S}=$ residual stress component of CTOD

The idea is that fracture occurs when the driving force for fracture, the CTOD terms in Eq. 65 , exceeds the resistance of the material to fracture, $\delta_{C}$. Values for the fracture toughness, applied stress, tensile properties, and pipe geometry are specified on the basis of pipeline operating conditions (e.g., pressure, temperature, imposed displacements) and pipe and weld requirements (e.g., diameter, thickness, strength, toughness). The remaining variables in Eq. 65 are the flaw depth, a, and the flaw length, $\ell$. Using the specified values, Eq. 65 is solved numerically for a as a function of $\ell$ using a Newton-Raphson iteration procedure. The computer program used for these calculations is given in Appendix A. 

The elastic component of CTOD is calculated using the simplified linespring model discussed in 2.2.3. The equations used in the calculations are:

$$
\begin{aligned}
& C^{C T O D_{E}}=K^{2}\left(1-v^{2}\right) / E^{-} \\
& K=t^{\frac{1}{2}}\left(\sigma_{0} g_{t}+m g_{b}\right)
\end{aligned}
$$

where $g_{t}$ and $g_{b}$ are given by:

$$
\begin{aligned}
g_{t}(\xi) & =\pi \xi^{\frac{1}{2}}\left(1.12+6.52 \xi^{2}-12.39 \xi^{4}+89.05 \xi^{6}\right. \\
& -188.61 \xi^{8}+207.39 \xi^{10}-32.05 \xi^{12} \\
g_{b}(\xi) & =\pi \xi^{\frac{1}{2}}\left(1.12-1.89 \xi+18.01 \xi^{2}-87.39 \xi^{3}\right. \\
& \left.+241.39 \xi^{4}-319.94 \xi^{5}+168.01 \xi^{6}\right)
\end{aligned}
$$

$\xi=\left(a+r_{y}\right) / t$ and $\sigma_{0}$ and $m$ are computed using Eqs. 27 through 33. For cracks longer than $96 \mathrm{~mm}(3.78 \mathrm{in})$, curvature must be accounted for in the compliance calculations. The curvature correction enters the line-spring model by replacing Eq. 26 with Eq. 47 and making the associated changes in Eqs. 28 through 33 . The revised equations are $28 c$ to $33 c$, in which the suffix $c$, and the subscript $c$ in the symbols $\alpha_{c}$ and $\beta_{c}$, stand for curvature.

$a_{12} \sigma_{0}+m\left\{a_{22}+\frac{(2 R)^{\frac{1}{2}}\left[12\left(1-v^{2}\right)\right.}{72\left(1-v^{2}\right) t^{\frac{3}{2}}}\right\}^{3 / 4}=0$

$\sigma_{0}=\alpha_{c} \sigma$

$m=-B_{c} \sigma$

$a_{c}=\left\{l /\left[t\left(1-v^{2}\right)\right]\right\}\left\{a_{22}+\frac{(2 R)^{\frac{1}{2}}\left[12\left(1-v^{2}\right)\right]^{3}}{72\left(1-v^{2}\right) t^{\frac{1}{2}}}\right\} / S$

$B_{c}=\left\{l /\left[t\left(1-v^{2}\right)\right]\right\} a_{12} / S=B$

$S=\left\{a_{11}+l /\left[t\left(1-v^{2}\right)\right]\right\}\left\{a_{22}+\frac{(2 R)^{\frac{1}{2}}\left[12\left(1-v^{2}\right)\right]^{3 / 4}}{72\left(1-v^{2}\right) t^{\frac{1}{2}}}\right\}-a_{12}^{2}$ 

In evaluating Eq. 66 , the plastic-zone correction, $r_{y}$, is added to the crack depth. The $r_{y}$ is calculated as in Eq. 17, but using depth and thickness in place of length and width, and inserting the appropriate stress ratio:

$$
\sin (\pi a / 2 t) / \sin \left(\pi\left(a+2 r_{y}\right)\right) / 2 t=\cos \left(\pi \sigma_{0} / 2 \bar{\sigma}\right) \text {. }
$$

The plastic zone size calculation is non-linear and self-consistent; both $(29-c)$ and (69) must be satisfied. The plastic zone enters (29c) through the parameters $a_{i j}$, which are actually functions of the effective crack length, $a+r_{y}$. These parameters are given as functions of a/t in Fig. 5, above, in the context of an elastic treatment of the ligament. As discussed in Section 2.4.1, yielding through the ligament must be considered in the calculations to obtain agreement with experiment. This is accomplished by replacing $a / t$ on the $x$-axis of Fig. 5 with $\left(a+r_{y}\right) / t$. Thus, the ligament behind the crack is treated as partially yielded, and having some plastic zone radius, $r_{y}$, before the applied stress reaches a certain value given by Eq. 34; above this stress, the ligament is fully yielded and CTOD increases according to Eq. 70. Before the ligament is fully yielded, no plasticity corrections to

$\ell$ are made.

The ligament yielding component of CTOD is given by:

$$
\operatorname{CTOD}_{L Y}=\left[2\left(e+2 r_{y}\right) / E\right][\sigma-(1-a / t) \bar{\sigma}]
$$

where $r_{y^{\prime}}$ is the strip-yield plastic zone solution given in Eq. 17.

The residual stress component of CTOD is estimated by assuming that the residual stresses result from weld shrinkage, and thus, are self-equilibrating. In this case, one can assume that yield-point stresses act over a distance comparable to the weld size or pipe thickness. If so, a displacement of $\sigma_{y} t / E$ will relieve the residual stress. An approximation of the contribution of such a residual stress to the driving force for fracture is to simply assume that the estimated displacement is all converted to CTOD:

$$
\mathrm{CTOD}_{\mathrm{RS}}=\sigma_{y} \mathrm{t} / \mathrm{E}
$$



Evaluating Eq. 71 using the ANGTS pipeline properties listed above, the result is a $C T O D_{R S}$ of $0.038 \mathrm{~mm}(0.0015 \mathrm{in})$.

Calculations of allowable flaw-size curves using the equations listed above are most conveniently done by computer. Several aids to study of the details of the calculation are given in Appendix A. The computer program, used to compute the allowable flaw-size curves for this study, is listed. A table gives values of $a_{11}, a_{12}, a_{22}, g_{j}$, and $g_{b}$ as functions of effective crack depth. Figure Al shows the behavior of Eq. 69, which relates $r_{y}$ to $\sigma_{0}$. The self-consistent plastic zone calculation is accomplished by finding the plastic zone size for which both (69) and $(29-c)$ are satisfied. As an aid to visualization of this solution process, Fig. Al shows $(29-c)$ and (69) plotted as curves on the same graph. The calculated $r_{y}$ value occurs where the two curves intersect. The advantage of doing these calculations by computer is clear. At each flaw length, several trial depths must be checked before the critical depth is found. For each trial depth, the plastic zone size must be calculated anew. Also listed in Appendix $A$ as an example is a hand calculation of critical flaw depth for a given flaw length. Computer results were used, instead of guesses, to reach the final step in both the plastic zone and critical depth iterations. Although the allowable flaw-size calculations discussed here are complex, the actual computer costs are insignificant compared to the manpower costs.

\subsubsection{Parameters}

The relative position of the allowable flaw-size curve depends on the parameters used to evaluate Eq. 65. For the ANGTS pipeline, the pipe grade, API $5 \mathrm{LX} \mathrm{70,} \mathrm{and} \mathrm{the} \mathrm{pipe} \mathrm{geometry,} 1220-\mathrm{mm}(48-\mathrm{in})$ diameter and 15.9-mm $(0.625-i n)$ wall thickness, have been specified, but a reliable data base on the tensile properties and the fracture toughness of the pipe and weld are not available. 

It is planned to operate the pipeline at $-4^{\circ} \mathrm{C}$, but the maximum allowable stress (or strain) normal to the girth welds is not known by the authors. It has been assumed that the pipeline will be in nearly continuous operation at a nearly constant pressure and thus, crack growth in service due to fatigue cycling has been neglected.

For the purposes of this investigation, the maximum credible stress level is assumed to be $90 \%$ of the specified minimum yield strength of the pipe, $434 \mathrm{MPa}\left(63 \times 10^{3} \mathrm{psi}\right)$. The following tensile properties are assumed for both the pipe and the girth welds:

$$
\begin{aligned}
& \sigma_{y}=482 \mathrm{MPa}\left(70 \times 10^{3} \mathrm{psi}\right) \\
& \bar{\sigma}=517 \mathrm{MPa}\left(75 \times 10^{3} \mathrm{psi}\right) \\
& \sigma_{u}=551 \mathrm{MPa}\left(80 \times 10^{3} \mathrm{psi}\right) \\
& E=208 \mathrm{GPa}\left(30.2 \times 10^{6} \mathrm{psi}\right) \\
& \nu=0.29
\end{aligned}
$$

The minimum fracture toughness at the service temperature is assumed to be $0.13 \mathrm{~mm}(0.005 \mathrm{in})$. It should be emphasized that this value exceeds the minimum results obtained in this investigation, but it is considered to be an achievable minimum for qualified welding consumables and procedures.

\subsubsection{Results and Discussion}

Allowable flaw-size curves have been developed using the fracture mechanics analysis model described Section 2.5.1. For the parameters listed in Section 2.5.2, the resulting curve, Fig. 28 , is considered to be the current best-estimate of the allowable flaw-size curve for the ANGTS pipeline.

The position of the curve is strongly affected by the parameters selected. A sensitivity study has been conducted to assess the influence of variations in fracture toughness, tensile properties, and stress levels on the allowable flaw sizes. The results are presented in the form of a reference curve 



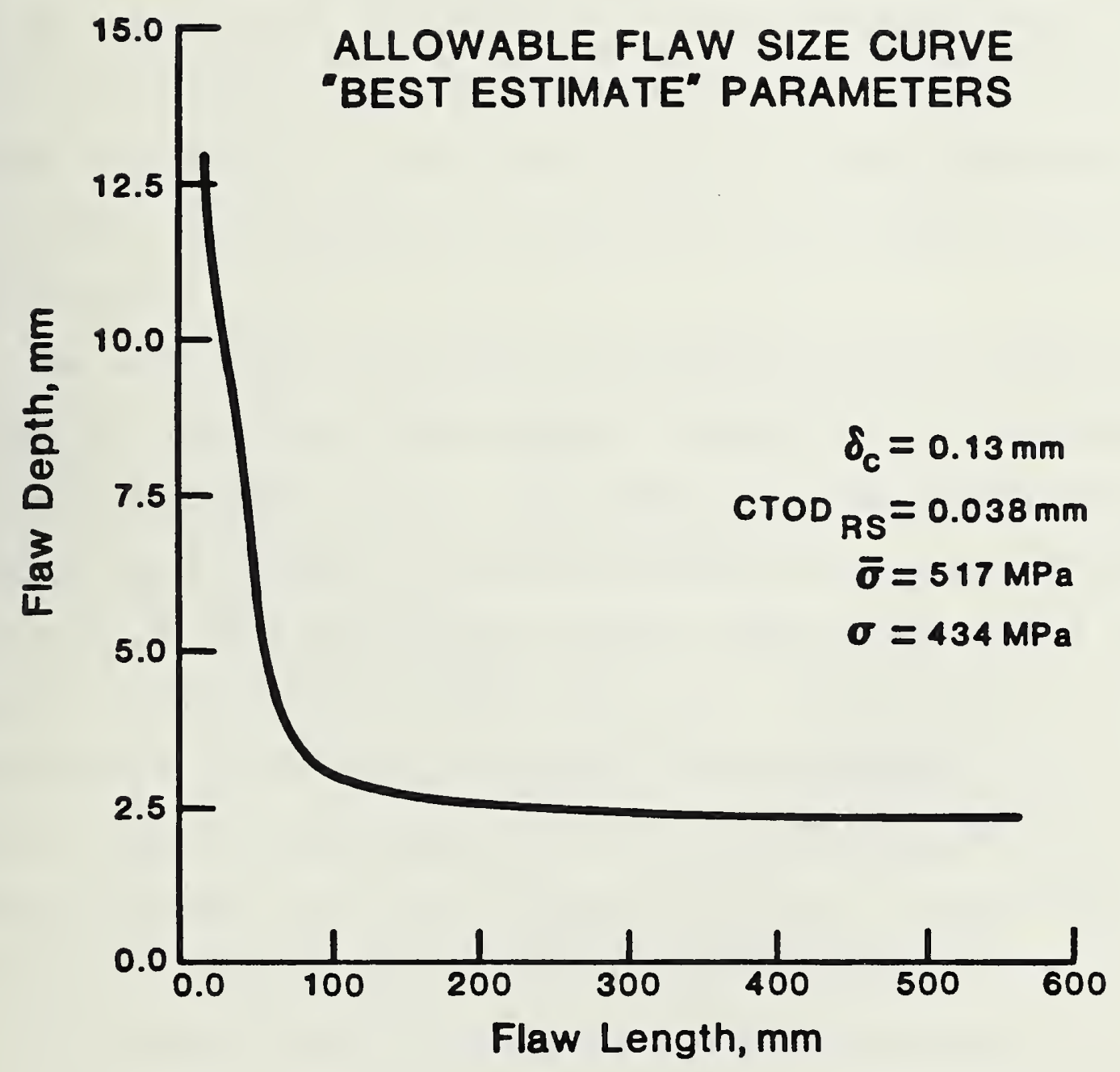

Fig. 28 - Best estimated allowable flaw-size curve. 

developed using the parameters listed in Section 2.5.2 and two additional curves developed using values of the variable under study below and above the reference value (Figs. 29-31).

The curves showing sensitivity to toughness variations are shown in Fig. 29. Note that by increasing the fracture toughness from $0.13 \mathrm{~mm}$ $(0.005-i n)$ to $0.25 \mathrm{~mm}(0.010-i n)$, the allowable flaw depths are more than doubled for flaws up to $125-\mathrm{mm}(4.92-$ in) 1 ong. For longer flaws, the increase in allowable depth decreases to about $30 \%$ for flaws that are $500-\mathrm{mm}$ (19.7-in) long.

The curves showing sensitivity to variations in applied stress are shown in Fig. 30. Note that a $10 \%$ increase in stress results in a substantial decrease in allowable flaw size. For cracks longer than $125 \mathrm{~mm},(4.92-i n)$ the al lowable depth decreases by more than $50 \%$ when the stress is increased from $90 \%$ of the specified minimum yield strength (SMYS) to $100 \%$ of SMYS, that is, from 434 to $482 \mathrm{MPa}\left(62.9\right.$ to $\left.69.9 \times 10^{3} \mathrm{psi}\right)$. The reason for this large sensitivity to applied stress is apparent from consideration of Eq. 70. Note that the $C T O D_{L Y}$ is proportional to $\sigma-(1-a / t) \bar{\sigma}$, and thus it is the relative difference between $\sigma$ and $\bar{\sigma}$ that influences flaw size. The same effect is seen in the curves depicting the influence of flow stress, shown in Fig. 31.

The allowable flaw-size curves developed herein are compared with allowable flaw sizes permitted under Appendix A 16th edition, of API 1104. Comparisons were made at the two toughness levels considered by API, that is, CTOD values of $0.13 \mathrm{~mm}(0.005 \mathrm{in})$ and $0.25 \mathrm{~mm}(0.010 \mathrm{in})$. The remaining parameters were those listed in Section 2.5.2. The results are presented in Figs. 32 and 33. At the $0.13-\mathrm{mm}(0.005-\mathrm{in})$ toughness level, Fig. 32, the allowable flaw-size curves developed herein are more restrictive than the API allowables at essentially all crack lengths. The differences are largest for crack lengths 


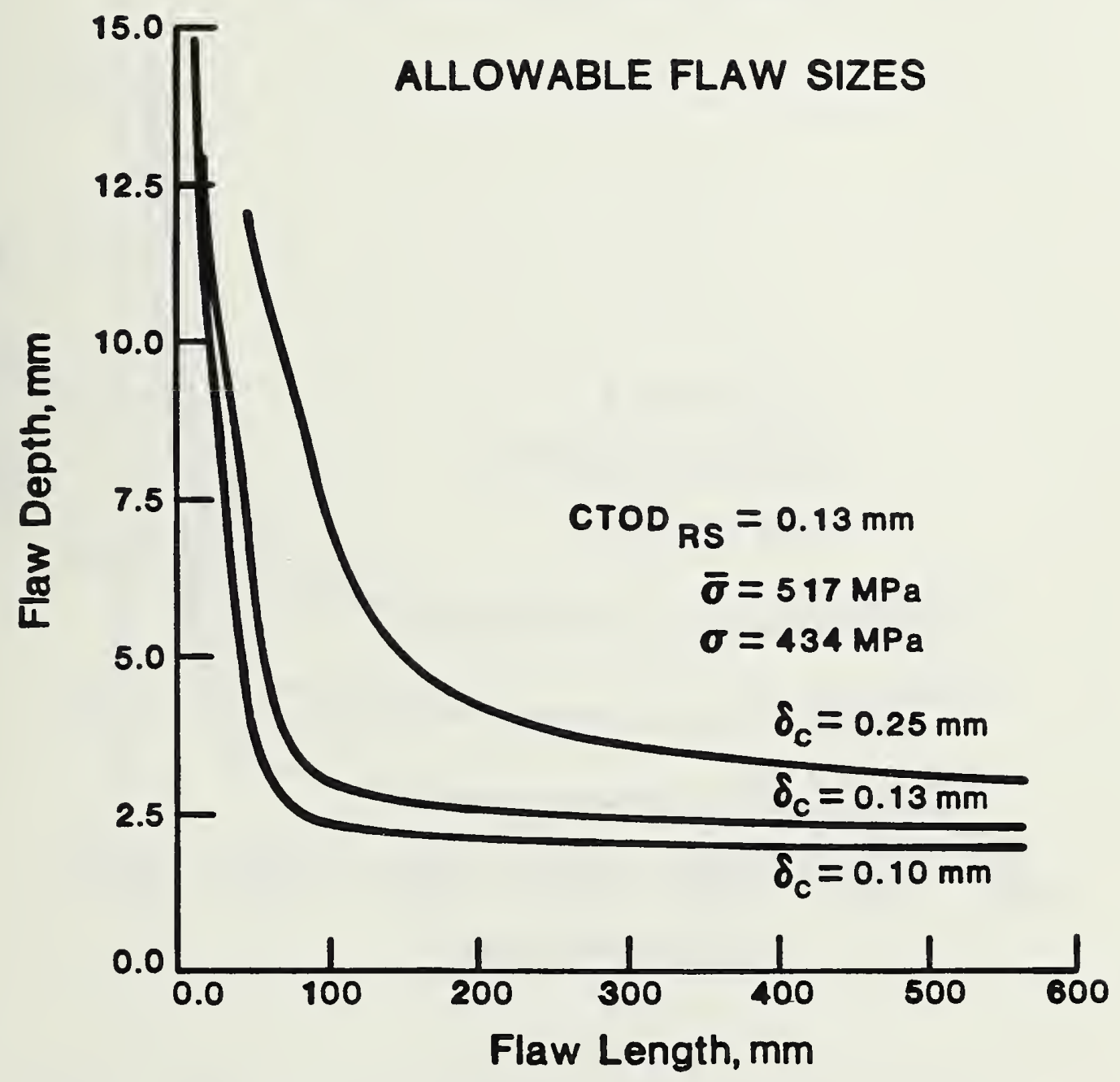

Fig. 29 - Effect of critical COD on the allowable flaw-size curve. 


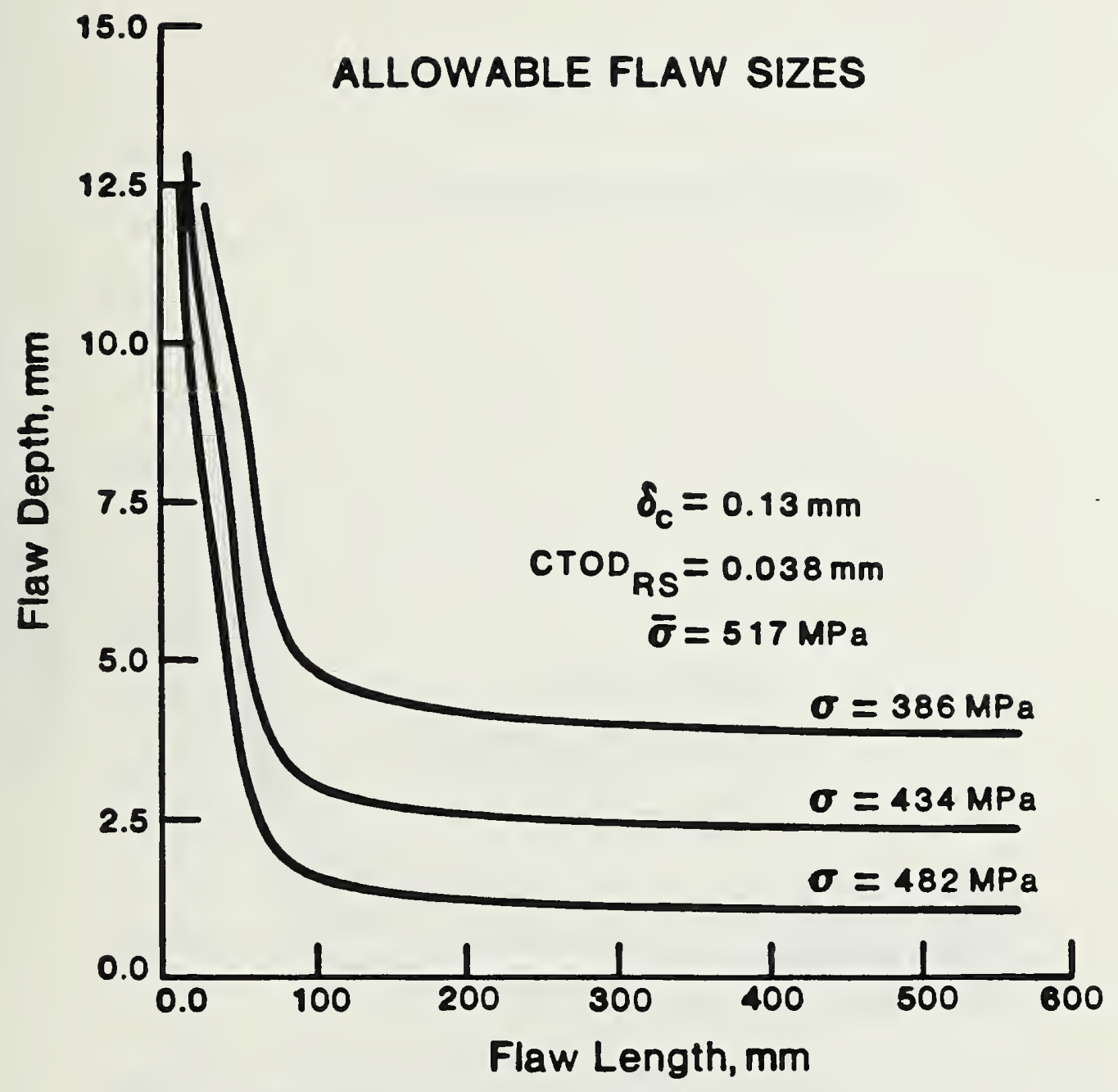

Fig. 30 - Effect of applied stress levels on the allowable flaw-size curve. 



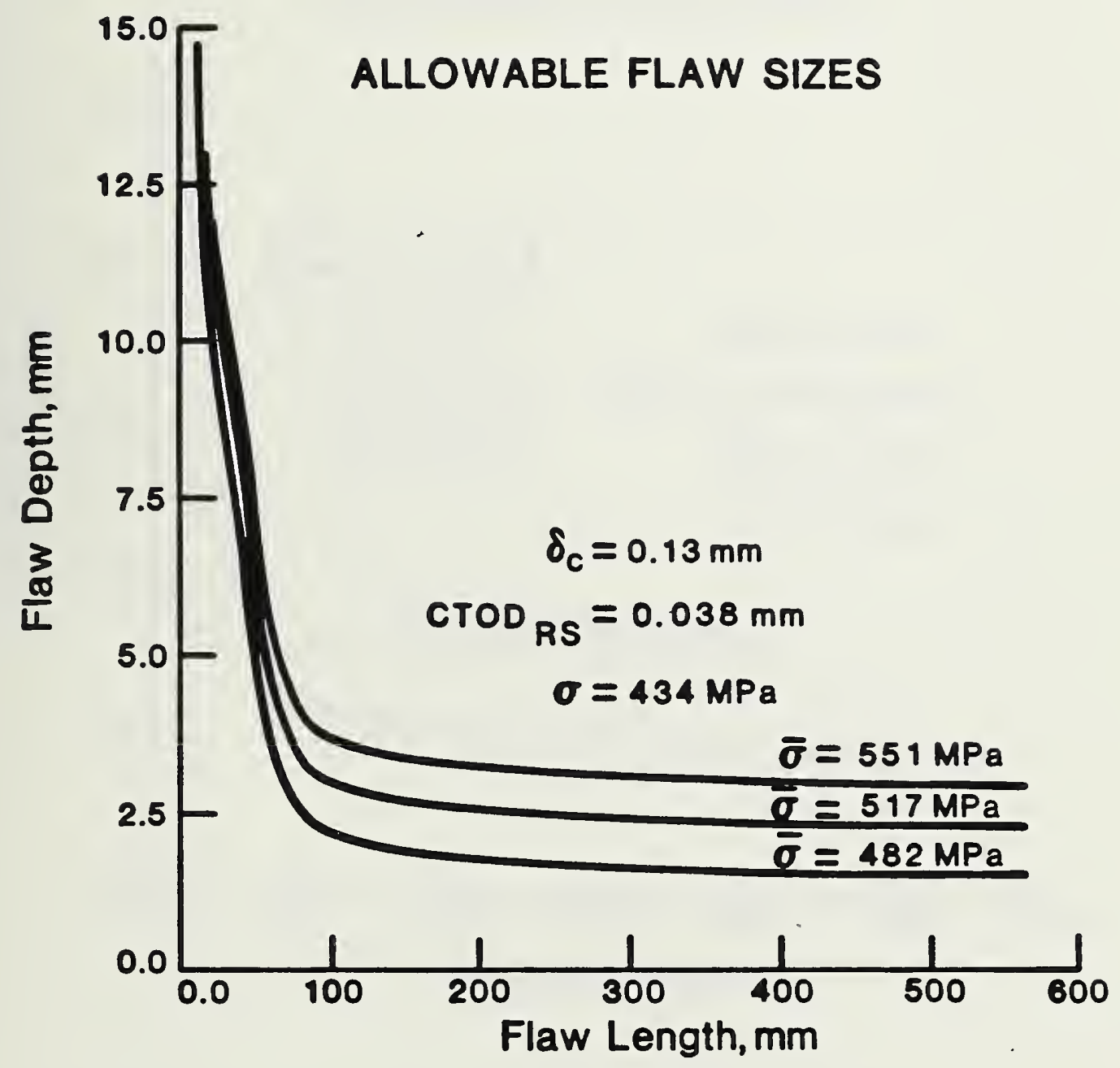

Fig. 31 - Effect of flow stress on the allowable flaw-size curve. 
. 


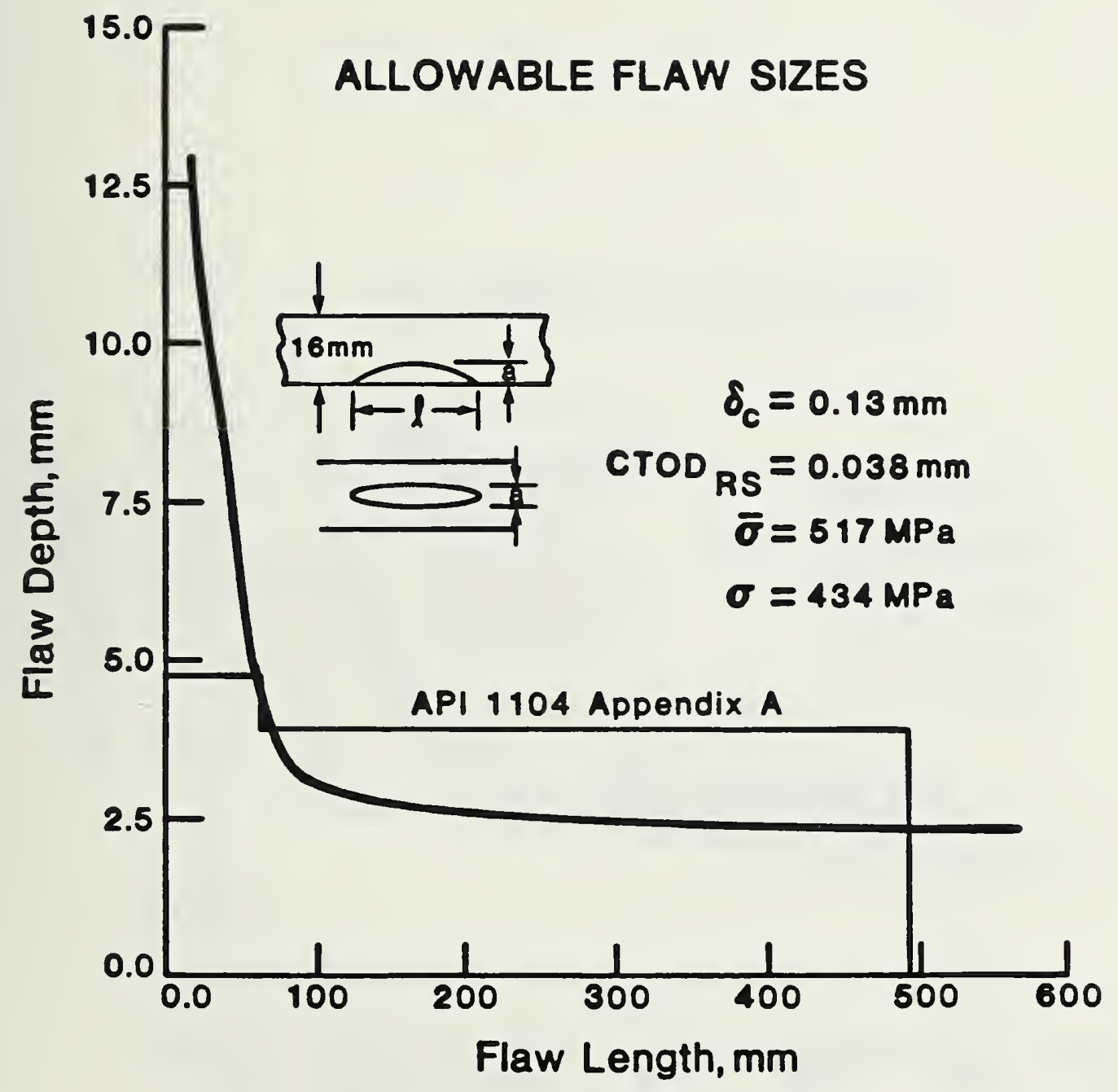

Fig. 32 - Comparison of allowable flaw-sizes with various criteria. Critical COD $=0.127 \mathrm{~mm}$. 



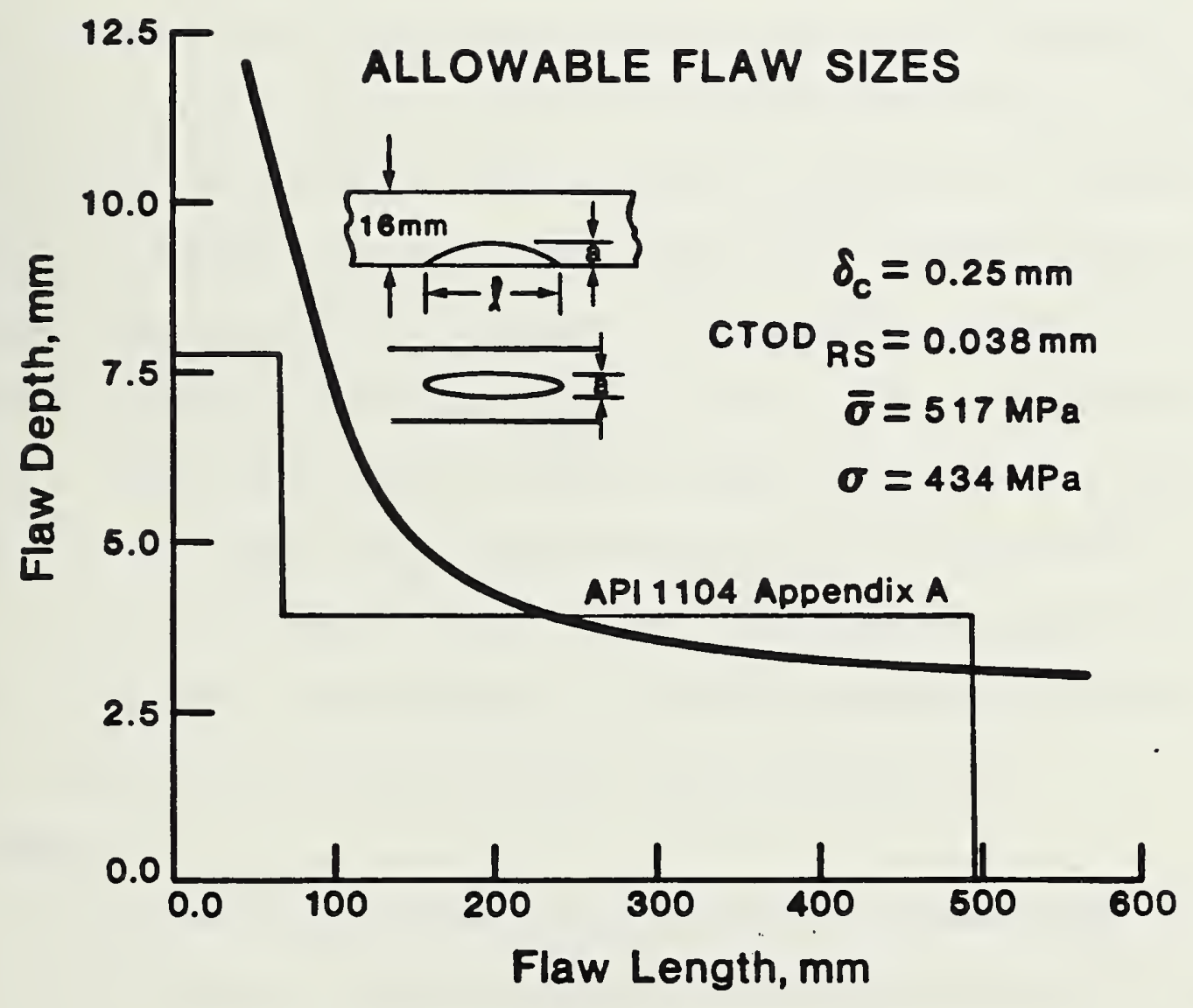

Fig. 33 - Comparison of allowable flaw sizes with various criteria. Critical $C O D=0.25 \mathrm{~mm}$. 

greater than $100 \mathrm{~mm}(3.94 \mathrm{in})$. For a flaw that is $250-\mathrm{mm}(9.84-\mathrm{in})$ 1ong, the allowable flaw depths are $2.5 \mathrm{~mm}(0.098 \mathrm{in})$ by the present method and $4.0 \mathrm{~mm}$ $(0.157 \mathrm{in})$ by the API method. At the higher toughness level, Fig. 33, the allowable flaw sizes developed using the API procedures are more restrictive for crack lengths up to $230 \mathrm{~mm}(9.06 \mathrm{in})$ and less restrictive for longer cracks.

The differences in allowable flaw sizes between the present approach and those of Appendix A of API 1104, 16th edition, result from differences in the treatment of ligament yielding, which is not explicitly addressed in Appendix A of API 1104, 16th edition. The assessment procedure and flaw-size allowables in Appendix A of API 1104, 16th edition resemble those of PD6493:1980, from the British Standards Institution ${ }^{8}$. Section 8.2.3 of PD6493:1980 accounts for ligament yielding using a flaw recategorization procedure. Flaws deep enough to produce ligament yielding are treated as through thickness flaws, whose characteristic size is the flaw length, not its depth. This procedure disallows some flaws, resulting in a more restrictive allowable flaw sizes. The treatment of ligament yielding in the present study is more extensive and more specific than that of PD6493:1980.

\subsection{SUMMARY}

A fracture mechanics analysis model, the pipe yielded-ligament model with through thickness plasticity correction, has been developed that relates allowable flaw sizes to applied stress level and weld toughness. For analys is purposes, the weld flaws have been treated as surface cracks. The fracture criterion used is the crack-tip-opening displacement (CTOD); that is, fracture occurs when the CTOD due to imposed strains (the applied CTOD) exceeds the fracture toughness (critical CTOD) of the girth welds. 

The validity of the model was confirmed for the case of surface cracks in tensile panels at stress levels below the net-section yield strength. The comparison of analys is and experiment was complicated by the fact that CTOD cannot be readily measured. Thus, it was necessary to make the comparison in terms of crack-mouth-opening displacement (CMOD). CMOD was measured with a clip-on displacement gage and is calculated using the flat plate yielded-ligament model with through thickness plasticity correction. The model further evaluated by analyzing the results of a series of tests on 900-mm (36-in) pipe conducted by the Welding Institute of Canada. Comparison of analysis, using the pipe yielded-ligament model with through-thickness plasticity correction, and experiment indicated that the line-spring model has acceptable accuracy for CTOD values up to $0.2 \mathrm{~mm}(0.0079 \mathrm{in})$, the range of interest in developing allowable flaw-size curves.

This pipe yielded-ligament model with through-thickness plasticity correcton has been used to calculate allowable flaw-size curves for operating conditions and girth-weld properties representative of the proposed ANGTS pipeline. The applicability of the model is limited to pipelines with maximum stresses in the longitudinal direction below the specified minimum yield strength of the pipe. It is further limited to pipelines where the yield strengths of the weld and base metal are similar. The required degree of similarity in yield strengths has not been established, but the desirability of higher yield strength in the weld metal has been clearly demonstrated. The use of allowable flaw-size curves can be considered for either general pipeline use or for site-specific engineering assessment. Allowable flaw-size curves generated using typical pipeline operating conditions and weldment properties are more conservative for long flaw lengths than those derived using the procedures of Appendix A of the 16th edition of API $1104 .^{2}$ 


\section{SHARP-FLAW DIMENSIONING}

C. M. Fortunko and R. E. Schramm

\subsection{Introduction}

In the evaluation of pipeline girth-weld quality on the basis of rational fitness-for-service criteria, ultrasonic inspection appears to be a better method than radiography, which has usually been used. Although radiography is well suited for assessing compliance with workmanship quality criteria, the relative insensitivity of radiography to the presence of sharp flaws makes it much less effective as a fitness-for-service inspection tool than ultrasonic inspection (cf. Appendixes $C$ and $E$ ). Furthermore, even though experience has shown that some information about the through-wall depth of blunt flaws can be inferred from densitometric analyses of radiographic data, recent work has shown that the reliability of sizing by such methods is very low for sharp flaws less than $0.25-\mathrm{mm}(0.0058-i n)$ thick, even when the most sophisticated microdensitometry techniques are employed. ${ }^{36}$ Densitometry techniques can provide information only on the through-wall radiographically projected dimension of a canted sharp flaw; they provide no information on overall through-wall flaw height. Therefore, such techniques cannot be reliably used to detect and measure cracks, incomplete fusion, and inadequate penetration. Conventional, high-frequency ultrasonic methods are sensitive to sharp flaws and can provide dimensional information under ideal conditions ( $c f$. Appendix D). However, as described in Appendix F, tests run on deliberately flawed pipeline welds used in this work showed an inability of state-of-the-art high-frequency methods to reliably perform the required flaw detection and sizing. This result confirmed conclusions in the literature that high-frequency approaches have fundamental limitations that limit their usefulness in a fracture mechanics approach to a fitness-for-purpose analys is of a welded structure. ${ }^{37}$ 

Recent theoretical and experimental investigations have shown that sharp flaws and cracks in butt welds can be sensitively detected using a new ultrasonic inspection technique. ${ }^{36-38}$ The new technique differs from most conventional ultrasonic nondestructive evaluation (NDE) methods in three important respects: ${ }^{39-43}$ First, the weld region is insonified using special ultrasonic probing signals: shear waves that are polarized parallel to the surface of the weldment ( $\mathrm{SH}$ waves). Second, the probing ultrasonic signals are generated and detected using the recently developed periodic-permanentmagnet (PPM) electromagnetic-acoustic transducers (EMATS). ${ }^{44,45}$ In contrast with the fluid-coupled piezoelectric transducers, the EMATs do not require intimate coupling and can operate efficiently over most unprepared surfaces. Third, the ultrasonic frequency of operation of the new inspection system is substantially lower $(0.5 \mathrm{MHz})$ than the frequency range used in conventional ultrasonic inspection of ferritic butt weldments $(2-10 \mathrm{MHz})$.

The lowering of the frequency of operation significantly reduces the sensitivity of the SH-wave EMAT system to minor perturbations in flaw surface detail: surface roughness and departure from planarity and branching. 46,47 If the frequency is sufficiently low, the amplitude of the signal scattered by the flaw can be unambigously related to the principal flaw dimensions. $40,48,49$ In addition, sharp flaws are more easily detected than blunt flaws of similar dimensions. Of course, these features are highly desirable from the fitnessfor-service point of view, which emphasizes the importance of the principal flaw dimensions and assumes that sharp flaws are inherently more critical than blunt flaws. ${ }^{4,50-52}$

The qualitative differences between ultrasonic scattering at low and high frequencies are illustrated in Fig. 34. (It is assumed that the flaw is planar and relatively long in the direction of the normal to the plane of 


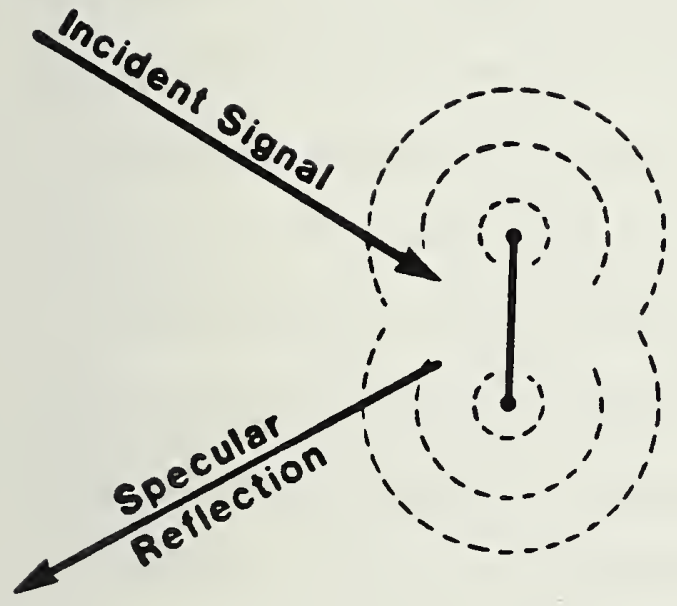

(a)

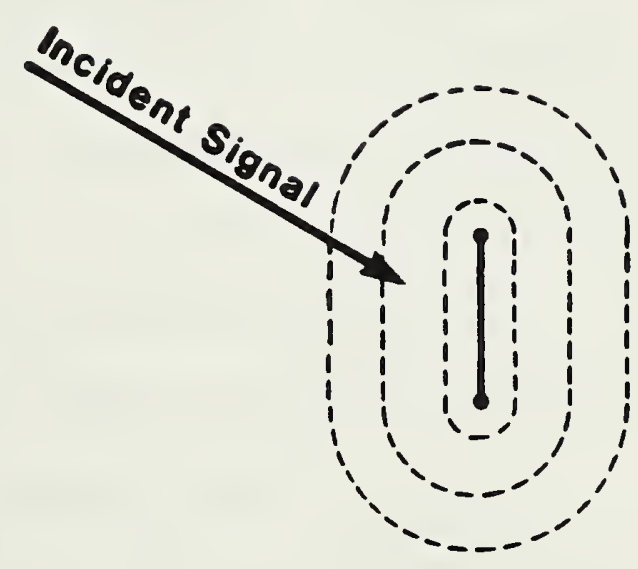

(b)

Fig. 34 - Ultrasonic scattering by two-dimensional, planar flaws:

(a) high-frequency region; (b) low-frequency region. 

reflection). At high frequencies (Fig. 34a), most of the incident ultrasonic energy from the face of the planar flaw is reflected specularly, that is, the angle of reflection with respect to the face normal equals the angle of incidence. Also, a significant portion of the incident signal is diffracted by the tips of the flaw, which act like ultrasonic line sources. If the surface of the flaw exhibits some roughness, some of the incident signal can be scattered incoherently (not shown). Furthermore, depending on the orientation of the flaw and the type of ultrasonic signal used (i.e., shear vs. longitudinal), significant mode conversions can also occur. As a result, the scattered ultrasonic field is very complicated and it is very difficult to infer the flaw dimensions from the ultrasonic data.

At low ultrasonic frequencies (Fig. 34b), the scattered ultrasonic fields are considerably easier to interpret because the specular reflection component becomes negligible and the tip diffractions are merged. Consequently, the scattered ultrasonic fields are much less sensitive to flaw orientation and surface roughness. ${ }^{47}$ In addition, at low ultrasonic frequencies, sharp flaws are detected more easily than blunt flaws of similar length and through-wall depth. The principal advantage, however, of operating at low ultrasonic frequencies is that the amplitude of the backscattered signal increases monotonically with the flaw dimensions.

In this report, emphasis is placed on the details of the experimenta? configurations and procedures. An experimental configuration is described that permits the ultrasonic interrogation of the entire weld zone from the outer surface of the pipe. It has a built-in self-calibration feature that automatically and consistently records the experimental data. This feature is needed to offset variations in the EMAT electromechanical coupling 


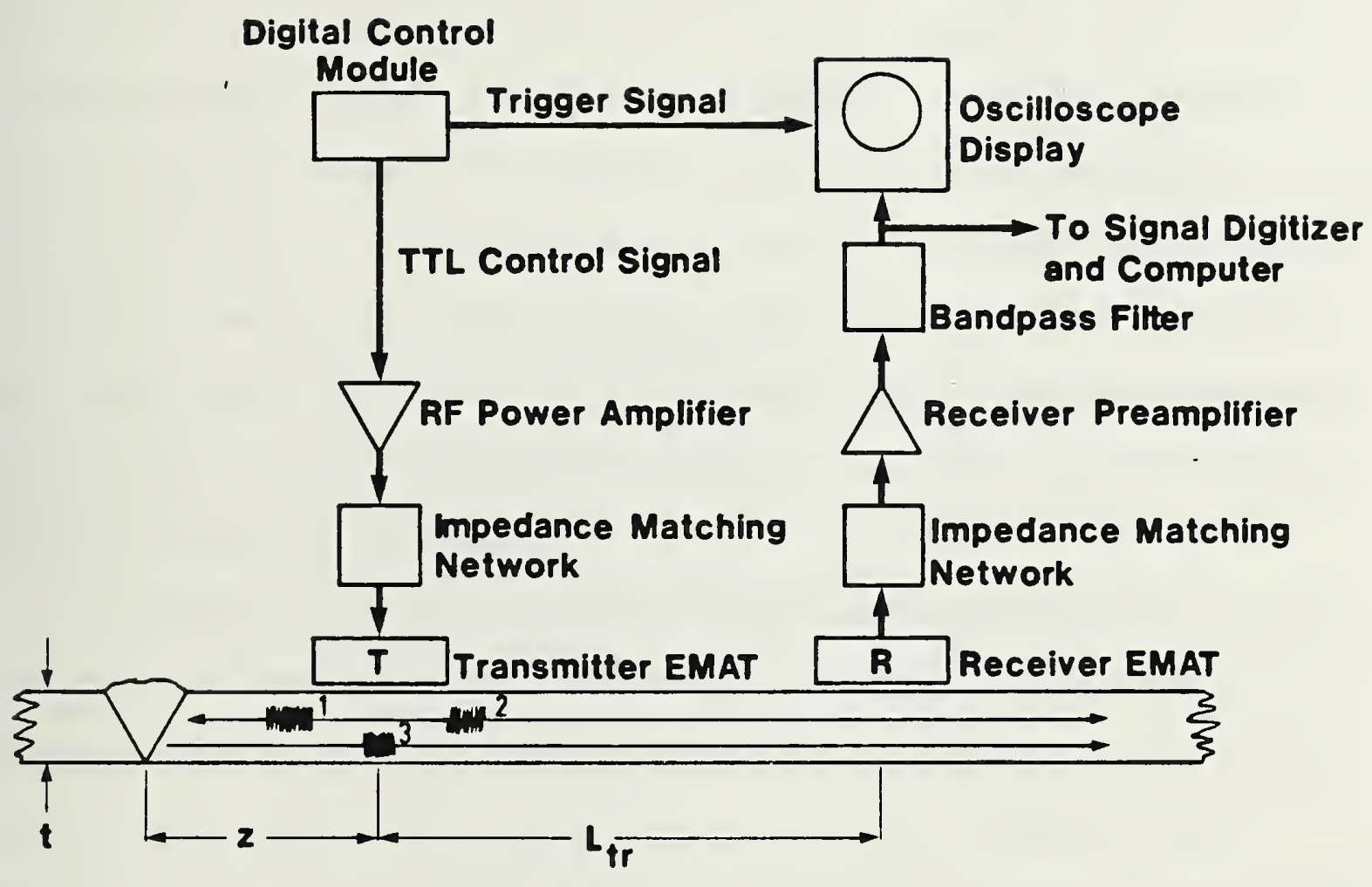

Fig. 35 - Low-frequency, SH-wave EMAT inspection configuration for pipeline girth welds. 

efficiencies, which may occur in practice as a result of variations in EMAT lift-off distance, material composition, pre-existing residual and applied stresses, and magnetic state. ${ }^{53}$

The physical principles governing the propagation of the special SH-wave probing signals in isotropic plates are discussed in detail. The discussion is confined to the special case of ultrasonically "thin" plates whose wall thickness does not substantially exceed the bulk ultrasonic wavelength $(7.4 \mathrm{~mm}$, 0.29 in at $454 \mathrm{kHz}$ ). This restriction is primarily dictated by the choice of a theoretical model used to describe the scattering of low-frequency SH-wave signals by planar flaws of different through-wall depth, position, and orientation with respect to the free surface. However, general scattering theories are found in Refs. 54 through 56.

Considerable attention is focused on experimental evidence that is used to validate the main features of the theory. In addition, experiments are described that establish the inherent sensitivity limitations of the system for different categories of surface and buried flaws. The results are summarized in a set of diagrams that are pertinent to a $454-\mathrm{kHz}$ SH-wave EMAT system operated on plate-like ferritic steel specimens $15.9-\mathrm{mm}(0.62-i n)$ thick. An inspection protocol, applicable to automated inspection of newiy fabricated pipeline girth welds, is also discussed.

\subsection{Inspection, Configuration, and Operation}

Recently, several ultrasonic systems have been developed specifically for the purpose of inspecting pipeline girth welds. ${ }^{41-44}$ However, the performance of the new systems is limited by uncertainties associated with coupling and the highly complex nature of ultrasonic scattering from flaws when the flaw dimensions of interest are large compared with the ultrasonic wavelength, but not large enough to permit high-resolution imaging. The low-frequency, 



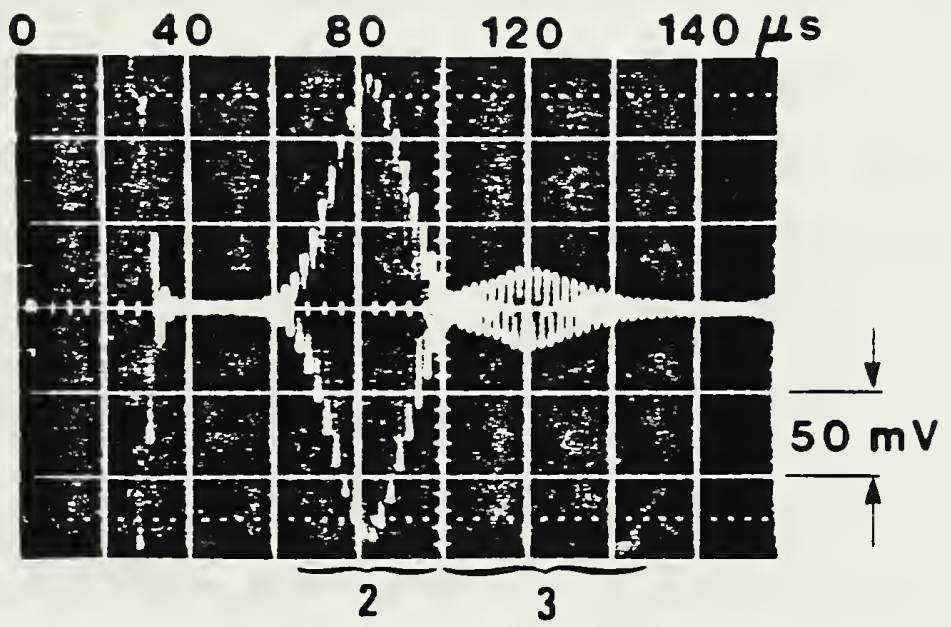

Fig. 36 - Detection of a surface flaw (inadequate penetration) in a 15.9-mm SMA girth weld using $454-\mathrm{kHz}$ SH waves. 

SH-wave EMAT system was then specifically designed to overcome most of the main shortcomings of conventional ultrasonic systems that use piezoelectric transducer elements, which typically operate at high ultrasonic frequencies (2-10 MHz).

Figure 35 shows an experimental configuration that has been found to be particularly useful in the detection and limited characterization of elongated flaws in pipeline girth welds. The configuration of Fig. 35 is composed of two identical SH-wave EMATs that are positioned on the outer surface of the pipe and aimed colinearly along the normal to the girth weld. The transmitter EMAT is located closer to the weld than the receiver EMAT. Because SH-wave EMATs are bidirectional, the transmitter EMAT generates two SH-wave signals of equal amplitude, which travel in opposite directions along the normal to the weld. The ultrasonic signal 1, traveling to the left of the transmitter EMAT, insonifies the weld region, while the ultrasonic signal 2, traveling to the right of the transmitter EMAT, passes directly beneath the receiver EMAT. In practice, signal 1 is used to interrogate the weld region, and signal 2 is used to calibrate the overall transduction efficiency of the system.

If a flaw is present in the weld, a portion of the probing signal 1 is backscattered in the direction of the receiver EMAT. The backscattered signal 3 arrives at the receiver EMAT after the reference signal 2 because it travels a longer distance. The two signals, 2 and 3 , are shown in the photo of Fig. 36 .

The signals in Fig. 36 were observed when an inadequate penetration flaw was insonified in a $1220-\mathrm{m}(48-i n)$ diameter, $15.9-\mathrm{mm}(0.62-$ in) thick API 5LX-70 SMA-welded pipe section. The center frequency of the ultrasonic signals was $454 \mathrm{kHz}$, corresponding to an ultrasonic (shear-wave) wavelength of $7.4 \mathrm{~mm}(0.29 \mathrm{in})$. In the photo, the first signal $(0-30 \mu \mathrm{s})$ is caused by 
direct electromagnetic interference between the transmitter and receiver EMATs. This signal carries no useful information. The second signal $(60-100 \mu \mathrm{S})$ is the reference signal 2; the third signal (100-140 $\mu \mathrm{S})$ is the backscattered signal 3 .

It has been determined that the amplitude of the reference signal 2 and the amplitude and shape of the backscattered signal 3 carry useful information about the dimensions, orientation, and position of the flaws within the weld. 38,39 Although it is not possible to reconstruct the shape of the flaw and determine its position and orientation from a single low-frequency ultrasonic measurement (because of poor spatial resolution at low frequencies), it is possible to obtain much useful quantitative information about these parameters from a series of independent measurements along the normal to the girth weld. In particular, by using appropriate signal processing techniques, it is possible to classify the flaws in terms of their position within the weld (i.e., surface vs. interior) and then to estimate their principal dimensions. ${ }^{38}$ However, to understand the principles of the ultrasonic data inversion procedures, one first requires a qualitative insight into the physical mechanisms governing the propagation and scattering of 10 frequency SH waves in isotropic plates.

\subsection{Theory of Horizontally Polarized Plate Wave Propagation}

When the frequency of operation is sufficiently low, it is often advantageous to represent the ultrasonic field in an isotropic plate as a modal expansion. 39,54 This representation is also approximately valid when the radius of curvature of the pipes is very large compared with the ultrasonic wavelength and when the observer is located sufficiently far from the transducer. Although clearly limited, the modal representation provides valuable quantitative insights that can be used to optimize the design of low-frequency, SH-wave inspection systems in terms of the important 

experimental parameters: plate thickness, frequency of operation, weld-to-EMAT spacing, and the receiver-EMAT-to-transmitter-EMAT spacing.

In an infinite isotropic plate of finite thickness, there are only a finite number of propagating, horizontally polarized plate waves and an infinite number of nonpropagating, horizontally polarized plate waves. Correspondingly, there are a finite number of plate waves polarized in the plane normal to the plate (Lamb waves) that can propagate and an infinite number that are cut off. 57,58 In this report, it is assumed for simplicity that SH-wave signals are not coupled to Lamb waves by elongated weld flaws, whose long dimension is parallel to the SH-wave polarization direction. This assumption is strictly true for two-dimensional geometries and is justified in practice by the inherent insensitivity the of SH-wave EMATs to ultrasonic waves polarized in the plane normal to the plate (Lamb and Rayleigh waves). ${ }^{59}$

In the coordinate system of Fig. 37, the transverse ultrasonic field distributions (particle velocity $v_{x}^{(n)}$ and stresses $T_{x z}^{(n)}$ and $T_{x y}^{(n)}$ ) of the $n^{\text {th }}$ propagating and nonpropagating $\mathrm{SH}$-wave plate waves are given by:

$$
\begin{aligned}
& V_{x}^{(n)}(y)=A_{n} \cos (n \pi y / t) \\
& T_{x z}^{(n)}(y)=\left(-B G A_{n} / \omega\right) \cos (n \pi y / t) \\
& T_{x z}^{(n)}(y)=\left(-n \pi G A_{n} / t j \omega\right) \sin n \pi J^{\prime} / t
\end{aligned}
$$

with

$$
B_{n}=\left[\left(\omega / v_{s}\right)^{2}-(n \pi / t)^{2}\right]^{\frac{1}{2}} \text { for } n=0,1,2, \ldots
$$

where: $t$ is the plate thickness, $v_{s}$ is the bulk shear wave velocity, $G$ is the shear modulus, $w$ is the angular frequency, $j=\checkmark-1, B_{n}$ is the propagation constant, $A_{n}^{2}=j \omega^{2} / 2 B_{y} G t \varepsilon_{n}$, and $\varepsilon_{0}=1$ and $\varepsilon_{n}=\frac{1}{2}$ for $n=1,2,3, \ldots$

Along the $z$ direction, the fields of the propagating and nonpropagating SH plate waves vary as:

$$
v^{(n)}(z, \tau), T_{x z}^{(n)} \text {, and } T_{x y}^{(n)}(z, \tau) \sim \exp \left[-j\left(\beta_{n} z+\omega \tau\right)\right]
$$



$\mathrm{SH}_{0}$
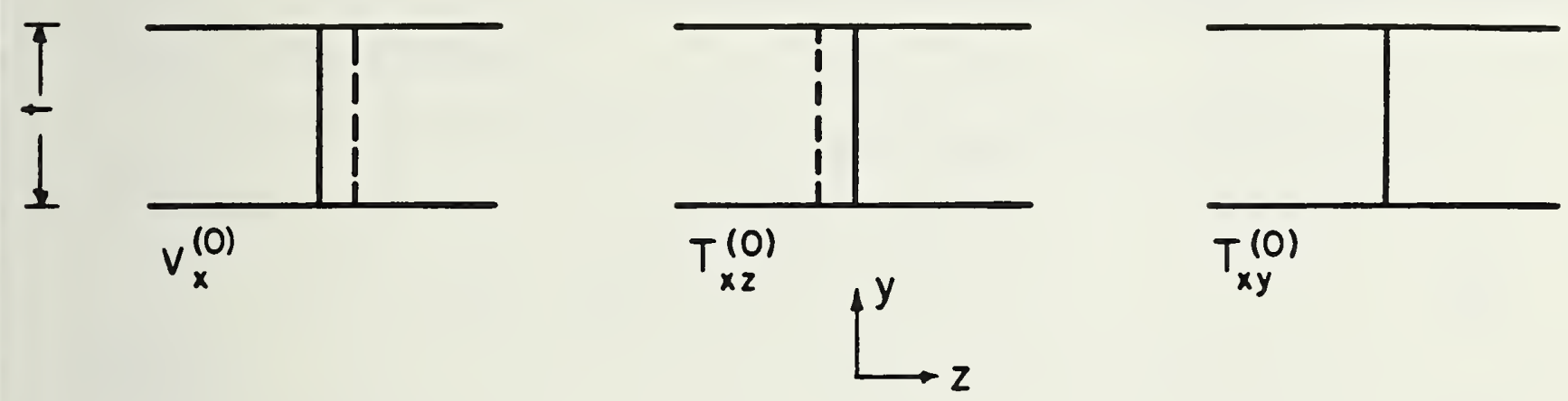

$\mathrm{SH}_{1}$
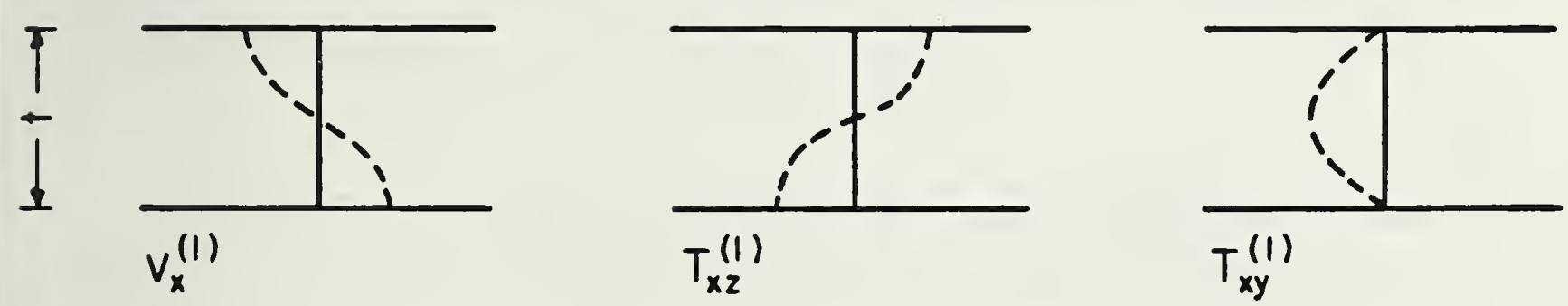

Fig. 37 - Transverse profiles of $\mathrm{SH}_{0}$ and $\mathrm{SH}_{1}$ plate waves. 

The particle velocity, $v_{x}$, and the two stress field components, $T_{x z}$ and $\mathrm{T}_{x y}$, for the two lowest order $\mathrm{SH}$ plate waves, the $\mathrm{SH}_{0}$ and $\mathrm{SH}_{1}$, are shown in Fig. 37. It is observed that the $\mathrm{SH}_{0}$ plate wave fields are uniform, but the $\mathrm{SH}_{1}$ and higher order plate wave fields exhibit sinusoidal variations along the transverse direction, $y$. The $\mathrm{SH}_{0}$ plate wave is also unique in that it carries no $T_{x z}$ stress component.

The phase and group velocities, $v_{p}$ and $v_{g}$, of the SH plate waves are given by:

$$
v_{p}^{(n)}=v_{s}\left[1-\left(f_{c}^{(n)} / f\right)^{2}\right]^{-\frac{1}{2}}
$$

and

$$
v_{g} v_{p}=v_{s}^{2}
$$

where $v_{s}$ is the bulk shear-wave velocity, $f$ is the wave frequency and the "cut-off" frequency of an $n^{\text {th }}$ SH plate wave is:

$$
f_{c}^{(n)}=n v_{s} / 2 t
$$

It follows from Eqs. 73 through 78 that each SH plate wave not only exhibits a different variation along the transverse direction, $y$, but also travels at a different velocity along the direction $z$. This observation is the basis for the quantitative procedures for flaw characterization and dimensioning from the ultrasonic scattering data obtained using the inspection system and experimental procedures described in the preceding section.

In this report, it is assumed implicitly that both the transmitter and receiver EMATs can only generate and receive the $\mathrm{SH}_{0}$ and $\mathrm{SH}_{1}$ plate waves with relative amplitudes of 1 to $1.6 .^{39} \mathrm{Although}$ the $\mathrm{SH}_{2}, \mathrm{SH}_{3}$, and $\mathrm{SH}_{4}$ plate waves can also propagate in a $15.9-\mathrm{mm}(0.625-\mathrm{in})$ thick plate at $454 \mathrm{kHz}$, they cannot be detected or generated efficientiy by the EMATs. Neverthless, their effect must be included in any calculation of the ultrasonic scattering by the flaws. 39 



\subsection{Theory of Scattering of Horizontally Polarized Shear Waves by Elongated}

Flaws

To gain a physical understanding of the scattering of SH plate waves by flaws, it is useful to use the concept of a reflection coefficient, $\Gamma$. Here, $\Gamma$ is defined as the ratio of the amplitudes of the incident and backscattered ultrasonic signals at the EMAT electrical terminals. For the two-dimensional case (no variations in $\vec{v}$ and $\vec{F}$ along $x$ and flaws of infinite length), a very useful relationship for $\Gamma$ has been derived by Auld. 59

$$
\Gamma=\left[4\left(P_{1} P_{2}\right)\right]^{-\frac{1}{2}} \int_{S_{F}}\left(\overrightarrow{v_{1}} \cdot \overleftrightarrow{T_{2}}-\overrightarrow{v_{2}} \cdot \overleftrightarrow{T_{1}}\right) \cdot \hat{n} d S
$$

where $\hat{n}$ is the inward-directed unit-normal to the surface, $S_{F}$, which surrounds the flaw. Subscript 1 is used to denote the ultrasonic field in the absence of the flaw, and subscript 2 is used to denote the ultrasonic field in the presence of the flaw, assuming that the EMATs 1 and 2. in Fig. 34 are used as transmitters. $P_{1}$ and $P_{2}$ are the electrical power levels delivered to the EMATs 1 and 2, respectively, when used as transmitters.

Equation 77 can be used to gain valuable insights. Consider the case of a two-dimensional, planar flaw shown in Fig. 38. In this case, Eq. 77 reduces to:

$$
\Gamma=j \omega / 4\left(P_{1} P_{2}\right)^{\frac{1}{2}} \int_{\frac{1}{2} S_{F}}\left(\Delta u_{1} \cdot T_{2}\right) \cdot \hat{n} d S
$$

where $\Delta u$ is the mode-III displacement jump across the face of the planar flaw and the integration is carried over only one-half of the surface, $S_{F}$.

In general, because several SH plate waves can propagate in a practical situation, the reflection coefficient $I$ can be rewritten as:

$$
\Gamma\left(z, L_{t r}\right)=j \omega / 4\left(P_{1} P_{1}\right)^{\frac{1}{2}} \sum_{n=0}^{\infty} \sum_{m=0}^{\infty} \exp \left(-j B_{n} z\right) a_{n} \Gamma_{n m} b_{m} \exp \left[-j B_{m}(z+L t r)\right]
$$





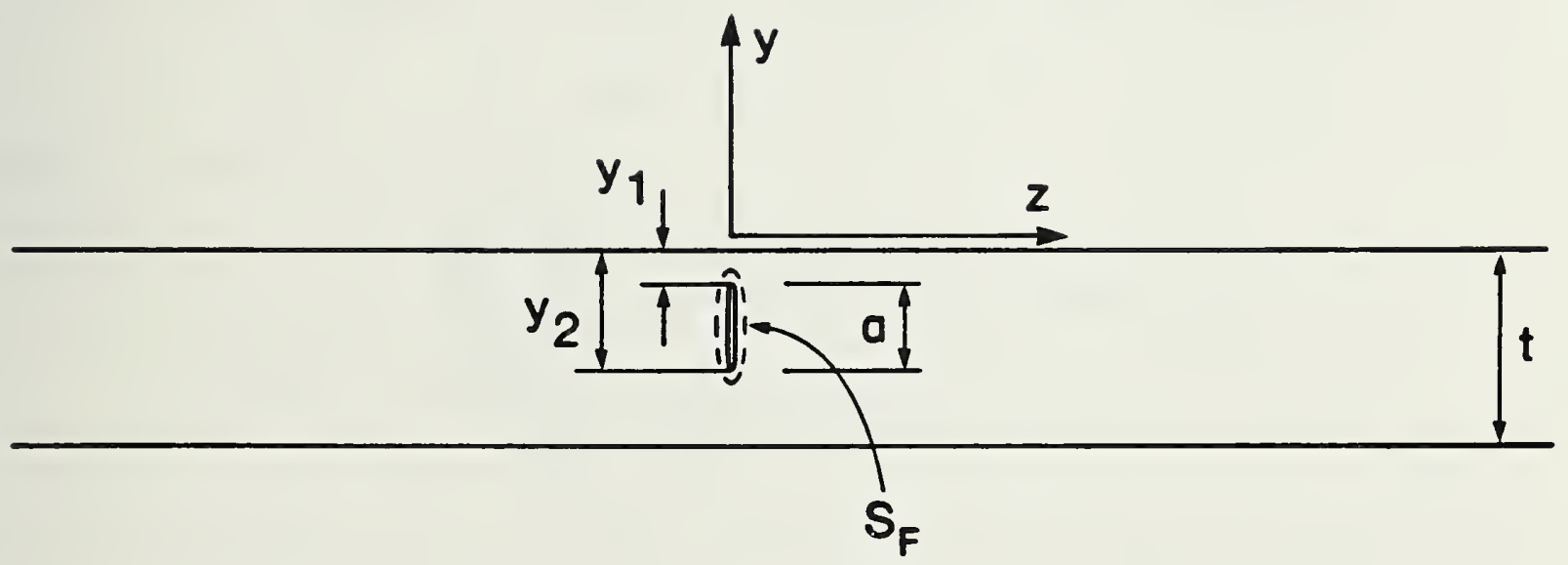

Fig. 38 - Planar flaw geometry. 

where $z$ is the distance between flaw and EMAT, $L_{t r}$ is the distance between EMATs, and the quantity $\Gamma_{n m}$ is defined as:

$$
\Gamma_{n m}=\int_{\frac{1}{2} S_{F}}(\overrightarrow{\Delta u}(n) . \leftrightarrow(m)) \cdot \hat{n} d S \quad .
$$

the quantities $a_{m}$ and $b_{m}$ express the relative transduction efficiencies of the

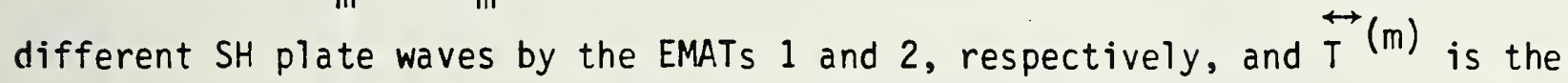
stress component $T_{x z}$ associated with the $m^{\text {th }}$ plate mode.

In practice, and as described in a preceding section, the ultrasonic scattering data (signal 3 in Fig. 35) is normalized using a reference signal (signal 2 in Fig. 35). This normalized signal, called the reflected signal amplitude ratio, $R_{s}$, is given by:

$$
R_{s}\left(z, L_{t r}\right)=\frac{\sum_{n=0}^{\infty} \sum_{m=0}^{\infty} \exp \left(-j \beta_{n} z\right) a_{n} \Gamma_{n m} b_{m} \exp \left[-j \beta_{m}\left(z+L_{t r}\right)\right]}{2 \mu t \sum_{\ell=0}^{\infty} a_{\ell} b_{\ell} \exp \left(-j \beta_{\ell} L_{t r}\right)}
$$

If the quantities $a_{n}, b_{n}, z$, and $L_{t r}$ are known and $R_{s}\left(z, L_{t r}\right)$ can be measured at a sufficient member of points, the unknown coefficients, $\Gamma_{\mathrm{nm}}$, can be found from Eq. 83. Equation 83 can be inverted if $a_{n}$ and $b_{n}$ vanish for $n$ or $m \geq N=M$. In general, $N \times N$ independent measurements are then needed but this number can be reduced by taking into account the symmetry of the matrix $\Gamma_{m n}$. Thus, if only the $\mathrm{SH}_{0}$ and $\mathrm{SH}_{1}$ plate waves are generated and detected by the EMATs (although more SH plate waves can propagate), only three independent measurements are needed. If signals carried by the $\mathrm{SH}_{0}, \mathrm{SH}_{1}$, and $\mathrm{SH}_{2} \mathrm{plate}$ waves are detected, then only six independent measurements are required. In practice, more measurements are available, but the size of the $I_{m n}$ matrix is determined by the number of $\mathrm{SH}$ plate waves that can be generated and detected by the EMATs. The larger the number $\mathrm{N}$, the more information is available for flaw characterization and dimensioning. 

Although a detailed treatment of the dependence of the coefficierits $\Gamma_{n m}$ on flaw size, shape, orientation, and position parameters is beyond the scope of this report, it is instructive to consider several cases of practical importance. For a more detailed treatment, the reader should consult Refs. $36,37,54-56,60$, and 61 .

The magnitudes and phases of the coefficients can be obtained using numerical methods for the vertically oriented surface and interior planar flaws of infinite length. ${ }^{39}$ The case of canted, planar flaws is treated in Refs. 40 and 56.

The numerical results for the coefficient $\Gamma_{00}$ for three different flaw types are shown in Fig. 39 as a function of the flaw through-wall depth, a. To illustrate the dependence of $\Gamma_{00}$ on the relative position of the flaw within a plate, the numerical calculations were carried out for edge (surface-connected), centered (buried) cracks, and eccentric (buried) cracks. The calculations were carried out for a case of current practical interest (wavelength, $\lambda,=7.4 \mathrm{~mm},(0.291 \mathrm{in})$; plate thickness, $t,=15.9 \mathrm{~mm}, 0.625 \mathrm{in})$.

It is interesting to compare the results for the relatively shallow $(a<\lambda / 2)$ surface and buried cracks. It is observed that $\Gamma_{00}$ for the shallow crack case initially exhibits a greater rate of increase than that for a shallow buried crack of the same through-wall depth. However, at a $\sim \lambda / 3$ for the surface crack and $a \sim \lambda / 2$ for the buried cracks, the rate of increase with $a / t$ is substantially less, and both coefficients tend linearly to 1 . Although the rate of increase of $\Gamma_{00}$ for a shallow buried crack is initially less than that for a shallow surface crack, for a $>\lambda / 2$, the results converge asymptotically. As will be seen later, the initial behavior of the coefficients determines the detectability thresholds of surface and buried flaws. 



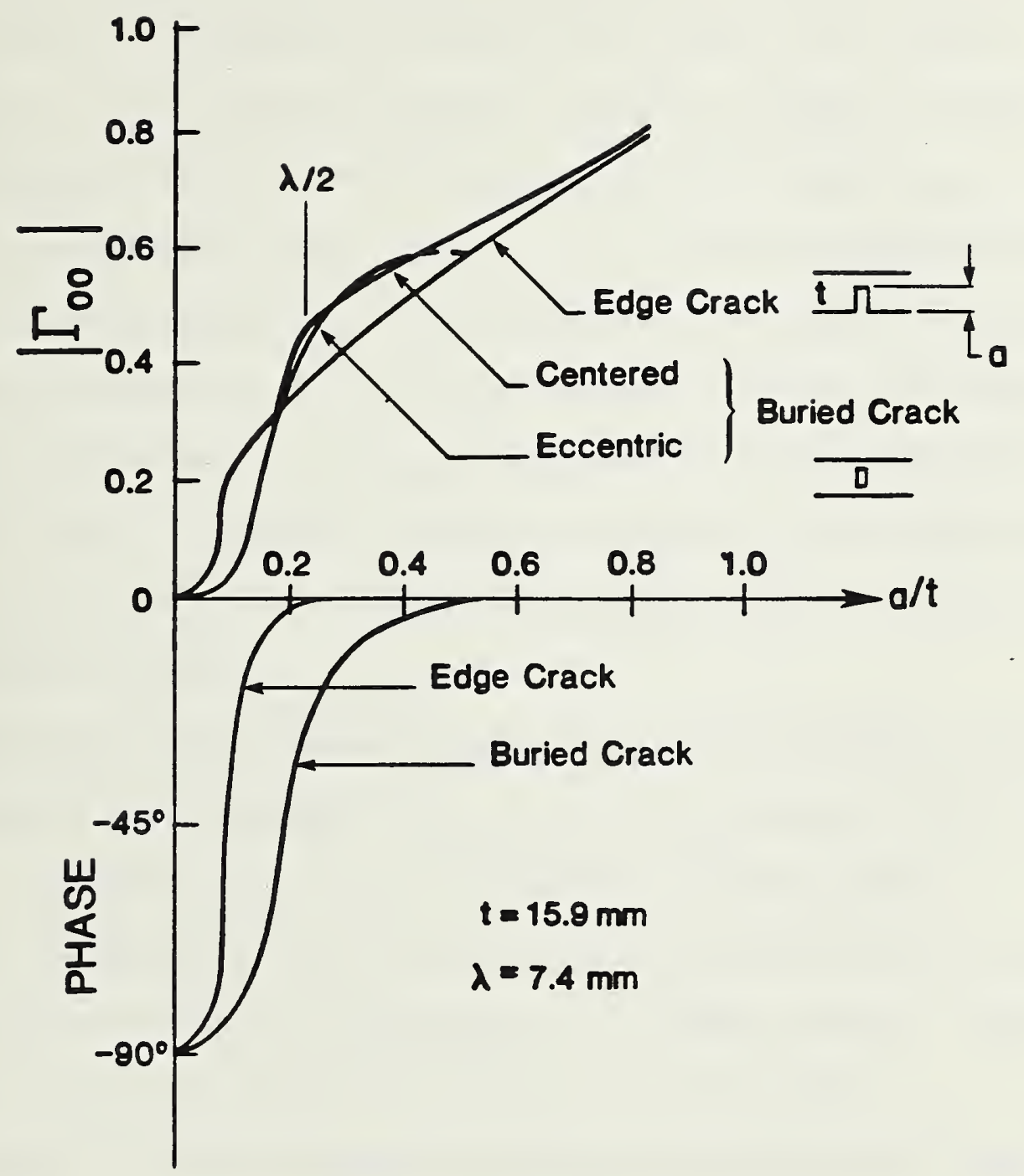

FLAW THROUGH-WALL DEPTH TO PLATE THICKNESS RATIO

Fig. 39 - Numerical results for the SH-wave plate mode scattering coefficient, $\Gamma_{00}$. 

It is also interesting to compare the behavior of $\Gamma_{00}$ with that of $\Gamma_{01}=\Gamma_{10}$ and $\Gamma_{11}$. The numerical results for $\Gamma_{01}$ and $\Gamma_{11}$ are shown in Figs. 40 and 41 . For relatively shallow, vertically oriented, surface cracks $(a \ll \lambda / 4)$ the magnitudes of the four scattering coefficients $\Gamma_{00}, \Gamma_{01}, \Gamma_{10}$, and $\Gamma_{11}$ are approximately equal. However, as the crack through-thickness dimension, $a$, is increased, $\Gamma_{00}$ and $\Gamma_{11}$ approach unity monotonically, but $\Gamma_{10}$ and $\Gamma_{01}$ reach a maximum at $a / t \sim 0.5$ and vanish for $a / t=1$. In addition, the signs of the coefficients $\Gamma_{01}$ and $\Gamma_{10}$ depend on which side of the plate contains the flaw. In contrast, the signs of $\Gamma_{00}$ and $\Gamma_{11}$ are always positive. These features can be used to classify flaws with respect to their relative position within the plate.

A comparison of the numerical results for buried, centered, and eccentric flaws shows that the magnitudes of $\Gamma_{01}, \Gamma_{10}$, and $\Gamma_{11}$ are not significant when a $<\lambda / 2$. By symmetry, $\Gamma_{01}=\Gamma_{10}=0$ for buried, centered cracks. Furthermore, the phases of $\Gamma_{00}, \Gamma_{10}, \Gamma_{01}$, and $\Gamma_{11}$ are $-90^{\circ}$ for very small through-thickness dimensions and approach $0^{\circ}$ for deeper cracks. Note that the phases for buried and surface cracks approach $0^{\circ}$ more rapidly.

An analysis of the above results indicates that significant results about the flaw characteristics and through-thickness dimensions can be obtained from the knowledge of the scattering coefficients, $\Gamma_{n m}$.

\subsection{Experimental Verification}

Although the main conclusions have been made on the basis of two-dimensional modeling, they have been found to be in very close agreement with experiments conducted on flaws of finite length and calibration standards. Thus, it can be expected that the general results outlined above can be used as a basis for rational inversion procedures for estimating flaw parameters from the available ultrasonic data. Because the magnitude and 



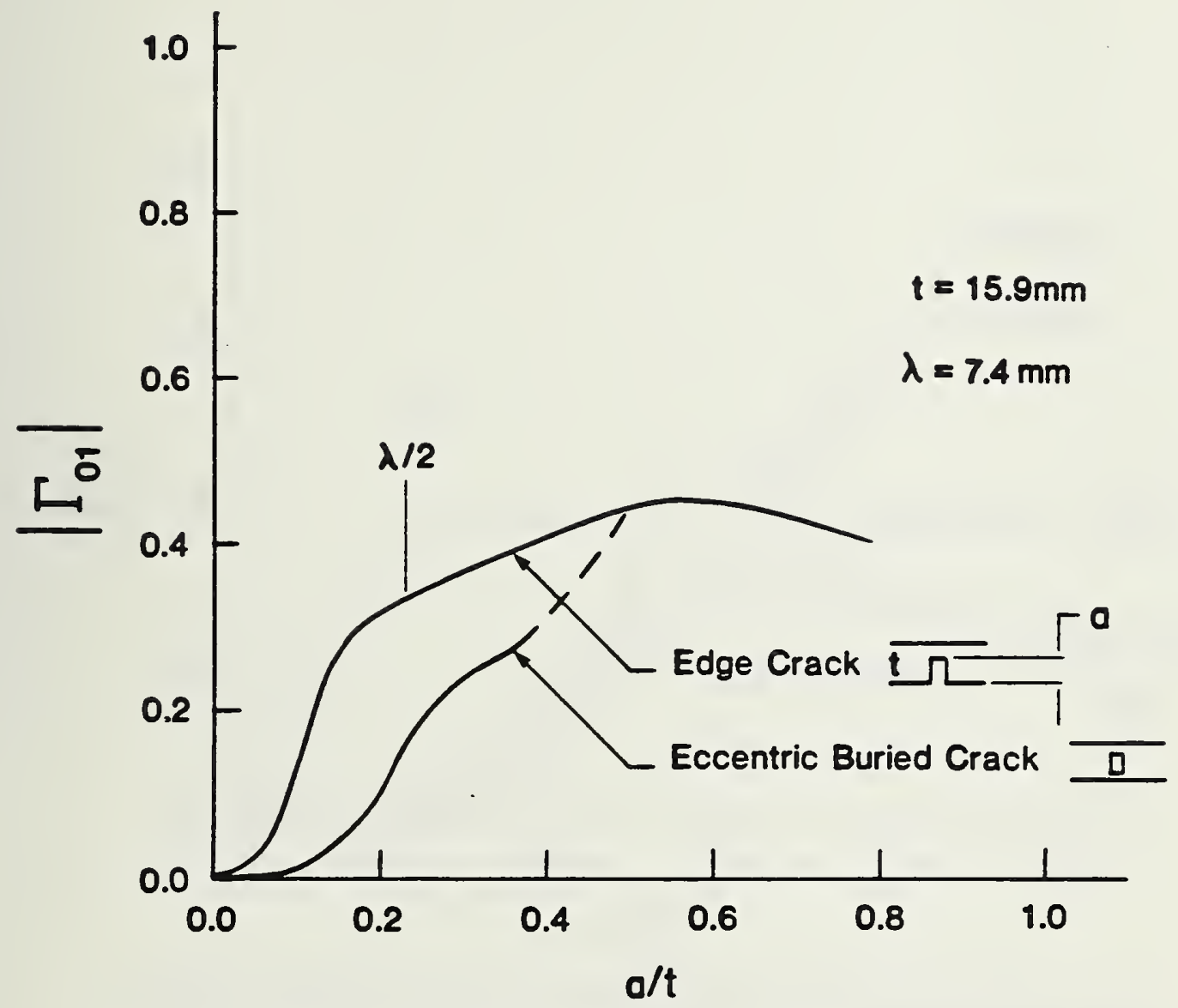

FLAW THROUGH-WALL DEPTH TO PLATE THICKNESS RATIO

Fig. 40 - Numerical results for the SH-wave plate mode scattering coefficient, $\Gamma_{01}$. 


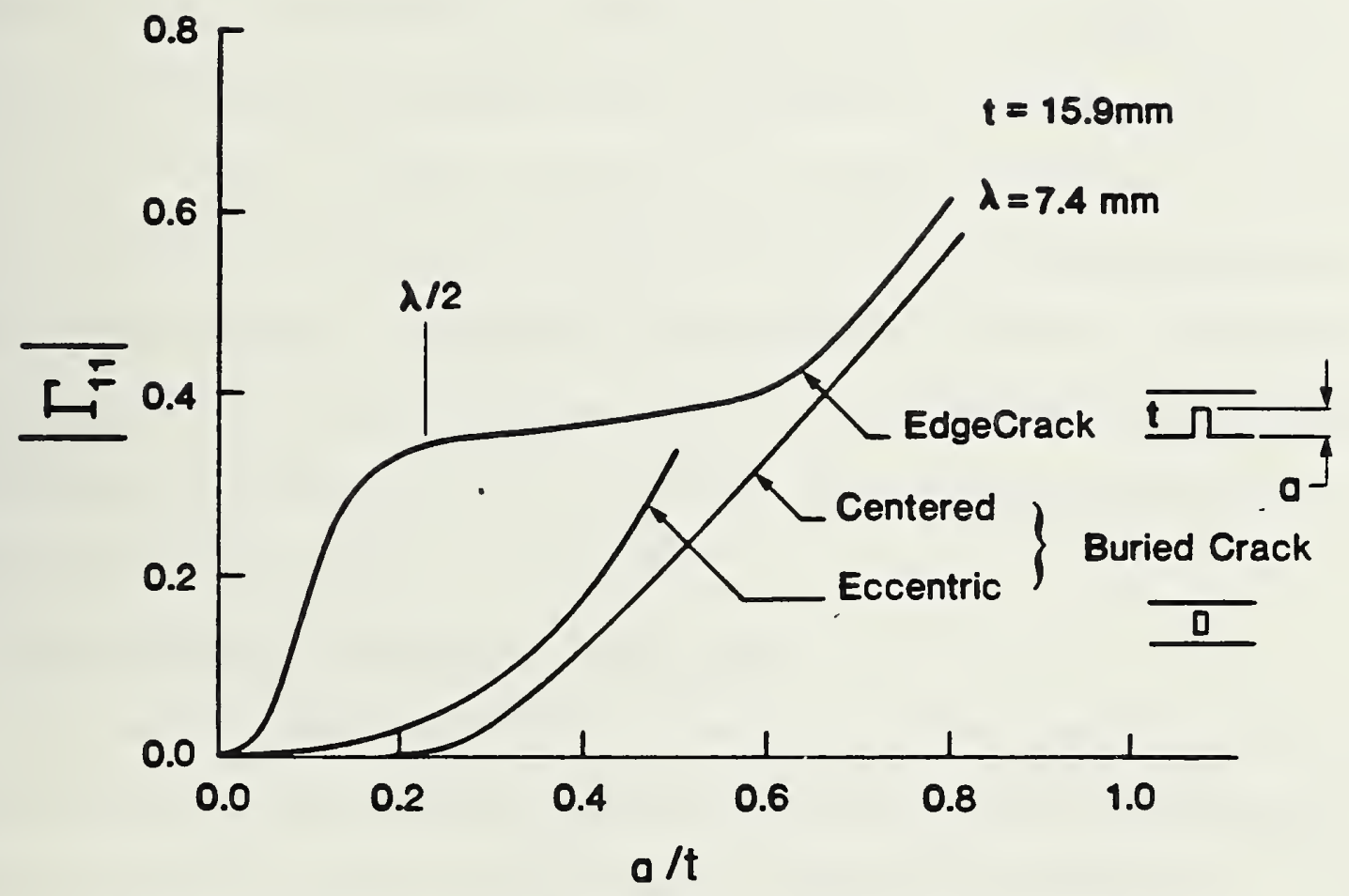

FLAW THROUGH-WALL DEPTH TO PLATE THICKNESS RATIO

Fig. 41 - Numerical results for the SH-wave plate mode scattering coefficient, $\Gamma_{1}$. 

phase of the scattered signals depend strongly on the position of the flaw within a plate, classification of flaws with respect to position must be performed first to select the appropriate set of sizing curves.

The theory of Eq. 83 has been directly compared with experimental results. Figure 42 shows a comparison of experimental results for surface notches in flaw-plate calibration specimens with a calculated response, using $a_{0}=b_{0}=$ $1, a_{1}=b_{1}=1.6, z=50 \mathrm{~mm},(1.97 \mathrm{in}) L_{t r}=210 \mathrm{~mm},(0.625 \mathrm{in}) \mathrm{f}=454 \mathrm{kHz}$, and $t=15.9 \mathrm{~mm}$. A slight correction for the effects of two-dimensional diffraction was made. Measurements were taken from the left ("triangles") and right ("squares") of the simulated flaws. It is seen that the experimental data adhere very closely to the calculated curve. Similar results were obtained for other classes of flaws. 62,63

\subsection{Detectability Limitations: Long Flaws}

In practice, the detectability of particular flaw types in a girth weld is not determined by ultrasonic-signal-to-electronic-noise considerations. Instead, the detectability limitations are determined primarily by: 1) flaw reflectivity as a function of flaw type, size, position, and orientation and 2) the presence of an ultrasonic background caused by transducer sidelobe radiation and surface irregularities. The ultrasonic background level caused by transducer sidelobe radiation can be reduced by using proper EMAT design techniques. However, the ultrasonic background level caused by topographical irregularities, for example, weld reinforcement, alignment mismatch ("high-low"), and "drop through," cannot be eliminated because, at low ultrasonic frequencies, it is not possible to resolve the flaw signals from the unwanted ultrasonic interferences.

The scattering of SH plate waves by certain surface irregularities (e.g., weld reinforcement and alignment mismatch) can be modeled analytically, but. 


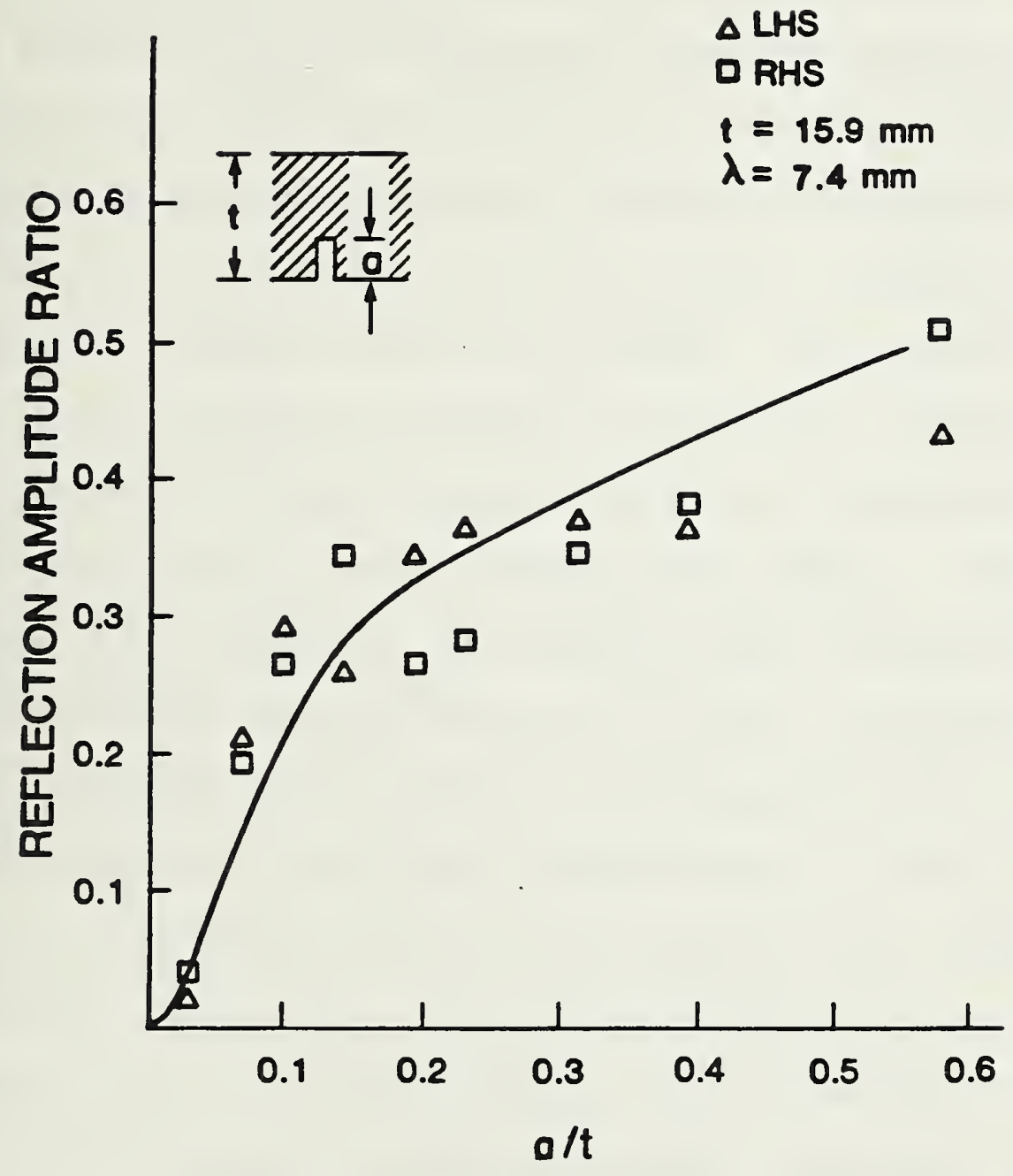

FLAW THROUGH-WALL DEPTH TO PLATE THICKNESS RATIO

Fig. 42 - Comparison of experimental and theoretical results for vertically oriented surface slots (flat-plate specimens 12-24). 

ultimately the results of such calculations must be verified experimentally. Here, a combined analytical-experimental approach is used to determine the principal factors limiting the sensitivity of the SH-wave EMAT system. For simplicity, the analysis is limited to the case of two-dimensional scatterers. Physically, this means that only flaws and surface irregularities longer than the transverse resolution width are considered. (Detectability of sharp flaws shorter than the ultrasonic beam width is discussed in Section 3.7).

To determine the reflectivities of very long, buried and surface flaws, a number of flat-plate calibration specimens were prepared. The specimens were made using 15.9-mm $(0.625-i n)$ thick pipeline steel (API-5LX-70) sections that were $1220-\mathrm{mm}(48-i n)$ long and $120 \mathrm{~mm}(4.7-i n)$ wide. To permit a systematic study of the effects of flaw position and orientation within a weld, four different categories of artificial flaws were used: 1) thin surface slots, 2) centered, buried slots, 3) off-center, buried vertical slots, and 4) off-center, canted slots. The surface and off-center slots were designed to simulate actual weld flaws (incomplete fusion, inadequate penetration, and cracks) in shielded-metal-arc (SMA) girth welds. The width of the slots was interitionally held below $0.4 \mathrm{~mm}(0.016 \mathrm{in})$, which is substantially less than the bulk shear wave wavelength $(7.4 \mathrm{~mm}(0.291 \mathrm{in})$ at $454 \mathrm{kHz}$, the inspection frequency). The artificial flaw dimensions are summarized in Table 5 , and the characteristic flaw dimensions, $A, B$, and $C$, are defined in Fig. 43.

The buried artificial flaws were made by filling the "V-grooves" in Fig. 43 using a combined tungsten-inert-gas (GTAW), gas-metal-arc (GMA) welding procedure. To simulate the effect of weld reinforcement, alignment mismatch ("high-low") and "drop through," additional flat-plate specimens were prepared. The maximum dimension for the three surface irregularities was $3.2 \mathrm{~mm}(0.126 \mathrm{in})$. 

Table 5 - Artificial Flaw Dimensions

\begin{tabular}{|c|c|c|c|c|}
\hline Specimen No. & $A(\mathrm{~mm})$ & $B(\mathrm{~mm})$ & $C(\mathrm{~mm})$ & Type of Slot \\
\hline $\begin{array}{l}1 \\
2 \\
3 \\
4 \\
5 \\
6 \\
7\end{array}$ & $\begin{array}{l}6.37 \\
5.88 \\
5.38 \\
4.88 \\
3.62 \\
2.38 \\
0.00\end{array}$ & $\begin{array}{l}1.58 \\
2.15 \\
1.95 \\
2.15 \\
3.12 \\
4.22 \\
5.48\end{array}$ & & $\begin{array}{l}\text { Off-center } \\
\text { vertical } \\
\text { slots } \\
\text { (buried) }\end{array}$ \\
\hline $\begin{array}{r}8 \\
9 \\
10 \\
11\end{array}$ & $\begin{array}{l}6.88 \\
6.58 \\
5.5 \\
3.62\end{array}$ & $\begin{array}{l}0.76 \\
2.50 \\
3.9 \\
7.45\end{array}$ & & $\begin{array}{l}\text { Centered } \\
\text { vertical } \\
\text { slots } \\
\text { (buried) }\end{array}$ \\
\hline $\begin{array}{l}12 \\
13 \\
14 \\
15 \\
16 \\
17 \\
18 \\
19 \\
20 \\
21 \\
22 \\
23 \\
24\end{array}$ & $\begin{array}{l}0 \\
0 \\
0 \\
0 \\
0 \\
0 \\
0 \\
0 \\
0 \\
0 \\
0 \\
0 \\
0\end{array}$ & $\begin{array}{l}0.50 \\
0.75 \\
1.03 \\
1.1 \\
1.5 \\
1.75 \\
2.18 \\
2.50 \\
3.18 \\
3.78 \\
5.2 \\
6.33 \\
7.47\end{array}$ & & $\begin{array}{l}\text { Vertical } \\
\text { edge } \\
\text { slots }\end{array}$ \\
\hline $\begin{array}{l}25 \\
26 \\
27 \\
28\end{array}$ & $\begin{array}{ll}\text { Full penetration weld } \\
6.07 & 0 \\
5.2 & 2 \\
3.33 & 5\end{array}$ & $\begin{array}{l}\text { (no defect) } \\
0.97 \\
2.55 \\
5.07\end{array}$ & $\begin{array}{l}0.75 \\
2.15 \\
4.3\end{array}$ & $\begin{array}{l}\text { Off-center } \\
\text { canted } \\
\text { slots }\end{array}$ \\
\hline
\end{tabular}





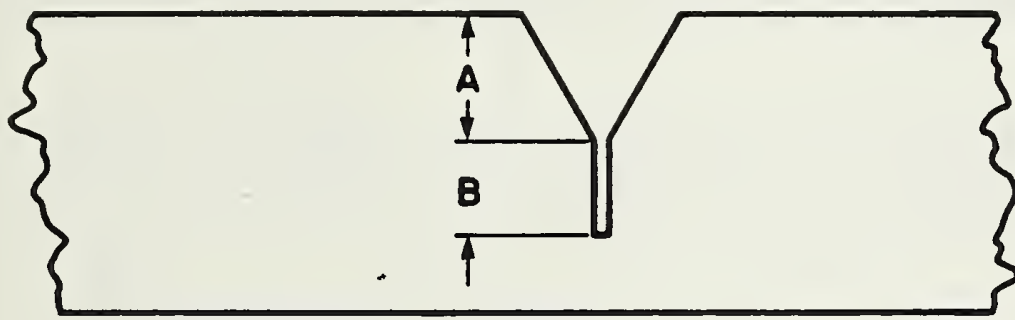

Vertical Flaw

a)

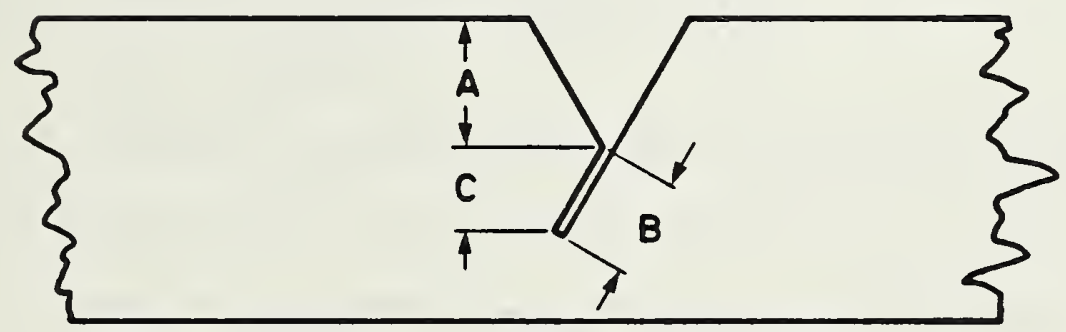

Canted Flaw

b)

Fig. 43 - Geometry of vertical slot (a) and canted slot (b) calibration specimens. The slot dimensions are summarized in Table 5. 

An examination of Eq. 83 shows that the reflection amplitude ratio, $R_{s}$, is strongly dependent on the flaw-to-EMAT spacing, $z$, and on the EMAT-to-EMAT spacing, $L_{t r}$. A similar result can be obtained for the reflection amplitude ratios of the three topographical irregularities: weld reinforcement, alignment mismatch ("high-low"), and "drop through." For example, in the case of weld reinforcement, the reflection amplitude ratio can be determined from:

$$
R_{S}=\frac{j \beta_{0}^{2} W_{w}{ }^{\top} \sum_{n=0}^{\infty} \sum_{m=0}^{\infty} a_{n} \exp \left(-j \beta_{n} z\right) \Lambda_{n m} b_{m} \exp \left[-j \beta_{m}\left(z+L_{t r}\right)\right]}{2 t \sum_{\ell=0}^{\infty} a_{\ell} b_{\ell} \exp \left(-j \beta_{\ell} L_{t r}\right)}
$$

with

$$
\left.\Lambda_{n m}=\frac{\sin \left(\beta_{n}+\beta_{m}\right) W_{W} / 2}{\left(\beta_{n}+\beta_{m}\right) W_{W}^{\prime} / 2}\left(\beta_{n} \beta_{m}{ }^{\varepsilon} \varepsilon_{m}\right)\right)^{\frac{1}{2}}
$$

where $w_{w}$ and $T_{w}$ represent the mean width and thickness of the weid reinforcement.

Assuming typical values of $W_{W}=20 \mathrm{~mm}(0.787), T_{W}=1.6 \mathrm{~mm}(0.063 \mathrm{in})$, $a_{0}=b_{0}=1, a_{1}=b_{1}=1.6$, and $a_{n}=b_{n}=0$ for $n \geq 2$, it can be shown that the value of $R_{s}$ does not exceed 0.12 when $t=15.9 \mathrm{~mm}(0.625 \mathrm{in})$ and $f=454$ $\mathrm{kHz} .{ }^{39}$ The above estimate has been verified experimentally using actual girth welds and flat-plate calibration specimens.

Figure 44 shows the variation of the amplitude reflection ratio as a function of the distance, $z$ (with $L_{t r}=210 \mathrm{~mm}, 8.27 \mathrm{in}$ ), obtained using a 15.9-mm (0.625-in) thick flat-plate calibration specimen designed to simulate a SMA weld reinforcement. It is apparent that the background signal ratio associated with the simulated weld reinforcement does not exceed 1.2 , in excellent agreement with the theory of Eq. 84. This observation is very significant, since the presence of the weld reinforcement is one of the principal factors in determining the ultimate sensitivity of this inspection system. 

Flaw-to-EMAT Distance, $z$, in

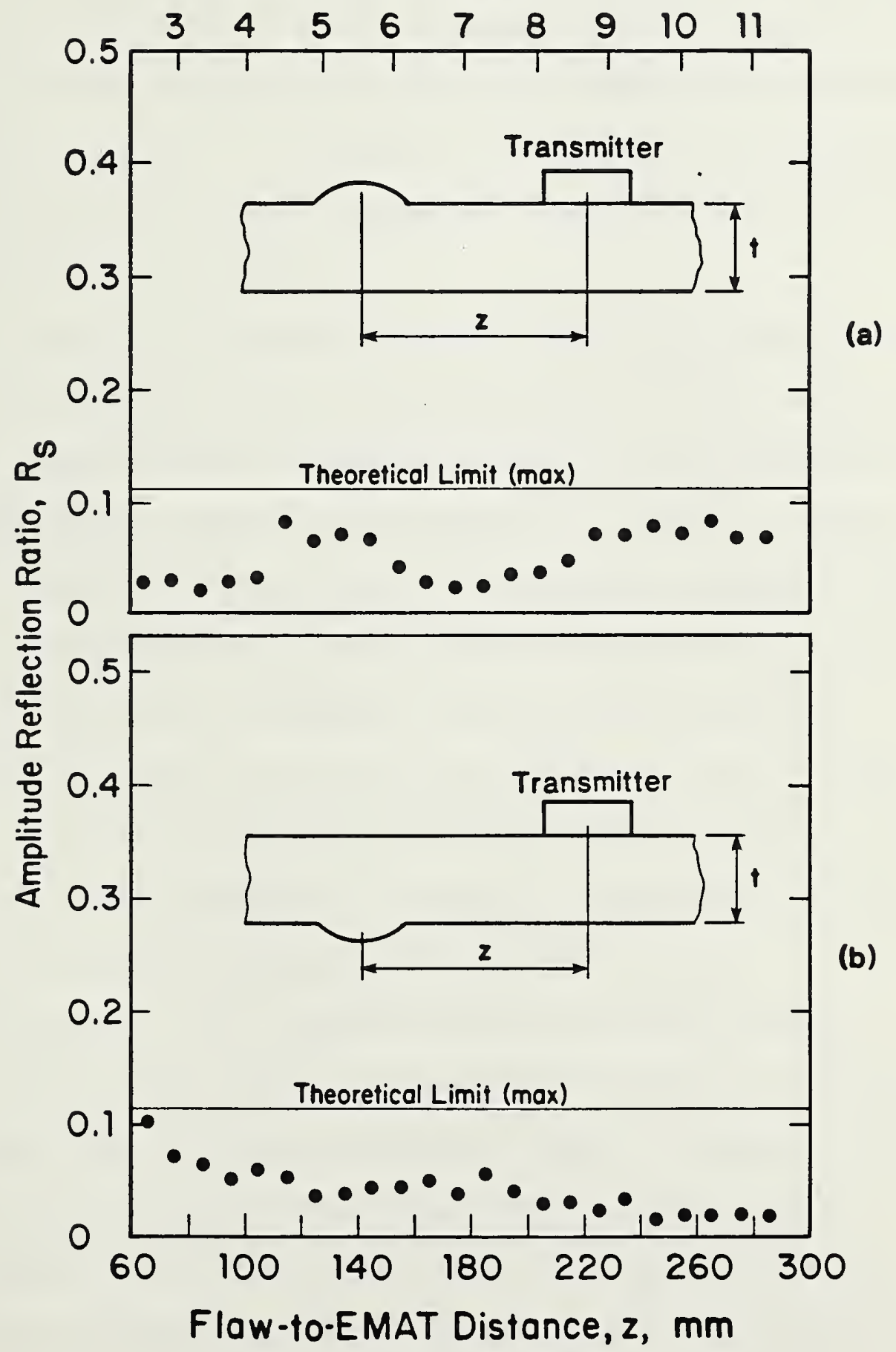

Fig. 44 - Detection of simulated weld reinforcement as a function of the transmitter-EMAT-to-welddistance, $z . L_{t r}=210 \mathrm{~mm}$. 

If vertically polarized plate waves (Lamb waves) were used to probe the weld, the reflected amplitude ratio associated with the weld reinforcement would be significantly larger. ${ }^{62,64}$ Physically, this is related to the fact that Lamb waves in a thick plate, which can support several propagating plate modes, are tightly bound to the surfaces and do not exhibit large displacements in the interior of a plate. ${ }^{58}$ In contrast, the $\mathrm{SH}_{0}$ plate wave displacements are uniform and the $\mathrm{SH}_{1}$, plate wave displacements exhibit only one null at the midplane of the plate (see Fig. 37).

Significant levels of ultrasonic background signals can also be caused by the presence of an alignment mismatch condition ("high-low") and "drop through" (at weld root). Figure 45 shows the measured values of the reflection amplitude ratio, $R_{s}$, at $454 \mathrm{kHiz}$ as a function of the distance, $z$, with $L_{t r}=210 \mathrm{~mm}$ (8.27 in) and $t=1.59 \mathrm{~mm}(0.023 \mathrm{in})$ for a $3-\mathrm{mm}(0.118-i n)$ alignment mismatch and a 3.2-mm (0.126-in) bead at the weld root. It is seen in Fig. 45 that the presence of a $2.8-\mathrm{mm}(0.110-i n)$ alignment mismatch condition causes a background signal level that is at least twice as large as that for a weld reinforcement or root bead of comparable thickness. For comparison, a plot of the coefficient $R_{S}$ as a function of $z$ is shown in Fig. 46 for a vertical surface slot 1.5-mm (0.059-in) deep and 100-mm (3.94-in) long.

An analysis using Eq. 83 in conjunction with calculated values of $\Gamma_{00}, \Gamma_{01}, \Gamma_{10}$, and $\Gamma_{11}$ shows that buried sharp flaws scatter less efficiently than surface sharp flaws of the same through-wall depth. Physically, this is caused by the fact that shallow surface flaws exhibit a larger mode III displacement jump (in Eq. 80) than buried flaws. This can be verified by using elastostatic expressions for $\Delta u_{x}$ in Eq. 82, which are valid when the ultrasonic wavelength is significantly longer than the flaw through-wall dimension a. ${ }^{40}$ In addition, shallow flaws lying near the midplane of the plate cannot efficiently scatter the $\mathrm{SH}$ plate waves and convert the $\mathrm{SH}_{1}$ plate waves to $\mathrm{SH}_{0}$ plate waves, and vice versa. This can also be verified directly 

Flow-to-EMAT Disfonce, $z$, in

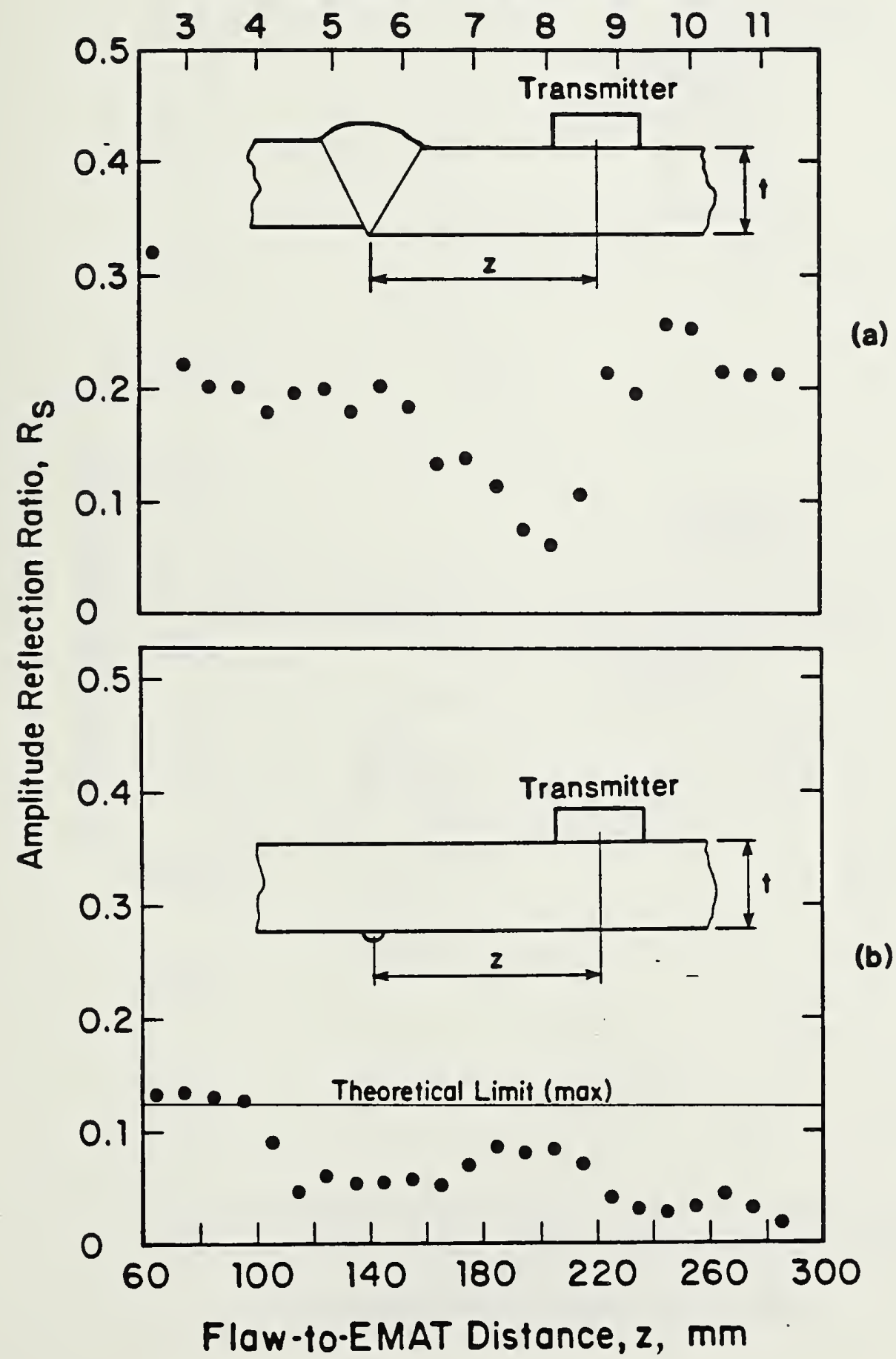

Fig. 45 - Detection of simulated 3-mm alignment mismatch (a) and "drop through" (b) as a function of the transmitter-EMAT-to-weld-distance, $z . L_{t r}=$ $210 \mathrm{~mm}$. 

Flow-to-EMAT Distance, $z$, in

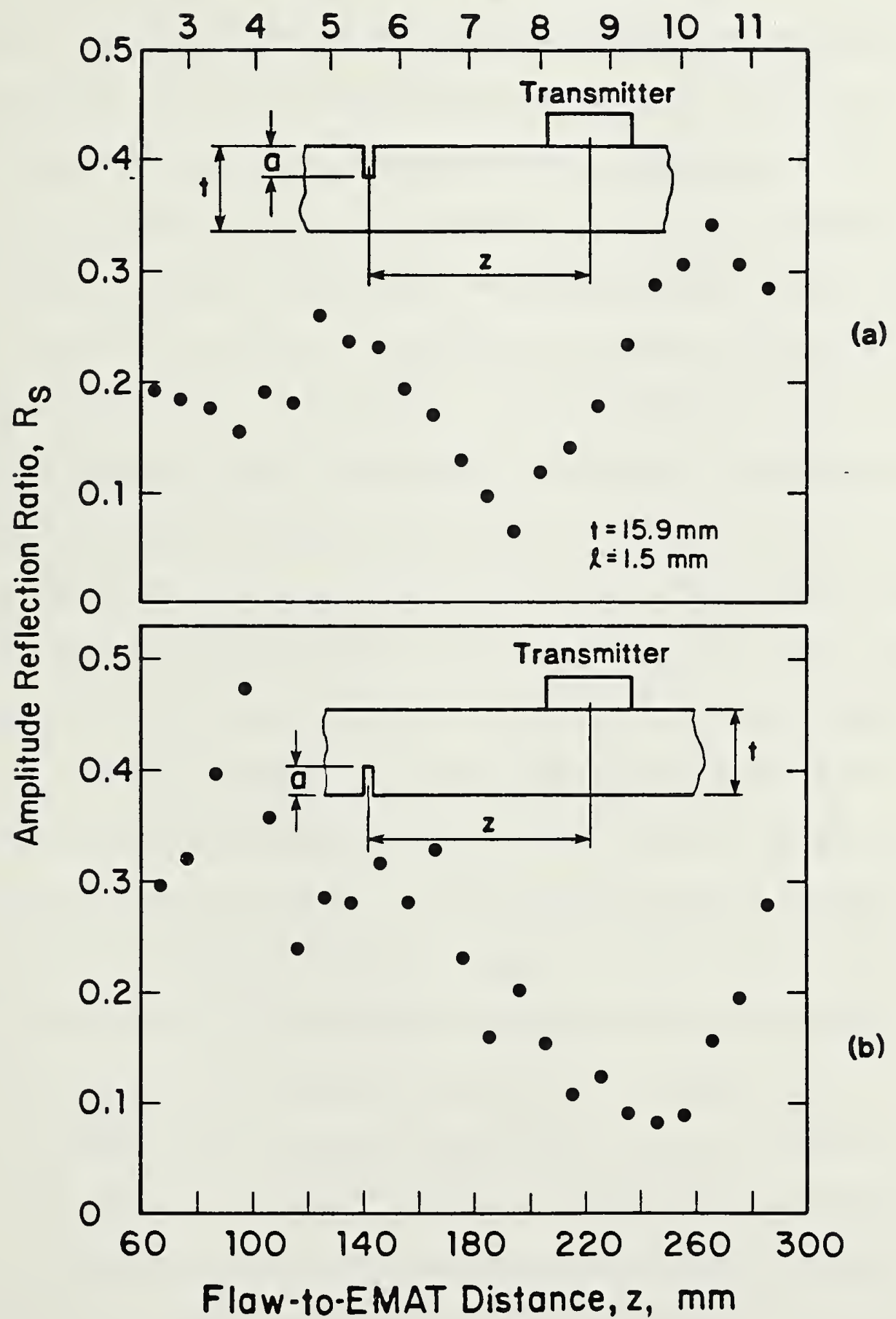

Fig. 46 - Detection of a 1.5-mm-deep surface slot as a function of the transmitter-EMAT-to-weld distance, $z$. (a) Slot in top surface of plate. (b) Slot in bottom surface of plate. $L_{t r}=210 \mathrm{~mm}$. (Flat-plate specimen 16.) 

by noting that $\Gamma_{01}, \Gamma_{10}$, and $\Gamma_{11}$ in Figs. 39 through 41 are negligible for such flaws. As a result, shallow buried flaws are generally more difficult to detect than surface flaws of similar dimensions.

It follows from Eqs. 83 and 84 and from the experimental data shown in Figs. 44 and 45 that the relative amplitudes and phases of the flaw and background ultrasonic signals are characteristically determined by the distances $z$ and $L_{t r}$ and by parameters describing the surface irregularity and the sources of the ultrasonic background. As a consequence, a particular selection of $z$ and $L_{t r}$ can result in significantly higher sensitivity to certain categories of flaws. For example, for certain EMAT-to-weld separation distances, significantly stronger reflections are obtained from flaws located near the root of the weld than from flaws located in other parts of the weld. ${ }^{39}$ As a result, in this particular configuration, flaws lying near the root of the veld may be easier to detect than flaws located in other parts of the weld. However, this difficulty can be corrected by devising a detection algorithm that utilizes ultrasonic data from different EMAT positions. In this way, a more uniform interrogation of the entire weld can be ensured. (The additional data are also needed for flaw characterization purposes.)

Equation 83 can be used to study the behavior of the flaw reflection signal as a function of the flaw position within the weld and the EMAT-to-EMAT and EMAT-to-weld separation distances, $L_{t r}$ and $z$. For simplicity, it can be assumed that the EMATs can only generate and receive signals carried by the $\mathrm{SH}_{0}$ and $\mathrm{SH}_{1}$ plate waves. This simplification is justified by the fact that the relative amplitudes $\left(a_{0}\right.$ and $\left.a_{1}\right)$ of the two plate waves are 1 and 1.6 , respectively. ${ }^{39}$ The relative amplitude of the $\mathrm{SH}_{2}$ plate wave $\left(\mathrm{a}_{2}\right)$ is approximately $0.3 .39,63$ 

To emphasize the influence of flaw position within the weld on the behavior of the flaw amplitude reflection ratio as a function of the separation parameters, $z$ and $L_{t r}$, it is convenient to fix the value of $L_{t r}$ such that $B_{0} L_{t r}=B_{1} L_{t r}$. This corresponds to the actual selection of the EMAT-to-EMAT spacing in the present experimental setup. Furthermore, it is convenient to assume that only three categories of sharp flaws need be considered: 1) surface flaws located near the top surface, 2) interior flaws located near the midplane, and 3) surface flaws located near the bottom surface of the weldment. The bottom surface corresponds to the surface opposite to the surface on which both EMATs are located.

Assuming that the surface flaws, which must be detected, are very shallow, it can be shown that the magnitudes of the scattering coefficients in Eq. 83, $\left(\Gamma_{00}, \Gamma_{11}, \Gamma_{01}\right.$, and $\left.\Gamma_{10}\right)$ are approximately equal. 39 However, the phases of the four coefficients are determined by which surface contains the flaw. If the flaw is located near the top surface, then the phases of $\Gamma_{00}, \Gamma_{01}, \Gamma_{10}$, and $\Gamma_{11}$ are identically equal. However, if the flaw is located on the bottom surface, then the phases of $\Gamma_{01}$ and $\Gamma_{11}$ are equal and opposite to the phases of $\Gamma_{01}$ and $\Gamma_{10}$. Finally, if the flaw is located near the midplane of the weldment, then $\Gamma_{01}, \Gamma_{10}$, and $\Gamma_{11}$ either vanish or are negligibly small, and only $\Gamma_{00}$ contributes strongly to the reflected signal.

The double summation in Eq. 83 can be evaluated graphically using a vector-diagram (phasor) representation. For simplicity, it is assumed here that the magnitudes of the four scattering coefficients $\left(\Gamma_{00} \Gamma_{01}, \Gamma_{10}\right.$, and $\Gamma_{11}$ ) are unity for the three categories of flaws. (This can be readily shown to hold in the elastostatic limit, which is valid when the flaw through-wall depth is a fraction of the ultrasonic wavelength, by using the linear elasticity solutions for $\Delta u_{x}$ to evaluate Eq. $82 .{ }^{65}$ ) Then, the amplitudes of 

the vectors (phasors) are determined by the SH plate wave amplitudes, $a_{n}$ and $b_{n}$, and the relative phases are determined by the differences between the SH plate wave propagation constants, $\beta_{n}$, and the EMAT-to-weld distance, $z$.

Figure 47 shows the evolution of the reflected signal amplitude for the three representative flaw categories as a function of the EMAT-to-weld distance, $z$. The vector diagrams for the case of surface flaws can be compared directly with the experimental results for surface slots shown in Fig. 46. For convenience, the vector diagrams are evaluated at six characteristic locations: 1) $\left.z=c, 2) z=\pi / 2\left(B_{0}-B_{1}\right), 3\right) z=\pi /\left(B_{0}-B_{1}\right)$, 4) $\left.z=3 \pi / 2\left(\beta_{0}-\beta_{1}\right), 5\right) z=2 \pi /\left(\beta_{0}-\beta_{1}\right)$, and 6$) z=5 \pi / 2\left(\beta_{0}-\beta_{1}\right)$. Physically, the above locations correspond to the following flaw-to-EMAT

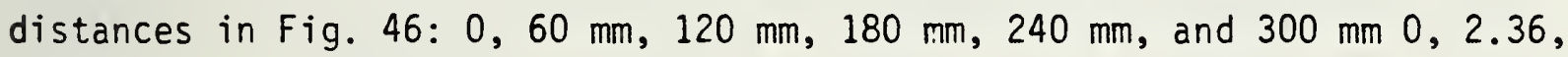
$4.72,7.09,9.45$, and $11.8 \mathrm{in})$. The vector diagrams are arranged vertically in the figure, such that the column on the extreme left corresponds to surface slots at the top surface, the center column corresponds to buried slots at the middle, and the column on the right corresponds to surface slots at the bottom surface.

In Fig. 47, the vectors corresponding to the four terms involved in evaluating the summation in Eq. 83 are represented by continuous lines. The resultant, corresponding to the actually observed signal amplitude, is represented by dotted lines.

A comparison of results shown in Fig. 47 with the experimental data in Fig. 46 show good quantitative agreement, except at $z=120 \mathrm{~mm}(4.72$ in) for the case of the surface slot in the top surface of the calibration specimen. The discrepancy at $z=120 \mathrm{~mm}(4.72 \mathrm{in})$ is attributed to the effect of signals carried by the $\mathrm{SH}_{2}$ plate wave, which has been excluded from the analysis. Similar results have been obtained for other calibration specimens, and the effect was observed for weld flaws. 39 
Figures 48 through 51 show the results of scattering measurements performed on calibration specimens containing surface and buried slots (see Table 12, Sec. 8) Figures 48 and 49 show the results for vertical slots at two particular EMAT-to-weld separation distances $z: 65 \mathrm{~mm}(2.56 \mathrm{in})$ and $100 \mathrm{~mm}(3.94 \mathrm{in})$. Figure 50 shows the results obtained for vertical slots whose centers coincide with the midplane of the calibration specimen. The cross section of a typical specimen is shown at the top of the figure). The dependence of the reflection amplitude ratio on flaw through-wall depth, a, is shown in the center of Fig. 50. It should be noted that this plot represents an average of results obtained from a wide range $(60-160 \mathrm{~mm}, 2.36-6.29 \mathrm{in})$ of separation distances, $z$. The actual behavior of the flaw-amplitude reflection ratio with the distance, $z$, is shown at the bottom of Fig. 50 . Characteristically, the amplitude reflection ratios in both plots (weld-crown up and weld-crown down) fall off monotonically with the distance, $z$. This effect is caused by the fact that flaws located near the midplane of the weldment are not strongly coupled to the antisymmetric SH plate waves. The monotonic decrease of the reflection amplitude with the distance, $z$, is caused by two-dimensional diffraction effects.

Figure 51 shows the results obtained for canted slots located above the midplane of the calibration specimens. The cross-sectional view of these calibration specimens is shown at the top of the figure. As in Fig. 50, the average amplitude reflection ratio is plotted as a function of the throughwall flaw depth, a. The evolution of the flaw signal with the distance, $z$, is shown at the bottom of the figure. In this case, note that the amplitude of the flaw signal does not decrease monotonically with the distance. This effect is caused by the fact that the canted flaws were not located at the midplane of the calibration specimen. As a result, they were weakly coupled to the $\mathrm{SH}_{1}$ plate waves, causing the interference phenomenon illustrated in Fig. 47. 


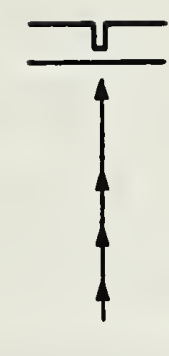

4

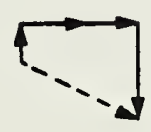

414

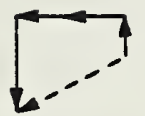

4

4

4

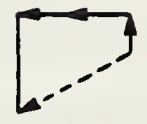

4

$\uparrow \downarrow 0 \mathrm{~mm}$

$60 \mathrm{~mm}$

$120 \mathrm{~mm}$

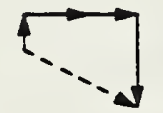

$180 \mathrm{~mm}$

$1+1$

$240 \mathrm{~mm}$

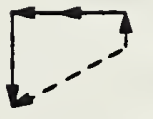

$300 \mathrm{~mm}$

Fig. 47 - Phasor diagram representation of the signals observed for different positions of the calibration slot within a plate at different transmitter-EMAT-to-weld-distances, $z$. $L_{t r}=210 \mathrm{~mm}$. 

The experimental flaw responses, shown in Figs. 48 through 51 , can be used in conjunction with the experimentally established ultrasonic background levels, shown in Figs. 44 and 45 , to determine the inherent flaw sensitivity limits of the low-frequency SH-wave EMAT system. The flaw sensitivity limits can be obtained approximately from the intersection points of the horizontal lines in Figs. 48 through 51, which correspond to the maximum expected background signal level and the measured flaw response curves. A more precise, statistical treatment would require additional experimental measurements and knowledge of the flaw distribution function. Thus, for vertically oriented flaws extending beyond the transverse resolution width of the ultrasonic beam, the minimum detectable flaw depths are $0.75 \mathrm{~mm}$ for vertical surface flaws and $2 \mathrm{~mm}(0.079$ in)for vertical buried flaws located the midplane of the weldment. For buried flaws, which are canted at $30^{\circ}$ with respect to the surface norma?, the corresponding minimum detectable through-wall flaw depth is approximately $3 \mathrm{~mm}(0.118 \mathrm{in})$.

\subsection{Detectability Limitations: Short Flaws}

Because the ultrasonic beam, which insonifies the weld region, has finite width, it is important to understand the relationship between the factors determining the transverse resolution of the SH-wave EMAT system and the inherent detectability limits for flaws of finite length. When the flaws are very long, their length, $\ell$, can be measured independently of the depth parameter, a, by scanning parallel to the welding direction. However, when the flaw is short, then it is not possible to determine $\ell$ and a unambiquously. Furthermore, the reflection amplitude ratio, $R_{S}$, is reduced (Eo. 83), thereby' increasing the minimum detectable flaw depth.

To understand the physical principles that determine the transverse resolution of the low-frequency SH-wave EMAT systen, it is necessary to take 



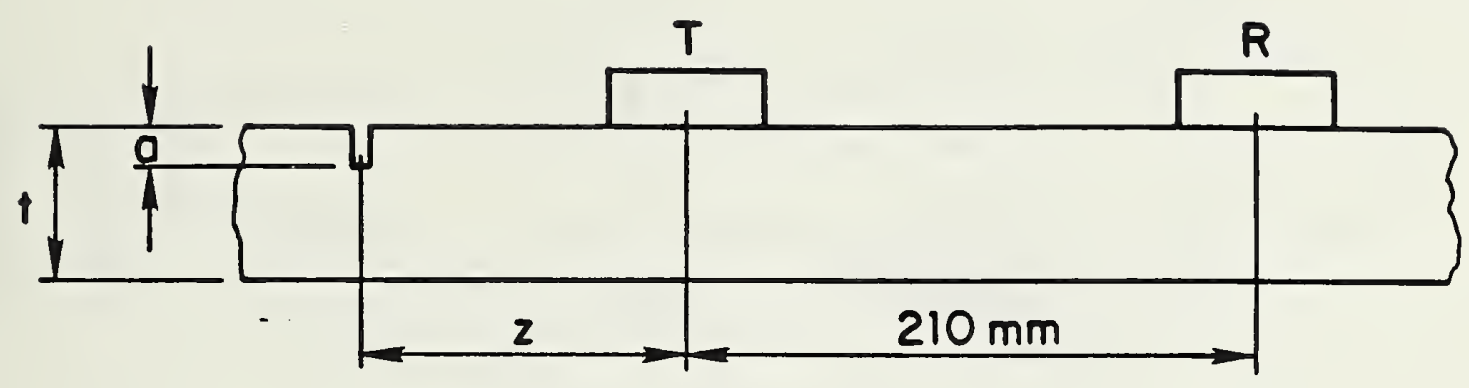

Flaw Depth, a, in
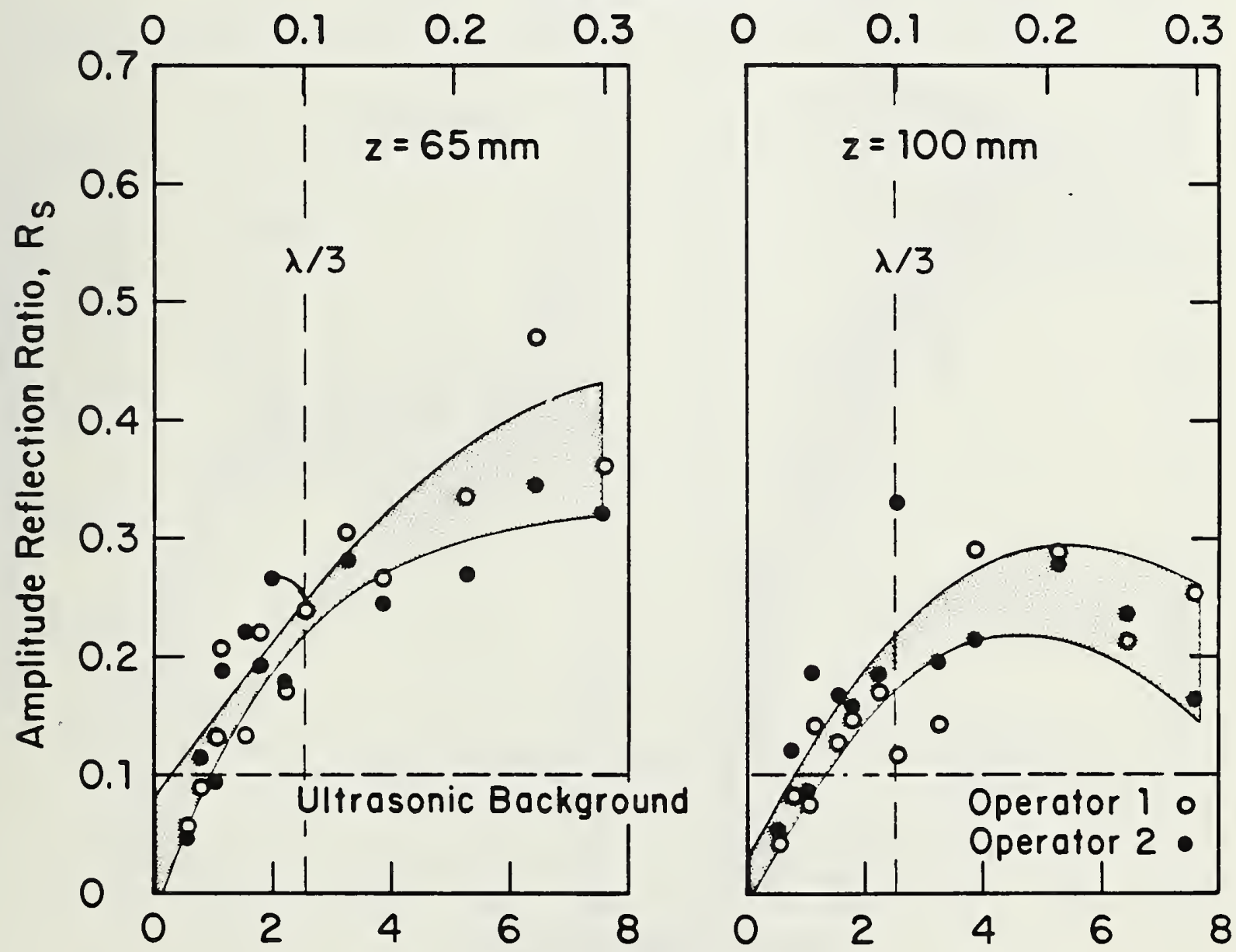

Flaw Depth, a, mm

Fig. 48 - Experimental calibration curves for sizing very long surface slots located on the top surface of plate for $z=65 \mathrm{~mm}$ and $z=100 \mathrm{~mm}$ (flat-plate specimens 12-24). 



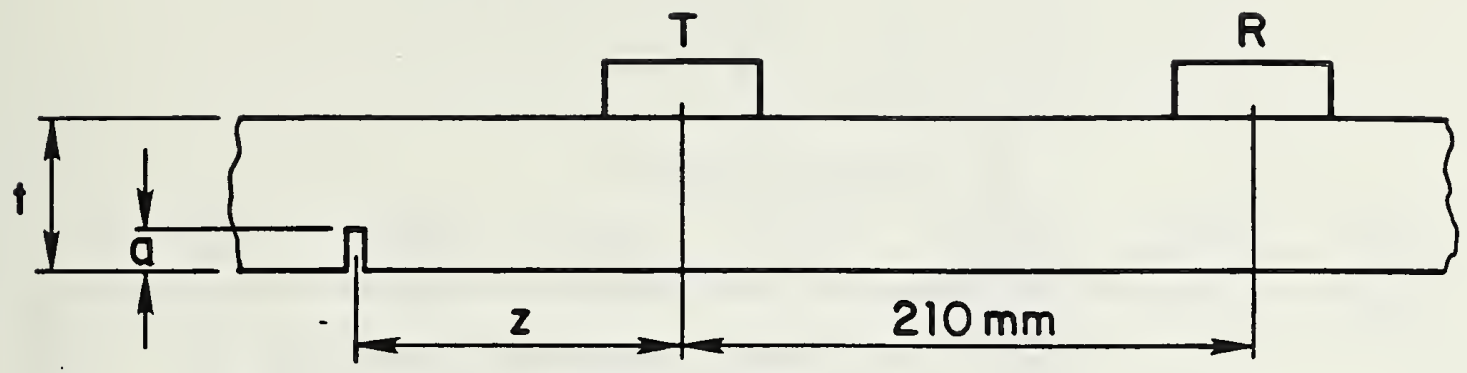

Flaw Depth, a, in
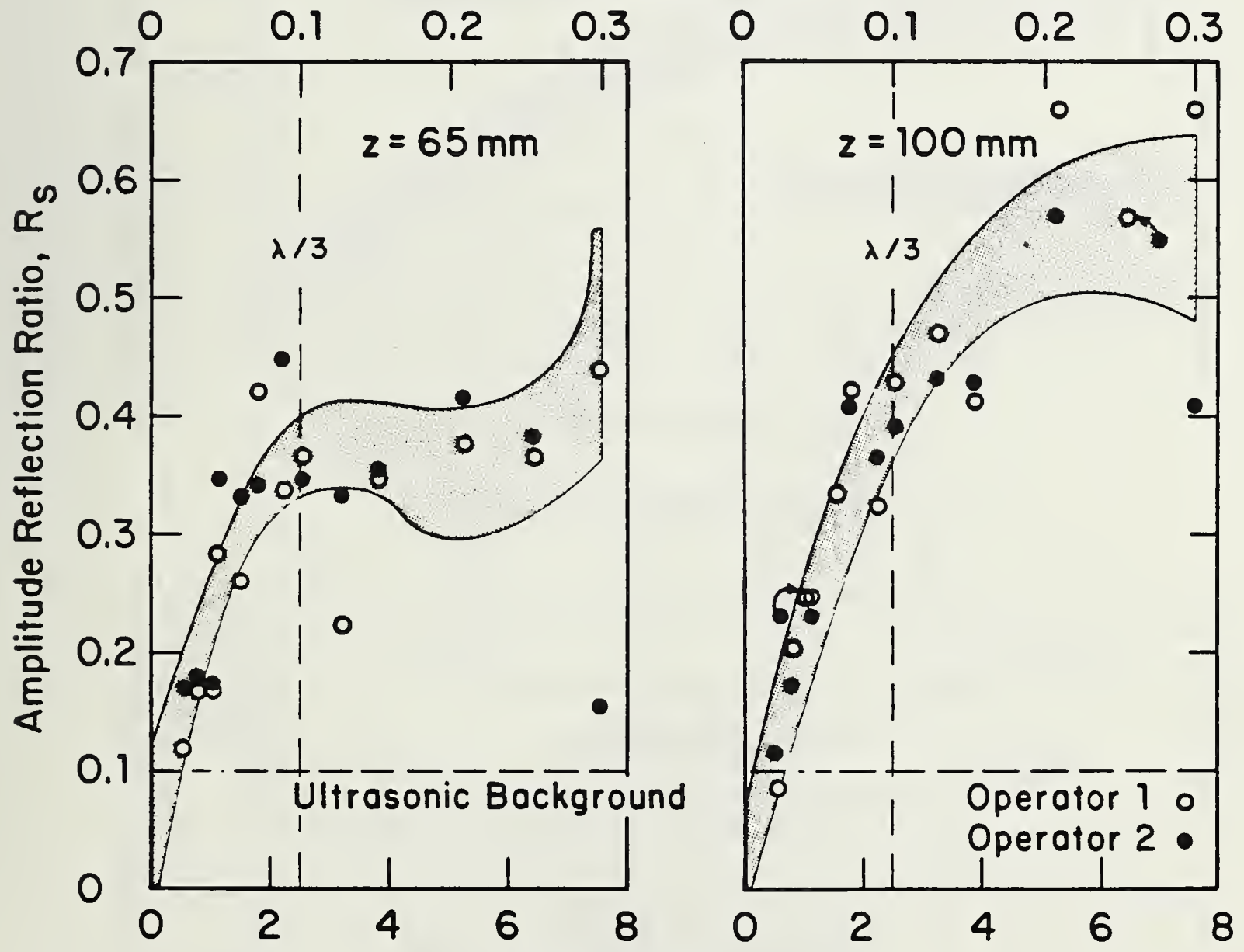

Flaw Depth, a, mm

Fig. 49 - Experimental calibration curves for sizing very long surface slots located on the bottom surface of a plate for $z=65 \mathrm{~mm}$ and $z=100 \mathrm{~mm}$ (flat-plate specimens 12-24). 



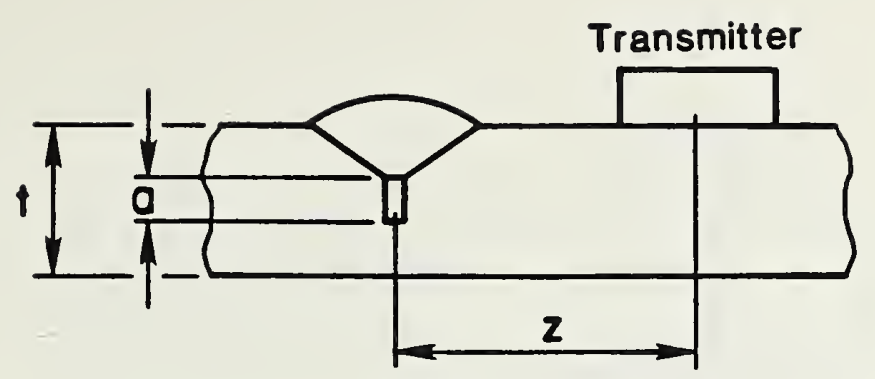

을

Flaw Depth, $a$, in

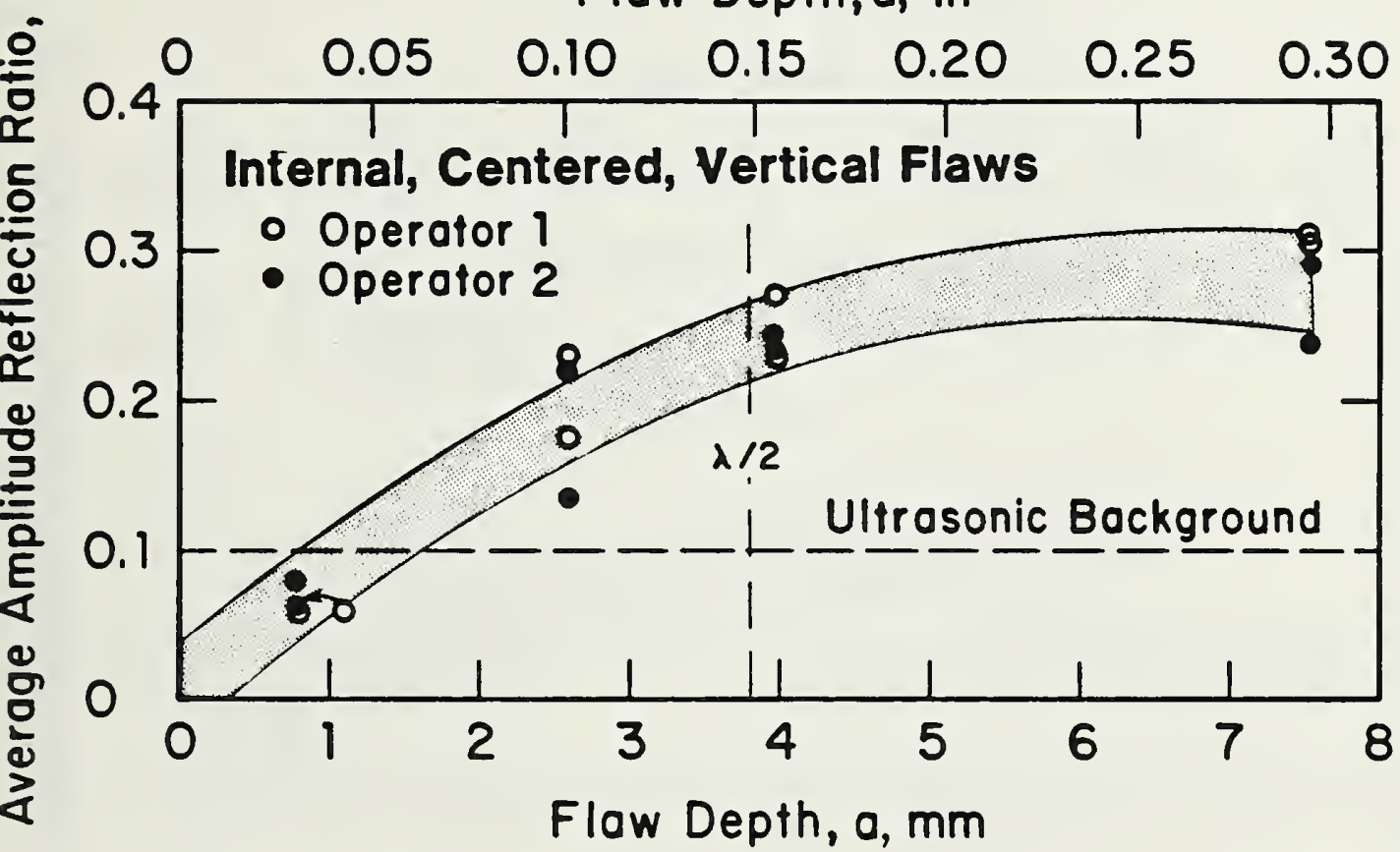

Flaw-ło-EMAT Distance, $z$, in

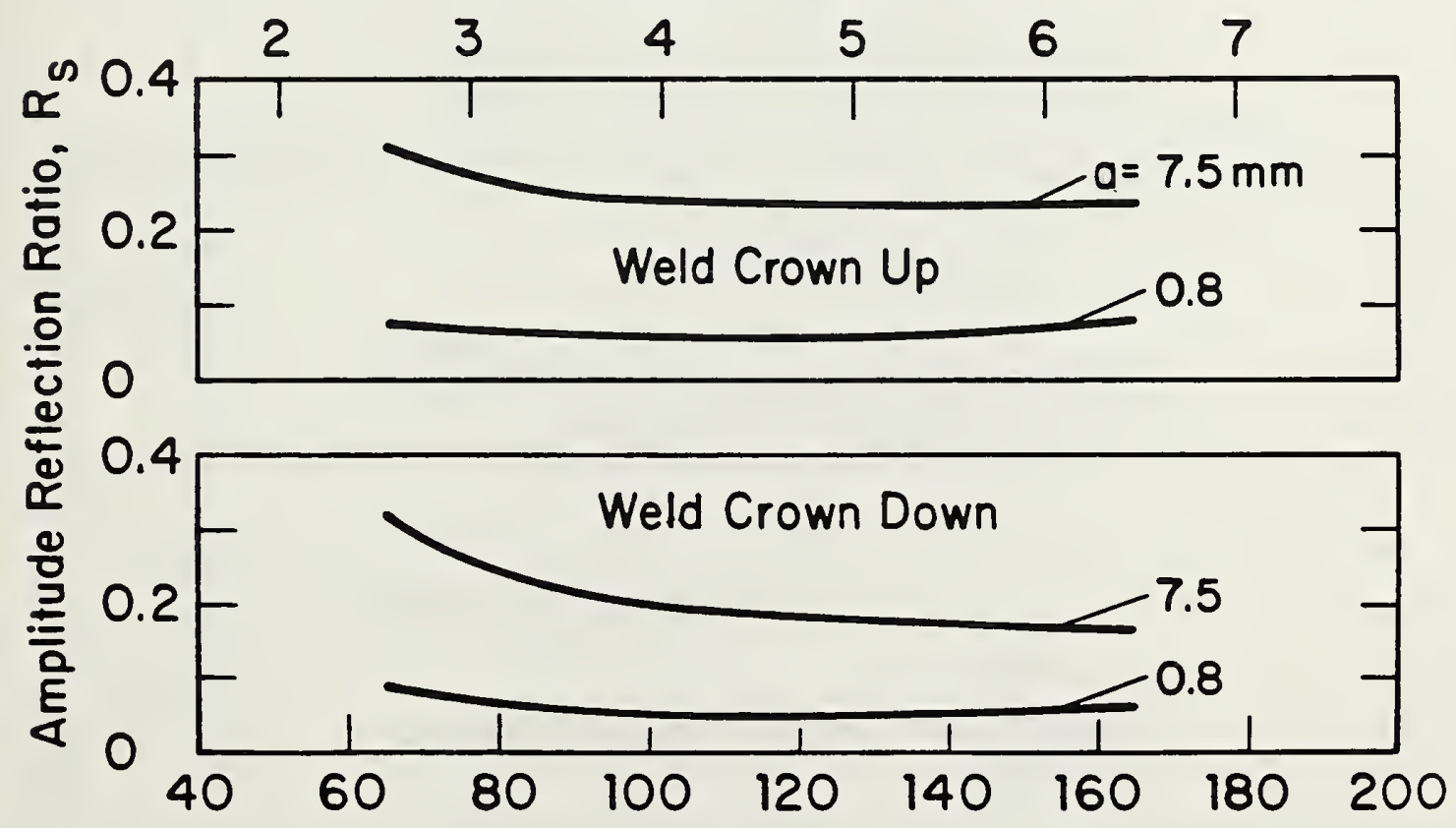

Flow-to-EMAT Distance, $z, \mathrm{~mm}$

Fig. 50 - Experimental calibration curve for sizing very long, vertical slots buried near the midplane of a plate and typical variations of the signal level

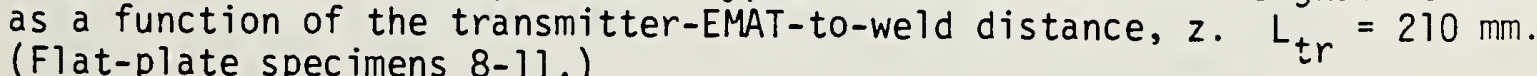




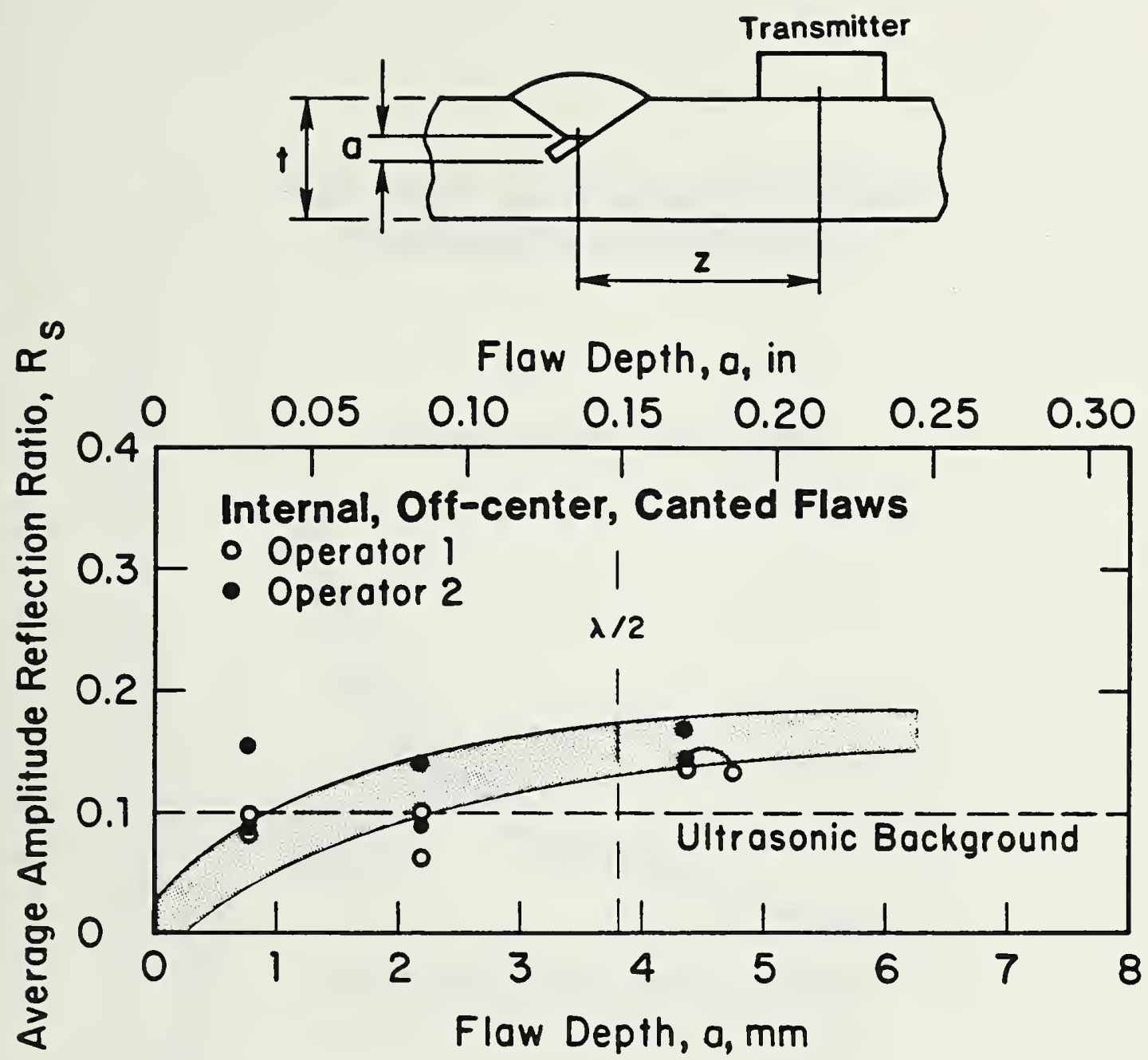

Flow-to-EMAT Distance, $z$, in

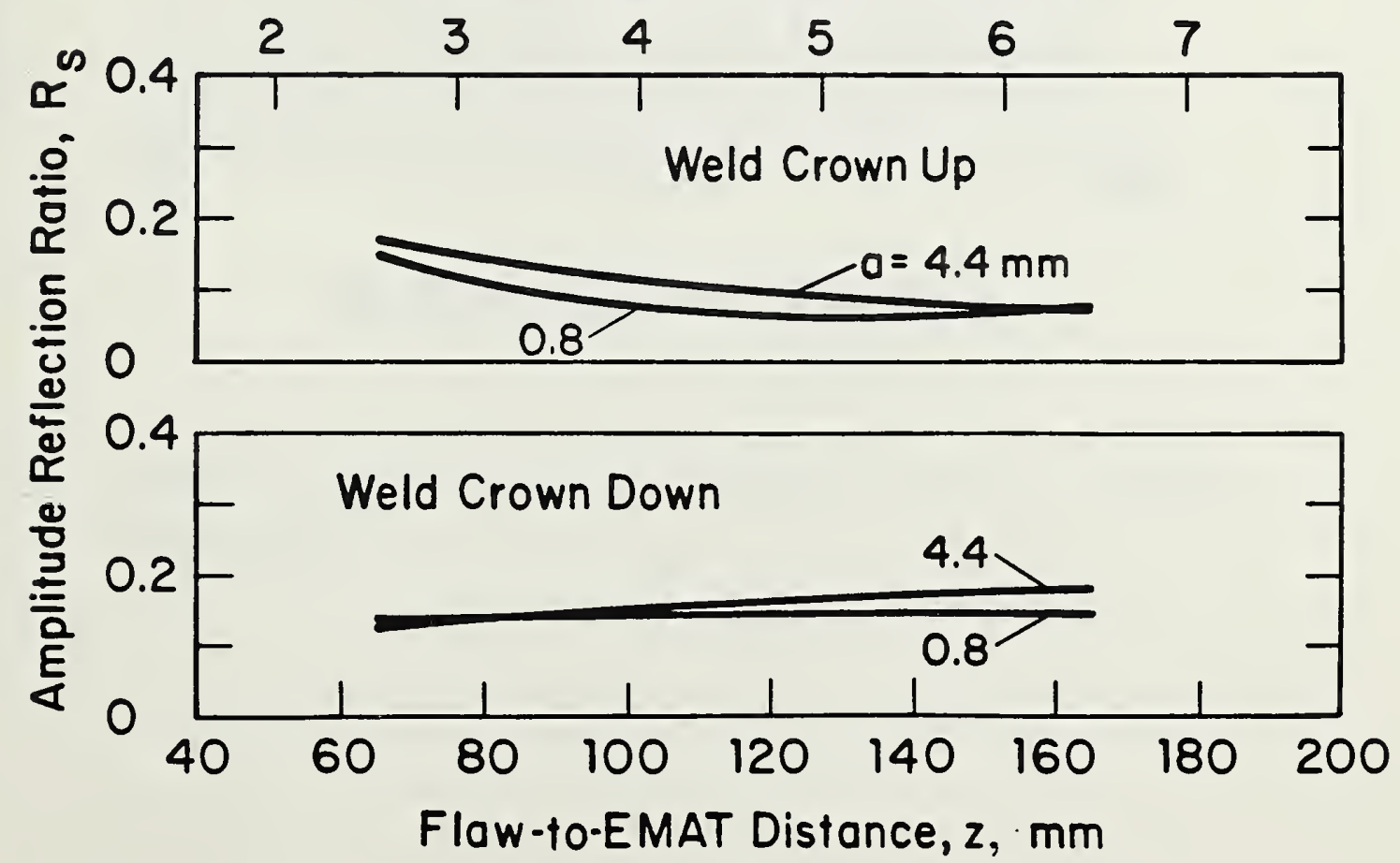

Fig. 51 - Experimental calibration curve for sizing very long canted $\left(30^{\circ}\right)$ slots buried near the midplane of a plate and typical variations of the signal level as a function of the transmitter-EMAT-to-weld distance, $z$. $L_{t r}=210 \mathrm{~mm}$. (Flat-plate specimens 25-23.) 



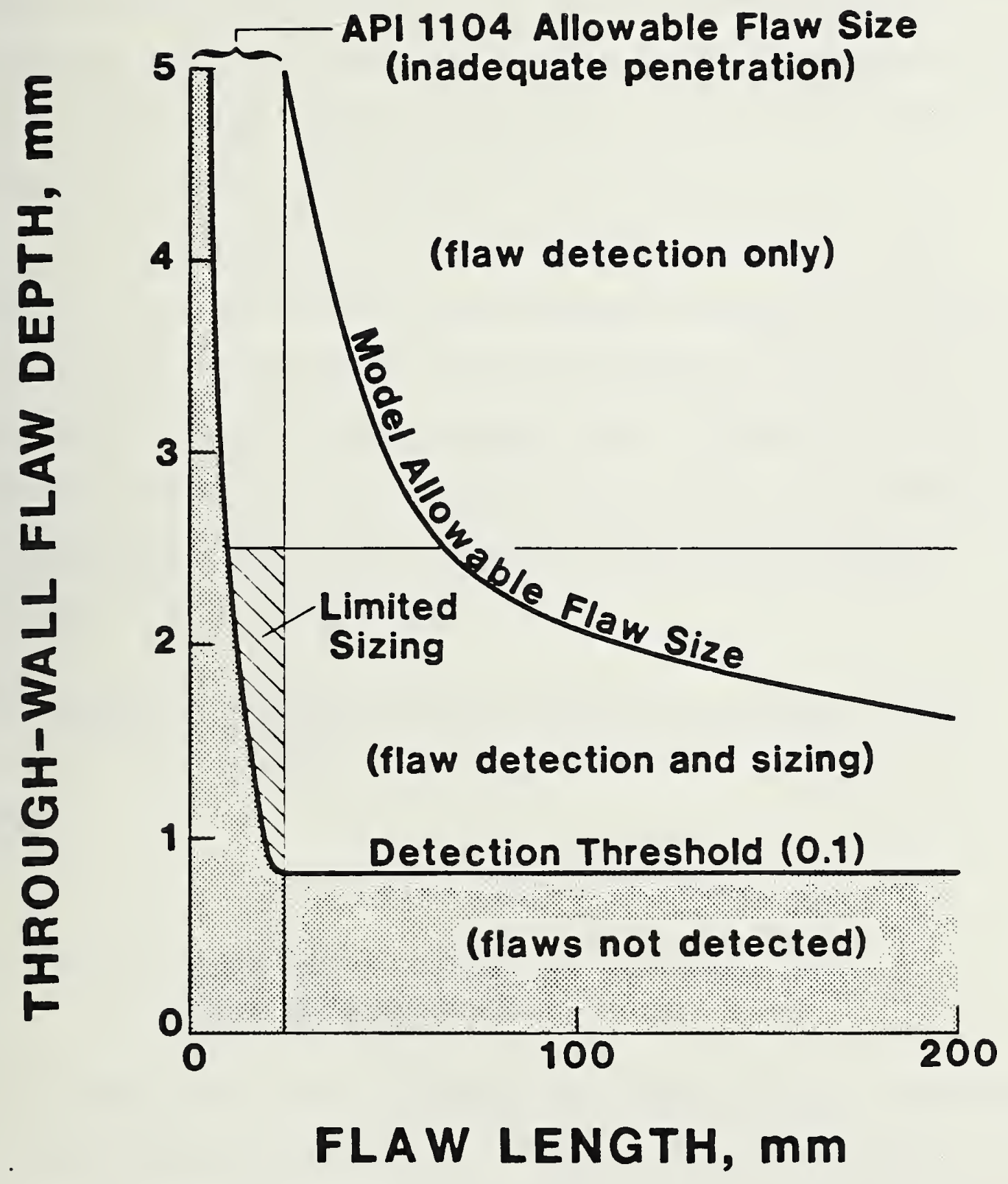

Fig. 52 - Summary of system performance characteristics for vertical surface flaws. 

into account the effects of two-dimensional diffraction. This can be accomplished by generalizing Eqs. 72 and 75 to three dimensions. If the transverse amplitude is Gaussian, as indicated by experimental evidence, then the ultrasonic velocity field distribution near the flaw is given by:

$$
\begin{aligned}
& v_{x}(x, y, z, \tau) \approx A_{n}(1+j)(2)^{-\frac{1}{2}}\left(\beta_{n} / 2 z\right)^{\frac{1}{2}} \cos (n \pi y / t) x \\
& \exp \left(-\beta_{n} w_{0}^{2} x^{2} / 2 \lambda z^{2}\right) \exp \left(-j \beta_{n} x^{2} / 2 z\right) \exp \left[-j\left(\beta_{n} z-\omega \tau\right)\right]
\end{aligned}
$$

where $w_{0}$ is the half-width of the ultrasonic beam near the transmitter EMAT. Corresponding expressions for the field quantities $T_{x z}^{(n)}$ and $T_{x y}^{(n)}$ can be obtained from Eq. 86 by applying Hooke's law. Equation 86 can now be directly substituted in Eq. 82. For elongated flaws, whose surface length, $\ell$, exceeds the through-wall depth a by a factor of 4 or more, and assuming $z \ll\left(z+L_{t r}\right)$, the result is:

$\Gamma=\frac{j \omega}{4\left(P_{1} P_{2}\right)} \sum_{n=0}^{\infty} \sum_{m=0}^{\infty} \exp \left(-j \beta_{n} z\right) a_{n} \Gamma_{n m}^{\prime} b_{m} \exp \left[-j \beta_{m}\left(z+L_{t r}\right)\right]$

where

$$
\Gamma_{n m}^{\prime} \approx \frac{\Gamma_{n m}}{j\left(\frac{\beta_{n}}{2 z}\right)^{\frac{1}{2}}\left[\frac{\beta_{m}}{2\left(z+L_{t r}\right)}\right]^{\frac{1}{2}}} \int_{\frac{-\ell}{2}}^{\frac{\ell}{2}} \exp \left(-j \beta_{n} x^{2} / 2 z\right) d x
$$

The integral in Eq. 88 is recognized as a Fresnel integral whose behavior can be deduced from the Cornu spiral. ${ }^{66}$ Specifically, the magnitude of the integral in Eq. 88 increases monotonically for $\ell<\left(4 \pi z / B_{n}\right)^{\frac{1}{2}} \approx(2 \lambda z)^{\frac{1}{2}}$ and then converges in an oscillatory manner to an asymptotic value. In writing Eq. 88, the effect of the exponential profile of the ultrasonic beam, expressed by the last term in Eq. 86, was neglected. This was done on purpose, to emphasize the fact that the transverse resolution of the SH-wave-EMAT system, $(2 \lambda z)^{\frac{1}{2}}$, 

increases at a significantly lower rate than the ultrasonic beam width, $2 \lambda t / \pi \omega_{0}$. From the above, it can be concluded that flaws that are longer than $(2 \lambda z)^{\frac{1}{2}}$, behave essentially as two-dimensional, planar flaws. At $454 \mathrm{kHz}$ and $z=50 \mathrm{~mm}(1.97 \mathrm{in}),(2 \lambda z)^{\frac{1}{2}} \approx 27.4 \mathrm{~mm}$ (1.078 in).

Because the form of the mode III displacement jump across the face of planar flaw is not generally known, it is not possible to obtain a closed-form expression for $\Gamma_{m n}^{\prime}$ as a function of all parameters describing a planar flaw. However, when the flaw length, $\ell$, is sufficiently short, it is possible to assume that the phases and amplitudes of the incident ultrasonic fields are constant over the face of the flaw. This assumption is equivalent to the Fraunhofer approximation. It is then possible to calculate the scattering coefficient $\Gamma_{00}$ approximately, it alone determines the detectability of planar flaws located near the midplane of the plate.

To evaluate $\Gamma_{00}$ using Eq. 82, a well-known, closed-form elastostatic solution, can be used that is valid for elliptical, buried cracks of length $\ell$ and "through-wall depth a: 50

$$
\int_{\frac{1}{2} S_{F}} \Delta u_{1}^{(0)} d S \approx \frac{8 \pi l a^{2}}{3 G \Phi\left(1-a^{2} / l^{2}\right)} \frac{1-v}{2-v} T_{1}^{(0)}
$$

where $v$ is the Poisson's ratio and $\Phi\left(1-a^{2} / \ell^{2}\right)$ is the complete elliptical integral of the second kind.

Equation 89 can be directly substituted into Eq. 82 . The result is:

$$
\Gamma_{00} \approx-j \frac{8 \pi^{2}}{3} \cdot \frac{1-v}{2-v}\left(\frac{\beta 0}{2 z}\right)^{\frac{1}{2}}\left[\frac{\beta_{0}}{2\left(z+L_{t r}\right)}\right]^{\frac{1}{2}} \frac{l a^{2}}{\lambda t \Phi\left(1-a^{2} / \ell^{2}\right)}
$$

The corresponding reflection amplitude ratio, $P_{s}$, for an elliptical flaw, buried near the midplane of the plate and canted at an angle, $\theta_{n}$, with respect to the plate surface normal, is given by: 


$$
R_{e 11}\left(z, L_{t r}\right) \approx \frac{-(1+j) \frac{8 \pi^{2}}{3} \frac{1-v}{2-v} \frac{l \partial^{2} \cos ^{2} \theta_{n}}{\lambda t(2 \lambda z)^{\frac{n}{2}} \Phi\left(1-a^{2} / \ell^{2}\right)} a_{1}^{2} \exp \left(-j \beta_{0} z\right) \exp \left[-j \beta_{0}\left(z+L_{t r}\right)\right]}{\sum_{n=0}^{1} a_{n} b_{n}\left[\beta_{n} L_{t r} / \beta_{0}\left(z+L_{t r}\right)\right]^{\frac{1}{2}} \exp \left(-j \beta_{n} L_{t r}\right)}
$$

For the range of experimental parameters stated previous $7 y$, and $\ell \sim 25 \mathrm{~mm}$ $(0.984 \mathrm{in}) \mathrm{a}=2.5 \mathrm{~mm}(0.098 \mathrm{in})$, the coefficient $R_{s}$ is 0.21 . This value is in excellent agreement with the experimentally observed value in Fig. 50. Thus, Eq. 19 can be used to estimate the detectability of sharp flaws that are shorter than $(2 \lambda z)^{\frac{1}{2}}$. To estimate the detectability of shallow surface flaws, the contribution of the scattering coefficients $\Gamma_{01}, \Gamma_{10}$, and $\Gamma_{11}$ must be included in the estimate of $R_{s}$. Since the function $\Phi\left(1-a^{2} / l^{2}\right)=\pi / 2$ at $a=\ell$ and tends monotonically to unity as $a / l$ tends to zero, the reflection amplitude ratio, $R_{s}$, for elongated, sharp flaws is directly proportional to $\&$ when $4 a<\ell<(2 \lambda z)^{\frac{1}{2}}{ }^{67}$ Equation 91 does not include a dependence on the beam width, $2 w_{0}$. This follows from the fact that the flaw is insonified by the far-field of the transmitter EMAT. This assumption holds when $\left(2 z / \beta_{0} w_{0} 2\right)^{2}$ > 1 , which is clearly satisfied when $z>50 \mathrm{~mm}(1.97 \mathrm{in}), \lambda=7.4 \mathrm{~mm}$, $(0.291$ in $)$ and $w_{0}=7.5 \mathrm{~mm}(0.295 \mathrm{in})$.

\subsection{System Performance Limitations and Standards of Acceptability}

The model-based results for flaws whose length cannot be resolved can be combined with the empirically derived results for two-dimensional, planar flaws that are longer than $(2 \lambda z)^{\frac{1}{2}}$, to construct a phenomenological model defining the limits of detectability for surface and buried flaws. It is convenient to present the detectability limits graphically with flaw depth, a, plotted as the $y$-axis and the flaw length, $\ell$, plotted as the $x$-axis. The detectability limits can then be directly related to current flaw acceptance standards based on the industry-wide such as those in Section 6 of API-1104 

flaw acceptance criteria based on fitness-for-service considerations. ${ }^{4,68}$ This is done in Figs. 52 and 53 for vertically oriented surface and buried planar flaws.

In Figs. 52 and 53 the allowable flaw sizes are defined by length vs. through-wall depth curves, except for Section 6 of the API-1104 Standard, ${ }^{2}$ which does not address the flaw through-wall depth. The allowable flaw-size curves were based on a yielded-ligament fracture mechanics model using arbitrary, but realistic, parameters to characterize the toughness of the materials and to estimate the stresses acting on the girth welds. ${ }^{68}$ These curves are used here only to illustrate the relationships between the capabilities of the SH-wave EMAT inspection system and different flaw acceptance criteria.

In Figs. 52 and 53 , the range of flaw sizes that can be detected is bounded by the heavy solid lines. The range of flaw sizes that cannot be detected is indicated by the shaded areas. It is interesting to note that the maximum flaw length allowable by Section 6 of the API-1104 Standard coincides with the resolution limit of the SH-wave EMAT system, $(2 \lambda z)^{\frac{1}{2}}$ when $z=50 \mathrm{~mm}$ $(1.97 \mathrm{in})$ and the operating frequency is approximately $500 \mathrm{kHz}$. Shorter flaws are considerably harder to detect. As a consequence, the $454-\mathrm{kHz}$ SH-wave EMAT system may also be appropriately used in conjunction with conventional, workmanship-based acceptance criteria, such as Section 6 of the API-1104 Standard.

It is interesting to observe (Figs. 48 and 49 ) that the amplitude of ultrasonic signals backscattered from surface flaws increases monotonically in the range $0<a<\lambda / 3$ and reaches a maximum when $a \sim \lambda / 3$. On the other hand, in the case of buried flaws (Fig. 50), the amplitude of the backscattered signals increases monotonically in the range $0<a<\lambda / 2$ and reaches a maximum value at $a \sim \lambda / 2$. When the above limits are exceeded, the interaction of the incident ultrasonic fields with the flaw becomes very complicated and the 



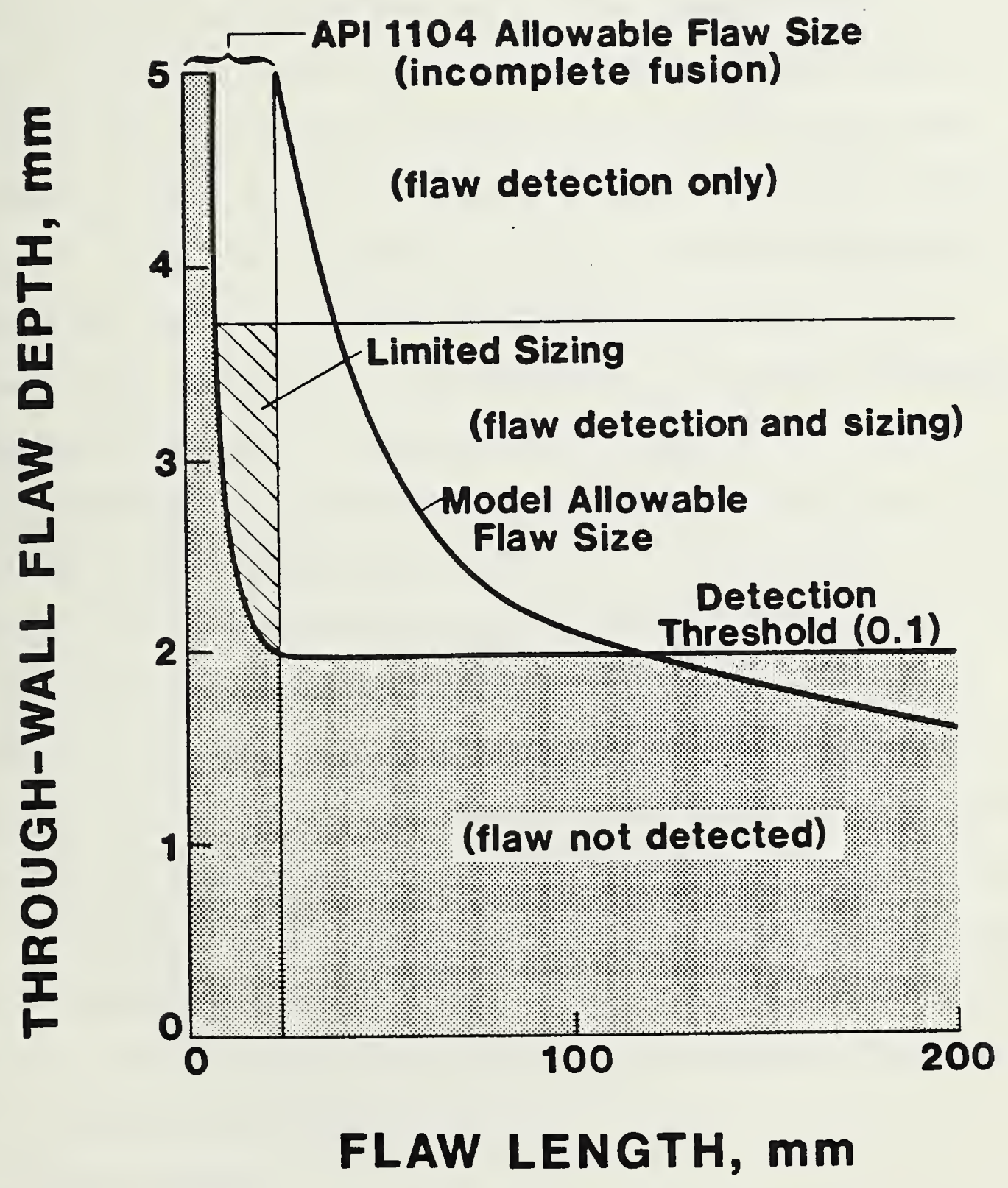

Fig. 53 - Summary of system performance characteristics for buried planar flaws. 

low-frequency (long-wavelength) inversion algorithms for flaw sizing become inoperative. Thus, $a<\lambda / 3$ for surface $f l$ aws and $a<\lambda / 2$ for buried flaws define the maximum flaw depths that can be sized. Deeper flaws can still be detected reliably, but cannot be sized unambiguously. These limits are indicated in Figs. 52 and 53 by horizontal lines parallel to the $x$-axis. The hatched areas in Figs. 52 and 53 represent a range of detectable flaws when the length $\ell<(2 \lambda z)^{\frac{1}{2}}$ and, therefore, cannot be determined independently of the through-wall depth, a. (At $454 \mathrm{kHz}$ the shear wavelength, $\lambda$, is approximately $7.4 \mathrm{~mm}(0.291 \mathrm{in})$. For comparison, the average through-wall depth of one SMA weld pass is approximately $3 \mathrm{~mm}, 0.118 \mathrm{in}$ ).

A consideration of the performance characteristics summarized in Figs. 52 and 53 reveals that the operational wavelength, $\lambda$, and the EMAT-to-weld distance, $z$, must be selected appropriately to assure the detection of flaws that are significant from the point of view of a particular acceptance standard for sharp flaws. The resolution of the system is reduced at longer wavelengths, but the range of flaw dimensions that can be sized is increased. In addition, the detectability limitations are determined principally by the mass-loading effects of the weld reinforcement and the presence of alignment mismatch. Consequently, consideration must be given to limiting the maximum dimensions of the weld reinforcement and alignment mismatch by employing appropriate process and tolerance controls.

\subsection{Inspection Protocnl}

The development of the low-frequency SH-wave EMAT system was guided by a need for a reliable ultrasonic inspection tool that would be capable of detecting significant planar flaws in pipeline girth welds. The detection of significant flaws necessarily involves a decision process based on a set of nondestructive measurements and a set of acceptance criteria. The output of 



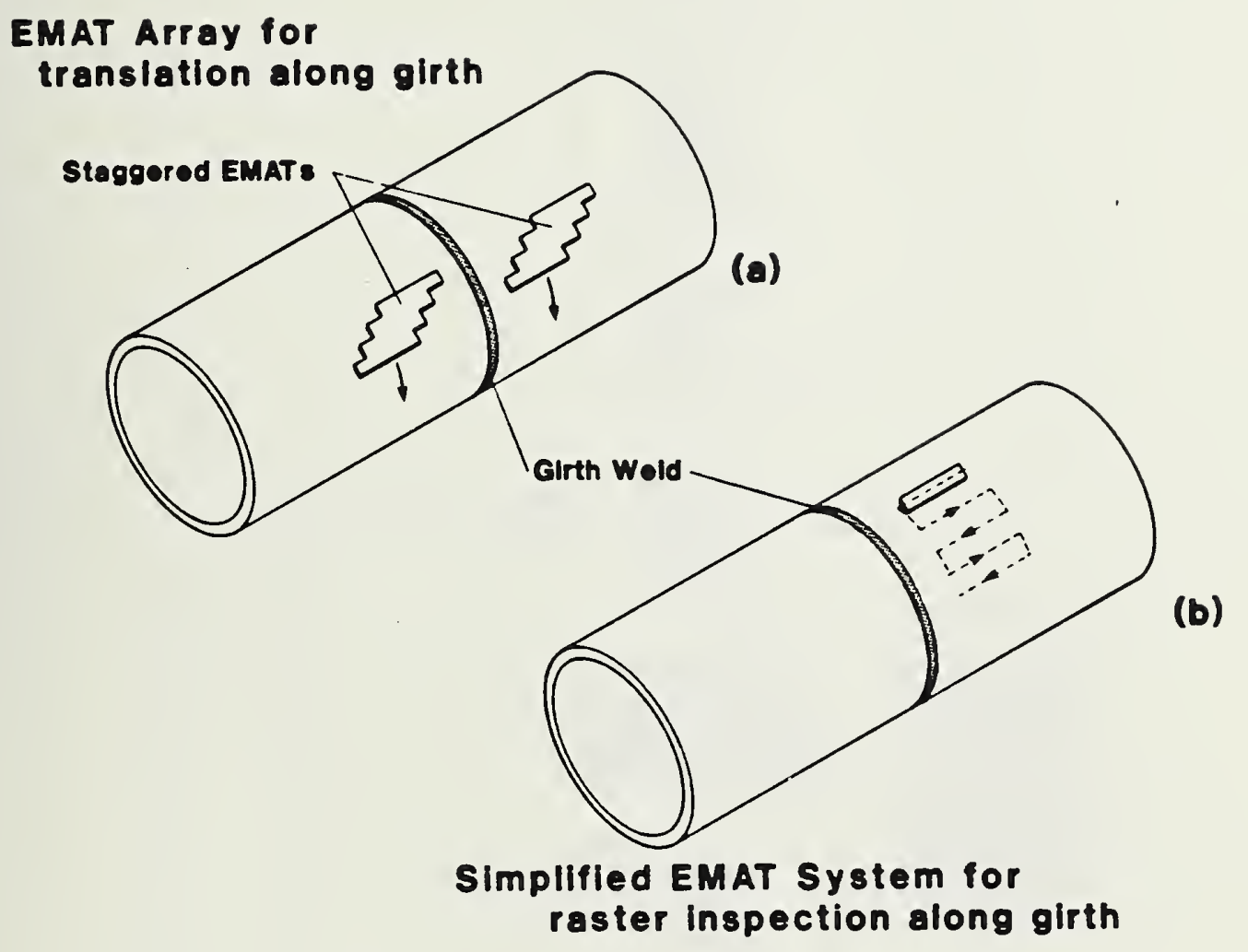

Fig. 54 - Two possible implementations of a practical SH-wave EMAT inspection system. 



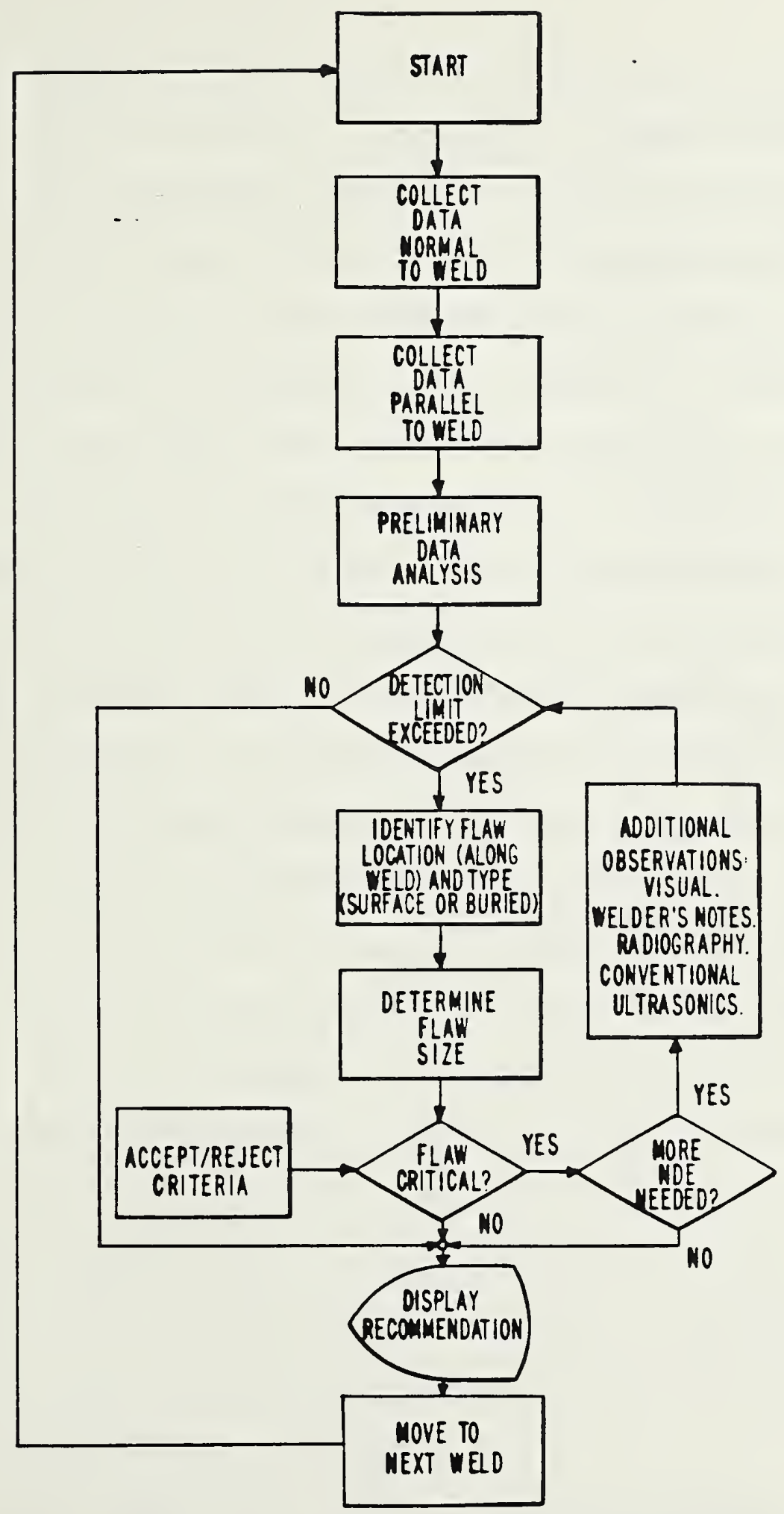

Fig. 55 - An inspection protocol for fully automated inspection of pipeline girth welds. 

the detection process is always a binary decision, yes or no, and an associated measure of confidence. The decision is based on models describing the nondestructive measurements, the failure processes and associated safety factors, and the knowledge of the statistical flaw distribution. 69

Acceptance criteria for sharp flaws are normally specified in terms of the principal length and through-wall depth dimensions. In addition, certain failure models treat surface flaws more severely than buried flaws. As a consequence, the inspection system must be able to classify the flaws in terms of their relative position within the weld and to estimate their principal dimensions. This can be done simultaneously using ultrasonic measurements from different locations along scan lines that are perpendicular and parallel to the pipe circumference. Two possible experimental configurations can be used to accomplish this task; they are illustrated in Fig. 54.

Figure 54a shows the preferred inspection configuration. The configuration of Fig. 54a uses two arrays of EMATs that are translated mechanically along the direction parallel to the girth weld. Each array is composed of a number of independent sets of EMATs that are positioned at different distances from the weld. A simplified inspection configuration is shown in Fig. 54b. The simplified configuration uses only one set of EMATs, as illustrated in Fig. 54, that is scanned along the girth weld in a "zig-zag" manner. In this case, the measurements are repeated on the opposite side of the weld. It is evident that both inspection configurations can perform the scattering measurements that are needed.

A flow chart, exemplifying a possible inspection protocol, is shown in Fig. 55. Because most girth welds are free of significant sharp flaws, the inspection protocol involves a preliminary "detection" threshold, which permits by-passing the decision process involving acceptance criteria. The 

protocol assumes that ultrasonic measurements are made, as illustrated in Fig. 55 and processed to compute the reflection amplitude ratio, $R_{S}$, for each measurement location. If a signal level is observed that exceeds the preliminary detection threshold, then the flaw is classified and measured using the theory developed in this report. The decision concerning the significance of the flaw can then be made. If the measure of confidence is low, then additional available information may be included in the decision process. The additional information may include visual observations, process control information (welder's notes), radiographic data, and conventional ultrasonic data. For this purpose, a subsidiary "loop" is included in the inspection protocol. At the end, the results of the decision processes are appropriately displayed and recorded. Permanent recording of the preprocessed low-frequency ultrasonic data is also envisioned.

Since the decision processes must include consideration of factors that cannot be adequately modeled or simulated in a laboratory environment, successful implementation of the proposed inspection protocol will involve considerable field experience. In addition, independent data describing the statistical distribution of flaws will be required from destructive assays.

\subsection{Summary}

Significant progress has been made in developing a low-frequency ultrasonic system optimized for inspection of girth welds in large-diameter pipelines. This system offers the following advantages: 1) rapid inspection rates, 2) elimination of transducer coupling agents, 3) insensitivity to weld reinforcements, 4) minimum dependence on operator skill and judgement, 5) full automatability, 6) tunability of the system to signal the presence of rejectable flaws based on any established fracture mechanics accept/reject criterion, and 7) permanent data recording. Other ultrasonic inspection 

systems provide comparable overall performance. In contrast to existing systems, the present system is designed to provide the specific information on flaw content and sizing required by the accept/reject criteria of a fitness-for-service analysis of flaw significance.

Phenomenological models of the ultrasonic measurement process have been devised to extract the dimension and position information from the ultrasonic data from planar flaws. In addition, the inherent performance limitations of the system have been established theoretically and empirically in a controlled laboratory environment. The results indicate that the new system offers considerable advantages over conventional ultrasonic and radiographic methods. In particular, it uses noncontacting electromagnetic-acoustic transducers whose efficiencies can be calibrated for each measurement. In addition, it operates at low ultrasonic frequencies, which reduce the complexity of data interpretation. At its present state of development, the SH-wave EMAT inspection system has the following deficiencies: 1) performance has not been demonstrated with real flaws under field conditions, 2) field proven hardware remains to be developed, 3) personnel trained in the system are not readily available, 4) equipment calibration standards remain to be developed, and 5) sensitivity of the system to detecting tight cracks closed by compression stresses has not been determined. 



\section{SIGNIFICANCE OF BLUNT FLAWS}

M. B. Kasen

\subsection{Introduction}

It has thus far been assumed that both sharp and blunt (nonplanar) flaws would be treated as significant and subject to an accept/reject criteria based on analytical fracture mechanics principles. But a number of studies have concluded that flaws such as porosity, slag, and arc burns have a very low probability of initiating cracks in tough weld metal and may be considered innocuous in a fitness-for-service analysis. ${ }^{70-76}$ It was therefore of interest to determine if this proposition could be defended in pipeline girth welds.

\subsection{Experimental Procedures}

A series of girth welds containing large contents of porosity, slag, and arc burns were fabricated in 1016-mm (40-in) API $5 L X-65$ pipe and in $1220 \mathrm{~mm}(48-i n)$ API $5 L X-70$ pipe of $15.9-\mathrm{mm}(0.625-i n)$ wall thickness. The objective was to obtain welds having flaw contents equal to or in excess of the worst case obtainable under field welding conditions. Welds meeting API 1104 workmanship quality were also produced. Automatic and manual (SMA) processes were represented, with fixturing, consumables, and personnel reflecting field procedures as much as possible. Welding parameters and joint designs, as reported by the fabricator, appear in Table 6. Typical welding electrode compositions are given in Table 7. Chemical composition of the base and weld metals are given in Table 1 (Section 2 ). Tensile properties of the weld metal and typical fracture toughness data on the welds are summarized in Table 8. A detailed discussion of toughness is given in Appendix H. Flaw contents obtained are illustrated in Fig. 56. 


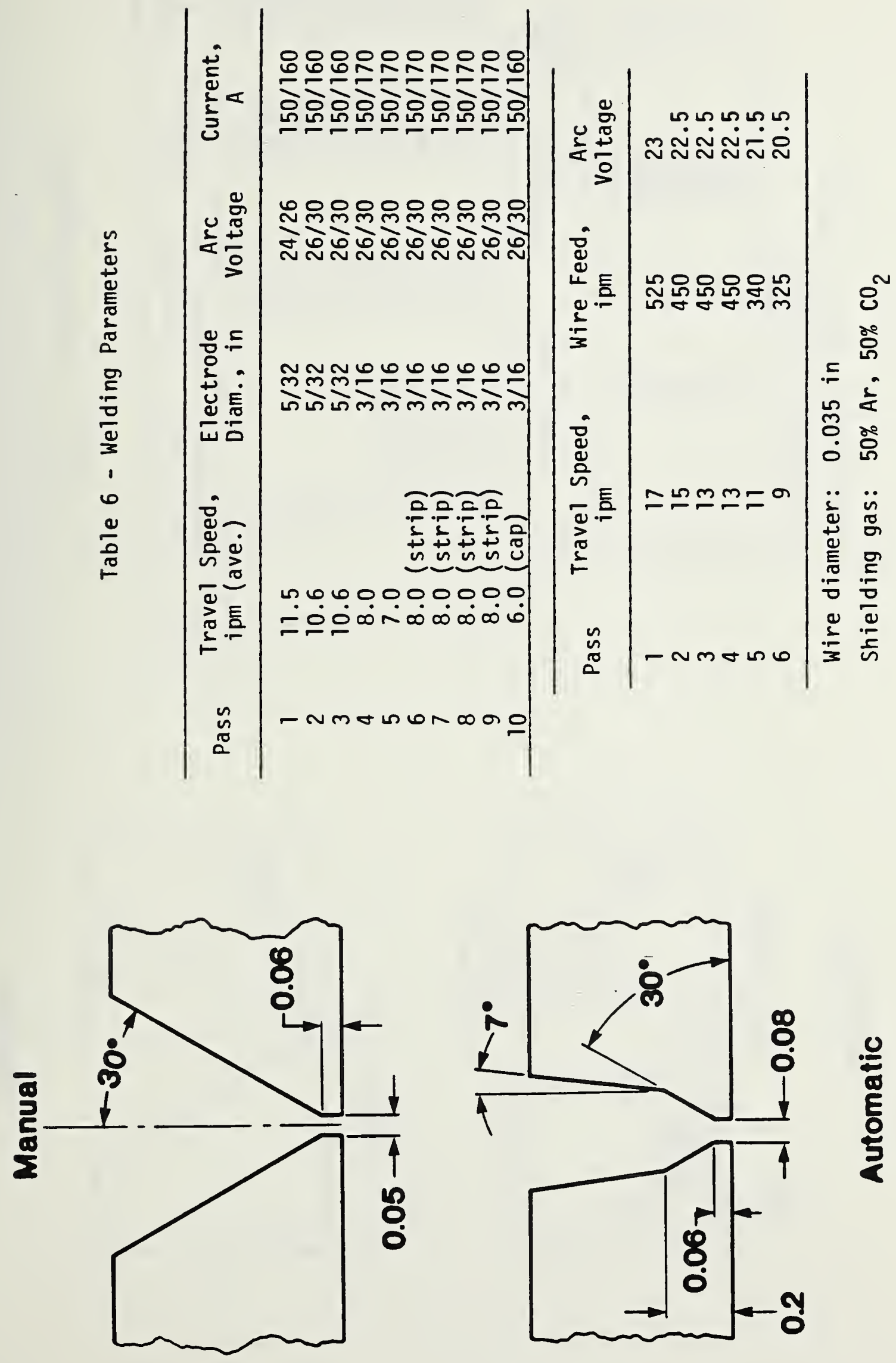


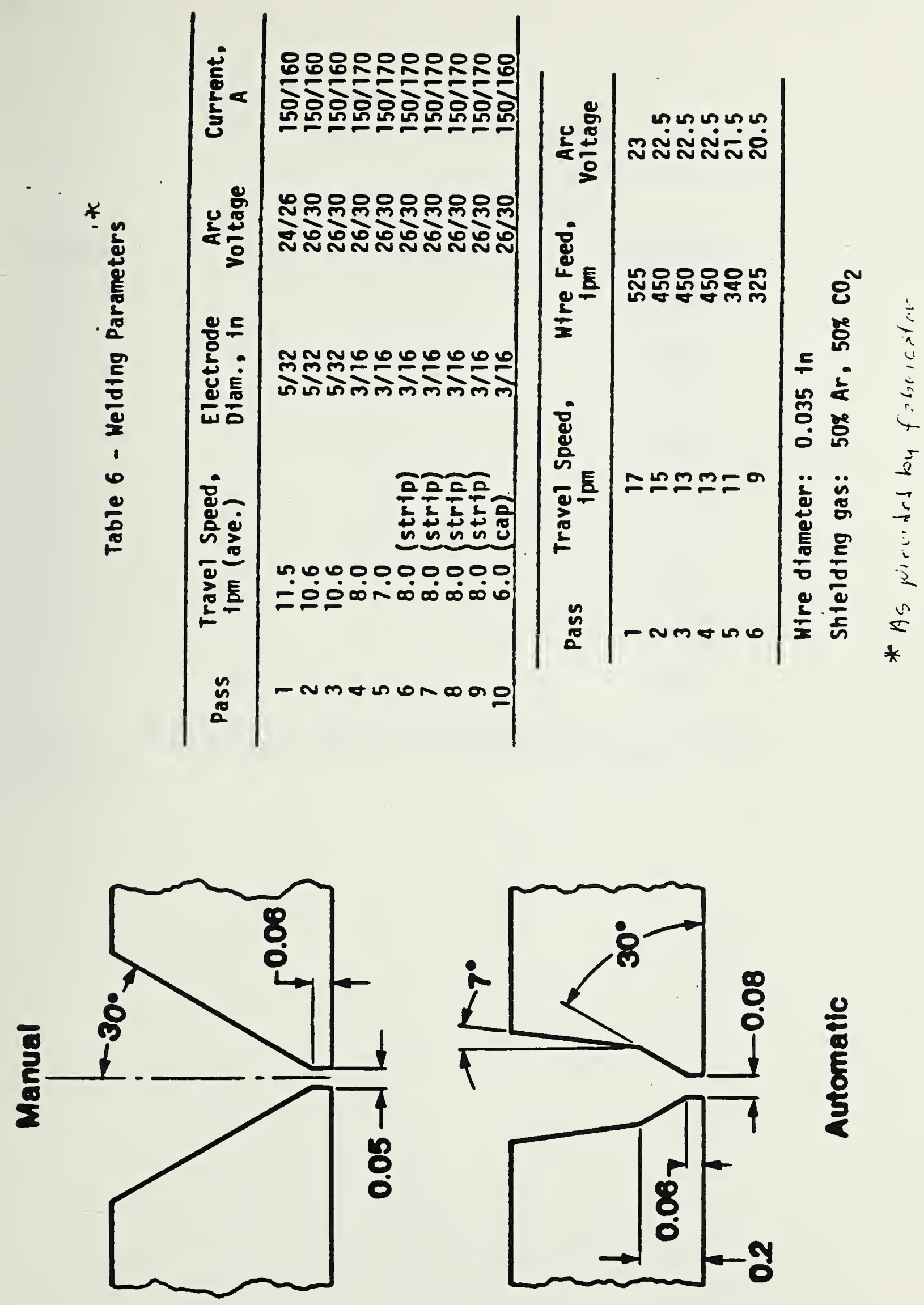

lable 7 - Typical Welding Electrode Compositions

Process

\begin{tabular}{ccc}
\hline Element & Shielded Metal-Arc* & Automat \\
\hline Mn & $0.47-0.51$ & 1.35 \\
Si & $0.07-0.10$ & 0.70 \\
Cr & $0.12-0.13$ & $\cdots$ \\
Ni & $1.03-1.10$ & 1.15 \\
Mo & 0.01 & $\cdots$ \\
V & $0.02-0.03$ & $-\cdots$ \\
C & $\ldots-.-$ & 0.10 \\
S & $\ldots$ & 0.015 \\
P & $\ldots$ & 0.015
\end{tabular}

Values reported by the fabricator in percent by weight

*AWS E8010G

TAWS E7OS-6 

Table 8 - Typical Weld Metal Properties

\begin{tabular}{|c|c|c|c|c|c|c|}
\hline Held Type & Mardness & \multicolumn{2}{|c|}{$\begin{array}{l}\text { Yield Strength at } \\
0.2 \% \text { offset, } 25^{\circ} \mathrm{C} \\
\mathrm{MPa} \quad \mathrm{Psi}_{\mathrm{S}} \times 10^{3} \\
\end{array}$} & \multicolumn{2}{|c|}{$\begin{array}{l}\text { Toughness, } \mathrm{COD}, \\
-18^{\circ} \mathrm{C} \quad(\mathrm{mm}) \\
0^{\circ} \mathrm{C} \\
\end{array}$} & $\begin{array}{l}\text { Critical coo, mm (in) } \\
\text { slow, Stable Crack } \\
\text { Growth }\end{array}$ \\
\hline Automatic & HRC 30 & 725.0 & 105.5 & $0.082(0.0032)$ & $0.094 \quad(0.0037)$ & $0.074(0.0029)$ at $0^{\circ} \mathrm{C}$ \\
\hline Manual & HRB 91 & 465.0 & 67.5 & $0.124(0.0049)$ & $0.0187(0.0074)$ & $0.079(0.0031)$ at $-18^{\circ} \mathrm{C}$ \\
\hline
\end{tabular}





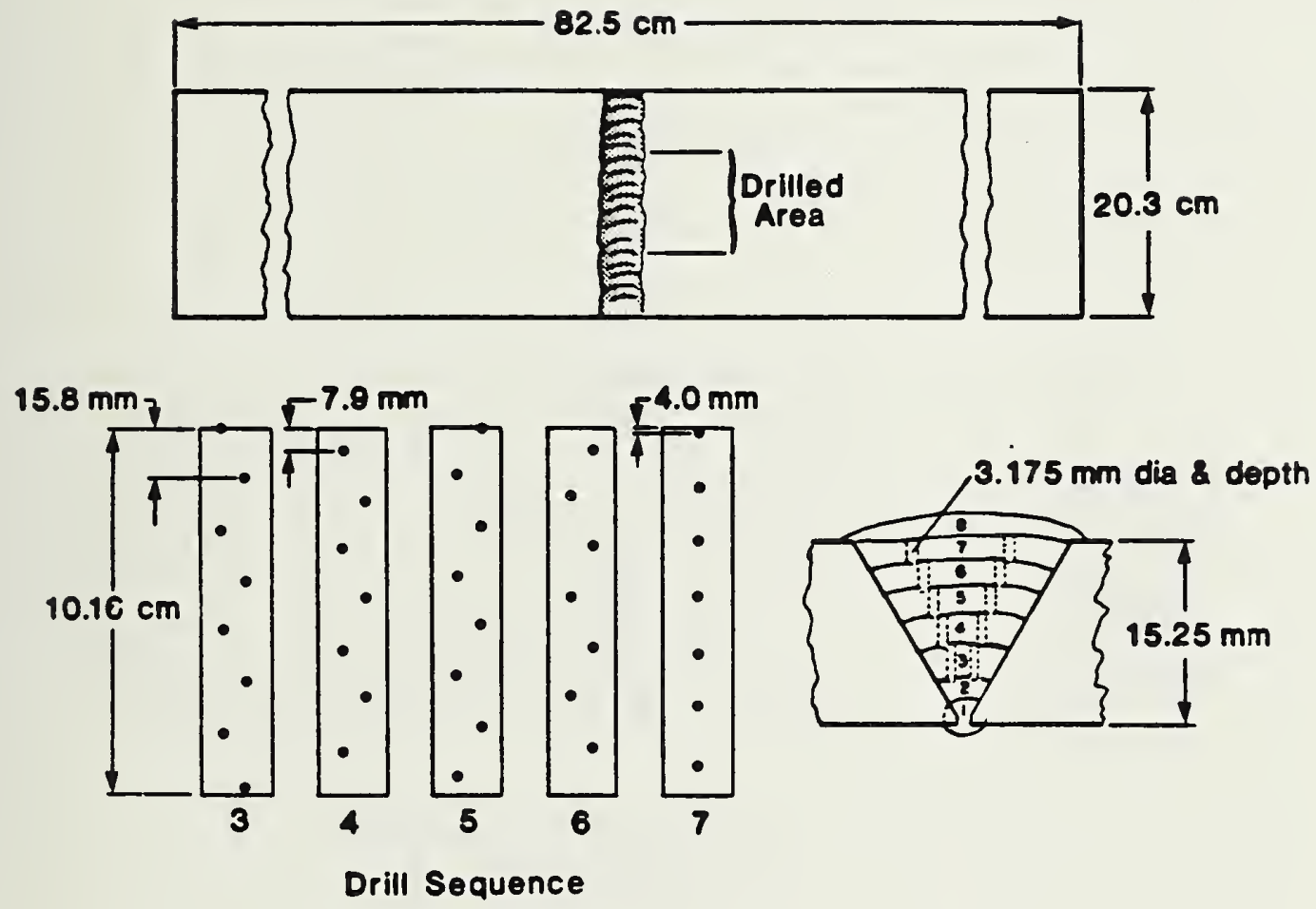

Fig. 57 - Drill pattern used to simulate buried porosity in manual (SMA) welds in API $5 L X-70$ pipe. 


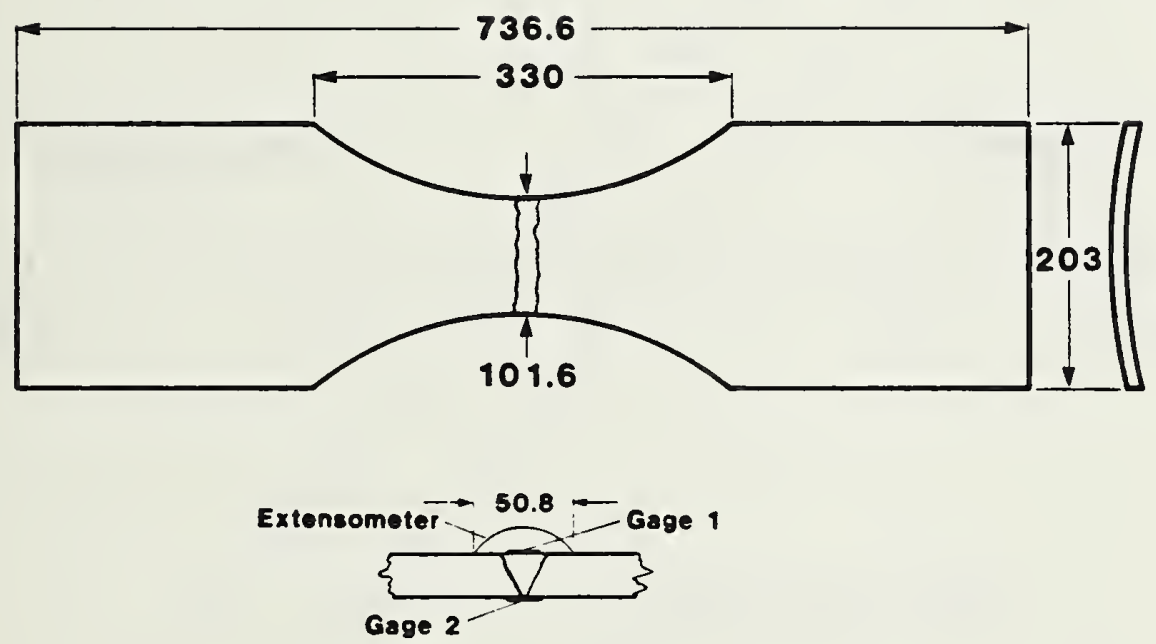

Fig. 58 - Specimen and instrumentation used for low-cycle fatigue studies of the significance of blunt weld flaws in crack initiation. Dimensions are in $\mathrm{mm}$. 



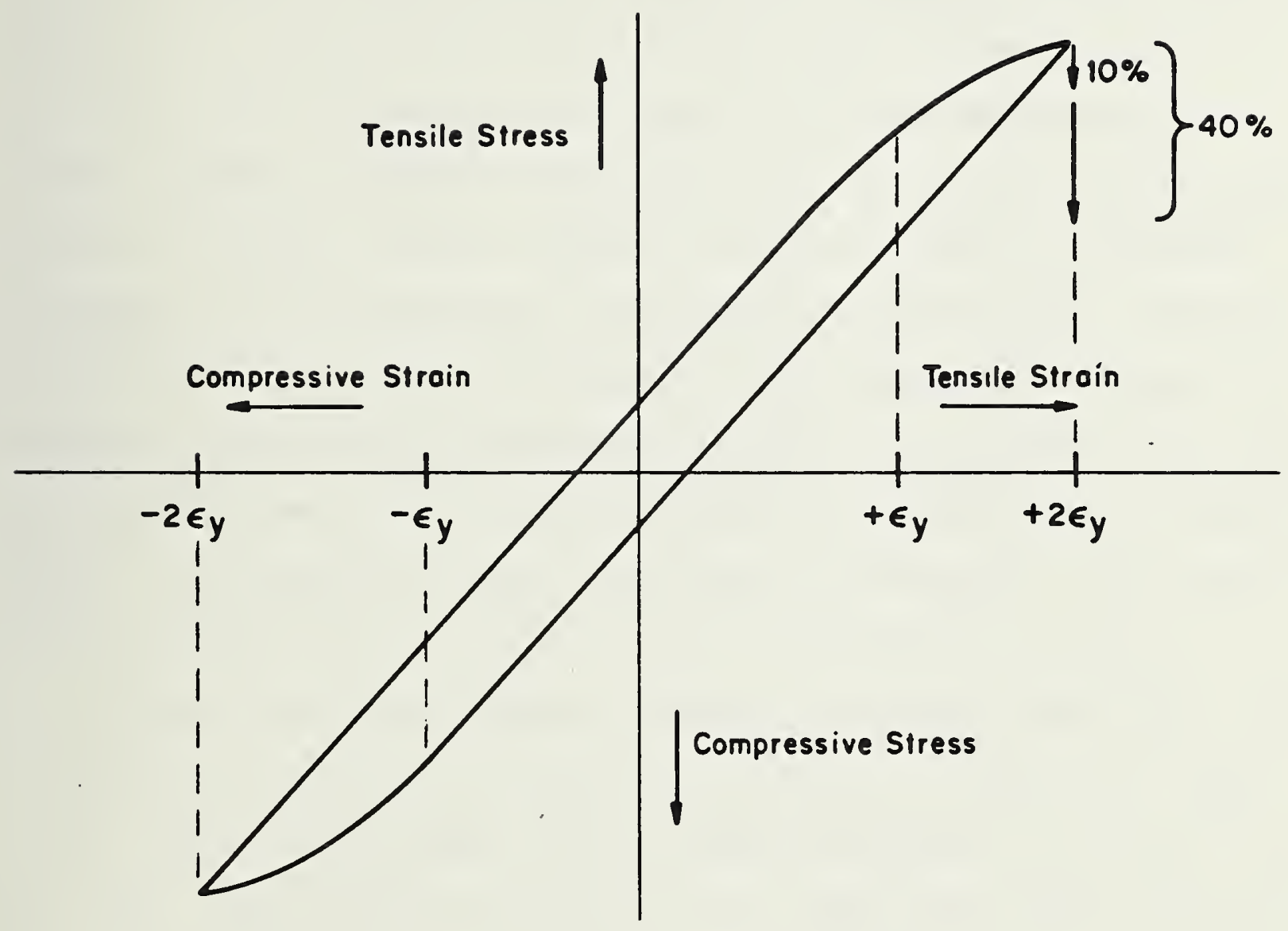

Fig. 59 - Schematic of hysteresis loops formed during low-cycle, straincontrolled fatigue test program. Crack initiation was defined as corresponding to a 10\% load drop to maintain the desired strain range. Specimens were broken in tension after a 40\% 1oad drop. Tests were run at strain levels just over yield $\left(\underline{t}_{\mathrm{y}}=\right.$ $\pm 0.0022)$ and at twice yield $\left( \pm 2 \varepsilon_{y}= \pm 0.009\right)$. 

A level of porosity obscuring 12 to $15 \%$ of the projected area of the weld radiographs was desired, since this level represents more than twice the obscuration level that would be practically allowable based on the probability of excess porosity interfering with radiographic detection of sharp flaws (see Section 6). This level was easily obtained during automatic welding by disturbing the shielding gas during deposition of two internal weld passes. Several attempts were made to produce this level of obscuration during manual welding by improper welding technique, excessive drying of the E8010G electrodes, or by fracturing the flux coating of the electrodes. Although a large amount of porosity was produced in a given pass, the final welds were found to contain far less than the desired level of obscuration. This flaw was therefore simulated by drilling a pattern of $3.175-\mathrm{mm}(0.125-\mathrm{in})$ diameter holes in five successive interior weld passes and overwelding. The procedure is illustrated in Fig. 57, and the result is illustrated in Fig. 56c.

Slag was produced by improper cleaning of an interior weld pass. As illustrated in Fig. 56b, this typically resulted in formation of "wagon tracks" due to slag entrapment from the E8010G electrodes at the sides of the weld bead. Lying adjacent to the fusion line, this type of slag residue has the highest probability of contributing to brittle fracture because of the stress concentration caused by the weld reinforcement. Arc burns were introduced at random on the heat-affected zones of sound welds after the welding had been completed. This simulated the worst case, because there was no annealing of the brittle martensite phase that forms under the burns due to rapid cooling.

Large, transverse weld specimens, having dimensions and instrumentation as illustrated in Fig. 58, were prepared from selected regions of the welded pipes. These specimens were subjected to fully reversed, strain-controlled, 

low-cycle fatigue, producing hysteres is loops as illustrated in Fig. 59. A temperature of $-2 \pm 1^{\circ} \mathrm{C}$ was maintained to simulate the lowest anticipated service temperature of the ANGTS line. This was accomplished by controlled metering of liquid-nitrogen vapor into a chamber surrounding the specimen. The cyclic rate was held to $0.2 \mathrm{~Hz}$ or less to minimize internal heating. The imposed strain was either slightly over weld-metal yield $( \pm 0.0022)$ or approximately twice weld-metal yield $( \pm 0.0045)$. The total strain range was, therefore, either 0.0044 or 0.0090 . This technique was selected because, although pipelines are not normally subjected to such severe cyclic loading, any flaw found innocuous under the imposed conditions can conservatively be considered innocuous in essentially statically loaded structures. ${ }^{77}$ A similar approach has been used to demonstrate the inherently innocuous nature of blunt flaws in pressure vessel weldments. 75,76

As cyclic testing proceeded, development of cracks in the weld region reduced the effective cross-sectional area, causing a drop in the load required to maintain the selected strain range. Monitoring of the load therefore provided an indication of the number of cyclic reversals required to initiate cracking in each weldment. A $10 \%$ load drop, corresponding to development of about a $10 \%$ cracked area in the specimen cross section, was used as the criterion for crack initiation. This criterion was selected because, once initiated, crack-growth rates were relatively rapid under the severe test conditions, regardless of flaw content. Cyclic straining was continued until a load drop corresponding to a $40 \%$ cracked area was obtained to permit the influence of flaw type and content on crack propagation to be studied. Testing was stopped at 3000 cycles if this criterion had not been reached. Specimens were then broken in tension. The desired strain range was initially established by strain gages located at the weld, as illustrated in 



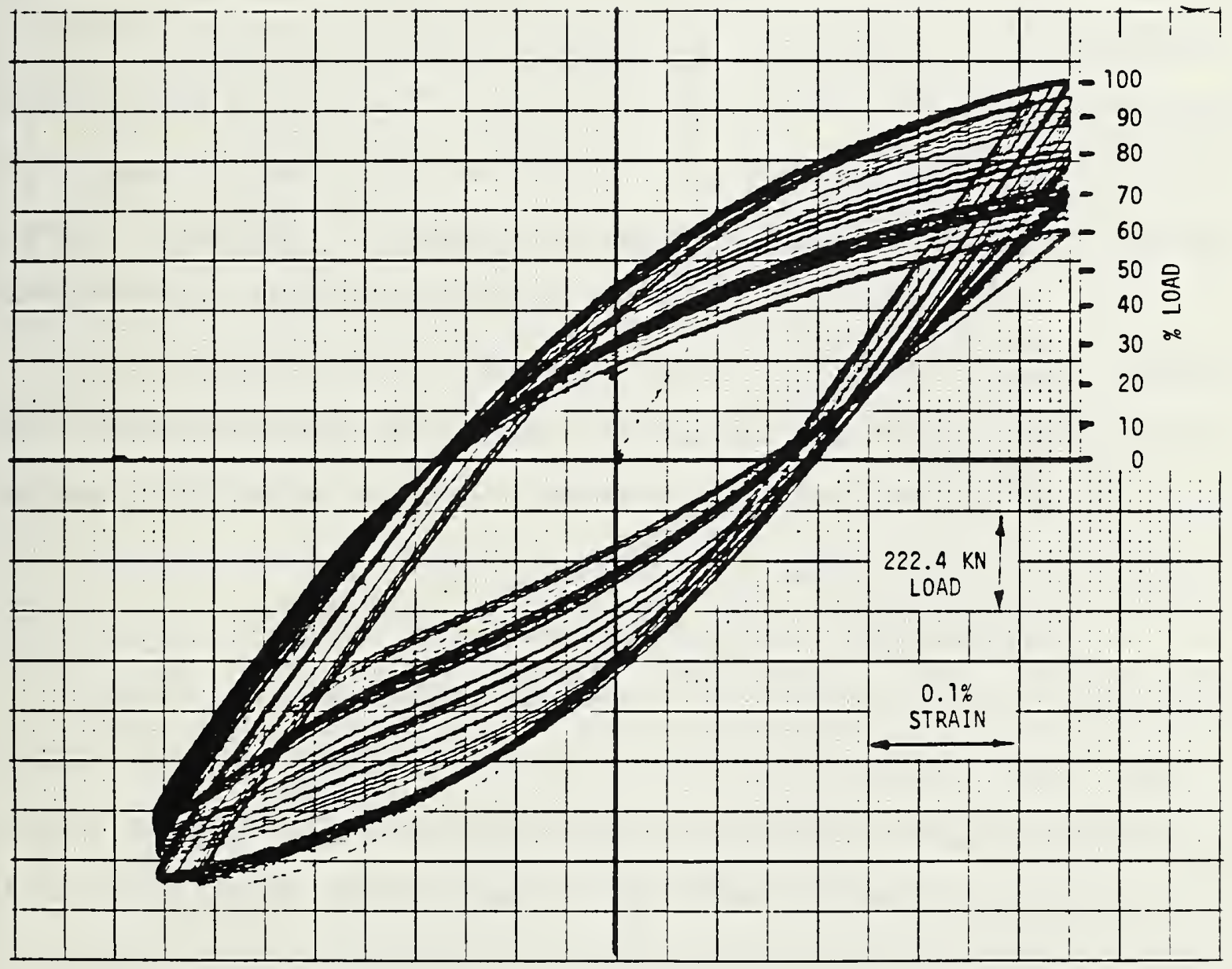

Fig. 60 - Hysteresis loop plot from extensometer on a sound manual weld tested at 0.0090 total strain with weld reinforcement removed. Specimen No. 95103. 

Fig. 58, while control of the 4.45-MN $\left(1 \times 10^{6} \mathrm{lbf}\right)$ servohydraulic test machine was provided by an extensometer spanning the weld region. Figure 60 illustrates the actual appearance of hysteresis loops produced by the extensometer and load cell during a test of a sound manual weld.

Specimen asymmetry due to pipe curvature produced a bending moment during compression, tending to cause failure by column buckling rather than in tension-compression. This was countered by development of the specimensupport system illustrated in Fig. 61 and by ensuring that the specimen halves were in good alignment prior to a test. The latter was achieved by slightly bending the specimens to remove the distortion caused by release of residual welding stresses when the specimens were removed from the pipe.

All the specimens were radiographed prior to testing. Several specimens were stereoradiographed after achieving the $40 \%$ load drop but before final fracture to determine if useful information could be obtained on the crack path relative to buried flaws. However, the cracked region could not be defined with a resolution justifying continuation of this procedure.

Weldments were tested with the weld reinforcement both intact and removed by flush grinding. Results with the reinforcement intact were of most interest in interpreting flaw significance, since this condition exists in the field. Removing the reinforcement forced failure through the flawed region and provided useful information on flaw interaction during crack initiation and propagation.

\subsection{Low-cycle Fatigue Results}

Results of tests conducted with the weld reinforcement intact are summarized in Table 9. Similar data for specimens tested with the reinforcement removed appear in Table 10. Results of tests on unwelded parent 



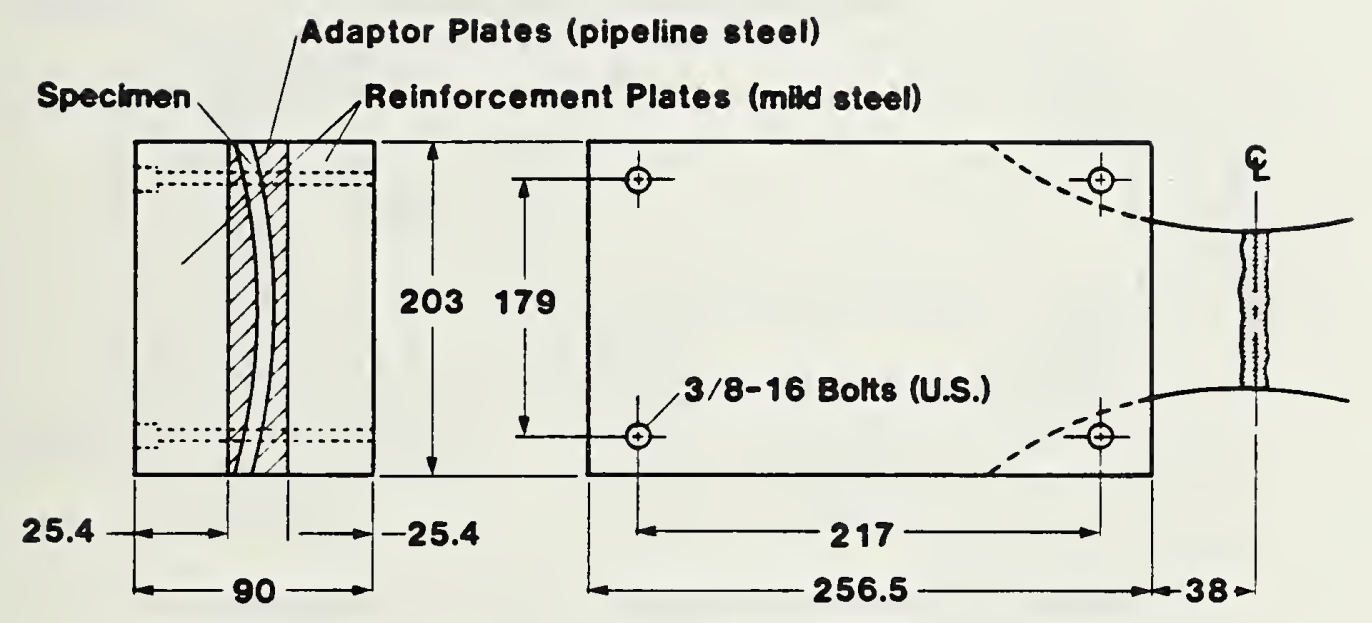

Fig. 61 - Specimen support system required to prevent failure in column buckling during low-cycle fatigue testing. Dimensions are in millimeters. 



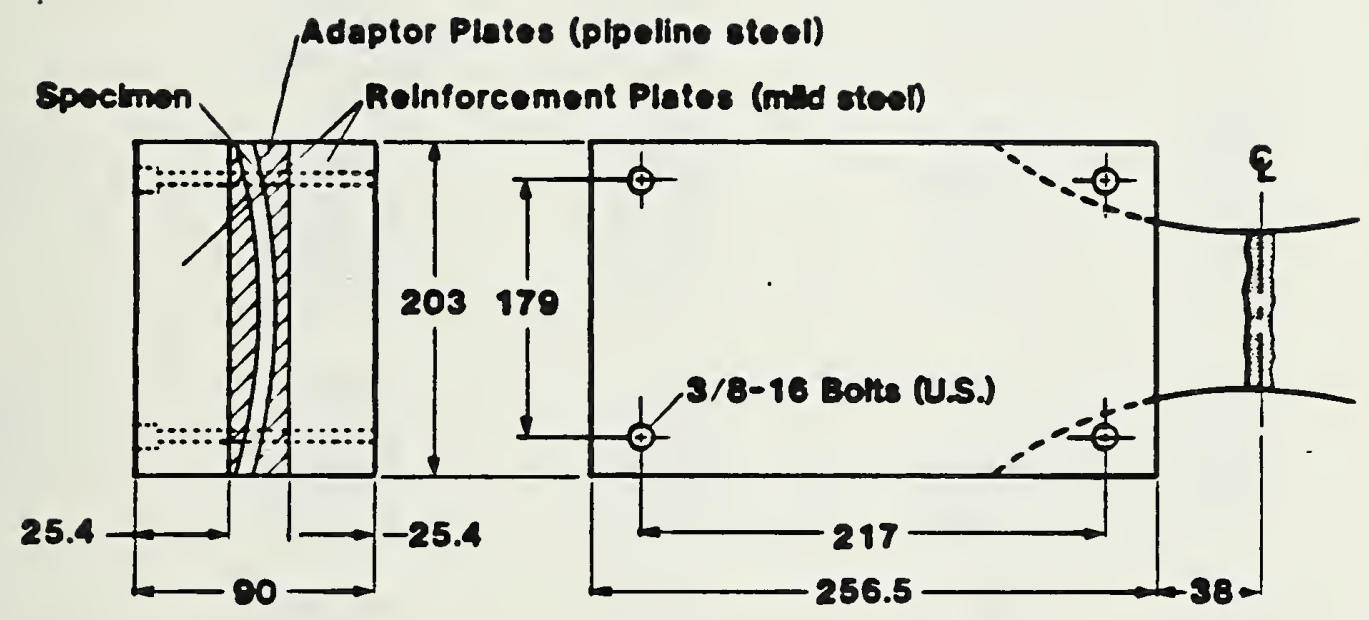

Fig. 61 - Specimen support system required to prevent failure in column buckling during low-cycle fatigue testing. Dimensions are in millimeters. 

Table 9 - Results of Low-Cycle Fatigue Program Conducted with Welds Having Reinforcement Intact and with Parent Pipe Materials

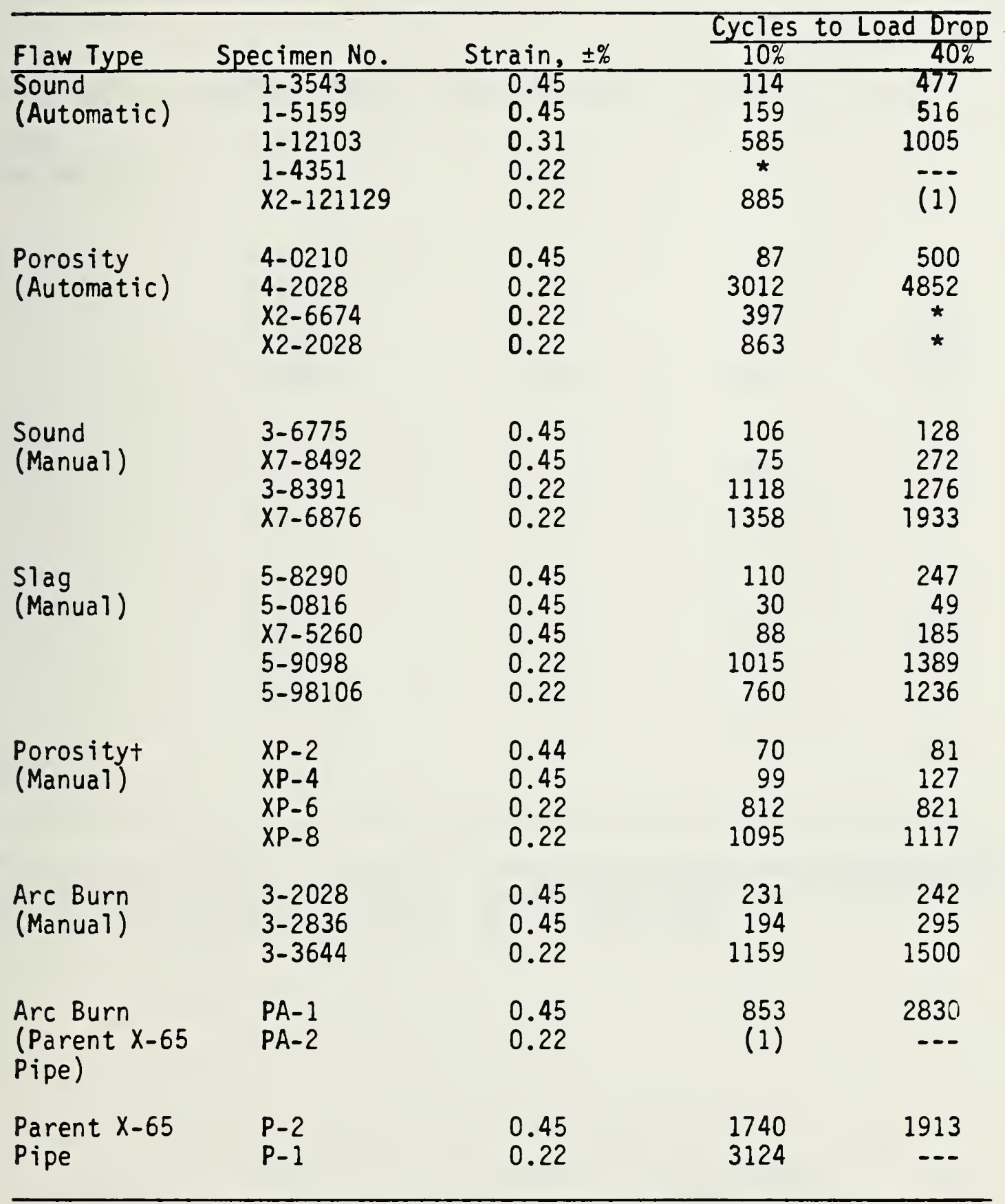

* Not achieved after 3000 cycles. Test discontinued. tArtificially introduced. See text. 

Table 10 - Results of Low-Cycle Fatigue Program Conducted with Welds Having Reinforcement Removed

\begin{tabular}{|c|c|c|c|c|}
\hline \multirow[b]{2}{*}{ Flaw Type } & \multirow[b]{2}{*}{ Specimen No. } & \multirow[b]{2}{*}{ Strain, $\pm \%$} & \multicolumn{2}{|c|}{ Cycles to Load Drop } \\
\hline & & & $10 \%$ & $40 \%$ \\
\hline $\begin{array}{l}\text { Sound } \\
\text { (automatic) }\end{array}$ & $\begin{array}{l}1-111119 \\
1-1119 \\
1-0311\end{array}$ & $\begin{array}{l}0.34 \\
0.22 \\
0.22\end{array}$ & $\begin{array}{l}120 \\
\star \\
+\end{array}$ & $\begin{array}{c}2130 \\
-.- \\
---\end{array}$ \\
\hline $\begin{array}{l}\text { Porosity } \\
\text { (automatic) }\end{array}$ & $\begin{array}{l}4-4452 \\
4-1220 \\
4-5563 \\
4-2836 \\
4-3644\end{array}$ & $\begin{array}{l}0.45 \\
0.45 \\
0.45 \\
0.22 \\
0.22\end{array}$ & $\begin{array}{r}155 \\
30 \\
117 \\
1026 \\
397\end{array}$ & $\begin{array}{r}165 \\
49 \\
276 \\
1525 \\
1505\end{array}$ \\
\hline $\begin{array}{l}\text { Sound } \\
\text { (manual) }\end{array}$ & $\begin{array}{l}3-7583 \\
3-95103 \\
3-0008 \\
5-4351\end{array}$ & $\begin{array}{l}0.45 \\
0.45 \\
0.22 \\
0.22\end{array}$ & $\begin{array}{r}81 \\
118 \\
1766 \\
2621\end{array}$ & $\begin{array}{c}136 \\
215 \\
2073 \\
\quad \neq\end{array}$ \\
\hline $\begin{array}{l}\text { Slag } \\
\text { (manual) }\end{array}$ & $\begin{array}{l}5-115123 \\
5-7179 \\
5-0008 \\
5-6371 \\
5-5563\end{array}$ & $\begin{array}{l}0.45 \\
0.45 \\
0.45 \\
0.22 \\
0.22\end{array}$ & $\begin{array}{r}26 \\
104 \\
250 \\
352 \\
132\end{array}$ & $\begin{array}{r}44 \\
198 \\
363 \\
442 \\
169\end{array}$ \\
\hline $\begin{array}{l}\text { Arc Burn } \\
\text { (manual) }\end{array}$ & $3-1018$ & 0.45 & 169 & 189 \\
\hline
\end{tabular}

* Not achieved after 2426 cycles. Test discontinued due to malfunction. +Not achieved after 1754 cycles. Test discontinued due to malfunction. FNot achieved after 3000 cycles. Test discontinued. 

pipe material and of parent material containing arc burns are included in Table 9. Figure 62 presents the data of Table 10 in graphical form, illustrating the number of cyclic reversals required to initiate cracking (10\% load drop) as a function of strain range. Figures 62 a through $c$ illustrate the results with individual flaw types, while all results are combined in Fig. 62d. Data of Table 10 do not appear in graphical form; however, the lower bound performance of this group tested with reinforcement removed is indicated by the dashed line on Fig. 62d.

These data show that some welds containing porosity or slag initiated cracking at lower cycles than did sound welds; others withstood higher cyclic loading. A minimum of 30 reversals at twice yield or about 450 reversals at just over yield were required to initiate cracking, regardless of the content of porosity, slag, or arc strikes. Comparison of the performance of welded specimens with that of unwelded plate illustrates that the strain discontinuity at the fusion line caused by the contour of the weld reinforcement was the dominating factor in lowering fatigue life in the welds. Weld failure occurred predominantly at this location, regardless of flaw content. The failure path was occasionally observed to pass through some of the porosity and slag. Neither crack initiation nor propagation was affected by the presence of arc burns in welded specimens. Arc burns initiated cracking only when they were introduced at the minimum cross section of unwelded plate. Here, cracking was observed to initiate at the strike after 853 cycles at twice yield (Table 9). No effect was detected at the lower strain level in arc-struck parent plate.

It has frequently been observed that the number of cycles defining lowcycle fatigue life, $N_{C}$, can be expressed as a function of total strain range, 


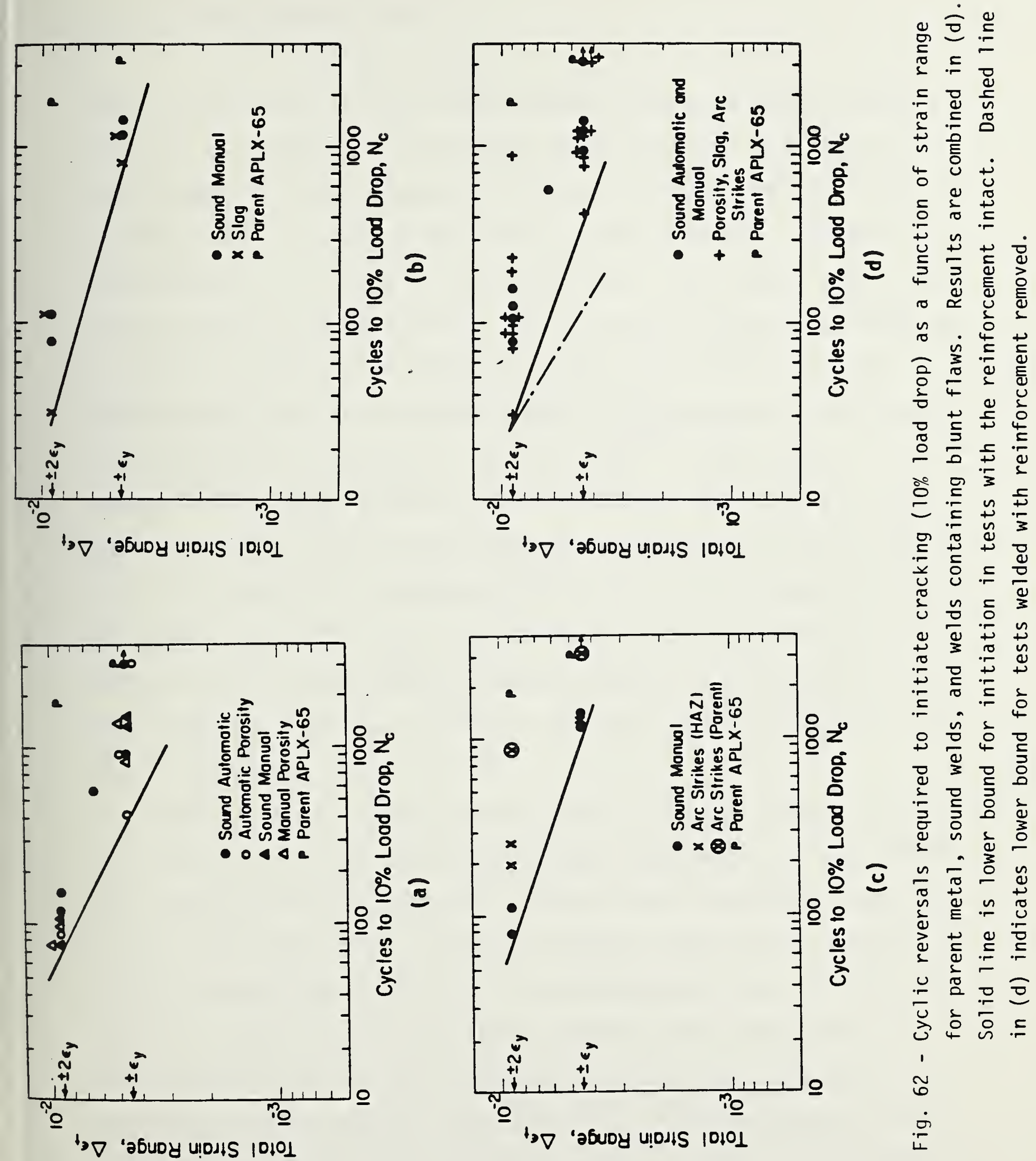
. 
$\Delta \varepsilon_{t}$, by a relationship of the form

$$
\Delta \varepsilon_{t} N_{c}^{m}=\text { constant }
$$

implying a straight line relationship between $\log \Delta \varepsilon_{t}$ and $\log N_{c}$, having a slope of $-m$. Gurney 77 has noted that results reported by a number of investigators for steel weldments of different configurations fall within a relatively narrow scatter band on such a plot, producing a slope of approximately $-1 / 3$. Figure 63 illustrates that a very similar result is obtained when the average number of cycles to crack initiation is plotted as a function of total strain range for manual welds tested with the reinforcement intact in the present program. Welds containing porosity and slag produced a relationship of the form $\Delta \varepsilon_{t} N_{c}^{0.3}=0.04$. However, the averaged data from the sound welds (including those containing arc burns) appeared to form a different population following the relationship $\Delta \varepsilon_{t} \mathrm{~N}_{\mathrm{C}}^{0.4}=$ 0.07 . This resulted in a divergence between the sound and flawed weld data at the higher $\left( \pm 2 \varepsilon_{y}\right)$ strain range, suggesting that the high porosity and slag content of the welds had somewhat reduced the cyclic life to crack initiation under this test condition. No effect of the flaw content is observed at the lower $\left( \pm \varepsilon_{y}\right)$ strain range.

Figure 64 , illustrating the average number of cycles to crack initiation as a function of total strain range for manual welds tested with the reinforcement removed, shows that forcing the failure to occur through the flawed weld interior rather than at the fusion line resulted in fewer cyclic reversals to initiate cracking than in tests with the reinforcement intact. In this test mode, it is seen that slag is a more significant flaw than porosity in reducing cyclic life to crack initiation. But, even under this severe test condition, 26 cyclic reversals were required to initiate cracking at twice yield, whereas 132 cycles were required at just over yield. This suggests 



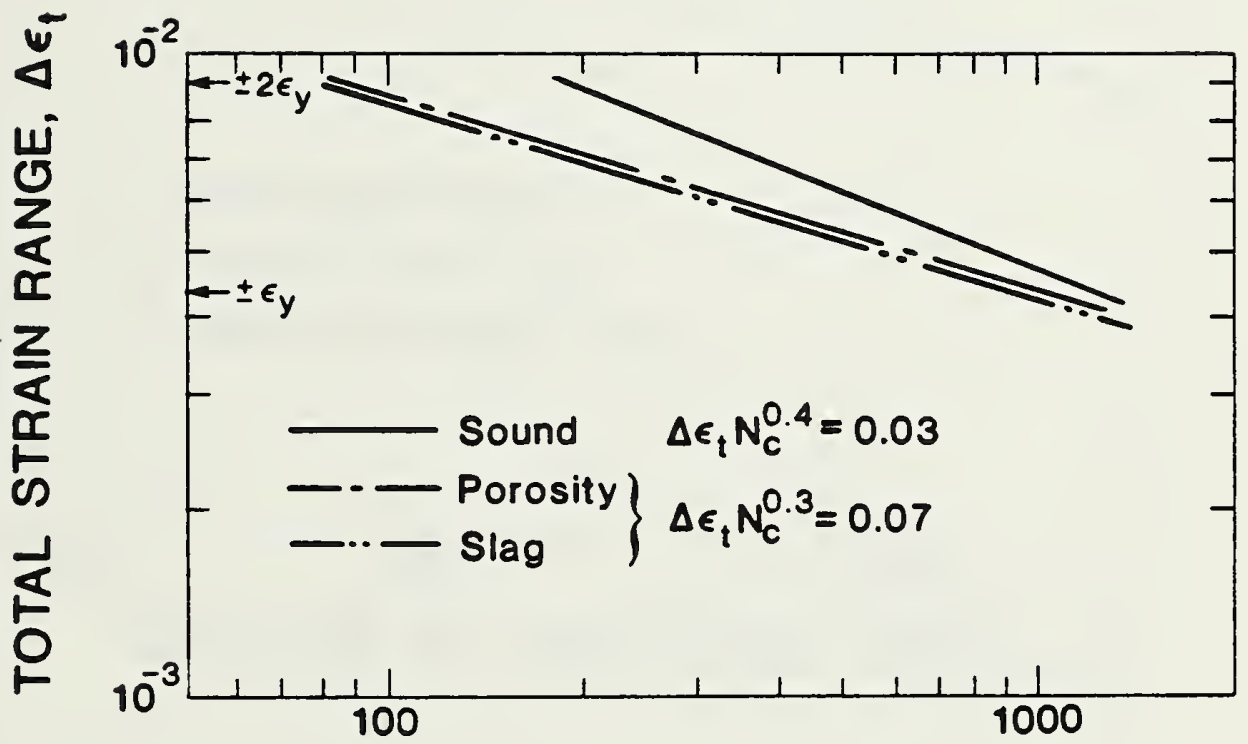

CYCLES TO $10 \%$ LOAD DROP, $\mathrm{N}_{\mathrm{C}}$

Fig. 63 - Averages of lifetime to crack initiation following Eq. 92 for specimens with weld reinforcement intact. 



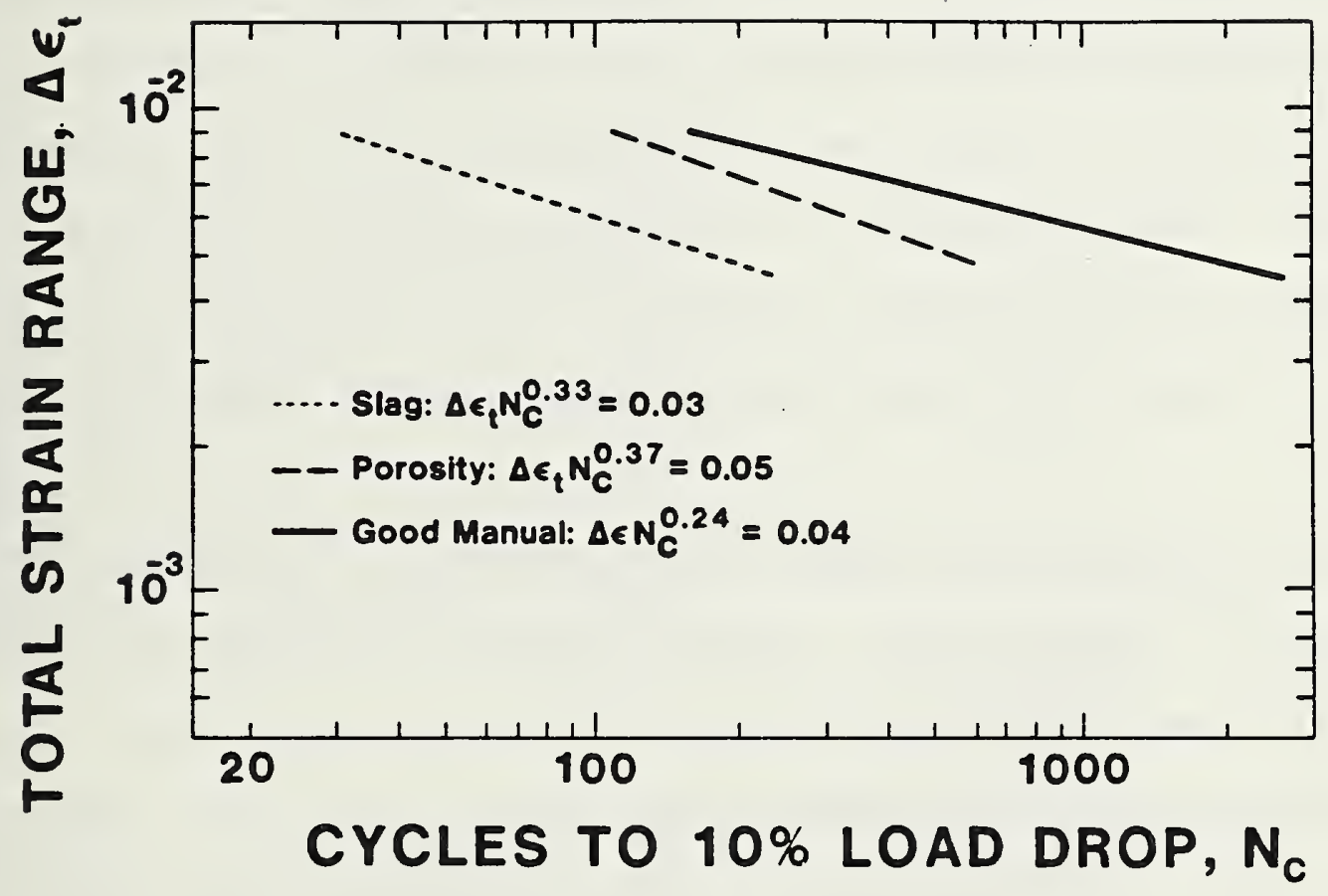

Fig. 64 - Averages of lifetime to crack initiation following Eq. 92 for specimens with weld reinforcements removed. 

that, even if the reinforcement were removed, porosity, slag or arc burns would pose little danger to the integrity of pipelines fabricated with welds having the toughness studied in this work.

\subsection{Fractographic Analys is}

Fractographic examination showed little flaw interaction at the lower strain level $\left( \pm \varepsilon_{y}\right)$. As shown in Fig. 65, the cracks in automatic welds containing a large amount of porosity were observed to propagate as single crack fronts with negligible initiation from individual pores. The higher strain $\left( \pm 2 \varepsilon_{y}\right)$ did cause cracks to form from individual pores in both the manual and automatic welds, with the cracks coalescing to form the fracture surface.

The presence of halos around slag stringers and micropores was noted on the fractured surfaces of all manual welds. This phenomena, illustrated in Fig. 66, is described in the literature as "fisheyes" 78,79 and is attributable to hydrogen embrittlement of the region adjacent to pores and surfaces to which hydrogen has segregated during the welding operation. Their appearance here suggests that hydrogen segregated in this manner is very stable, because the specimens were tested one and one half years after welding. The mechanism of fisheye formation is not well understood; however, lack of fatigue indications on the fisheye surface is indicative of a pop-in type of crack, perhaps reflecting a stress-induced diffusion of hydrogen from its segregated site into the surrounding weld metal during loading. It is generally assumed that fisheyes only form in weld metal stressed beyond yield; ${ }^{78}$ however, this has not been firmly established. Whatever their cause, fractographic analys is indicated that the fisheyes contributed relatively little to fatigue crack initiation unless they penetrated the specimen surface. For example, the main 


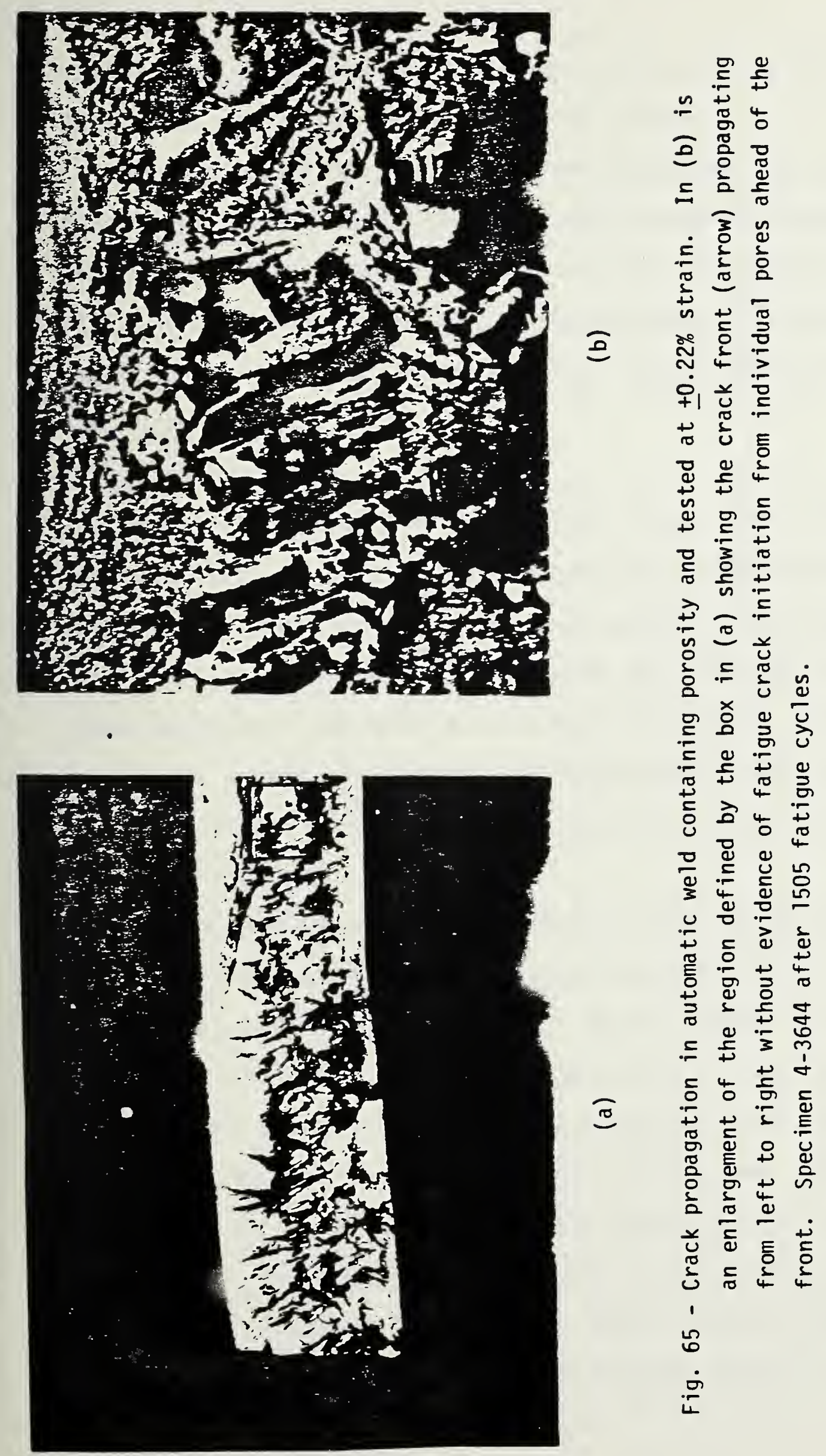

fatigue crack in the specimen illustrated in Fig. 66b initiated and propagated in a region remote from the large internal fisheye.

In addition to the fatigue work described above, two specimens each representing sound automatic welds and welds containing automatic and manual porosity at levels shown in Fig. 56 were pulled to failure in tension at -2 $\pm 1^{\circ} \mathrm{C}$. Results, given in Table 11 , showed no effect of the flaw content on the static tensile strength. All welds failed through the heat-affected zone of the API $5 L X-70$ pipe.

\subsection{Discussion}

These results show that the probability of crack initiation from porosity, slag, and arc burns during low-cycle fatigue was very low in the manual and automatic pipeline girth welds tested in this program. Although some reduction in life to crack initiation was noted when welds containing large quantities of porosity and slag were tested at the highest strain range $\left( \pm 2 \varepsilon_{y}\right)$, the geometrical discontinuity formed by the weld reinforcement was found to dominate the fatigue failure mode in all tests conducted with the reinforcement intact.

This is consistent with the findings of others on the relative effect of the geometrical discontinuity created by the reinforcement as compared with that of buried blunt flaws. $72,76,77,80$ In the absence of brittle fracture initiation, the primary structural effect of such flaws is to reduce the effective weld cross section. However, porosity content causing a 12 to $15 \%$ radiographic obscuration will reduce the cross-sectional area by only about $1 \%$ in welds of the thickness studied in the present work, ${ }^{77}$ whereas the weld reinforcement itself typically contributes 5 to $8 \%$ to the thickness of the weld region. Therefore, a negligible effect could be predicted for circumstances where the weld-metal yield strength matches or exceeds that of 

the parent pipe material. Table 9 shows that this was the case in the present study.

Even with the reinforcement removed to force failure through the highly flawed weld metal, the welds showed a high tolerance to fatigue crack initiation from the blunt flaws. Because strain levels were well above yield, these data indicate that the probability of crack initiation from such flaws would be negligible if such welds were subjected to the essentially static loading conditions of operating pipelines.

Therefore, the results of this study suggest that porosity, slag, and arc burns are innocuous as fracture initiation sites in girth welds fabricated with materials of the toughness equal to or exceeding that listed in Table 8. The validity of this conclusion has not been experimentally demonstrated for welds of lower toughness. However, as noted by Harrison, 71,72 the rounded, blunt shapes of such flaws and their inherently small critical through-wall dimensions (see Section 5) make them size-for-size less harmful than cracks of equal dimensions. He observes that if the critical flaw size is so small as to indicate serious risk of fracture initiation from porosity and slag, it is doubtful that the selected material has a toughness appropriate for the application. Harrison concludes that porosity and slag required no special consideration in regard to brittle fracture. This concept has been accepted by Commission $X$ of the International Institute of Welding (IIW) following the recommendations of an IIW Working Group on Significance of Defects. ${ }^{72,73}$ This Group, representing Japan, England, and Belgium, based its conclusions on: 1) the examination of approximately one hundred cases of fatigue by brittle fracture in ferritic steel weldments, during which no instance of fracture initiation at porosity or slag was observed, 2) results of tests at many laboratories demonstrating that such flaws have little or no effect on static 

failure load, and significantly less effect on fatigue life than the surface profile and, 3) consideration of theoretical elastic and fracture mechanics analysis. The group concluded: "Provided materials of adequate notch ductility or fracture toughness for the particular service application have been used to tolerate the smallest planar defects which can reliably and practically detected, porosity, slag inclusions, and surface irregularities, in amounts up to which they can be clearly identified as the only defects present, will have no significance on failure by brittle fracture".$^{72}$

The present work appears to substantiate these general conclusions with one exception: The detection of fisheye crack formation from the surface of entrapped slag in welds made with the E8010G high-hydrogen electrodes suggests that it might be prudent to reserve judgement on the potential significance of slag until the conditions for formation of fisheyes are better defined. Although such cracks were developed during low-cycle fatigue in the present work, they are found on tensile, bend test and similar fracture surfaces produced at low strain rates. ${ }^{87}$ The possibility of their formation as the result of pipe-laying stresses or other imposed static loads cannot, therefore, be ruled out at the present time. Should this occur, fisheyes may constitute a signficant flaw in weld metal of lower toughness than that studied in this program.

The probability of arc burns contributing to fracture is related to the probability of crack initiation in the hard martensitic region produced by a burn and to the probability that such a crack is of critical size for fracture propagation in the parent material. As a first approximation, the carbon equivalent (CE) of the parent material might be taken as a measure of the former probability, because this parameter is controlled in the selection of pipeline material to limit the martensite content and hardenability in the 

heat-affected zone of the weldment. A typical formula for pipeline construction might be

$$
C E=C+M n / 6+\left(C r+M_{0}+V\right) / 5+N i+C u / 15
$$

yielding a CE value of 0.414 for the API $5 L X-65$ material used for the arc-burn studies. A typical maximum allowable CE value for $16-\mathrm{mm}(0.63-\mathrm{in})$ thick pipe would be 0.40; consequently, it is concluded that the arc-burn significance tests were conducted under a conservative criterion from a hardenability point of view. Despite these conditions, no cracking was observed in any of the arc burns, either before or after testing.

Even assuming cracking was present, the literature suggests that propagation would be unlikely owing to the high toughness of current pipe materials. Fearnehough and Jones 81 have noted that a number of tests conducted in the United States and England have demonstrated that defect failure in modern pipeline materials is primarily controlled by plastic collapse phenomena and that the critical defect size is, therefore, associated with tensile properties. Results of the study to develop appropriate accept/reject curves for current pipeline materials (see Section 2) support this view in concluding that a model based on ligament yielding is most appropriate. These considerations suggest that the controlling parameter for partial wall defects is flow stress on the remaining pipe wall. Lumb and Fearnehough ${ }^{82}$ have analyzed a large number of accidental pipeline failures and data from experimental burst tests by this criteria, noting that in no case was failure was found to initiate from flaws having depths less than $20 \%$ of the wall thickness for applied stresses equal to the specified minimum yield strength of the flawed material. This corresponds to a tolerable flaw depth of about $3 \mathrm{~mm}(0.12 \mathrm{in})$ for pipe of the thickness studied in the present work. The experimentally determined relationship between arc-burn width and depth 



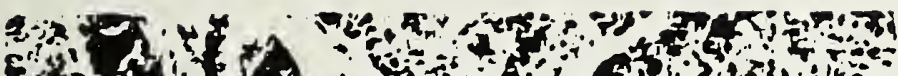
(f)

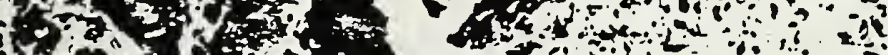

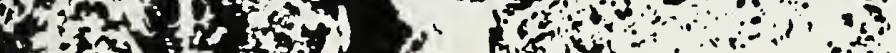

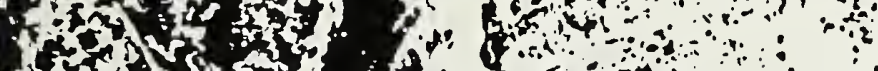
Fod

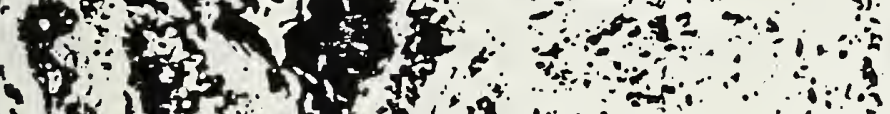

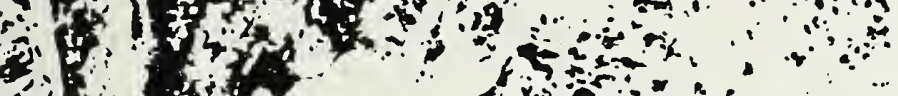

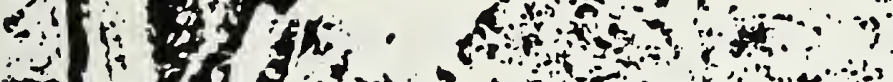

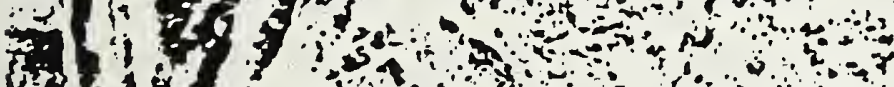
4) 1, 1. ?

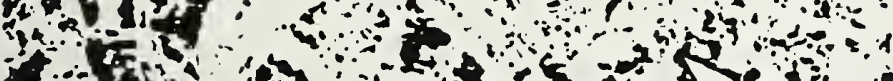
सfol

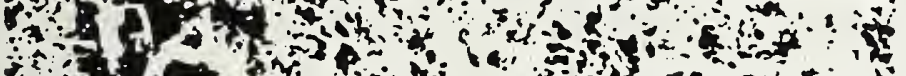
(1) 10 a

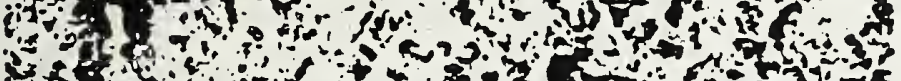

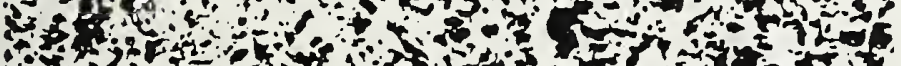

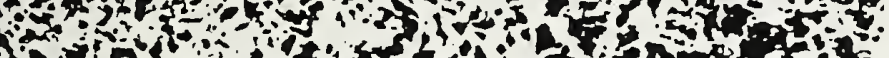

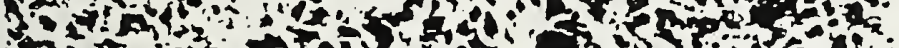
20

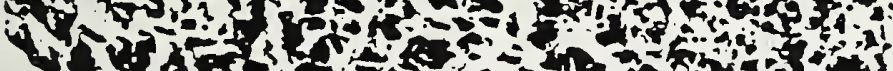

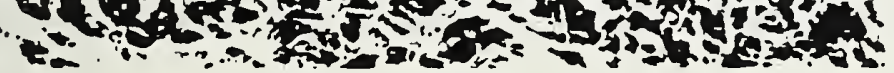
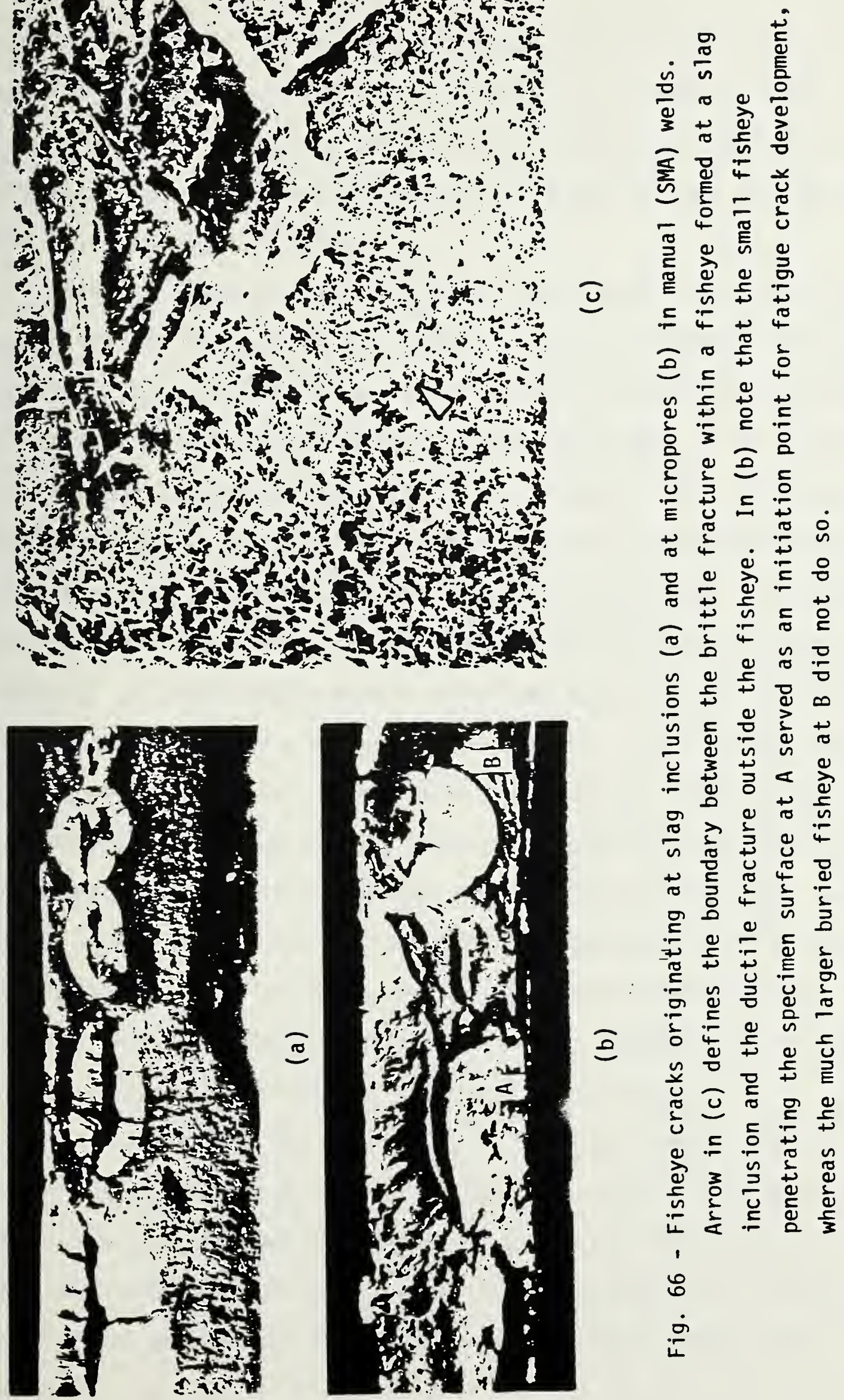

(see Fig. 69) shows that by this criterion a 12-mm $(0.5-i n)$ wide arc burn would be acceptable, even if it were treated as a crack of equal dimension. Such a large arc burn is unlikely to occur in practice, substantiating the conclusion about the innocuous nature of this type of flaw in pipe materials that fail by plastic collapse.

Figure 67 combines the results of the present low-cycle, straincontrolled fatigue program on pipeline girth welds tested (reinforcement removed) with the results of a similar low-cycle fatigue program conducted by the British Welding Institute (BWI) in their assessment of the significance of porosity and slag on the performance of pressure vessel weldments. ${ }^{74,75}$ Specimen sizes, flaw contents, and parent-metal yield strengths were very similar in both programs. The Welding Institute study was conducted at $25^{\circ} \mathrm{C}$ over a total strain range of approximately 0.0009 (below specimen yield strength) to 0.0045 (approximately twice specimen yield strength), with cyclic strain applied between a preset maximum and zero. In contrast, the present study investigated flaw significance under higher total strains of approximately 0.0044 and 0.0090 , produced by fully reversed loading at $-2^{\circ} \mathrm{C}$. Therefore, the present work extends the Welding Institute data to higher strain levels and to lower fatigue life. The Welding Institute data reflect cyclic life to a $60 \%$ load drop, whereas the present data reflects a $40 \%$ drop; however, this difference in failure criteria is insignificant in view of the high crack propagation rates at the higher strain levels.

As noted in Fig. 67, co-plotting of the data suggests a change of slope in the $\log \Delta \varepsilon_{t}-v s .-\log N_{c}$ plot in the vicinity of $10^{3}$ cycles where the total strain range has reached about 0.007 . This is consistent with the observation that the fatigue failure mode changed from propagation of a single fatigue crack front through the flawed welds at the lower strain range $\left( \pm \varepsilon_{y}\right)$ 



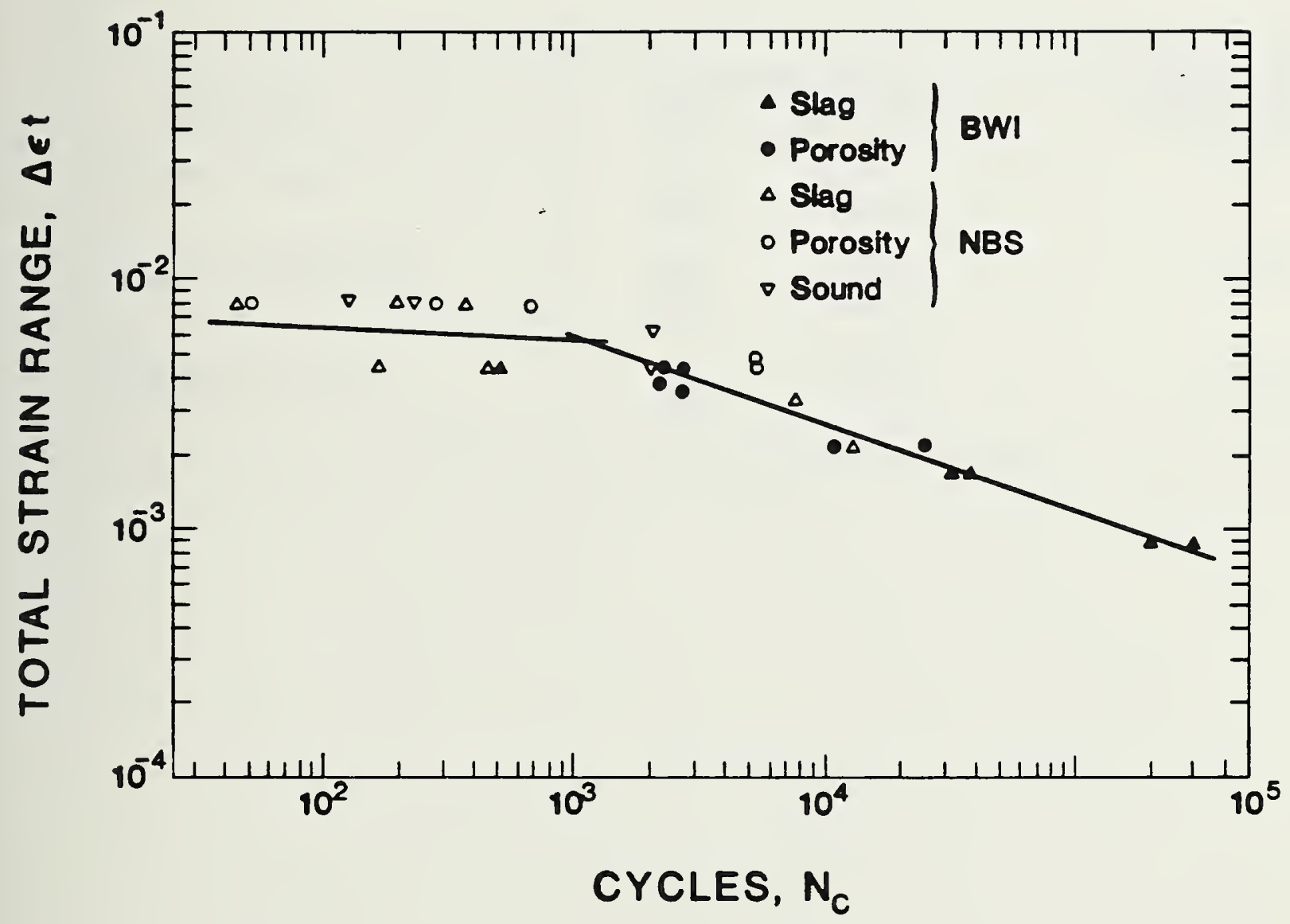

Fig. 67 - Combined results of the NBS low-cycle fatigue study and results published by the BWI in a similar study. ${ }^{74,75}$ Change in slope of the number of cyclic reversals to develop cracks in 40 to $60 \%$ of the cross-sectional area. $N_{c}$, as a function of total strain range is attributed to a change in the failure mode at high strain levels. All data are from welds with the reinforcement removed. 
Table 11 - Ultimate Stress for Welds Pulled to Failure in Tension at $-3^{\circ} \mathrm{C}$

\begin{tabular}{lccc}
\hline Condition & \multicolumn{2}{c}{ U1timate Stress } & $\begin{array}{c}\text { Radiographic } \\
\text { Obscuration }\end{array}$ \\
\hline SPa & psi $\times 10$ & None \\
Automatic Porosity & 651 & 94.4 & \\
& 648 & 94.0 & $\sim 15 \%$ \\
Manual Porosity & 665 & 96.4 & \\
& 651 & 94.4 & $\sim 12 \%$ \\
\hline
\end{tabular}



to multiple initiation and coalescence of cracks, from individual flaws at the higher strain range $\left( \pm 2 \varepsilon_{y}\right)$. Newman and Gurney ${ }^{83}$ have also noted that multiple initiation of fatigue cracks is usually observed at high stresses, leading to rapid crack coalescence. Thus, the conclusion of Harrison et al. ${ }^{75}$ that extrapolatinginformation on flaw significance established under high cycle conditions to endurances of $10^{2}$ cycles on a strain basis may be incorrect for highly flawed welds; a valid extrapolation may be confined to endurances above $10^{3}$ cycles. It also suggests that the constant in Eq. 92 determined from the $\Delta \varepsilon_{t}-v s .-N_{c}$ curve above $10^{3}$ cycles may not be a valid indication of failure ductility on the first loading cycle for flawed welds.

\subsection{Summary}

The probability of fracture initiation from buried slag and porosity or from arc burns has been assessed in manually and automatically welded pipeline girth welds. Highly flawed welds and sound welds were subjected to fully reversed, strain-controlled, low-cycle fatigue at strain levels just above weldment yield and at twice yield. The primary criterion for flaw sensitivity was a comparison of the number of cyclic reversals for crack initiation in the flawed welds and in the sound welds. Additional information on the contribution of the flaws to crack initiation was obtained from fractographic analysis of tests conducted with the weld reinforcement removed.

Porosity and slag had no discernable effect on the cyclic life to initiation at the lower strain level. There was evidence of some reduction in cyclic life in the flawed welds, in particular welds containing slag, at the higher strain level. But, even under this most severe test condition, a minimum of 30 cyclic reversals was required to initiate cracking. Arc burns played no part in crack initiation in any of the weldments, regardless of test condition. A comparison between the performance of flawed welds, sound 

welds, and unwelded pipe material showed that the geometrical discontinuity formed by the weld reinforcement dominated the crack initiation process, regardless of flaw type or content.

Fractographic examination showed no initiation from individual pores at the lower strain level, but such initiation was observed at the higher strain level. Fisheye cracks surrounding micropores and slag inclusions were observed on the fracture surface of all manual welds tested, indicating localized segregation of hydrogen to such flaws during welding with the highhydrogen cellulosic electrodes. Such flaws appeared to have a minimal effect on cyclic life to crack initiation, unless they penetrated the surface.

Because the strain levels were above yield and because the flaw levels were equal to or in excess of those anticipated under worst-case field conditions, these data indicate that the probability of crack initiation from such flaws in statically stressed pipelines containing realistic flaw contents is vanishingly remote. Note that the validity of this conclusion has been demonstrated at only one level of weldment toughness and hardenability. Particularly in the case of arc burns, it cannot be assumed that the conclusions reached in this study can safety be extrapolated to weldments of lower toughness. 



\section{INTRINSIC LIMITATIONS ON DIMENSIONS OF BLUNT FLAWS}

M. B. Kasen, G. E. Hicho, and R. C. Placious

\subsection{Introduction}

Flaws of a three-dimensional, blunt configuration, such as porosity, slag and arc burns, presently account for the majority of remedial welding under workmanship criteria. This partially reflects the sensitivity of radiography to detection of such flaws. Section 4 of this report considered the more general question of the significance of such flaws, assessing their influence on the mechanical stability of weldments under fatigue conditions. We here consider the question of how blunt flaws might be measured, assuming they are considered significant in a fracture mechanics analysis.

Poor sensitivity and diffuse reflection from the flaw periphery limit the detection and measurement of the through-wall depth of porosity and slag by ultrasonic means. Radiography is able to detect such flaws, but gives direct information only on their projected dimensions. Through-wall flaw depth can be estimated by scaling the radiographic density of the flaw to a known density difference--for example, to the density difference caused by a radiographic penetrameter shim of known thickness or to density differences produced by a step wedge exposed and developed under the same conditions as the radiograph of interest. Such an approach was used in applying fracture mechanics principles to assessing the need for remedial welding of flawed girth welds in the Trans-Alaska $0 i l$ Pipeline. ${ }^{4}$ However, intrinsic and human variables plus uncertainties about conditions under which the field radiographs were made introduced large uncertainties into the measurements. This led to extreme conservatism in assessing blunt flaw significance. Subsequent analysis of the procedures used ${ }^{84}$ has shown that some sources of inaccuracy could be ameliorated by improving control over the radiographic inspection 

process, and procedures using advanced state-of-the-art electronic techniques could be used to reduce the subjectivity of such measurements. 36 Unfortunately, such improvements would increase the cost of pipeline inspection while providing relatively modest gains in measurement accuracy.

It was therefore of interest to consider more cost-effective ways of assessing blunt-flaw through-wall dimensions. One such approach is based on the rationale that the depth dimension of porosity and slag should be related to the radiographically projected dimensions and that the maximum flaw depth should be intrinsically limited to the depth of the weld pass in which the flaws occur. The Trans-Alaska pipeline work ${ }^{4}$ had shown that the depth of arc burns could be correlated with burn widths measured from field radiographs. The present work sought to confirm the validity of the principle for porosity and slag.

\subsection{Experimental Procedures}

Pipeline segments containing porosity and slag were cut out circumferentially adjacent to the weld reinforcement. The segments were taken from the welds fabricated as detailed in Table 5 and were selected to provide examples of isolated flaws. Samples containing the flaws were then radiographed normal to the weld and in the plane of the pipe, so as to reveal both projected flaw length (or width) and flaw depth (through-wall flaw dimension). Slag in the form of "wagon tracks" in the manual welds required sectioning of the welds down the centerline and studying each half independently. The samples of porosity were obtained from automatic welds. Several methods were investigated for measuring the flaws; the method finally selected was an image-enhanced television projection of the flaw images at enlargements up to four diameters. Correlation of flaw dimensions with that of the weld-pass depth was established by measuring pass depth directly from 

polished and etched weld cross sections. It was recognized that the true flaw size is somewhat larger than that revealed by the projected dimensions; however, the latter was used because it is the criteria used in all flaw measuring from radiographs, as required for a fracture mechanics analysis. Thirty-nine specimens containing slag and ten containing porosity were studied.

\subsection{Results}

The experimentally determined relationship between the through-wall depth, $D_{f}$, of porosity and slag and the thickness of the weld pass, $H$, in which the flaws occur is shown in Fig. 68. Similar data illustrating the relationship between the radiographically projected dimensions are illustrated in Fig. 69.

Both techniques confirmed that the through-wall depth of porosity and slag was essentially confined to a dimension equal to or less than that of the depth of the weld pass in which they occur. Flaw depth was also confined to a dimension less than that of the average weld-pass thickness measured between the plate surfaces. The widths of slag stringers, $w_{f}$, were always less than slag depth, whereas the length of the pores, $L_{f}$, was equal to or larger than their depth. The projected length and depth of pores appeared to be linearly related for very small pores, but this relationship broke down as the pore size approached that of the weld-pass depth. Slag depth could not be predicted from slag width.

Fractures of the porous weld illustrated in Fig. 70 provides additional evidence that porosity in an underlying pass is not likely to be extended into a successive sound pass during multipass welding. Here, the succeeding pass has cut into the porous region of the underlying pass, but the quality of the successive pass was not affected. The difficulty experienced in producing 


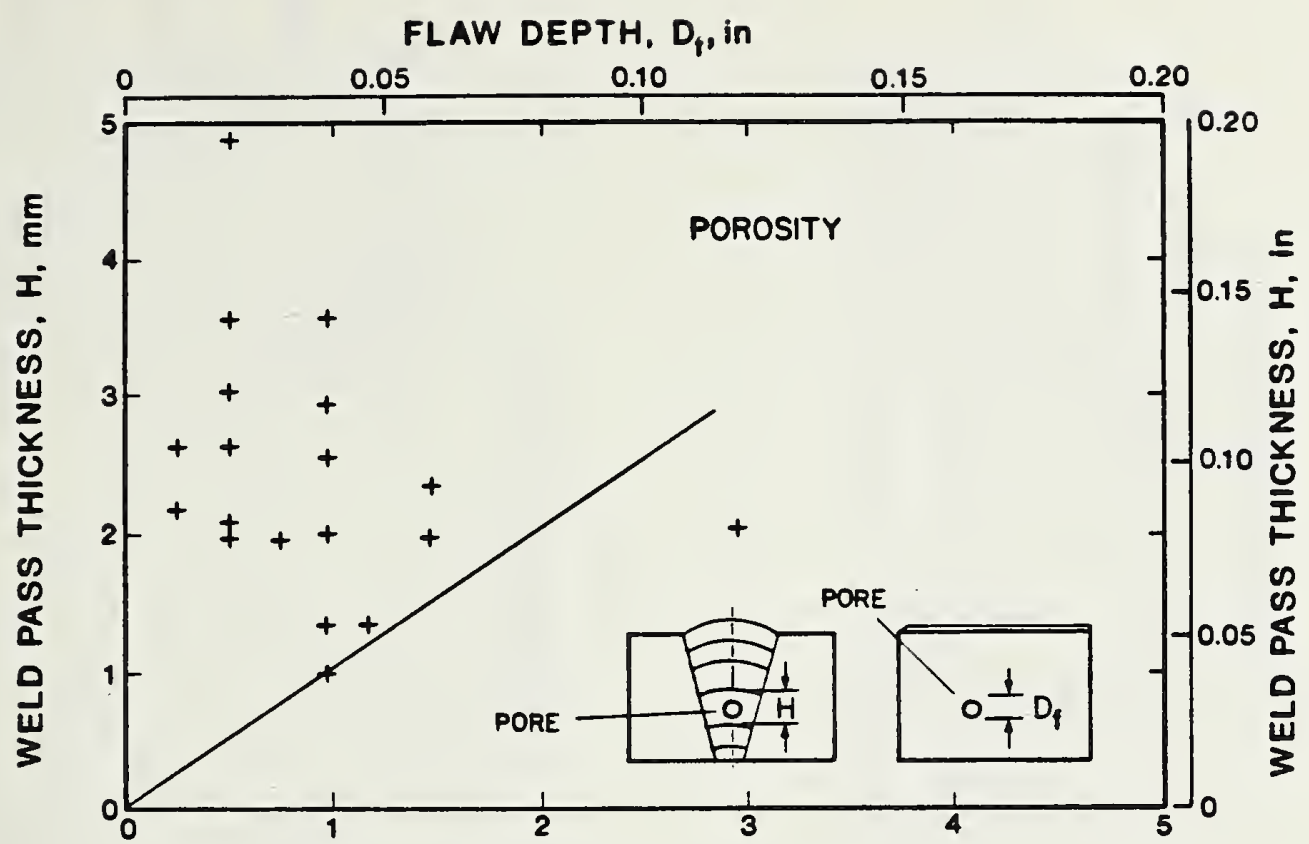

FLAW DEPTH, $D_{f}, \mathrm{~mm}$

FLAW DEPTH, $D_{f}$, in

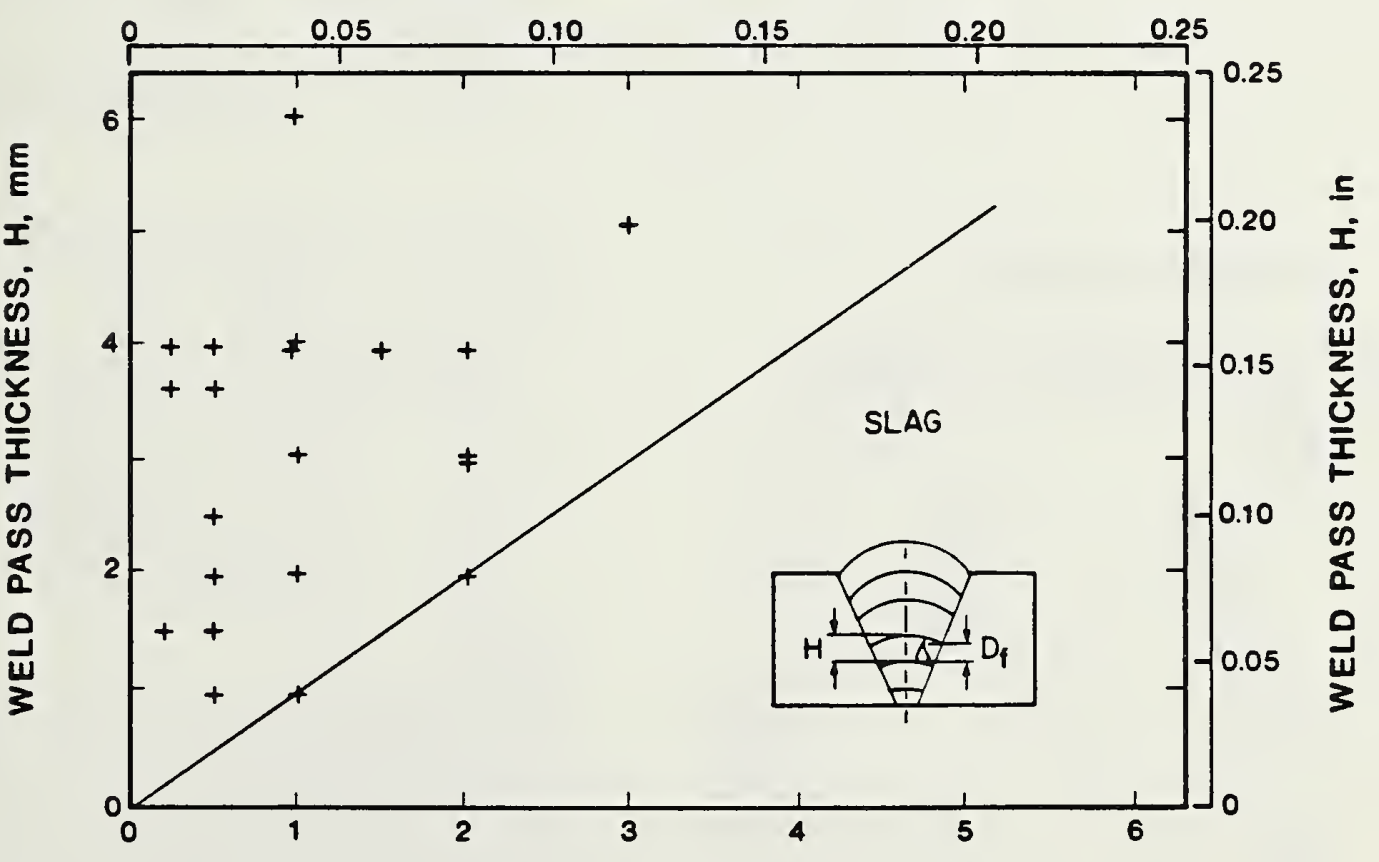

FLAW DEPTH, $D_{f}, \mathrm{~mm}$

Fig. 68 - Experimentally determined relationship between the through-wall depth of porosity and slag and the thickness of the weld pass in which the flaw occurred. 





high levels of buried porosity during manual welding (see Section 4) suggests that this assumption is generally valid.

Results of prior studies on the inherent depth limiations of arc burns are illustrated on Fig. 69c. ${ }^{4}$ In practice, the arc-burn depth is taken as the intercept between the burn diameter (or maximum width) on the ordinate of this figure and the lower bound of the scatter band depicting total burn depth. Arc burn length is measured directly from the radiograph.

\subsection{Summary}

It has been shown by direct measurement on automatic and manual welds that the maximum through-wall depth of slag and porosity is intrinsically limited to a maximum dimension equal to the depth of the weld pass in which such flaws occur. It was found that the depth of porosity or slag could not be predicted from the projected dimensions of such flaws on a radiograph.

These results confirm that characterizing the average weld-pass thickness associated with a specified welding procedure can provide an upper limit to the through-wall dimensions of porosity and slag, should such information be desired in a fracture mechanics analysis. It is probable that a more precise measurement would rarely be justified in practice, because it is probable that most flaws having depths equal to that of the average typical pipeline girth-weld-pass thickness would likely be acceptable.

These present results complement previous studies demonstrating an inherent limitation to the through-wall depth of arc burns based on burn width. 



\section{PRACTICAL LIMITATIONS ON BLUNI FLAW CONTENT \\ USING RADIOGRAPHIC INSPECTION \\ M. B. Kasen}

Although the studies described in Section 4 of this report indicate that blunt flaws have a low probability of initiating brittle fracture, their presence may interfere with detection of significant, sharp flaws during the inspection process. This is much less of a concern when ultrasonic techniques are used, because the proximity of blunt and sharp flaws should enhance rather than diminish the flaw signal. But when radiography is used as the inspection method, the high radiographic density produced by blunt flaws can mask the presence of sharp flaws unless limits are placed on the permissable size and distribution of blunt flaws.

Slag does not present a problem in this context because of its localized nature in the weld. However, a primary concern is the possibility that excessive scattered porosity can mask the presence of sharp flaws, such as lack of fusion, incomplete penetration, or cracks, when radiography is used as the primary inspection tool. Studies by the British Welding Institute ${ }^{71}$ have suggested that obscuration must be limited to less than $10 \%$ of the projected radiographic area to avoid this possibility. The British Standards Institution has, therefore, recommended limiting the permitted obscuration to less than $5 \%$ for ferritic steel weldments having a Charpy $V$-notch energy absorption of not less than $40 \mathrm{~J}(29 \mathrm{ft}-1 \mathrm{bf})$ at the minimum service temperature. For materials of lower toughness, porosity should be assessed as a planar flaw on the basis of flaw interaction. ${ }^{8}$ The Working Group on the Significance of Defects under Commission $X$ of the International Institute of Welding has reached a similar conclusion in proposing acceptance levels for 
defects that may fail by brittle fracture. 73 This group recommended accepting porosity contents up to $5 \%$ of the projected area of the radiograph for welds in ferritic steel having yield strengths up to $482 \mathrm{MPa}\left(70 \times 10^{3} \mathrm{psi}\right)$, provided that the weld metal has a minimum Charpy energy absorption of $41 \mathrm{~J}$ (30 ft-lbf) at the minimum service temperature. In other cases of lower toughness, $3 \%$ obscuration would be acceptable. These would appear to be reasonable figures, because welds containing less than this level of porosity are easily obtained by good practice, and the probability of $5 \%$ obscuration masking sharp flaws in tough weld metal is very low. Since Section 4 has shown that there is a negligible effect on crack initiation due to a 13 to $15 \%$ obscuration by porosity, a 3 to $5 \%$ level would also appear to be conservative from a performance point of view.

Several procedures have been suggested for correlating the percentage of radiographic obscuration due to porosity in welds with porosity content by volume. 71,85 However, such methods are, for the most part, tedious and timeconsuming and provide more detailed information than is required for field interpretation of weld quality. If a $5 \%$ maximum obscuration level is chosen, a reasonable means of distinguishing between the appearance of a weld containing porosity at a $3 \%$ and at a $5 \%$ obscuration level should be sufficient. One possibility is to compare the radiograph with a series of sketches illustrating the appearance of porosity distributions covering the range of interest. Such a series, based on similar sketches currently used in API 1104 and in other weld-quality standards, is shown in Fig. 71. These illustrations suggest that, if an acceptance level of $4 \%$ were specified, an interpreter might err one percentage point higher to a $5 \%$ level, but would be much less likely to err $2 \%$ to a $6 \%$ obscuration level. This is because the human eye is much more capable of judging small differences in obscuration at low obscuration levels than at high levels. 

The importance of the dot distribution size in the reference standard is illustrated in Fig. 71. Here, it appears that the percentage obscuration increases from top to bottom, whereas in fact each sketch has the same five percent obscuration. This difference in visual perception is caused by a progressively finer dot distribution from top to bottom among the sketches, illustrating that reference sketches containing more simulated large pores than would be likely in the radiographs would give the interpreter a conservative bias. 



\section{Simulated Porosity}

\section{Obstruction}

$3 \%$ $\vdots \bullet \because \bullet \bullet \bullet \bullet \bullet \bullet \bullet \bullet \bullet \bullet$
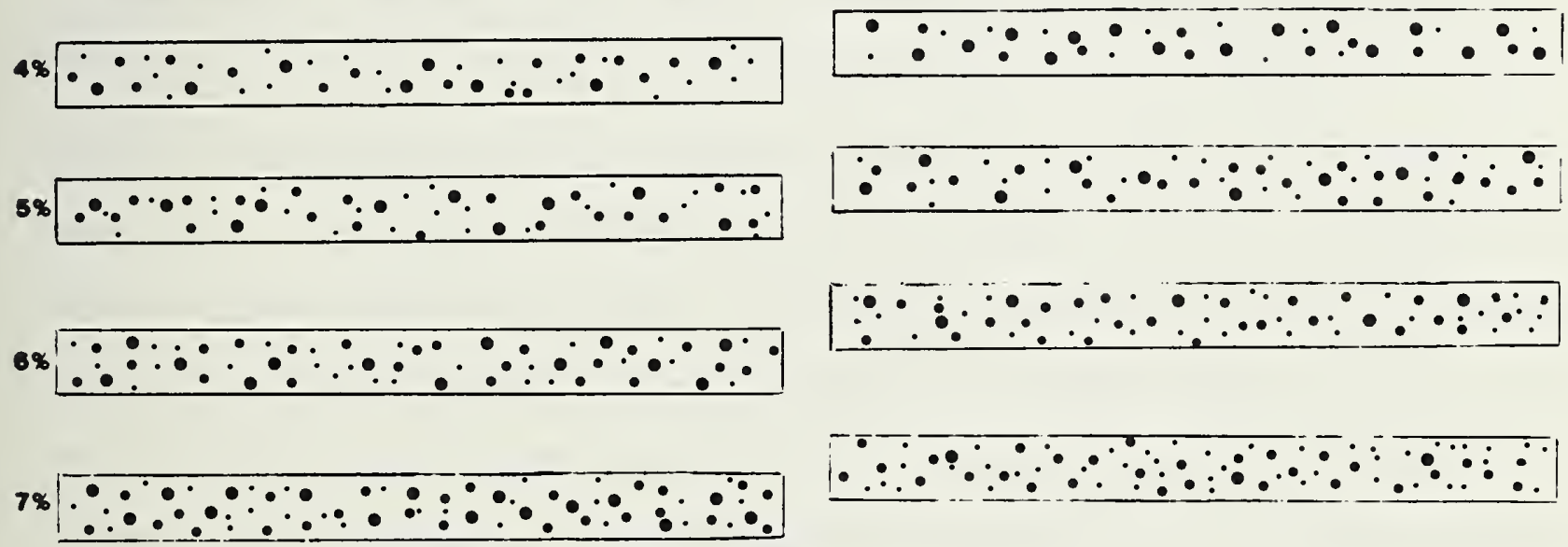

Fig. 71 - Possible development of a radiographic reference standard for establishing limits to permissible obscuration due to scattered porosity. Series (a) illustrates the relative ease of distinguishing percentage differences in obscuration in the 3 to $7 \%$ range. Series (b) illustrates that the eye is biased toward a higher degree of obscuration as the size of the scattered porosity decreases. Each of the sketches in (b) contains $5 \%$ obscuration. 



\section{CONCLUSIONS}

Studies have been conducted to establish fitness-for-service criteria for large-diameter pipeline girth welds.

1. A fracture mechanics analys is model that relates allowable flaw sizes to applied stress level and fracture toughness has been developed, verified experimentally, and used to calculate allowable flaw-size curves for the proposed ANGTS pipeline. The model is based on a yielded-ligament principle and incorporates a trhough-thickness plasticity correction. Experiments on surface cracks in tensile panels, welded pipe segments, and large-diameter $(900 \mathrm{~mm}, 36-$ in) pipes confirmed the validity of this model. The applicability of the model is limited to pipelines with maximum stresses in the longitudinal direction below the specified minimum yield strength of the pipe. It is further limited to pipelines where the yield strengths of the weld and base metal are similar. The degree of similarity in yield strengths has not been established, but the desirability of higher yield strength in the weld metal has been clearly demonstrated. The use of allowable flaw-size curves can be considered for either general pipeline use or for site-specific engineering assessment. Allowable flaw-size curves generated using typical pipeline operating conditions and weldment properties are more conservative for long flaw lengths than those derived using the procedures of Appendix $A$ of the 16 th edition of API $1104 .^{2}$

2. Radiography has serious deficiencies as an inspection tool in a fitness-for-service analysis. Experience and laboratory studies have shown that it is relatively insensitive to significant sharp flaws in a fracture mechanics evaluation. Through-wall flaw dimensioning from radiographs has also been found to be inaccurate. Ultrasonic methods are sensitive to sharp 

flaws and are more adaptable to measuring flaws. However, conventional systems are too slow and complex for routine pipeline inspections. In this study, a new ultrasonic inspection system was developed for this task. The system utilizes electromagnetic transducers (EMATs) to generate long-wavelength shear (SH) waves. Unlike conventional techniques, this approach does not require contact between the ultrasonic transducer and the pipeline. The long-wavelength, low-frequency signal simplifies flaw detection because the signal scales monotonically with increasing through-wall dimension in the range of interest. Specifically, it has been shown that vertically oriented $0.75-\mathrm{mm}(0.0295-\mathrm{in})$ deep and $25-\mathrm{mm}(0.984-\mathrm{in})$ long surface flaws and 2-mm $(0.782-i n)$ deep and $25-m m(0.984-i n)$ long interior flaws can be reliably detected and measured. Detectability limits for shorter flaws have been determined and related to representative workmanship and al ternative acceptance standards. Furthermore, it has been demonstrated that the flaw signal is relatively unaffected by differences in type, orientation, and roughness of the flaws. The use of $\mathrm{SH}$ waves enhances sensitivity to sharp flaws oriented perpendicular to the surface, while providing relatively low sensitivity to blunt flaws. This facilitates detectability of the most significant flaws in a fitness-for-purpose analysis. The system is much less sensitive to the presence of weld reinforcements than are conventional systems. It is adaptable to full or partial automation, and it increases the reliability of flaw detection and sizing because it depends less on operator skill. At its present state of development, the SH-wave EMAT inspection system has the following deficiencies: 1) performance has not been demonstrated with real flaws under field conditions, 2) field proven hardware remains to be developed, 3) personnel trained in the system are not readily available, 

4) equipment calibration standards remain to be developed, and 5) sensitivity of the system to detecting tight cracks closed by compression stresses has not been determined.

3. The presence of large quantities of buried porosity and slag, or of arc burns in manual welds has been shown to have a negligible effect on the low-cycle fatigue life of the pipeline girth welds evaluated in this study. A similar result has been shown for porosity in automatic welds. Because the applied strains were well in excess of yield strengths, and the flaw levels were in excess of those anticipated under worst-case field conditions, these results indicate that the probability of crack initiation from such flaws would be negligible if such welds were subjected to the essentially static loading conditions of operating pipelines. These results suggest that such blunt flaws may be considered innocunus as fracture initiation sites in girth welds fabricated with materials of the toughness studied in this program. The validity of this conclusion has not been experimentally demonstrated for welds of lower toughness; however, the conclusion is consistent with that of others who have addressed the problem of blunt flaw significance in weldment failure. It is emphasized that these conclusions pertain only to buried porosity and slag and to arc burns not associated with cracks. Porosity breaking the surface or arc burns containing cracks should be treated as equivalent-sized sharp flaws.

For welds having substantially lower toughness than that evaluated in this program, blunt flaws may be conservatively treated as sharp flaws of equal dimensions. This work has shown that the maximum through-wall dimension of porosity and slag is intrinsically limited by the depth of the weld pass in which they occur or to flaw-width criteria in the case of arc burns. The 

upper limit to flaw depth provided by these criteria can therefore be conservatively used in a fracture mechanics analysis, eliminating the need for measuring the through-wall depth of flaws in the field.

Low-cycle fatigue caused fisheye cracks to form adjacent to micropores and slag in all welds made with E8010G cellulosic electrodes, even though testing was performed $1 \frac{1}{2}$ years after welding. This indicated a stable, residual hydrogen content in the weld metal. Available data indicate that such flaws can also occur if static loads approach or exceed weld-metal yield, suggesting the possibility that such flaws could develop during the pipe-laying operation subsequent to the inspection process. Except when they penetrated the surface, such cracks did not appear to affect the fatigue strength significantly, susgesting that they are basically innocuous flaws in weldments of the toughness tested. However, it cannot be assumed that cracking from this source will remain innocuous in weld metal of lower toughness. Conditions under which fisheye cracks develop in high-hydrogen electrode pipeline welds deserve future study. 



\section{RECOMMENDATIONS}

\subsection{Implementation of Inspection Methods}

The practical implementation of a fitness-for-service inspection system for a pipeline involves many factors outside the scope of this report. Nevertheless, it is instructive to consider the technical factors that might influence the manner in which the inspection system is integrated into an overall quality control program. Three possible options, based solely on perceived technical merit, are listed in Table 12.

\subsubsection{Option I}

The first option assumes that all of the welds will be inspected by an automated ultrasonic system to provide the basic flaw detection and measuring capability required for the fitness-for-service analysis. It is also envisioned that some fraction of each welder's production be inspected by conventional radiography to provide control over workmanship, serviceability of welding equipment, and quality of consumables. Since welds inspected by radiography would also be inspected by ultrasonic means, radiography would not be used to establish an accept/reject criterion for weld quality. This option is technically preferable because it differentiates between the purpose of each inspection technique and uses each in the area of its greatest strength.

Documentation provided by Section 3 of this report suggests that an ultrasonic system based on low-frequency SH waves generated by electromagnetic 

transducers (EMATS) would provide a promising option for flaw detection and measuring with the best adaptability to automation. Such a system will not respond to the presence of blunt flaws unless they are very large or locally concentrated. This is a distinct advantage because, as documented in Section 4 of this report, blunt flaws may be considered intrinsically innocuous, from a fitness-for-service point of view, if minimum toughness levels are maintained. There is no possibility of sharp flaws being obscured by the presence of blunt flaws, because proximity of the two flaw types will enhance the ultrasonic signal.

This option assumes that blunt flaws not detected by the ultrasonic inspection method can be neglected, based on the work described in Section 4. Strictly speaking, this assumption has only been validated for weld metal of the toughness studied in this program; however, the conservatism built into the present investigation, along with the conclusions reached by others who have studied the problem, suggests that the assumption would probably be valid for any reasonable minimum toughness specified for pipeline construction. Additional conservatism arises from the realization that slag, which this work has shown to be the most likely of the blunt flaws to initiate fracture, is relatively easily detected by ultrasonic means due to its solid nature.

\subsubsection{Option II}

Option II considers the case where Option I is premature for existing technology. Here, radiography is assumed to be the main inspection system, and ultrasonic methods are used selectively to measure the through-wall depths of sharp flaws that are detected by radiography. Flaw length would be measured directly from the radiographs. This option is less technically desirable because of the relatively poor sensitivity of radiography to sharp 

flaws, which are the most significant from a fitness-for-service point of view. Again, it would appear that development of a portable, manual variant of the ultrasonic system described in Section 3 of this report would be very usefur in this option.

Some restriction must be placed on the allowable content of blunt flaws when radiography is used as the primary inspection tool. Section 6 has discussed the limitations imposed on porosity content due to obscuration concerns. The extent to which large individual pores reduce the weld crosssectional area and, therefore, reduce the weldment tensile strength must also be considered in some circumstances. This is unlikely to be a problem in girth welds made in heavy wall line pipe where the average weld pass thickness (and therefore the maximum through-wall dimension of a pore) is a small percentage of total wall thickness. But it could become a problem where thinner wall pipe is used or where welds are fabricated with automatic processes producing deep, narrow weld passes. Such concerns can be avoided by limiting the allowable dimensions of individual pores to that of a permissible sharp flaw based on a fracture mechanics accept/reject criteria, assuming pore depth to be equivalent to that of the weld pass.

In addition to limits on the quantity and size of allowable porosity, consideration must also be given to its distribution. A localized cluster of porosity exceeding the obscuration limit could conservatively be treated as a single crack of length equal to the maximum cluster dimension and having a depth equal to that of the average weld pass. Since a linear distribution of porosity is frequently found to be associated with incomplete penetration, 86 it would be prudent to treat closely spaced groups as an equivalent-sized single crack. 

In view of the tolerance of tough weld metal to relatively large sharp flaws (see Section 2), it is unlikely that applying a fracture mechanics assessment to the significance of porosity will result in appreciable remedial welding.

Where it is desired to do so, limitations on the allowable length of entrapped slag may also be conservatively established by considering the slag inclusion to be a sharp flaw of the length indicated on the radiograph to have a through-wall dimension equal to that of the average weld pass depth.

As with porosity, this procedure is not expected to contribute substantially to the amount of remedial welding required where weld metal of appropriate toughness is used. Should it be desirable to place a,limitation on acceptable slag width, the present work suggests that a rational limitation would be the same as for slag depth. Although Fig. 68b (Section 5) shows that it is not possible to determine slag depth from projected width, it indicates that the maximum width will not exceed that of the weld pass depth.

Excessively wide slag width may, therefore, be taken as an indication of an excessively deep weld pass. Note that applying this approach to the manual girth welds studied in the present work would result in a practical limitation of $3 \mathrm{~mm}$ on slag width and depth--the same as has been proposed as upper limits on these parameters under the brittle fracture criteria in the British Standard Institution Document PD 6493:1980. ${ }^{87}$

Where it is deemed necessary to analyze the significance of arc burns on a fitness-for-service basis, they may also be treated as equivalent-sized sharp flaws having lengths equal to that of the burns and having through-wall depths scaled from the maximum burn widths by use of Fig. 68c (Section 5). A precedent for this approach was established by the Trans-Alaska Pipeline (TAPS) work. ${ }^{88}$ 

These suggested limitations on the content of blunt flaws when inspection is conducted by radiography are summarized in Table 13.

\subsubsection{Option III}

This option assumes that appropriate ultrasonic methods for sizing planar flaws are not available and that all inspection criteria must depend on information obtained by radiographic techniques. This option is least technically desirable, since sharp flaws are not easily detected and sharp-flaw depth cannot be accurately measured using radiography. Unlike blunt flaws, one cannot, in general, assume an inherent limitation on the through-wall dimension of sharp flaws. The only alternative is to limit sharp flaw length by the criteria of existing workmanship standards.

On the other hand, the suggested treatment of blunt flaws presented under Option II and detailed in Table 13 are equally relevant to this option. Since the majority of current remedial welding is done to remove blunt flaws having sizes in excess of that permitted by workmanship codes, this proposed alternative criteria for acceptability of blunt flaws could substantially reduce pipeline construction costs by providing a more rational approach to deciding when remedial welding is justified to remove such flaws. 



\subsection{Implementation of Fitness-for-Service Criteria}

A fracture mechanics approach to assessing the significance of weld flaws to pipeline integrity can be applied in several ways, depending on the objective of the user. Its use in determining the technical basis for requiring remedial welding in the event of code violations revealed during a postconstruction audit has already been demonstrated during the TAPS program. However, the full potential of the approach requires applying the principles before and during pipeline construction to reduce the need for remedial welding consistent with pipeline structural integrity and safety and, hence, to reduce construction cost.

The latter philosophy can be implemented in two basic ways: One is to establish accept/reject criteria for weld flaws before the construction begins. This would require defining the maximum applied and residual stresses and strains for designated portions of a line where such judgments can be effectively made and combining that information with a characterization of the minimum fracture toughness of the weld metal, as deposited by the consumables and processes selected for the line. Allowable flaw-size curves based on such data could then be routinely applied during construction. Procedures assuring maintenance of minimum weld metal fracture toughness during construction would probably be required. Consideration might be given to generating a series of allowable flaw-size curves reflecting differences in weld-metal toughness, which would permit welds of lower than expected toughness to be accepted, provided that the increased restrictions on flaw size associated with the lower toughness were not exceeded. Advantages of this generalized approach are overall economy and simplicity of field implementation. The criteria established by this approach may be overly conservative for some portions of the line; however, this can be minimized by proper selection of line segments. 

Alternatively, a specific-site approach may be selected, either in conjunction with the generalized approach or independently applied. This involves an engineering critical assessment of welds at highly stressed locations, locations where the welds are subjected to unusually low temperatures or where other environmental factors must be taken into consideration. Since toughness, stress, and allowable flaw size are related by the fracture mechanics model, allowable flaw-size curves can be constructed to address any desired set of conditions. It would be necessary to generate appropriate accept/reject criteria reflecting the specific prevailing conditions. Such an approach would probably result in requests for variances from accepted code requirements. Therefore, at the start, it might prove useful to generate a family of allowable flaw-size curves covering the variables anticipated during construction. Availability of such a family might significantly facilitate implementing either the generalized or specific-site approach.

When qualifying weld metal to a fracture toughness criteria, it should be recognized that weld metal deposited with high-hydrogen, cellulosic electrodes will initially have a low toughness due to the high-hydrogen content in the weld deposit. Toughness will increase with aging time after welding, because the hydrogen content is lowered by diffusion. ${ }^{89}$ Therefore, qualifying conditions should include a delay time subsequent to welding, selected to represent the toughness at the time of maximum weldment stress in the line. Since residual hydrogen content is affected by a number of factors, it may be necessary to characterize the change in toughness with aging time for a particular welding procedure. 

Since residual hydrogen content is affected by a number of factors, it may be necessary to characterize the change in toughness with aging time for a particular welding procedure. 

Table 12 Technical Options for Field Implementation

\begin{tabular}{|c|c|c|c|c|c|}
\hline \multirow[b]{3}{*}{ Option } & \multicolumn{5}{|c|}{ Fitness-for-Service Criteria } \\
\hline & \multicolumn{2}{|c|}{ NDE Method } & \multicolumn{2}{|c|}{ Flaw Assessment } & \multirow{2}{*}{$\begin{array}{c}\text { Workmanship } \\
\text { Criterion }\end{array}$} \\
\hline & Primary & Backup & Sharp & Blunt & \\
\hline \multirow[t]{2}{*}{ I } & Automated & None & e vs. a & None & $10 \% R T$ \\
\hline & UT (EMAT) & & & & \\
\hline \multirow[t]{2}{*}{ II } & Conventional & Manual & e vs. a & $L_{f}$ vs. $D_{f}$ & RT \\
\hline & RT & UT (EMAT) & & & \\
\hline \multirow[t]{2}{*}{ III } & Conventional & None & e only & $L_{f}$ vs. $D_{f}$ & RT \\
\hline & RT & & & & \\
\hline
\end{tabular}

*Assume $D_{f}$ limited to average pass thickness. 

Table 13 - Suggested Limitations on Allowable Content of Blunt Flaws

\section{POROSITY}

\section{Quantity}

Less than $5 \%$ radiographic obscuration

$\underline{\text { Size }}$

Equivalent single crack*

Distribution

Clusters: Over 5\% obscuration treat as equivalent single crack*

Linear: Treat groups separated by less than twice wall thickness as equivalent single crack*

$\underline{\text { SLAG }}$

Treat as equivalent single crack*

ARC BURNS

Treat as equivalent single crack*

*Through-wall depth inherently limited to average weld pass depth. 



\subsection{Future Research}

\subsubsection{Fracture Mechanics}

Procedures for the use of elastic-plastic fracture mechanics for the derivation of allowable flaw-size curves are in the early stages of development. Further work is recommended to increase confidence in the analytical results. Future research needs in the areas of analytical model development, model verification, and implementation are summarized below.

Analytical Model Development The present analytical model presented is a relationship among stress, flaw size, and toughness. For applicability to situations where the strains exceed yield, a relationship among strain, flaw size, and toughness is needed. Further developments should be incorporated into the strain-based model: First, the behavior of small flaws in a large section (such as large-diameter pipe) needs to be modeled to account for gross-section yielding. Second, the differing stress-strain relationships of the weld and the base plate should be incorporated into the model. Third, the influence of residual stresses on fracture at high strain levels should be assessed.

Model Verification The model verification studies in the present program were limited to the analysis of results of large-diameter-pipe tests conducted as part of an independent program. Direct observations of the spread of plasticity are needed for proper modeling of the plastic-zone circumferentially cracked pipe in bending. Further tests are needed to verify the curvature correction proposed herein. And finally, verification of the 

fundamental assumption of the fracture mechanics approach would greatly contribute to confidence in the analysis, that is, verification that large-diameter pipe does fracture at a critical value of CTOD measured in a fracture toughness test.

Implementation. Given an allowable flaw-size curve, an implementation strategy is needed to ensure that the assumptions used in the derivation are adhered to during pipeline construction. Improvements in the CTOD testing method are needed, particularly for evaluating weld-metal toughness. A statistical sampling procedure is needed to ensure that the yield strength and toughness of the pipeline girth welds exceed the values used to establish the allowable flaw-size curves.

\subsubsection{Inspection}

Recent studies have shown that it is feasible to increase substantially the detection sensitivities of the SH-wave EMAT system with respect to planar flaws that are canted with respect to the through-thickness direction. Examples of such flaws include cracks in the heat-affected zone (HAZ) and incomplete fusion. To improve the detectability of such flaws, the present SH-wave EMAT system would have to be augmented by an additional SH-wave EMAT that would be sensitive to the $\mathrm{SH}_{2}, \mathrm{SH}_{3}$, and $\mathrm{SH}_{4}$ plate waves. It can be shown theoretically that these three plate waves are scattered much more strongly by canted planar flaws than the dominant $\mathrm{SH}_{0}$ and $\mathrm{SH}_{1}$ modes. ${ }^{90}$ The technology for constructing such transducers is now available. ${ }^{91}$

Another topic that requires further quantitative investigation is the effect of residual and applied residual stresses on the functioning of the SH-wave EMATs. It is believed that under certain circumstances, the efficiencies and pure-mode characteristics of the periodic-permanent-magnet 

EMATs may be significantly degraded. As a consequence, the EMAT performance characteristics should be verified experimentally using a pipe section welded by approved procedures and subjected to realistic membrane and residual stresses. Current understanding of how SH-wave EMATs function on a surface under stress is very limited.

It is believed that additional work is needed in the area of signal analysis. For example, accurate estimation of the SH-plate wave scattering coefficients could be obtained by nonlinear least-squares fitting of the measured signal amplitudes as function of the distances separating the EMATs from the weld along scan lines that are perpendicular to the pipe girth. Then, the confidence levels in the ultrasonic measurements would be improved. Also, better estimates of flaw shape, cant angle, position within the weld, and other relevant characteristics would be obtained. The least-squares fitting approach is particularly appropriate since an accurate, predictive model for the flaw scattering amplitudes is now available.

Finally, it is believed that construction of a fieldable prototype of the 454-kHz SH-wave EMAT system is necessary. The fieldable system would be needed to establish the influence of specific environmental conditions and welding practices on the inherent limitations of SH-wave EMATs and suggest possible improvements. The prototype system would be evaluated as a limited section of pipeline that has been fully characterized using other available nondestructive testing (NDT) procedures, including ultrasonics, radiography, and penetrant and visual examination. 



\subsubsection{Metallurgy}

Metallurgical parameters affecting the toughness of weldments in pipeline steels deserves further study. The sensitivity of weld-metal toughness to variations in chemical composition of the filler material, to variations in welding procedure, and to the interaction of such parameters should be better defined to increase confidence in the assumption of an existing minimum toughness level in the fitness-for-service analysis. American Welding Society (AWS) classifications of electrodes used in pipeline construction presently give electrode producers wide latitude in selecting a chemistry that will produce weld metal meeting the minimum mechanical properties of the specification. This is because, up to now, weld strength has been the dominating consideration. For example, the Al'S specification for type E8010G electrodes commonly used in manual welding of API $5 L X-65$ and API $5 L X-70$ pipelines establishes minimums for $\mathrm{Mn}, \mathrm{Si}, \mathrm{Cr}, \mathrm{Ni}, \mathrm{Mo}$, and $\mathrm{V}$, but requires that only one of these elements meet the minimums in order to comply with the specification. It is expected that this compositiona? latitude will, in itself, cause a substantial variation in weld metal toughness; however, the extent to which this is true is presently unknown. An equal uncertainty exists with regard to the variation in toughness produced by a given electrode composition at different heat input levels. These uncertainties now require that each manufacturer's proprietary electrode be evaluated for toughness under a specific welding procedure. This is a tedious and expensive process. Resolving these uncertainties will permit establishment of parameters necessary for prequalifying electrode types on the basis of toughness, thus reducing cost while maintaining weldment reliability. 

The present study indicates a negligible probability of fracture initiation from arc burns in contemporary pipeline materials. However, a relationship between arc-burn significance and toughness, hardenability, and other metallurgical parameters has not been established. It would be desirable to do so to provide increased confidence in the findings of the present study. For example, it would be valuable to determine if any circumstances exist under which excessive deterioration of heat-affected-zone toughness could significantly exacerbate the significance of arc burns.

The conditions under which fisheye cracks can form in weld metal deposited by the shielded-metal-arc process should be determined. It is of particular importance to determine the probability of their formation as a result of pipe-laying stresses because, should this occur, significant flaws could be introduced into the welds after the inspection process. The literature suggests that this is a distinct possibility, because such flaws have been most often observed during bend testing where the weld metal has been strained beyond yield. The study should correlate the probability of fisheye formation with the hydrogen content of the electrodes and should establish the required postweld heating conditions required to eliminate their formation, should they prove to be a problem in pipeline construction. 



\section{ACKNOWLEDGMENTS}

This study was supported by the Materials Transportation Bureau (MIB) of the U.S. Department of Transportation. Mr. Lloyd Ulrich, Alaska Natural Gas Pipeline Project, (MTB) Office, Alaska Natural Gas Pipeline Projects, was contract manager. Support for the nondestructive inspection studies was partially supplied by the Welding Research Council, Dr. Glenn Oyler, Research Director.

We particularly appreciated the cooperation of the Northwest Alaskan Pipeline Company, Salt Lake City, Utah. The continued support of Mr. Edward B. Clark of that company was deeply appreciated.

Many people made essential contributions to the present study. We gratefully acknowedge the cooperation of Nova, an Alberta Corporation, in providing data from their large-scale test program. The girth welds investigated in this study were fabricated by the H. C. Price Company, Dewey, Oklahoma, under the direction of Mr. Richard Gwin and Mr. Steve Madden. Artificially flawed manual we?ds were prepared by the Lincoln Electric Company, Cleveland, Ohio, under the direction of Mr. Jerry E. Hinkel. Lowcycle fatigue studies were performed at the Denver facility of the Martin Marietta Corporation by Mr. John Shepic and Mr. Richard Gibb. Radiographic support was provided by the Rocky Flats Division, Rockwell International Corporation, under the direction of Mr. Jack Summers. Calibration specimens required for the ultrasonic study were prepared by Dr. Jerry E. Jones, Colorado School of Mines, Golden, Colorado.

Many NBS people contributed significantly to this study. We particularly acknowledge the contributions of J. David McColskey, Don Harne, Doug Chrisholm, Mel Jadah, Mike Lauriente, Bill Carpenter, and Dr. Richie Mikesell to the success of the experimental program. We are grateful to Ms. JoAnne Wilken for preparation and typing. 

10. NOTATION

10.1 Roman Letter Symbols

a

$a_{0}, a_{1}$

$a_{m}, b_{m}$

$a / t$

$a_{11}, a_{12} a_{22}$

$A_{n}$

$A_{1}$

$\mathrm{A}_{2}$

c

$c_{00}, c_{20}, c_{02}$

$D_{f}$

E

$f$

$f_{C}$

$f_{W}$

$\mathrm{F}$

$F_{b}$

$\mathrm{F}_{\mathrm{c}}$

$g_{b}, g_{t}$ crack depth

relative amplitude of plate wave

relative transduction efficiencies of a plate wave by

transmitter and receiver EMATs, respectively

normalized crack depth

edge crack compliance constants

normalization constant for $n^{\text {th }}$ SH plate mode

gross-section area in the plane of the crack

net-section area in the plane of the crack

contour surrounding crack tip at a point along crack froret

coefficients in Kobayashi's solution for CMOD of an

elastic surface crack

blunt flaw through-wall dimension

Young's modulus

wave frequency

cutoff frequency of SH plate waves

finite width correction of Newman

force per unit length

boundary correction factor for computing stress-intensity

factor of surface cracks

closing force on the ligament of a surface crack

constant for computing stress-intensity factor of an edge crack 



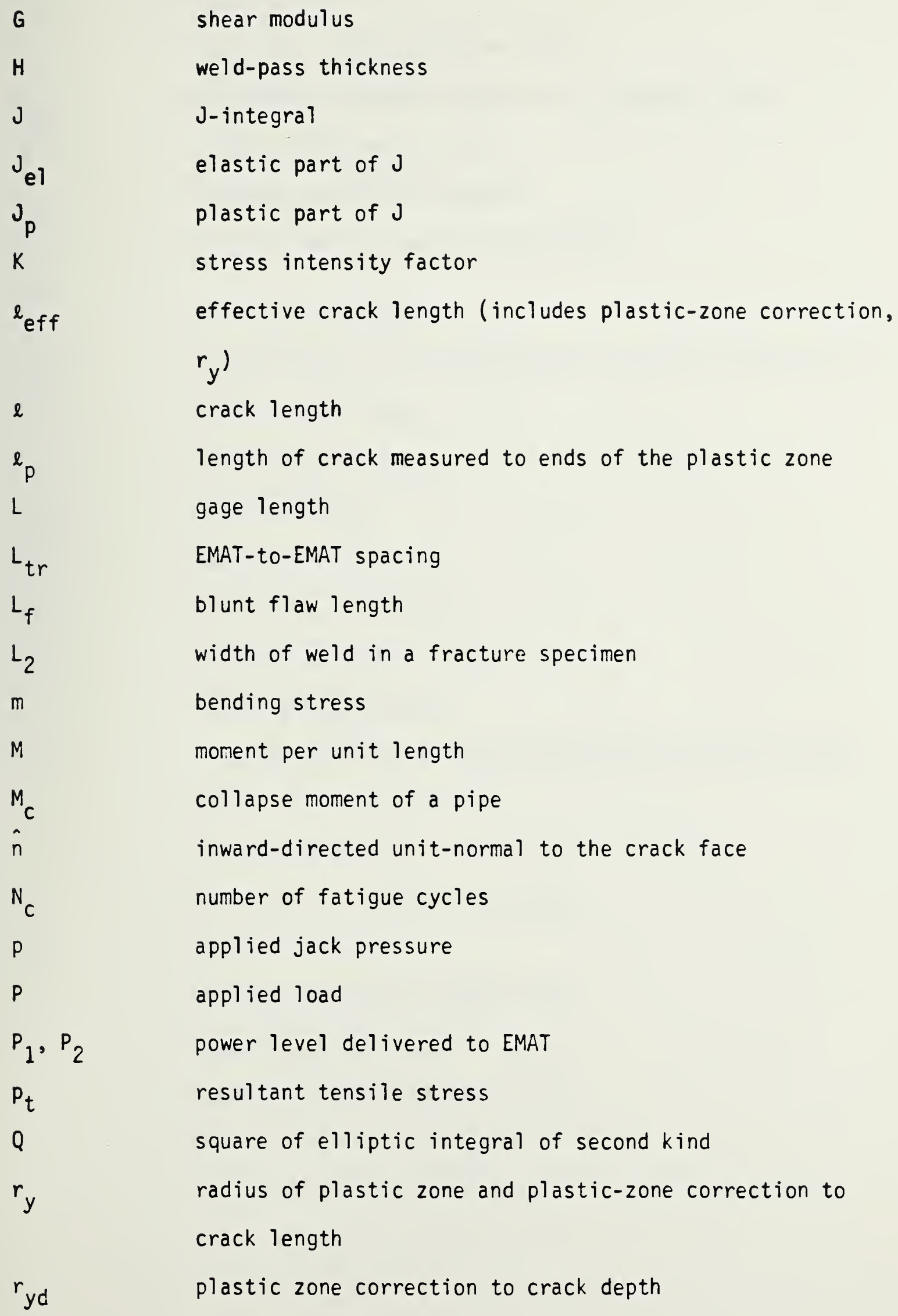





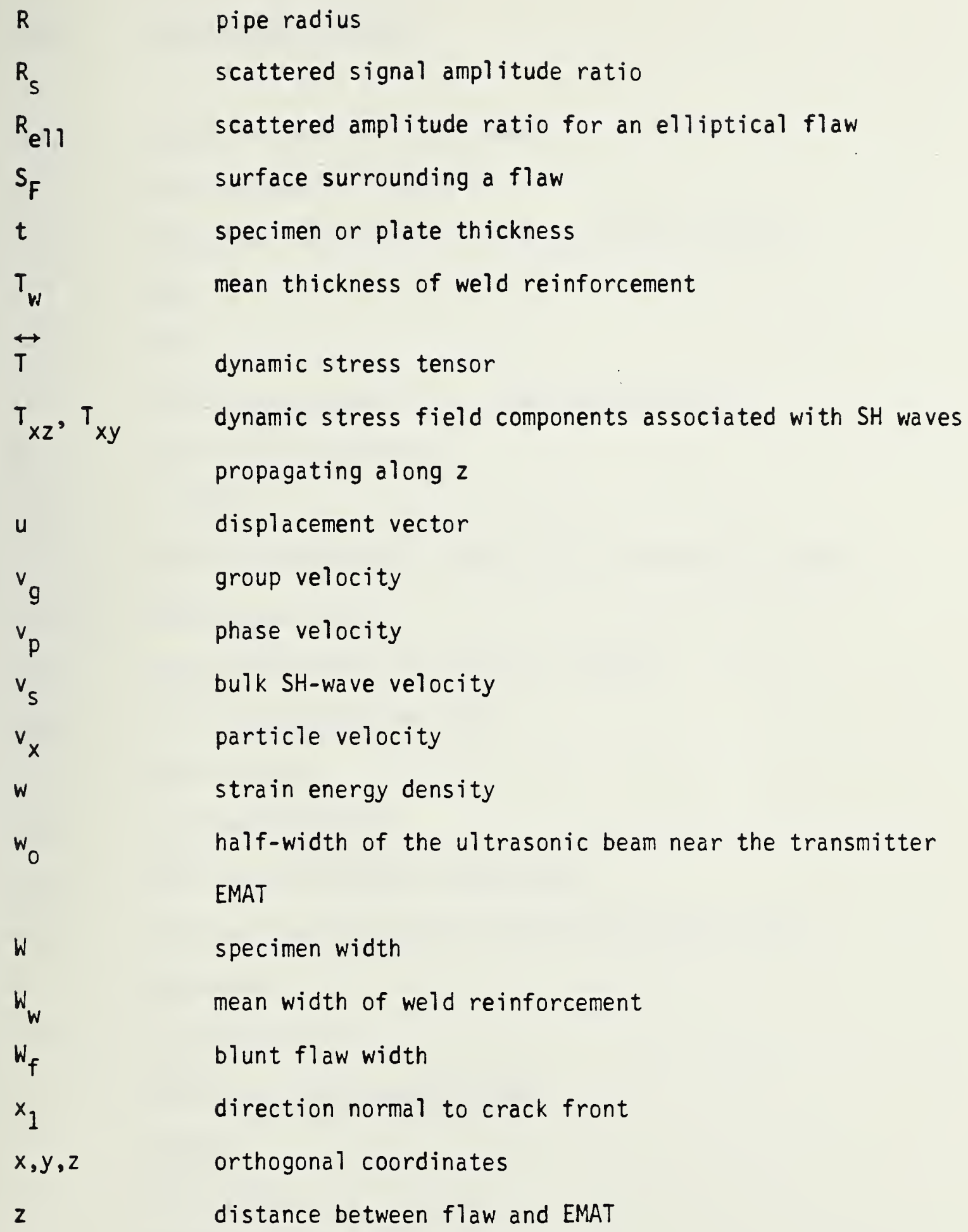





\subsection{Greek Letter Symbols}

$\alpha, \beta, \theta \quad$ angles or angular displacements

$B_{n} \quad$ wave propagation constant

$\Gamma$ reflection coefficient of EMAT ultrasonic signals

$\delta \quad C M O D$

$\delta_{c} \quad$ CTOD

$\Delta^{\prime} \quad$ remote displacement in a center-cracked panel

$\Delta_{c} \quad$ edge crack compliances

$\Delta_{0} \quad 1 / 2$ CMOD

$\Delta u \quad$ mode III displacement jump across the face of a crack

$\varepsilon_{L} \quad$ gage-length strain

$\Delta \varepsilon_{t} \quad$ total strain range in low-cycle fatigue

${ }_{\text {NSY }} \quad$ strain at net-section yield

Ey yield strain

$\varepsilon_{y y} \quad$ component of strain

$\theta_{c} \quad$ edge crack rotational compliance

on angle of elliptical flow with plate surface normal

$\lambda$ wavelength

$\checkmark \quad$ Poisson's ratio

p extent of Dugdale plastic zone

o stress

$\bar{\sigma} \quad$ flow stress

$\sigma_{c} \quad$ closing stress

$\sigma_{L Y} \quad$ applied stress at which ligament yield of a surface crack occurs

${ }^{\sigma}$ NSY net-section yield stress 



$\begin{array}{ll}\sigma_{0} & \text { average tensile stress } \\ \sigma_{u} & \text { ultimate tensile strength } \\ \sigma_{x y} \sigma_{y z} & \text { shear stresses } \\ \sigma_{y} & \text { yield stress } \\ \tau & \text { time } \\ \emptyset & \text { angular position along crack front } \\ \Phi & \text { elliptic integral of the second kind } \\ \omega & \text { angular frequency }\end{array}$

\subsection{Acronyms and Similar Abbreviations}

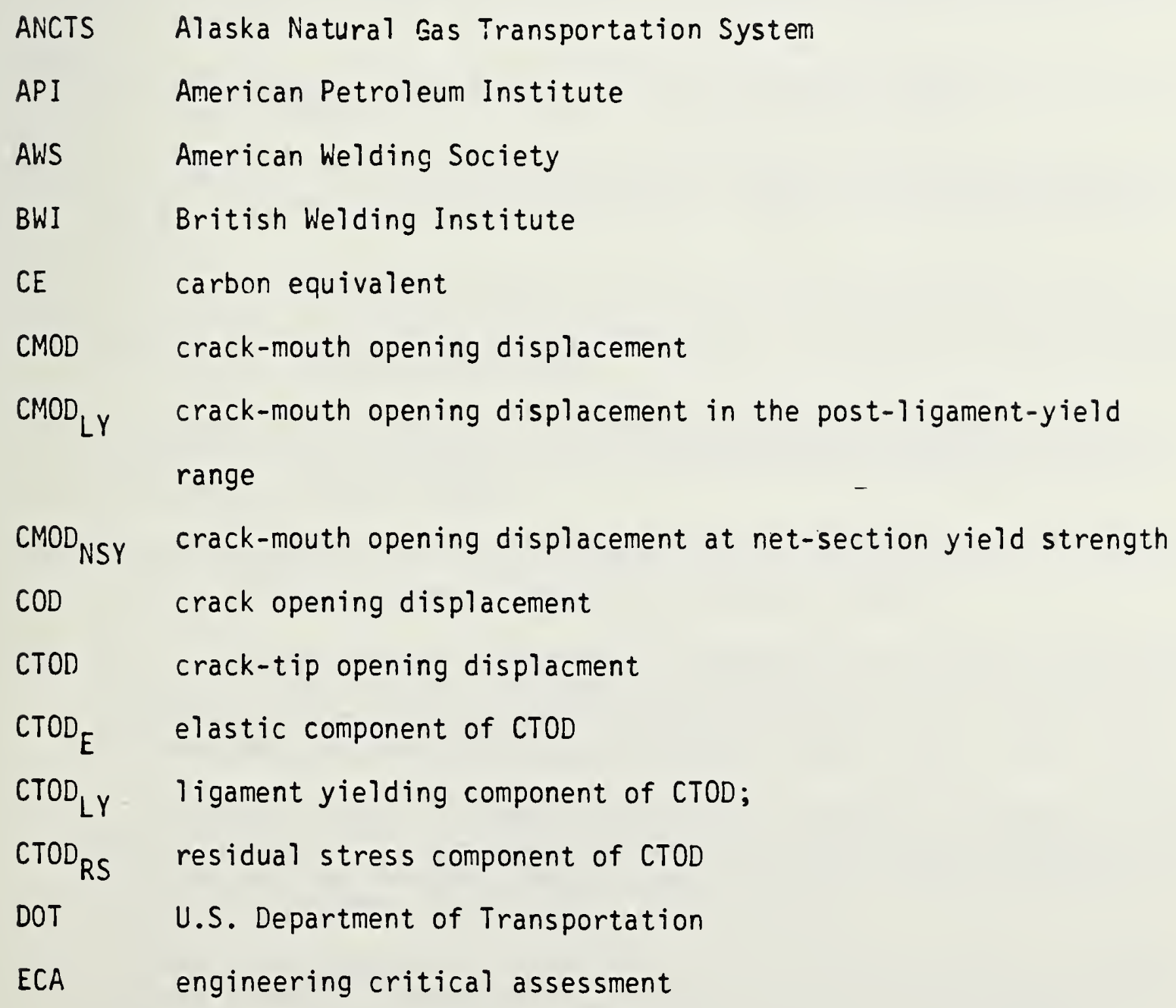





\section{REFERENCES}

1. "Minimum Federal Safety Standards for Liquid Pipeline," Code of Federal Regulations, Title 49 Transportation, Part 195, Office of the Federal Register, National Archives and Records Service, General Services Administration (revised 1 Cct. 1975). Available like a journal in libraries.

2. "Standard for Welding Pipelines and Related Facilities," 15th \& 16th Edition, API Standard 1104, American Petroleum Institute, New York (July 1983).

3. "Consideration of Fracture Mechanics Analysis and Defect Dimension Measurement Assessment for the Trans-Alaska Oil Pipel ine Girth Welds," NBSIR 76-1154, National Bureau of Standards, Gaithersburg, Maryland (Oct. 1976).

4. R. P. Reed, H. I. McHenry, and M. B. Kasen, "A Fracture Mechanics Evaluation of Flaws in Pipeline Girth Welds," Weld. Res. Counc. Bul1: 245, (Jan. 1979).

5. A. A. Butchman, "Trans-Alaska Crude 0il Pipeline" (petition for waiver of girth weld defects), Fed. Regist. 42 119, 31512 (1977).

6. J. W. Barnum, "Trans-Alaska Crude 0il Pipeline" (decision on petition for waiver of girth weld regulations), Fed. Regist. 41233 52933-52949 (1976).

7. "Draft British Standard Rules for Derivation of Acceptance Limits for Defects in Fusion Welded Joints," Document 75/77081 DC, British Standards Institution, London (Feb. 1976).

8. "Guidance on Some Methods for the Derivation of Acceptance Levels for Defects in Fusion Welded Joints," PD 6493 (1980), British Standards Institution, London (1980).

9. "Specifications for Field Welding of Carbon Steel Pipelines," BS 4515 (1969), British Standards Institution, London (1969).

10. M. M. P. Carne and J. D. Harrison, "Proposals for a Fracture Mechanics Based Criterion for the Acceptance of Defects in Pipeline Girth Welds," presented at the American Welding Society International Conference, Pipeline Welding and Inspections, Houston, Texas (Sept. 21-22, 1982).

11. A. G. Glover, and R. I. Coote, "Alternative Inspection Methods and Defect Acceptance Standards for Large Diameter Gas Pipelines," presented at the American Welding Society International Conference, Pipeline Welding and Inspection, Houston, Texas (Sept. 21-22, 1982).

12. "Method of Assessment for Defects in Fusion-Welded Joints with Respect to Brittle Fracture," Japan Welding Engineering Society Standard WES 2805-1980, The Japan Welding Engineering Society, Tokyo (1980). 

13. E. L. Von Rosenberg and C. P. Royer, "Pipel ine Welding Standards," presented at the American Helding Society International Conference, Pipeline Welding and Inspection, Houston, Texas (Sept. 21-22, 1982).

14. "A Critical Evaluation of Numerical Solutions to the 'Benchmark' Surface-flaw Problem," Exp. Mech., 20, No. 8, 253-264 (1980).

15. J. C. Newman, Jr. and I. S. Raju, "Analyses of Surface Cracks in Finite Plates under Tension or Bending Loads," NASA Technical Paper 1518 (1979). Available from NTIS, Springfield, Virginia.

16. J. G. Merkle, "A Review of Some of the Existing Stress Intensity Factor Solutions for Part-through Surface Cracks," ORNL-TM-3983, U.S. Atomic Energy Commission (Jan. 1973).

17. J. C. Newman, Jr., "Predicting Failure of Specimens with Either Surface Cracks or Corner Cracks at Holes," NASA TN D-8244 (1976). Available from NTIS, Springfield, Virginia.

18. G. R. Irwin, "Plastic Zone near a Crack and Fracture Toughness," Proceedings Seventh Sagamore Ordance Materials Research Conference, Vol. IV., Syracuse University Press, Syracuse, New York, 63-78 (1960).

19. D. S. Dugdale, "Yielding of Steel Sheets Containing Slits," Mech. Phys. Solids, 8, 100-108 (1960).

20. G. R. Irwin, "Characterization of Part-through Cracks in Tension," The Surface Crack: Physical Problems and Computational Solutions, ed. J. L. Swedlow, ASME, New York, 1-10 (1972).

21. R. B. King, "Elastic-Plastic Analys is of Surface Flaws Using a Simplified Line-Spring Model," Eng. Fract. Mech., in press.

22. A. S. Kobayashi, "Crack Opening Displacement in a Surface Flawed Plate Subjected to Tension or Plate Bending," Proceedings of the Second International Conference on Mecharical Behavior of Materials, American Society for Metals, Metals Park, Ohio, 1073-1077 (1976).

23. A. S. Kobayashi, "Crack Opening Displacement in a Surface Flawed Plate Subjected to Tension or Plate Bending," Document No. D-180-19446-1, The Boeing Aerospace Company, Seattle (1976).

24. J. R. Rice and N. Levy, "The Part-through Surface Crack in an Elastic Plate," J. Appl. Mech., 39, 185-194 (1972).

25. J. R. Rice, "The Line-Spring Model for Surface Flaws," The Surface Crack: Physical Problem and Computational Solutions, ed. J. L. Swedlow, ASME, New York, 171-185 (1972).

26. D. M. Parks, "The Inelastic Line. Estimates of Elastic-Plastic Fracture Mechanics Parameters for Surface Cracked Plates and Shells," ASME 80-C2/PVP-109, ASME, New York (1980). 

27. R. R. Lockett and D. M. Parks, "The Line Spring Model for J Analys is of Surface-Cracked Plates and Shells," presented at the Second International Symposium on Elastic-Plastic Fracture Mechanics, Philadelphia (1981).

28. M. Shiratori and T. Miyoshi, "Evaluation of the $J$ Integral for Surface Cracks," presented at the Second International Symposium on Elastic-Plastic Fracture Mechanics, Philadelphia (1981).

29. D. M. Parks, "Inelastic Analys is of Surface Flaws Using the Line Spring Model," Proceedings of the Fifth International Conference on Fracture, Pergamon, Oxford, 2589-2598 (1981).

30. R. B. King, Y. W. Cheng, and D. T. Read, "J-Integral Analys is of Surface Flaws in Pipeline Steel Plates," presented at the Second International Symposium on Elastic-Plastic Fracture Mechanics, Philadelphia (1981).

31. Y. W. Cheng, H. I. McHenry, and D. T. Read, "Crack Opening Displacement of Surface Cracks in Pipeline Steel Plates," presented at the ASTM 14th Symposium on Fracture Mechanics, Los Angeles (1981).

32. P. T. Heald, G. M. Spink, and P. J. Worthinton, "Post Yield Fracture Mechanics," Mater. Sci. Eng., 10, 129-136 (1972).

33. R. deWit and J. H. Smith, "Development of Some Analytical Fracture Mechanics Models for Surface Defects in Plates of Ductile Metals," Continuum Modeling of Discrete Systems, University of Waterloo Press, Ontario, Canada (1980).

34. H. Tada, P. C. Paris, and G. R. Irwin, The Stress Analys is of Cracks Handbook, Del Research Corporation, Hellertown, Pennsylvania (1973).

35. R. Coote, Nova Corporation, Calgary, Canada, private communication.

36. S. A. Wenk, "Radiographic Video Technique for Sizing Defects in Pipe Welds," Mater. Eval.., 39, No. 8, 748-751 (1981).

37. P. A. Doyle and C. M. Scala, "Crack Depth Measurement by U1 trasonics," U1 trasonics, 16, No. 3, 164-170 (1978).

38. C. M. Fortunko and R. E. Schramm, "Ultrasonic Nondestructive Evaluation of Butt Welds Using Electromagnetic-Acoustic Transducers," Weld. J., 61, No. 2, 39-46 (1982).

39. C. M. Fortunko, R. B. King, and M. Tan, "Nondestructive Evaluation of Planar Defects in Plates Using Low-Frequency Shear Horizontal Waves," J. Appl. Phys., 53, No. 5, 3450-3458 (1982).

40. C. M. Fortunko, "Ultrasonic Detection and Sizing of Two-Dimensional Defects at Long Wavelengths," Appl. Phys. Lett., 38, No. 12, 980-982 (1981).

41. N. Nielsen, "P-Scan System for Ultrasonic Weld Inspection," Br. J. NDT, 23, No. 3, 63-69 (1981). 

42. A. deSterke, "Automated U1trasonic Inspection of Circumferential Welds in Pipelines," Met. Constr., 12, No. 3, $42-46$ (1981).

43. W. C. Minton, "Ultrasonic Inspection of Pipeline Welds," in Proceedings 1980 Pipel ine Welding and Inspection Conference, ed. D. Weir, American Welding Society, Miami, 65-76 (1980).

44. R. F. Lumb, "Nondestructive Testing of Pipelline Girth Welds in the 1980's," Br. J. NDT, 24, No. 7, 197-207 (1982).

45. C. M. Fortunko, C. F. Vasile, and R. B. Thompson, "Electromagnetic Transducers for Weld Inspection," presented at 37th National Fall Conference of The American Society for Nondestructive Testing, Detroit, Michigan (Oct. 3-6, 1977).

46. C. F. Vasile and R. B. Thompson, "Excitation of Horizontally Polarized Elastic Waves by Electromagnetic Transducers with Periodic Magnets," J. Appl. Phys., 50, No. 4, 2583-2588 (1979).

47. M. deBilly, F. Cohen-Tenondji, G. Quentin, K. Lewis, and L. Adler, "Ultrasonic Evaluation of Geometrical and Surface Parameters of Rough Defects in Solids," J. Nondestruct. Eval., 4, 244-261 (1980).

48. B. A. Auld and S. Ayter, "Perturbation Method for Analyzing the Effect of Uitrasonic Echo Returns on Rough Surfaces in Material Cracks and Voids," 1980 IEEE U1trasonics Symposium Proceedings., ed. B. R. McAvoy, IEEE, New York 852-856 (1980).

49. J. M. Richardson and R. K. Elseley, "Extraction of Low-Frequency Properties from Scattering Measurements," 1979 IEEE U1trasonics Symposium Proceedings, ed. B. R. McAvoy, IEEE, New York, 336-341 (1979).

50. B. Budiansky and J. R. Rice, "On the Estimation of a Crack Fracture Parameter by Long Wavelength Scattering," J. Appl. Mech., 45, No. 6, 453-454 (1978).

51. C. D. Lundin, "The Significance of Weld Discontinuities - A Review of Current Literature," Welding Research Council Bulletin 222, Welding Research Council, New York (1976).

52. M. B. Kasen and R. P. Mikesell, "Interim Report on the Significance of Blunt Flaws in Pipeline Girth Welds," Proceedings of International Conference on Pipeline and Energy Plant Piping, ed. N. F. Eaton, Pergamon, Toronto, 329-336 (1980).

53. R. B. Thompson, "The Relationship between Radiating Body Forces and Equivalent Surface Stresses: Analys is and Application to EMAT Design," J. Nondestruct. Eval., 1, 79-86 (1980).

54. S. K. Datta, C. M. Fortunko, and R. B. King, "Sizing of Surface Cracks in a Plate Using SH Waves," 1981 IEEE U1trasonics Symposium Proceedings, ed. B. R. McAvoy, IEEE, New York, 863-867 (1981). 

55. S. K. Datta and A. H. Shah, "Scattering of SH Waves by Embedded Cavities," Wave Motion, in press.

56. S. K. Datta, A. H. Shah, and C. M. Fortunko, "Diffraction of Medium and Long Wavelength Horizontally Polarized Waves by Edge Cracks," J. Appl. Phys., 53, No. 4, 2895-2903 (1982).

57. J. Krautkramer and $H$. Krautkramer, Ultrasonic Testing of Materials, Second Edition, Springer-Verlag, Berlin, 338-343 (1977).

58. W. J. Pardee and R. B. Thompson, "Half-Space Radiation by EMATs," J. Nondestruct. Eval., 1, 150-181 (1980).

59. B. A. Auld, "General Electromechanical Reciprocity Relations Applied to the Calculation of Elastic Wave Scattering Coefficients," Wave Motion, 1 , 3-10 (1979).

60. N. N. Egorov and A. V. Kharitonov, "Diffraction of Shear Normal Modes by a Surface Crack in an Elastic Plate," Sov. Phys. Acoust., 25, No. 1, 34-37 (1979).

61. R. B. King and C. M. Fortunko, "Extended Variational Solution for Scattering from Flaws in Plates," J. Appl. Phys. 53, No. 5, 3459-3460 (1982).

62. R. B. Thompson, "A Model for Electromagnetic Generation of Rayleigh and Lamb Waves," IEEE Trans. Sonics Ultrason. 20, No. 4, 340-346 (1973).

63. C. M. Fortunko and R. E. Schramm, "An Analys is of Electromagnetic-Accustic Transducer Arrays for Nondestructive Evaluation of Thick Metal Sections and Weldments," in Proceedings 1982 Review of Progress in Quantitative NDE, eds. D. 0. Thompson and C. E. Chimenti, Plenum, New York (1983), in press.

64. R. B. Thompson, S. A. Alers, and M. A. Tennison, "Application of Direct Electromagnetic Lamb Wave Generation to Gas Pipeline Inspection," Proceedings 1972 Ultrasonics Symposium, ed. A. deKlerk, IEEE, New York, 91-94 (1972).

65. H. Tada, P. C. Paris, and G. R. Irwin, The Stress Analysis of Cracks Handbook, Del Research, Hellertown, Pennsylvania, 5.1-8.1 (1973).

66. M. Born and E. Wolf, Principles of Optics, Pergamon, Oxford, 430-433 (1975).

67. J. C. Newman, Jr., "A Review and Assessment of the Stress-Intensity Factors for Surface Cracks," in Part-through Crack Fatigue Life Prediction, ed. J. B. Chang, ASTM STP 687, American Society for Testing and Materials, Philadelphia, 16-42 (1979).

68. R. B. King, and Y. W. Cheng, Fracture and Deformation Division, National

Bureau of Standards, Boulder, Colorado, private communication. 

69. J. M. Richardson and A. G. Evans, "Accept-Reject Decisions for Structural Ceramics: Application to Failure from Voids," J. Nondestruct. Eval., 1, No. $1,37-52$ (1980).

70. J. D. Harrison, "Low Cycle Fatigue Tests on Welded Joints in High Strength Steels," Proceedings, Conference on Welded Structures, Welding Institute, Cambridge (1971).

71. J. D. Harrison, "The Basis for a Proposed Acceptance Standard for Weld Defects, Part 1: Porosity," Met. Const. Br. Weld. J., 4, 99-107 (1972a).

72. J. D. Harrison, "The Basis for a Proposed Acceptance Standard for Weld Defects, Part 2: Slag Inclusions" Met. Const. Br. Weld. J., 4, 262-268 (1972b)

73. F. M. Burdekin, University of Manchester, personal communication.

74. C. R. Boulton, "Acceptance Levels of Weld Defects for Fatigue Service," Weld. J., 56, 13-22 (1977).

75. J. D. Harrison, G. L. Archer, and C. F. Boulton, "Significance of Defects in Pressure Vessels under Fatigue Loading," Tolerance of Flaws in Pressurized Components, The Institution of Mechanical Engineers, London, 255-270 (1978).

76. Y. Ishii and K. Iida, "An Analys is of Intermediate Cycle Fatigue Strength of Defective Welded Joints," Trans. Jap. Weld. Soc., 3, 1-8 (1972).

77. T. R. Gurney, Fatigue of Welded Structures, 2nd Edition., Cambridge University Press, Cambridge, England (1979).

78. N. Bailey, "Fisheyes, Hydrogen Embrittlement, and Removal, "Weld. Inst. Res. Bul1 1., 15, 369-372 (Dec. 1974).

79. A. Matting and M. Neitzel, "The Influence of Weld Defects on the Fatique Behavior of Welded Constructions," Br. Weld. J., 14, No. 1, 3-12 (1967).

80. G. L. Archer, "Research on the Significance of Defects in Weld Regions of Pressure Vessels," Proceedings Conference on Pressure Vessel Standards, The Impact of Change, The Welding Institute, London, 100-106 (1972).

81. G. D. Fearnehough and D. G. Jones, "An Approach to Defect Tolerance in Pipelines, "paper ERS E. 199, Proceedings Conference on Defect Tolerance of Pressure Vessels, paper ERS E.199, Institution of Mechanical Engineers, Great Britain (1978).

82. R. F. Lumb and G. D. Fearnehough, "Toward Better Standards for Field Welding of Gas Pipelines," Weld. J. Res. Suppl., No. 54, 63-s-71-s (1975).

83. R. P. Newman and T. R. Gurney, "Fatigue Tests on $\frac{1}{2}$ in. Thick Transverse Butt Welds Containing Slag Inclusions: lst Interim Report," Br. Weld. J., 11 , No. 7, 341-352 (1964). 

84. R. C. Placious, D. A. Garrett, M. B. Kasen, and H. Berger, "Dimensioning Flaws in Pipel ine Girth Welds by Radiographic Methods, "Mater. Eval., 39, 755-560 (1981).

85. J. Norrish and D. C. Moore, "Porosity in Arc Welds and its Effect on Mechanical Properties," Proceedings Second Conference on the Significance of Defects in Welds, Welding Institute, Cambridge (1968).

86. J. D. Harrison, "The Basis for a Proposed Acceptance Standard for Weld Defects, Part 1: Porosity," Metal. Const. Brit. Weld. J., 4, 97-107 (1972).

87. "Guidance on Some Methods for the Derivation of Acceptance Levels for Defects in Fusion Welded Joints, PD6493, British Standards Institution, London (1980).

88. R. P. Reed, H. I. McHenry, and M. B. Kasen, "A Fracture Mechanics Evaluation of Flaws in Pipeline Girth Welds," Weld. Res. Counc. Bull. 245, Welding Research Council, New York (Jan. 1979).

89. P. Tait and D. M. Haddrill, "Fracture Toughness of Some Mild Steel Manual Metal-Arc Weld Deposits," Weld. Met. Fabr., 370-375 (1970).

90. S. K. Datta, University of Colorado, Boulder, Colorado (private communication.

91. R. B. King and C. M. Fortunko, "Determination of In-Plane Residual Stress in Plates Using Horizontally Polarized Shear Waves," J. App. Phys., 54, 3027-3035, (1983). 



\section{BIBLIOGRAPHY OF RELEVANT NBS PUBLICATIONS}

\subsection{Fracture Mechanics}

"Consideration of Fracture Mechanics Analysis and Defect Dimension Measurement Assesssment for the Trans-Alaska 0il Pipeline Girth Welds," Vols. I and II, NBSIR 76-1154, National Bureau of Standards, Boulder, Colorado (1976).

Y. W. Cheng, R. B. King, D. T. Read, and H. I. McHenry, "Post Yield Crack-Opening Displacement of Surface Cracks in Steel Weldments," submitted for publication in Fracture Mechanics, Fifteenth Symposium, American Society for Testing and Materials, Philadelphia.

Y. W. Cheng, H. I. McHenry, and D. T. Read, "Crack-Opening Displacement of Surface Cracks in Pipeline Steel Plates," to be published in Fracture Mechanics, Fourteenth Symposium, STP 793, American Society for Testing and Materials, Philadelphia.

R. deWit and J. H. Smith, "Development of Some Analytical Fracture Mechanics Models for Pipelien Girth Welds," Fracture Mechanics, Twelfth Conference, STP 700, American Society for Testing and Materials, Philadelphia, 513-528 (1980). 

R. B. King, Y. W. Cheng, D. T. Read, and H. I. McHenry, "J-Integral Analys is of Surface Cracks in Pipel ine Steel Plates, "to be published in Elastic-Plastic Fracture Mechanics, Second International Symposium, American Society for Testing and Materials, Philadelphia.

H. I. McHenry, D. T. Read, and J. A. Begley, "Fracture Mechanics Analys is of Pipeline Girth Welds," Elastic-Plastic Fracture, STP 668, American Society for Testing and Materials, Philadelphia, 632-642 (1980).

D. T. Read and H. I. McHenry, "Strain Dependence of the J-Contour Integral in Tensile Panels," Advances in Fracture Research, Pergamon, New York (1980).

R. P. Reed, H. I. McHenry, and M. B. Kasen, "A Fracture Mechanics Evaluation of Flaws in Pipeline Girth Welds, "Bulletin 245, Welding Research Council, New York (1979).

12.2 Inspection and Dimensioning of Flaws

S. K. Datta, C. M. Fortunko, and R. B. King, "Sizing of Surface Cracks in a Plate Using SH Waves," Proceedings, 1981 Ultrasonic Symposium, ed. B. R. McAvoy, IEEE, New York, 863-867 (1981).

C. M. Fortunko, "Ultrasonic Detection and Sizing of Two-Dimensional Defects at Long Wavelengths," Appl. Phys. Lett., 38, No. 12, 980-982 (1981).

C. M. Fortunko, R. B. King, and M. Tan, "Nondestructive Evaluation of Planar Defects in Plates Using Low-Frequency Shear Horizontal Waves," J. Appl. Phys., 53, No. 5, 3450-3458 (1982). 

C. M. Fortunko and R. E. Schramm, "An Analys is of Electromagnetic-Acoustic Transducer Arrays for Nondestructive Evaluation of Thick Metal Sections and Weldments," Proceedings, 1982 Review of Progress in Quantitative Nondestructive Evaluation, eds. D. 0. Thompson and C. E. Chimenti, Plenum Press, New York (1983), in press.

C. M. Fortunko and R. E. Schramm, "Ultrasonic Nondestructive Evaluation of Butt Welds Using Electromagnetic-Acoustic Transducers," Weld. J., 61, No. 2, 39-46 (1982).

M. B. Kasen, G. E. Hicho, and R. C. Placious, "Inherent Through-Wall

Depth Limitations on Blunt Flaws in Welds," accepted for publication in Weld $\mathrm{J}$.

R. B. King and C. M. Fortunko, "Extended Variational Solution for Scattering from Flaws in Plates," J. Appl. Phys., 53, No. 5, 3459-3460 (1982).

R. C. Placious, "Radiographic Inspection of Pipeline Welds," Pipeline Welding and Inspection, American Welding Society, Miami, 51-63 (1980).

R. C. Placious, D. A. Garrett, M. B. Kasen, and H. Berger, "Dimensioning Flaws in Pipeline Girth Welds by Radiographic Means," Mater. Eval. 39, 755-760.

\subsection{Significance of Blunt Flaws}

M. B. Kasen, "Significance of Blunt Flaws in Pipeline Girth Welds," accepted for publication in Weld. J. 

M. B. Kasen and R. P. Mikesell, "Interim Report on the Significance of Blunt Flaws in Pipeline Girth Welds," Pipeline and Energy Plant Piping: Design and Technology, Welding Institute of Canada, Pergamon Press, Toronto, 329-336 (1980).

M. B. Kasen and R. P. Mikesell, "Effect of Blunt Flaws Studied in Pipel ine Girth Welds," Oil Gas J. (Technol.), 155-159 (March 30, 1981)

12.4 General

M. B. Kasen and C. M. Fortunko, "Fitness-for-Purpose Criteria for Pipel ine Girth Welds," presented at Pipeline Welding and Inspection Conference, American Welding Society, Houston, Texas (Sept. 21-22, 1982).

R. P. Reed, "Goal: Better Standards for Pipel ine Welds," Weld, Des. Fabr., 68-71 (1981).

C. Smith, "Putting It All Together--Alaskan Pipel ine Weld Criteria," Dimensions, , 3-5 (1980). 



\section{APPENDIXES}

\section{Appendix A - Details of Calculation of Allowable Flaw-Size Curves}

The following computer program was used to calculate the allowable flawsize curves presented in this report. Each curve required approximately $40 \mathrm{~s}$ of processor time on a modern large-scale scientific computing facility. 

PROSRAM YIELIG (INPUT, QUTPUT $=11000, T A P E 88=11000, T A P E I, T A P E 2)$

REAL NU

NOTATION:

H IS CRACK DEPTH

C IS CRACK HALF LENGTH

T IS PLATE THICKNESS

$R$ IS PIPE RAOIUS

W IS PLATE FULL hIDTH

CI IS $2 * C I H$

AI IS H/T

DELTAZ IS (EFFECTIVE CRITICAL CTOD) * I H I YIELDST

E IS YOUNG TS MDOULUS

NJ IS POISSONTS RATIC (***** REAL NUHBER $* * * * * *)$

STRESS IS APPLIED STRESS

YIELDST IS YIELD STRENGTH

$S$ IS STRESSIYIELDST

A() IS ARRAY CF CRACK DEPTH VALUES

FL() IS ARRAY OF CRACK LENGTH VALUES

COM.YON PI, DELTA2, C1, S

COMMON IGEJMI HS C, T, R

CCMMON /MATPR/ YIELDST, E, NU

DIMENSION FL $(100), A(100)$, LMESS(8)

$P I=3.141592654$

PRINT 1

FORMATS* PROGRAM TO CALCLATE AND PLOT A VS L CURVES USING THE YIEL

IDED LIGAMENT MCDEL.*,//1)

PRINT 2

FDRMATI* CRITICAL EOD, YIELI STKENGTH, ANE APPLIED STRESS ARE NJW

IREAD IN.*I

READ $(1, *)$ CODCR, YIELDST, STRESS

IF (EOF(I).NE.O) GOTO 9979

CODRES $=0.0015$

YSKSI = YIELDST / 1000.

SKSI - STRESS 11000 .

SET UP AND MARK THE PLOT

PLOTTING SUBROUTINES FROM THE FOISSPLAF (REG. TRADE MARK)

PACKAGE ARE USED IN THIS PROGFAM.

CALL ID(FDAVE READ, $\times 38533$ A VS L\$\$,100)

CALL CJMPRS

CALL NJBRDR

CALL IREA2C (2,2, 2.)

CALL HNSPEC $(88, \neq F I L E \neq)$

CALL ENDPL $(0)$

CALL RESET (ANOBRDRF)

CALL TITLEI FALLOHABLE FLAH SIZES\$, 100,

1 EDEFECT LENGTH, INS $*, 100$,

2 DEFECT DEPTH, INS $\$ 100$,

$36.0,6.01$ 
CALL GRAF $(C .0,5.0,25.0,0.0,0.1,0.6)$

ENCDDE(80, 410, LMESS) CODCR

410 FORMATIFERITICAL CTOD = *,F 7.3,*IN.S*I

CALL MESSAG(LMESS,100,1.6, 5.5)

ENCDOE $(80,415$, LMESS $)$ CDDRES

FORMATIFRES. STRS. CTOD RED. = \#F,4,*IN.S*I

CALL MESSAG(LMESS, 100,1.6, 5.0)

ENCODE $(80,420$, LMESS) YSKSI

FORMATIFFLOW STRENGTH $=\neq, F 5.1, \neq$ KSI.\$\$1

CALL MESSAG(LAESS, 100,1.6, 4.5)

ENCOCE $(80,430$, LMESS) SKSI

Fo FJRMATIEAPPLIED STRESS $=\#, F 5.1, *$ KSI.S\#)
CALL MESSAG(LMESS,1OC, $1.6,4.0)$

430 FJRMATI FAPPLIED STRESS $=\#, F 5.1$
CALL MESSAGILMESS, $10 C, 1.6,4.0)$

PRINT 10, CCDCR, YIELDST, STRESS

FORMATI * CRITICAL COD = *, FI2.6,* IN*,I,

1 * YIELL STRENGTH $=$ *,F12.0,* PSI*,1,

2 * APPLIED STRESS = *, FI2.C,*PSI**I

HERE WE SPECIFY A STEEL PIPELINE, 48 INCHES IN DIAMETER

$$
\begin{aligned}
E & =30.2 E 6 \\
N U & =0.29 \\
R & =24 . \\
& =2 . \\
T & =.625
\end{aligned}
$$

EFFECTIVE CRITICAL COD IS CRITICAL COD LESS RESIDUAL STRESS EFFECT. CODCR = CODCR - CODRES

DELTAZ = E CODCR/H/YIELDST

NPTS $=100$

RELLMAX $=0.15$

$S=$ STRESS / YIELDST

DO 1000 IPT $=1$, NPTS

CI = RELLMAX * FLOATINPTS - IPT) / FLDATINPTS)

$C=W C 1 / 2$.

THREE CANJIDATE VALUES of FIEST TRIAL VALUE of al are calculated

AISIMP $=10-S+D E L T A 2 / 2 . / C I$

AILY - 1. - S

AINSY $=(1 .-5) / C 1$

AINSY = AMINI(AINSY,1.0)

ALTR = AMINI (AISIMP, 0.99999*AINSY)

DEVTARG $=1.0 E-06$

STEPSZ $=1.00$

DEYPREV $=1.0 E 100$

DO 3 OC IIT $=1,15$

DEVVAL = DEV (AITR)

DEVSIZ = ABS (DEVVAL)

IF(LEVSIZ . GT. DEVPREV) GO TO 280 

DEVREL - ABS(DEVVAL/DELTAZ)

IF (DEVREL. LT. DEVTARG) GOTO 312

AITRN - AITR - STEPSZ * DEVHALIDDEVDA(AITR)

IF(AITRN.GT.AINSY.OR.AITRN.LT. $(A I L Y / 2,1)$ GOTO 280

AITR = AITRN

DEVPREV = DEVSIZ

IF (STEPSZ •LT. 1.0$)$ STEPSZ=AMINI $(1.3 * S T E P S Z, 0.5 *(S T E P S Z+1.0))$ GOTO 279

280 STEPSZ = STEPSZIZ.

DEVPREV $=1.0 E 100$

$A I T R=0.75 * A L L Y$

CONTINJE

CONTINJE

AFAIL $=\triangle I T R * T$

FLFAIL $=* C I$

PRINT 306, FLFAIL, AFAIL

FORMATI FLAH DEPTH ITERATION FAILED TO CONVERGE AT*, /,

* LENGTH $=$ \#7.2, DEPTH = \#,F5.2I

312

$A 1=A I T R$

$F L(I P T)=* C I$

$A(I P T)=T * A I$

IF(A).GT.0.75) GOTJ 1020

1000

CONTINJE

$I L A S T=I P T$

DJ $104 J I=1$ I ILAST

PRINT 1025, FL(I), A(I)

$11 C 25$ FORMATI2E14.5)

1040 CONTINJE

c

$c$

CALL CJRVE(FL, A, ILAST,O)

CALL FRAME

CALL ENDPL(-1)

GOTO 4

CALL DJNEPL

CALL EXIT

END

FUNCTIJN DEV(AI)

REAL N'S

COMMON PI, DELTA2, C1, S

COMMCN IGEJMI H, $C$, $T$, $R$

COMAEA IRATPR/ YIELOST, E, NU

$H=T * A l$

$\sim=2$. $* P I * R$ 

FOR THIS VERSION, ALWAYS USE SIMPLIFIED LINE SPRING

USE OF CURVEATURE IN SLS DEPENDS ON CRACK LENGTH

IFIH .GT. 0.0$)$ GJ TO 100

CALL NEWMAN(H, C, T, W, F, O)

$S L Y=1 .-A L$

COD-J FACTOR M SET TO 1 IN DENOMINATOR DF

TERY ON RIGHT OF NEXT STATEMENT.

DELELAS = SLY $\$ S L Y * F * F * P I * H / C / W$

IF (S . GT. SLY) GOTO 200

COD-J FACTOR M SET TO I IN DENCMINATJR OF

2 ND TERY ON RIGHT OF NEXT STATEMENT.

DEV = DELTAL - S*S*F*F*PI*HIOI\#

RETLRN

CONT INSE

STRESS - $S$ * YIELDST

CALL LSJCYIELDST, E, NU, H, C, T, R, STRESS, C.O, CTOD,CMOD,

A STAPLY,STCLOSI

DELELAJ = CTOD $\#$ /W/YIELDST

IF (STAPLY .GT . O.J) SLY = STAPLY/YIELDST

IF (STAPLY .GT. 0.0 ) GO TO 200

DEV = DELTAZ - DELELAS

GO TO 300

CONTINJE

$C P I=(2 . / P I) * A S I N(\operatorname{SIN}(P I * C 1 / 2.) / \operatorname{COS}(P I *(1,-(1,-5) / A I) / 2)$.

RYI $=0.5 *(C P I-C I)$

DEV = DELTAZ - DELELAS - $2 . *(C I+R Y I) *(S-S L Y)$

CONTIVUE

WPITE $(2,310)$ S, STRESS, STAFLY, SLY

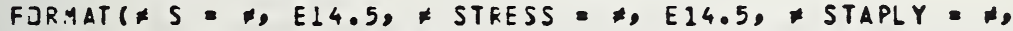

A EI4.5, $\#$ SLY $=\#, E 14.51$

WRITE $(2,315) \mathrm{H}, \mathrm{C}$

FORPAT ( $F=\#, E 14.5, \neq C=\ldots, E 14.5)$

HRITE(2,320) DELTA2, DELELAS, DEV

FOKMAT ( D DLLTA2 = *, E14.5, \#JELELAS * E, E14.5, \#DEV = \#,E14.5) END

FUNETION DDEVDALA)

$Y=\operatorname{DEV}(A)$

$A M_{1}=A * 0.99-1.0 E-03$

IF $A M . L T .0 .0) \quad A M=0.0$

$Y M=D E V(A M)$

DDEVDA $=(Y-Y M) /(A-A M)$

END 

SUBROUTINE NENMAN(H, C, T, H, F, Q)

CEMMON PI, DELTAZ, CI, S

REAL MI, M2, $M 3$

CSAY - C

FORYULAS SET UP FOR $C>H$

IF (H.GT. C) $\mathrm{C}=\mathrm{H}$

$A R G=(P I / 2) *.(2 . * C / \|) * S O R T(H / T)$

$F Y=1.15$ ORT $(\operatorname{COS}(A R G))$

ANGULAR FACTOR = FPHI = I AT ROOT

FPHI - 1.

G FACTOR = 1 AT RDOT

$G=1$.

$\mathrm{MI}=1.13-0.09 *(\mathrm{H} / \mathrm{C})$

$M 2=-0.54+0.89 /(0.2+H / C)$

$M 3=0.5-1 . /(0.65+H / E)+14 . *(1 .-H / E) * 24$

$F=(M I+M 2 *(H / T) * * 2+M 3 *(H / T) * 4) * F P I * G * F W$

$Q=1 .+1.464 *(\mathrm{H} / \mathrm{C}) * 1.65$

$C=$ CSAV

RETJRN

END

SUBROLTINE LSJ (SFLJH,E,YU,H,C,T,R,ST,SB, CTUD,CHOD,STAPLY,STCLOS) REAL NJ, NU7, NU8, KF

DATA (PI $=3.14159265)$

SIMPLIFIED LINE SPRING MCCEL FOP CALEULATION LF APPLIED CTOC FOR

SURFACE FLAN AS A FUNCTION OF STRESS FDR ELASTIC CASE HITH RDOT PLASTIC ZONE CORRECTION.

NOAENCLATJRE

HPHYS = PHYSIEAL CRACK DEPTH

H EFFECTIVE CRACK DEPTH

T PLATE THICKNESS

CPHYS - PHYSICAL CRACK HALF-LENGTH

- EFFECTIVE CEACK HALF-LENGTH

- plate flill hidTH

ST - APPLIED TENSILE STRESS

SB = APPLIED BENDING STRESS

SFLOW - FLOW STRENGTH

E YOUNGTS MODULUS

NU PUISSONTS RATIO

LLY = FLAG FOR LIGAMENT YIELDING

ISSUME PRCPORTIONAL LOADING. LET BJNT = HIS

BONT = SB/ST

C **** HERE THE CALCULATION BEGINS *****

HPHYS $=H$

HSAV = H

CPHYS = C

C LLY IS FLAG INDICATING LIGAYENT YIELCING. LLYKO MEANS N.J LY. 

$L L Y=-100$

PROCEDURE TO SEE IF LIGAMENT IS FULLY YIELDEO OR NOT

MAXIMLIM EFFECTIVE VALLE OF H IS H * $(T-H) / 2$

AT THIS H VALUE, MAXIMUH CLOSURE STRESS HAS BEEN REACHED

MAXIMUM CLDSURE STRESS, AT FULL LIGAMENT YIELD, IS SCMAX

MAXIMUM CLOSURE STRESS OCCURS HHEN SFLOW IS APPLIED ON

THE WHOLE LIGAMENT

SCHAX $=S F L O H *(T-H) / T$

MAXIMUY ALLOHED EFFECTIVE H EXTENDS HALFHAY ACROSS THE LIGAMENT $H=H+(T-H) / 2$.

STAPLY IS APPLIED STRESS AT FIRST DCCURENCE OF FULL LIGAMENT YIELD

CALL CALCI (H,C,T,R,NU, D2,03,04,D,NU7,NUB)

STAPLY $=$ SCMAX*D/ (NJT*(O4+NUE)-NU8*03*BONT)

IFI ST . GT. STAPLY, GO TO 52

C LIGAMENT IS NOT FULLY YIELDED

C SET STAPLY = - 1000. TD INDICATE THAT THE LIGAMENT HAS NOT FULLY

C YIELOED

STAPLY $=-1$ COO

CALCJLATIDN OF ROJT PLASTIC ZONE STARTS HERE

SAVE STARTING VALUE OF H, NO PLASTIC ZONE, AS HSAV

H STARTS AT H IS HSAV, AND INCREASES

UNTIL THE CORRECT PLASTIC ZONE SIZE

IS REACHED BY AN ITERATIVE CALCULATION

NOMENCLATJRE: HSAY IS VALUE CF H BEFOKE ENTERING ITERATION

HSTART IS VALLE OF H AT START OF ITERATION

HCI IS VALUE OF H AT START OF CUERENT ITERATION

BEEIN ITERATION

DELTAH IS SMALL INCREMENT OF H

$H=H J \triangle V$

HSTART $=H$

DO $50 \quad 11=1,30$

$H C I=H$

OELTAH $=.01 * H$

IF (DELTAH .LT. 0.0001) DELTAH $=0.0001$

$H=H+D E L T A H$

CALL CALCI (H,C,T,R,NU, O2,C3,04,D,NU7,NU8)

CALL CLOSUR(O2,03, Q4,D, NU7,NLB,ST, SB, STCLOS,SBCLOS)

STCLOS, SBCLOS ARE TENSILE ANL BENDING CLOSURE STRESSES FOR H-DELTAH

save THESE VALUES OF TENSILE AND BENDING CLOSURE STRESSES

STCSAV=STCLOS

SBCSAY S SBCLOS

C ALTERNATE PROCEDURE IS TO CALCLLATE CLOSURE STRESS FROM THE

C PLASTIC ZCNE SIZE. THE IDEA IS TO FCRCE CONSISTENCY.

C CLOSURE STRESS FROM PLASTIC ZONE SIZE IS SCLOSPZ

CALL SCPZ(H,HSTART, T, SFLOH, SCLOSPZ)

C SCLOSPZ IS ALTERNATE CLOSURE STRESS FOR H+DELTAH

SCP ZSAV = SCLOSPZ

$H=H-D E L T A H$

CALL CALCI(H,C,T,R,NU, $02,03,04, D, N J 7, N U 8)$

CALL CLOSUR (O2,03,I4,D,NUT,AUB,ST,SB, STCLJS,SBCLOS)

CALL SEPZ(H,HSTART,T, SFLOH, SCLOSPZ) 

IC OSICOH, DS3CDH ARE DERIVATIVES OF CLOSURE STRESS, MOMENT MRT H DSTCLH = (STCSAV-STSLOS) /DELTAH

DSBCDH = (SBCSAV-SBCLOS)/DELTAH

OSCP ZOH= (SCPZSAV-SCLOSPZ) /DELTAH

C MAKE THE INDICATED CORRECTION TO H

$H=H+(S T C L O S-S C L O S P Z) /(D S C P Z D H-D S T C D H)$

IF(HCI. LT. 0.000001$)$ GD TO 50

IFI ABS(CH-HEI)/HEI) .LT. O.0CI) GOTO 55

50 CONTINJE

C ITERATION COMPLETE. H IS EFFECTIVE CRACK LENGTH PEINT 51, H, C

51 FORMATI ITERATION DN PLASTIC ZONE SIZE FAILED TO GONVERGE.\#, a $\# H=F, F 7.4, F C=F, F 7.41$

52 COTO 55
52 CONTINJE

C LIGAMENT IS FULLY YIELDED. H=HPHYS $+(T-H) / 2$

C MERE Je CALCULATE THE CMOD ANJ CTOD UP TO LIGAMENT YIELDING

IC THESE ILL BE ADDED TO THE CONTRIBUTION GALCULATEO BY THE YIELDED

IG LIGAMENT MJDEL LATER

$L L Y=100$

S3APLY = SONT $\#$ STAPLY

CALL CLOSUR (O2,J3,J4,D,NU7,NUU, STAPLY,SBAPLY, STCLLY, SBCLLY)

STCLOS $=$ STCLLY

SBCLOS = SBCLLY

ST = STAPLY

$S B=S B A P L Y$

C CTOD ACCFUING AFTER LIGAMENT YIELDS HILL BE CALCULATED ELSEHHERE CONTINJE

C ME NJW HAVE A PLASTIC-ZONE-CORRECTED CLOSUEE STRESS STCLCS

C A PLASTIC-ZONE-CORRECTED CLOSURE MOMENT SBCLCS

C NOH GALCULATE DELTA AND THETA, TO GET COD S

$D E L T A=40 * C *(S T-S T: L O S) / E$

USE CUKVED PLATE EXPRESSION FOR LONG CRACKS, FLAT PLATE

EXPRESSIOV FOR SHORT GRACKS

IF $(2 . * C . G T \cdot 0.025 * 2.0 * P I * R)$ GO TO $7 C$

THETA $=(S B-S B C L J S) * 8 . *(1 .+N U) * C /(3,+N U) / E / T$

GO TO 80

THETA = SORT(R/T) * (2.13.)*(13.*(1.-NU*NU))*0.75)

- * (SB-SBCLOS)/E

CONTIVUE

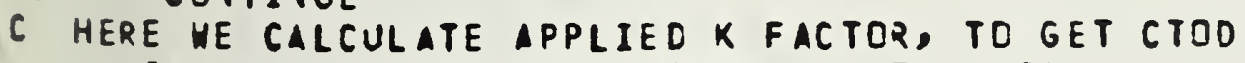

CALL KEALCISTCLOS, SBCLOS, H, T, KF)

CTOD - KF $\# K F(1,-N U * N U) / E / S F L O H$

CMUOD=DELLTA+THETA*T

$H=$ HPYYS

ENO 

SUBRDUTINE SCPZ(H,HSTART,T,SFLOH, SCLOSPZ)

THIS SUBROJTINE CALCULATES ALTERNATE CLOSURE STRESS SCLOSPZ

FROM PLASTIC ZJNE SIZE

$P I=3.1415927$

HPLAS=HSTART+2.*(H-HSTART)

IF (HPLAS OLT. 1.OE-10) ED TO 10

$O=S I N(P I * H S T A K T / 2 . / T) / S I N(P I * H P L A S / 2 . / T)$

IF(O.GT. 0.9997$)$ GO TO 10

$S C L O S P Z=(2$ * $\$ F L O H / P I) * A C O S(0)$

RETURN

SCLOSPZ $=0.0$

END

SUBROJTINE CALCI(H,C,T,R,NU, $02,03,04, D, N U 7, N U 8)$

REAL NU, NUT, NUB

DIMENSION $X(13), Y(19), Z(13)$

DATA (PI $=3.14159265)$

DATA.

a) $x(1)=1.9761)$,

a $\quad x(2)=11.487)$,

a $x(3)=7.7086)$,

a) $\quad x(4)=13.0143)$

a) $\quad x(5)=280.121)$

al $\quad x(6)=-1097.72)$,

af $\quad x(7)=3418.98)$,

a) $\quad x(8)=-7686.92)$,

af $\quad x(9)=12794.1)$,

a) $\quad x(10)=-13185$.$) ,$

a) $\quad x(11)=7868.27)$,

a $x(12)=-1740.25)$,

a $\quad x(13)=124.136)$

DATA

A) $Y(1)=1.97351$,

a) $Y(2)=-2.2166)$,

a) $Y(3)=21.6051)$,

a) $Y(4)=-69.3133)$,

al $Y(5)=196.31$,

a) $Y(3)=-406.261)$,

al $Y(7)=644.935)$,

a) $Y(8)=-408.957)$,

a $\quad Y(7)=-159.693)$,

a $(r(10)=-988.988)$,

a) $Y(12)=4266.551$,

a) $Y(12)=-2997.14)$,

a) $Y(13)=-6050.78)$,

a) $Y(14)=8855.36)$,

a $Y(15)=3515.43)$,

al $Y(16)=-11744.1)$,

a) $Y(17)=4727.98)$,

al $Y(18)=1695.61)$,

A $Y(17)=-845.896$ ) 



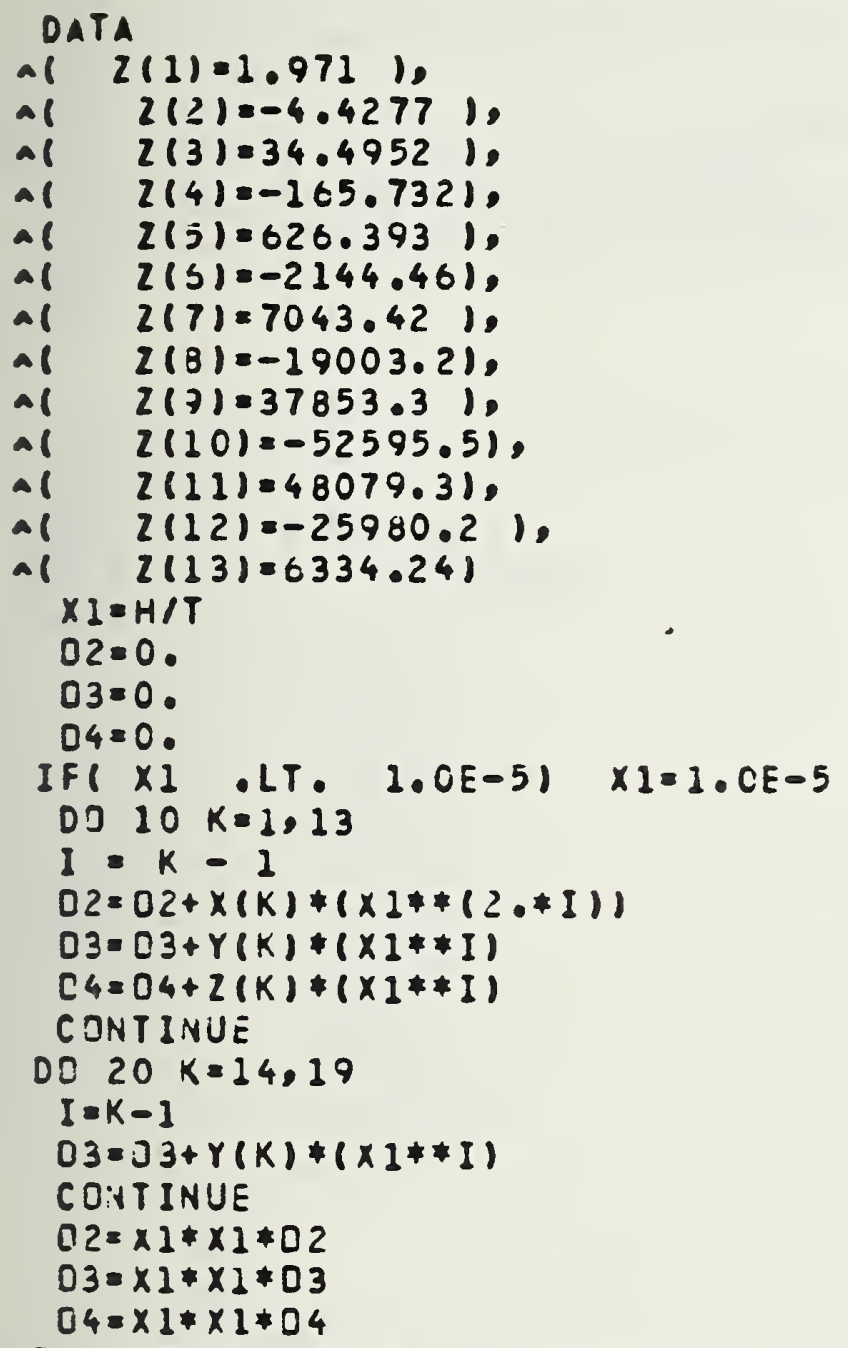

IN THE NOTATILN DF KINGTS PAPER DN THE SIMPLIFIED LINE SPRING MODEL, $C 02=A 11 \quad 03=A 12 \quad 04=A 22$

( $N U=N U, N U T=(C / T) *(2)(1-N U * N U))$

$N U T=(こ / T) * 2 . /(1,-N U * N U)$

C THE QJANTITY NB DEPENJS ON THE CRACK LENGTH. FOR CRACKS LESS

IC THAN 2.5 OF THE CIR:UMFERENCE, WE USE THE FLAT PLATE EXPRESSION.

C FOR LONGER CRACKS, THE EXPRESSION FOR CURVEATURE IS USED IF $(2 . C \quad . G T . .025 * 2 . * P I * R)$ GJ TO $3 C$ NUB $=(C / T) \div 2.13 . /(1 .-N U) /(3 .+N U)$

601340

130

NU8 $=$ SORT(R/T)* $(1.16.) /(13 .(1 .-N U * N U)) * .25)$ $D=(02+N U 7) *(U 4+N U 8)-03 * 03$

END 

SUBROLTINE CLOSUR $(02,03,04, D, N U 7, N U B, S T, S B$, STCLOS, SBCLLS) REAL N.J7, NUB

C CLOSURE STRESS, SIGMA SUB C, IS STCLOS

C CLOSURE MOYENT, M SUB 6 , IS SBCLOS

$S T C L O S=(N U 7 *(C 4+N J 8) * S T-N U E * 03 * S 9) / D$

$S B C L C S=(-N U 7 * 03 * S T+N U B *(O 2+N(U 7) * 5 B) / D$

END

SUBRDUTINE KCALC(STCLOS,S3CLOS,H,T, KF)

REAL KF

DIMENSION U(7), V(7)

DATA (PI $=3.141592654)$

DATA $(J(1)=1.12)$,

- $\quad(U(2)=6.52)$,

- $(U(3)=-12.39)$,

- $(U(4)=89.05)$,

a $(U(5)=-188.61)$,

a $(U(6)=207.39)$.

A $(U(7)=-32.05)$

DATA (V(1) = 1.12),

- $\quad(V(2)=-1.89)$,

- $(v(3)=18.01)$,

- $(V(4)=-87.39)$,

- $(V(5)=241.9)$,

a $(v(6)=-319.94)$,

- $(V(7)=168.01)$

$X_{1}=H / T$

$61=0.0$

$62=0.0$

D) $10 k=1,7$

$I=k-1$

$G 1=G I+U(K) *(x) *(2 * I))$

$G 2=62+V(K) *(x) * 1)$

10 CDNTINJE

$K F=(S T C L O S * G I+S B C L O S * G 2) * S Q R T(P I * X 1) * 5 O R T(T)$

RETURN

END 

Example allowable flaw-size calculation

1. Input parameters

$$
\delta_{c}=0.125 \mathrm{~mm}
$$

$$
\begin{aligned}
\text { CTOD }_{\text {RS }} & =0.038 \mathrm{~mm} \\
\bar{\sigma} & =517 \mathrm{MPa}
\end{aligned}
$$$$
\sigma=434 \mathrm{Mpa}
$$$$
E=2.08 \times 10^{5} \mathrm{MPa}
$$

$v=0.29$

$\mathrm{R}=610 \mathrm{~mm}$

$$
t=15.9 \mathrm{~mm}
$$

2. Short flaw, use (28) - (33)

Long flaw, use $(28-c)-(33-c)$

Dividing line is $2 c=0.025 \times 2 \pi \times R=96 \mathrm{~mm}$

3. Critical condition: $\delta_{C}=C T O D_{E}+C T O D_{L Y}+C T O D_{R S}$

Therefore we demand $C T O D_{E}+C T O D_{L Y}=0.087 \mathrm{~mm}$

4. Procedure: for every $\ell$, find a so that

$$
C T O D_{E}+C T O D_{L Y}=0.087
$$

4.1 Choose $\ell=201.1 \mathrm{~mm}$ for this example. From 2, above, we see that we have the long-flaw case.

4.2 Choose a trial a value, $2.75 \mathrm{~mm}$.

(The first trial a value is arbitrary, but the better the choice, the fewer iterations needed).

4.3 Calcualte $C T O D_{E L}+C T O D_{L Y}$ for this a value.

4.3.1 Check for full igament yielding.

$$
\begin{aligned}
& 4.3 .1 .1 \text { Using }(14), \\
& \sigma_{c}=(1-a / t) \bar{\sigma}=427.6 \mathrm{MPa}
\end{aligned}
$$



4.3.1.2 At full ligament yielding, a $+2 r_{y}=t ; r_{y}=6.575 \mathrm{~mm}$;

$$
\begin{aligned}
& a_{e f f}=a+r_{y}=9.32 \mathrm{~mm} \\
& a_{e f f} / t=0.586
\end{aligned}
$$

To apply $(29-c)$ we need $\alpha_{c}$ from $(31-c)$, which requires $S$ from $(33-c)$.

In $(31-c)$ and $(33-c)$ we need $a_{11}, a_{12}$, and $a_{22}$ :

From Table $A 1, a_{11}=3.136 ; a_{12}=1.746 ; a_{22}=0.993$

Applying $(33-c)$ we find

$$
\begin{aligned}
S & =\left\{3.136+\frac{201.1}{\left.15.9\left(1-0.29^{2}\right)^{-}\right\}}\left\{0.0993+\frac{(2.610)^{\frac{1}{2}}\left[12\left(1-.29^{2}\right)^{3 / 4}\right.}{72\left(1-29^{2}\right) 15.9^{\frac{1}{2}}}\right.\right. \\
& -(1.746)^{2} \\
& =\{3.136+13.809\}\{0.993+0.8018\}-3.0485 \\
& =27.364
\end{aligned}
$$

Applying $(31-c)$, using values from the previous step where needed, we get

$$
\alpha_{c}=13.809\{0.993+0.8018\} / 27.364=0.9057
$$

Applying $(29-\mathrm{c})$, we find

$$
\begin{aligned}
\sigma_{c}= & 0.9057 \times 434=393.1 \mathrm{MPa} \\
& 4.3 .1 .3 \text { We note that } \sigma_{0}<\sigma_{c} \text {, therefore the ligament is not } \\
& \text { fully yielded. } \\
& \text { Therefore, we know }
\end{aligned}
$$

$$
\begin{aligned}
& C{ }^{2} D_{L Y}=0 \\
& r_{y}<6.575 \mathrm{~mm}
\end{aligned}
$$

4.3.2 Fird the vlaue of $r_{y}$ for which $(29-c)$ and (69) are satisfied.

4.3.2.1 Guess initial value of $r_{y}$ :

$$
\text { let } r_{y}=4.164 \mathrm{~mm}
$$

4.3.2.2 Apply (29-c).

$$
\begin{aligned}
& r_{y}=4.164 \\
& (a / t)_{\text {Effective }}=0.4355
\end{aligned}
$$



From Table A1, $a_{11}=0.8989 ; a_{12}=0.5917 ; a_{22}=0.3955(33-c)$ gives:

$S=\{0.8989+13.809\}\{0.3955+0.8018\}-.3501$

$$
=17.260
$$

(31-c) gives: $\alpha_{c}=13.809\{0.3955+0.8018\} / 17.260$

$$
=0.9579
$$

$(29-c)$ gives: $\sigma_{0}=.9579 \times 434=415.7 \mathrm{MPa}$

$$
\text { 4.3.2.3 Apply (69). }
$$

$$
\begin{aligned}
& \frac{\sin (0.2717)}{\sin (1.0944)}=\frac{.2683}{.8887}=0.3019 \\
& \operatorname{arc} \cos (0.3019)=1.2641 \\
& \sigma_{0} 416.1 \mathrm{MPa} \\
& 4.3 .2 .4 \quad \sigma_{0} \text { from }(29-\mathrm{c}) \text { agrees with } \sigma_{0} \text { from }(69) \text { to better } \\
& \text { than } 1 \text { part in } 1000 . \text { This is sufficient for a hand } \\
& \text { calcualtion. Therefore } r_{y}=4.164 \text { for the trial a value, } \\
& a=2.75 \mathrm{~mm} .
\end{aligned}
$$

\subsubsection{Apply (66)}

We need $g_{t}$ and $g_{b}$

From Table A1, we have $g_{t}=2.7187 ; g_{b} 1.5561$

We al ready have $\sigma_{0}=415.9$, averaging results of 4.3 .2 .2 and 4.3.2.3.

To get $m$, we apply $(32-c)$, then $(29-c)$ using vlaues calculated above we have

$$
B_{C}=13.809 \times 0.5917 / 17.260=0.4734
$$

From $(29-c), m=-0.4734 \times 434=-205.4 \mathrm{MPa}$ 

In applying (66), we convert to meters to retain consistent units:

$$
\begin{aligned}
K & =(.0159)^{\frac{1}{2}}[415.9 \times 2.7187-205.4 \times 1.5561] \\
& =102.3 \mathrm{MPa} / \mathrm{m}
\end{aligned}
$$

$$
\begin{aligned}
& \text { 4.3.4 Apply (11) } \\
& \mathrm{CTOD}_{E}=8.913 \times 10^{-5} \mathrm{M} \\
& =8.913 \times 10^{-2} \mathrm{~mm} \\
& =.08913 \mathrm{~mm}
\end{aligned}
$$

4.3.5 Our calcualted CTOD is within $2.4 \%$ of the critical vlaue. This is sufficiently accurate for a hand calculation.

5. Therefore, for $\ell=201.1 \mathrm{~mm}$, we have found $\mathrm{a}=2.75 \mathrm{~mm}$, and we have one point on the allowable flaw-size curve. 



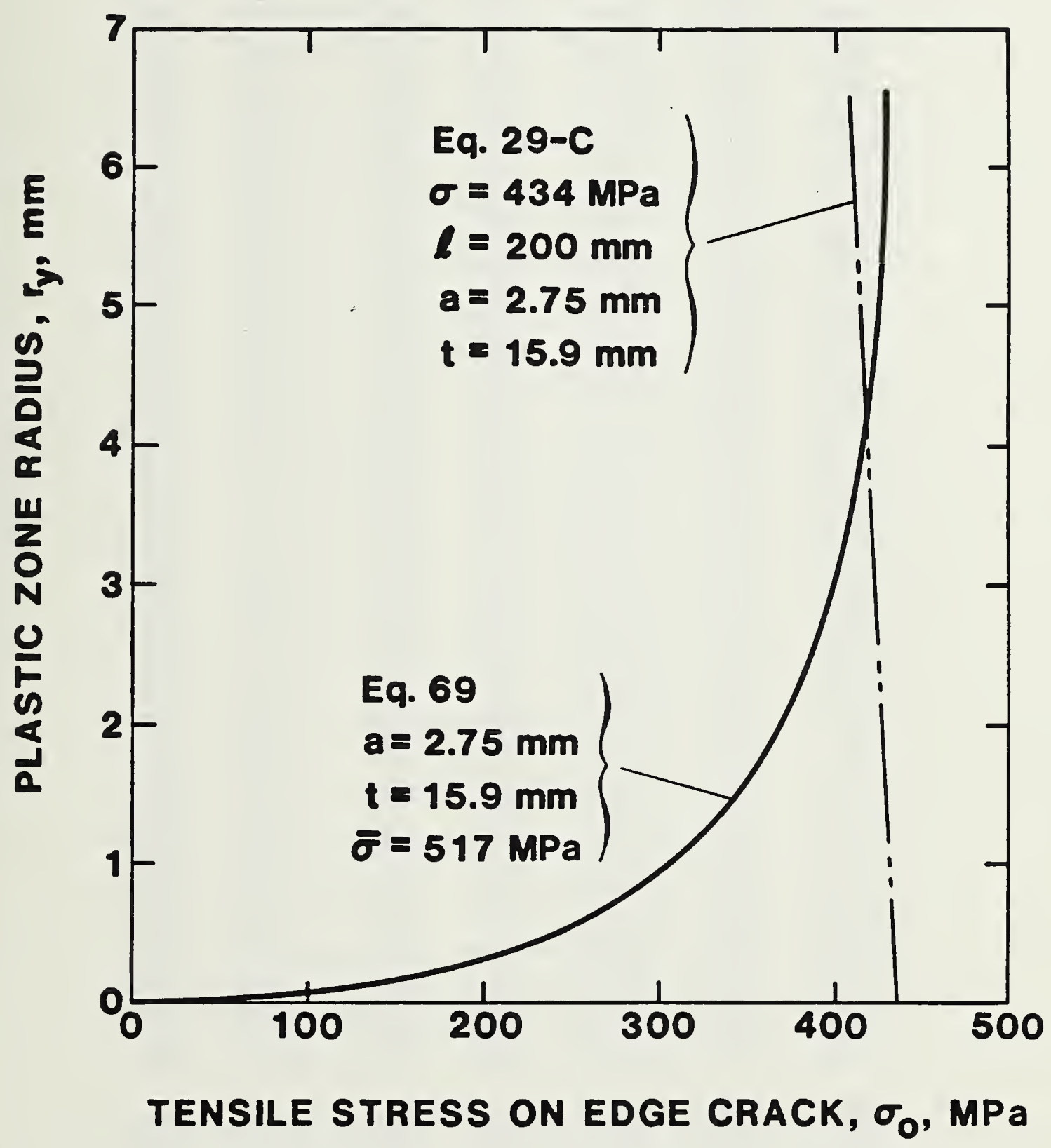

Figure Al. Plot of plastic zone radius, $r$, against tensile stress on equivalent edge crack, $\sigma_{c}$, for two diffefent equations. The intersection of the two curves is the plastic zone radius for the specified input parameters. 

Table Al. Parameters used in calculation of allowable flaw size curves.

EFFECTIVE

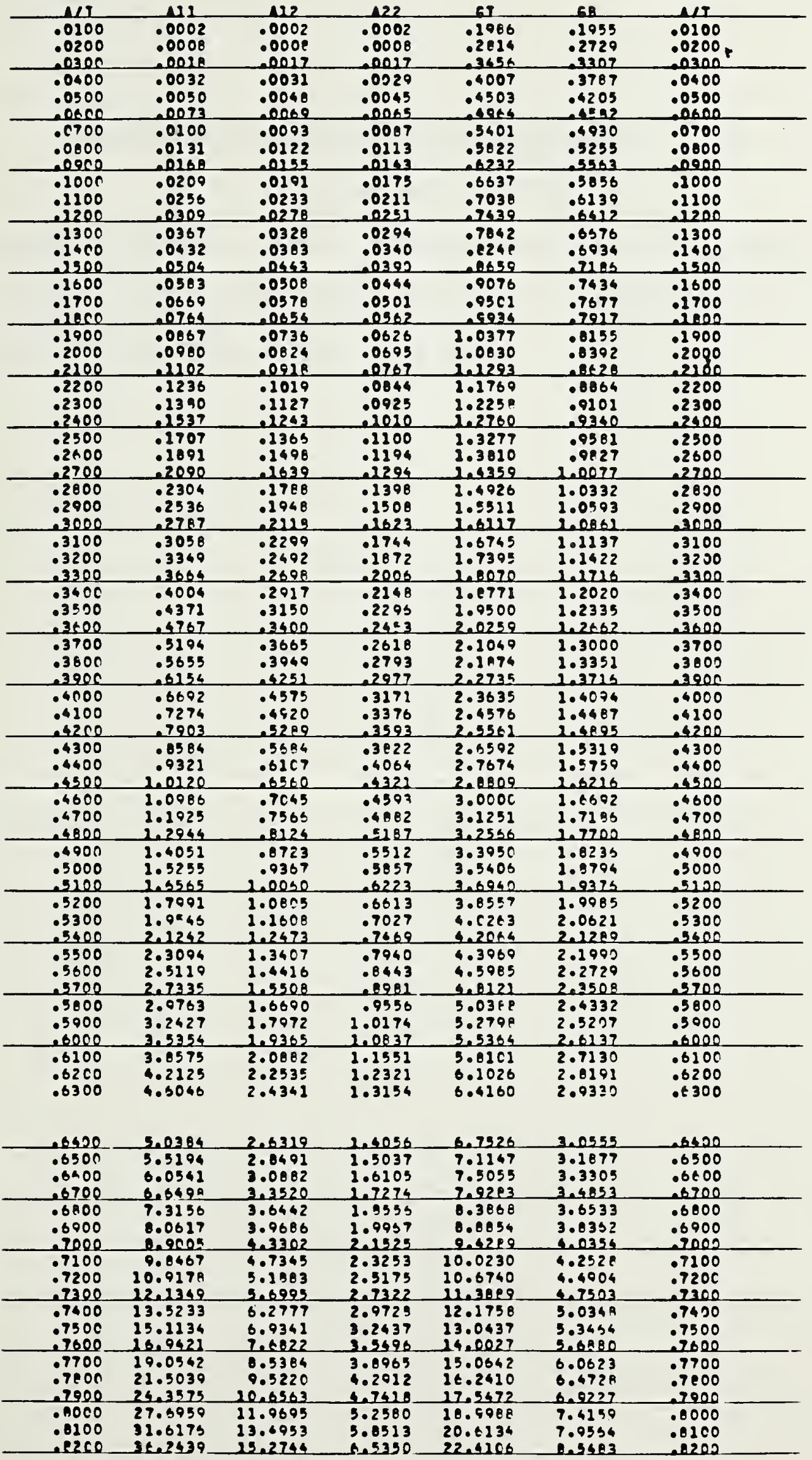





\section{APPENDIX B - General Research on Corrosion}

J. Kruger and R. P. Wei

This appendix contains research summaries presented in the quarterly progress reports. This research was conducted prior to the emphasis on ANGTS and, in some cases, is only partially completed. 

TO:

Department of Commerce

National Bureau of Standards

Washington, D. C.

Attention: Dr. J. Kruger, Chief

Corrosion and Electrodeposition Section

FROM: $\quad$ Dr. R. P. Wei, Consultant

3148-B East Boulevard

Bethlehem, PA 18017

SUBJECT: Test Methods for Evaluating Environmentally Assisted Cracking Under Stress

REFERENCE: NES Order No. 809451 (April 6, 1978)

DOT/OPSO PrOgram - DOT-AS-70039

Background and Scope of Work

Transmission pipelines are exposed to a broad range of chemical environments, both in terms of corrosive species that are present in soils (such as carbonates, chlorides, nitrates, etc.) and of aggressive species that are transported within the pipelines (such as hydrogen, hydrogen sulfide and water/water vapor as impurities, ammonia, etc.). These environments, acting in concert with operating stresses (both static and cyclic stresses) and residual stresses, can cause cracks to initiate and grow, and result in subsequent failure (leakage or rupture). In addition to these external environments, hydrogen that might be present in the steel (introduced during pipeline fabrication and processing, or by corrosion or cathodic charging during service) can also lead to cracking. Quantitative information is needed, therefore, to assess the safety and reliability of pipelines during service.

As a part of the referenced DOT/OPSO Program, more specifically as a part of. Task III of the Program on Effects of Environmental and Stress Conditions on Defect Enlargement, the following tasks were undertaken: 

(1) Assess existing knowledge in terms of tost methods for evaluating environmentally assisted cracking under stress (stress corrosion cracking and corrosion fatigue) for transmission pipeline steels and weldments.

(2) Based on (1) and preliminary experiments at NBS, recommend a testing program to be carried out in part by NBS in second year of DOT project to develop appropriate test methods for assessing susceptibility of transmission pipeline steels and weldments to environmentally assisted cracking under stress.

(3) Collaborate with NBS personnel to identify research needed in this area to bridge the gap between test methodology and prediction of service performance, and to ensure a proper collection of field data.

This report summarizes work carried out under these three tasks. It is important to recognize that this work represents only an assessment of the technological base for evaluating the effects of environmental and stress conditions on defect enlargement. Such a technological base is necessary, but by and of itself is not sufficient, for ruling on waiver requests. As such, questions relating to waiver requests were not included as a part of this work.

To place this work in perspective, it is useful to briefly consider the test methodology within the context of the overall problem of service life estimation (or prediction). Service life estimation begins with a proper structural analysis, which provides quantitative descriptions of stresses (including residual stresses) at critical locations and includes stress analyses of cracks that might be present initially or that might develop during service. Projected service 
loading and environmental conditions are then defined, with due consideration for variations in actual service experience. Characterizations of material properties and responses are then made in light of the projected service loading and environmental conditions. Finally, the various information is synthesized through suitable modeling and used in estimating service life. The reliability of this estimate reflects the uncertainties in the supporting analyses and the projected service conditions, and depends on the appropriateness and reliability of the material characterization data. It will depend also on how well the entire estimation procedure has been supported by prior service experience and by experimental verification:

Suitable test methods for evaluating environmentally assisted cracking of linepipe steels and weldments under stress, therefore, must properly reflect the anticipated service for the material and must be evaluated in terms of the intended end use of the test results. The choice of test methods often reflects engineering judgements regarding the relative importance of crack initiation as opposed to crack growth in determining service life, and with respect to failure mechanisms (e.g., stress corrosion cracking vs. corrosion fatigue). Distinction must be made also between those tests that are designed for screening purposes and those that can provide information for quantitative life estimations, and between scientifically and technologically oriented test methods.

Existing Test Methods and Their Assessment.

Current test methods that can be used for evaluating environmentally assisted cracking of linepipe steels and weldments under stress include: 

1. "Constant-strain" or "constant-displacement" tests (e.g., U-bend test, c-ring test, bent-beam test, etc.) [1-3].

2. Constant-load tests using smooth and notched tensile specimens [4].

3. Constant strain rate tests [5-7]:
a. Smooth tensile specimens.
b. Precracked cantilever specimens.

4. Fracture mechanics based test's using precracked specimens (subjected to either constant load or constant crack opening displacement) [8-10].

5. Fatigue tests using smooth and notched specimens.

6. Fracture mechanics based fatigue tests using precracked specimens $[11,12]$.

Test methods 1 to 4 are designed for evaluating stress corrosion cracking response (i.e., environmentally assisted cracking under "static" loads), while test methods 5 and 6 pertain to fatigue or cyclically varying loads.

Test methods 1,2 and 5 are considered, by now, to be traditional methods for evaluating stress corrosion cracking and corrosion fatigue response of materials, and are often described as "initiation" tests. These tests measure the time to produce fracture of the specimens that have been loaded in prescribed manners and exposed to the appropriate chemical environment. Some of the tests (such as the U-bend test) are suitable primarily for screening purposes. Other tests provide threshold stresses for stress corrosion cracking and for corrosion fatigue. These stresses are then utilized in conventional design analysis, principally to define stress levels below which failures are not to be expected. Although the tests involve crack initiation and growth, 

crack growth is not explicitly taken into account in the interpretation or in the use of the data. While these methods may be suitable for screening purposes, there are fundamental problems associated with their use in developing information for quantitative service life estimation. Their reliability and utility as a part of a predictive procedure remain to be established.

Development of test method 3 is based principally on the observation by Parkins and his coworkers that a critical strain rate is associated with stress corrosion cracking susceptibility [5-7]. The test methods have been used principally as a research tool and mostly in short-term tests lasting up to one (1) week. In addition, the interpretation of results from slow bend tests of precracked cantilever specimens as "average" crack growth rate versus beam deflection rate must be seriously questioned [5-7]. Specifically, the beam deflection rate itself is a function of the crack growth rate (among others), and cannot be accepted, therefore, as a suitable measure of the driving force for crack growth. Because of this fundamental objection and because of the short term nature of the tests, these methods are not considered to be suitable for use in developing data for quantitative life estimations.

Methods 4 and 6 are fracture mechanics based test methods that are of potential value in providing data for quantitative life estimations. A tentative test method for measuring fatigue crack growth rat.es has been released by the American Society for Testing and Materials recently [12]. Recommended test methods for stress corrosion cracking and for corrosion fatigue are under development, but are not yet available. These methods presume the presence of cracks and, therefore, treat the growth of these cracks during service. Two 

separate approaches are currently in use; one involving cracking threshold and the other involving crack growth kinetics [10]. These methods have been used with some success for high-strength aerospace materials. Their utility in terms of the lower strength materials (such as linepipe steels) remains to be established. In addition, it has been recognized that there are a number of nonsteady-state phenomena associated with environmentally assisted cracking, and that these phenomena can affect the proper interpretation and the utility of experimental results [10]. For example, erroneously high values for stress corrosion cracking threshold or fatigue threshold, or unconservative crack growth rates can be obtained when the nonsteady-state phenomena are not properly taken into account during testing and data analyses [10]. Further development work is needed before these test methods can be used to provide reliable quantitative data for life estimation.

A large number of test methods have been developed over the years and are in use for evaluating environmentally assisted cracking of materials under stress. Many of these test methods have been designed for screening or research purposes, and are not suitable, therefore, for providing data for use in service life estimation procedures. Other test methods (such as the fracture mechanics based methods) require further development and verification before their data can be relied upon for quantitative service life estimation. It was recommended that further development and verification of test methods be made, with particular attention to their applicability for the quantitative evaluation of environmentally assisted cracking of linepipe steels and weldments under stress.

Test Method Evaluation and Development

To develop appropriate test methods for assessing the susccptibility 

of transmission pipeline steels and weldments to environmentally assisted cracking under stress, it is useful to consider the types of cracking problems that might be of concern.

(1) Initiation of crack or crack growth from notch-like and crack-like flaws.

(2) Propagation of cracks.

If the flaws or cracks are internal to the material or weldment, service environments are not expected to have an effect on crack initiation or propagation. If, on the other hand, the flaws or cracks are near to the interior or exterior surfaces of the linepipes, crack initiation and propagation could be adversely affected by the external service environment. Internal flaws or cracks that are exposed to "trapped" hydrogen in the steel or weldment are to be treated as flaws exposed to external aggressive environments. Test methods can be devised to simulate and evaluate initiation and propagation from internal flaws (such as porosities, inclusions, etc.) and cracks by using inert test environments. For external flaws and cracks, similar test methods, incorporating the appropriate environments, are to be used. Broad categorization of test methods is indicated in the following table, where notches are to be used to simulate flaws (other than cracks).

\begin{tabular}{|c|l|l|l|}
\hline \multirow{4}{*}{ MODE } & ORIGIN & ENVIRONMENT & LOADING \\
\hline \multirow{3}{*}{ Initiation } & \multirow{2}{*}{ Notches } & Inert & Static/Cyclic \\
\cline { 3 - 4 } & \multirow{2}{*}{ Cracks } & Aggressive & Static/Cyclic \\
\cline { 3 - 4 } Crack & Inert & Static/Cyclic \\
\cline { 3 - 4 } Propagition & \multirow{2}{*}{ Cracks } & Aggressive & Static/Cyclic \\
\cline { 3 - 4 } & & Inert & Static/Cyclic \\
\cline { 3 - 4 } & & Aggressive & Static/Cyclic \\
\hline
\end{tabular}



Specific choices of test methods, to a large extent, depend on engineering judgement as to the most probable mode of failure. If, for example, stress corrosion crack initiation is judged to be important, then tests of "smooth" or "notched" specimens exposed to aggressive environments under static loads would be selected. Heretofore, stress corrosion cracking has been considered to be an important problem for linepipe steels and weldments, and most of the current test methods therefore reflect this judgement.

To assess some of the current test methods (particularly to reproduce past experience); and to explore other test methods and to examine the importance of other cracking modes, a number of exploratory experiments have been carried out on a X-65 steel plate ${ }^{\dagger}$. These experiments included slow strain rate tests on "smooth" specimens (performed at NBS-Gaithersburg) [5] and fracture mechanics based fatigue crack propagation tests (performed at Lehigh University) at room temperature. The slow strain rate test results are summarized in the following table, and the fatigue crack propagation results are summarized in Figures 1 to 3.

Stress

at

Failure

Spec. No.

Environment

Strain

Rate
Reduction

in area

(pct)
1

2

3

3

4

5

6
$\mathrm{CO}_{3} / \mathrm{HCO}_{3}$

Air

$\mathrm{CO}_{3} / \mathrm{HCO}_{3}$

$\mathrm{CO}_{3} / \mathrm{HCO}_{3}$

Distilled $\mathrm{H}_{2} \mathrm{O}$

$\mathrm{CO}_{3} / \mathrm{HCO}_{3}$
$1 \times 10^{-6} / \mathrm{s}$

$1 \times 10^{-6} / \mathrm{s}$

$1.6 \times 10^{-7} / \mathrm{s}$

$1.4 \times 10^{-7} / \mathrm{s}$

$1 \times 10^{-6} / \mathrm{s}$

$7.2 \times 10^{-6} / \mathrm{s}$
80,500

78,700

81,400

81,300

79,700

81,300 strain

0.213

0.218

0.185

0.204

0.179

0.212
75.68

77.4

73.5

74.4

66.0

76.1

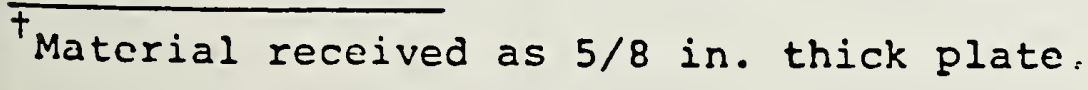



STRESS INTENSITY FACTOR RANCE, $\Delta K$ (ksi $\sqrt{\text { in. }}$ )

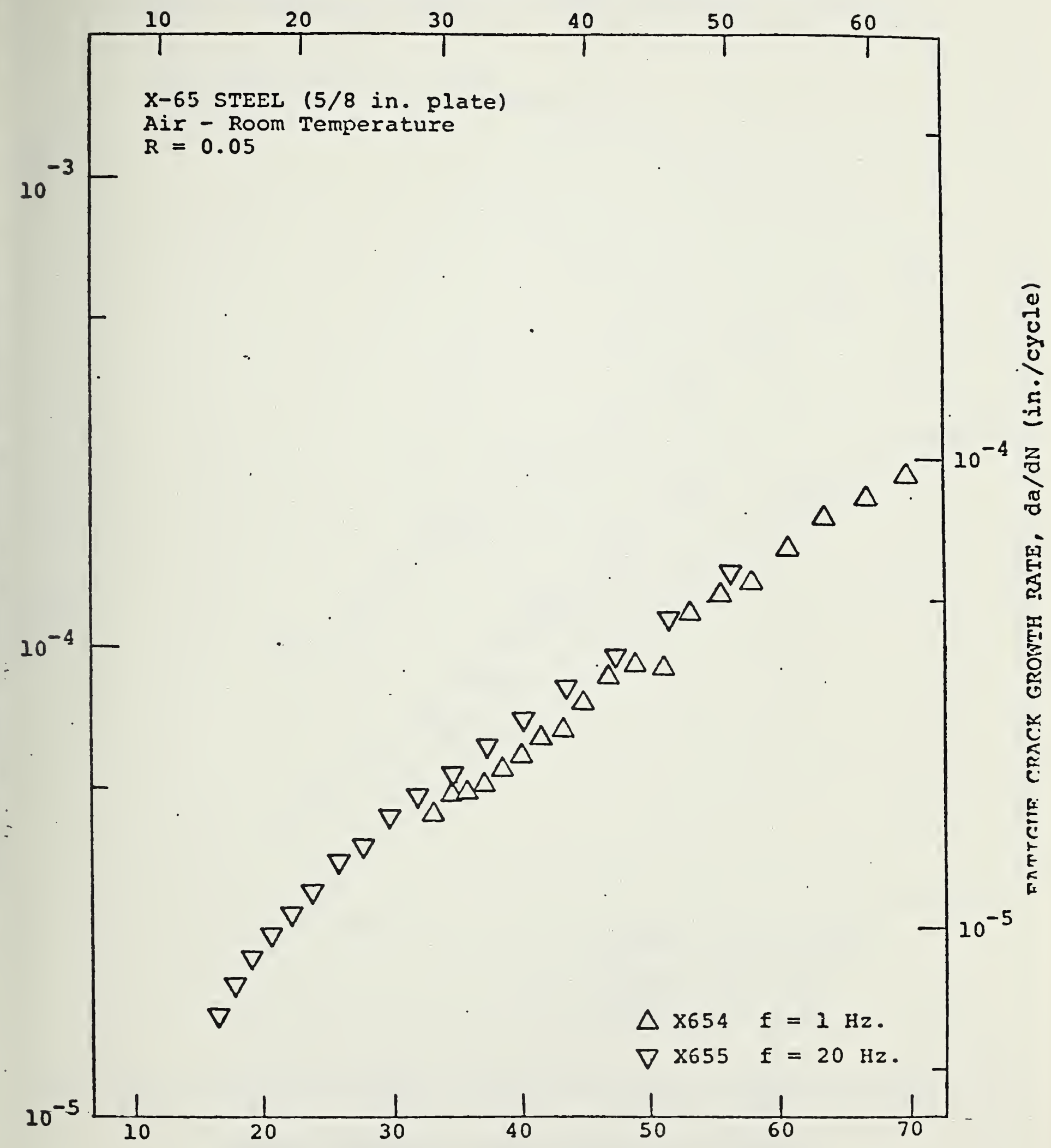

STRESS INMENSITY EACTOR RANGE, $\triangle K$ (MPa $\sqrt{m}$ )

Figure 1: Rinetics of Fatigue Crack Growth in X-65 Steel in Air at foom Temperature. 

STRESS INTLNSITY FACTOR RANGE, $\Delta K$ (ksi $\sqrt{\text { in. })}$

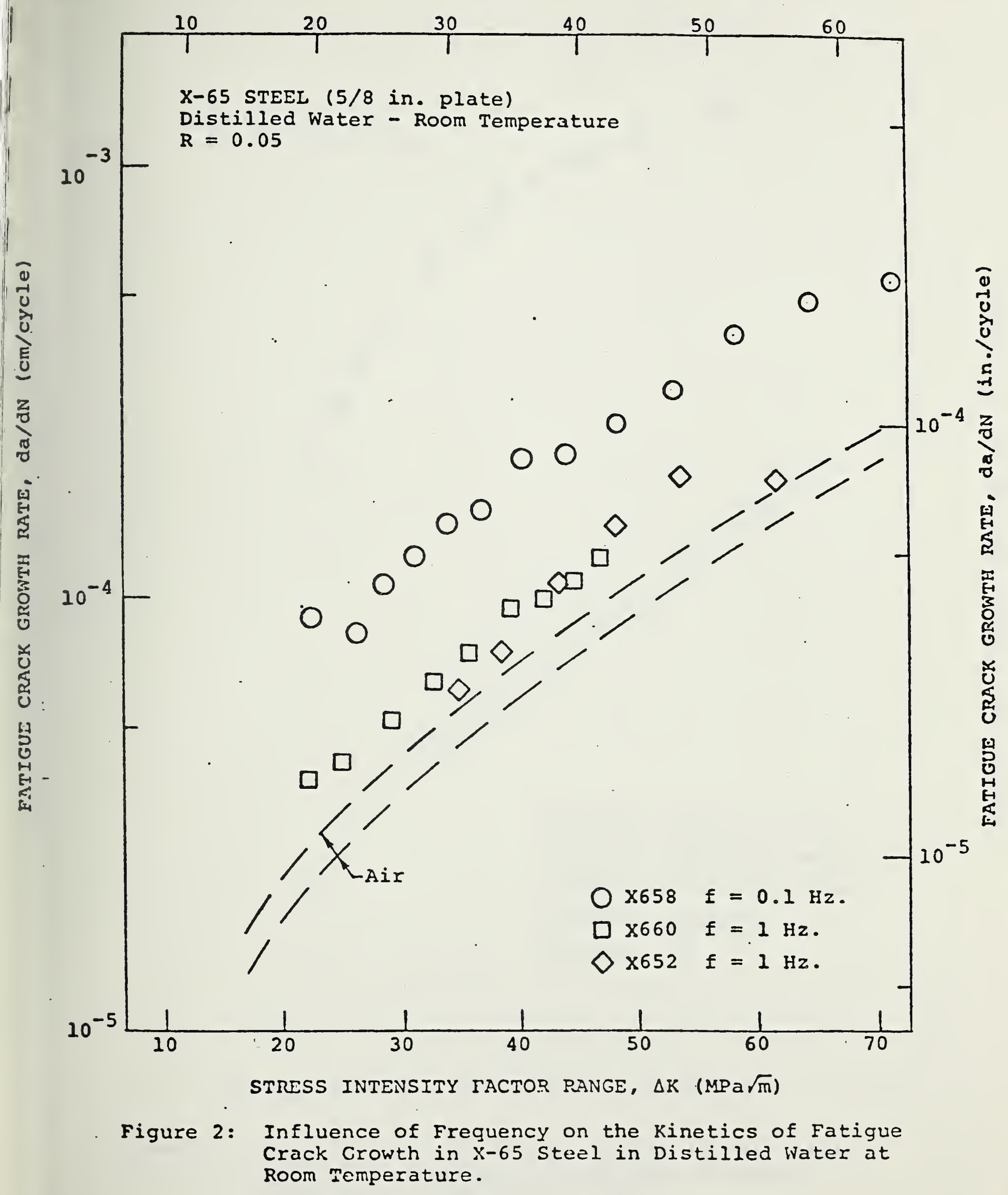



Slow strain rate tests were carried out in three environments: air, distilled water and carbonate/bicarbonate $\left(\mathrm{CO}_{3} / \mathrm{HCO}_{3}\right)$ solution, under open circuit conditions, at room temperature. These results indicate that for these environments, no appreciable stress corrosion cracking susceptibility (low reduction in area) was observed. Work in the literature $[5,7]$ suggests that specimens polarized in $\mathrm{CO}_{3} / \mathrm{HCO}_{3}$ solutions may be more susceptible to stress corrosion cracking failure, and such conditions should be examined in future tests.

Fatigue crack propagation tests were carried out at room temperature in air (at 1 and $20 \mathrm{~Hz}$ ), distilled water (at 0.1 and $1 \mathrm{~Hz}$ ), carbonate / bicarbonate solution under open circuit conditions $\left(\mathrm{CO}_{3} / \mathrm{HCO}_{3}\right.$ at $1 \mathrm{~Hz}$ ) and high-purity hydrogen sulfide (at $1.33 \mathrm{kPa}$ or 0.19 psia, and $1 \mathrm{~Hz}$ ) [12]. The specimens are oriented in the longtransverse (TL) direction [12]. Sinusoidally varying loads, at a load ratio (ratio of minimum to maximum load in a given cycle) of 0.05 , were used. Data in air (Figure 1) show little effect of cyclic load frequency, and are used as reference data. Results in distilled water (Figure 2) indicate an effect of this environment on fatigue crack growth. These data are consistent with the response for other low alloy steels [13-15], and reflect embrittlement by hydrogen that is produced by the water-steel surface reactions $[15,16]$. The observed frequency dependence is consistent with surface reaction control of this embrittlement $[15,16]$. Crack growth data in all four environments, at $1 \mathrm{~Hz}$, are shown in Figure 3 . These results indicate severe embrittlement by the low pressure $(1.33 \mathrm{kPa})$ hydrogen sulfide $[19,20]$, and an inhibiting effect of $\mathrm{CO}_{3} / \mathrm{HCO}_{3}$-under open circuit conditions. The stress intensity $(K)$ levels for environmentally assisted fatigue crack growth are believed to be well below the 

threshold $\mathrm{K}$ level for stress corrosion cracking [13]. Taken in conjunction with the recognition that stress fluctuations do occur in pipelines during normal operation [17,18], environmentally assisted fatigue crack initiation and propagation must be considered as a significant potential cause for failures of transmission linepipes.

Based on these and other results, it is recognized that steels that appear to be immune to stress corrosion cracking (static load) may be highly susceptible to environmentally assisted fatigue crack growth (corrosion fatigue). The discrepancy between the apparently low stresses associated with field failures of linepipes and the high stresses required to induce stress corrosion failure in the laboratory [5-7] suggests that corrosion fatigue should not and could not be ruled out as a possible cause for failure in service. As such, environmentally assisted crack initiation and propagation, under both static and cyclic loading conditions, must be considered and assessed. A testing program to incorporate both loading conditions is proposed.

\section{Proposed Test Program}

To further evaluate test methodology for assessing environmentally assisted crack initiation and propagation, the following tests should be considered:

- Slow strain rate tests (monotonic loading) of smooth and notched specimens to assess conditions for stress corrosion crack initiation.

- Slow strain rate cycling (fatigue) tests of smooth and notched specimens to assess conditions for corrosion fatigue crack initiation.

- Static load (or wedge force load) tests of precracked (fracture mechaniss type) specinens to determine stress corrosion 

cracking threshold $\mathrm{K}$ ( $\mathrm{K}_{\mathrm{Iscc}}$ ) for appropriate environmental conditions, and to determine the kinetics of crack growth under these conditions.

- Cyclic load (fatigue) tests of precracked (fracture mechanics type) specimens to determine the threshold $\Delta K$ for fatigue crack growth for appropriate environmental conditions, and to determine the kinetics of crack growth under these conditions. A range of loading variables should be considered; these include frequency, load ratio, hold-time, waveform, etc. In determining crack growth threshold and crack growth kinetics, care-.. ful distinction should be made between steady-state and nonsteadystate crack growth $[10,12]$. The impact of nonsteady-state growth on the measurement of rates and threshold $\mathrm{K}$ levels must be recognized $[10,12]$.

To complement data that have already been developed under the preliminary testing program, the following series of tests are recommended to complete test method evaluation under the referenced program:

(1) Cyclic loading of smooth and notched specimens to assess conditions for crack initiations in fatigue, and to determine the importance of this failure mode in relation to the performance of linepipe steels and weldments.

(2) Laboratory evaluation of test methods for determining $\mathrm{K}_{\text {Iscc }}$ and of conditions for the initiation of stress corrosion crack growth. Modified wedge-opening-load (decreasing K) specimens are to be used. Two initial $K$ levels $(30$

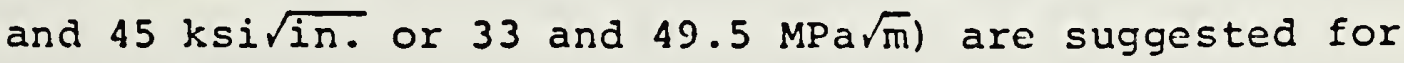
examination. Tests in the polarized and unpolarized con- 

ditions should be considered.

(3) To assess the aggressiveness of field conditions, and to establish potential correlation between laboratory and field data, in-ground tests of modified wedge-openingload specimens (see item (2) above) at NBS test sites are recommended. Initial $\mathrm{k}$ levels that conform to those used in the laboratory tests are suggested. A minimum of two different test sites should be considered.

Summary

A review has been made of currently available test methods for assessing the susceptibility of transmission pipeline steels and weldments to environmentally assisted cracking under stress. Preliminary experiments have also been carried out to reproduce past experiences with some of the test methods, and to explore other test methods and potential failure modes. It is recognized that the validity and usefulness of any test method depend on its ability to simulate service conditions and environments, and to reflect the mode of failure. It is believed that, at this time, no test method satisfies this requirement in relation to service performance of linepipe steels and weldments. Even if such methods exist, data from the tests would represent only a part of the necessary information in considering waiver requests, but would not be sufficient. The usefulness of this type of data must be established and documented on the basis of careful correlation with service performance.

This study has also shown that corrosion fatigue (environmentally assisted fatigue crack initiation and propagation) is a potentially important failure mode in linepipe steels and weldments. The ability to initiate and propagate cracks at relatively low stress levels in 

fatigue suggests that corrosion fatigue might provide a better explanation for some of the service failures. It is recommended that recognition be given to the potential for corrosion fatigue induced failure of linepipe steels and weldments in service, and that recordings of cyclic loads should be included in the collection of field data.

\section{References}

1. ASTM G 30 - 72, Standard Recommended Practice for Making and Using U-Bend Stress Corrosion Test Specimens, Annual Book of ASTM Standards, Part 10, 1975, pp. 703-711.

2. ASTM G 38 - 73, Standard Recommended Practices for Making and Using the C-Ring Stress-Corrosion Cracking Test Specimen, Annual Book of ASTM Standards, Part 10, 1975, pp. 751-759.

3. ASTM G 39 - 73, Standard Method for Preparation and Use of Bent-Beam Stress-Corrosion Specimens, Annual Book of ASTM Standards, Part 10, 1975, pp. 760-769.

4. Craig, H. I., Sprowls, D. O., and Piper, D. E., "Stress-Corrosion Cracking", Hancbook of Corrosion Testing and Evaluation, W. H. Ailor, Editor, Wiley, 1971, pp. 231-290.

5. Parkins, R. N., "The Controlling Parameters in Stress-Corrosion Cracking", 5th Symp. Line Pipe Research, American Gas Association, Cat. No.'I30174, 1974, U1-40.

6. Berry, W. E., "Stress Corrosion Cracking Laboratory Experiments", 5th Symp. Line Pipe Research, American Gas Association, Cat. No. L30174, 1974, pp. V1-43.

7. Parkins, R. N. and Greenwell, B. S., "The Interface Between Corrosion Fatigue and Stress-Corrosion Cracking", August/Sept. Metal Science, 1977, pp. 405-413.

8. Brown, B. F. and Beachem, C. D., Corrosion Science, Vol. 5, 1965, pp. 745-750.

9. Novak, S. R. and Rolfe, S. T., "Modified WOL Specimen for KIscc Environmental Testing", Journal of Materials, JMLSA, Vol. 4, No. 3, 1969, pp. 701-728.

10. Wei, R. P., Novak, S. R., and Williams, D. P., "Some Important Considerations in the Development of Stress Corrosion Cracking Test Methods", Materials Research and Standards, MTRSA, Vol. 12, No. 9,1972 , p. 25 . 

11. Wei, R. P., "Fracture Mechanics Approach to Fatigue Analysis in Design", JEMT, Vol. 100, 1978, Pp. 113-120.

12. ASTM E647-78T, "Tentative Test Method for Constant-Load-Amplitude Fatigue Crack Growth Rates Above $10^{-8} \mathrm{~m} / \mathrm{cyclc}$ ", Annual Book of ASTM Standards, Vol. 10, 1978.

13. Barsom, J. M., Corrosion Fatigue, ed. O. Devereux, A. J. McEvily and R. W. Staehle, NACE, Houston, Texas, p. 424, 1972.

14. Gallagher, J. P. and Wei, R. P., Corrosion Fatigue, ed. O. Devereux, A. J. McEvily and R. W. Staehle, NACE, Houston, Texas, P. 409, 1972 .

15. Pao, P. S., Wei, W. and Wei, R. P., Environment-Sensitive Fracture of Engineering Materials, ed. Z. A. Foroulis, TMS-AIME, New York, New York, pp. 565-580, 1979.

16. Simmons, G. W., Pao, P. S. and Wei, R. P., Met. Trans. A, Vol. 9A, August 1978, pp. 1147-1158.

17. Vosikovsky, O. and Cooke, R. J., Int. J. Pres. Ves. \& Piping, Vol. 6, 1978, pp. 113-129.

18. "Environmentally Induced Cracking of Natural Gas and Liquid Pipelines", Vols. 1 and 2, Final Report, Contract \#DOT-OS-60519, ASL Engineering, Inc., 495 South Fairview Ave., Goleta, CA, 33017, Dec., 1977.

19. Brazill, R., Simmons, G. W. and Wei, R. P., J. of Engr. Matls. \& Tech., Vol. 101, July 1979, pp. 199-204.

20. Nelson, H. G., Effect of Hydrogen on Behavior of Materials, TMSAIME, A. W. Thompson and I. M. Bernstein, eds., 1976, Pp. 602-E11. 
Subtask 2: Enviounmental Assisted Cracking Under Stress

Principal Investigator: Dr. Jerome Krliger, (301) 921-2094

Progress: This is the final report on this subtask (formerly Task $3 A$ ). Objectives and Aporoach

The objectives and approach of subtask 2 were as follows:

(1) Assess existing knowledge in terms of test methods for evaluating environmentally assisted cracking under stress (stress corrosion cracking and corrosion fatigue) for transmission linepipe stecls and weldments.

(2) Based on (1) and preliminary experiments at iNBS, recommend a testing program to develop appropriate test meihods for assessing susceptibility of transmission linepipe steels arid weldments to environmentally assisted cracking under stress.

(3) Identify research needed in this area to tridge the gap between test melinouicgy and prediction of service performance, and to ensure a proper coliection of field data.

To carry out the assessment of existing knowledge and to participate in objectives (2) and (3), Prof. R. R. Hei of Lehigh liniversity was engaged as a consultant. Prof. Wei produced preliminary assessment in 1978 and submitted a final report in nctober 19\%. His final report, which is attached as Appendix A, contained, besides an assessment of the problem, recommendations for some measurements to be carricd cut at NBS. It also contained the results of corrosion fatigue measurements carried out at Leinigh.

\section{Results}

The followir:g test results were obtained from the preliminary experiments carried out at NBS and Lehigh:

(1) Slow Strain iate Tests (NBS) - Tests have been carried out in threc environnients, air, distilled water and carbonate/bicarbonate $\left(\mathrm{CO}_{3} / \mathrm{HCO}_{3}\right.$ solutions. " The following table gives the results found for pipe steel at room temipcratures: 

$\begin{array}{rrr}\text { Spce. No. Envirument Strain } & \text { Stress } \\ \text { Rate } & \text { (psi) }\end{array}$

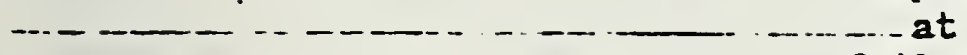

failure

$1 \quad \mathrm{CO}_{3} / \mathrm{HCO}_{3} \quad 1 \times 10^{-6} / \mathrm{s} \quad 80,500 \quad: 213 \quad 75.6 \%$

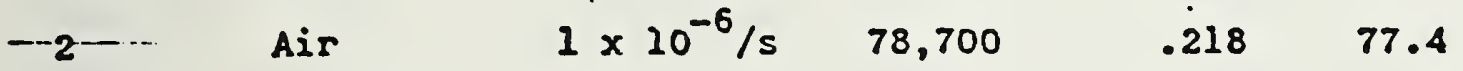

$-3-\mathrm{CO}_{3} / \mathrm{HCO}_{3}-$

$1.6 \times 10^{-7} / \mathrm{s} \quad 81,400 \quad .185 \quad 73.5$

$4 \mathrm{CO}_{3} / \mathrm{HCO}_{3}$

$1.4 \times 10^{-7} / \mathrm{s}$

81,300

$.204 \quad 74.4$

5 Distilled $\mathrm{H}_{2} \mathrm{O}$

$1 \times 10^{-6} / \mathrm{s} \quad 79,700$

.17966 .0

$6 \mathrm{CO}_{3} / \mathrm{HCO}_{3}$

$7.2 \times 10^{-6} / \mathrm{s}$

81,300

$.212 \quad 76.1$

These results indicated that for these environments no appreciable stress corrosion susceptibility (low reduction in area) was observed.

(2) Fracture Mechanics Fatique Tests (Lehigh) - Tests on precracked pipe steel specineri showed a three-fold enhancement. of crack growth rate in distilled water at $1 \mathrm{~Hz}$.

Based on these results and recommendations of the secord ile report, tests were sarried out that produced the following results:

(1) Tapered Pin Loaded Fracture Mechanics Specimens

One specimen loaded to $49.5 \mathrm{MPa} / \mathrm{m}$ ( $45 \mathrm{ksi} / \mathrm{in}$ ) and immersed in $1 \mathrm{~N}$ $\mathrm{CO}_{3} / \mathrm{HCO}_{3}$ solution at room temperature. Solution was changed every month. SSpecimen when broken apart revealed no crack growth. One specimicn loaded to $49.5 \mathrm{MFa}$ 'ill (45 ksi /in) polarized at $650 \mathrm{mV}$ SCE in $1 \mathrm{~N} \mathrm{CO}_{3} / \mathrm{HCO}_{3}$. Specimens when broken apart $r \in v e a l e d$ no crack growth. 

These results and the results from the preliminary tests all indicate no failures can be detected for static fracture mechanics tests or static and cyclic slow strain rate tests. Only cyclic fracture mechanics testing as described in Appendix A appears to reveal the possibility of susceptibility tc failure. It is this sort of testing regime that is most closely tied to actual pipeline experience.

In addition to the ahove tests some field tests were initiated in late 1979. These involved the burial of tapered pin loaded fracture mechanics specimens at two test sites, one at NBS in a soil, that is not particularly corrosive and one at Patuxent, Md in a soil that is highly corrosive and contains sulfides. The specimens buried at these sites were as follows:

(1) Three specimens buried at. NBS site (12/4/79)

$$
\begin{aligned}
& \text { Two loaded to } 49.5 \mathrm{MPa} / \mathrm{m}(45 \mathrm{ksi} / \mathrm{in}) \\
& \text { One loaded to } 33.0 \mathrm{MPa} / \mathrm{m} \text { (30 ksi } / \text { in) }
\end{aligned}
$$

(2) Three specimens buried at Patuxent site (12/13/79)

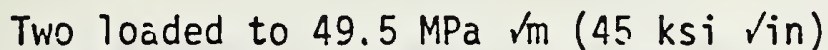

One loaded to $33.0 \mathrm{MPa} / \mathrm{m}(30 \mathrm{ksi} / \mathrm{in})$

It is planned to remove these specimens in one year after burial to determine if any crack growth has occured.

Conclusions

Using the above described test results and the assessment by Prof. Hei, the following conclusions can be drawn:

(1) No test method currently available is callable of simulating service conditions and ellvironments to allow a raiiable assessment of the effect of environment on the failure by cracking of linepipe steels and weldmerts.

(2) If such methods were to be developed, data from these tests would represent orily part of the necessary information in considering waiver requests, but would not be sufficient without establishing a correlation between this test data and service performance.

(3) Corrosion fatigue (cyclic stressing) is a potentially important failure mode in linepife steels and weldmenis. Therefore, recordings of cyclic loads should be included in the collection of field data.

(4) Greater attention should be paid to the role of cycilic stressing in the environmentally assisted failure (cracking) of linepipe steel weldments. More research is needed in this area. 

APPENDIX C - Radiographic Evaluation of the Significance of Weld Defects

M. B. Kasen

The primary questions that must be addressed in any fitness-forpurpose assessment of weld quality are: What flaws are significant in a given structure? At what dimensions do such flaws become critical, endangering the integrity of the structure? How good is the chosen NDE method for detecting and sizing such flaws?

Other than visual inspection, radiography is the most commonily used method for examining the quality of a weld. While undoubtedly effective in judging the quality of a weldors performance, it is necessary to question if radiography is equally effective in assessing the quality of welds on a fitness-for-purpose basis. Table I presents a breakdown of the types of weld flaws detected by radiography under field conditions. Although illustrated for a pipeline, the relative distribution of flaw types is probably typical of most manual welding operations. Most significant is the eight-to-one preponderance of blunt or three dimensional flaws. This will surprise no one familiar with radiography, because the ability of radiography to reveal a flaw is proportional to the difference in radiographic absorption of the flaw as compared to that of the surrounding metal. This difference is much higher for pores, inclusions and arc strikes than for sharp, two dimensional flaws such as cracks or lack of fusion. Furthermore, the detection of blunt flaws is independent of the orientation of the radiographic source relative to the weld, while the probability of detecting a sharp flaw is 

highly orientation dependent. It is easily demonstrated that a through crack along the fusion line of a manual weldment made with the usual $60^{\circ}$ included angle joint preparation has a high probability of being missed by the interpreter under the best of radiographic conditions. It can therefore be concluded that the series of welds surveyed on Table I contain a much higher percentage of sharp, cracklike flaws than was actually observed.

This creates two problems. The most important is that many cracklike flaws, which have the highest probability of initiating weldment failure, go undetected. The second is that a substantial amount of money is spent to repair blunt flaws which may have a very low probability of initiating failure.

The fitness-for-purpose approach to assessing flaw significance in a given structure has been described in other papers in this series. One of the initial applications of this approach was to weldments of the Alyeska oll pipeline. Here it was necessary to assume that all flaws detected by radiography were significant as crack initiators and that those exceeding existing workmanship criteria required dimensioning and assessing by fracture mechanics criteria. This approach was nonconservative in assuming that the radiographs were revealing all the significant flaws and very likely over conservative in having to assume that blunt flaws were the equivalent of sharp flaws of equal dimension. This reflected the difficulty of assessing stress concentrations for blunt flaws. Furthermore, difficulty in assessing the through-wall dimensions of blunt flaws from the radiographs necessitated the assumption that such flaws were twice the estimated size. 



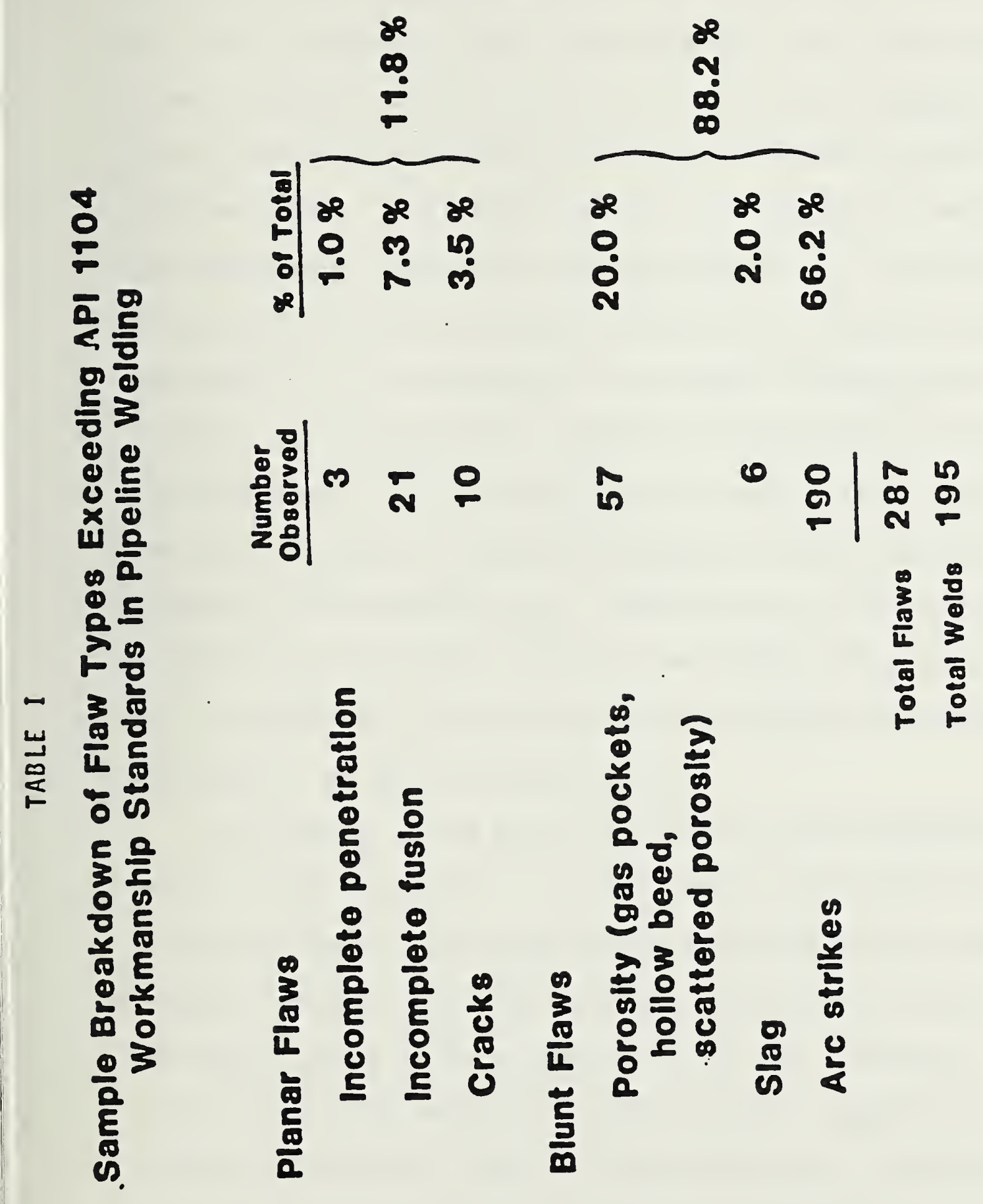



The current fracture mechanics approach attempts to provide the basis for a more realistic method. It is assumed that radiography will very likely remain the primary NDE technique, but it also assumes that other techniques such as ultrasonics will provide a backup method for flaw sizing and may eventually supplant radiography as the primary inspection method. The current approach also continues to assume that two-dimensional, planar flaws are significant in all structures, requiring dimensioning and analysis by analytical fracture mechanics techniques. But the assumption is also made that blunt flaws may be innocuous in some structures, depending on the stresses and toughness of the weld metal, allowing permissible flaw content to be established on the basis of technical engineering judgment rather than by techniques requiring precise dimensioning. The advantage of this approach is significant cost saving if repair of noncritical flaws can be minimized and a significant increase in structural integrity by emphasizing the detection of potentially dangerous flaws.

The approach to the blunt flaw portion of this program has been reviewed in the literature.' In brief, it is assumed that flaws which can be shown to have a low probability of initiating cracks in welds subjected to stress regimes far more severe than those in service can be rationally judged to be innocuous under șervice conditions. The specific approach has been modelled after similar research by the British Welding Institute in a study of flaw significance in welded pressure vessels. ${ }^{2}$ Weldments representing the parent materials, welding processes, procedures and consumables used in the actual structure and containing much higher levels of porosity, slag and arc strikes than 

would rationally be permitted under field conditions, are subjected to fully-reversed, low-cycle strain-controlled fatigue at strain levels slightly above yield of the weld metal and twice the strain at yield. Care is also taken to perform the testing at credible field temperatures. Al though weldments in pressure vessels or in the pipelines of current concern are not subjected to service stresses and strains of this magnitude, any flaw which can be shown to be innocuous under these severe conditions will have such a low probability of initiating flaws under essentially static conditions as to justify limiting their content on pragmatic reasons without incurring the complexities of the analytical fracture mechanics approach. ${ }^{3}$

In the present work, a comparison is made between the number of cycles to crack initiation in pipeline girth welds made in AP5LX-65 line pipe by manual (SMA) cellulosic electrodes and by an automatic process. In addition to sound welds meeting the API 1104 workmanship criteria, very badly flawed welos were prepared containing porosity levels giving 15:. radiographic obscuration, continuous slag and arc strikes imposed after the welding operation. Specimens containing a 4 inch length of weldment were fatigued in a transverse manner at strains slightly over yield $( \pm 0.22 \%)$ and at twice yield $( \pm 0.45 \%)$ while maintaining the temperature at $-2 \pm 1^{\circ} \mathrm{C}$. Crack initiation was monitored by observing changes in the load required to maintain the strain range. A $10 \%$ drop in load, representing development of a $10 \%$ cracked area, was taken as the criterion for crack initiation. Tests were continued until a $40 \%$ load drop ( $40 \%$ cracked area), was achieved so as to provide information on crack propagation. Specimens were th:n broken in tension for fractographic analysis. 

The results obtained from 37 specimens representing parent metal as well as sound and flawed weldments, tested with the reinforcement cap both intact and machined off, appear in Figs. 1 and 2. From Fig. 1 , it is seen that approximately $800 \mathrm{cyclic}$ reversals were required for crack development when the weld cap was intact, regardless of whether the welds were sound or contained very large amounts of porosity, slag or arc strikes. Even for strain reversals equal to twice yield, about 30 cyclic reversals were required. Removing the weld cap forces failure through the flawed welds, decreasing the fatigue life to initiation. But even here, about 150 cyclic reversals were required at just over yield and about 25 at twice yield for crack initiation.

- The data plotted on Fig. 2 relate more closely to field conditions, as the weld reinforcement cap is intact in all cases illustrated. This figure also examines the effect of specific flaw types. The performance of the unwelded, parent material is also illustrated.

The top plot illustrates that the automatic welds containing $15 \%$ porosity by projected area (about 3\% by volume) performed equally well as did welds free of porosity or other flaws. The center plot illustrates that the presence of slag somewhat decreases the cyclic life to crack initiation; nevertheless, about 750 reversals were required at just above the yield and about 30 at twice yield to achieve initiation. The lower plot illustrates that arc strikes had no effect on cyclic life. In no case were weld failures observed to initiate from such flaws. Arc striking the parent plate was likewise ineffective in initiating fracture at the $\pm 0.22 \%$ strain level. It was necessary to strain the arc struck parent material at $\pm 0.45 \%$ before any effect was noted. 



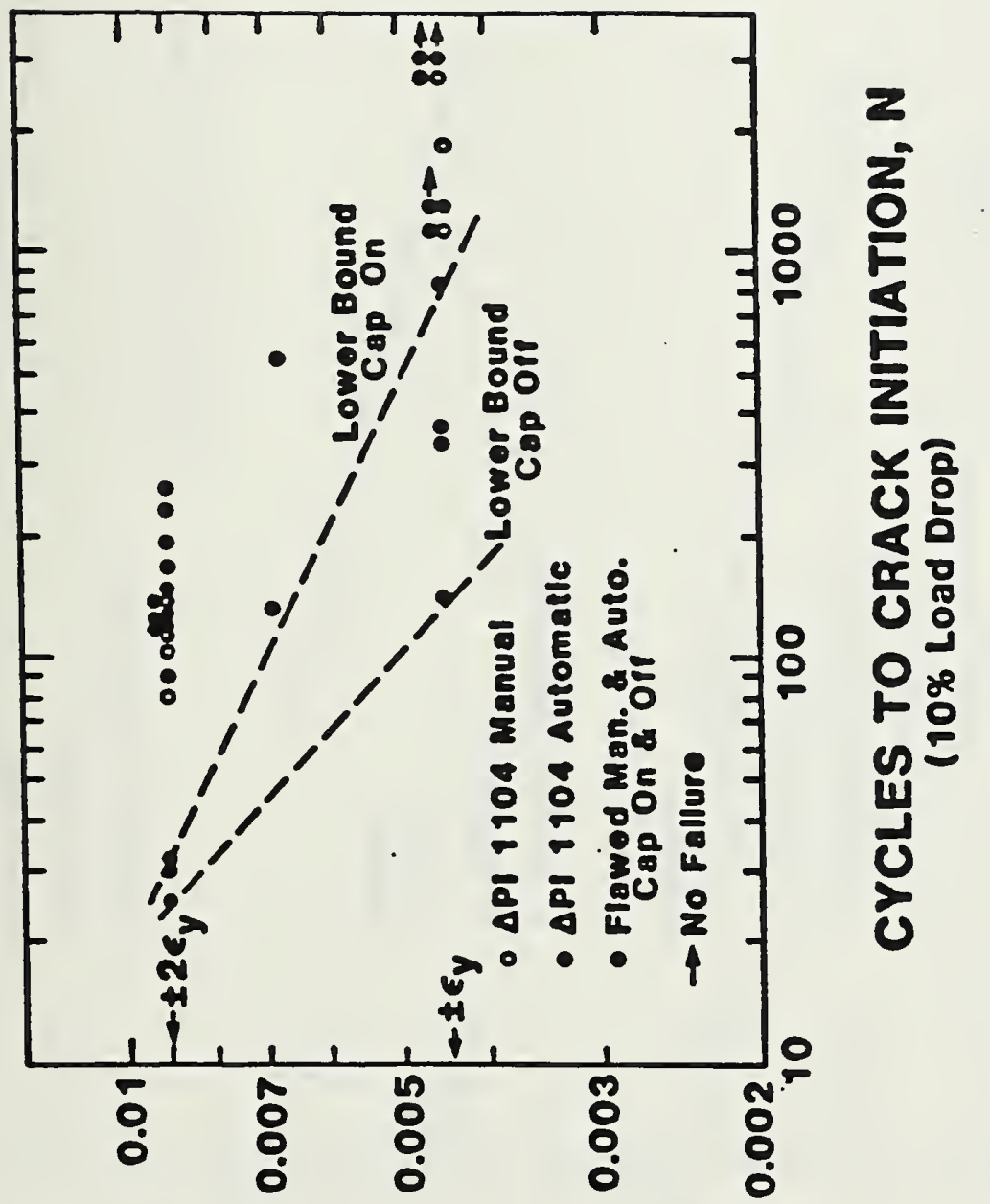

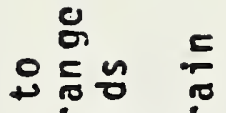
$2=n \frac{2}{2}$ $\sim \Sigma \equiv$ u

nा

28020

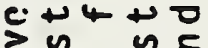
a 0 的 ᄂ4 U일

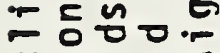
$u \cdot r=0$

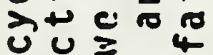
4 든 $45=\pi \dot{0}$ $0+\frac{1}{2}$ in ป 인 E $c=0 n: 0$ $0-0>-1$

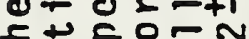
选药 둔 O-DEE 든 구응두

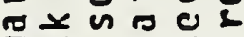

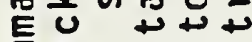

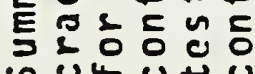
n $u 40+0$

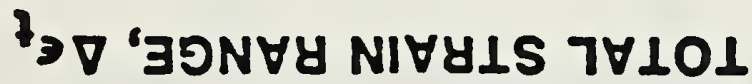





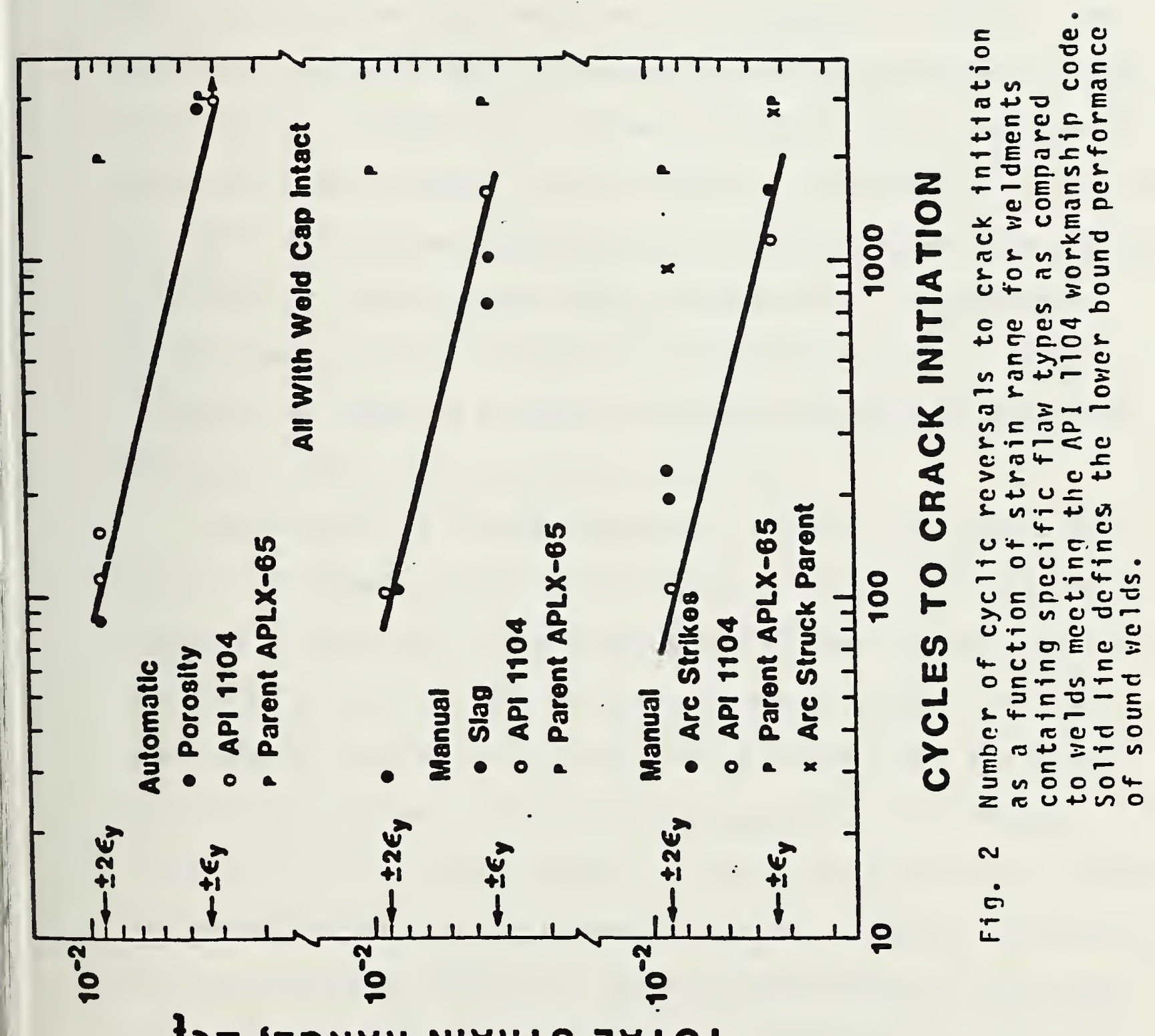

$I^{3}$ 'ヨอN 

These data add credibility to the argument that blunt flaws have a very low probability of initiating fallure in an operating pipeline, particularly when it considered that the permissible flaw level would be limited in practice to substantially less than in the test series and that line is unlikely to see more than one or two deformations to strains above yield and that these would not be accompanied by reversal. The strongest case can be made for automatic porosity and manual arc strikes. Slag presents a slightly more complex problem because fractographic evidence shows that failures with this type flaw were influenced by the development of hydrogen-assisted cracking originating either from the slag inclusions or from micropores within the weld metal. It therefore appears that, to the extent that slag contributes to crack initiation, it does so by providing a surface for hydrogen segregation rather than from the stress concentration due to slag shape.

Returning to the original questions, available data suggest that porosity in automatic welds and arc strikes in manual welds are not significant in weld metal having the toughness of that studied. The probability of failure from slag also appears remote; however, the associated hydrogen cracking arising from the use of cellulosic electrodes needs further analysis. These results suggest that it is probably unnecessary to dimension such flaws in order to establish a valid fitnessfor-purpose criteria for their content in a weld. Finally, we conclude that radiography is effective in detecting the presence of such flaws, but is of very questionable effectiveness in the detecting or dimensioning of two-dimensional, planar flaws of the type that have a much higher probability of initiating weld failure. It remains for other NDE systems such as ultrasonics to perform that task. 
References

1. Kasen, M. B. and Mikesell, R. P., "Interim Report on the Significance of Blunt Flaws in Pipeline Girth Welds", Pipeline and Energy

- Plant Piping: Design and Technology, Pergamon Press, 1980, 329.

2. Boulton, C. F., "Acceptance Levels of Weld Defects for Fatigue Service", Welding J., 56, 1977, 13-22.

3. Gurney, T. R., Fatigue of Welded Structures, 2nd Ed., Cambridge Univ. Press, Cambridge, England, 1979. 

Appendixes $B$ and $C$ of this document are available on request. 



\title{
APPENDIX D - Defect Characterization and Dimensioning of Cracks \\ in Welds by the Ultrasonic Diffraction Method
}

\author{
S. Golan ${ }^{\star}$
}

\section{ABSTRACT}

The possibility of applying an ultrasonic diffraction method for dimensioning of crack-like defects in welds was investigated. A feasibility study was carried out and optimum test conditions were established using a series of test specimens with narrow slits. A series of welded specimens with in-welded cracks were tested. The possibility of using a quantitative diffraction technique for nondestructive examination of pipeline welds in field conditions is discussed.

\section{INTRODUCTION}

With the conventional ultrasonic pulse-echo technique, flaw size is related to echo-amplitude. Amplitude, however, being strongly affected by many factors ${ }^{1}$, is rather insensitive to size. Consequently, the pulseecho technique is not suitable for quantitative measurements. In welds, quantitative evaluation becomes even more complicated by the anisotropy and inhomogeneity inherent in welded materials and by geometric factors. The inability of the pulse-echo technique, which is based on reflectivity, to serve as a quantitative tool provided the impetus for development of techniques based on other phenomena of interaction between the flaw and the acoustic field, such as scattering, diffraction, interference, etc. It seems that the most promising technique to develop into a practical and economical quantitative testing tool for field work is the diffraction technique. ${ }^{1-6}$ Figure 1 shows an example of ultrasonic sizing of slits. More such curves are given in reference 1 . It is especially useful for dimensioning of crack-like defects. This technique has three main advantages over some other emerging techniques: flaw size is computed using only time measurements, which can be made more accurate and more reliable than amplitude measurements; conventional pulse-echo equipment can be used; and operators familiar with the pulse-echo technique can easily adopt the diffraction technique.

- Permanent address, Israel Institute of Metals, Israel. This report was prepared while the author was on a temporary assignment to the NBS Office of Nondestructive Evaluation. 



\section{BASICS OF THE DIFFRACTION TECHNIQUE}

When an ultrasonic wave impinges on the tip of a crack, waves of shear $(S)$ and compressional ( $P$ ) modes are scattered from the tip in a circular form. Figure 2. shows a photoelastic pattern of the scattered acoustic field from the tip of a narrow slit in a glass block. From the time interval between the entering of the incident wave and emerging of the tip-diffracted wave the depth of the tip can be computed. ${ }^{-5}$ Most reliable results are obtained when time delay is measured from a conveniently chosen reference signal. Figure 3 shows a diffraction triangle formed by the transmitted, the diffracted and a compressional surface wave which serves as reference. 1 Equation 1 gives the relationship between time delay and depth of the tip when a $P$ surface wave is used as a reference.

$c_{2}^{2}-c_{1}^{2} \gamma^{2}+4 \cos \alpha\left[c_{1}(\Delta+1)-c_{2}^{2}(\sin \alpha) \gamma\right]-4 \cos ^{2} \alpha\left[(\Delta+1)^{2}-c_{2}^{2}\right]=0$

$\alpha \quad$ - Angle of incident beam

$C_{1}, C_{2} \quad-$ Mode coefficients for impinging and diffracted beams

$C=1$ - For a compressional beam

$c=\frac{v_{c}}{V_{s}} \quad$ - For a shear beam

$V_{s} \quad-\quad$ Shear wave velocity

$v_{c} \quad$ - Compressional wave velocity

$Y=\frac{y}{a} \quad$ - Depth of source of diffraction; nondimensional

$\Delta=\frac{\delta}{a} \quad$ - Depth of source of diffraction; nondimensional

$2 \delta$

- Time delay expressed in units of length

$2 a$

- Distance between transducers

$y$

- Depth of source of diffraction.

From this general equation specific equations can be derived for different modes of transmitted and diffracted waves and for various diffraction triangles. ${ }^{1,2}$ If the reference signal is a bottom reflection and the diffracted and reference signals are maximized (see Figure 4), the relationship between time-delay and depth for a single or double transducer technique is given in Equation 2. 



$$
y=T-\delta \cos \alpha
$$

\section{T - thickness of material.}

\section{OPTIMUM CONDITIONS FOR DIMENSIONING OF CRACKS}

The diffraction techniques can be used to dimension cracks open to the front or back surfaces as well as internal cracks, provided a tip-diffracted signal can be detected. This, however, is quite often very difficult. The tip-diffracted wave usually has a low amplitude, and high amplification is necessary to reveal the signal. This, in turn, forms a noisy background which might blur the tip signal. The noise signals come from various sources; scattering from grains and inclusions, mode conversion, scattering from the crack surface, back wall reflection, surface waves, side loops, internal reflections from the wedge, and electronic noise. Figure 5 shows a typical oscilloscopic trace in the background of a signal diffracted from the tip of a fatigue crack. 5 The crack depth can be sized with sufficient accuracy and reliability only if the tip diffracted signal can be clearly detected and unambiguously identified among the spurious signals. As has been shown in this and some other works ${ }^{1-5}$ under certain conditions signal-to-noise ratio can be considerably improved and the tip signal can be detected. By analyzing the oscilloscopic trace, the tip signal can be in many cases identified.5,6

Three parameters determine the detectability of a signal; amplitude, resolution, and dynamic response. Dynamic response is defined as the drop in amplitude caused by displacing the transducer from its maximum amplitude position (see Figure 6). When the dynamic response is high, a sharp drop in amplitude will be observed when the transducer is slightly displaced. This parameter is a very strong crack indicator.

All three parameters are mode and angle dependent. On a goniometer the angle-amplitude dependence was determined for the PP mode (incident and diffracted waves of the compressional mode) $)^{3}$. It was found that maximum amplitude is obtained when the angle between the transducers approaches $180^{\circ}\left(\alpha \rightarrow 90^{\circ}\right)$.

In former works ${ }^{3-4,6}$ the PP mode was primarily used in order to prevent interfering signals of the $P$ mode. In later work ${ }^{6}$ it was found that, by using the SS mode and angles $\alpha$ in the range of $40^{\circ}$ to $50^{\circ}$, higher amplitudes are obtained. The dynamic response is higher ${ }^{6}$ because of the smaller angles and, because of the lower velocity of shear waves, resolution is almost doubled. By choosing an angle between $40^{\circ}$ and $50^{\circ}$ interfering compressional mode signals do not appear. An additional improvement in amplitude is achieved by using one instead of two transducers. This has additional advantages: it is more convenient to handle one transducer; no alignment between the transducers is necessary; and the dead zone is smaller, as it is not 1 imited by wedge geometry. ${ }^{6}$ The high amplitude noise which appears when one transducer is used can be minimized by chosing a wedge which filters out the internal reflections. 7 

For the feasibility study a series of specimens with a simulated internal crack in welds was prepared. The "inwelded crack" was formed by incomplete penetration of a butt weld. The location, size and orientation of the "crack" was controlled by the bevel geometry of the welded plates (see Figure 7, 8 and 9). In order to achieve minimum crack width $(0.00$ to $0.06 \mathrm{~mm})$, minimum deformation and, as closely as possible, a straight line of the tip, the plates were welded and cooled to room temperature while clamped in a fixture. The material used was 25.4-mm low-carbon steel plates. Specimens of 22-mm width were cut from the welded plates. The specimens were machined, polished and etched to reveal the "crack" and the weld cross section. In order to check the ability of the diffraction method to dimension normal as well as inclined internal cracks in the as-welded condition, four types of specimens were prepared: as-welded with normal "cracks"; machined to remove the excess of the welding, with normal "cracks"; and as-welded and machined with $45^{\circ}$ "cracks" (see Figures 8 and 9 ). Measurements were made with the two-transducer technique, $45^{\circ} 5-\mathrm{MHz}$ shear waves, equilateral diffraction triangle, and a back-wall reflection signal as reference. (The $45^{\circ}$ angle is within the range of good detectability). Because of the many advantages of the one-transducer technique an initial attempt was made to employ it. This technique was, however, abandoned because of high-amplitude interfering signals reflected from the "crack" surface (see discussion below).

The setup and the transducer arrangement are shown in Figures 10 and 11 . Heasurements were taken after the amplitude was maximized by slight lateral movements of the transducers. Results were verified by measurements from four different transducer locations, with direct and double-skip beams (see Figure 12), and from both sides of the specimen. In Table 1 a schematic presentation of the different beam paths and direct and ultrasonic measurement results are given in $x, y$ coordinates. The coordinate system is shown in Figure 8 . The $x$ coordinate was determined from the positions of the transducers. The scatter of the $x$ measurements was within $\pm 2 \mathrm{~mm}$. The $x$ data are arithmetic averages of five measurements. In Figure 13 values of depth (y coordinate) made by direct and by ultrasonic measurements are compared. The differences are not necessarily deviations but, perhaps, true differences in the depth of the tip at the outer surface (measured directly) and at the middle of the specimen (measured ultrasonically).

\section{DISCUSSION}

Al though it was shown in several works ${ }^{-5}$ that accurate and reliable sizing of cracks with the diffraction technique is feasible, testing of welds in materials less than $25 \mathrm{~mm}$ in thickness under field conditions presents special difficulties which have not yet been completely resolved. This can be seen in Table 1 where, in many places, measurements were not possible. Some of the difficulties are described below and schematically presented in Figure 14. Because of the coarser grains, the directional structure and the microimperfections usually present in weld material, the ultrasonic noise from the weld is more severe than in the parent material. This might be improved by using a lower frequency. 

The advantage of using the one-transducer technique is obvious. This technique, however, might cause misleading results because of multiple reflections from the crack surface. Because of the crown of the weld, scanning of the weld with a direct beam is limited and often not possible and doubleskip beam paths must be employed. This increases noise and attenuation.

The high-amplitude back-wall reflection often masks the low-amplitude tip-diffracted signal and resolution becomes difficult and often impossible, especially in thinner material.

Some of the difficulties mentioned above might be diminished by changing testing parameters such as frequency, diffraction triangle, mode combination, transducer and wedges. Further investigation to diminish interfering factors is recommended. 

Table 1

Ultrasonic Measurements

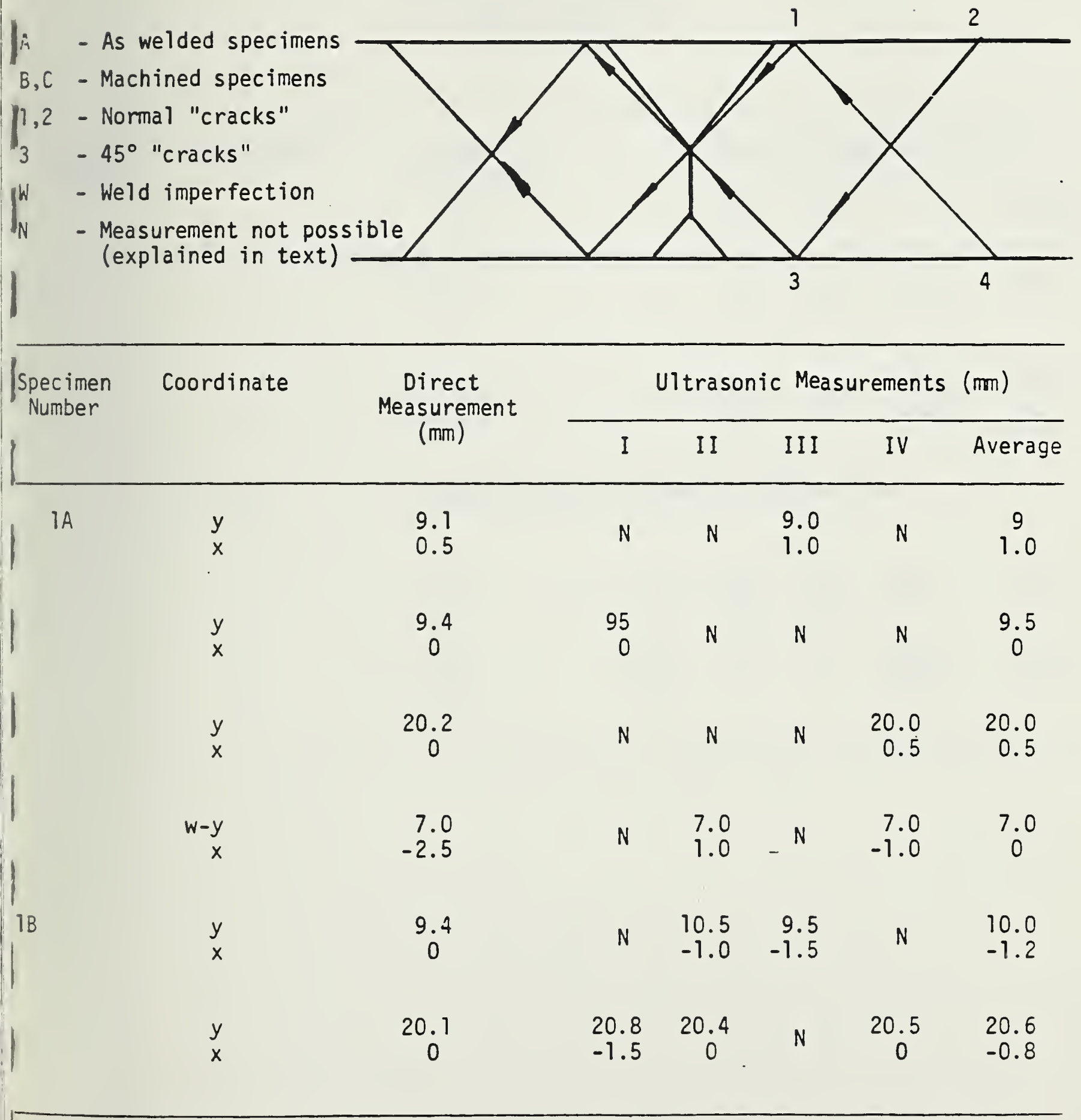



Table 1

Ultrasonic Measurements

(Continued)

Specimen Coordinate

Number
Direct

Measurement

(mm)
Ultrasonic Measurements (mm)

I II III IV Average

$\begin{array}{llcc}11.0 & 11.0\end{array}$

0

19.6

$-8.4$

19.4
$\quad-8.5$

20.0
$N \quad-8.0$

19.7

$-8.3$

$w-y$
$x$

Several points from weld imperfections

$3 C$

$\begin{array}{cc}y & 11.4 \\ x & 0 \\ y & 18.8 \\ x & 18.4\end{array}$

$\begin{array}{crr}10.6 & 10.7 & 11.5 \\ 0 & 1.5 & 1.0\end{array}$

10.7

10.9

0

18.7

8.0

N

N

18.5

0.6

18.4

8.0

8.0

18.6

8.0 

Table 1

Ultrasonic Measurements

(Cont inued)

\section{Specimen Coordinate}

Number
Direct

Measurement $(\mathrm{mm})$

Ultrasonic Measurements (mim)

I II III IV Average

$\begin{array}{ccccc}N & 8.8 & 9.1 & 9.5 & 9.1 \\ & 2.0 & 1.0 & 0 & 1.5\end{array}$

$\begin{array}{ccccc}N & 8.8 & 9.1 & 9.5 & 9.1 \\ & 2.0 & 1.0 & 0 & 1.5\end{array}$

9.6
0

18.8
0

19.7
0.5

N

N

19.0
0.0

19.4

0.3

12.1
0

18.4
0

N N N

N

N

11.7
0

$\begin{array}{ccc}11.2 & 11.0 \\ 0 & 0\end{array}$

11.5
0.5

11.2

0.2

18.1
0

$\begin{array}{rr}18.3 & 17.4 \\ -1.0 & 1.5\end{array}$

18.5
$N \quad-0.5$

18.1
0

$2 c$

$\begin{array}{cc}y & 11.0 \\ x & 0 \\ y & \\ x & 18.0 \\ w-y & 0 \\ x & \end{array}$

9.8
1.5

9.4

1.2

$\begin{array}{ll}\mathrm{N} & 9.0 \\ & 1.0 \mathrm{~N}\end{array}$

$\begin{array}{ccc}17.9 & N\end{array}$

N

17.9
0

$\begin{array}{ll}7.0 & 7.0 \\ N \quad 3.0 & 2.5\end{array}$

7.0
3.5

7.0 



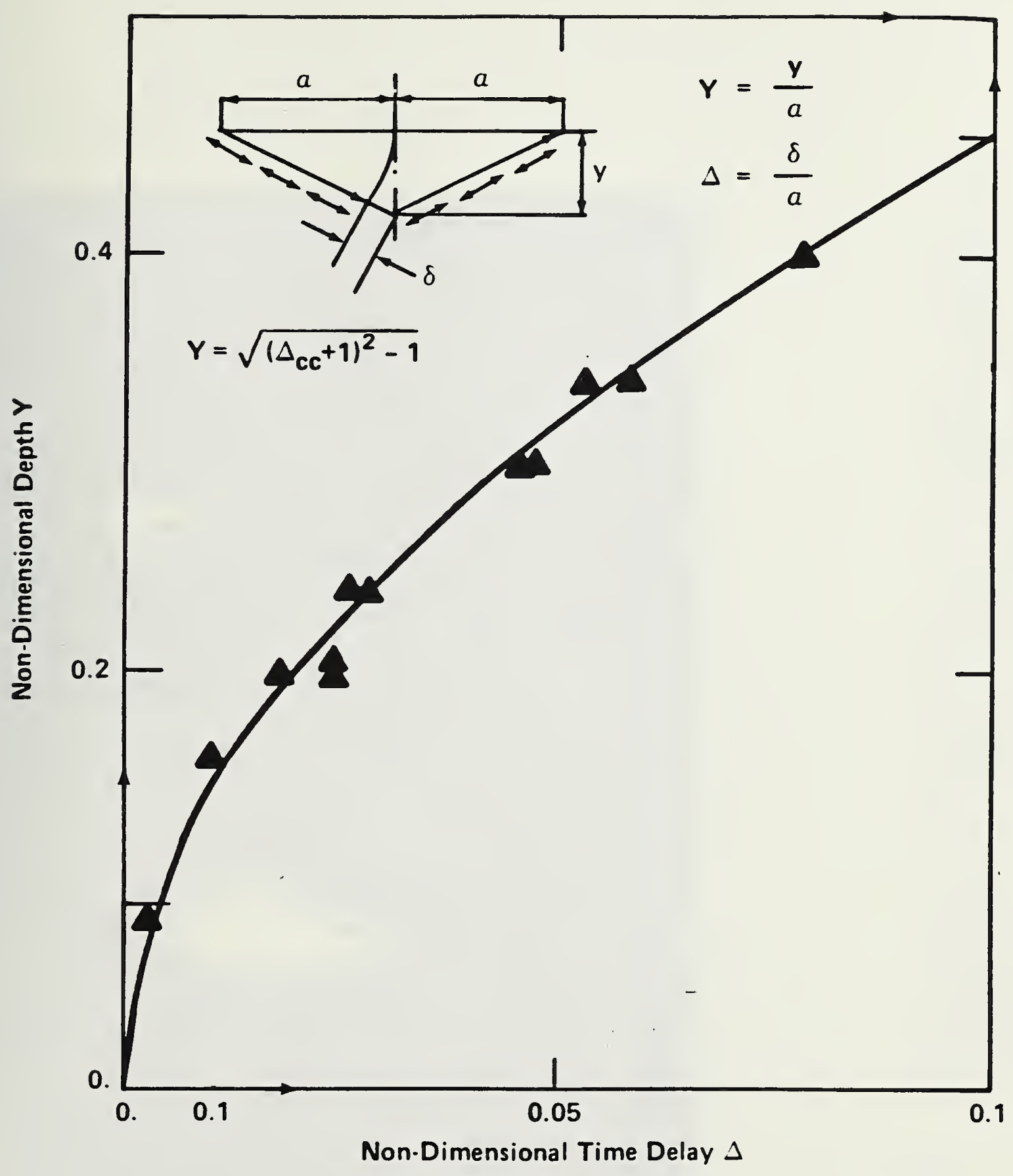

Figure 1. Sizing of Slits With The Double Transducer Technique PP array (compressional impinging, compressional diffracted waves). 




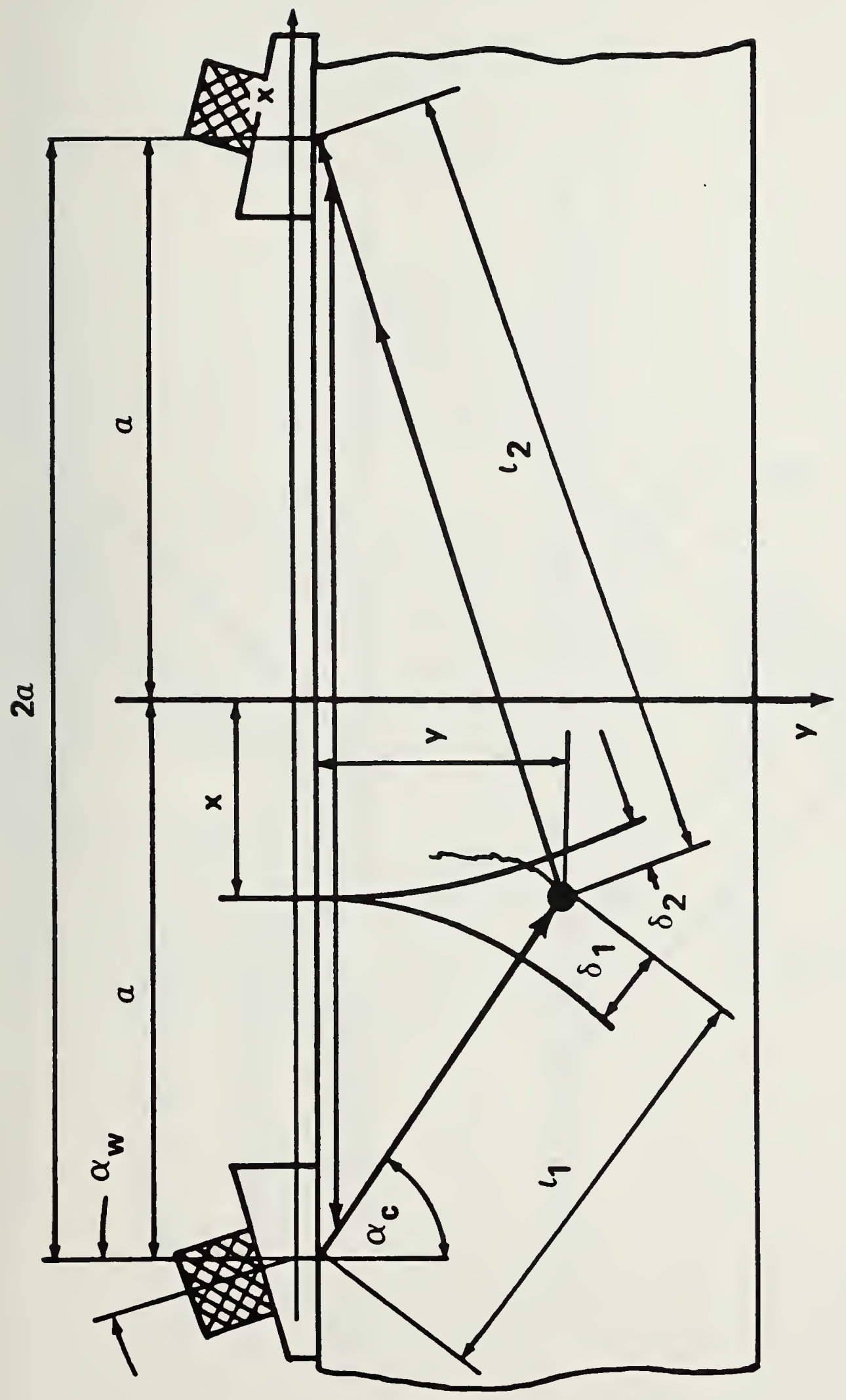

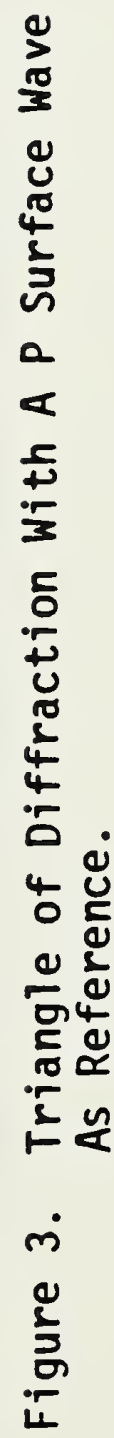




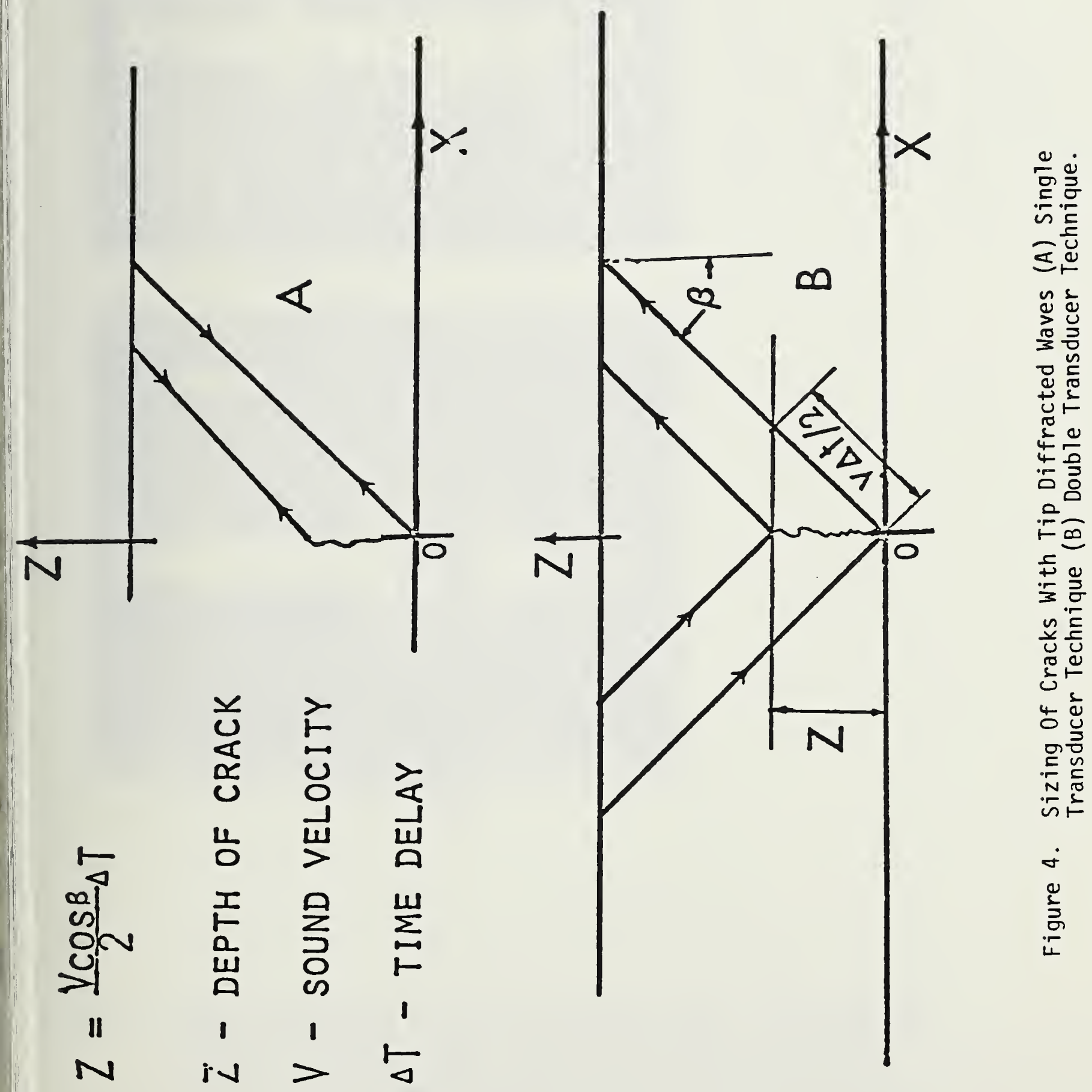


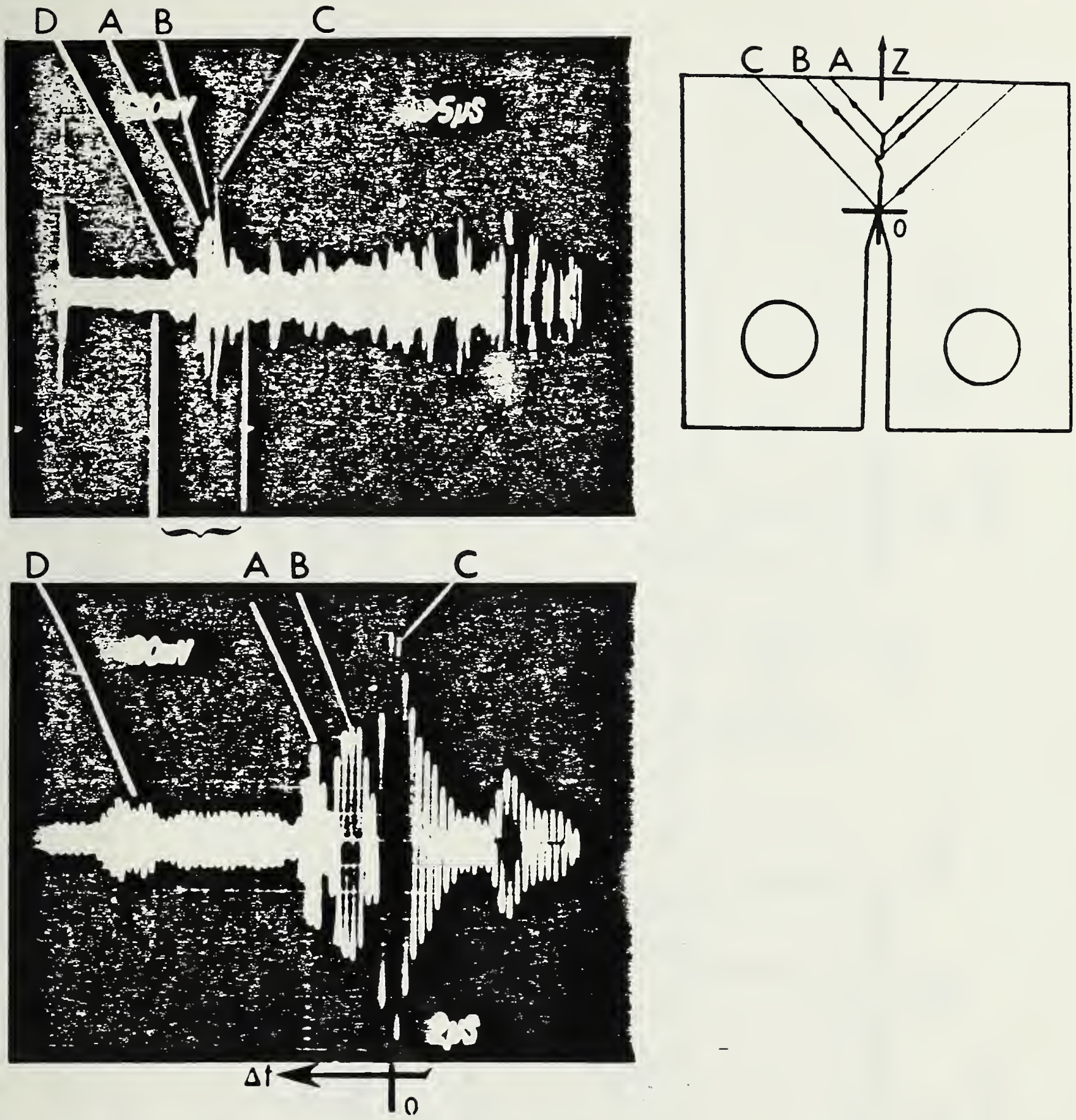

Figure 5. Noisy Background of the Oscilloscope Trace. The expanded Section Contains: (A) A Signal Diffracted From The Tip of The Crack (B) Signals From Protrusions On The Crack Surface (C) A Signal Diffracted From The Tip of The Notch and (D) Noise Signals From The Material. (C) Is The ZeroReference Point. Time iT Is Measured From (C) To The Left. 


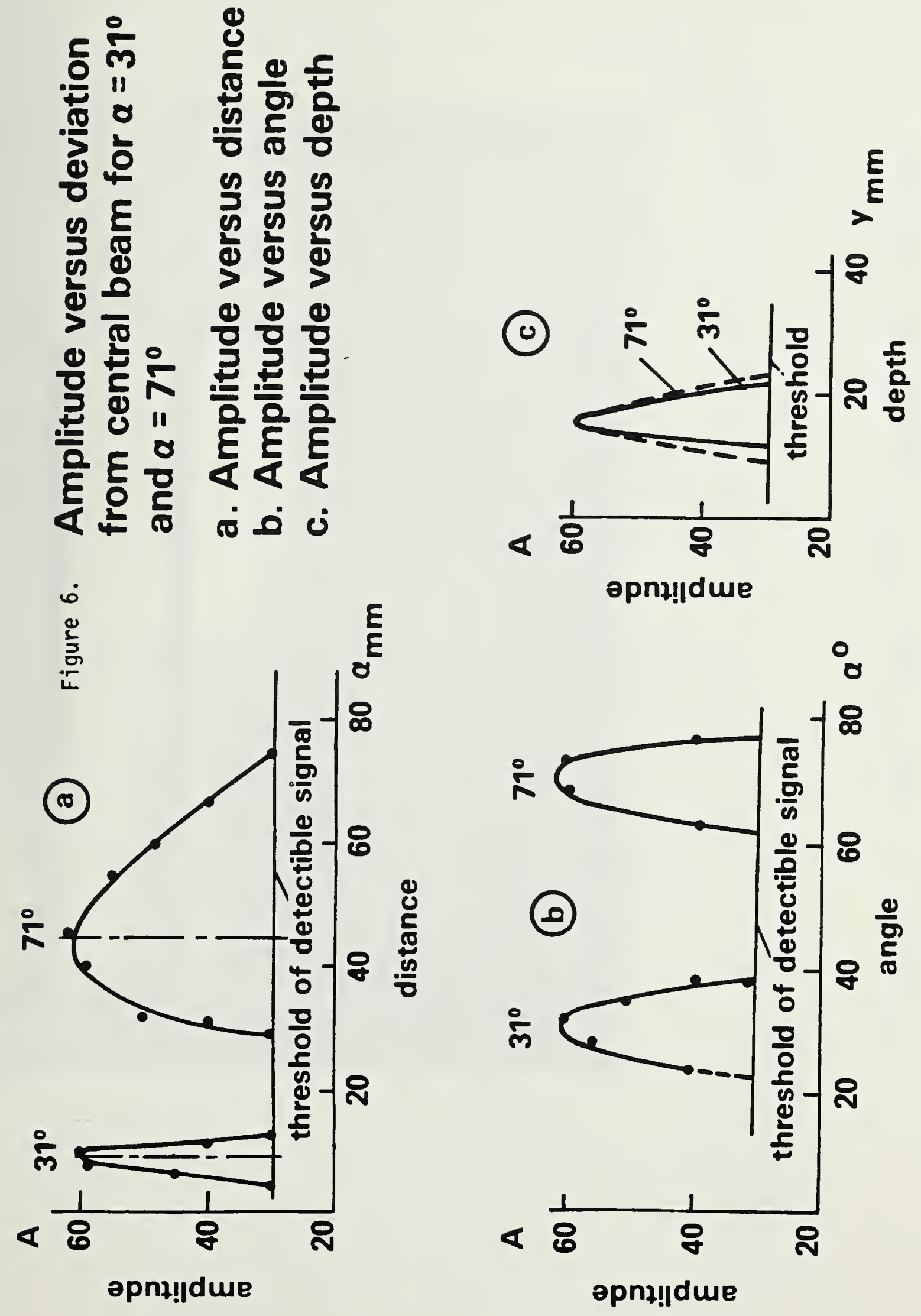



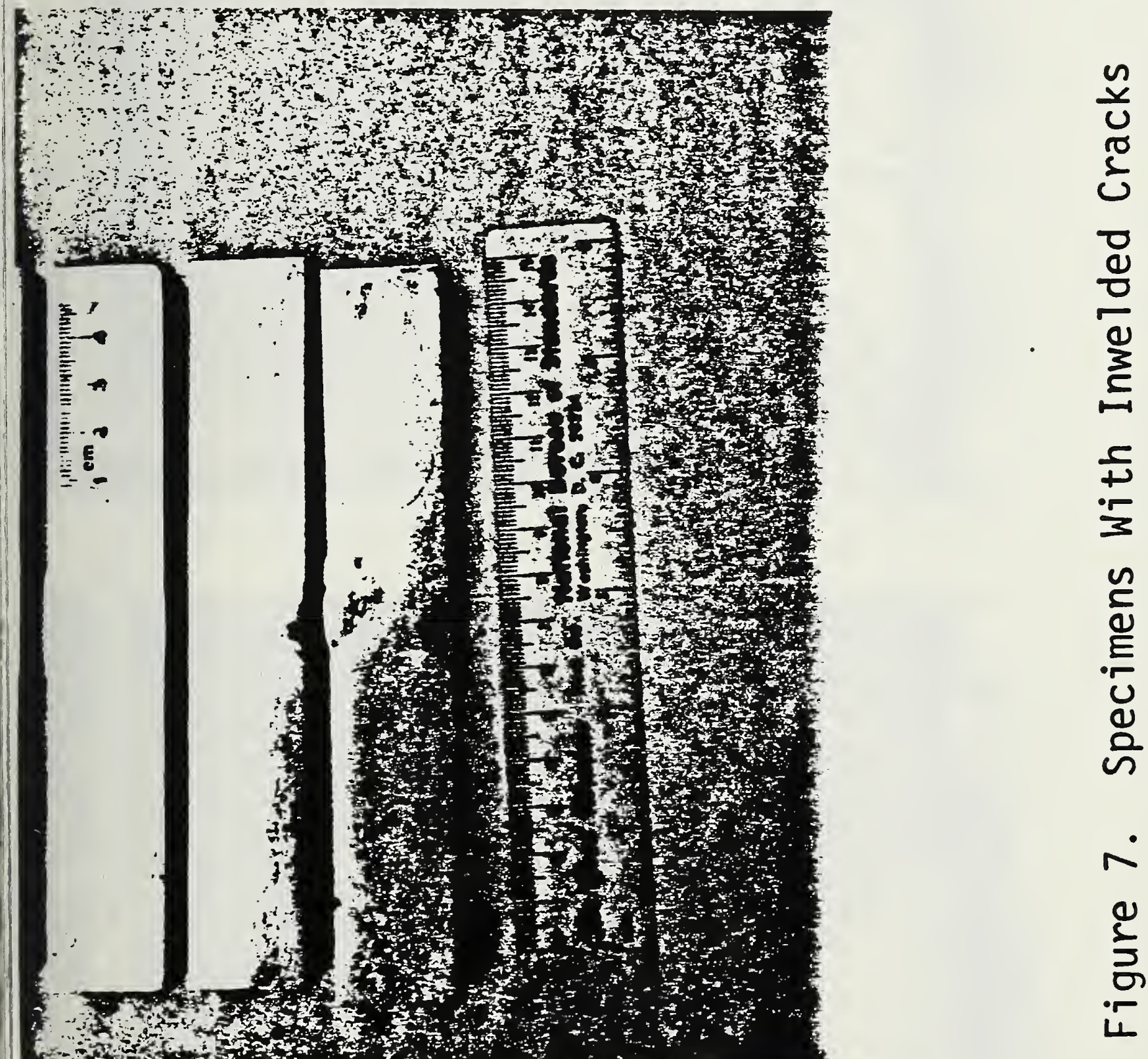





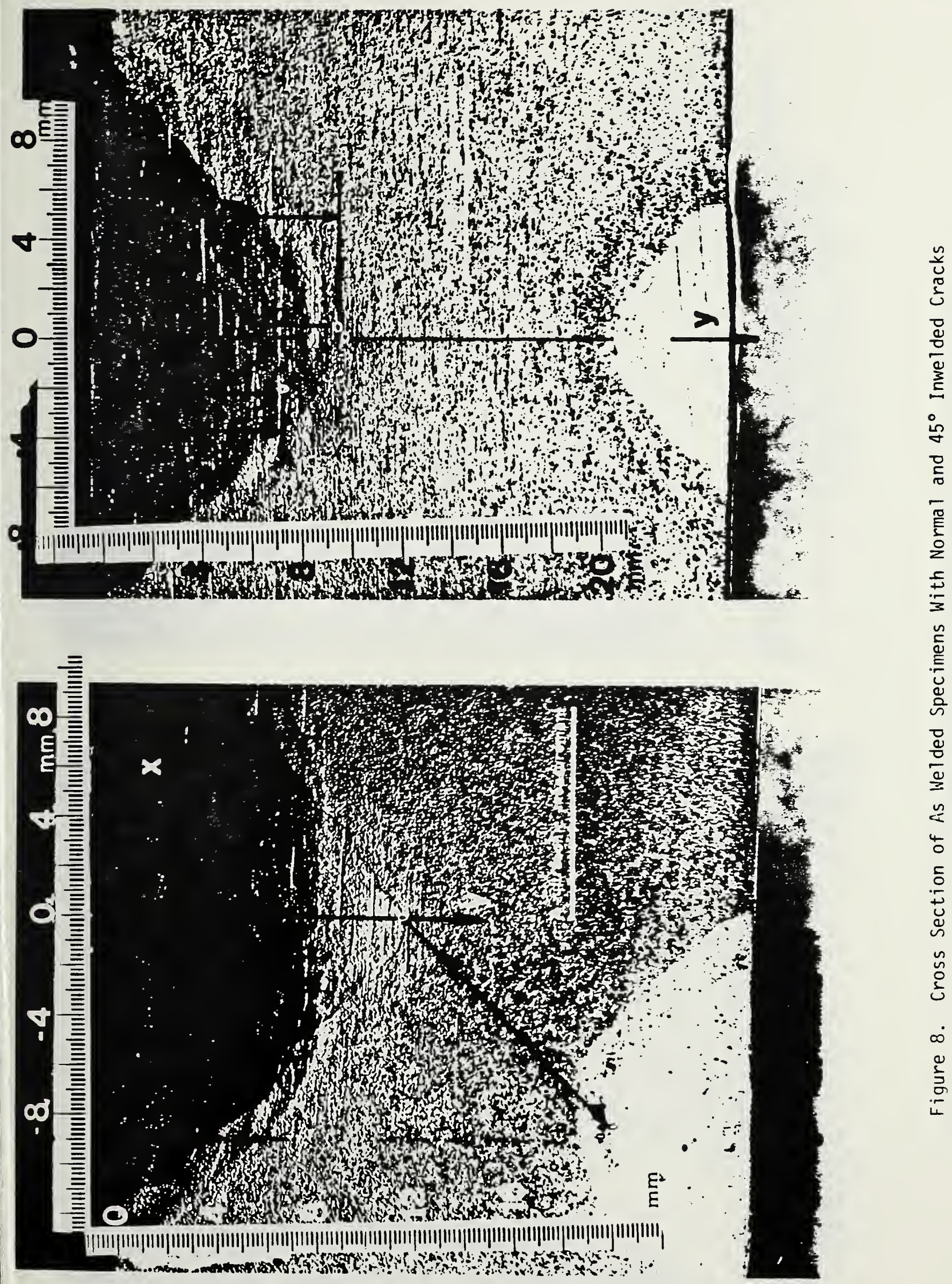





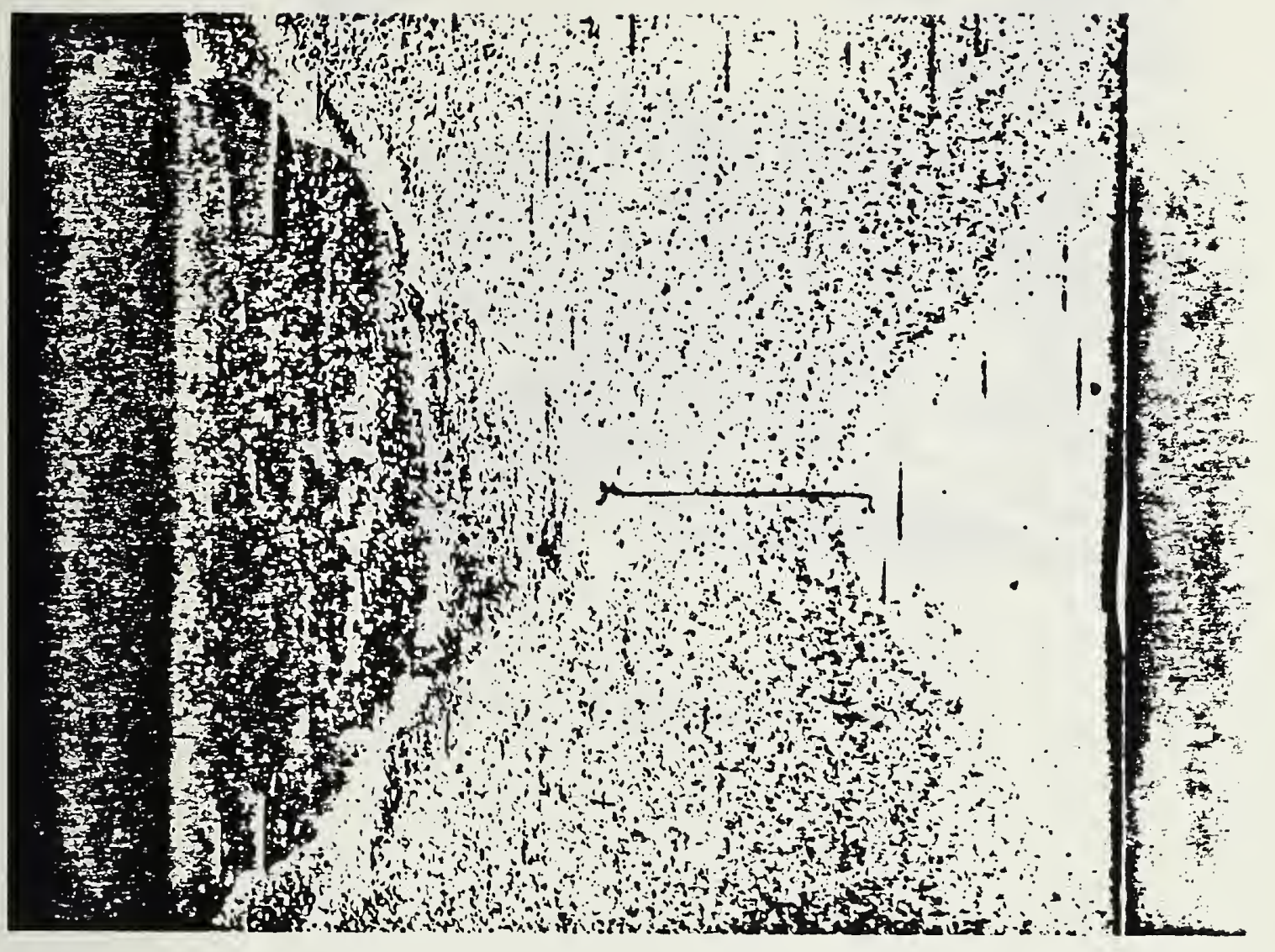

年

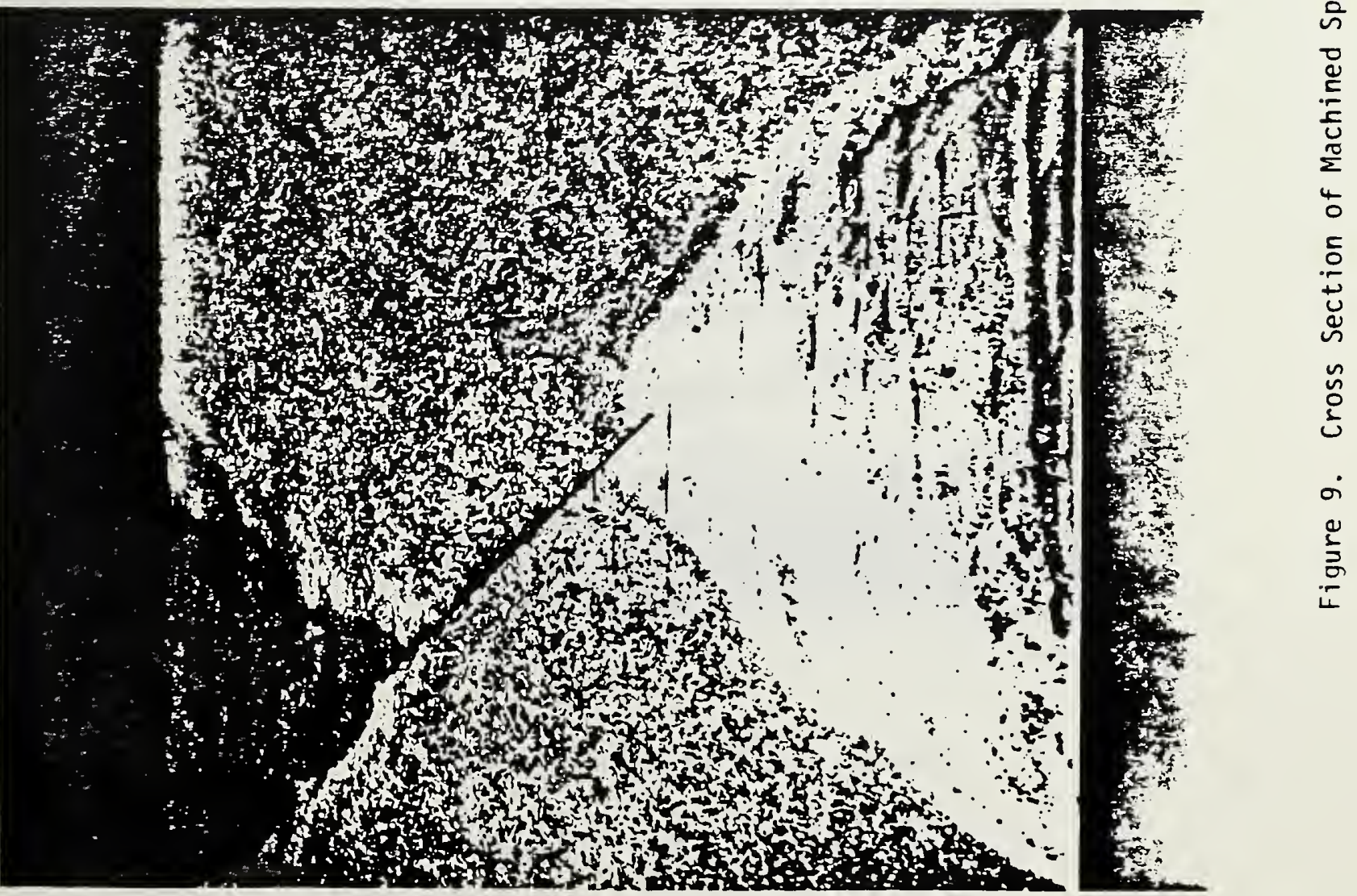





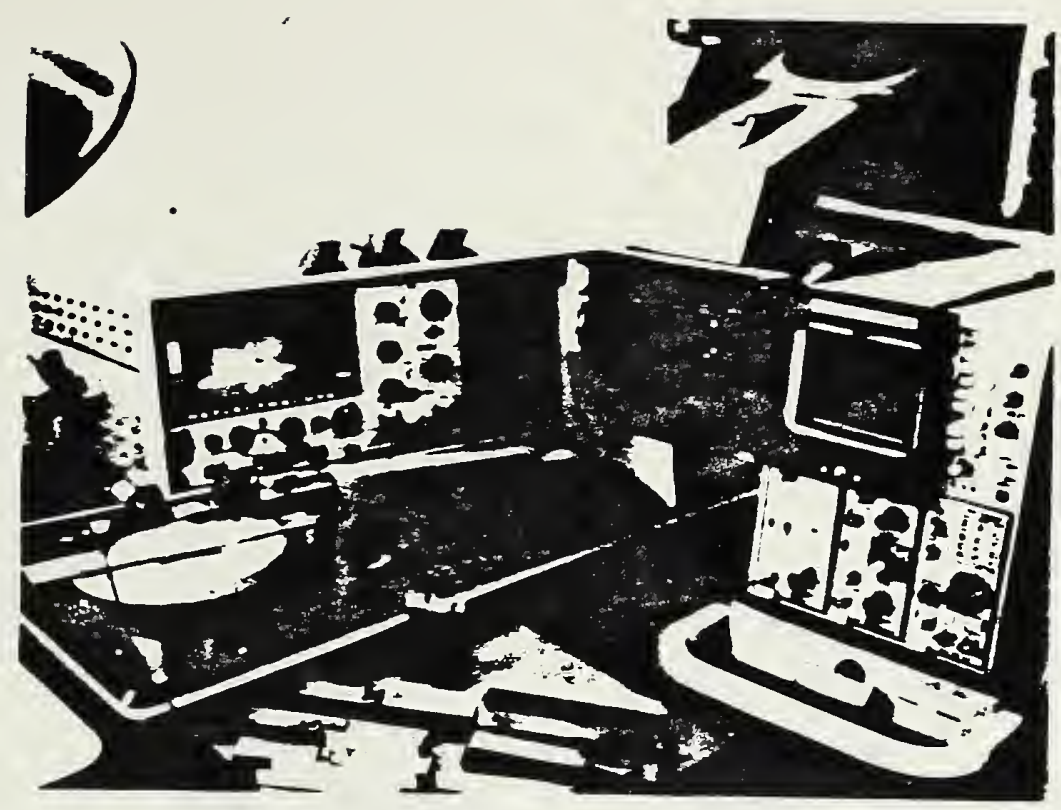

Figure 10. Experimental Set-Up

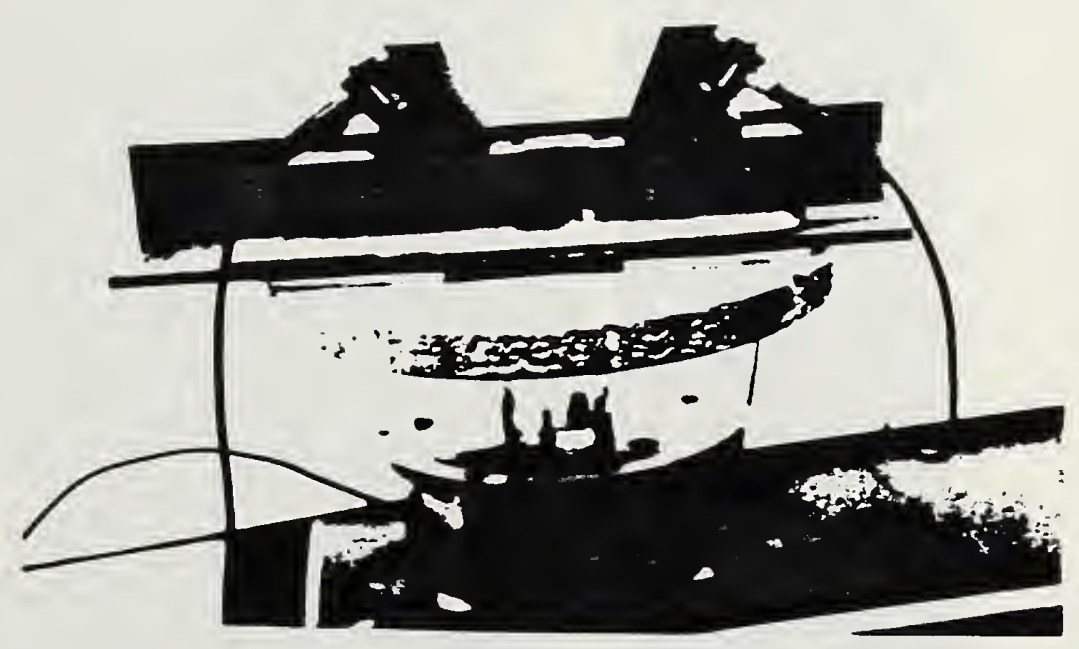

Figure 11. Transducer Arrangement 


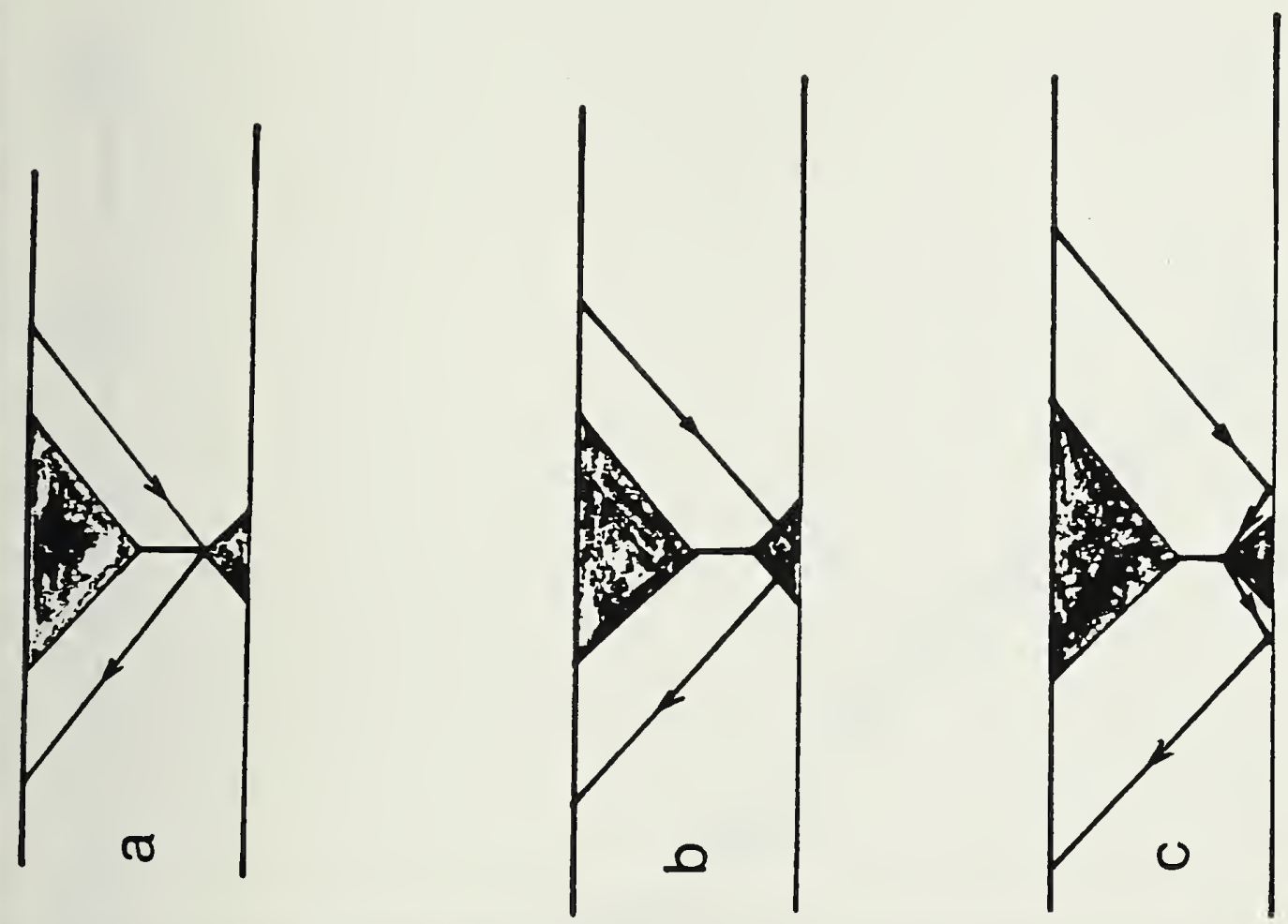

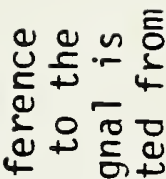

व

它ठ

응

웡

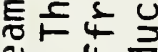

வ

+ $\dot{E}$

엉든 응

$=\infty+5$

5 으문 万人

- 145

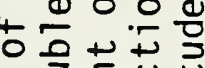

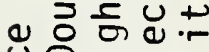
은 ᄂU一⿻上 U

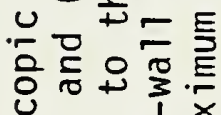

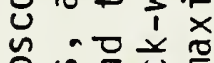

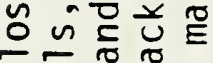
= -5 5

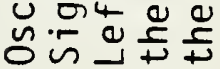

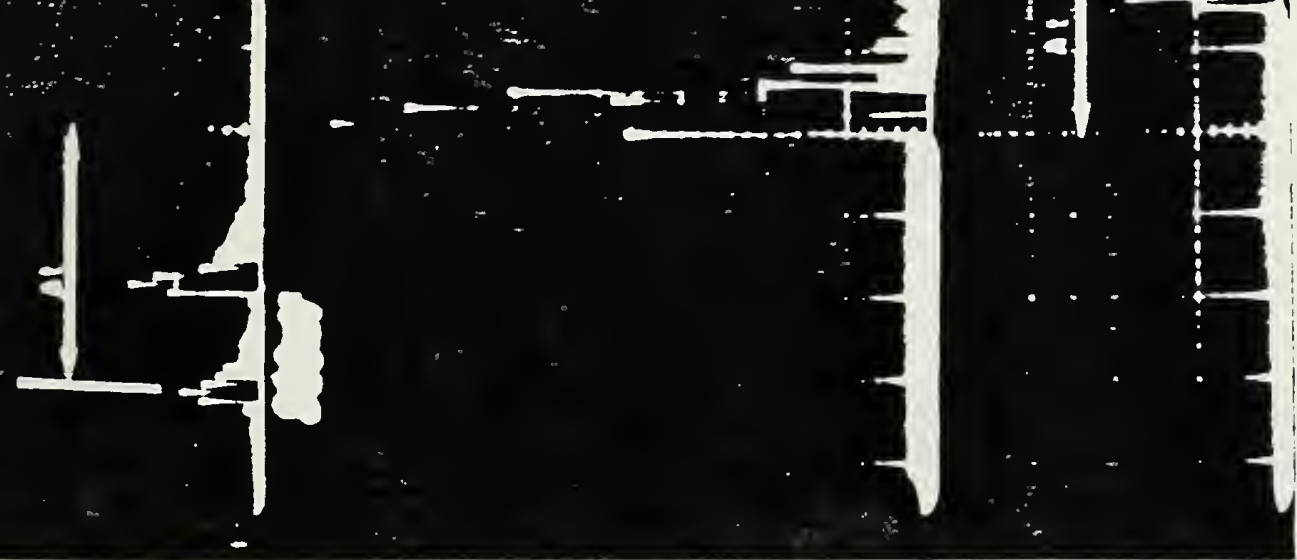

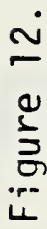





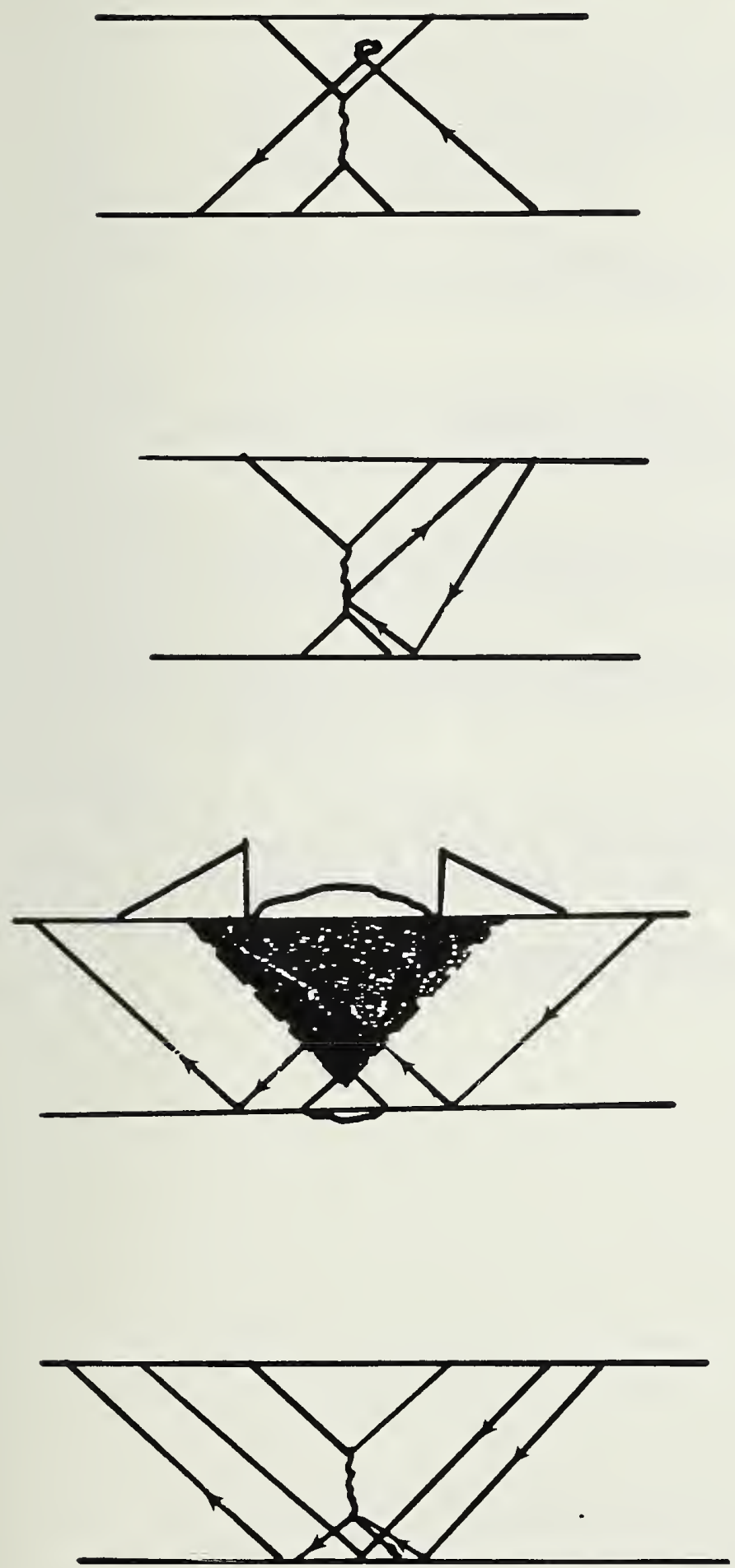

Poor resolution. The strong backward signal mask the weak diffracted signal.

Large dead zone because of weld crown when a direct beam is used.

Multiple reflections from crack surface when one transducer is used.

Noise from grains and

small imperfections. 

1. Golan, S., "Sizing of Cracks with Scattered Ultrasonic Waves," Proc. of First International Symposium on Uitrasonic Materials Characterization, National Bureau of Standards, Gaithersburg, MD, June 1978 (in press).

2. Silk, M. G. and Lidington, B. N., "Defect Sizing Using an U1trasonic Time Delay Approach," British J. NDT, 17, 33-36 (1975).

3. Silk, M. G., "Sizing Crack-like Defects by Ultrasonic Means," Chapter 2 in Research Techniques in Nondestructive Testing, 3 , R. S. Sharpe, Ed., Academic Press, New York (1977).

4. Golan, S., Baruch, J. and Yaroh, S., "Sizing of Cracks with Time Delay of Scattered UItrasonic Waves," Israel Institute of Metals - Technion, Internal Report 014-146 (1977).

5. Golan, S., Adler, L. and Cook, V., "Ultrasonic Diffraction Technique for Characterization of Fatigue Cracks," to be published.

6. Y. R. Birchack and C. G. Gardner, "Comparative U1trasonic Response of Machined Slots and Fatigue Cracks in 7075 Aluminum," Materials Evaluation, 34, 275-280 (1976).

7. Krautkramer, UItrasonic Testing of Materials, Second Edition, Springer-Verlag, 186-189 (Berlin 1969). 



\title{
APPENDIX E - Flaw Characterization
}

\author{
R. C. Placious
}

Progress, Aug 1, 1979 - Nov 30, 1979:

Radiographic activities for this period were directed primarily toward the evaluation of manual versus automatic processing of radiographs. The measurement standard for defect dimensioning was designed by, and is presently being fabricated at, NBS/Gaithersburg. The Rocky Flats group will use this device in connection with pipeline specimens from the weldments fabricated at $H$. C. Price Co. in Dewey, OK. With this arrangement, Rocky Flats will produce four film standards for defect dimensioning. Further details on this activity are given in the letter and the memorandum which are appended to this report as Attachments 1 and 2. Work on the assessment of defects in field radiographs will proceed following completion of the film standards.

Other radiographic work at NBS on existing weld specimens containing defects is continuing. One of the most serious problems in pipeline radiography is crack detectability. For example, a series of NBS radiographs showed thiat a crack as wide as 0.005 in. and having a depth of about one weld pass is not ordinarily detectable if the crack is approximately parallel to the sidewall chamfer. Such cracks can usually be detected only if the $x$-ray beam is properly oriented. How to best reveal these "hidden" cracks is undergoing continued study since this is regarded as a critically important part of this project. Multiple exposures represent one possible answer.

The report presenting guidelines for assessing field radiographs is still being assembled. There are many possible approaches toward developing a guide for evaluating existing radiographs and suggestions from OPSR regarding format would be very beneficial. NBS would rather not generate recommendations which are not useful or which are rapidly outdated. The intent is that recomnendations for useful image-quality indicators will make it possible for inexperienced individuals to interpret defect size with acceptable accuracy in the absence of full information regarding exposure and processing conditions.

Ultrasonic activities for this period were largely concerned with the low signal-to-noise ratio inherent in time-delay diffraction measurements. In order to optimize the experinental set-up with respect to shear-wave angle, a series of measurements were taken to characterize the forward and back scattering from cracks. The results were that 

optimum signal is obtained for $47^{\circ}$ shear-wave backscatter (pulse-echo). For forward scatter (pitch-catch, two-transducer technique) the optimum angles are $47^{\circ}$ and $57^{\circ}$ for scatter from the top and bottom of the crack, respectively. However, these amplitudes are well below that for backscatter.

Measurements were taken on intentionally-cracked weld specimens using the two-transducer technique with a tuned pulser/receiver, $5-\mathrm{MHz}$, $45^{\circ}$ shear-wave transducers, and a $50-\mathrm{MHz}$ oscilloscope. The results were mixed. Where measurements could be taken, the measurements correlated fairly well with actual size measurements, typically within a few tenths of a millimeter. However, in many instances the measurements could not be made due to excessive noise and spurious signals from grain boundaries. In some of the specimens the weld crown was removed prior to testing. This appeared to improve the ability to make measurements, but did not necessarily improve the accuracy of results. Some results are given in Table 1.

Some further experiments were performed on more field-oriented equipment using the pulse-echo (one-transducer) technique. A 5-MHz, duel-element transducer was used with a portable, broadband, pulser/ receiver with integral digital thickness readout. This system provides optimum spacial resolution, easily detecting discontinuities within 1 millimeter of specimen walls, and direct readout of depth of discontinuity. Measurements taken on simulated cracks (elox notches) agreed with actual values within about $0.2 \mathrm{~mm}$ on notches from 3 to $10 \mathrm{~mm}$ deep in specimens of carbon steel bar stock and X-65 pipe. These results are given in Table 2.

Plans, Dec 2, 1979-Jan. 31, 1980:

As soon as the radiographic film standards become available, the Rocky Flats group will be asked to evaluate representative defects, both artificial and natural. Both field and laboratory radiographs will be involved. An attempt will be made to also include a second organization in the evaluation.

Efforts to define better ways of revealing cracks will continue. In this connection, the primising developments in real-time radiography will be followed.

Uitrasonic measurements, using both the one-transducer and the twotransducer techniques, will be made on samples containing continuous lack-of-fusion (samples cut from pipe weld specimen 4-7482). These measurements will be correlated with actual defect sizes obtained by sectioning or from direct measurements made on the sides of the samples. 

Table 1 - Some Results of Crack S1zing Measurements in Weld Specimens Using the Two-Transducer Time-Delay Technique

Specimen Number

Coordinate Actual Location Measured by Ultrasonics (mm) Location

(mm)

$I$

II

III
IV Average

$1 \mathrm{~A}$

(Cracks normal to surface, weld crown on)

$\begin{array}{lcll}Y & 9.1 & N & N \\ X & 0.5 & & \\ Y & 9.4 & 9.5 & N \\ X & 0 & 0 & \\ Y & 20.2 & N & N \\ X & 0 & & \end{array}$

N

9.0 N

9. 0

N

1.0

1.0

$\mathrm{N}$

N N

9.5

N N

N

$20.0 \quad 20.0$

0.50 .5

1B

(Cracks normal, crown removed)
$Y$

$\mathrm{X}$

$Y$.

$\mathrm{X}$
9.4

0

20.1

0

\section{N}

10.5

$-1.0$

20.4

$-1.5$

0

9.5
-1.5
$N$

N

10.0

$\mathbb{N}$

$\begin{array}{cr} & -1.2 \\ 20.5 & 20.6 \\ 0 & -0.8\end{array}$

\begin{tabular}{|c|c|c|c|c|c|c|c|}
\hline $\begin{array}{l}3 A \\
\text { (Cracks } 45^{\circ} \text { to } \\
\text { surface, crown on) }\end{array}$ & $\begin{array}{l}Y \\
X \\
Y \\
X\end{array}$ & $\begin{array}{l}11.1 \\
0 \\
19.6 \\
-8.4\end{array}$ & $\mathbf{N}$ & $\begin{array}{l}N \\
19.4 \\
-8.5\end{array}$ & $\begin{array}{l}\mathbf{N} \\
\mathbf{N}\end{array}$ & $\begin{array}{l}11.0 \\
0 \\
20.0 \\
-8.0\end{array}$ & $\begin{array}{l}11.0 \\
0 \\
19.7 \\
-8.3\end{array}$ \\
\hline $\begin{array}{l}3 \mathrm{C} \\
\text { (Cracks } 45^{\circ}, \\
\text { crown removed) }\end{array}$ & $\begin{array}{l}Y \\
X \\
Y \\
X\end{array}$ & $\begin{array}{l}11.0 \\
0 \\
18.8 \\
8.4\end{array}$ & $\begin{array}{l}10.6 \\
0 \\
18.7 \\
8.0\end{array}$ & $\begin{array}{l}10.7 \\
1.5 \\
N\end{array}$ & $\begin{array}{l}11.5 \\
1.0 \\
N\end{array}$ & $\begin{array}{l}10.7 \\
0 \\
18.5 \\
8.0\end{array}$ & $\begin{array}{r}10.9 \\
0.6 \\
18.6 \\
8.0\end{array}$ \\
\hline
\end{tabular}

$Y$ depth below top surface

$X$ lateral position relative to arbitrary reference

$\mathrm{N}$ measurement not possible 

Table 2 - Results of Ultrasonic Heasurements of F.lox Notches in $X-65$ Pipe

Notch Actual Depth (mm) Measured Depth (mm)

I II

III

Aṿerage

A $\quad 9.9$

10.0

9.7

9.8

9.8

B $\quad 7.4$

8.0

7.3

7.2

7.5

C $\quad 5.3$

5.4

5.0

4.8

5.1

D $\quad 3.0$

2.8

3.2

3.1

3.0 

Mr. Wayne Stump

Mr. J.L. Summers

Rocky fiats Plant

Energy Systems Group

P.0. Box 464

Golden, CO 80401

Dear Wayne and Jack:

We have performad a series of tests with the radiographic exposures you made for us. I would like to suggest the following relative to your measurement standard. We now feel quite sure that 10 minute manual processing gives a film response nearly equal to automatic processing (Kodak $X$-0itat). We do not know - the reverse situation i.e., what temperature and time cycle in an automatic processor gives a response similar to 5 or 8 minute manual processing. Rather than take the time to do this I propose that you proceed with making up four film standards using a portion of one of the pipe sections you received from Kasen. Plan on making up these standards according to the following recipe:

Exposure $\stackrel{\mu}{\pi} \mathrm{T}, 220 \mathrm{kVCP}, 5 / \mathrm{AA} / 10$ - Automatic processing at recommended time/temperature cycle.

Exposure $\frac{\pi}{\pi} 2,220 \mathrm{kVCp}, 5 / \mathrm{AA} / 10$, Automatic processing at reduced time/temperature cycle

Exposure $\ddot{\pi}, 170 \mathrm{kVCP}, 5 / \mathrm{AA} / 10$, Autoriatic processing at reconvended time/temp. cycle.

Exposure $\# 4,170 \mathrm{kVCP}, 5 / \mathrm{AA} / 10$, Automatic processing at reduced time/temp. cycle.

This will yield four standards for defect assessment and hopefully provide us with information on contrast change due to kilovoltage and/or processing variations. With the available budget constraints this is probably about all we can hope to accomplish.

The concept of reduced automatic processing is an attempt to sinulate the film response for manual processing at its recominended time and temperature. One might achieve the reduced temperature in the automatic processor (say e.g. $70^{\circ} \mathrm{F}$ ) by disconnecting the heater (or pulling the wall plug) at the close of business one day and processing the next day before the temperature has risen. John Callinan (Kodak) says the $X$-0ilat will run $O K$ at $74^{\circ} \mathrm{F}$ but $70^{\circ} \mathrm{F}$ might be closer to the latitude achieved in manual processing.

Attachment 1 

The necessary filn density needed in the 4 film standards is about 2.2 or 2.3 in the lightest area of the image. That is - the density at point $A$ in my sketch (attached) of the proposed step wedge to be used. I will now discuss this step wedge.

Nine pieces of .010" shim stock, cut as indicated and containing a series of holes on one side should be placed on a piece of the pipe from Kasen. This will give us .090"of added material which should then approximate the average thickness of the weld including the crown. The contrast produced by the 9 steps and the 9 hole images are what you will use to develop your film standard. Note that these contrast changes will be going in the same direction as the image of a real defect in the weld region.

If this all sounds complicated - or if you see a serious problell with the procedure - could we plan on disclissing it at the St. Louis meeting? I will be there from Monday noon unt il liednesday late afternoon. I am looking forward to discussing this with you. If you prefer, I could supply the shim stock step wedge for you.

Sincerely,

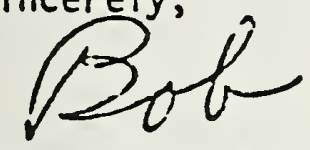

Robert C. Placious, Physicist Radiation Physics Division Center for Radiation Research

Enclosure

Stated

cc:

L. Mordfin

M. B. Kasen

D. Polansky 


October 24, 1979

MEMORANDUP FOR Dr. M. B. Kasen $\times 3.58$

Cryogenics Division

NBS, Boulder

From: Or. R. C. Placious

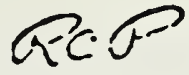

Radiation Physics Div. NBS, Washington, D. C.

Subject: Pipeline Meeting at Boulder, $\mathrm{CC}$ on Oct.ober 25, 1979

Flaw Assessment (Primarily geared to old program)

1. Rocky Flats is making up 4 film standards to evaluate our field audiographs (made at HC Price and CO. by Cleveland X-Ray). Details of Film Standards.
(a) $170 \mathrm{kV}$, Automatic Processing at Standard Cycle
(b) $220 \mathrm{kV}$, Automatic Processing at Standard Cycle
(c) $170 \mathrm{kV}$, Automatic Processing at Reduced Teriperature
(d) $220 \mathrm{kV}$, Automatic Processing at Reduced Temperature

These standards will provide data on variatiors in depth assessment due to kilovoltage and autonatic versus manual processing. (c) and (d) are

- designed to provide the kind of latitude obtainable in manual processing. Note: Kasen has copy of letter to Wayne Stump with details of above.

2. NBS, Washington, D.C. is evaluating effect of film type on defect assessment. This will be done by use of a scanning microdensitometer. The completion of (1) and (2) vill provide us with much of the information needed to (a) Provide DOT wi th the information needed to evaluate a waiver request; (b) Permit NBS to make recommendations to DOT on improving field radiographic prosedures; and $(c)$ Place limits on accuracy obtainable using film contrast to assess flaw depth.

3. Other Activities.

(a) We are trying to determine probabilities of crack detection by radiography and ultrasonics.

(b) We are cooperating with the ultrasonics group at NGS-Wash. on the a) ternatives to radiography.

4. He have Dan Polansky at NBS now. He brings long experience in radiographic NDT with him. He is presently very active in high energy real time radiography and is expected to provide valuable help in assessing the capabilities of this technology in pipeline radiography in the field. He is also Chairman of ASTII E-7 which has responsibilities for all consensus - tandards in IIDT.

Attachment 2 



\section{FLAW CHARACTERIZATION}

Subtask 1: Radiography

Principal Investigator: Dr. Robert C. Placious, (301) 921-2201

Progress: This progress report addresses the suggested procedures for evaluating waiver requests on the basis of present state-of-the-art field radiography. We propose a scope for the radiographic record that should accompany the waiver application. The evaluation of dimensional inaccuracies that arise, as a result of the radiographic techniques used, can only be made if this information is available. A radiographic record could well take the form shown in Figures 1 and 2. The detailed information for evaluating flaws defined by field radiography and stated by the record will certainly include graphs of the type shown in Figure 3. (Note that the figures are of a preliminary nature and thus, in the interest of expediency, it was not felt that they needed to be prepared professionally.) Improved radiographic procedures woula inclide specifications such as:

a. Shims, if used, shall include actual thickness identification numbers (as penetrameters do).

b. Portable $x$-ray sources shall be referenced to a prototype unit which has had its output calibrated ( $k \mathrm{~V}, \mathrm{~mA}$, time).

c. Elastic belts shajl be required to maintain weld/film contact etc. 

[TOBE SUBAITTED WUTH ANY WRIVER REQUEST)

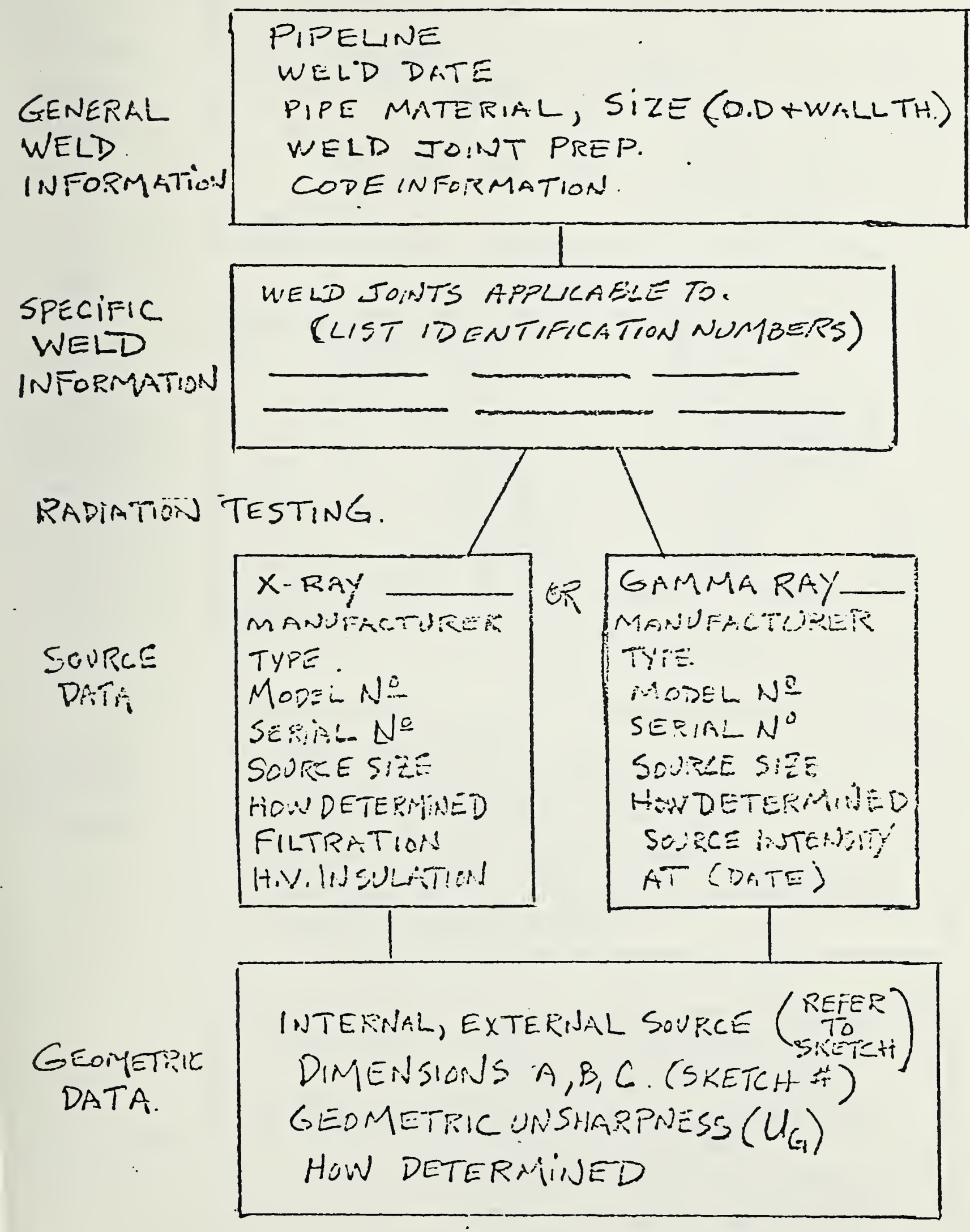

309

FlG I 



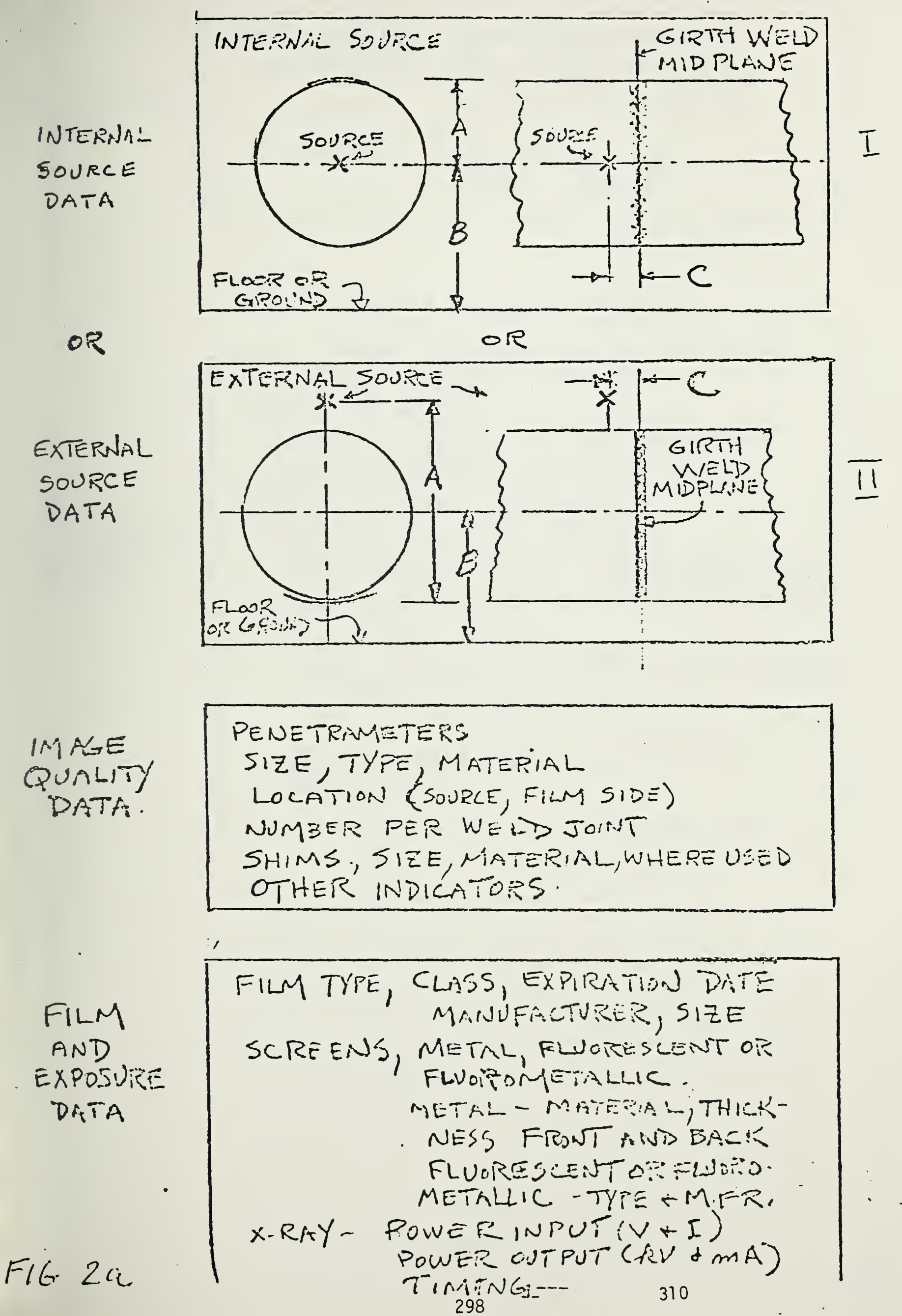





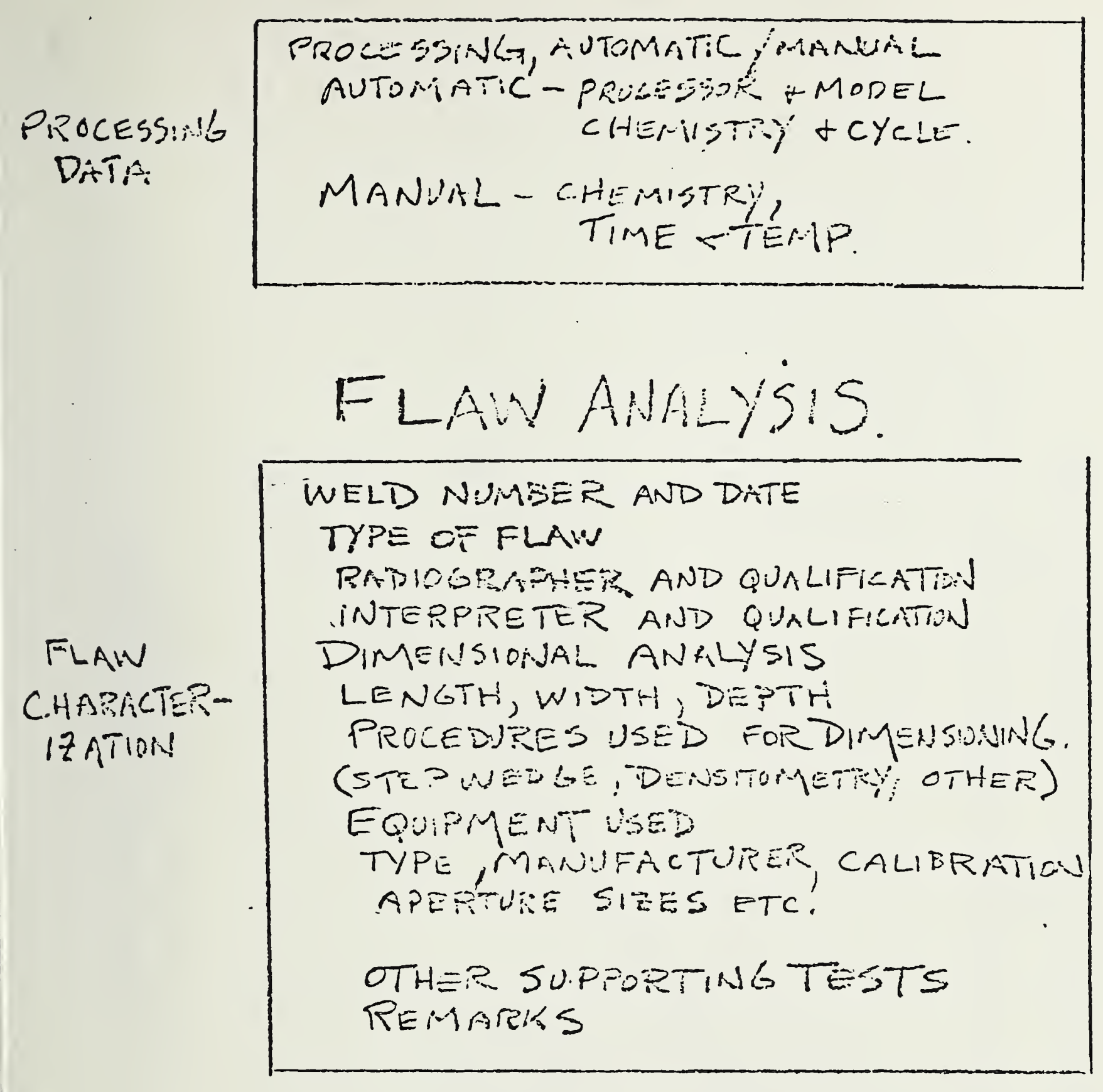

311

$F 16,2 b$ 



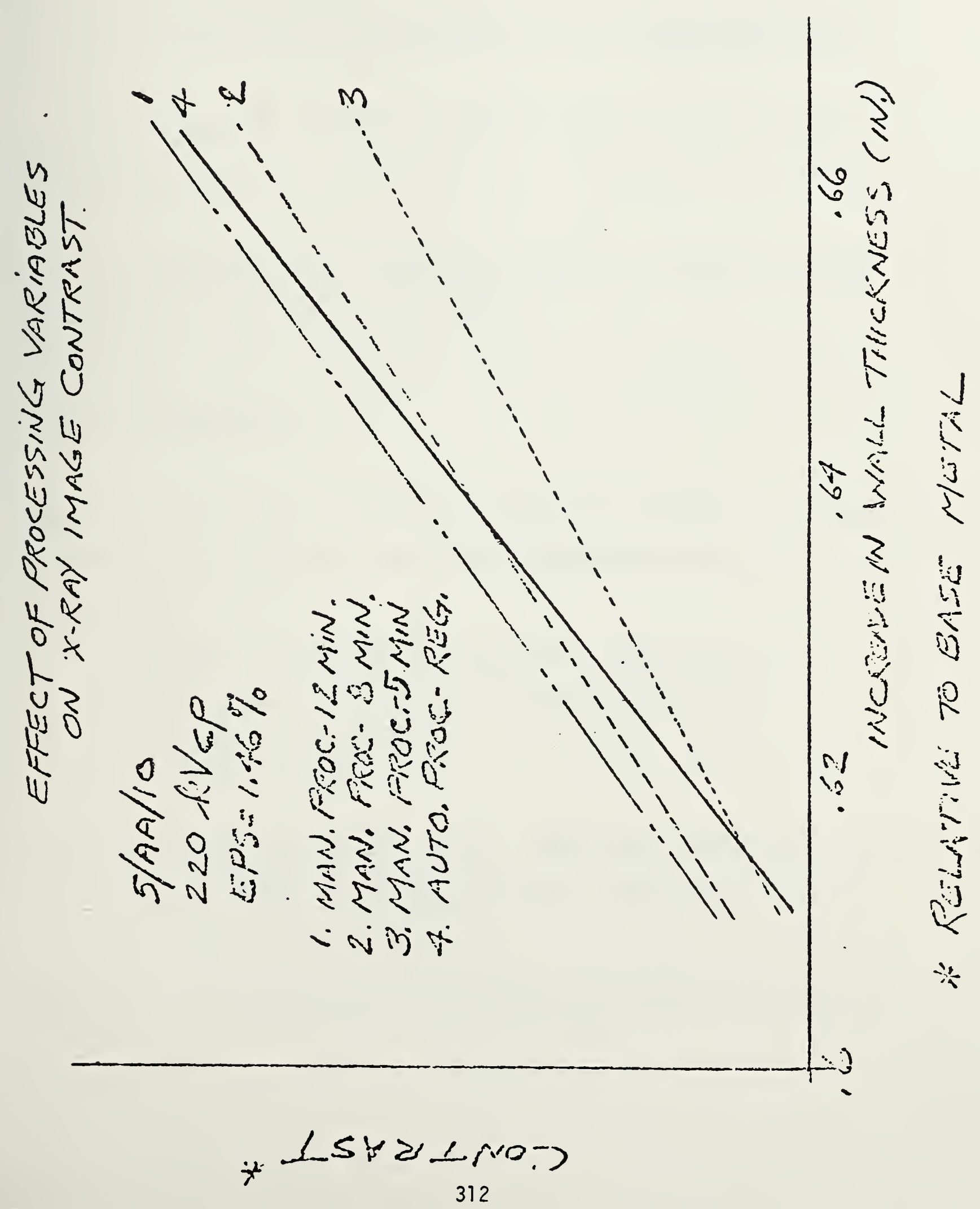



Plans: Plans call for a continuation of present efforts to acquire data which relate to probable inaccuracies in flaw analysis due to $x$-ray variables, processing variables, and operator skill and training. These efforts seek to further define:

a. The contents and the completeness of the radiographic record.

b. The detailed information needed to evaluate the data on the radiographic record.

c. The changes needed in field techniques or procedure to improve image quality and, therefore, flaw analysis.

Subtask 2A: U1trasonics

Progress: The following interagency report has been issued, which summarizes the NBS work on the ultrasonic time-delay diffraction technique for sizing defects:

S. Golan, "Defect Characterization and Dimensioning of Cracks in Welds by the U1trasonic Diffraction Method," NBSIR 80-1983, March 1980.

A copy of this report is appended hereto. Additional copies may be ordered from the Natiunal Technical Information Service, Springfield, VA 22161.

Plans: There are no plans for continuing this work at the present time. 



\section{FLAW CHARACTERIZATION}

Subtask 1: Radiography

Principal Investigator: Dr. Robert C. Placious, (301) 921-2201

Progress: Figures $1,2 a$ and $2 b$ of the last progress report presented $a$ tentative listing of the essential elements needed to evaluate a pipeline weld flaw detected on a field radiograph. Figure 3 of that report indicated how the evaluation would be carried out in cases where documentation of the radiographic procedure was unavailable or only sketchily available.

Efforts during the present reporting period continued to examine the influences of various $x$-ray parameters on radiographic contrast. The last progress report provided data on the variation of contrast with metal thickness, with processing conditions as a parameter. During the present reporting period the effects of kilovoltage changes on contrast were examined. See the figure on the next page. A number of graphs of this kind will be needed to perform a proper evaluation of flaw depth. Dot has now been provided with two such graphs.

Plans: Efforts to characterize the influences of various $x$-ray parameters on radiographic contrast will continue, using data from NBS studies and from external sources. Problems relating to $x$-ray film type variability, and to kilovoltage versus manual processing variability, are among those remaining to be addressed. 



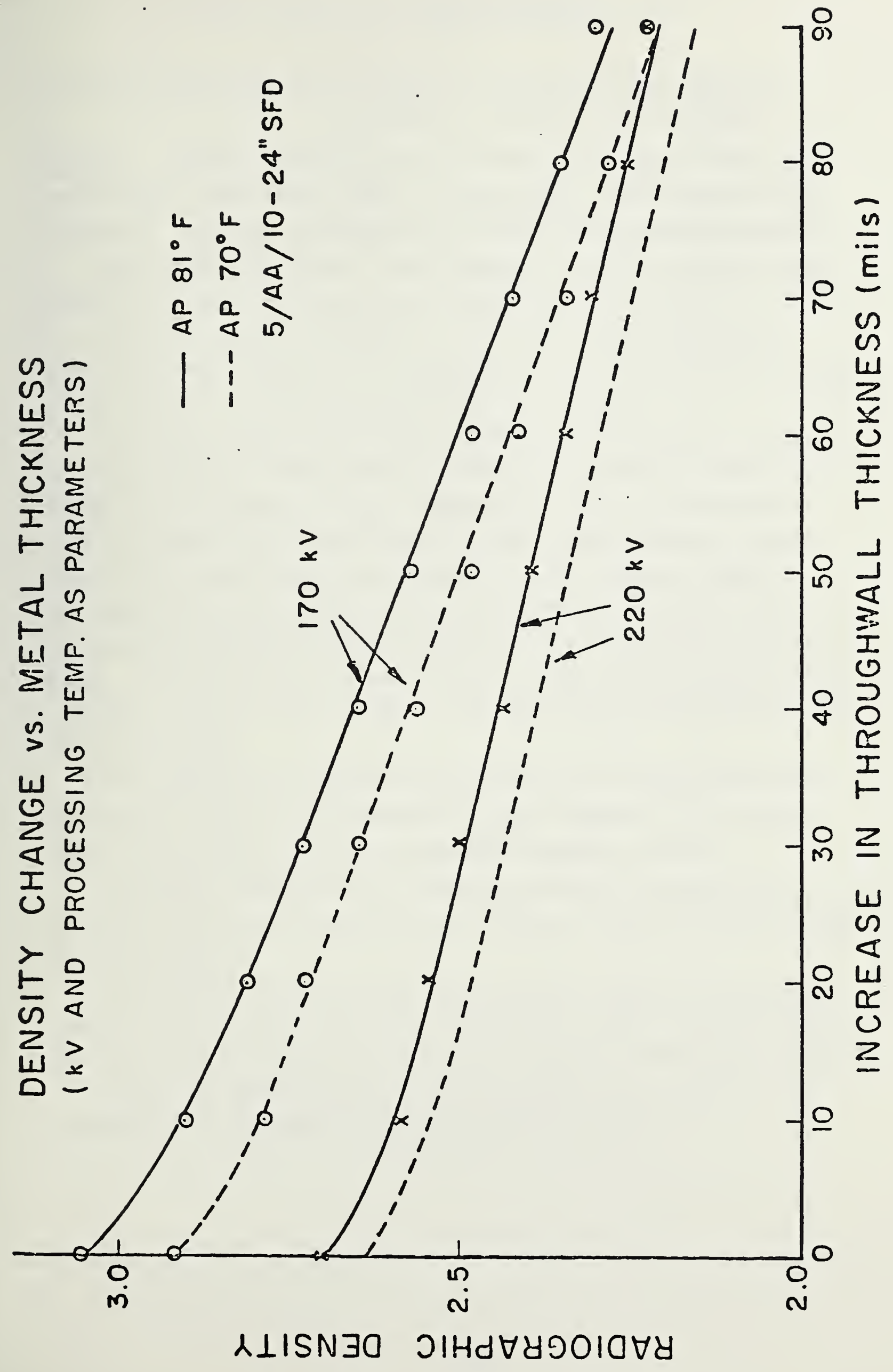



Principal Investigators: Dr. Robert C. Placious, (301) 921-2201

Mr. Daniel J. Chwirut, (301) 921-3646

Progress: The NBS interaction with the Rocky Flats group (RF), on radiographic dimensioning flaws, is continuing. (The tests are described in the letter furnished on PP. 28-30 of the Seventh Report).

Radiographs were made of metallographic samples containing high porosity and slag from the pipeline section welded at the H. C. Price Co. (See, for example, Figure 1.) It is anticipated that threedimensional radiography will be required in order to obtain an accurate analysis of the volume of the flaws in these damples.

An in-house assessment of the ultrasonics effort on this project has led to the conclusion that the time-delay diffraction technique holds the most promise for this application. Most of the NBS work on this technique has been carried out with in-house funds al though some of the results have been included in these progress reports. An interagency report summarizing the NBS effort was prepared and - is presently undergoing detailed technical review prior to publication. Plans: The RF phase of the radiographic effort will be completed and, upon NBS approval of the exposures, RF will make up film standards for flaw measurements.

The tomographic information obtained from the three-dimensional radiographic work will be provided to other memebers of the project team to facilitate more precise fracture mechanics analysis.

Ultrasonic examinations of samples containing continuous lack of fusion (which were deferred due to delays in procuring angle wedges) will be started.

An effort will be initiated to assess the effects of weld-bead removal, and the relative merits of single vs. double skip techniques, for flaw depth sizing by the ultrasonic time-delay diffraction approach.

Copies of the NBS report on the time-delay technique will be furnished as attachments to the next progress report. 


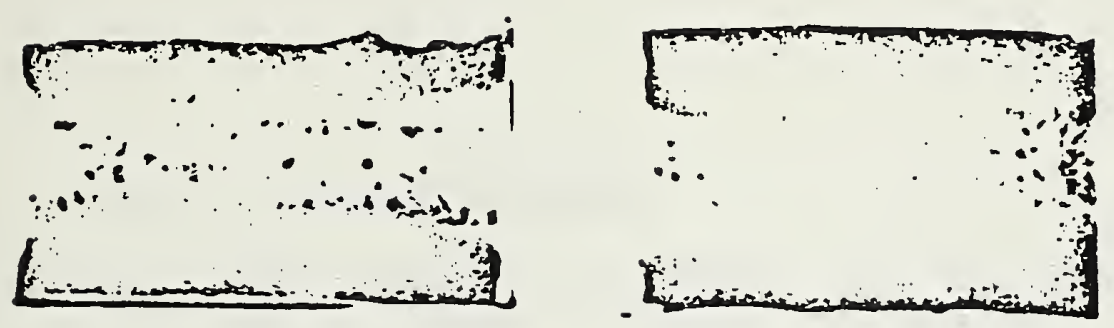

High

Contrast

a Film
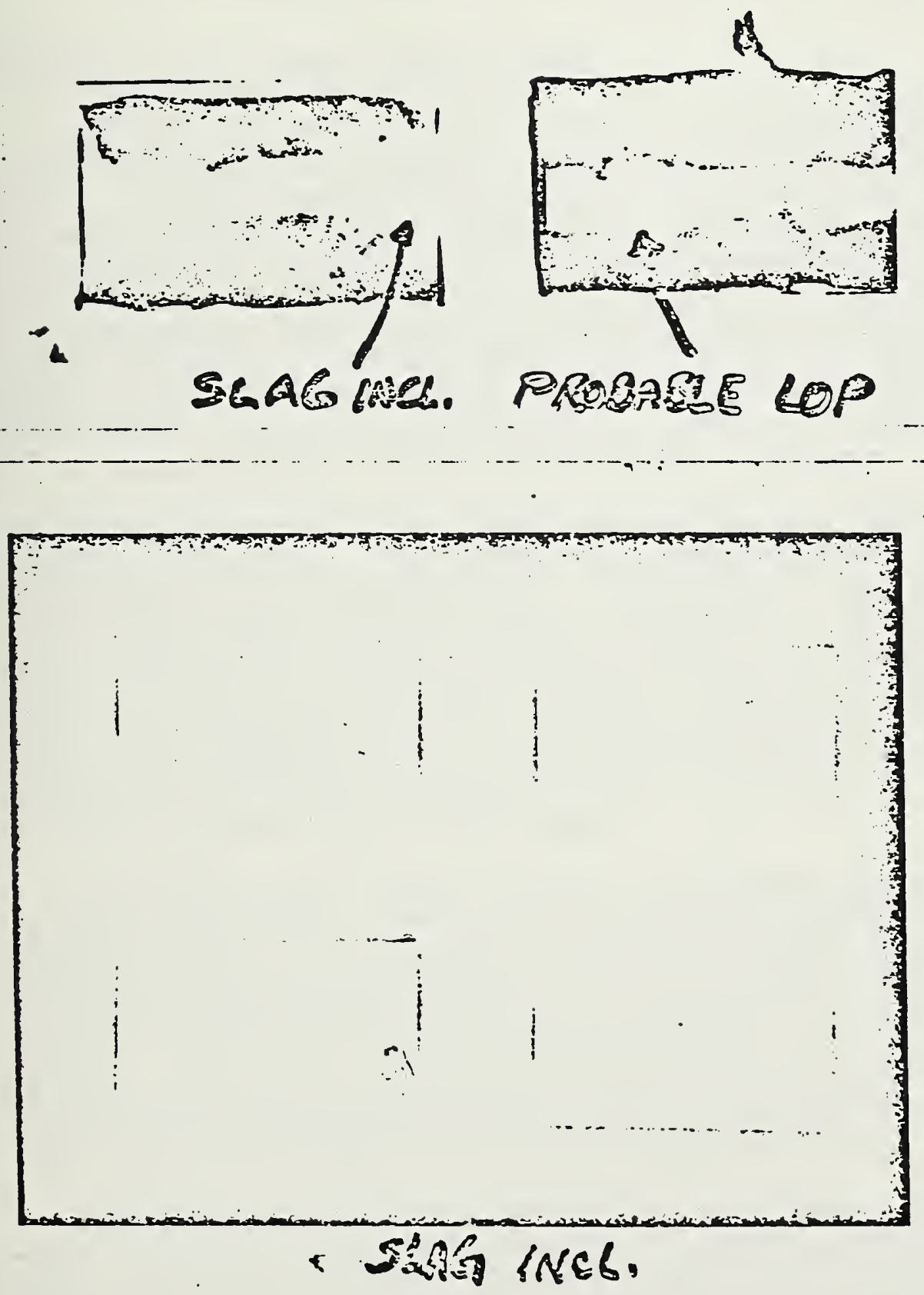

Horma 7

Contrast

Film

Fig.1. Polaroid Pictures of Radiographs of Metallographic Samples. Quantitative Determination of Degree of Porosity May Require Special Radiographic Procedure ( 3 Dimensional Radiography) 



\section{FLAW CHARACTERIZATION}

(Principal investigators: Dr. Robert C. Placious, 301-921-2201, and Mr. Daniel J. Chwirut, 301-921-3646)

Progress, Aug 1, 1979 - Nov 30, 1979:

Radiographic activities for this period were directed primarily toward the evaluation of manual versus automatic processing of radiographs. The measurement standard for defect dimensioning was designed by, and is presently being fabricated at, NBS/Gaithersburg. The Rocky Flats group will use this device in connection with pipeline specimens from the weldments fabricated at $\mathrm{H}$. C. Price Co. in Dewey, OK. With this arrangement, Rocky Flats will produce four film standards for defect dimensioning. Further details on this activity are given in the letter and the memorandum which are appended to this report as Attachments 1 and 2. Work on the assessment of defects in field radiographs will proceed following completion of the film standards.

Other radiographic work at NBS on existing weld specimens containing defects is continuing. One of the most serious problems in pipeline radiography is crack detectability. For example, a series of NBS radiographs showed that a crack as wide as 0.005 in. and having a depth of about one weld pass is not ordinarily detectable if the crack is approximately parallel to the sidewall chamfer. Such cracks can usually be detected only if the $x$-ray beam is properly oriented. How to best reveal these "hidden" cracks is undergoing continued study since this is regarded as a critically important part of this project. Multiple exposures represent one possible answer.

The report presenting guidelines for assessing field radiographs is still being assernbled. There are many possible approaches toward developing a guide for evaluating existing radiographs and suggestions from OPSR regarding format would be very beneficial. NBS would rather not generate recommendations which are not useful or which are rapidly outdated. The intent is that recommendations for useful image-quality indicators will make it possible for inexperienced individuals to interpret defect size with acceptable accuracy in the absence of full information regarding exposure and processing conditions.

Ultrasonic activities for this period were largely concerned with the low signal-to-noise ratio inherent in time-delay diffraction measurements. In order to optimize the experinental set-up with respect to shear-wave angle, a series of measurements were taken to characterize the forward and back scattering from cracks. The results were that 

optimum signal is obtained for $47^{\circ}$ shear-wave backscatter (pulse-echo). For forward scatter (pitch-catch, two-transducer technique) the optimum angles are $47^{\circ}$ and $57^{\circ}$ for scatter from the top and bottom of the crack, respectively. However, these amplitudes are well below that for backscatter.

Measurements were taken on intentionally-cracked weld specimens using the two-transducer technique with a tuned pulser/receiver, $5-\mathrm{MHz}$, $45^{\circ}$ shear-wave transducers, and a 50-MHz oscilloscope. The results were mixed. Where measurements could be taken, the measurements correlated fairly well with actual size measurements, typically within a few tenths of a millimeter. However, in many instances the measurements could not be made due to excessive noise and spurious signals from grain boundaries. In some of the specimens the weld crown was removed prior to testing. This appeared to improve the ability to make measurements, but did not necessarily improve the accuracy of results. Some results are given in Table 1.

Some further experiments were performed on more field-oriented equipment using the pulse-echo (one-transducer) technique. A $5-\mathrm{MHz}$, duel-element transducer was used with a portable, broadband, pulser/ receiver with integral digital thickness readout. This system provides optimum spacial resolution, easily detecting discontinuities within 1 millimeter of specimen walls, and direct readout of depth of discontinuity. Measurements taken on simulated cracks (elox notches) agreed with actual values within about $0.2 \mathrm{~mm}$ on notches from 3 to $10 \mathrm{~mm}$ deep in specimens of carbon stee 1 bar stock and $X-65$ pipe. These results are given in Table 2.

Plans, Dec, 1, 1979-Jan. 31, 198ח:

As soon as the radiographic film standards become available, the Rocky Flats group will be asked to evaluate representative defects, both artificial and natural. Both field and laboratory radiographs will be involved. An attempt will be made to also include a second organization in the evaluation.

Efforts to define better ways of revealing cracks will continue. In this connection, the primising developments in real-time radiography will be followed.

Ultrasonic measurements, using both the one-transducer and the twotransducer techniques, will be made on samples containing continuous lack-of-fusion (samples cut from pipe weld specimen 4-7482). These measurements will be correlated with actual defect sizes obtained by sectioning or from direct measurements made on the sides of the samples. 

Table 1 - Some Results of Crack Sizing Measurements in Weld specimens Using the Two-Transducer Time-Delay Technique

Spec1men Number

Coordinate Actual Location Measured by Ultrasonics ( $\mathrm{mm}$ ) Location

(mm)

$\mathbf{I}$

II

III

IV Average

$1 \mathrm{~A}$

(Cracks normal

to surface, weld crown on)
9.1 1

0.5

9.4

0

20.2

0
N

$9.5 \quad N$

0

N

N
9.0

1.0

N N

1.0

9.5

N

20.020 .0

$0.5 \quad 0.5$

$1 B$

(Cracks normal,

crown removed)

$\begin{array}{lcccccc}\mathrm{Y} & 9.4 & \mathrm{~N} & 10.5 & 9.5 & \mathrm{~N} & 10.0 \\ \mathrm{X} & 0 & & -1.0 & -1.5 & & -1.2 \\ \mathrm{Y} & 20.1 & 20.8 & 20.4 & \mathrm{~N} & 20.5 & 20.6 \\ \mathrm{X} & 0 & -1.5 & 0 & & 0 & -0.8\end{array}$

$\begin{array}{lrrrrrr}Y & 11.0 & 10.6 & 10.7 & 11.5 & 10.7 & 10.9 \\ X & 0 & 0 & 1.5 & 1.0 & 0 & 0.6 \\ Y & 18.8 & 18.7 & N & N & 18.5 & 18.6 \\ X & 8.4 & 8.0 & & & 8.0 & 8.0\end{array}$

\section{$3 \mathrm{~A}$}

(Cracks $45^{\circ}$ to surface, crown on)

$\mathrm{Y}$

$Y$

$\mathrm{X}$

$\mathrm{X}$

$$
11.1
$$

0

19.6

$-8.4$
N

$\begin{array}{cc}11.0 & 11.0 \\ 0 & 0\end{array}$

\section{$3 C$}

(Cracks $45^{\circ}$,

crown removed)

$\longrightarrow$

$\begin{array}{lccccrr}\mathrm{Y} & 11.0 & 10.6 & 10.7 & 11.5 & 10.7 & 10.9 \\ \mathrm{X} & 0 & 0 & 1.5 & 1.0 & 0 & 0.6 \\ \mathrm{Y} & 18.8 & 18.7 & \mathrm{~N} & \mathrm{~N} & 18.5 & 18.6 \\ \mathrm{X} & 8.4 & 8.0 & & & 8.0 & 8.0\end{array}$

7 depth below top surface

$X$ lateral position relative to arbitrary reference

$\mathrm{N}$ measurement not possible 

- Table 2 - Results of Ultrasonic lleasurements of F.lox Notches in $\mathrm{X}-65$ Pipe

Notch Actual Depth (mm)

$\begin{array}{llllll} & & \text { I } & \text { II } & \text { III } & \text { Average } \\ \text { A } & 9.9 & 10.0 & 9.7 & 9.8 & 9.8 \\ \text { B } & 7.4 & 8.0 & 7.3 & 7.2 & 7.5 \\ \text { C } & 5.3 & 5.4 & 5.0 & 4.8 & 5.1 \\ \text { D } & 3.0 & 2.8 & 3.2 & 3.1 & 3.0\end{array}$



Mr. Wayne Stump

Mr. J.L. Summers

Rocky Flats Plant

Energy Systems Group

P.0. Box 464

Golden, CO 80401

Dear Wayne and Jack:

We have performed a series of tests with the radiographic exposures you made for us. I would like to suggest the following relative to your measurement standard. He now feel quite sure that 10 minute manual processing gives a film response nearly equal to automatic processing (Kodak $X$-0ilat). He do not know - the reverse situation i.e., what temperature and time cycle in an automatic processor gives a response similar to 5 or 8 minute manual processing. Rether than take the time to do this I propose that you proceed with making up four film standards using a portion of one of the pipe sections you received from kasen. Plan on making up these standards according to the following recipe:

Exposure $\stackrel{\#, 2}{\pi}, 20 \mathrm{kVCp}, 5 / \mathrm{AA} / 10$ - Automatic processing at recommended time/temperature cycle.

Exposure $\pi 2,220 \mathrm{kVCp}, 5 / \mathrm{AA} / 10$, Automatic processing at reduced time/temperature cycle

Exposure $\div 3,170 \mathrm{kVCP}, 5 / \mathrm{AA} / 10$, Automatic processing at reconinended time/temp. cycle.

Exposure $\pi 4,170 \mathrm{kVCp}, 5 / \mathrm{AA} / 10$, Automatic processing at reduced time/temp. cycle.

This will yield four standards for defect assessment and hopefully provide us with information on contrast change due to kilovoltage and/or processing variations. With the available budget constraints this is probably about all we can hope to accomplish.

The concept of reduced automatic processing is an attenipt to simulate the film response for manual processing at its reconinended time and temperature. One might achieve the reduced temperature in the automatic processor (say e.g. $70^{\circ} \mathrm{F}$ ) by disconnecting the heater (or pulling the wall plug) at the close of business one day and processing the next day before the temperature has risen. John Callinan (iojak) says the $X-00^{\circ}$ at will run $O K$ at $74^{\circ} \mathrm{F}$ but $70^{\circ} \mathrm{F}$ might be closer to the latitude achieved in manual processing. 

The necessary filn density needed in the 4 film standards is about 2.2 or 2.3 in the lightest area of the inage. That is - the density at point $A$ in my sketch (attached) of the proposed step wedge to be used. I will now discuss this step wedge.

Nine pieces of .010" shim stock, cut as indicated and containing a series of holes on one side should be placed on a picce of the pipe from Kasen. This will give us .090"of added material which should then approximate the average thickness of the weld including the crown. The contrast produced by the 9 steps and the 9 hole images are what you will use to develop your film standard. Note that these contrast changes will be going in the same direction as the image of a real defect in the weld region.

If this all sounds complicated - or if you see a serious problem with the procedure - could.we plan on disclissing it at the St. Louis meeting? I will be there from Monday noon until llednesday late afternoon. I am looking forward to discussing this with you. If you prefer, I could supply the shim stock step wedge for you.

Sincerely,

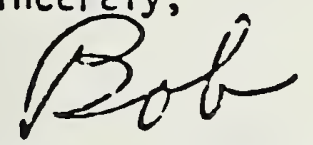

Robert C. Placious, Physicist

Radiation Physics Division

Center for Radiation Research

Enclosure

Stated

cc:
L. Mordfin
M. B. Kasen
D. Polansky 


October 24, 1979

MERORANDUPi FOR Dr. M. B. Kasen $\times 3.5 .58$

Cryogenics Division

NBS, Boulder

From: Dr. R. C. Placious

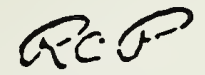

Radiation Physics Div.

NBS, Washington, D. C.

Subject: Pipeline Meeting at Boulder, co on October 25, 1979

Flaw Assessment (Primarily geared to old program)

1. Rocky flats is making up 4 film standards to evaluate our field audiographs (made at HC Price and Co. by Cleveland X-Ray).

Details of Film Standards.

(a) $170 \mathrm{kV}$, Automatic Processing at Standard Cycle

(b) $220 \mathrm{kV}$, Automatic Processing at Standard Cycle

(c) $170 \mathrm{kV}$, Automatic Processing at Reciuced Teriperature

(d) $220 \mathrm{kV}$, Automatic Processing at Reduced Teniferature

These standards will provide data on variatiors in depth assessment due to kilovoltage and automatic versus manual processing. (c) and (d) are

- designed to provide the kind of latitule obtainable in manual processing. Note: Kasen has copy of letter to Wayne Sturip with details of above.

2. NBS, Washington, D.C. is evaluating effect of film type on defect assessment. This will be done by use of a scanning microdensitometer. The completion of (1) and (2) 1111 provide us with much of the information needed to (a) Provide DoT wi th the information needed to evaluate a vaiver request; (b) Permit IIBS to make recommendations to DOT on improving field radiographic procedures; and (c) Place limits on accuracy obtainabie using film contrast to assess flaw depth.

3. Other Activities.
(a) He are trying to determine probabilities of crack detection by radiography and ultrasonics.
(b) We are cocperating with the ultrasonics group at NBS-Wash. on the alternatives to radiography.

4. We have Dan Polansky at NBS no:t. He brings long experience in radiographic NDT with him. He is presently very active in high energy real time radiography and is expected to provide valuable help in assessirg the capabilities of this technoloyy in pipeline radiography in the ficld. lle is also Chairnan of ASTil E-7 which has responsibilities for all consensus - tandards in IIDT. 

APPEDNIX F - Ultrasonic Examination of Girth Welds in 40-Inch, X-65 Line Pipe Specimens

W. C. Minton 


TIP Report No. 1

October 2, 1978

Page 2

\subsection{SUMMARY}

All or portions of five girth welds in specimens of 40-inch, $X-65$ line plpe were examined ultrasonically by manual techniques. Numerous ultrasonic reflectors were recorded. The recorded information from the examinations was plotted and attempts made to separate geometric reflectors, such as those originating at the inside reinforcement or root of the weld, the external. reinforcement or crown of the weld, high-low, and other nondetrimental conditions from reflectors present in the weld due to flaws which must be evaluated in accordance with API Standard 1104 for Welding Pipelines and Related Facilities. The attempts were only partly successful with standard, wide-beam ultrasonic examination techniques.

The examinations were first performed in the as-welded condition. Following this, a selected portion of one weld was ground flush, first on the inner surface and then on both surfaces, in order to record the effect of removal of these geonetric reflecting conditions. The results clearly indicated the interpretation difficulties encountered in attempting to dis-. criminate between geometric and detrimental reflectors in the as-welded condition.

Recommendations are made for improvements in resolution so that geometric reflectors may be distinguished from detrimental flaws.

A Search Unit Tracking and Recording System (SUTARS) semi-mechanized ultrasonic examination was perforped on three of the test welds. A reduction in time required to examine a weld was obtained. A preliminary conputer evaluation of a portion of the SUTARS data verified the results of the allmanual ultrasonic examinations.

\subsection{DESCRIPTION OF SPECIMENS}

The specimens examined were numbered 4 through 8 and consisted of sections of 40-inch outside diameter (OD), X-65 line pipe (average wall thickness $0.65 \mathrm{inch}$ ). Each of the specimens was at least 2 feet in length on elther side of the girth weld, which joined the sections approximately at the center. Some of the specimens had been welded manually; others had been welded by automatic methods. Both automatic and manual weids were examined by the same ultrasonic procedure.

\subsection{EQUIPMENT AND PROCEDURES}

3.1 Manual Ultrasonic Examination

A manual ultrasonic examination was performed in accordance with 

Trip Report No. 1

October 2, 1978

Page 3

Southwest Research Institute (SwRI) Procedure SwRI-NDT-800-58, Revision 0, - copy of which is attached in Append1x A.

3.1.1 The ultrasonic instruments used were conventional pulse-echo flaw detectors, Sonic Instruments type FTS Mark I. These Instruments can be operated either from self-contained batteries or from alternating current line.

3.1.2 The search units consisted of the following:

(a) Straight-beam: 3/8-inch diameter, $5.0 \mathrm{MHz}$;

(b) Angle beam: 3/8-inch diameter, $2.25 \mathrm{MHz}$.

These were used with plastic, low-noise angle beam wedges to produce sound beam angles in steel of $45^{\circ} \pm 2^{\circ}$ or $60^{\circ} \pm 2^{\circ}$.

3.1.3 Reference blocks used for screen distance calibration were half-round mild steel test blocks or IIW test blocks.

3.1.4 The basic calibration block used to adjust equipment for all examinations was .604 inch thick mild steel and contained side-drilled hole reflectors as shown in Figure 1.

3.1.5 Couplant material was Sonogel II, a commercial product.

3.1.6 The welded specimens were supported on wooden cradles about one foot off the floor, a position similar to that which would be found along a pipeline right-of-way prior to burial of a welded line.

\subsection{Semi-Mechanized Search Unit Tracking and Recording System}

This consisted of the SwRI-developed equipment shown schematically in Figure 2. The function of the SUTAR System is to replace the laborious manual recording of each datum point produced during the ultrasonic scanning of welds. The equipment consists of a conventional ultrasonic flaw detector modified so that selected information fron the examination can be recorded in a format suitable for computer evaluation. In addition, the coordinates of the search unit positions during the scanning of the weld are continuously recorded in a similar format.

\subsection{MANTAL EXANINATIONS}

\subsection{Lamination Scans}

A preliminary examination of the naterjal adjacent to the weld was performed using straight-beam $\left(0^{\circ}\right) 5.0 \mathrm{MHz}$ examination frequency. The purpose of this examination was to detect and record any laminar conditions 

Trip Report No. 1 October 2, 1978

Page 4

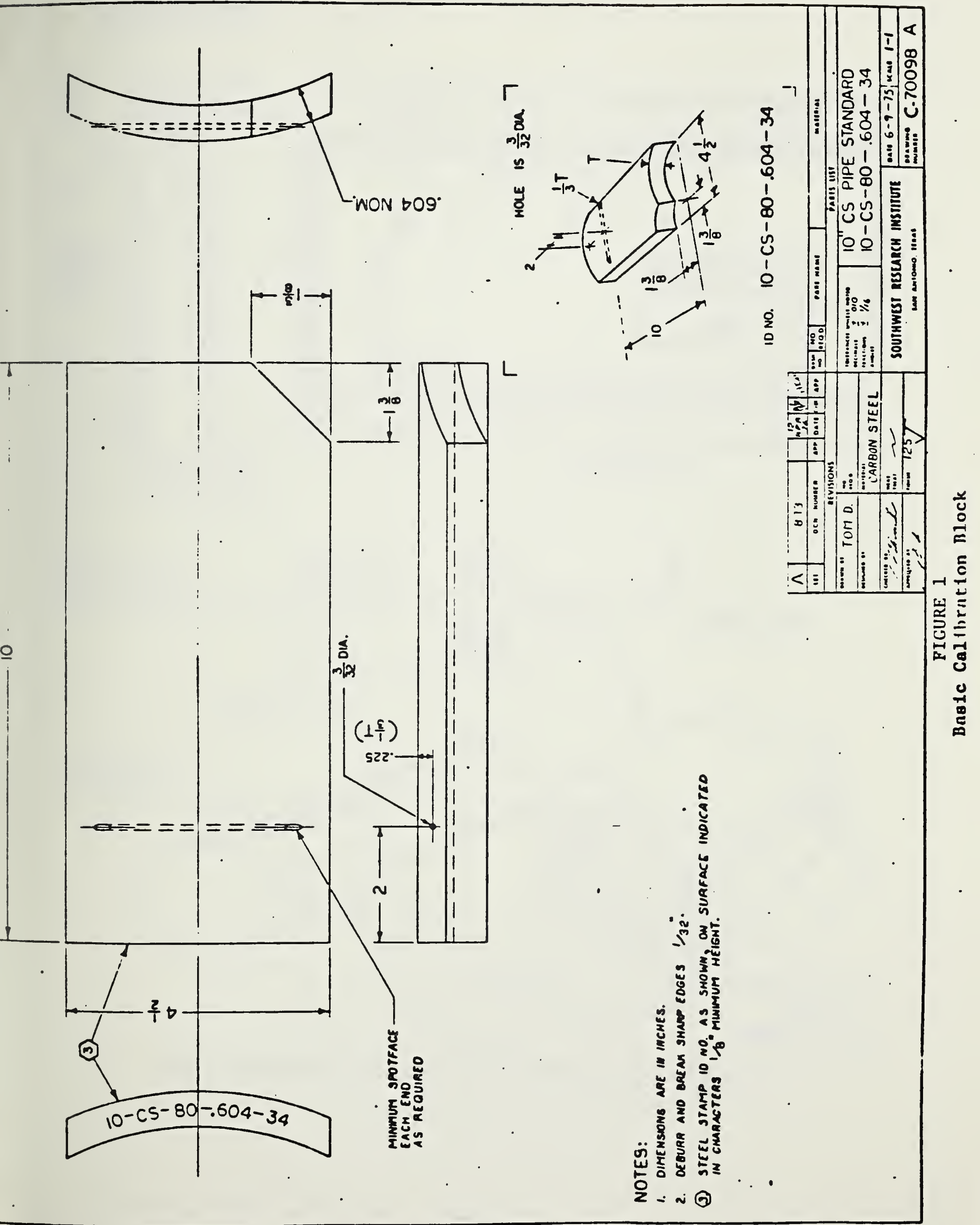



Irip Report No. 1

October 2, 1978

Page 5

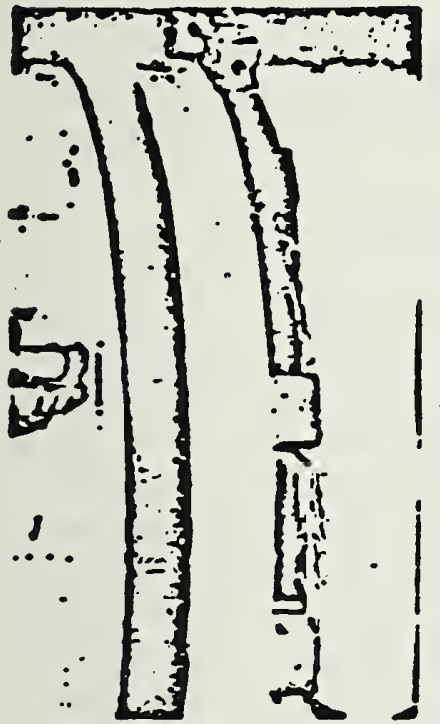

Search Unit and

Receiving Array

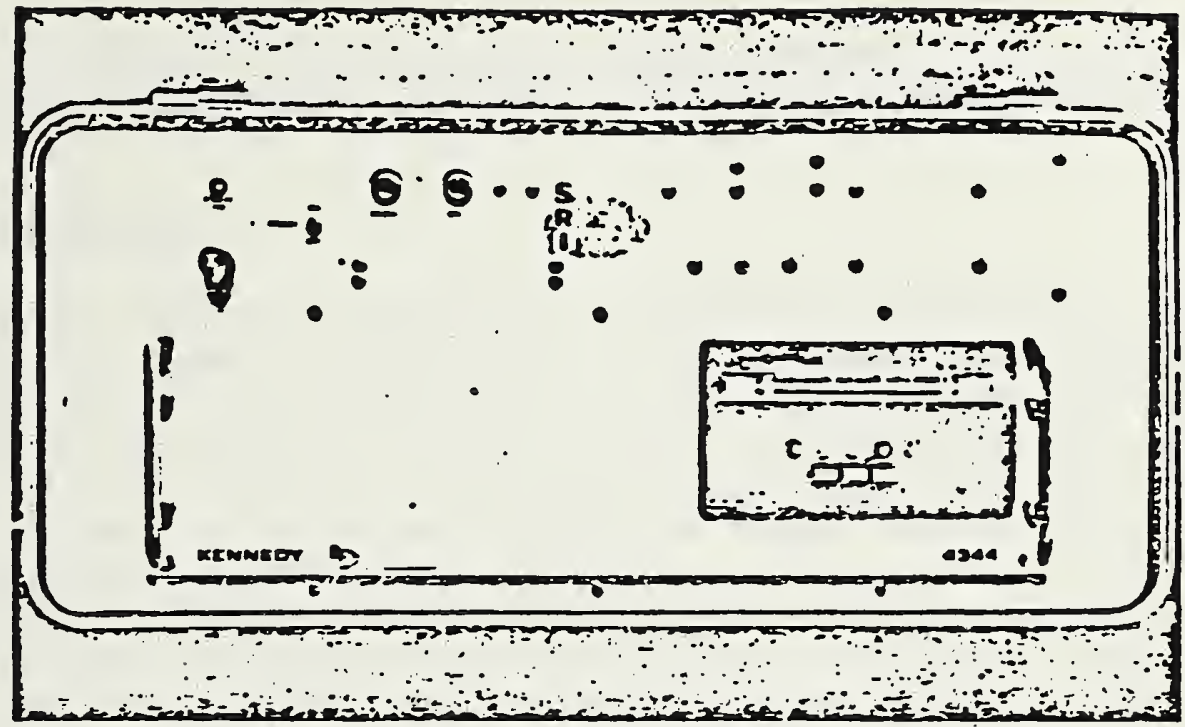

Tape Recorder

4-TRACK DIGITAL TAPE RECORDER
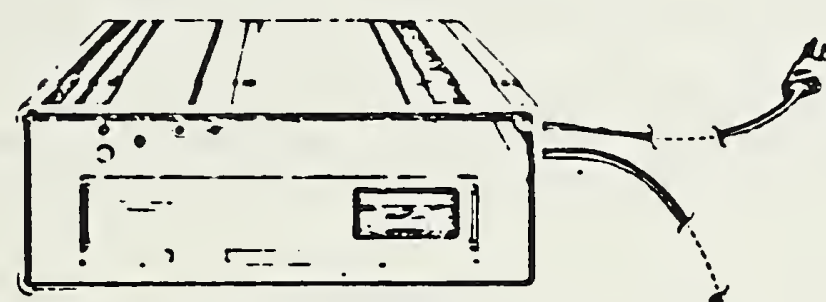

2-200 B:ETERS

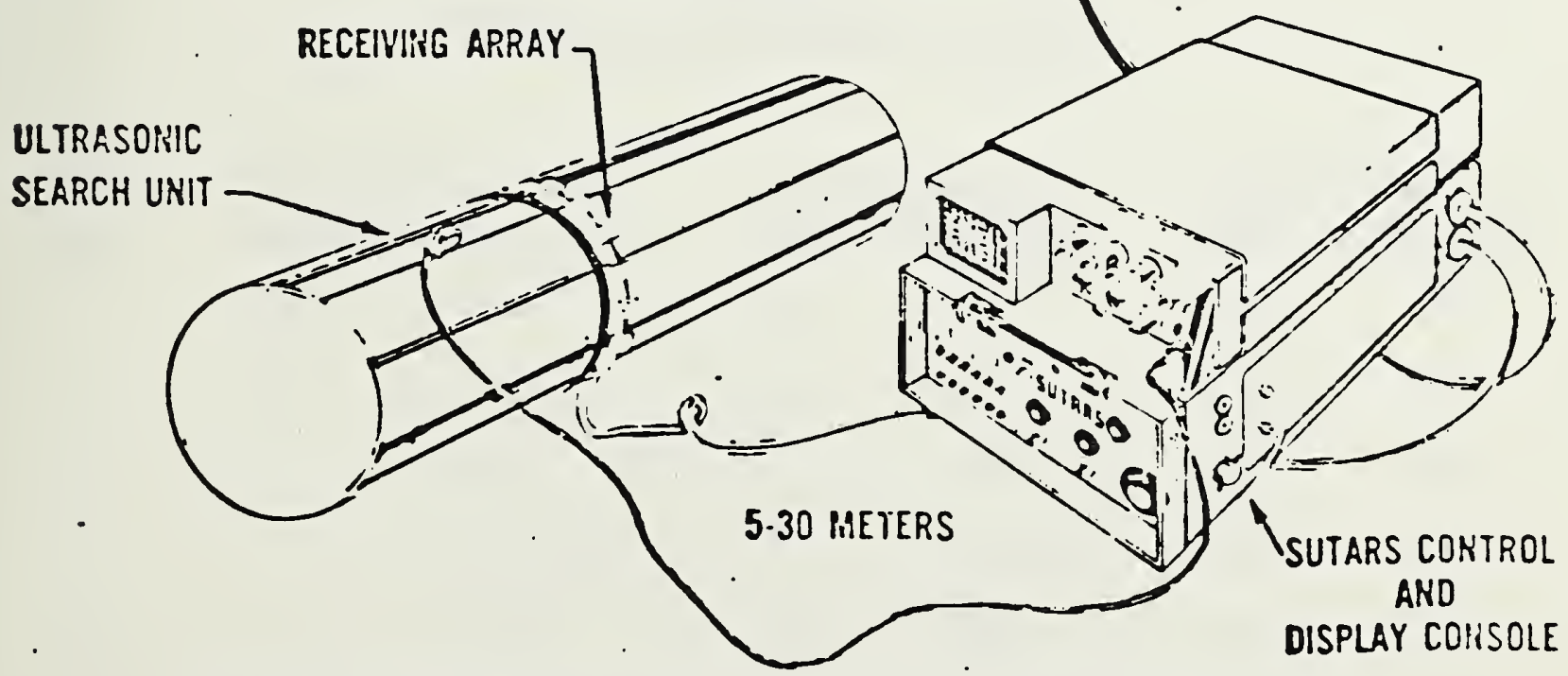

Figure 2. Schematic Arrangement of SUTARS Data Acquisition Subsystem 

Trip keport No. 1

October 2, 1978

Page 6

In the steel plate from which these sections of pipe were made. Such laminar conditions can Interfere with other ultrasonic examinations conducted from or through those areas.

\subsection{Weld Examinations}

The original intent was to use $45^{\circ}$ and $60^{\circ}$ shear wave examinations Introduced from both directions perpendicular to the weld axis for detection of flaws in the plane of the weld, and to use $45^{\circ}$ shear waves from both sides of the weld directed circumferentially for detection of discontinuities not orfented with the weld axis. Table 1 shows the examinations which were actually completed. Because of the time required to record the extremely large number of indications exceeding the threshcld recording level, it was not feasible to examine 100 percent of all welds with the scans originally planned. The data recorded consisted of the search unit position coordinates and the indicated sound path distance for those indications exceeding 20 percent of the amplitude of the reference level. This reference level was established by constructing a distance amplitude curve utilizing the sidedrilled hole targets in the basic reference block. The manual examination ultrasonic data are attached in Appendix B, together with the data sheets showing the instrument adjustments for each of the scans.

Each of the indications recorded was plotted manually to show its position in the cross section of the weld. The flaw plots are attached in Appendix $C$.

TABLE I

Ultrasonic Examination Coverage

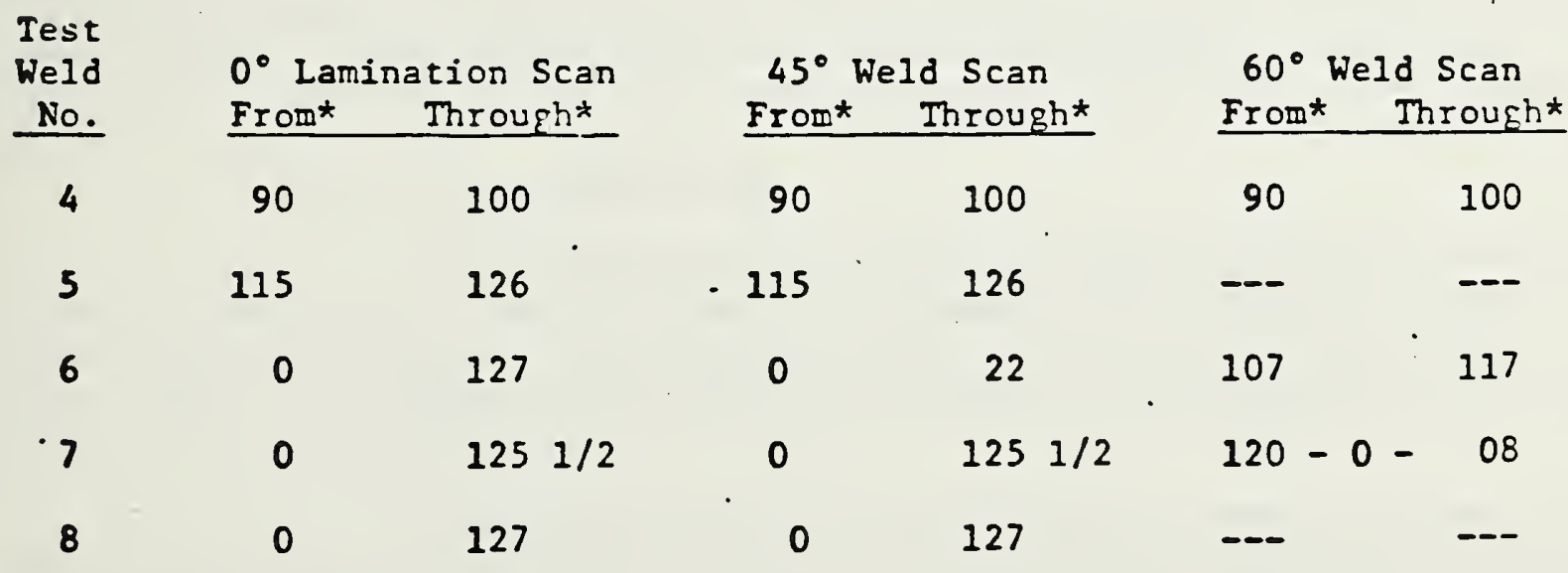

*Numerals refer to $x$-ray film stations. 

Trip Report No. 1

October 2, 1978

Page 7

\subsection{SUTARS EXAMINATIONS}

Those welds examined by SUTARS received the same scans as the other manual examinations. In both cases, the search unit was manipulated by hand and the data consisting of search coordinates, amplitude of ultrasonic indications and the sound beam path distances were recorded. The difference with SUTARS is that the data were recorded on a digital magnetic tape recorder for later processing by means of a computer program. Specimens Nos. 6, 7, and 8 were examined using SUTARS.

\subsection{RESULTS}

\subsection{Manual Examinations}

\subsubsection{Test Weld No. 4}

The lamination scan with $0^{\circ}$ sound beam showed six small laminar conditions in the base material adjacent to the examined portion of the weld. All six of the laminar conditions were judged too small to affect the results of the weld examinations appreciably since none was as large as the sound beam.

As indicated in Table 1 , the examination coverage was approximately 10 inches of weld extending from radiographic station 90 to radiographic station 100 . This area was chosen since radiographs previously made showed side-wall lack of fusion in this region. Both $45^{\circ}$ and $60^{\circ}$ examinations were conducted from both sides of the weld. At radiographic station 95-1/2, a concentrated examination was performed using both $45^{\circ}$ and $60^{\circ}$. Attached in Appendix $C$ are cross-section plots of these indications.. The side-wall lack of fusion indicated on the radiographs was shown with $60^{\circ}$, but had surprisingly low amplitude. It was not clearly shown using the $45^{\circ}$ sound beam, however. The majority of the ultrasonic indications proved to be due to the root bead geometry conditions and external weld crown irregularities.

\subsubsection{Test Weld No. 5}

The lamination scan with $0^{\circ}$ sound beam showed two small laminar conditions in the base material adjacent to the examined portion of the weld, which was a 10-inch sector between radiographic stations 115 and 125. These two laminar conditions were too small to appreciably affect the results of weld examination.

Weld examination of Test Weld No. 5 was conducted with $45^{\circ}$ sound bcam only from both directions. Cross-section plots of the ultrasonic indications show high amplitude root area reflectors from the root geometry and low amplitude indications of the slag condition which radiographs had shown in this weld. One of the apparent slag indications (Indication 

Trip Report No. 1

October 2, 1978

Page 8

No. 5 on Data Sheet 1306) located at radlographic station 122 was plotted to its half amplitude points. This plot indicates that the apparent flaw 1s trapped slag between passes near the side wall of the weld.

\subsubsection{Test Weld No. 6}

The lamination scan with $0^{\circ}$ sound beam showed nine laminar conditions in the base material adjacent to the examined portion of this weld. All nine of the laminar indications were small spots, too small to affect the results of the weld examinations appreciably.

The areas examined in Weld No. 6 consisted of $45^{\circ}$ scans from radiographic stations 0 to 22. Also, $45^{\circ}$ and $60^{\circ}$ scans were performed In the area from radiographic stations 107 to 117 . Cross-sectional plots of the indications seen with the use of $45^{\circ}$ and $60^{\circ}$ angle beams are attached In Appendix $C$. The plots indicate that a variety of conditions were picked up by the ultrasonic beam. The $60^{\circ}$ scans from both directions showed numerous geometric indications due to weld crown irregularities, root reinforcement, and surface irregularities in the parent material as well as flaw indications such as lack of penetration or side wall lack of fusion. Centered at $x$-ray station 112, a lack of penetration flaw visible in the radiograph of this weld showed a high amplitude indication. However, other indications due to root bead reinforcement geometric reflector conditions also produced high amplitude indications in the same region.

\subsubsection{Test Weld No. 7}

The lamination scan using $0^{\circ}$ sound bean showed 12 laminar indications in the base material adjacent to the butt weld in this pipe. With one exception, all of these were only spot indications with negligible effect on the examination of the weld itself. The exception was from radiographic stations 18-1/2 through $20-1 / 8$ and extending to slightly over 2 inches from the upstream side of the weld. A lamination was detected in this area approximately 0.18 inch below the $O D$. Severity of this lamination was great enough to prevent weld examination with angle beams from the upstream direction of this nearly 2-inch-long area. There was also a visible large open surface lap about $I$ foot long near.this position. This visible condition extended longitudinally on the surface of the base material upstream from the weld.

This weld was scanned completely with a $45^{\circ}$ angle beam around the circumference of the pipe from both the upstream and downstream sfdes. In addition, scans with $60^{\circ}$ angle beam were conducted from radiographic stations 120 through 0 to 08 from both upstream and downstream sides. Additional concentrated $60^{\circ}$ scans were performed at selected locations in this area. To determine the effect of the internal and external weld bead reinforcement on the ultrasonic results, a small area near radiographic location 3 was first ground flush on the inside surface and then on the outside surface. Following each of these grindings, the areas were reexamined to 

Trip Report No. 1

October 2, 1978

Page 9

determine the effect. These examinations are shown in the plots of indications In Appendix $C$. The results show that grinding the beads flush produces a marked increase in the signal amplitude from the known root crack condition in the area which was ground flush as well as eliminating the interference of the root and crown reinforcement reflectors.

A relatively unused technique for crack depth determination was tried in the flush-ground area. This technique, called the "precursor method," consists of determining the difference in path lengths of a sound beam reflected from the corner formed by the intersection of a crack with the surface and the weak reflection of that beam due to crack-tip diffraction. As shown in Figure 3, this difference should be proportional to the crack depth. The depth thus indicated at radiographic station 3-1/8 was 0.021 Inch in this test weld.

\subsubsection{Test Weld No. 8}

The lamination scan with $0^{\circ}$ sound beam showed that this base material was nearly free of laminar conditions adjacent to the weld. One small spot--a laminar reflector smaller than the sound beam--was located.

A $45^{\circ}$ scan was performed completely around this test weld from the upstream and downstrear sides. Most of the reflectors recorded proved to be produced by the weld crown reinforcement geometry. A crosssection plot of these indications is attached in Appendix C. Six of the eight indications which plot approxinately mid-wall in the weld actually can be shown to be due to mode conversion and redirection of the sound beam to the crown surface, since the indications can be manually damped on that surface.

\subsection{SUTARS Examinations}

:- - The magnetic recording tapes on which the data from the SUTARS examinations were acquired were retained by SwRI. In accordance with the contract, the final processing of these tapes to extract the data and evaluate the examination information was deferred awaiting authorization of this effort by the National Bureau of Standards (NBS). A portion of data for Test Weld No. 6 was processed and is attached in Appendix D.

\subsection{CONCLUSIONS}

\subsection{Manual Examinations}

7.1.1 The time required for complete manual ultrasonic examination of line pipe welds proved to be greater than anticipated. The neccsity to consider and evaluate reflectors which produced anplitudes as low as 20 percent of the reference level required that the search unit coordinates and 

Tr1p Report No. 1

October 2, 1978

Page 10

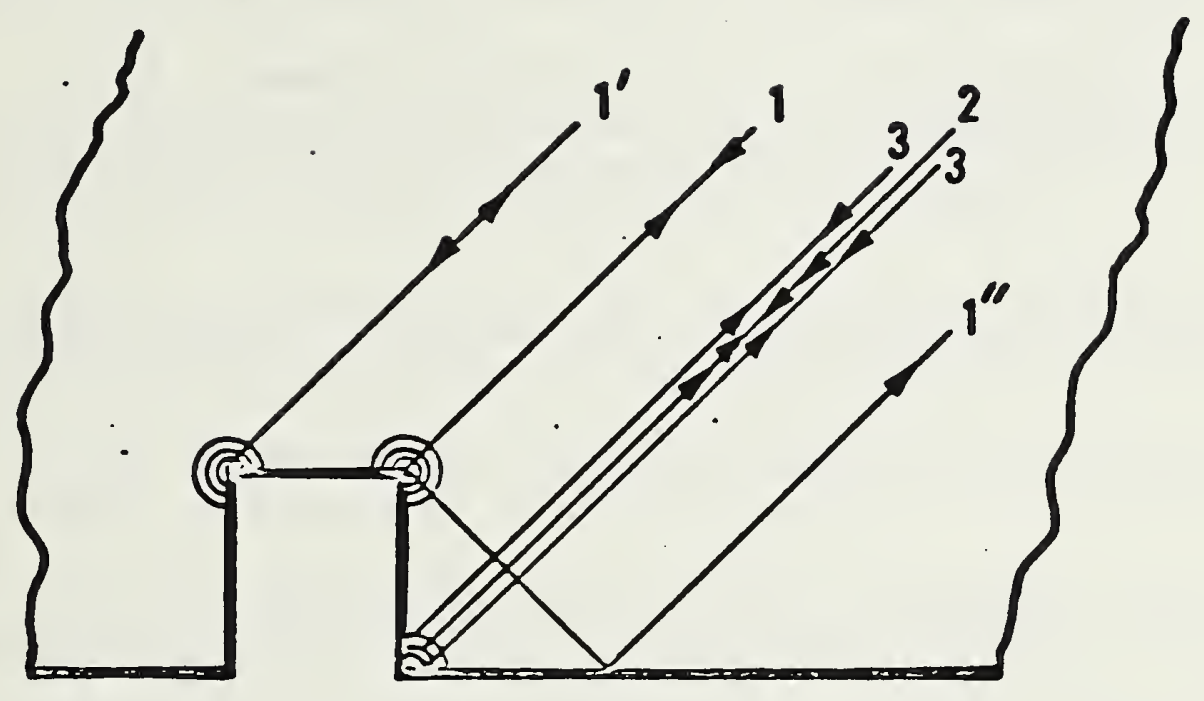

A. Components of Sound Beam Reflected and Refracted from a Notch or Crack

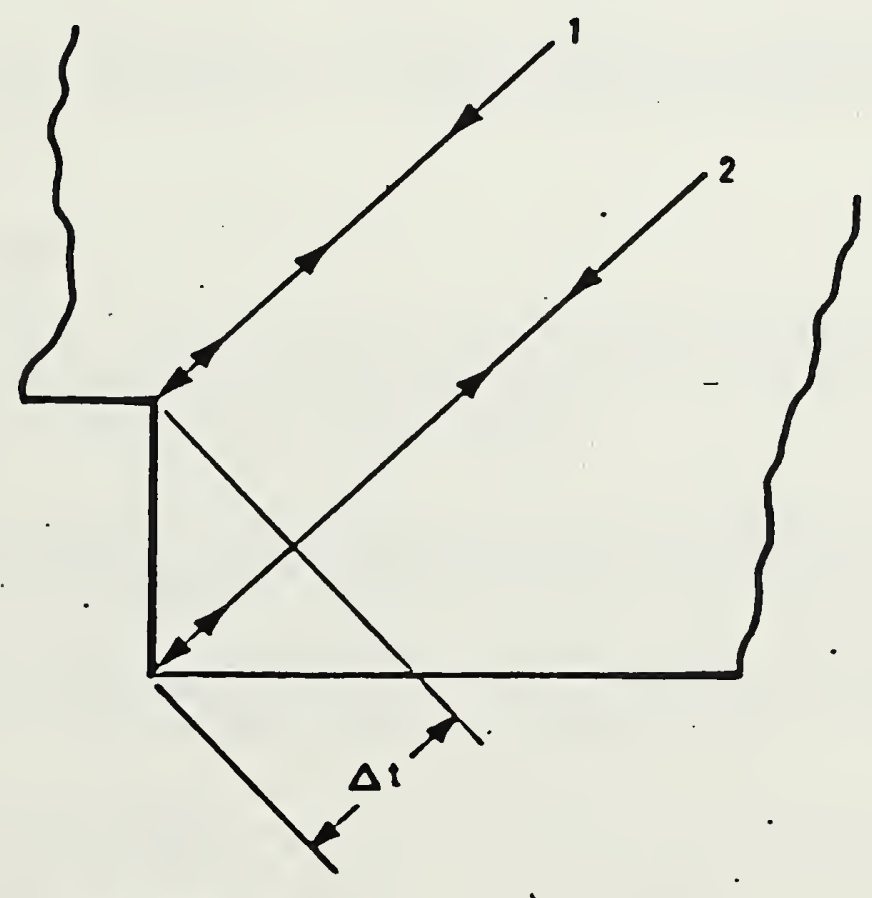

B. Sound Beam Components Used for Determining Crack Depth 

Tr1p Rंeport No. 1

October 2, 1978

Page 11

other data from all such reflectors be recorded. Root bead and weld crown reinforcements--so called geometric reflectors--constituted large and variable Interference which was present at nearly all locations along each of the welds.

\subsubsection{Geometric interference occurs at the same transducer.} coordinates and together with indications of real defects, and is essentially Inseparable from such defect indications with the conventional wide-beam transducers which were employed during these examinations.

7.1.3 Removal of weld beads by grinding eliminates the problem and wight prove feasible in special cases or for examinations of localized areas, but is considered impractical for routine work.

7.1 .4 A $45^{\circ}$ sound beam angle is more sensitive than $60^{\circ}$ to geometric reflectors as well as to most real defects.

7.1.5 The "precursor" method, where applicable, shows promise for improved determination of the depth of detected flaws.

7.1.6 Mode conversion interference was not an appreciable factor with manual welds, but did occur with one of the test welds which was welded automatically. This appeared to be due to a small internal weld preparation edge, which seryed as a mode converting surface. Such a condition would be avoidable with a slightly different edge preparation.

7.1.7 Collimatic:, focusing, or other high-resolution techniques are needed for better separation of real flaws and geometric features in welds such as these.

\subsection{RECOMMENDATIONS}

8.1 The SUTARS data acquired during these examinations should be processed:completely, evaluated, and compared with the manually acquired data from each weld examined by both methods.

8.2 Higher-resolution methods, such as collimated, focused, or concentrated beam transducer techniques need to be developed for better separation and identification of geometric and detrimental flaw indications.

8.3 Adaptive learning methods offer additional possibilities for reducing the data to eliminate geometric indications.

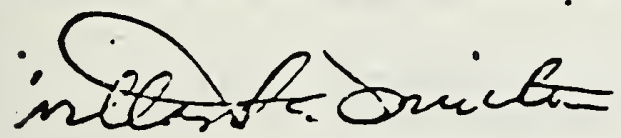

Willard C. Minton

Technical Consultant

Department of Research and Development

Quality Assurance Systems and Engineering.

Division 


\section{SOUTHWEST RESEARCH INSTITUTE}

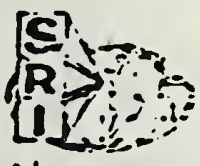

OPERATING PROCEDURE

s

MANUAI ULTRASONIC EXAMINATION OF LINE PIPE TELDS

Page 1 of 23

SwPI-NDT-600-58

Revision 0

August 1978

\section{SWRI-NDT-800-58}

\section{$1.0 \quad$ PURPOSE}

This procedure provides the technical information and detailed steps necessary to ensure a complete and accurate manual ultrasonic examination of pressurc piping welds and the adjacent base material, in accordance with applicable Codes.

\subsection{SCOPE. AND APPLICATIO:}

Piping welds and the adjacent base wateriel in the nominal thicknes: range of greater than 0.3 to 2.0 inches shall be examined in accordance with this procedure.

lianual, contact, pulse-echo stear-wave angle bean and longitudiral fiave straight bear. ultrasonic techniques shall be utilized for the exarination of piping welds and adjacent base waterial.

Welds to be exanined shall ba circupierential and longitucinal pipe welds or as specified in the applicable SwRI Exanination Plan.

2.1 Applicabie Documents

The following document forms a pirt of this procedure as applicable:

(1) API STD 1104, Standard fur Welding Pipelines and Related Facilities, Fourta:nth Ecition.

3.0 RESPONSIBIIITY

(1) The Director of the Department of Assurance Systems and Engineering ijvision, shall be responsible for the preparation, review, appro:al, and control of this procedure.

(2) The examiner shall be responsib?e. for implementing the requirements of this procedure.

4.0 CODF AND PROCEDURE RF.OUIREMFNIS

Not applicable. 



\section{SOUTHWEST RESEARCH INSTITUTE}

SwRI-NDT-800-58

Revision 0

August 1978

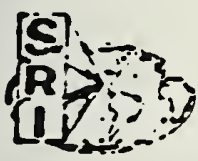

OPERATING PROCEDURE

Page 2 of 23

\begin{tabular}{lll}
\hline PERSONNEL AND FOUIPMENT \\
\hline
\end{tabular}

Personnel performing examinations in accordance with this procedure shall be certified in accordance with SwRI NQAP 11-1, "Special Process Control."

\subsection{Reference Block}

Reference bloclis used for screen distance calibration and verification shall be either of the following:

(1) SwRI Half-Round (Sketcl, 1)

(2) Alts Type DC (Sketch 2)

(3) IIW (Sketch 3) or DSC

The Shri llalf-Round or Als Type DC reference block shall be of the same basic material as the production material, e.g., stainless steel or carbon steel.

5.3 Basic Calibration Block for Circunferential, Longitudinal, Support Attachment, and Branch Pipe Connec:ion lields

Side-drilled basic calibration hole reflectors shall be placed in a block manufactured fron material equivalent to the production material. The calibration material shall be detemined by the production piping material to which the search unit is applied.

The basic calibration blocl: thickness shall be deternined by the thickness of the production piping material to which the search unit is applicd. When a basic calibration block of the same thickness as the production piping material is not avallable and where the production piping material thicliness is 1 inch or less, the basic calibration block thickness shall be within $25 \%$ of the production material.

An example of a basic calibration block is attached in sketch 4 . 



\title{
SOUTHWEST RESEARCH INSTITUTE
}

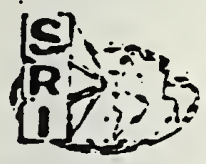

\author{
OPERATING PROCEDURE
}

SwRI-NDT-800-58

Revision 0

August 1978

Page 3 of 23

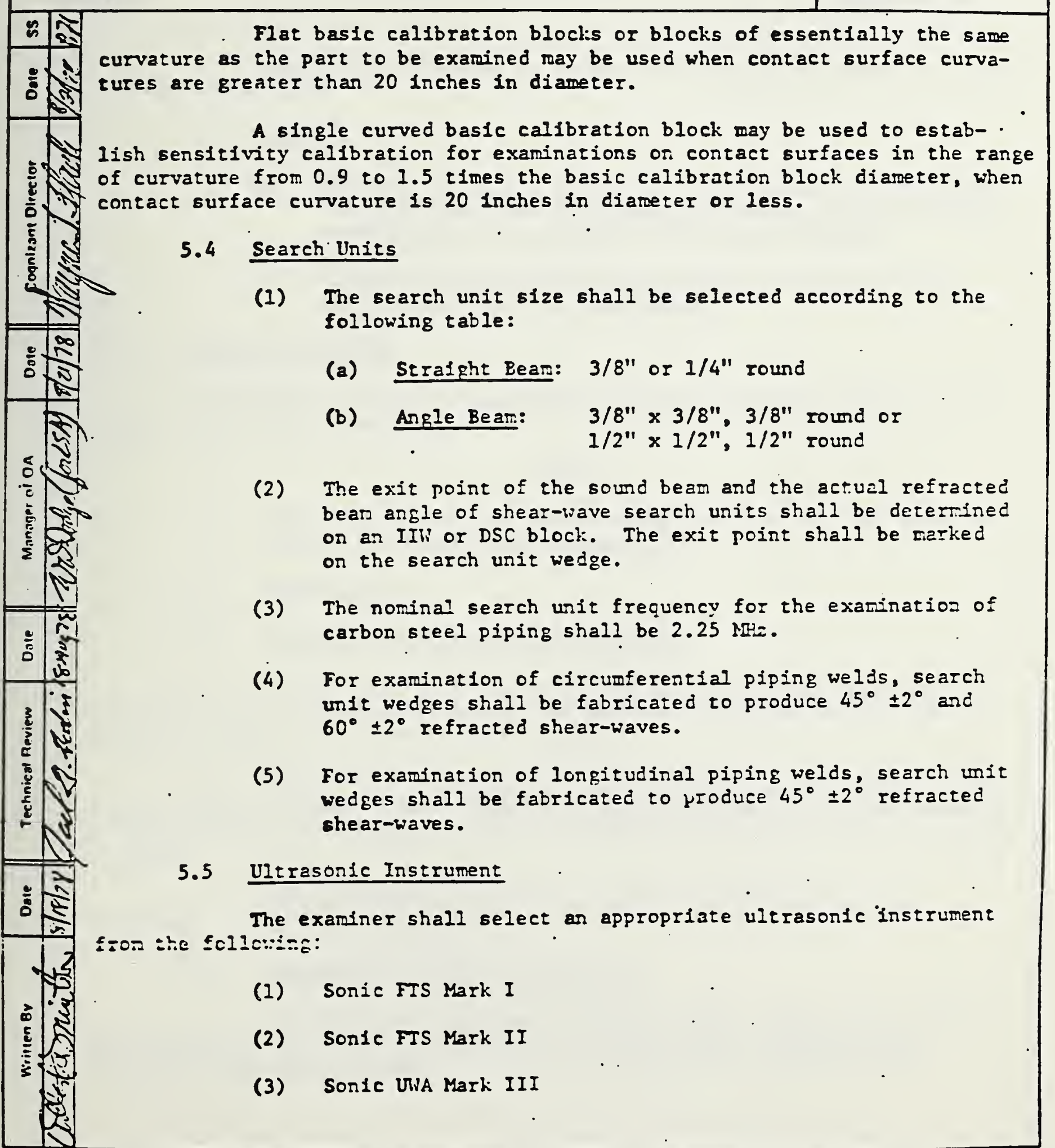





\title{
SOUTHWEST RESEARCH INSTITUTE
}

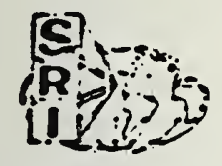

\author{
OPERATING PROCEDURE
}

SwRI-NDT-800-58

Revision 0

August 1978

Page 4 of 23

\begin{abstract}
\&
\&

calibration sticher.

Each Instrument shall be aligned and display a current aligntent
\end{abstract}

5.6 Couplant

(1) Sonogel, UItragel, Echogel or other connercial couplants shall be used when performing ultrasonic calibrations and examinatlons in accordance with this procedure.

(2) Couplant materials used for examinations shall be the came as used for the calibration.

6.0 CAIIBRATION METHOD

The complete ultrasonic examination system calibration shall be performed prlor to the exarination.

\section{NOTE}

The "REJECI" control shall be raintained in the "0" position during calibration and examination.

\subsection{Calibration}

\subsubsection{Straight-Bean Distance Calibration}

In all cases, the screen distance chosen shall be the shortest applicable size to include at least $1 / 4 t$ beyond the thickest production material to which the search unit is applied.

Observing back reflections from the applicable reference block, adjust. "MATERIAI CAIIBRATION," "RANGE," and "DELAY" controls of the instrument to obtain the required linear sound path distance, displayed along the screen baseline. following: $1.0^{\prime \prime}, 2.5^{\prime \prime}$, and 5.0".

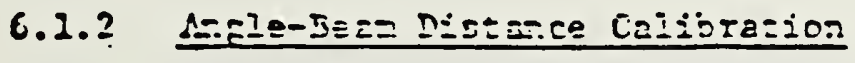

In all cases, the screen distance chosen shall be the shortest applicable size which will include at least $1 / 8$ vee path past the anticipated examination range. 



\section{SOUTHWEST RESEARCH INSTITUTE}

SwRI-NDI-800-58

Revision 0

August 1978

\section{OPERATING PROCEDURE}

Page 4 of 23

क्: Each instrument shall be aligned and display a current alignment
calibration stlclier.

\subsection{Couplant}

(1) Sonogel, Oltragel, Echogel or other comercial couplants shall be used when performing ultrasonic calibrations and examinations in accordance with this procedure.

(2) Couplant materials used for examinations shall be the same as used for the calibration.

6.0 CAIIBRATION NETHOD

The complete ultrasonic examination system calibration shall be performed prior to the exarination.

\section{NOTE}

The "REJECI" control shall be maintained in the "O" position during calibration and exarination.

\subsection{Calibration}

\subsubsection{Straigint-Beam Distance Calibration}

In all cases, the screen distance chosen shall be the shortest applicable size to include at least $1 / 4 t$ beyond the thickest production material to which the search unit is applied.

Observing back reflections from the applicable reference block, adjust "MATERIAI CAIIBRATION," "RANGE," and "DELAY" controls of the instrument to obtain the required linear sound path distance, displayed along the screen baseline. following: $1.0^{\prime \prime}, 2.5^{\prime \prime}$, and $5.0^{\prime \prime}$.

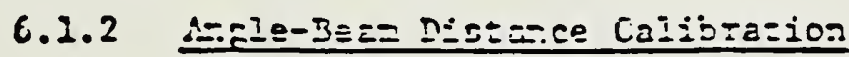

In all cases, the screen distance chosen shall be the shortest applicable size which will include at least $1 / 8$ vee path past the anticipatcd examination range. 



\title{
SOUTHWEST RESEARCH INSTITUTE
}

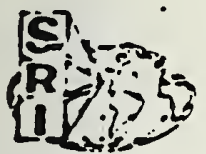

\author{
OPERATING PROCEDURE
}

SwRI-RIDT-800-58

Revision 0

August 1978

Page 5 of 23
\&

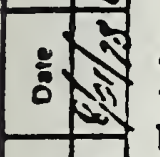

7

adjust "MATERIAI CAIIBRATION," "RANGE," and "DELAY" controls of the instrument to obtain the required linear sound path distance, displayed along the screen baseline.

Screen distances shall be selected from the following sizes: $2.5^{\prime \prime}, 5.0^{\prime \prime}$ and $20.0^{\prime \prime}$.

- When a single instrument is used for both $45^{\circ}$ and $60^{\circ}$ exacinations, the screen distance calibration shall be conducted in the followmanner:

(1) The screen distance size shall be determined by the angle beam search unit requiring the Jonger examination range.

(2) Position the $45^{\circ} \pm 2^{\circ}$ search unit on the appropriate reference block, observe and record all verification block entries as reguired on the appropriate SwRI Instrument Calibration Record.

(3) Position the $60^{\circ} \pm 2^{\circ}$ search writ on the appropriate reference block and, without changing the "ILATERIAI CALIBRATION," "RANGE," or "DELAY" cOntrols, observe and record all verification block entries as required on the appropriate SwRI Instrument Calibration Record.

(4) No attempt shall be made to compensate for any slight delay difference observed between $45^{\circ} \pm 2^{\circ}$ and $60^{\circ} \pm 2^{\circ}$ screen distance calibrations. This difference, if any, Instead shall be considered when resclving indications.

\subsubsection{Angle-Beam Distance Ampljtude Correction}

If a curved block is used, distance amplitude correction (DAC) curves for the examination of circumferential welds shall be constructed by utilizing the responses from the holes oriented perpendicular to the axis of the basic calibration block for butt welds and parallel to the axis for icagitudiral sear. weics.

\subsubsection{Naterial 1 Inch or Less in Thickness}

\section{$45^{\circ}$ and $60^{\circ}$ DAC}





\section{SOUTHWEST RESEARCH INSTITUTE}

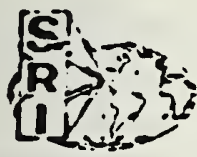

OPERATING PROCEDURE

SWRI-NDT-800-58

Revision 0

August 1978

Page 6 of 23
का श्चै
Position $45^{\circ} \pm 2^{\circ}$ search unit to obtain maximum response from the hole and position producing the highest auplitude from the following:
Hole
Vee Path Positions
$1 / 2 \mathrm{~T}$
$2 / 8,6 / 8,10 / 8$
(2) Adjust instrument gain controls to obtain the prirary reference response at $75 \% \pm 5 \%$ of FSH and mark this amplitude on the screen. The gain controls shall not be adjusted once the primary reference response has been established.
(3) Position the search unit to obtain maximum response fror the remaining positions and mark each amplitude on the screen.
(4) Join these points with a smooth curved line, the length of which shall not extend more than $1 / 8$ vee path.
beyond the last qualified calibration point.

(5)

Repeat steps (1) through (4) using a $60^{\circ} \pm 2^{\circ}$ search unit.

\section{NOTES}

If the configuration of the weld is such that a $45^{\circ} \pm 2^{\circ}$ full node examination covers the entire weld voluze and the $60^{\circ} \pm 2^{\circ}$ search unit sound bean is directed into the weld root on the straight pass, indications detected beyond the $6 / 8$ vee path while conducting $60^{\circ} \pm 2^{\circ}$ examinations shall not be recorded.

If the configuration of the weld is such that a $45^{\circ} \pm 2^{\circ}$ vee path examination falls to cover the entire weld volume and the $60^{\circ} \pm 2^{\circ}$ search unit sound beam is directed into the weld root on the straight pass, and $a$ $62^{\circ} \pm 2^{\circ}$ full vee exanisision covers the entire weld volume, Indications detected in the calibrated area shall be recorded.

*full screen height 



\section{SOUTHWEST RESEARCH INSTITUTE}

OPERATING PROCEDURE

SWRI-NDT-800-58

Revision 0

August 1978

Page 7 of 23

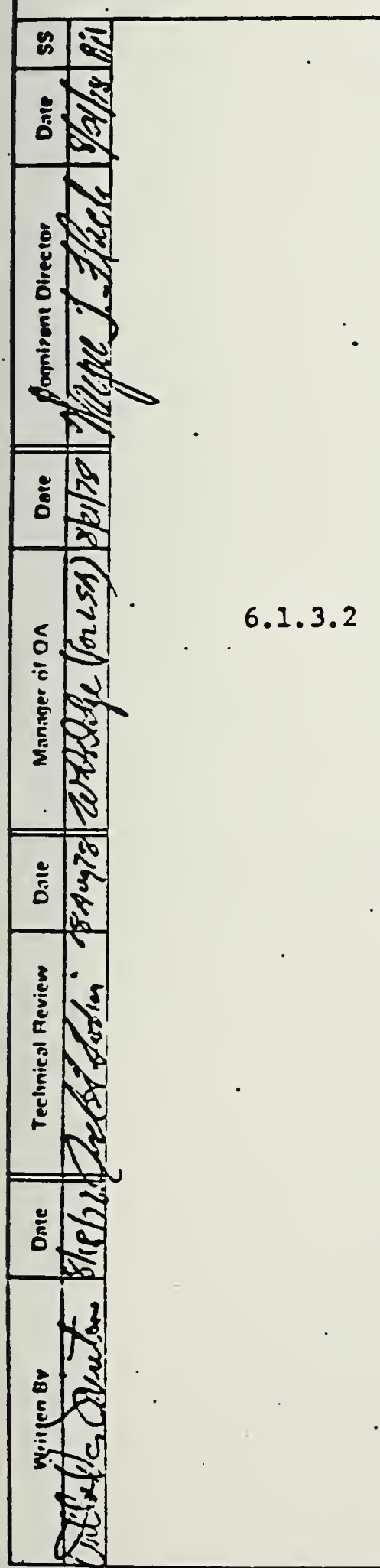

\section{EXCEPTIONS}

If the configuration of the weld is such that the $60^{\circ} \pm 2^{\circ}$ search unit sound beam is not directed into the weld root on the straight pass, a $14 / 8$ vee path callbration shall be accomplished with $45^{\circ} \pm 2^{\circ}$ search unit. Indications detected in the calibrated area shall be recorded.

If the configuration of the weld is such that a $45^{\circ} \pm 2^{\circ} 14 / 8$ vee path exarination and a $60^{\circ} \pm 2^{\circ} 10 / 8$ vee path examination fail to cover the entire weld volume, $14 / 8$ calibration shall he accomplished with both $a 45^{\circ} \pm 2^{\circ}$ and $a 60^{\circ} \pm 2^{\circ}$ search unit and indicatlons detected in the calibration area shall be recorded.

\section{Material Greater Than I Inch But Not Over 2 Inches in In Ihichiness}

$45^{\circ}$ and $60^{\circ} \mathrm{DAC}$

(1) Position the $45^{\circ} \pm 2^{\circ}$ search unit to obtain maximus response from the hole and the position producing the highest amplitude fror the following:

\begin{tabular}{|c|c|}
\hline Hole & $45^{\circ}$ Vee $P$ \\
\hline $\begin{array}{l}1 / 4 T \\
3 / 4 T\end{array}$ & $\begin{array}{l}7 / 8,9 / 8 \\
3 / 8,5 / 8\end{array}$ \\
\hline
\end{tabular}

(2) Adjust Instrument gain controls to obtain the prinary reference response at $75 \% \pm 5 \%$ of $\mathrm{FSH}$ and mark this aplitude on the screen. The gain controls shall not be adjusted once the primary reference response has been established.

(3) Posftion tine search luit is obteis zaxinum response from the renaining positions and mari: each amplitude on the screen.

(4) Join these points with a smooth curved line, the length of which shall not extend rore than $1 / 8$ vee bejond the last qualified caljbration point. 



\section{SOUTHWEST RESEARCH INSTITUTE}

SwRI-1JDT-800-58

Revision 0

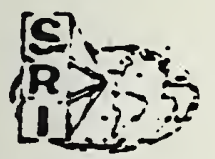

OPERATING PROCEDURE

August 1978

Page 8 of 23

Repeat steps (1) through (4) using a $60^{\circ} \pm 2^{\circ}$ search unit utilizing the following positions:

Hole

$1 / 4 \mathrm{~T}$

$3 / 4 \mathrm{~T}$ $60^{\circ}$ Vee Path Positions

$7 / 8$

$3 / 8,5 / 8$

\section{EXCEPTIONS}

If the configuration of the weld is such that a $45^{\circ} \pm 2^{\circ}$ full vee examination fails to cover the entire weld volume a $9 / 8$ vee calibration shall be accorwlished with a $60^{\circ} \pm 2^{\circ}$ search unit.

If the configuration of the weld is such that the $60^{\circ} \pm 2^{\circ}$ search unit bear is not directed into the welc root, a $13 / 8$ vee calibration shall be accomplished with a $45^{\circ} \pm 2^{\circ}$ search urit.

\subsection{Calibration Verification}

\subsubsection{Sweep Range and DAC Curve Verification}

Sweep range and DAC curve calibration shall be verffied on the appropriate reference and basic calibration block:

(1) At the start of a series of exacinations

(2) With any substitution of search unit cable

(3) With any substitution of power source

(4) At least every 4 hours during the examination

(5) At the finish of a series of examinations

(6) At any t1me when, in the opinion of the examiner, there Is dorts 25 to the valicity of the caitbration.

\subsubsection{Celibration Chazges}

(1) Perform the following if any point on the DAC curve has decreased more than $20 \%$ or $2 \mathrm{~dB}$ in amplitude, or any point on the DAC has moved on the sweep line more than 5\% of full screen width: 



\section{SOUTHWEST RESEARCH INSTITUTE}

SwRI-NDT-800-58

Revision 0

$\left\{\begin{array}{l}C^{7} \\ R=0 \\ 1]\end{array}\right.$

OPERATING PROCEDURE

August 1978

Page 9 of 23

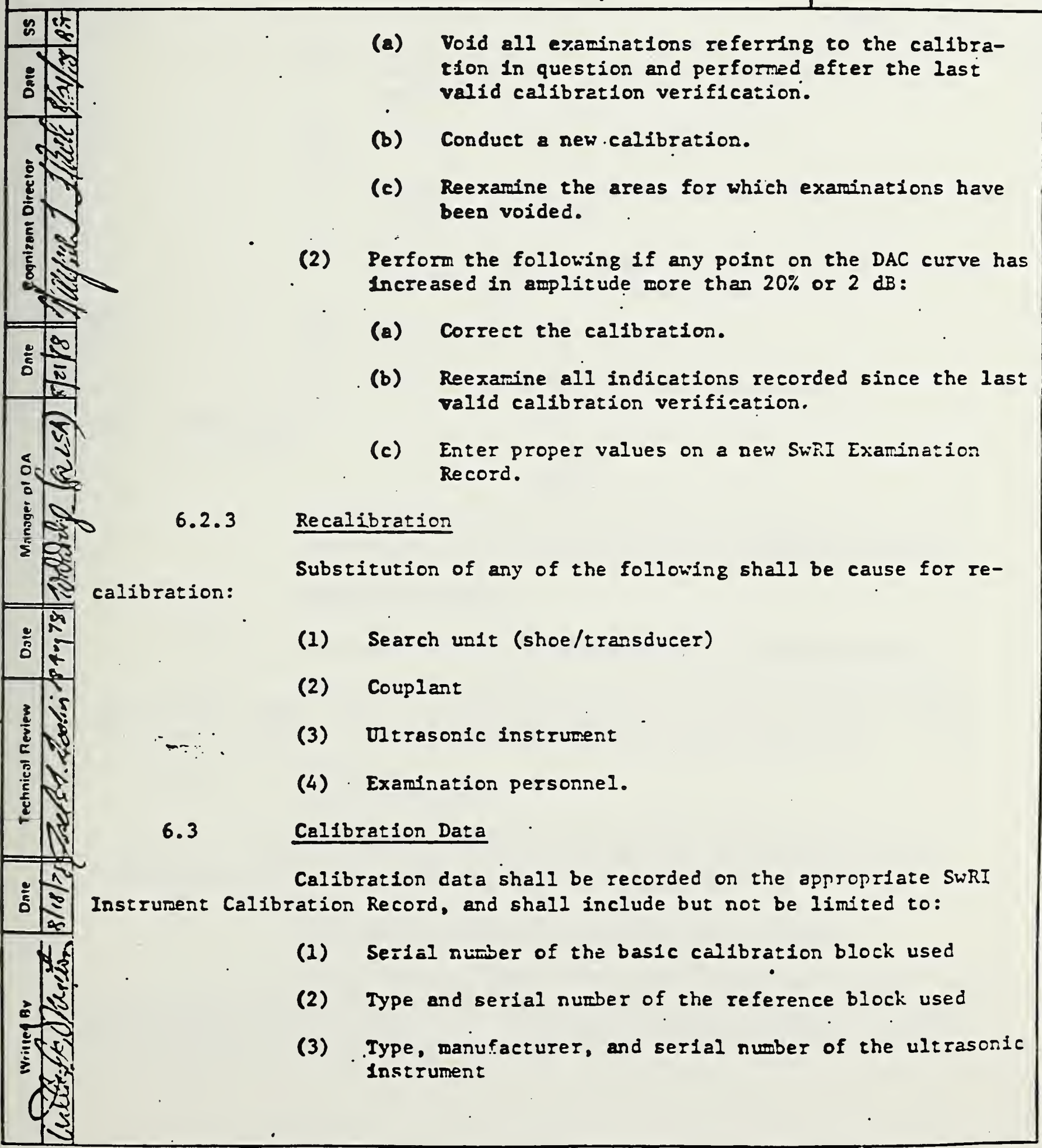





\section{SOUTHWEST RESEARCH INSTITUTE}

SWRI-NDT-800-58

Revision 0

August 1978

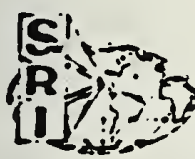

OPERATING PROCEDURE

Page 10 of 23

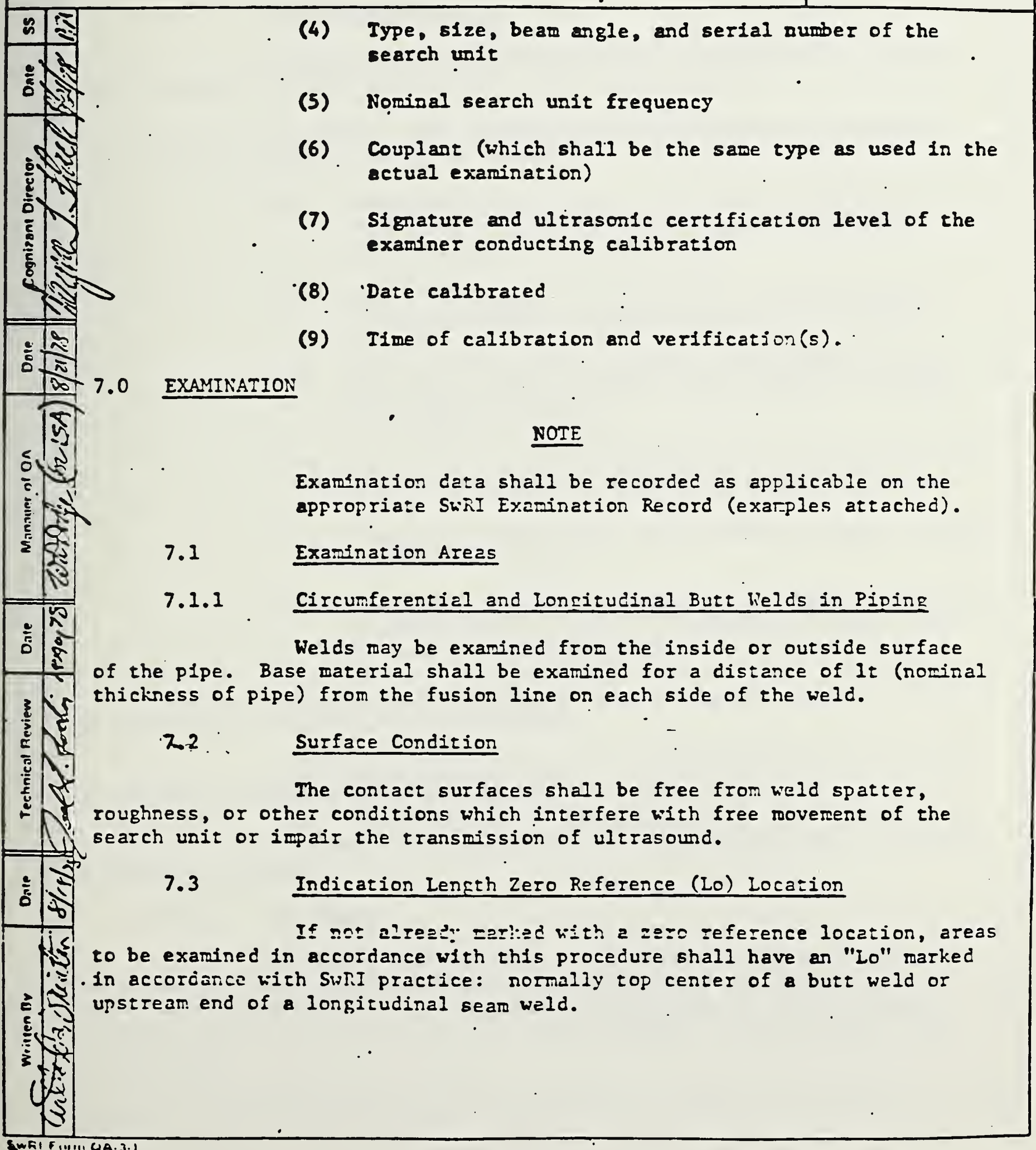





\section{SOUTHWEST RESEARCH INSTITUTE}

SWRI-INDI-800-58

Revision 0

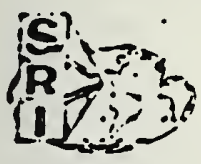

OPERATING PROCEDURE

August 1978

Page 11 of 23

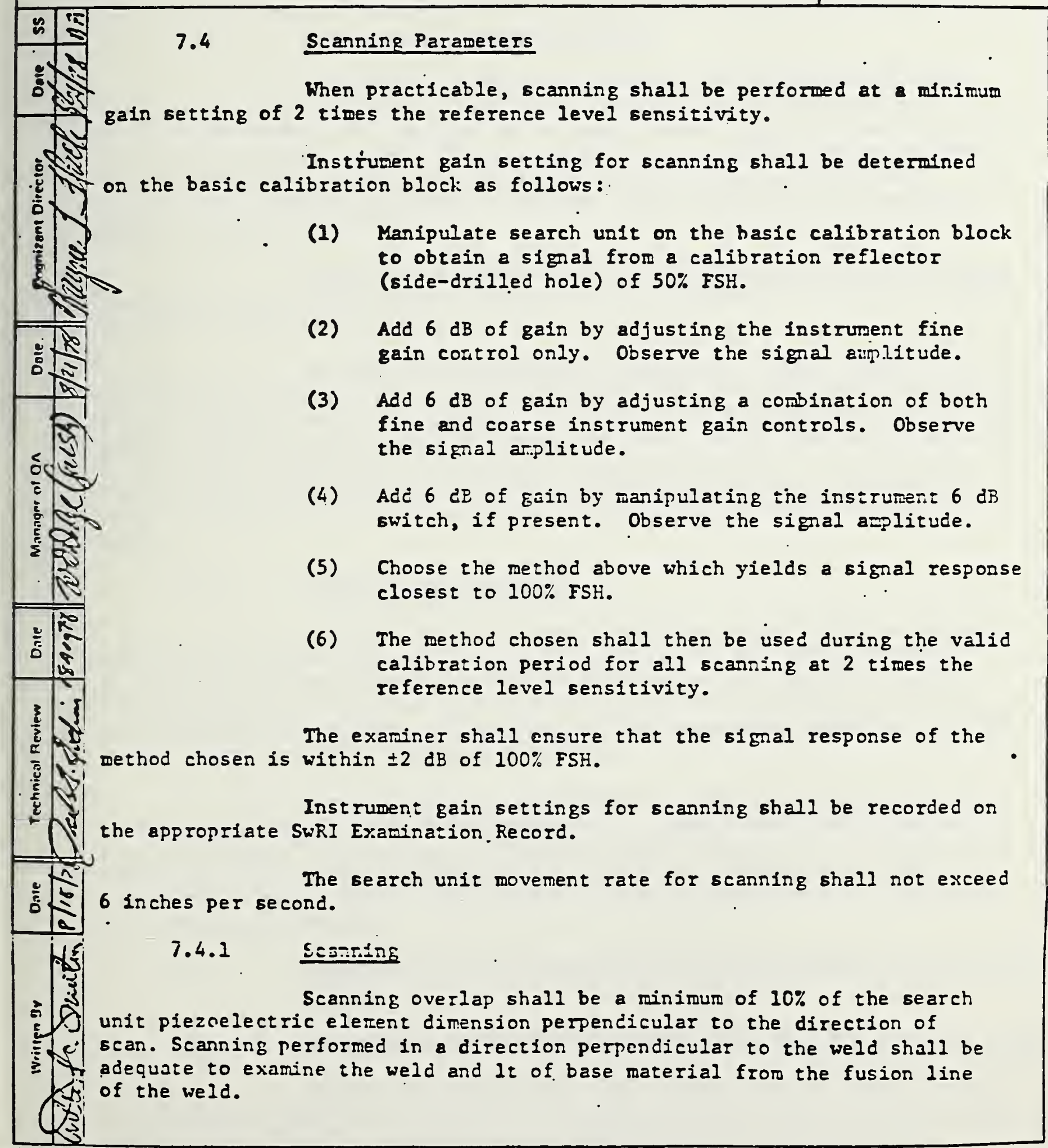





\section{SOUTHWEST RESEARCH INSTITUTE}

SWRI-NDT-800-58

Revision 0

[ST

August 1978

OPERATING PROCEDURE

Page 12 of 23

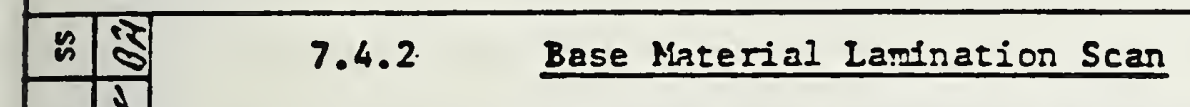

A lamination scan using straight beam Iongitudinal waves shall be performed before the angle-bear. exarinaticn. This examination shall cover as wuch as practical of the area through which the angle beam is later to be passed. Screen distance callbration for this examination shall be conducted in accordance with Paragraph 6.1.1. Scanning sensitivity shall be as required to maintain the first back reflection at an amplitude between $50 \%$ and $90 \%$ of FSH.

Intermediate echoes having an amplitude equal to or greater than $50 \%$ of the initial back reflection and accompanied by a $50 \%$ loss of back reflection shall be recorded.

To record an intermediate indication, a back reflection signal shall be obtained from an indication-free area and the instrument gain control adjusted until this signal is at $75 \% \pm 5 \%$ of FSH; then record the interDediate indication when its amplitude is eçual to $50 \%$ of the initial back reflection and accompanied by a $50 \%$ loss of back reflection. If total loss of back reilection accomparies the internediate echo, the area of total loss of back reflection shall be recorded on the appropriate SwRI Examinarion Record.

\subsubsection{Thichness Measurements}

In conjunction with the larination scan, thichess measurements shall be taken acjacent to Lo for longitudinal and circumferential welds (on the centerline of the weld if feasible and at one point in the base material on both sides of the weld). If these reasurements cannot be taken adjacent to Lo, then the location of the measurements shall be recorded on the appropriate SwP.I Examination Record.

Screen distance calibration for this examination shall be conducted in accordance with Paragraph 6.1.1. Measurements shall be taken by placing the $0^{\circ}$ search unit on the appropriate position on the examination surface and observing the position of the back wall reflection on the instrument screen. These measurements shall be recorded on the appropriate SwRI Examination Record.

\section{5 \\ Examination of Circumferential and Longitudinal Butt Welds in Piping}

7.5.1

Angle-Beam Examination for Indications Parallel with the Weld

Angle beam examinations for circumferential welds shall be accomplished using $45^{\circ} \pm 2^{\circ}$ and $60^{\circ} \pm 2^{\circ}$ refracted shear waves from both sides of the weld. For this examination, the sound-bcar shall be directed 



\section{SOUTHWEST RESEARCH INSTITUTE}

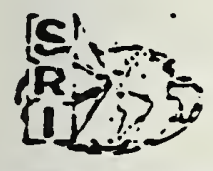

OPERATING PROCEDURE

SwRI-NDT-800-58

Revision 0

August 1978

Page 13 of 23
\&

perpendicularly into the weld to detect indications which are parallel to the weld. Calibration for these examinations shall be in accordance with Paragraphs 6.1.2 and 6.1.4.

Angle beam examinations for longitudinal welds shall be accomplished using a $45^{\circ} \pm 2^{\circ}$ refracted shear wave from both sides of the weld. For this examination, the sound-beam shall be directed perpendicularly Into the weld to detect indications which are parallel to the weld.

\subsubsection{Angle-Beam Examination for Indications Pernendicular to the Weld}

An angle-beam examination for indications perpendicular (transverse) to the weld shall be conducted on each wold using a $45^{\circ} \pm 2^{\circ}$ shear wave. This examination shall be conducted by placing the search unit adjacent to the weld with the beam directed along the weld. The entire length shall be scanned with the search unit sound bean directed in this manner. The search unit shall then be turned $180^{\circ}$ and the scan repeated. Calibration for these examinations shall be in accordance with Paragraphs 6.1 .2 and 6.1 .4 .

\subsection{Postexamination Cleaning}

Arrangerents shall be made with the customer for postexamination removal of couplant materials.

\subsection{RECORDING CRITERIA}

Ultrasonic reflectors producing a response $50 \%$ or greater of the reference level shall be recorded on the appropriate-SwRI Examination Record.

Indications producing a response $20 \%$ or greater of the reference level and suspected by the examiner to be other than geometrical in nature shall be recorded and investigated by a Level II or Level III exariner to the extent necessary to determine the shape, identity, and location of the reflector.

Indications $100 \%$ or greater of the reference level shall be investigated by a lcvel II or Level III examiner tc the extent sesessary to determine the

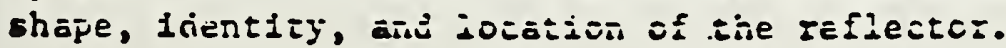

Indications $20 \%$ or greater of the reference level investigated and found to be other than geometrical in nature shall be reported to the customer for evaluation.

Any scanning, limitations shall be recorded. 



\title{
SOUTHWEST RESEARCH INSTITUTE
}

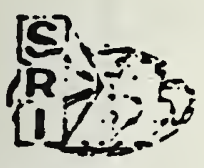

\author{
OPERATING PROCEDURE
}

SWRI-LIDT-800-58

Revision 0

Auguse 1978

Page 14 of 23

\subsection{RECORDS}

The customer shall receive copies of documents generated in accordance with this procedure in the exardnation report.

Permanent documents generated in accordance with this procedure shall be stored and retained as a portion of the examination report. The examination report shall be stored by the Manager of Support Services, Quality Assurance Systems and Engineering.Division, in the Data Storage Facility for the period specified by the contractual agreement with the customer.

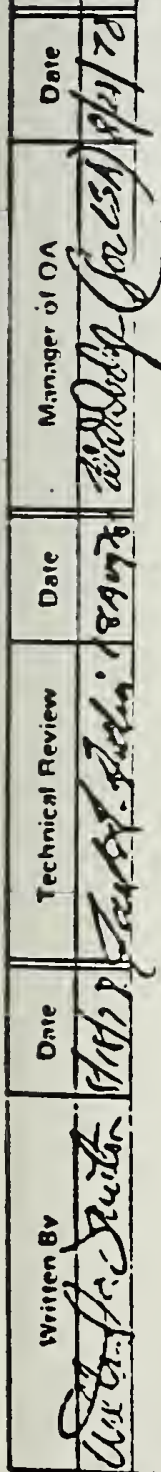




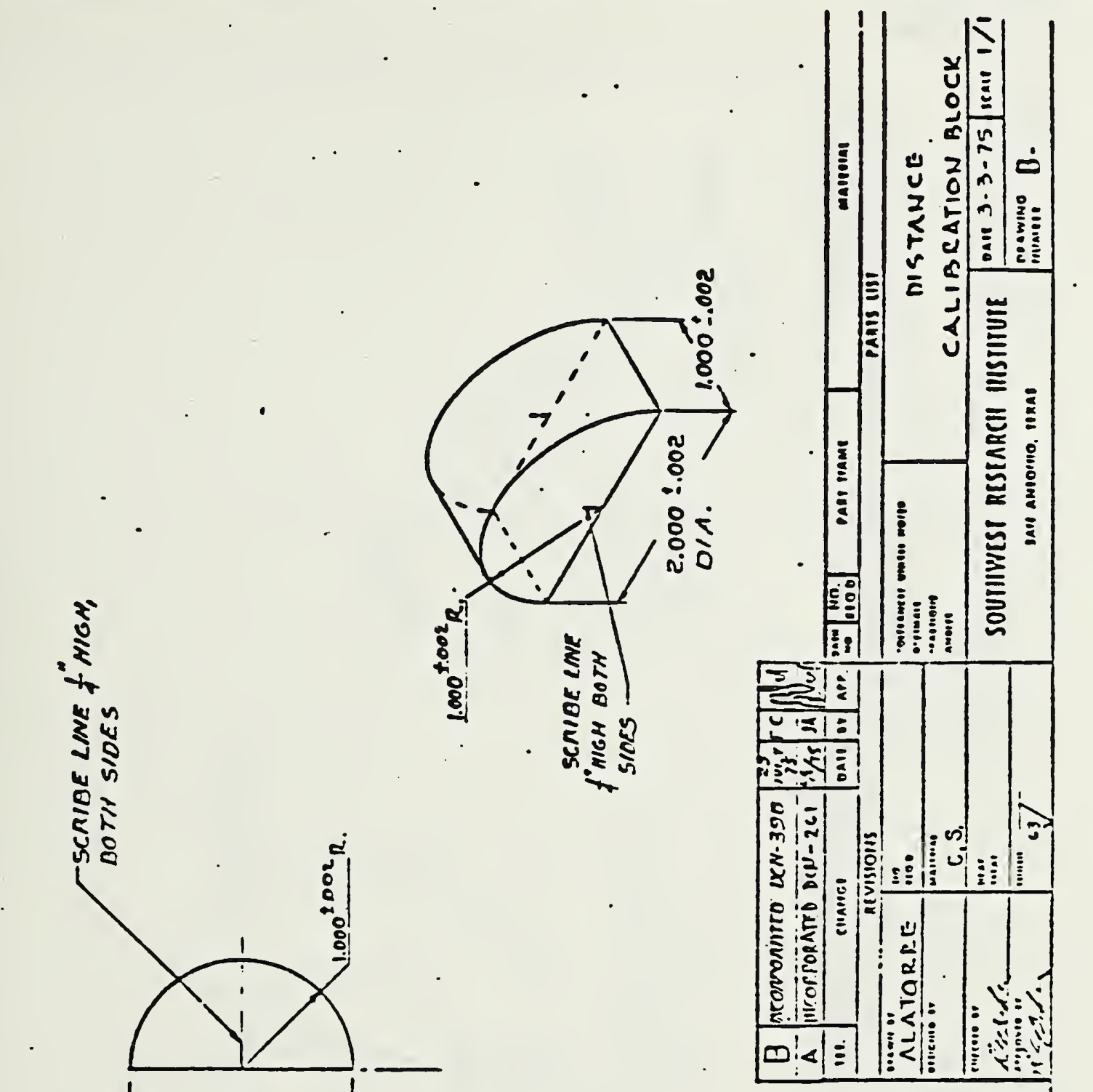

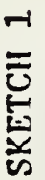



Append1X A - SwRI-NDT-800-58

Revision 0

August 1978

Page 16 of 23

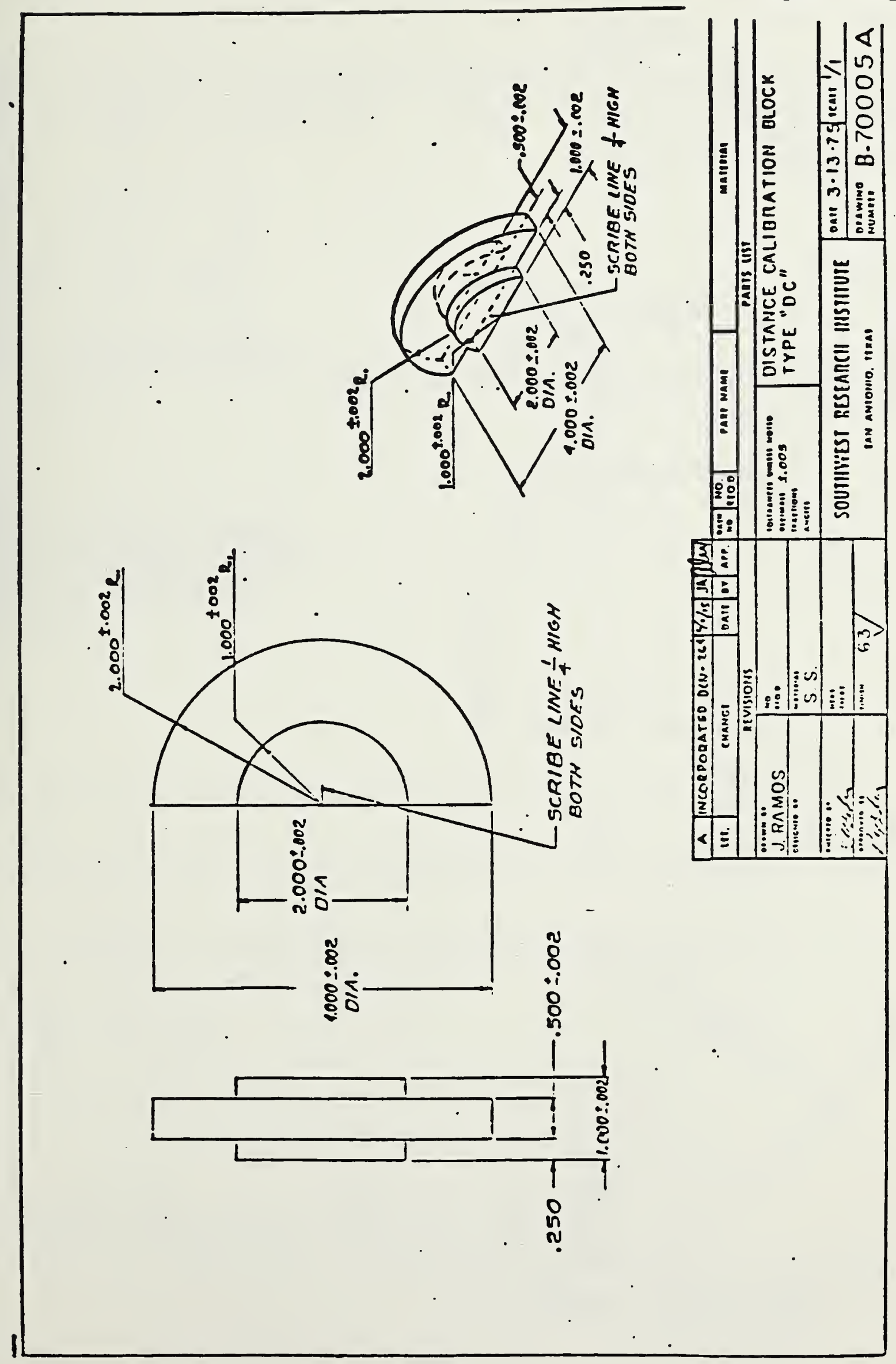





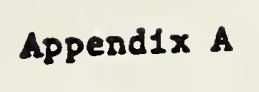

SWRI-NDI-800-58

Revision 0

August 1978

Page 17 of 23 .

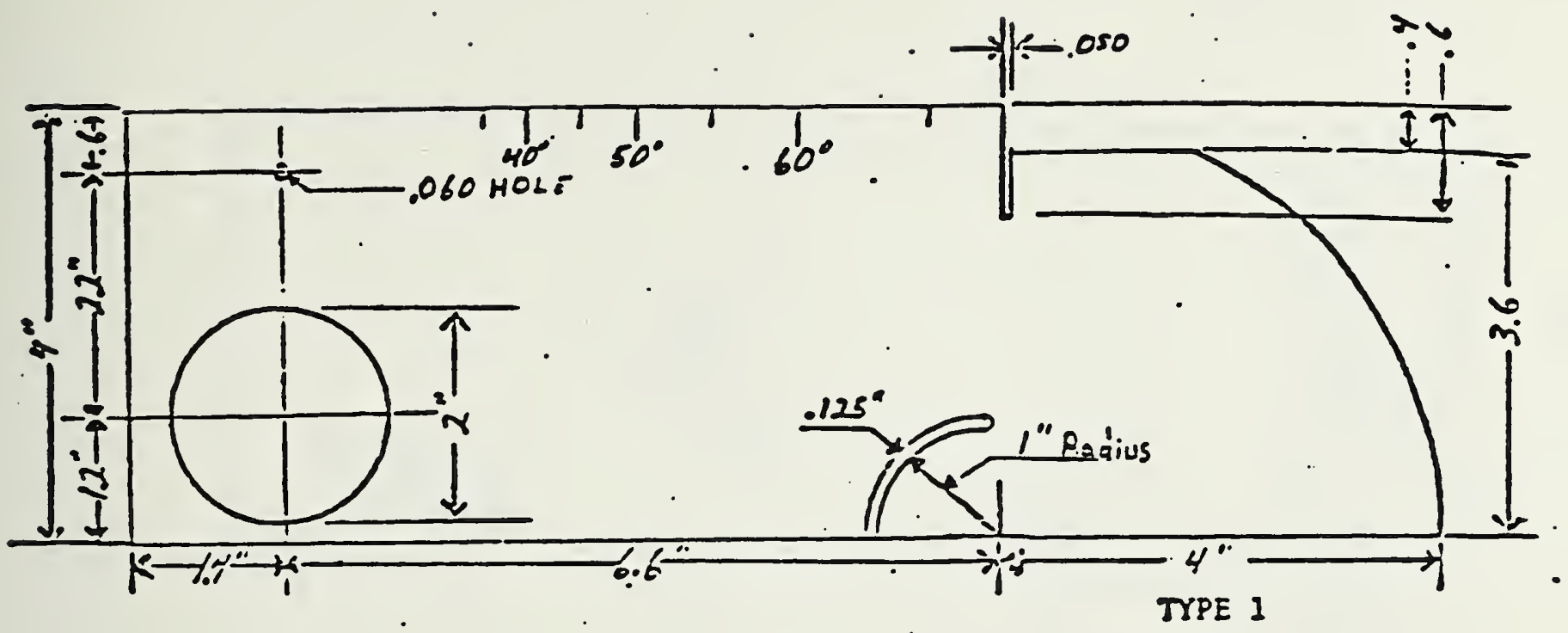

NOTE:

OTHER IIH APPROIED REFERENCE

BLOCKS VIITH SLICHTI: DIFFERENT

DINENSICNS OR DISTANEE CAII-

BPATION SLOT FERTLEES ARE

FENU:USEIEIE.

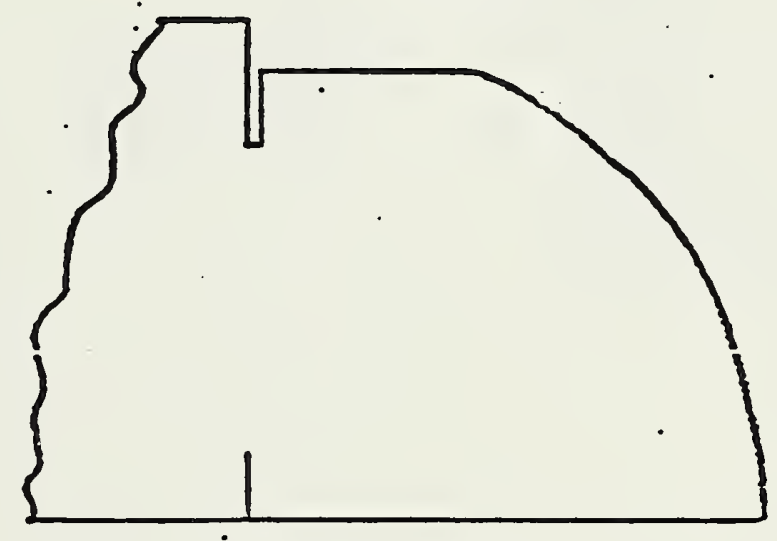

TIPE 2
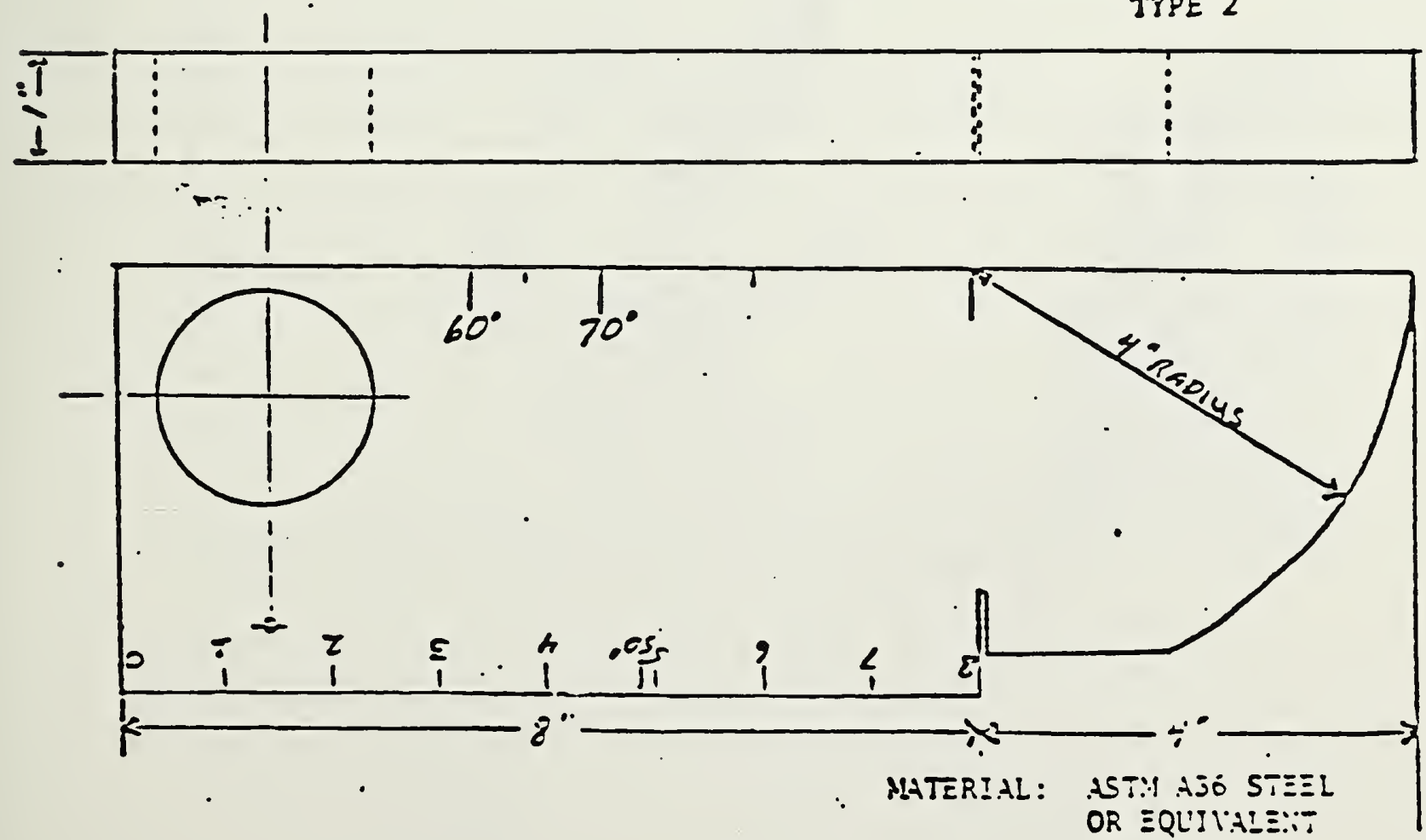

Page 18 of 23

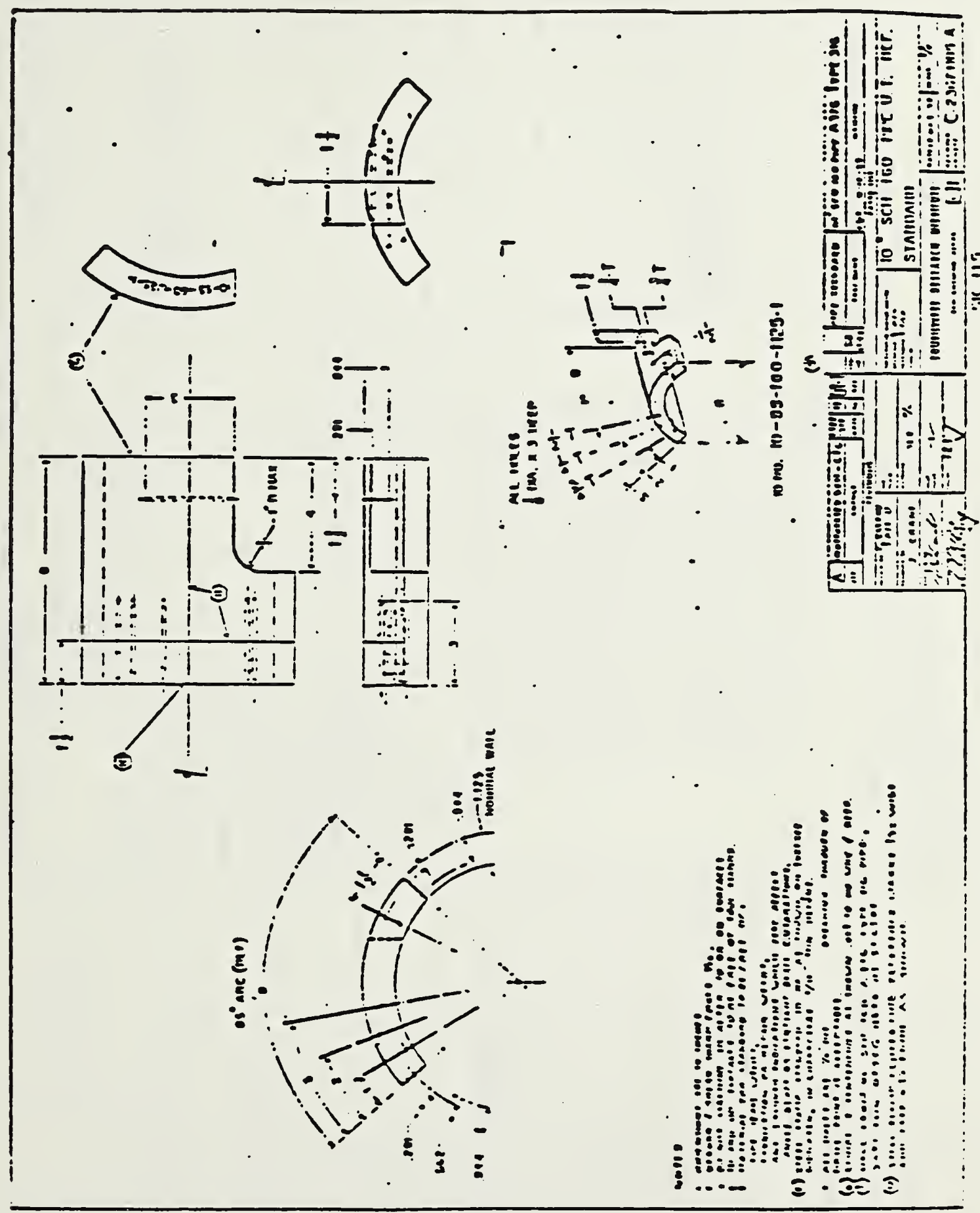

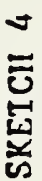





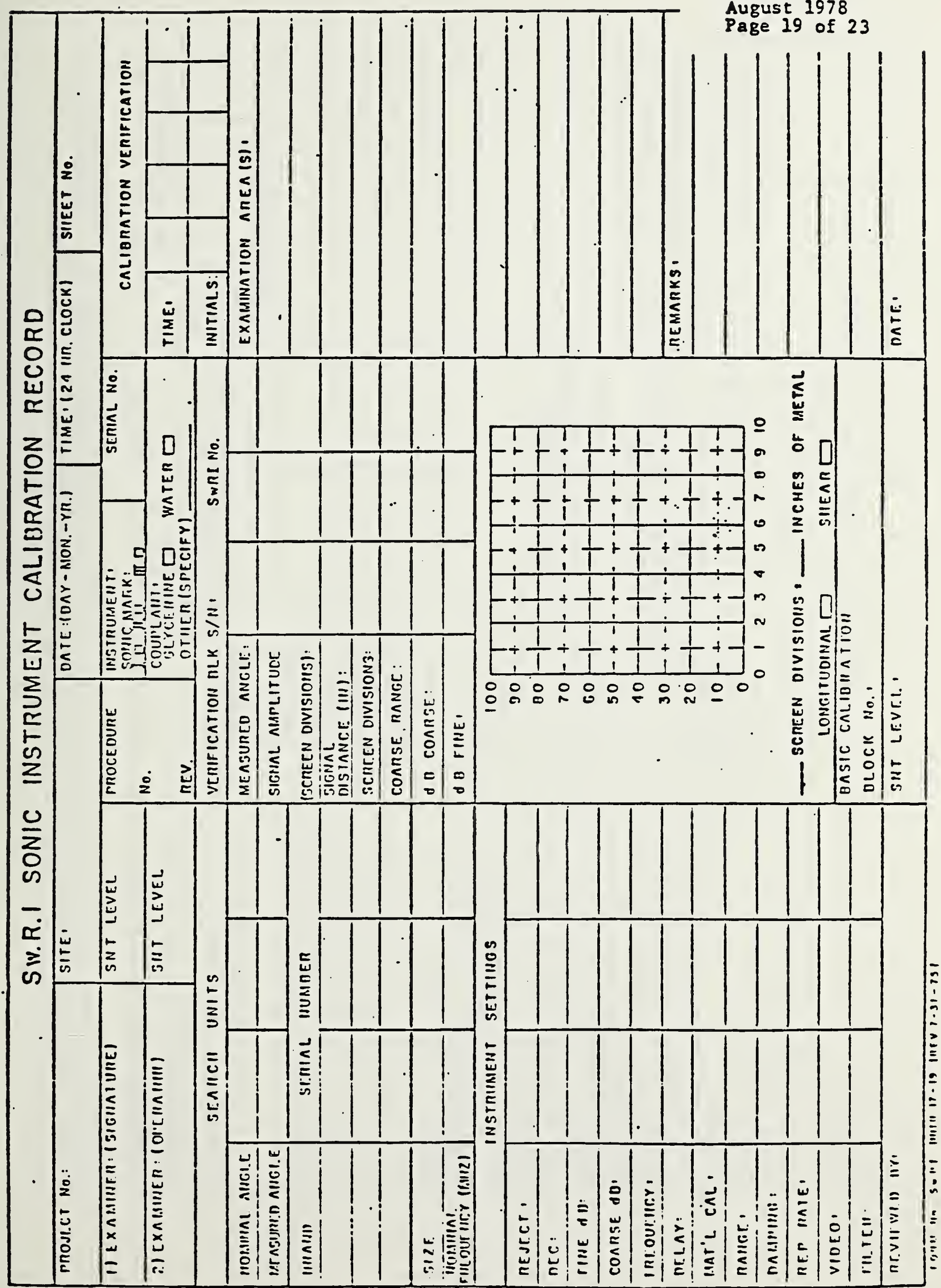





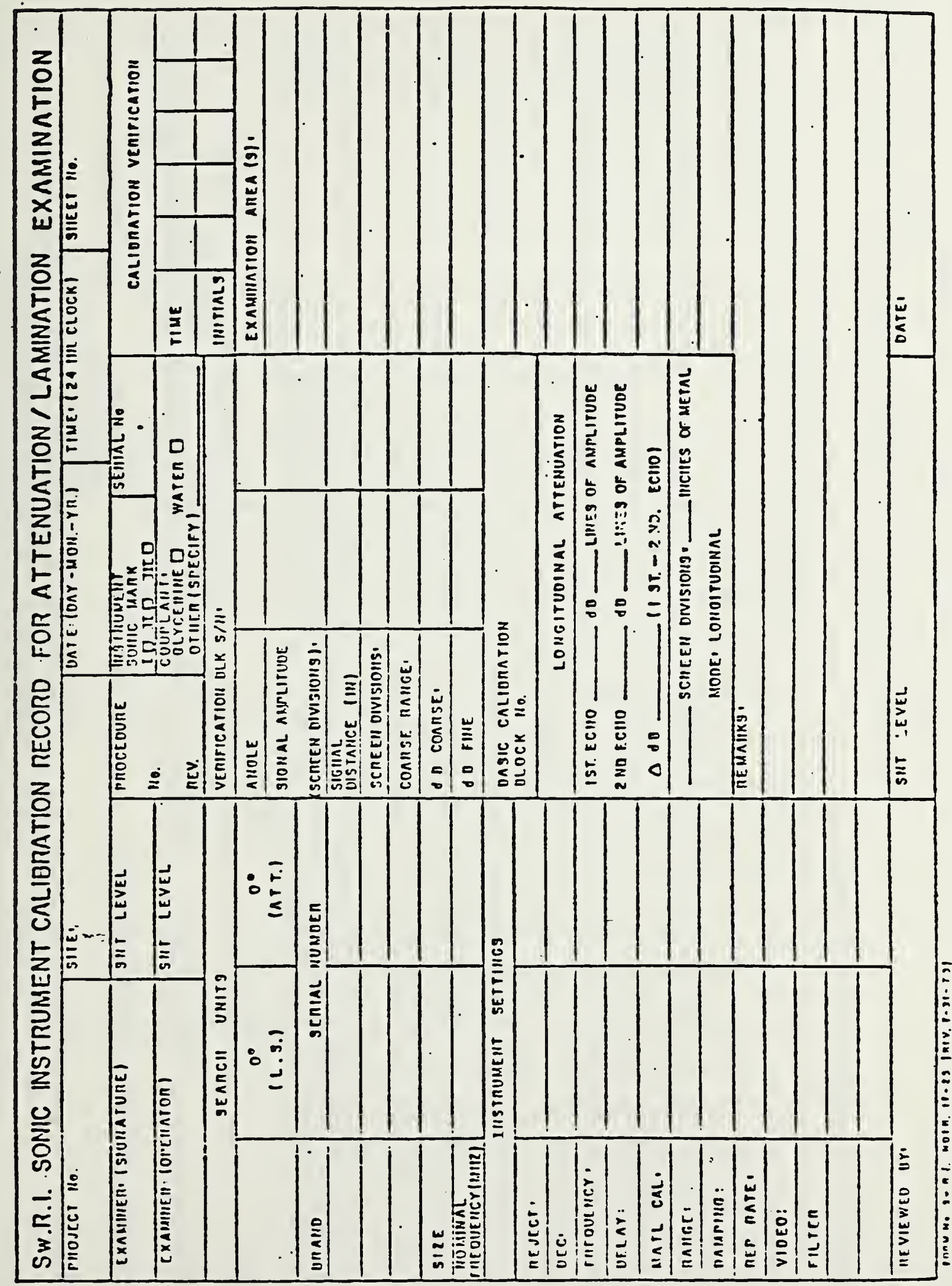



Append1x A SwRI-NDT-800-58

Revision 0

August 1978

Page 21 of 23

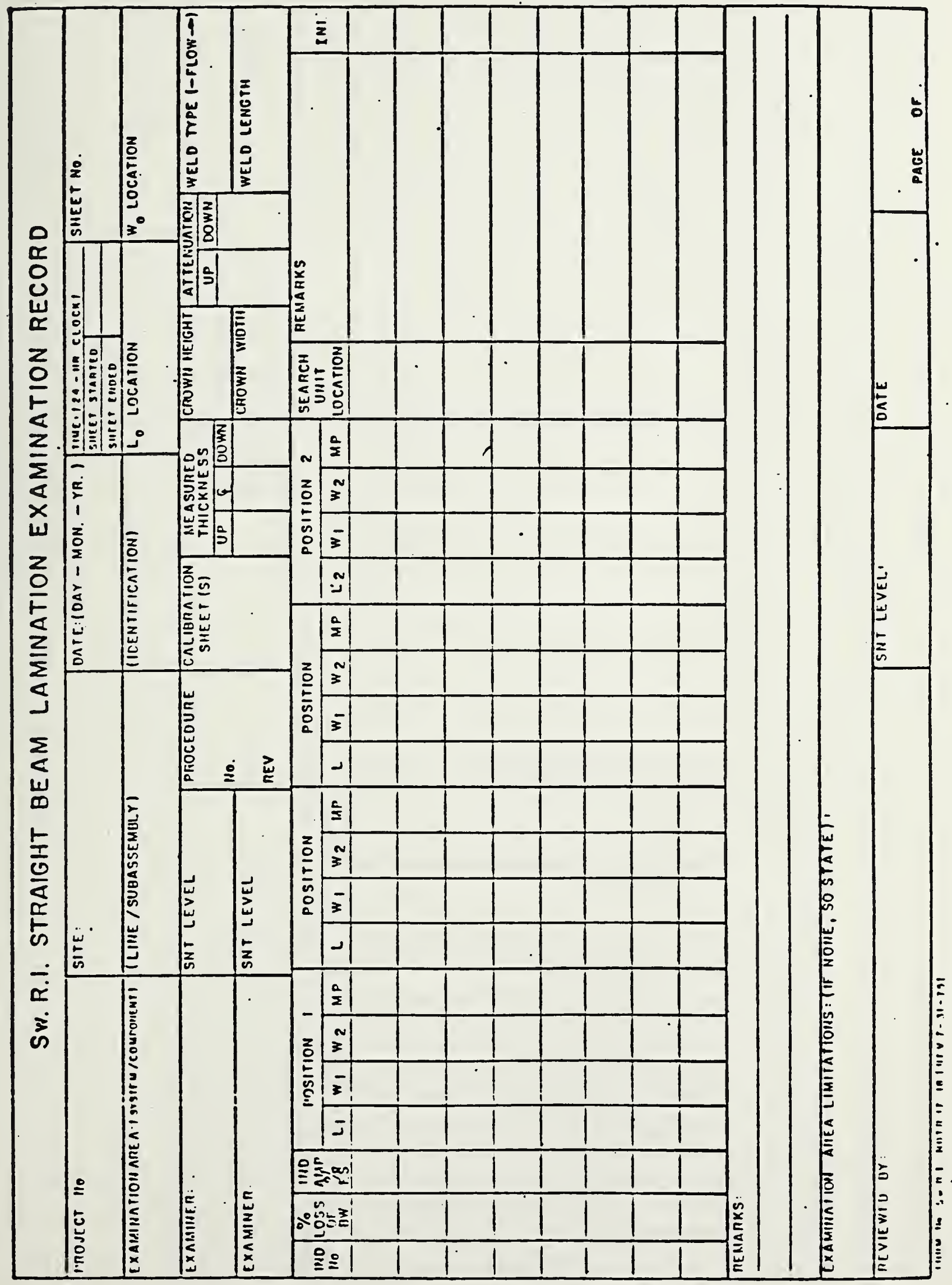



Append1x A SwRI-NDT-800-58

Revision 0

August 1978

Page 22 of 23

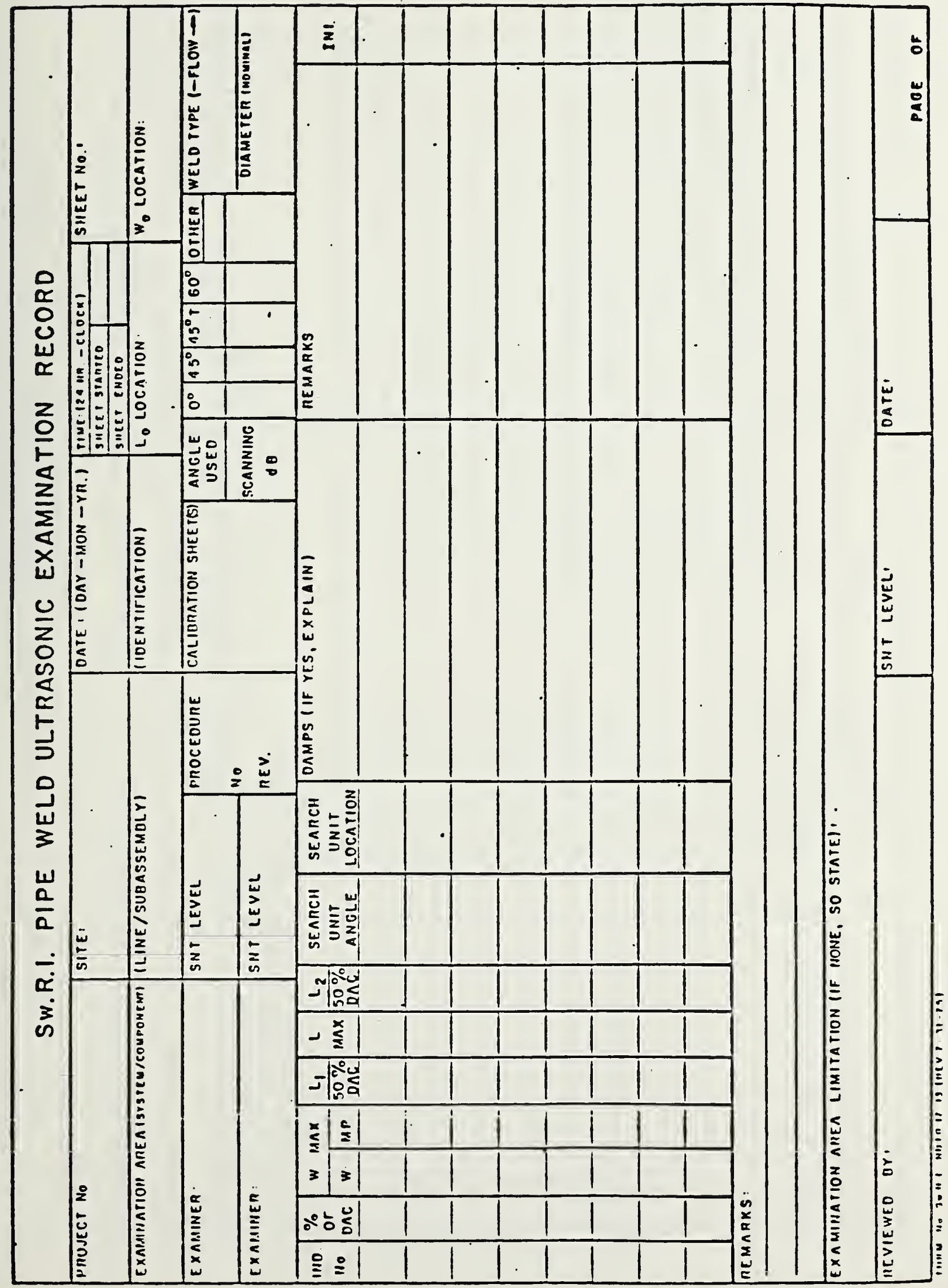



Append1X A, SWRI-:NDI-300-53

Revision 0

Augus: 1978

Paze 23 of 23

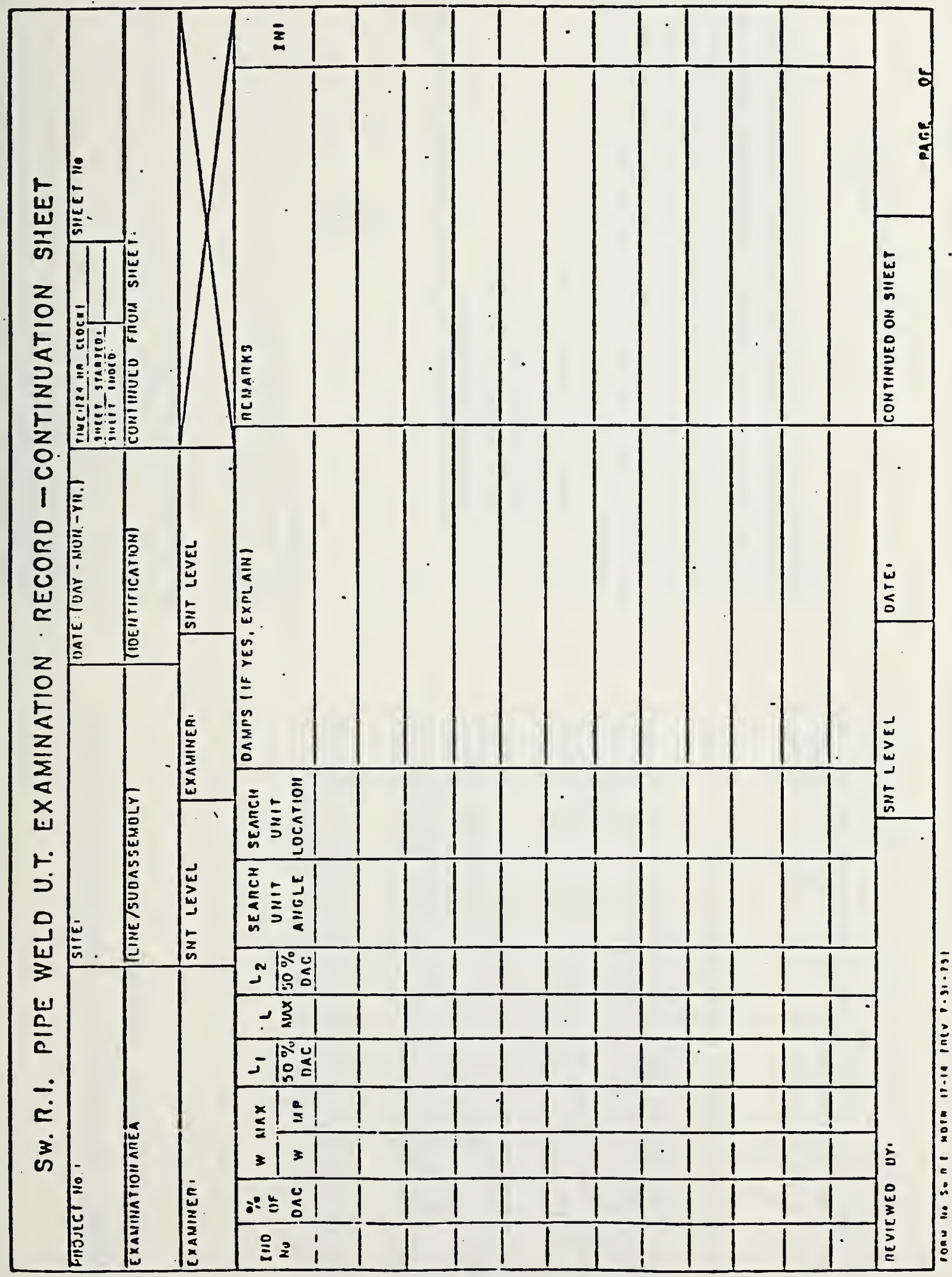





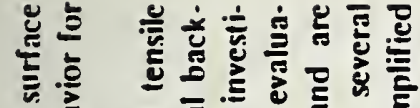
每

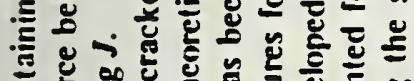

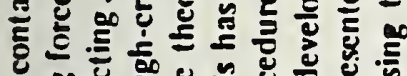

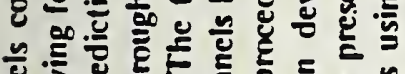

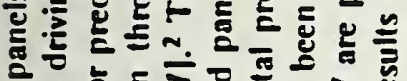

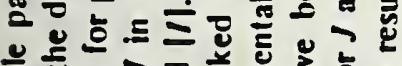

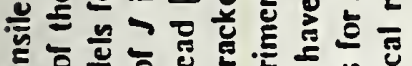
(1)

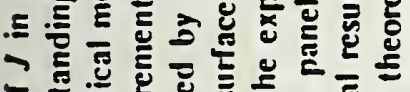

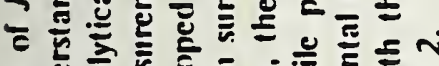

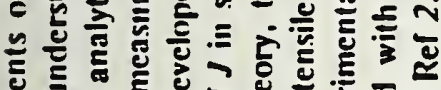

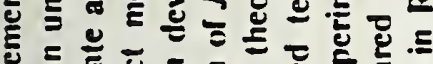

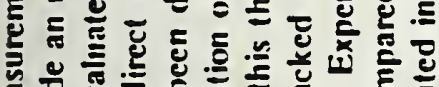

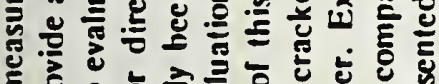

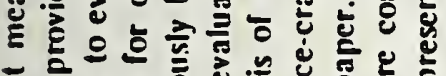
可

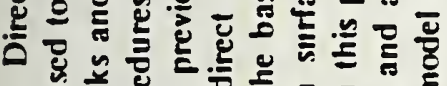

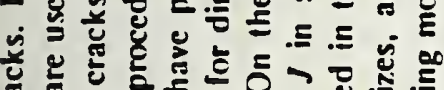

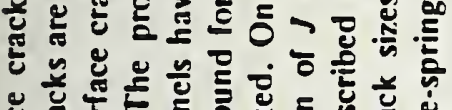

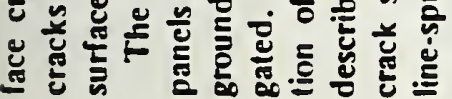

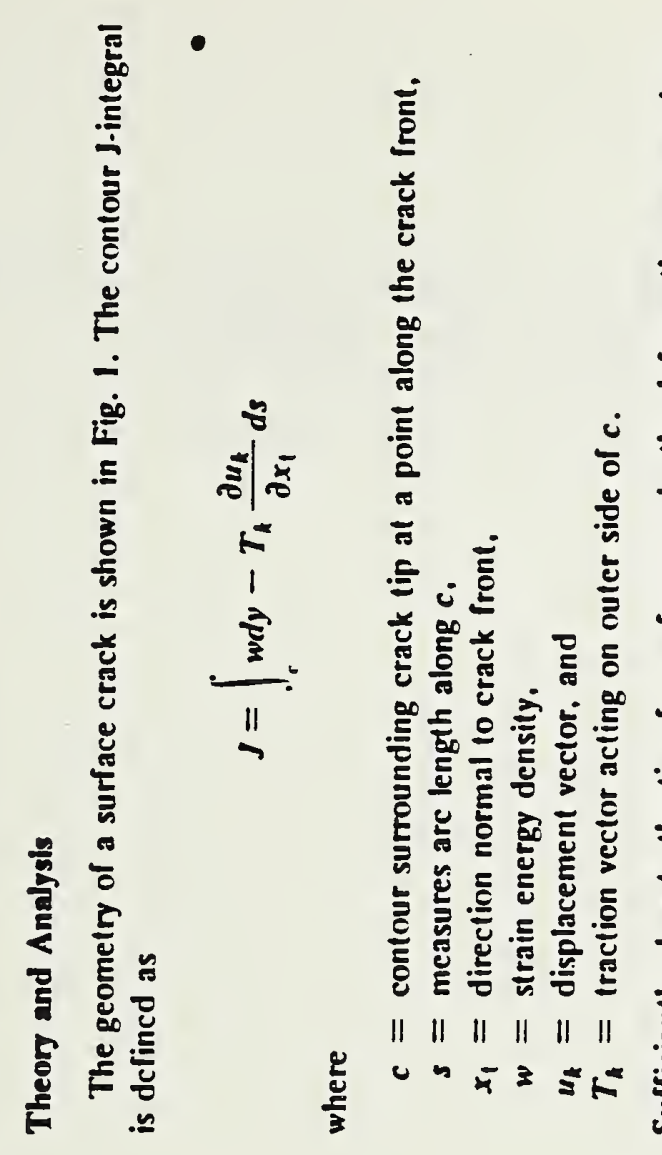

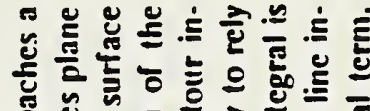

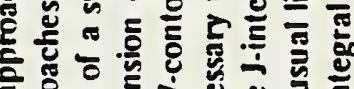

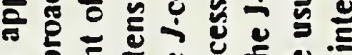

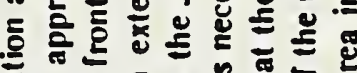

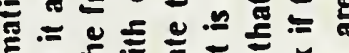

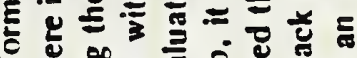

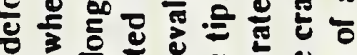
等

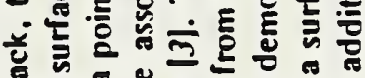

政言

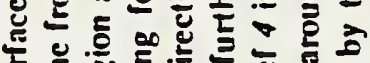

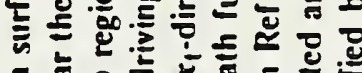

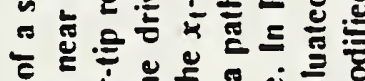

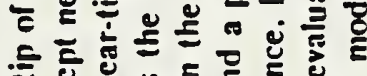

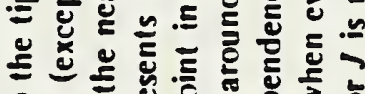
s.

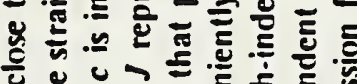

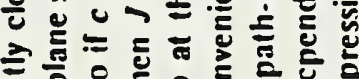

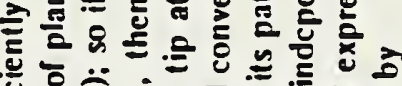

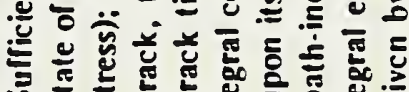

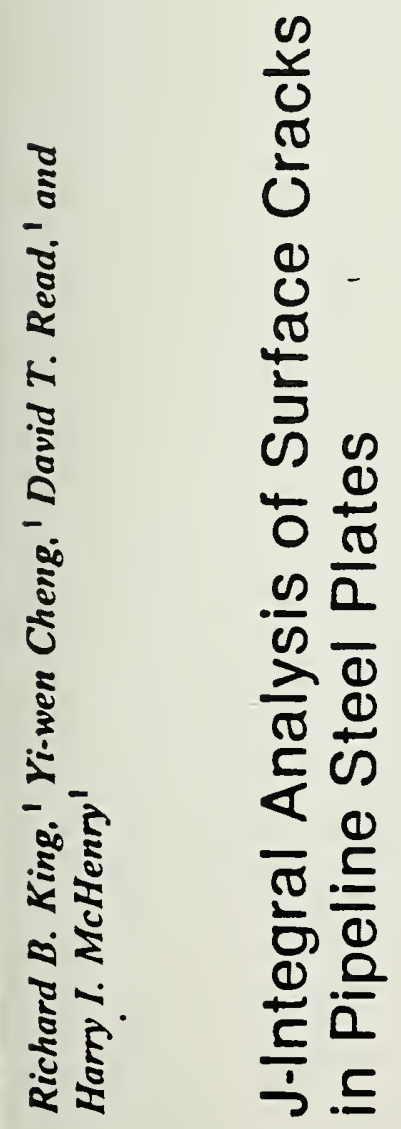

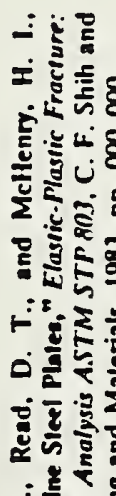

$\frac{1}{3} \times$

s.t.

the $\therefore$

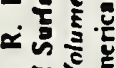
$\therefore$ ㅎํ ज完

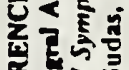

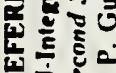

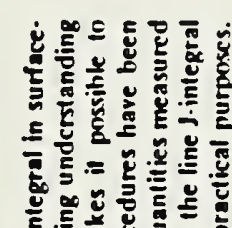

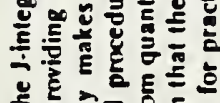

至

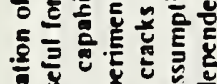

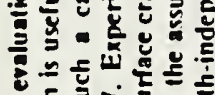

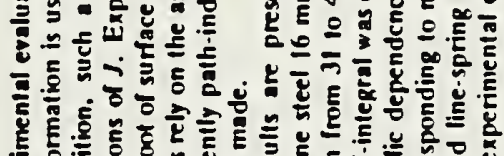

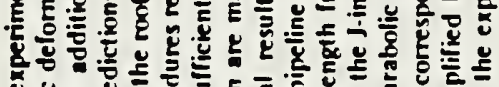

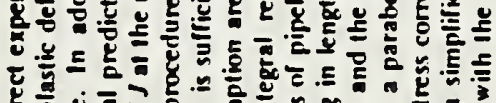

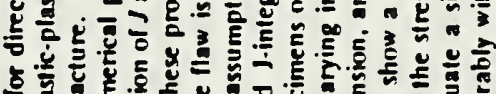

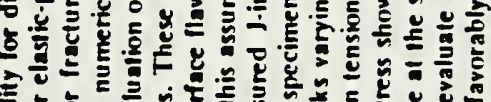

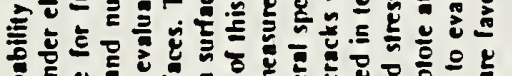

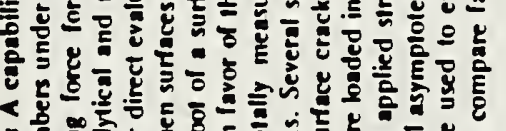

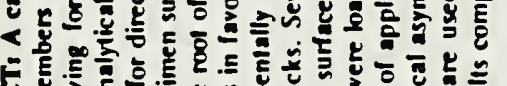

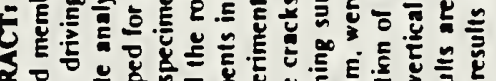

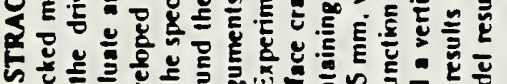

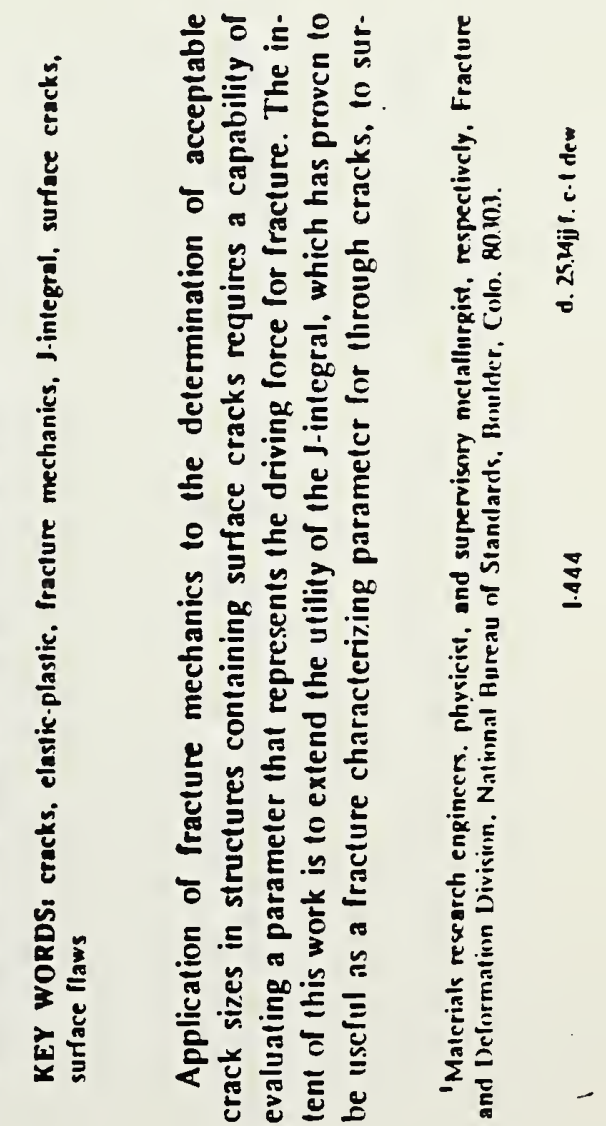





\section{APPENDIX G - J-Integral Analys is of Surface Cracks in Pipeline Steel Plates}

Richard B. King, Yi-wen Cheng, David T. Read, and Harry I. McHenry 



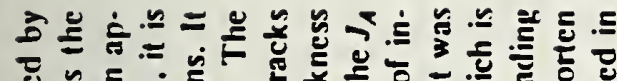

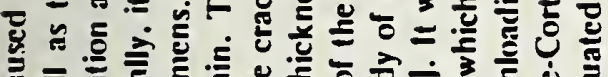

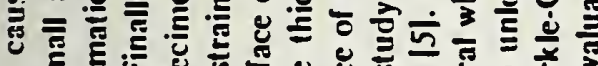

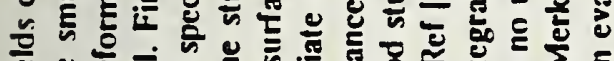

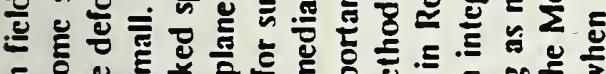
б

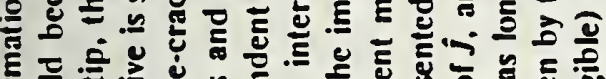

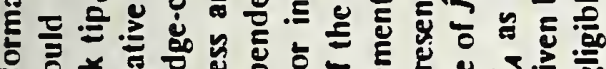

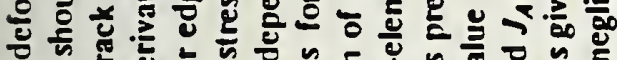

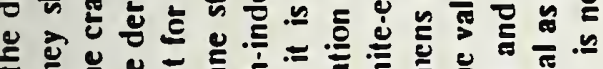

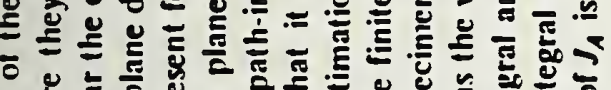

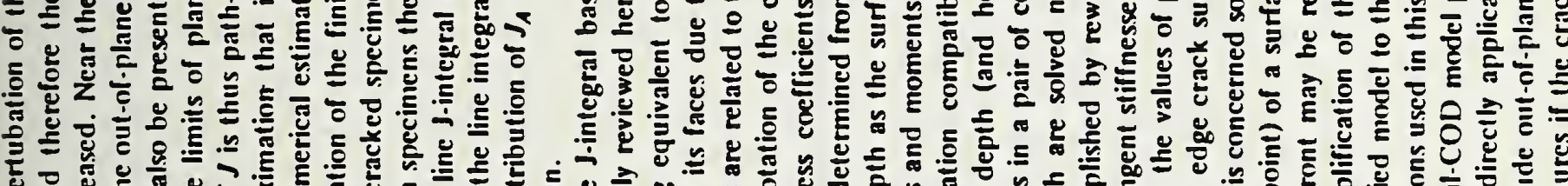

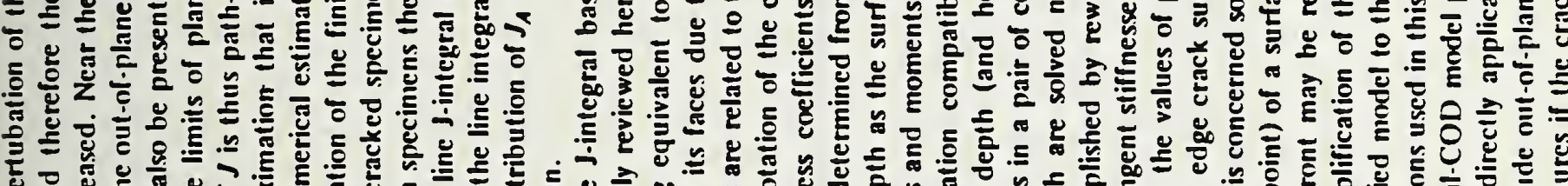

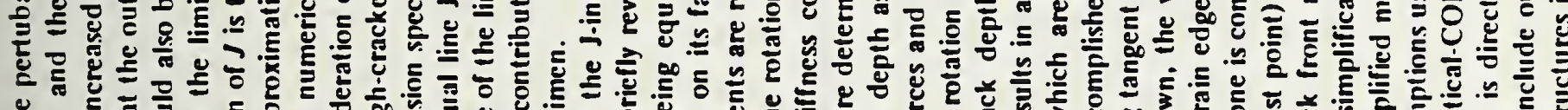

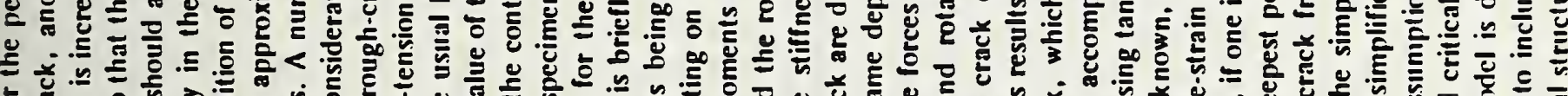
¿ б巳巳

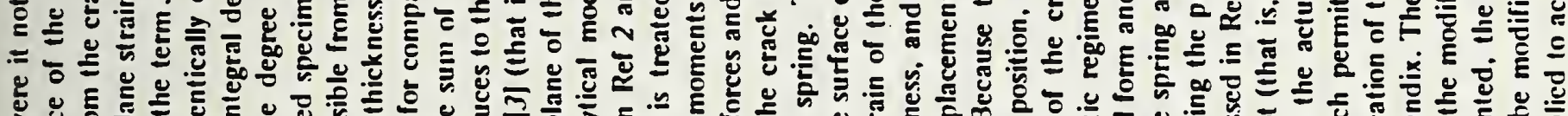
范

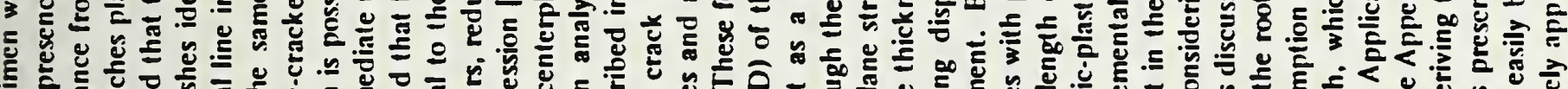

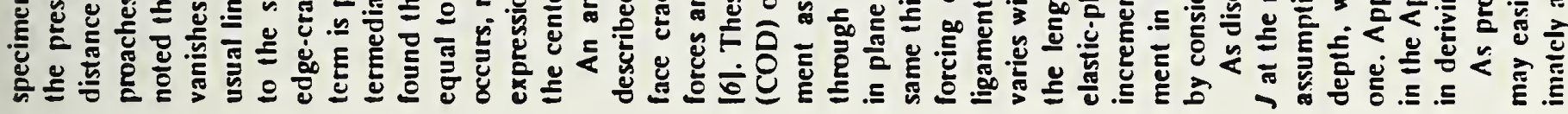

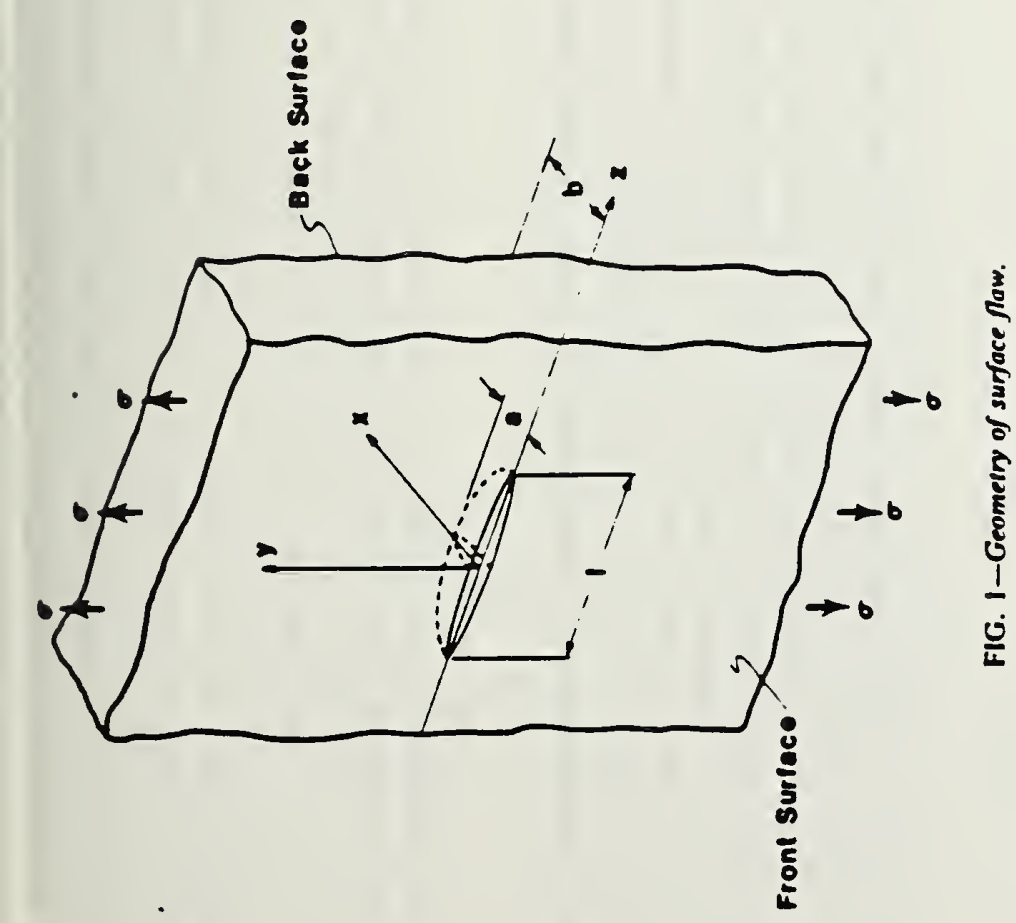

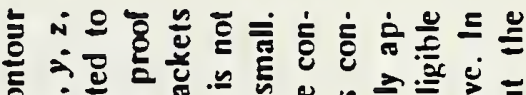

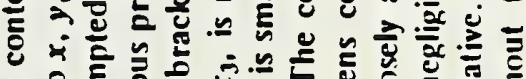

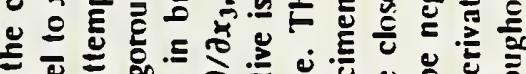

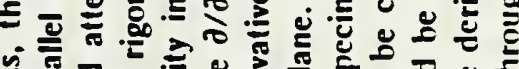

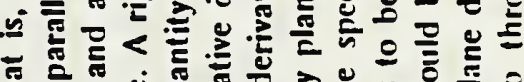

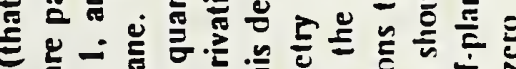

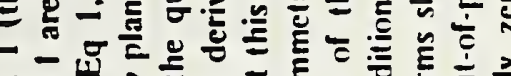

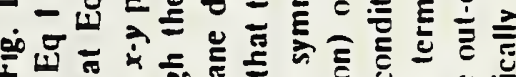

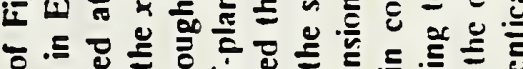

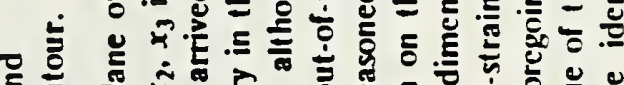

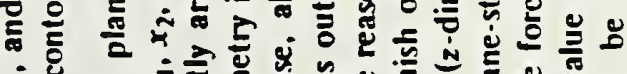

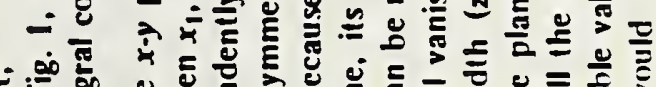

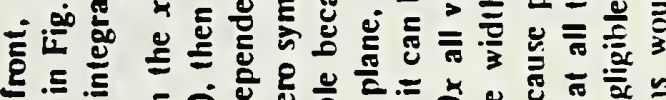

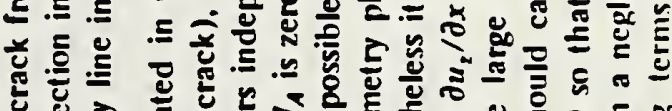

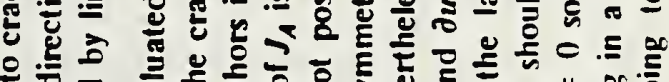

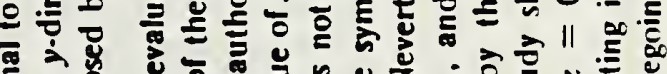

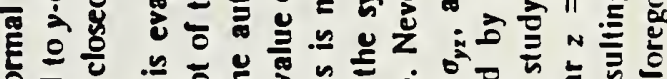

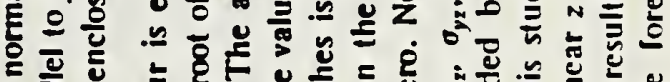

西 若 



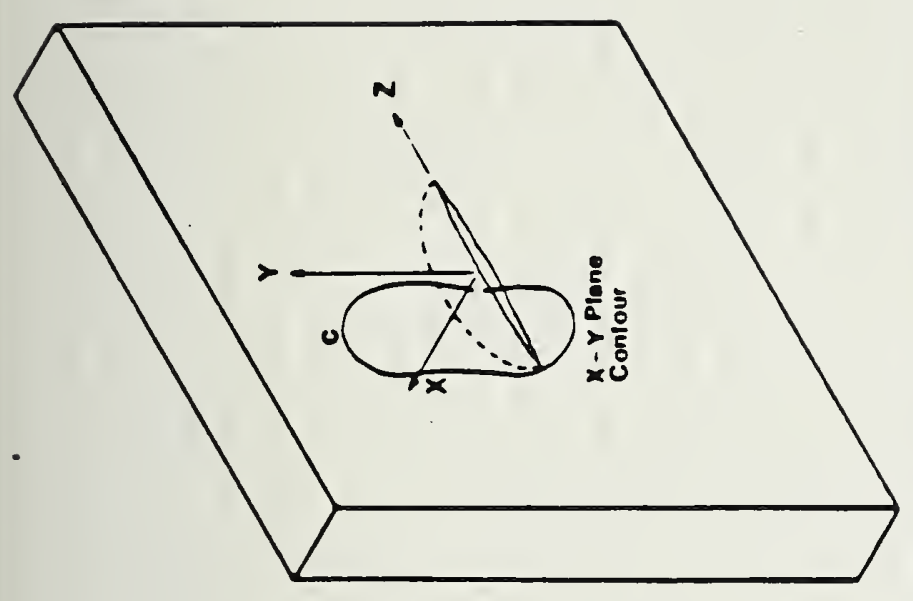

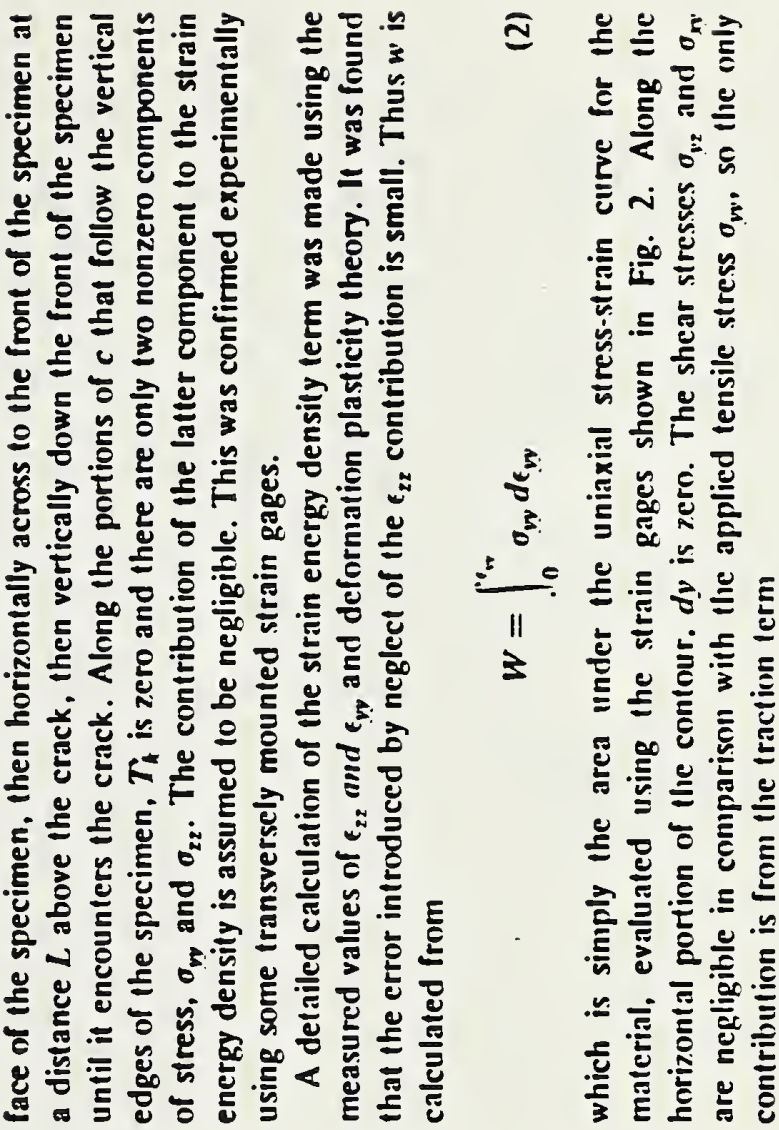

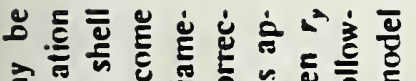

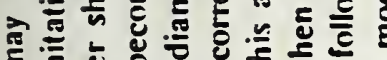

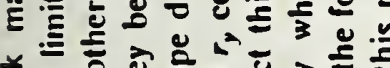

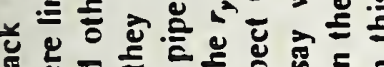
讨政 น

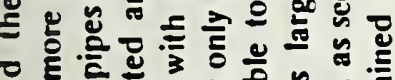

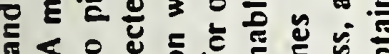

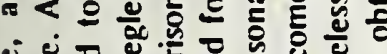

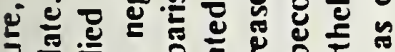

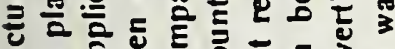

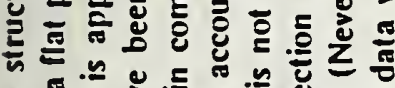

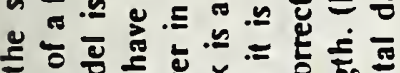

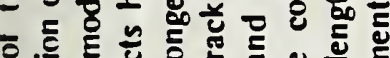
б 额: = 兰

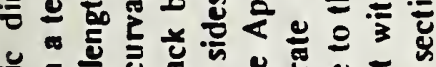

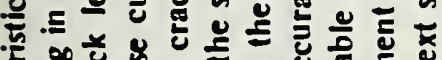

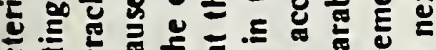

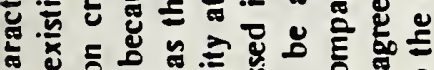
斻

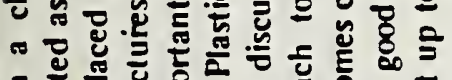

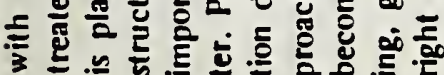

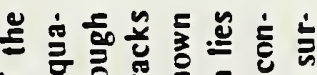

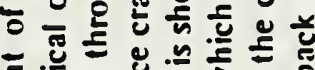

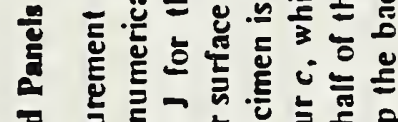
ปั

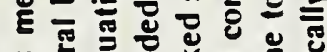

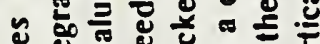
흘 혼

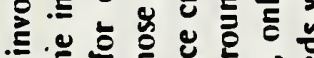
ত क्ष 들

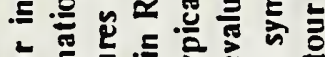
늘

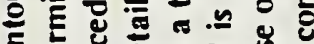
항 원 خั

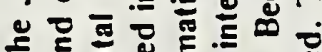
¿ E E Eั

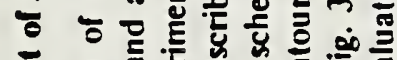

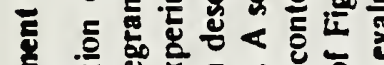
帚兘 满

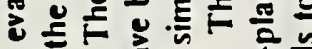
들

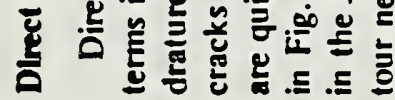

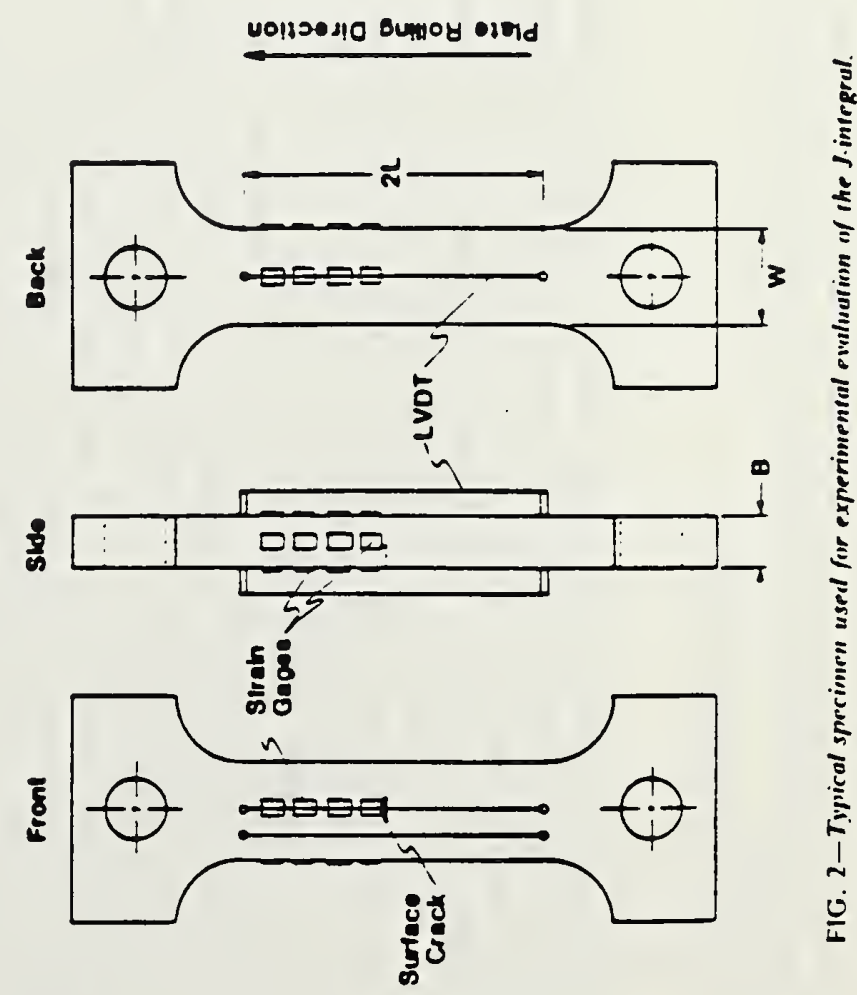





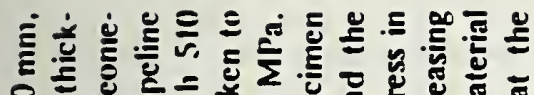
\&

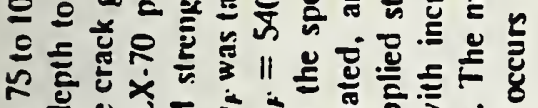

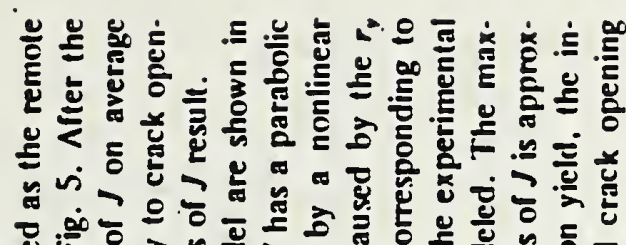
E 론

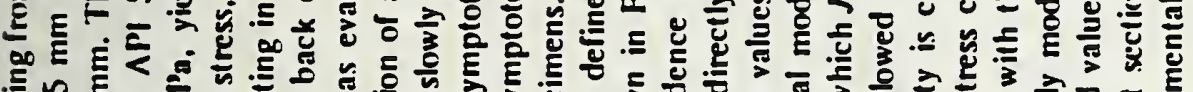

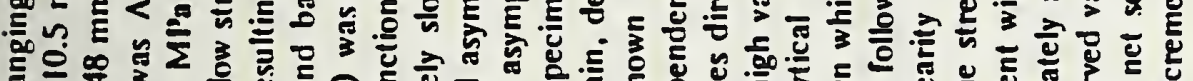
론을

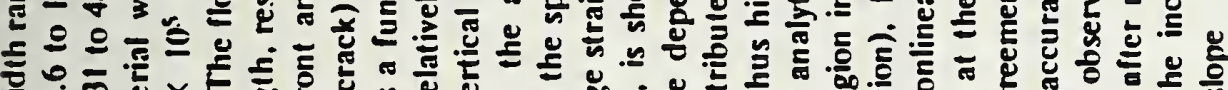

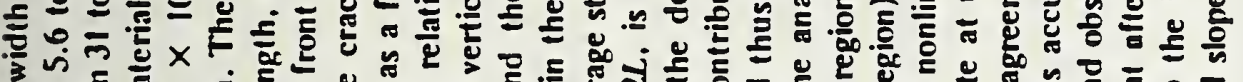

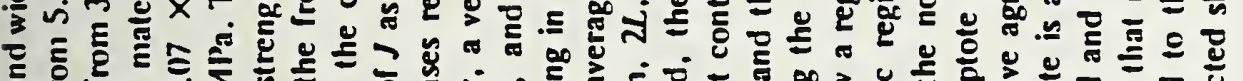

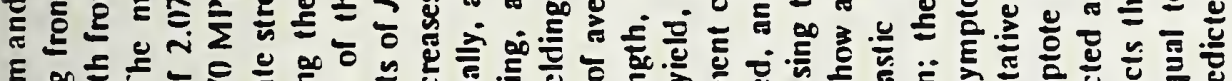

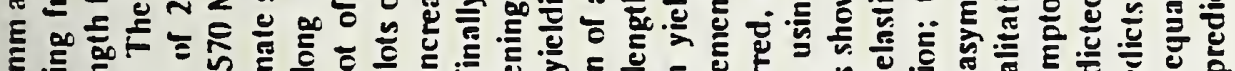

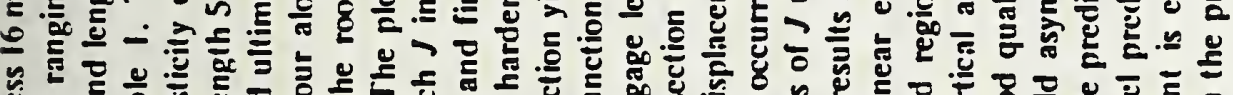
论

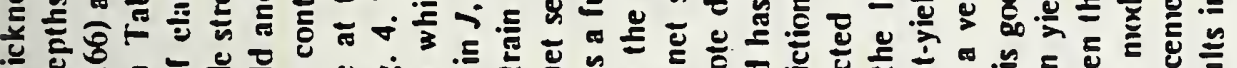

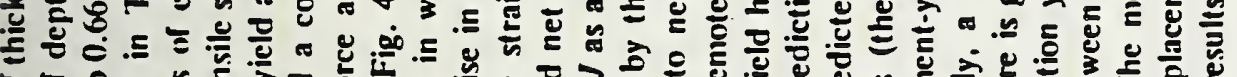

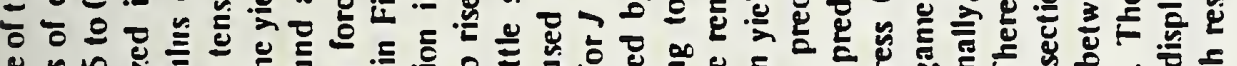

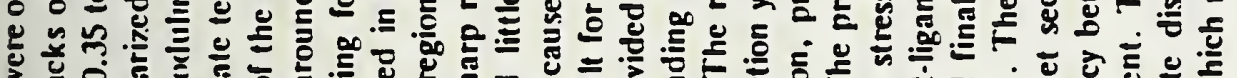

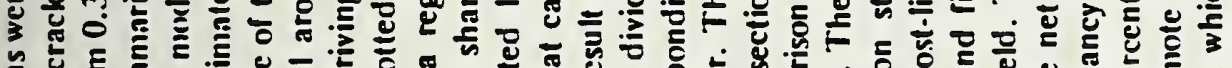

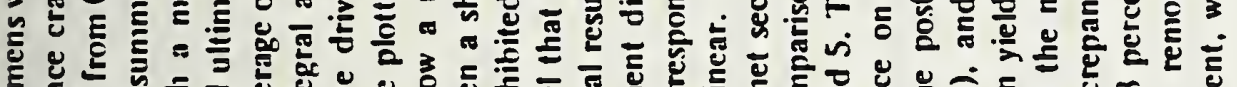

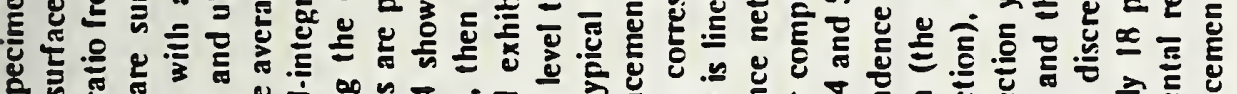

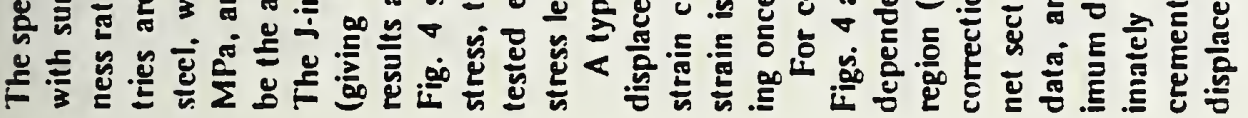

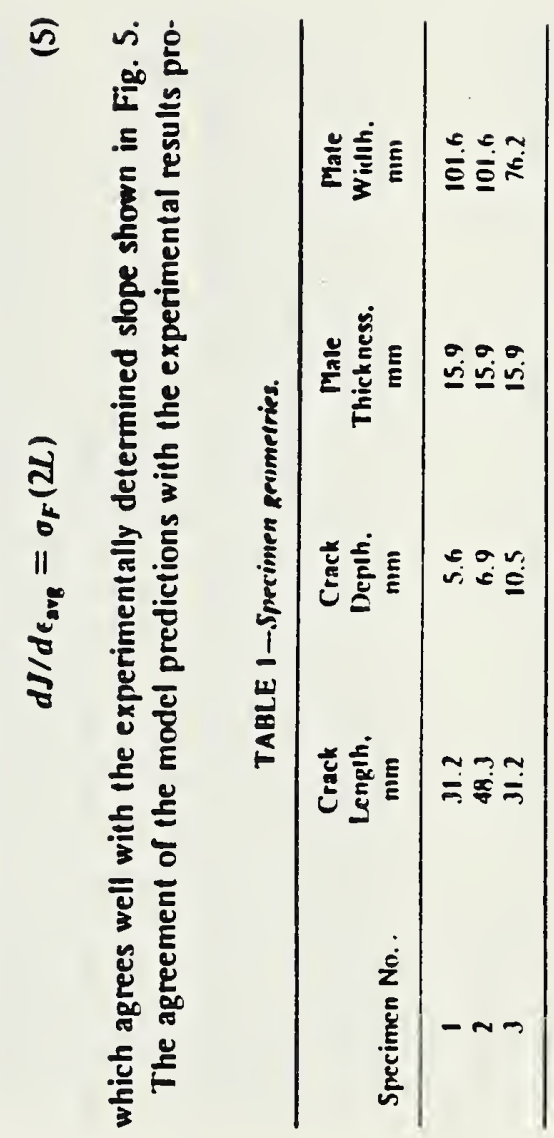

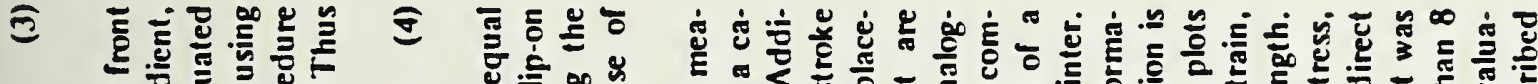

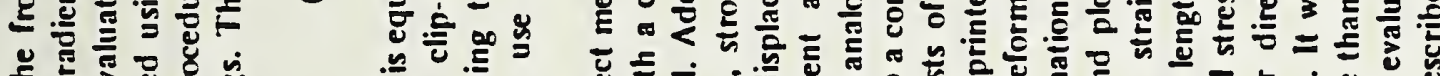

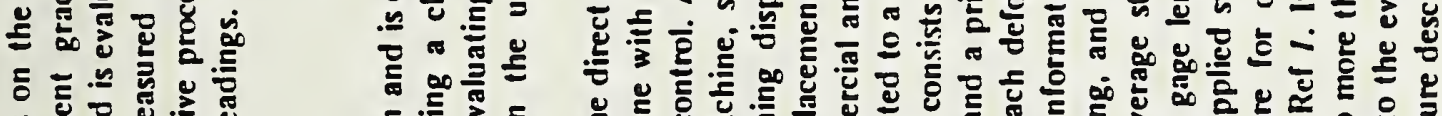

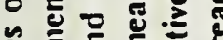

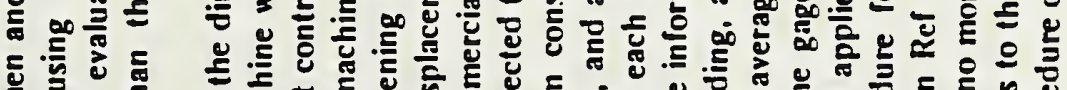

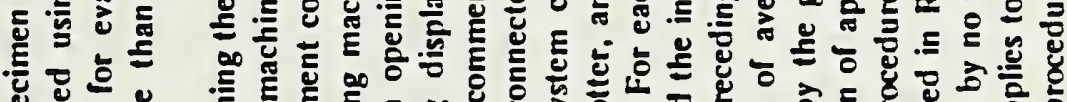

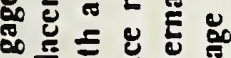
E 产突

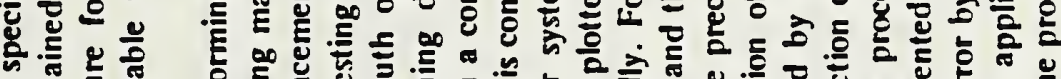

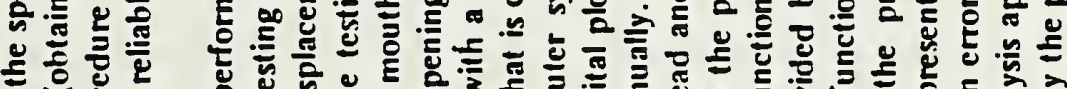

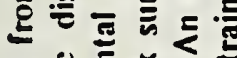

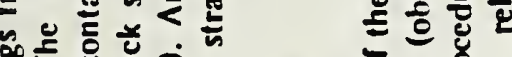

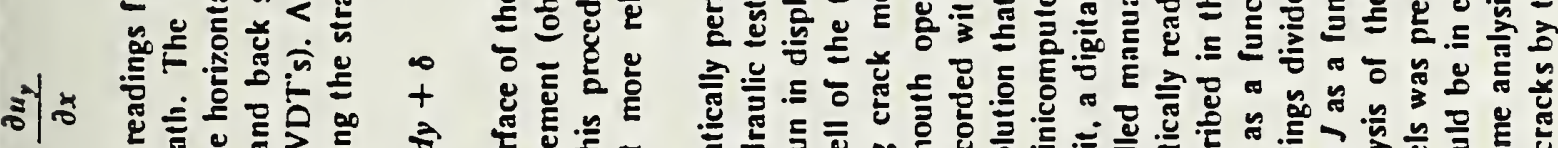

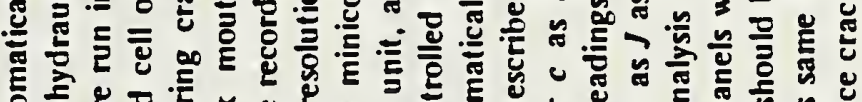

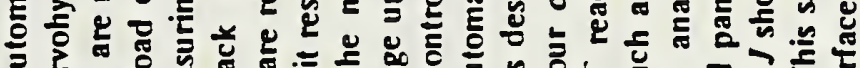

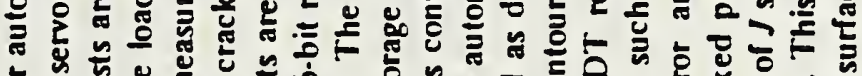

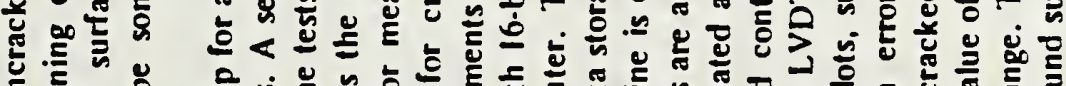

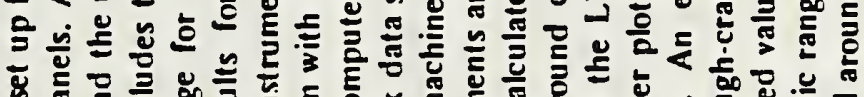

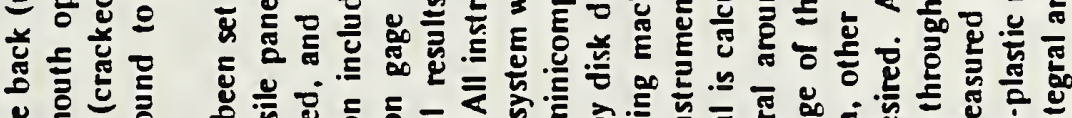
E 는

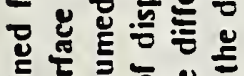

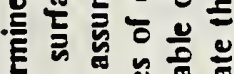

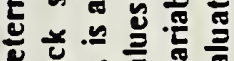

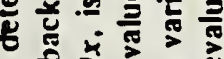

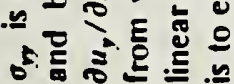

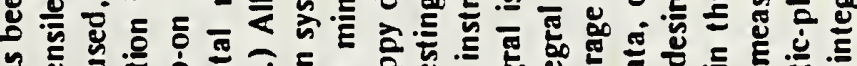

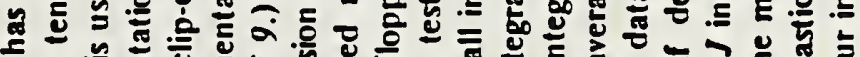
ธํㅡㄹ \&

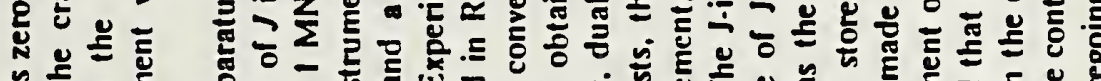
은

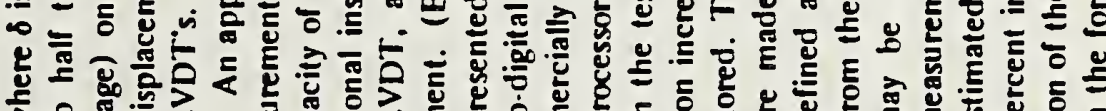

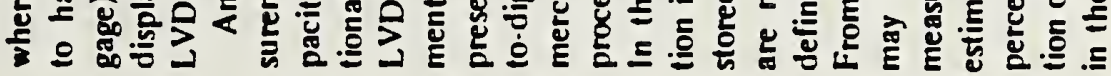

$\bar{x}$

这. ..

气

อั

过.

巳 :

它

至

..

远

놀

를

这

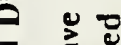

롤

言 苞

造 $\frac{E}{\bar{E}}$

区

ㅎํㄴ

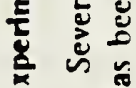





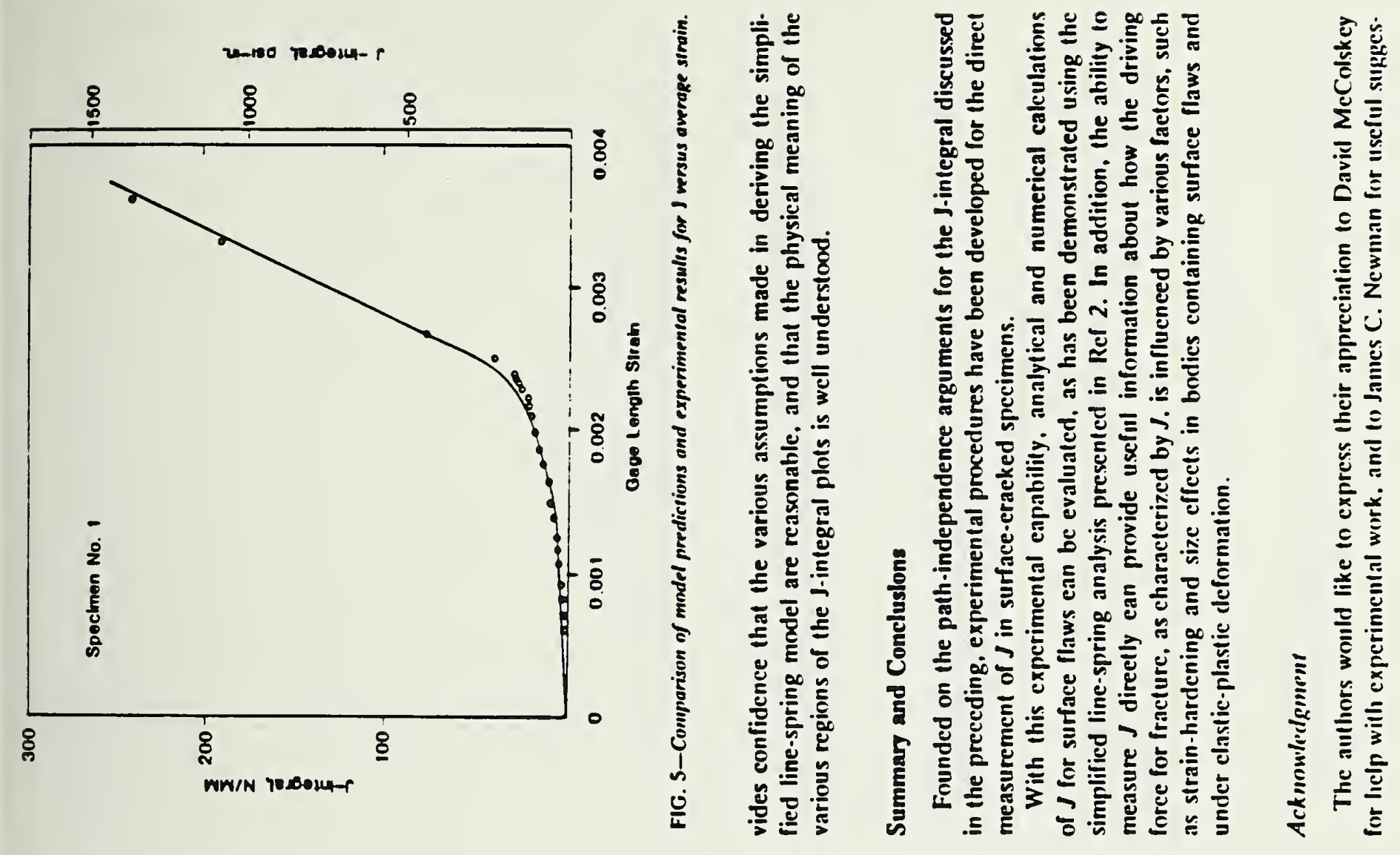

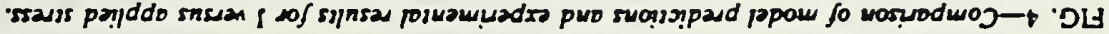

-an 'sesule

- an 'seguls

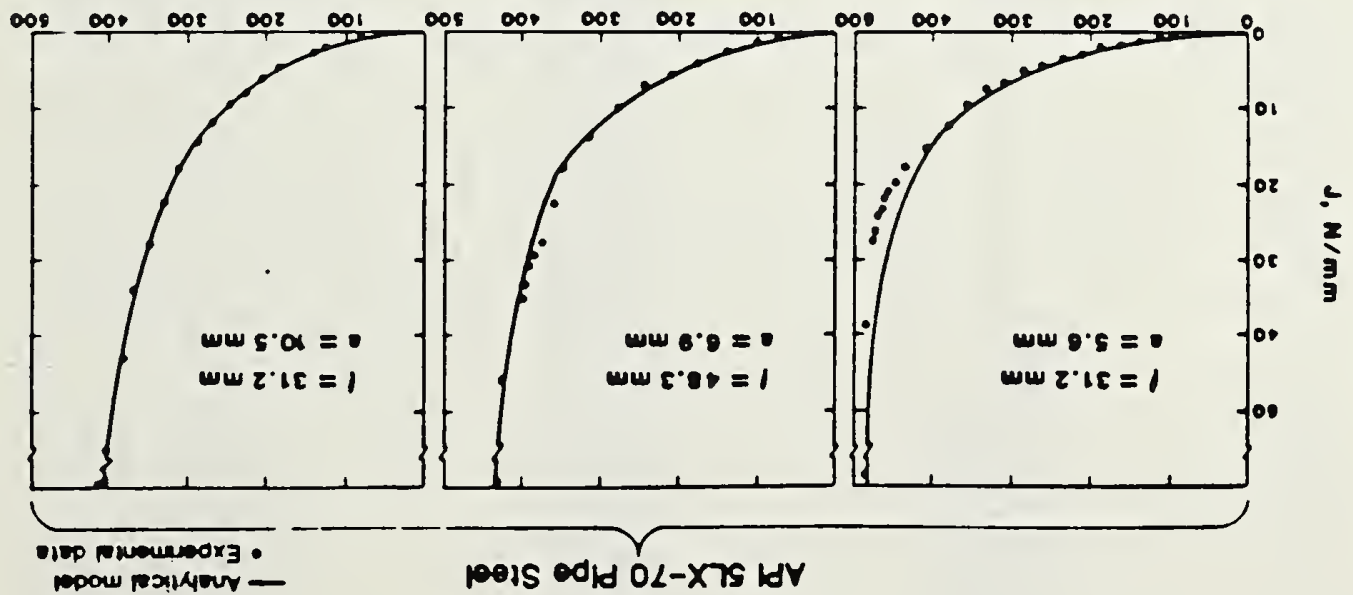




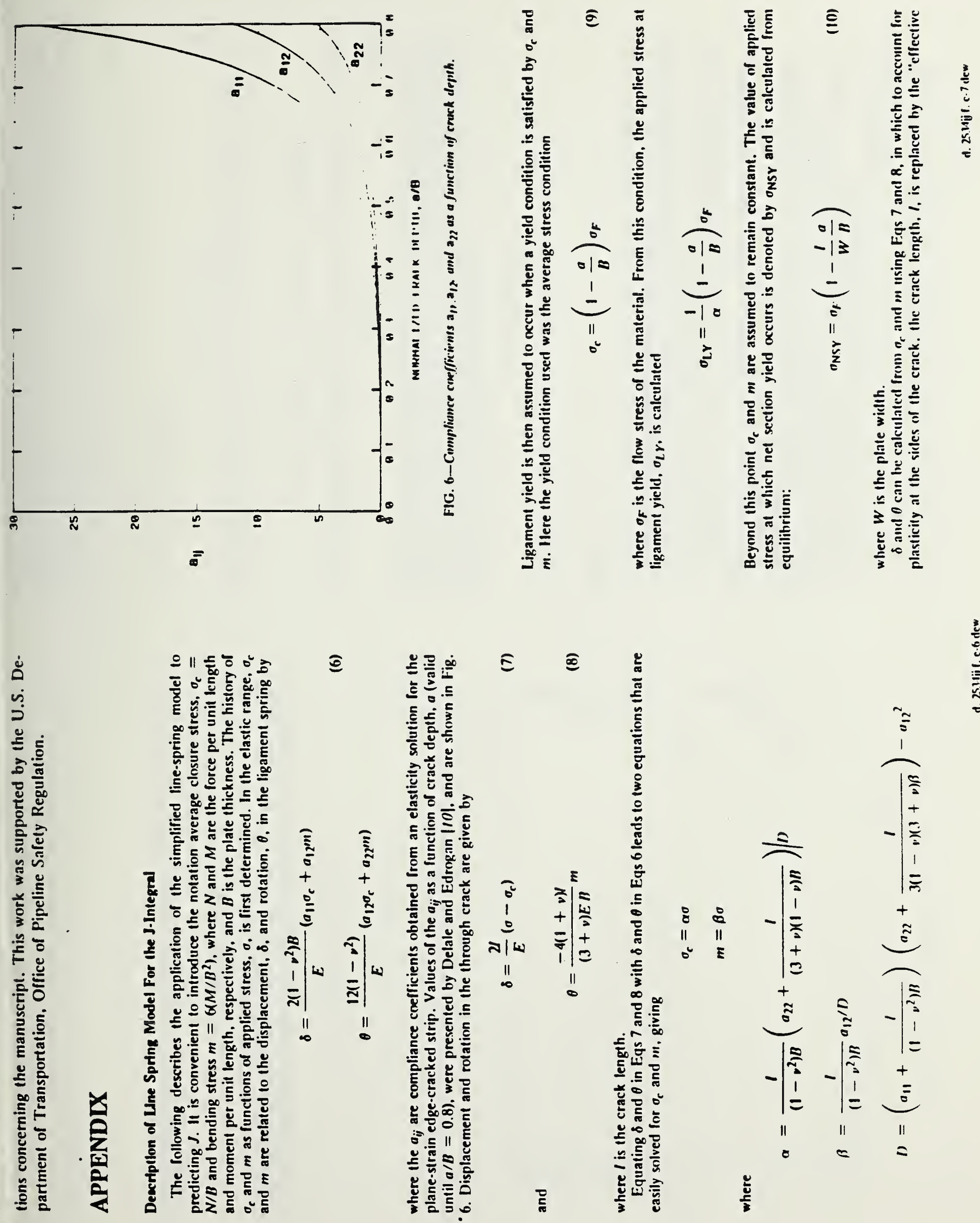


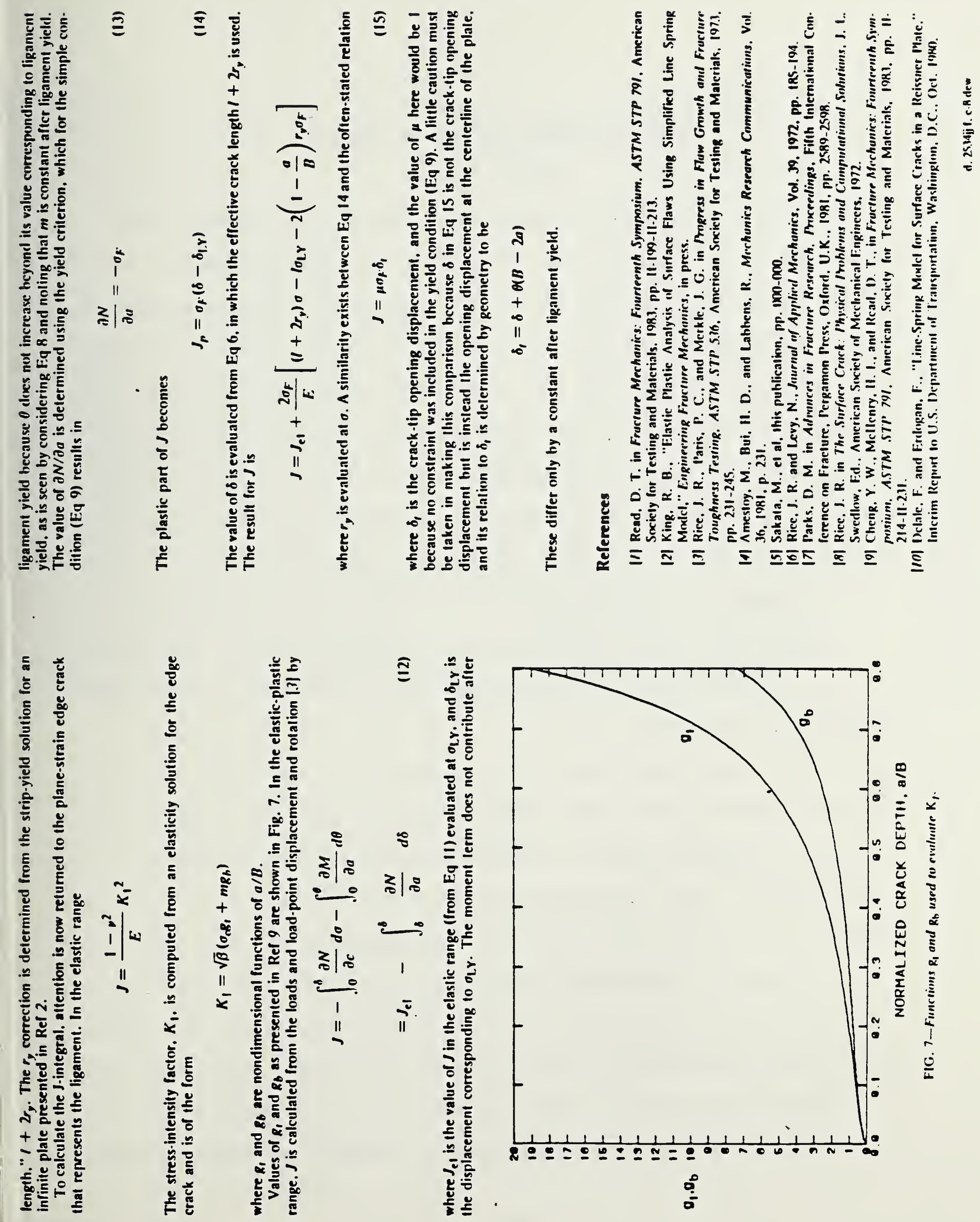



\section{APPENDIX H - Metallographic Analysis}

\section{H. I. McHenry}

Metallographic examinations were carried out on sections representative of the automatic gas-metal-arc (GMA) girth weld and the manual shielded-metal$\operatorname{arc}($ SMA) girth weld. As shown in Fig. 1, the automatic weld contains seven passes, including a root pass, a hot pass, fill passes, and the reinforcement pass. The weld is narrow with the fusion line lying generally normal to the plate surfaces (except for the reinforcement pass). The base plate microstructure, shown in Fig. 2, is heavily banded and consists of alternate layers of polygonal ferrite and nonpolygonal ferrite (acicular ferrite or low-carbon bainite). The banded microstructure originating from chemical segregation in the ingot is a result of the plate rolling operations prior to pipe fabrication. The plate midthickness region (Fig. 2b) exhibits thicker nonpolygonal ferrite layers than regions near the plate surfaces.

The manual SMA weld, shown in Fig. 3, contains eight passes, including a root pass, a hot pass, fill passes, and the reinforcement pass. Starting at the root pass, successive weld passes become wider, and the fusion line lies at a substantial angle to the plate surfaces. A region containing a large slag particle can be seen just below the reinforcement pass. Throughout the cross section of the base-metal microstructure, shown in Fig. 4 a, is a heavily banded region (Fig. 4b) located at the plate midthickness. A large delamination can be observed (Fig. 4) in the midthickness of the base plate. This delamination extends from the weld fusion line through the heat-affected zone (HAZ), terminating in the base metal (Fig. 5a) and occurs along the boundary between the polygonal and nonpolygonal ferrite phases (Fig. 5b). 




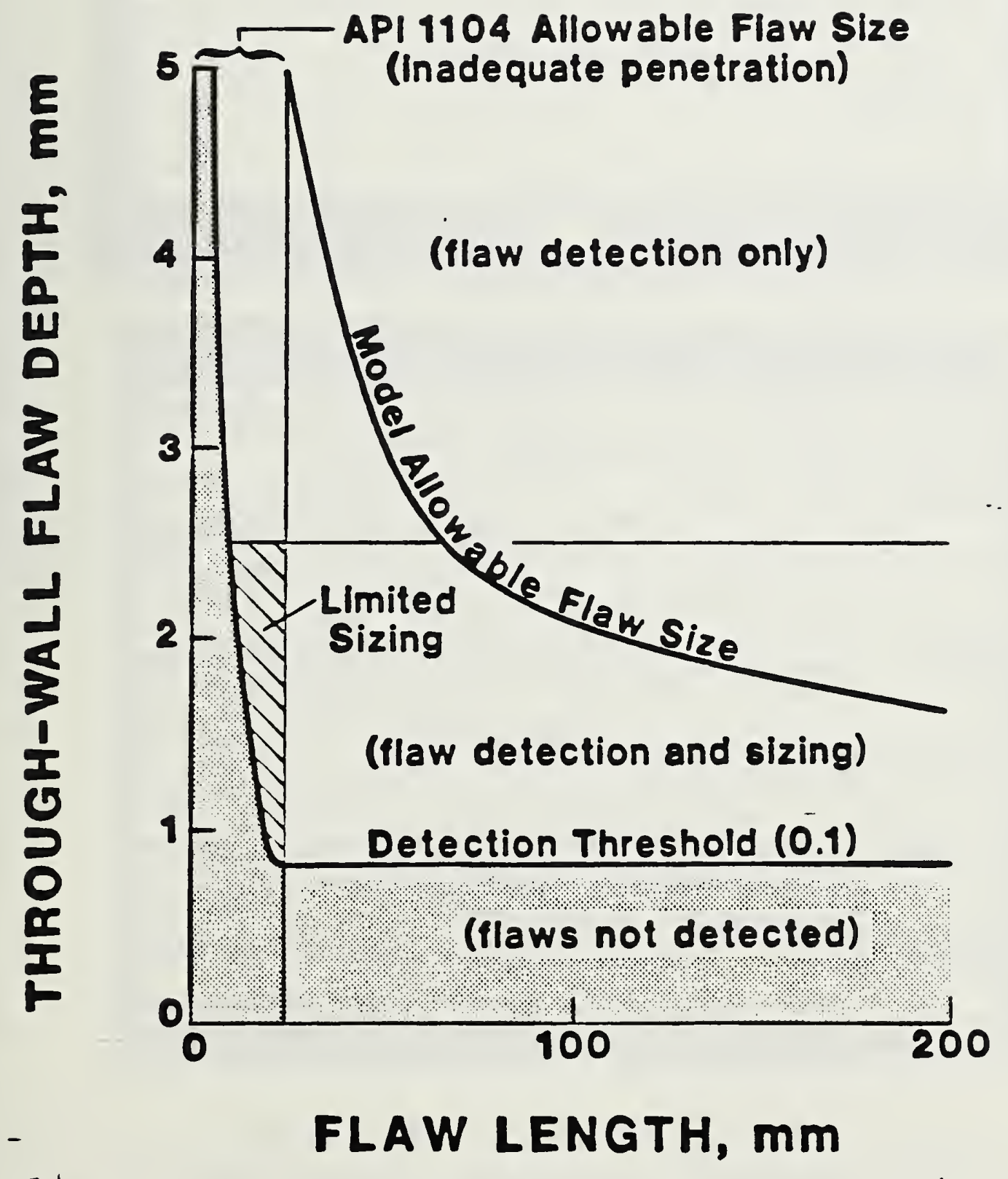

$5 i$

Fig. 66. Surmary of system performance characteristics for vertical surface flaws. 


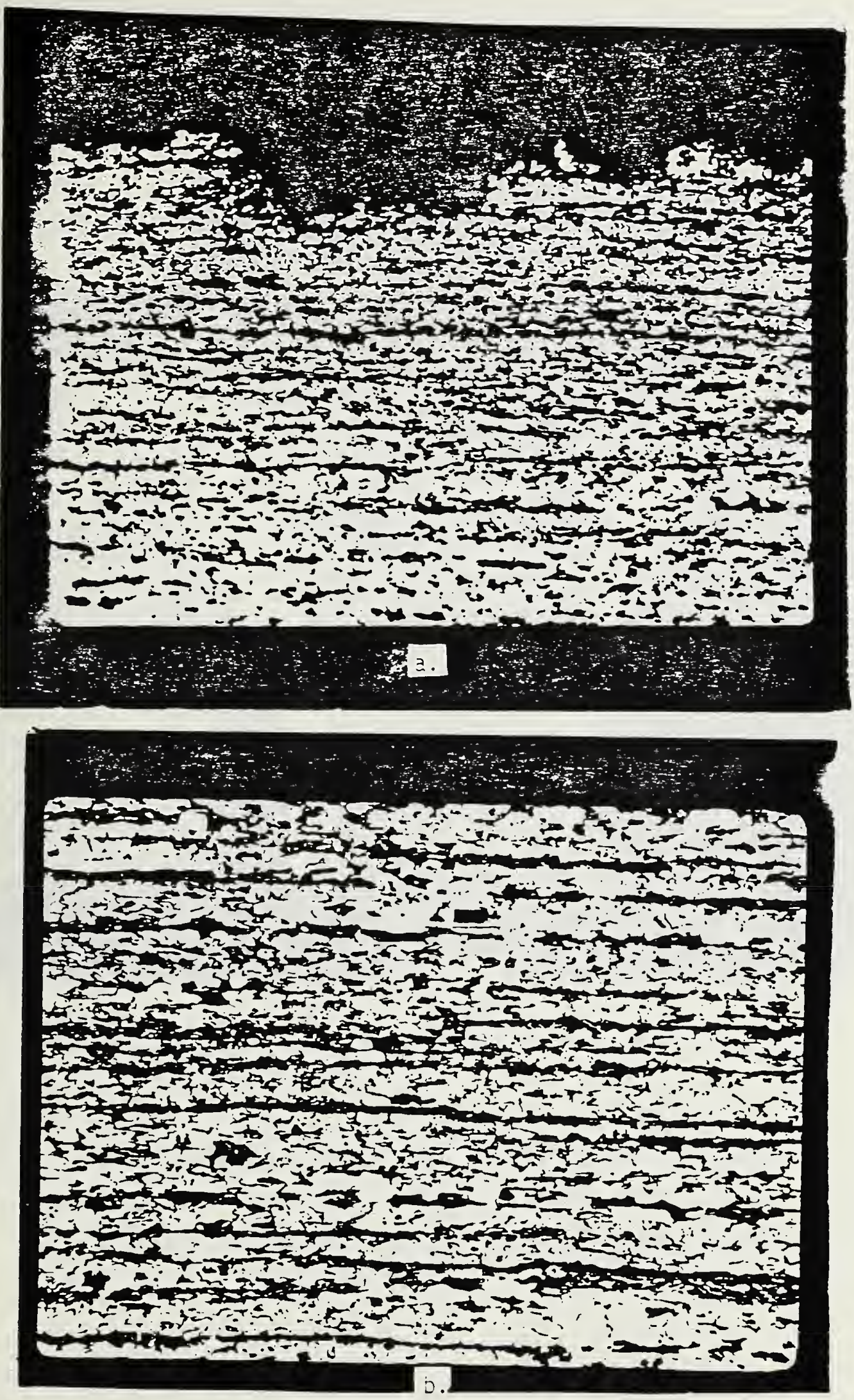

Fig. 2 - Photomicrograph of base plate A from plate W1-1. Light etching phase is polygonal ferrite; dark etching is nonpolygonal ferrite. Plate rolling direction is $\longrightarrow$. (a) Near outside plate surface.
(b) Near plate midthickness.
Etch:
5\% nital. Magnification:
$200 x$. 


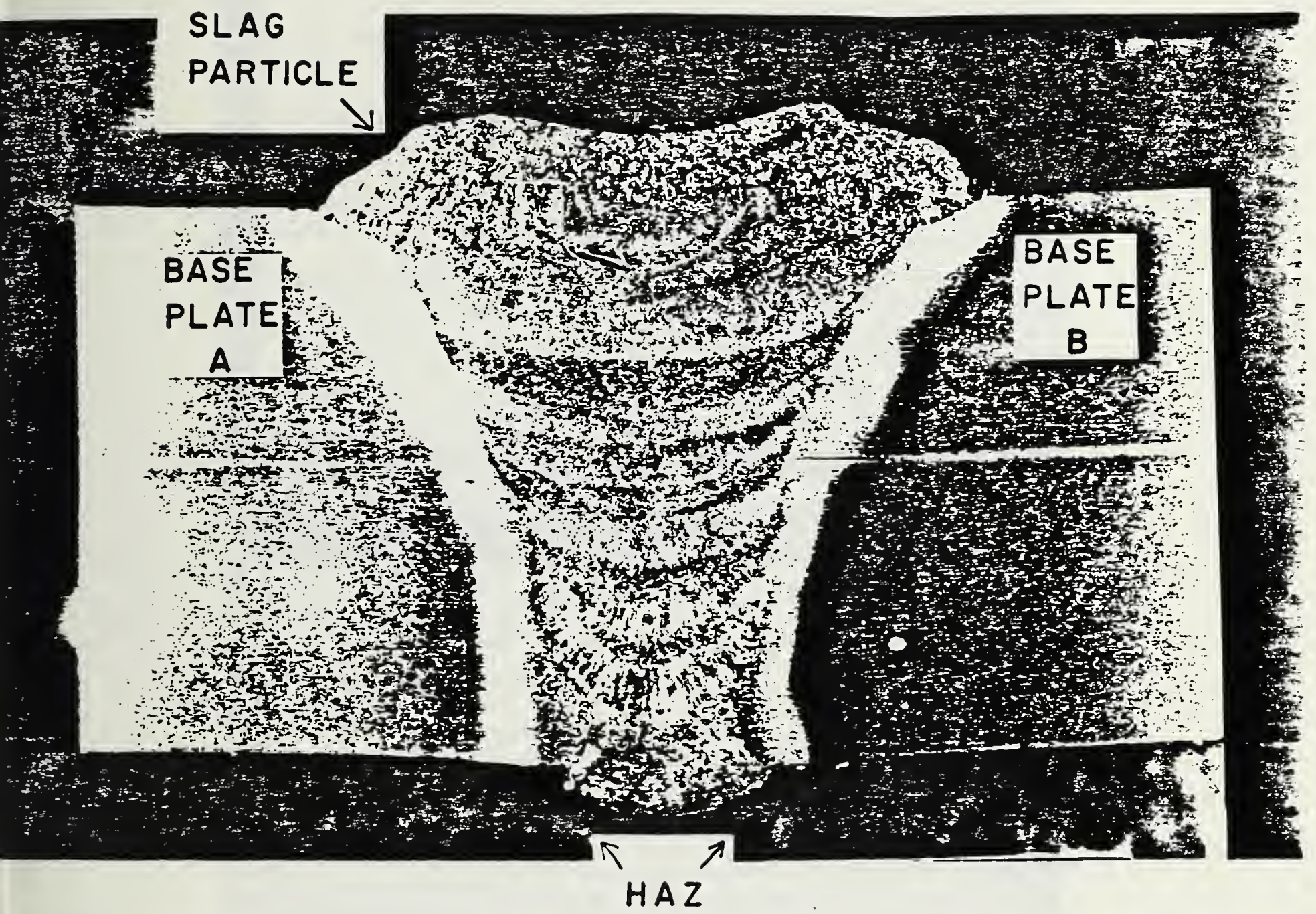

Fig. 3 - Profile section of manual SMA girth weld in plate W4-2. Cross section shows base metal, HAZ, and weld metal. Plate rolling direction is $\rightleftarrows$ Etch: $5 \%$ nital. Magnification: $5 X$. 

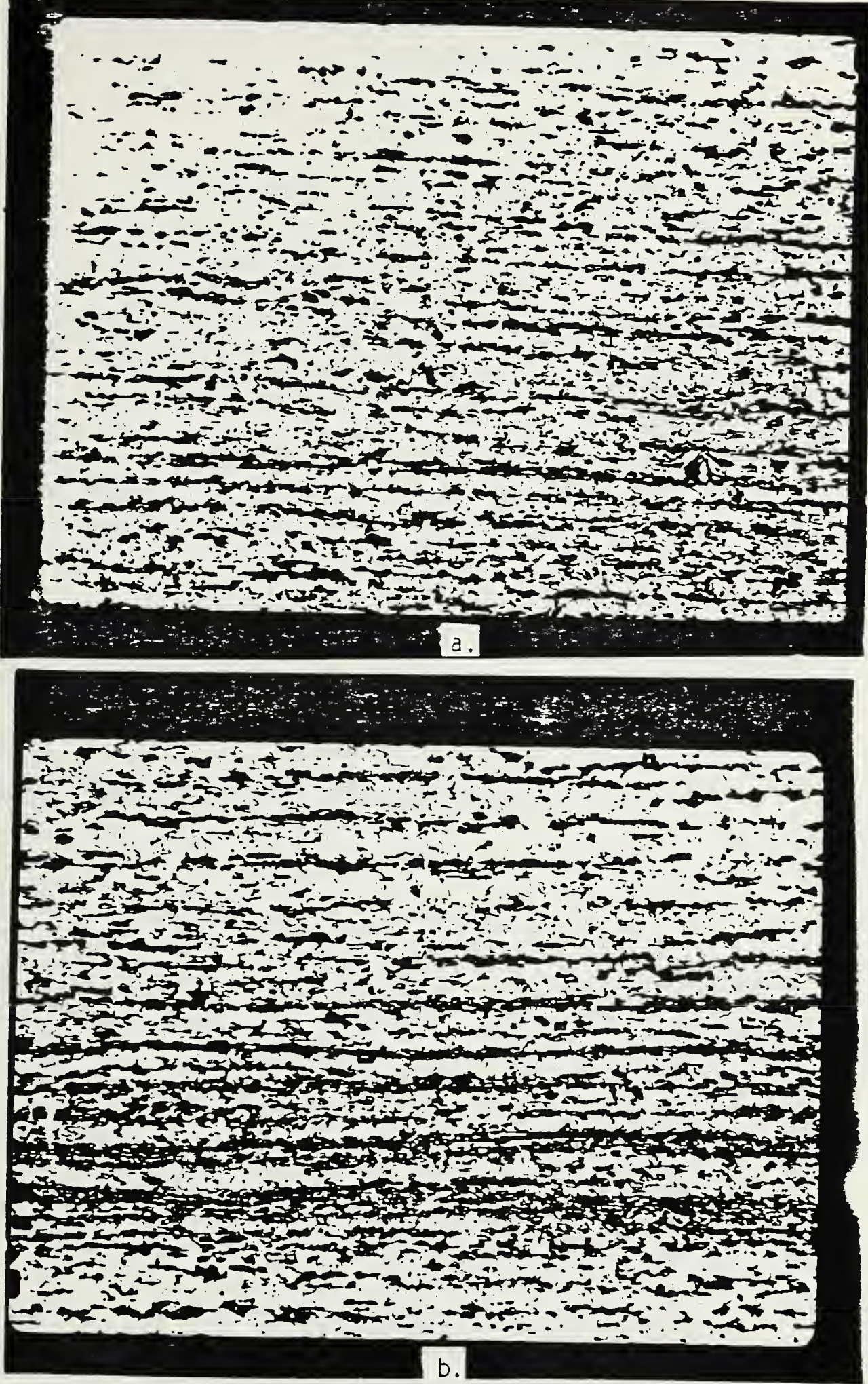

Fig. 4 - Photomicrographs of base plate B from plate W4-2. Light etching phase is polygonal ferrite; dark etching phase is nonpolygonal ferrite. Plate rolling direction is $\longrightarrow$. (a) Near outside plate surface. (b) Near plate midthickness. Etch: 5\% nital. Magnification: $200 x$. 



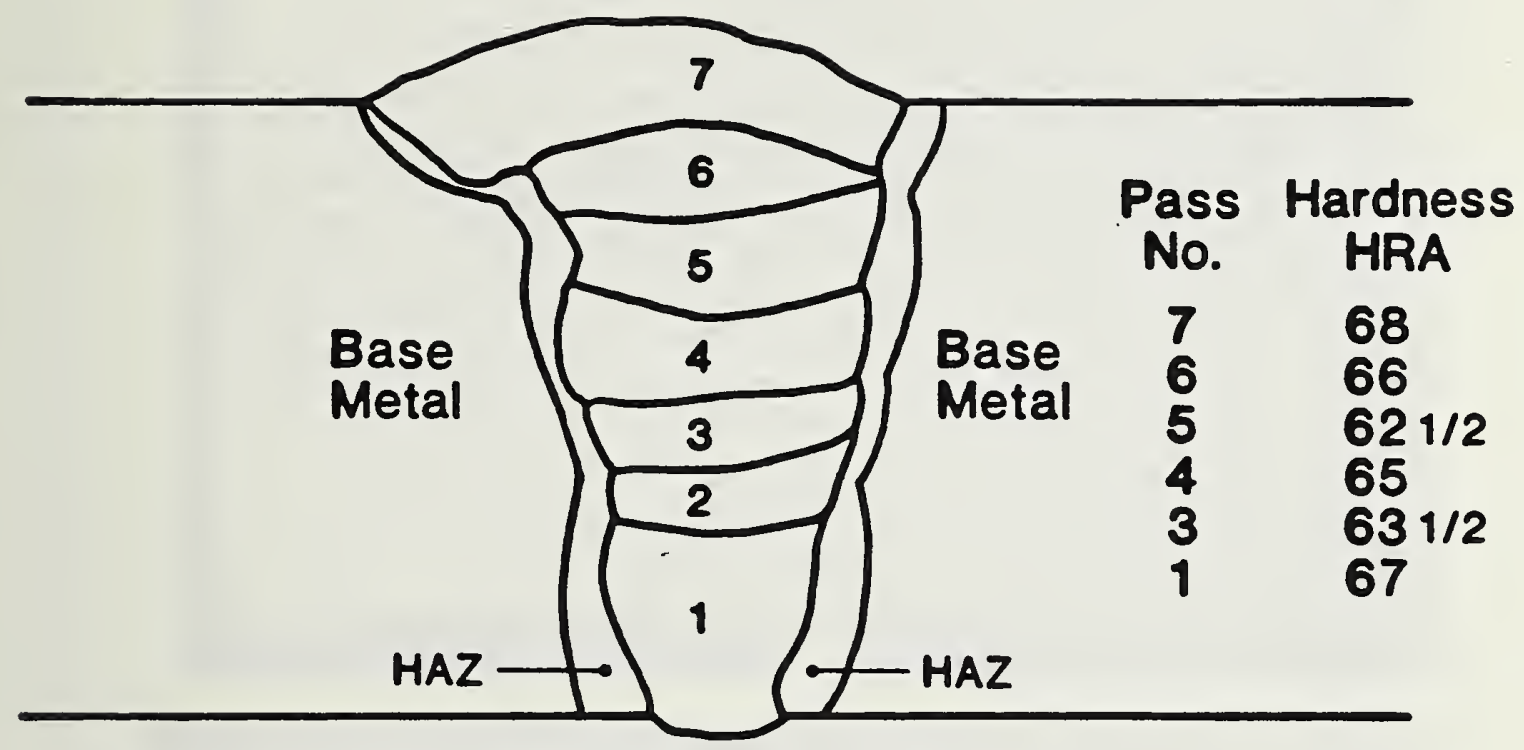

Automatic GMA Weld, Plate W1-1

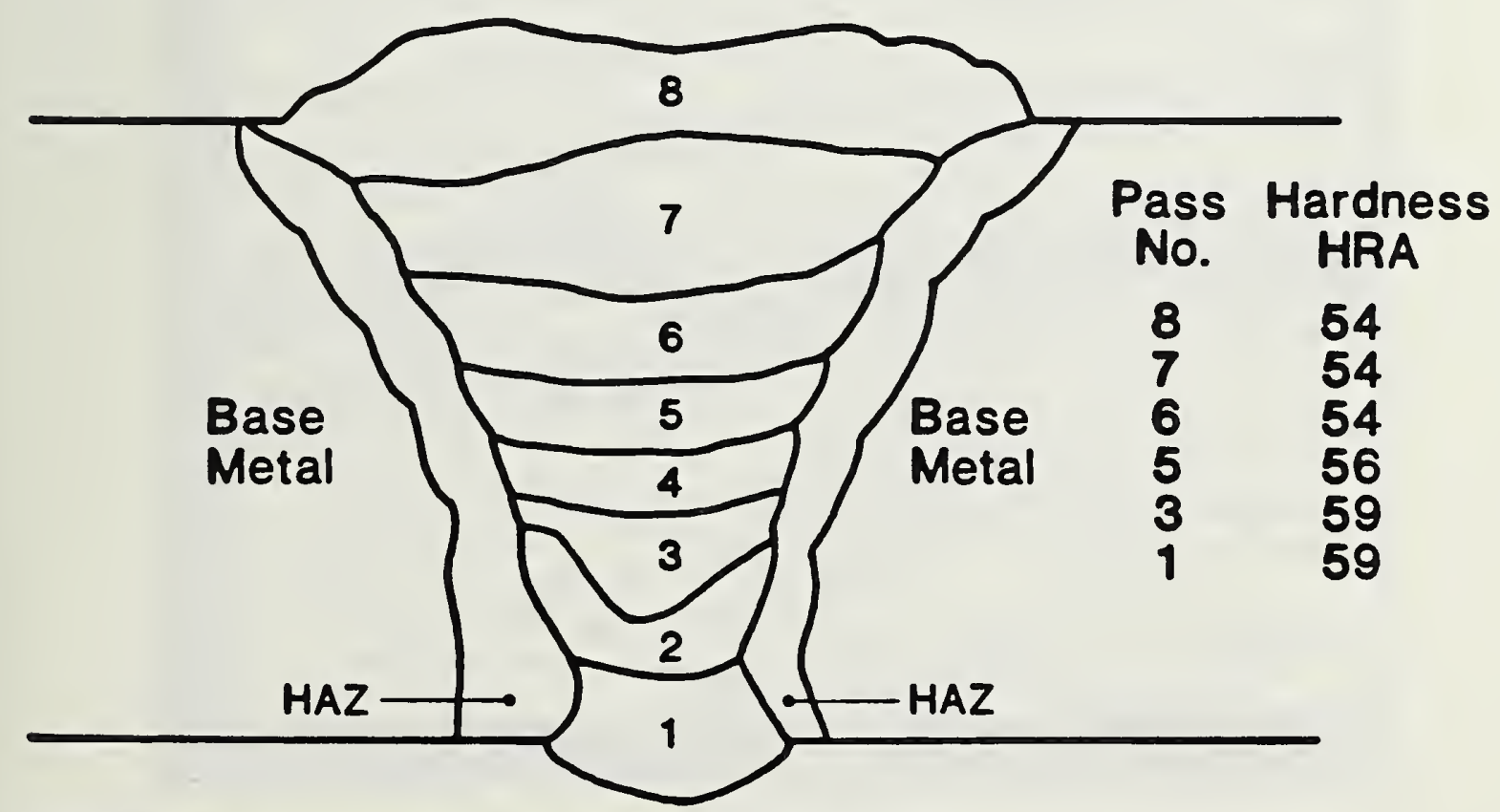

Manual SMA Weld, Plate W4-2

Fig. 6 - Profile schematics of automatic and manual girth welds. 


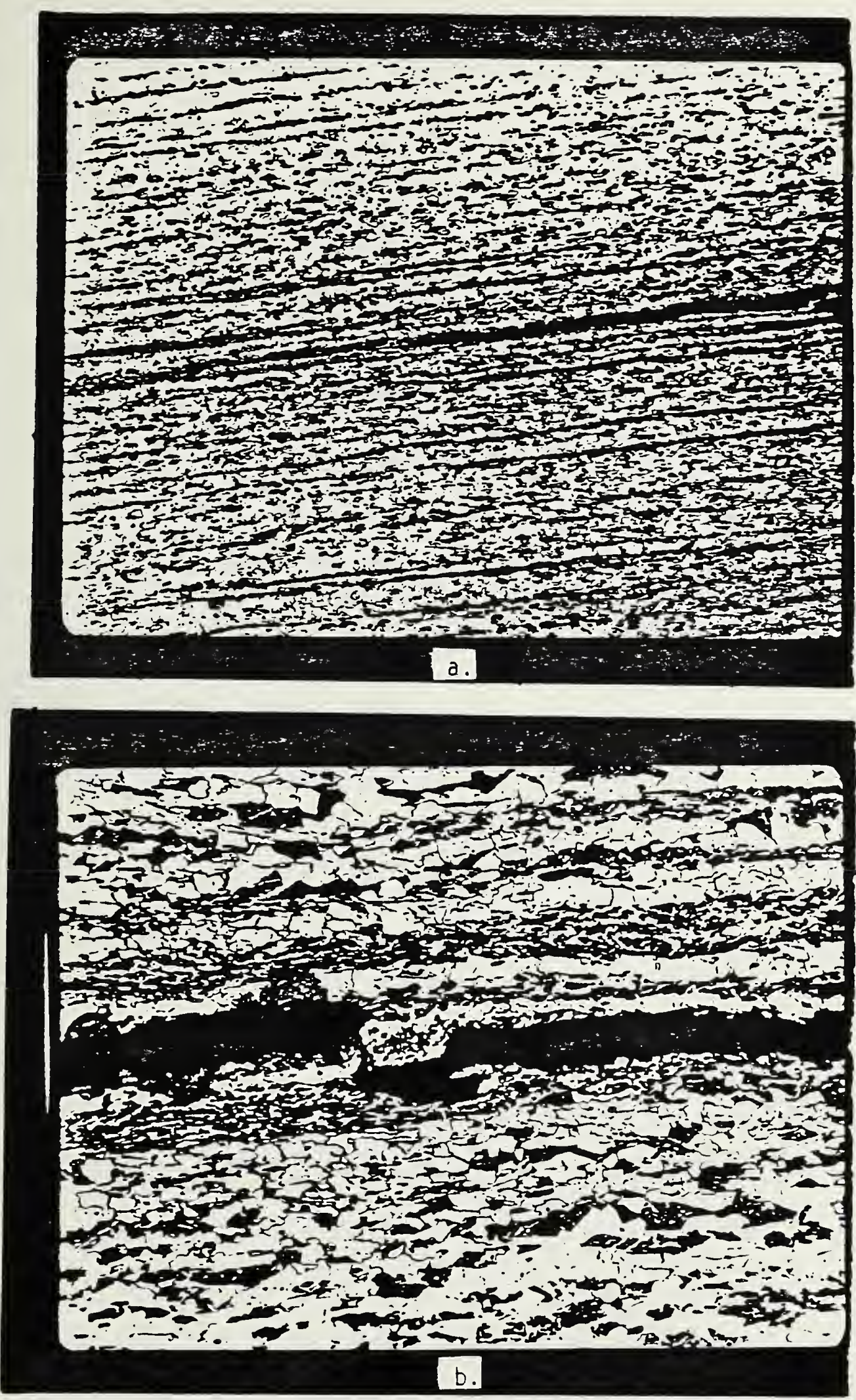

Fig. 5 - Photomicrograph of base metal delamination in plate W4-2.

(a) End of delamination in base metal; magnification: 100x.

(b) Location of delamination along boundary between polygonal

ferrite and nonpolygonal ferrite phase; magnification: 500X.

Etch: $5 \%$ nital. 
Table 1 - Hardness Data Summary

\begin{tabular}{|c|c|c|c|c|c|}
\hline \multirow{3}{*}{$\begin{array}{l}\text { Weld } \\
\text { Type }\end{array}$} & \multicolumn{4}{|c|}{ Average HRA Values } & \multirow{3}{*}{$\begin{array}{l}\text { Weld } \\
\text { Metal }\end{array}$} \\
\hline & \multicolumn{2}{|c|}{ Base Metal } & \multicolumn{2}{|c|}{$H A Z^{\star}$} & \\
\hline & A Side & B Side & A Side & B Side & \\
\hline Automatic & 57 (HRB 93) & 57 (HRB 94) & $62(\operatorname{HRC} 23)$ & 62 (HRC 23) & $65 \frac{1}{2}($ HRC 30$)$ \\
\hline Manua 7 & 57 (HRB 93) & $56 \frac{1}{2}($ HRB 92) & $60($ HRC 20) & $60($ HRC 20) & 56 (HRB 91) \\
\hline
\end{tabular}

* Converted from Vickers hardness numbers. 



\section{Hardness Tests}

Profile sections from each girth-weld type containing weld metal, HAZ, and base metal were prepared for hardness surveys. Profile schematics of the two types of girth welds identifying the weld-pass boundaries are shown in Fig. 6. Vickers hardness measurements and Rockwell A Scale hardness measurements were taken throughout the base metal, $H A Z$, and selected individual weld passes. The average hardness numbers were converted, where necessary, to the Rockwell A Scale, HRA, for comparison. The hardness values for individual weld passes are indicated in Figure 6 and the summary of all hardness results are given in Table 1.

The average hardness of the HAZ in the automatic weld is intermediate, lying between the lower base-metal hardness and the higher weld-metal hardness. For the manual weld, the average HAZ hardness is somewhat higher than the base-metal or weld-metal hardness. Furthermore, the average HAZ and weld-metal hardness values for the automatic weld are higher than the average values for the manual weld. These observations are consistent with the observation that the automatic weld is generally narrower than the manual weld, indicating either lower average heat input, higher average welding rates, or a combination of both, resulting in less tempering of the automatic weld HAZ and a higher cooling rate for the automatic weld metal.

\section{Tensile Properties}

The tensile properties of the weld metal from both weld types were determined at $-101{ }^{\circ} \mathrm{C}$ and $+24^{\circ} \mathrm{C}$. The strength properties at intermediate temperatures were determined by interpolation between the two temperatures. Standard ASTM subsize round tensile specimens, $0.635-\mathrm{cm}$ diameter with a 2.54-cm gage length, were prepared such that the reduced gage length section contained only weld metal with the specimen axis parallel to the weld axis. 

The gage-length section of specimens from the automatic weld generally sampled weld passes 4 and 5, whereas specimens from the manual weld generally sampled weld passes 5 and 6 .

The tensile tests at $-101^{\circ} \mathrm{C}$ were carried out inside an environmental chamber in which the atmospheric temperature was controlled by balancing cooling from liquid nitrogen and heating from electric resistance heaters. The specimen temperature was measured with a thermocouple attached to the gage-length section, and the test temperature was controlled to $\pm 3^{\circ} \mathrm{C}$ during the tests. The test machine crosshead speed was $0.13 \mathrm{~mm} / \mathrm{min}$ up to yielding and $0.76 \mathrm{~mm} / \mathrm{min}$ after yielding. The tensile properties for each girth-weld type and of the API $5 L X-70$ base material are sumarized in Table 2.

The manual-weld test specimens were taken from two welds; the automaticweld test specimens were all taken from a single weld. The results of the tensile tests for the manual-weld specimens from both plates at the two test temperatures indicate good weld uniformity, and thus the measured tensile properties can be used as representative of each weld type. The average ultimate tensile strength and yield strength of the automatic weld increased $15 \%$ and $11 \%$, respectively, as the test temperature was lowered from $+24^{\circ} \mathrm{C}$ to $-101^{\circ} \mathrm{C}$. For the manual weld, the average strength properties increased about $21 \%$ for the same change in test temperature. The tensile ductility parameters, percent elongation and percent reduction in area, were not found to be strongly temperature dependent for these two test temperatures.

\section{Fracture Toughness}

It was anticipated that these weldments would have sufficient plasticity so that slow, stable crack extension would occur within the temperature range of interest, and therefore, elastic-plastic fracture toughness parameters would be needed for the flaw-size analyses. Crack-tip-opening displacement 



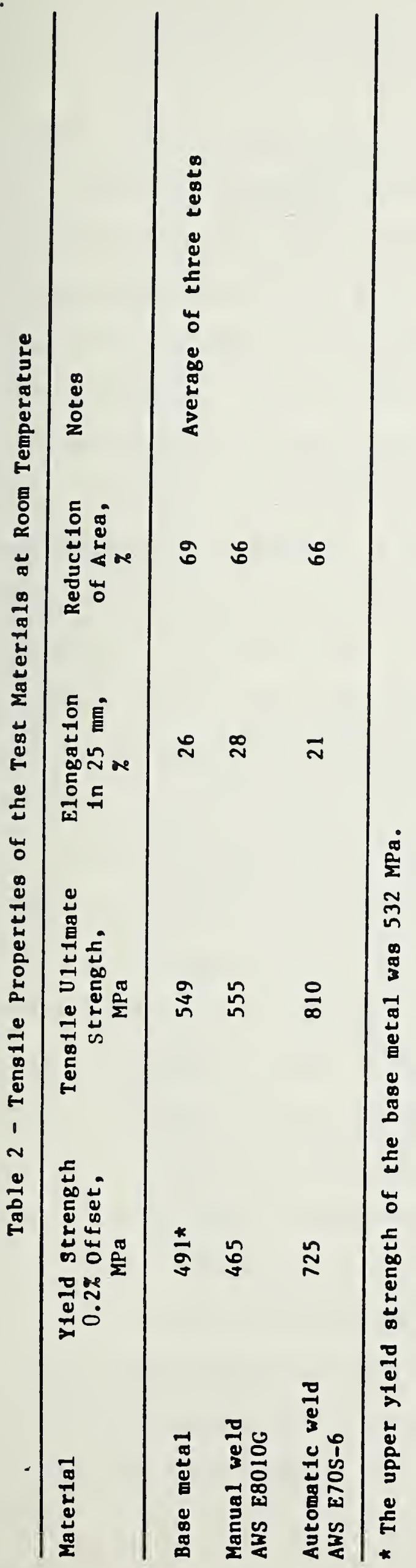



(CTOD) tests were carried out over the temperature range of +24 to $-101^{\circ} \mathrm{C}$. The temperature dependence of the fracture toughness was determined for the weld metal and HAZ regions of both weld types. An estimate of the lowest temperature at which reproducible, slow, stable crack growth would occur was made from the temperature dependence data and a multiple specimen resistance curve approach ${ }^{1,2}$ and was used to determine the critical CTOD at the initiation of stable crack growth.

All fracture tests were carried out in three-point bending on specimens of the preferred geometry, as described in British Standard BS5762:1979. ${ }^{1}$ In the weld-metal specimens, the machined notches were oriented parallel to the weld axis and centered totally within the weld. For the HAZ specimens, the machined notches were oriented parallel to the weld axis and centered so that the final crack location after precracking would place the central portion of the fatigue crack front in the HAZ.

The introduction of fatigue precracks into weld metal and HAZ specimens presents a special problem because of the requirements on the straightness of the fatigue crack front. ${ }^{3}$ Typically, the transverse residual stress pattern associated with multipass welds in plates and pipe (e.g., from surface to surface through the plate thickness or pipe-wall thickness) changes from tensile stresses near each surface to a balancing compressive stress in the interior. Fatigue crack growth in this stress environment often results in two areas of growth near the surfaces in the residual tensile stress region and little or no growth in the residual compressive stress region, as shown in Fig. 7a. There is some evidence that measured fracture toughness properties result from specimens exhibiting this bimodal fatigue crack shape are higher than from specimens with a straighter crack shape, particularly in the elasticplastic toughness region. 4 



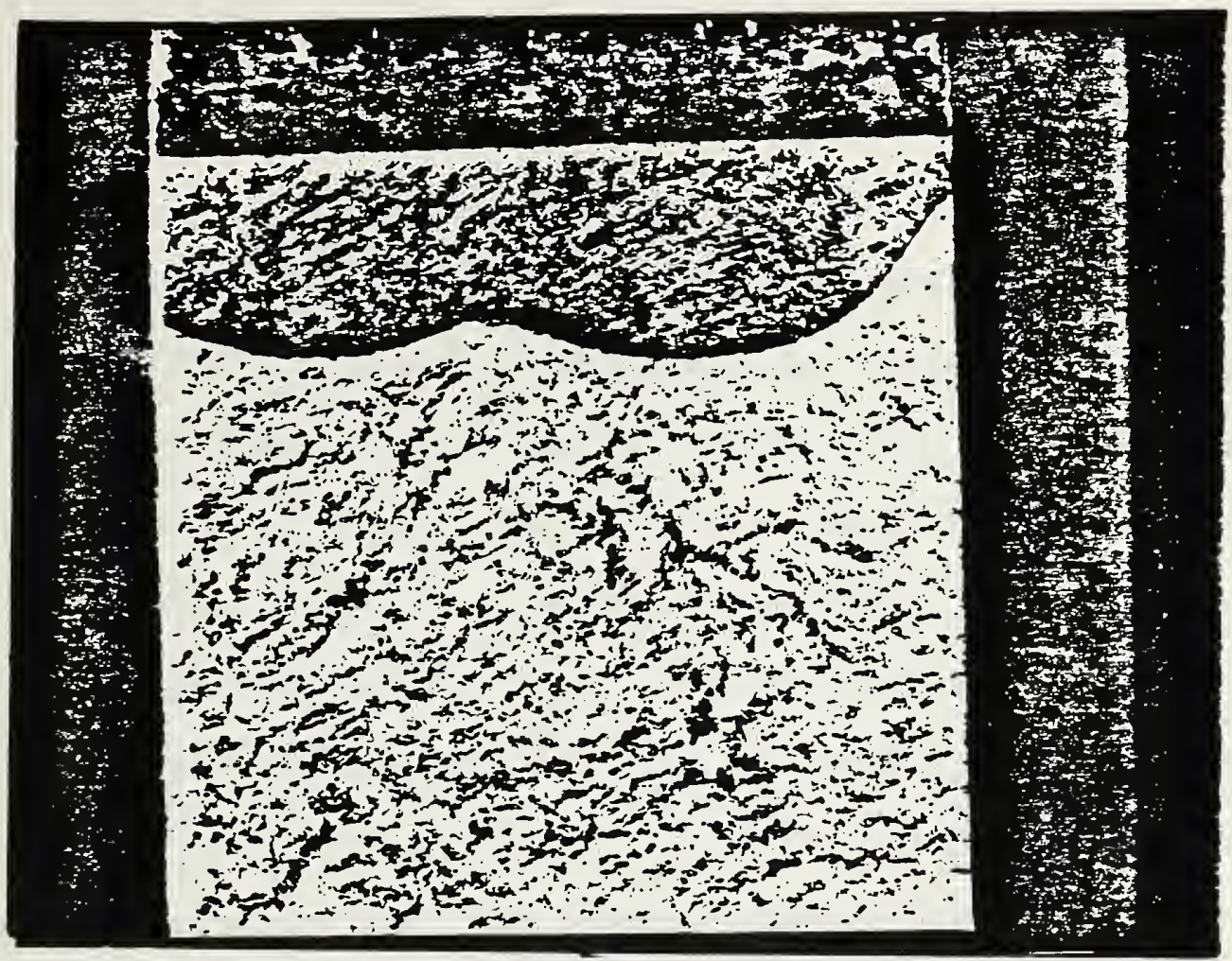

a.

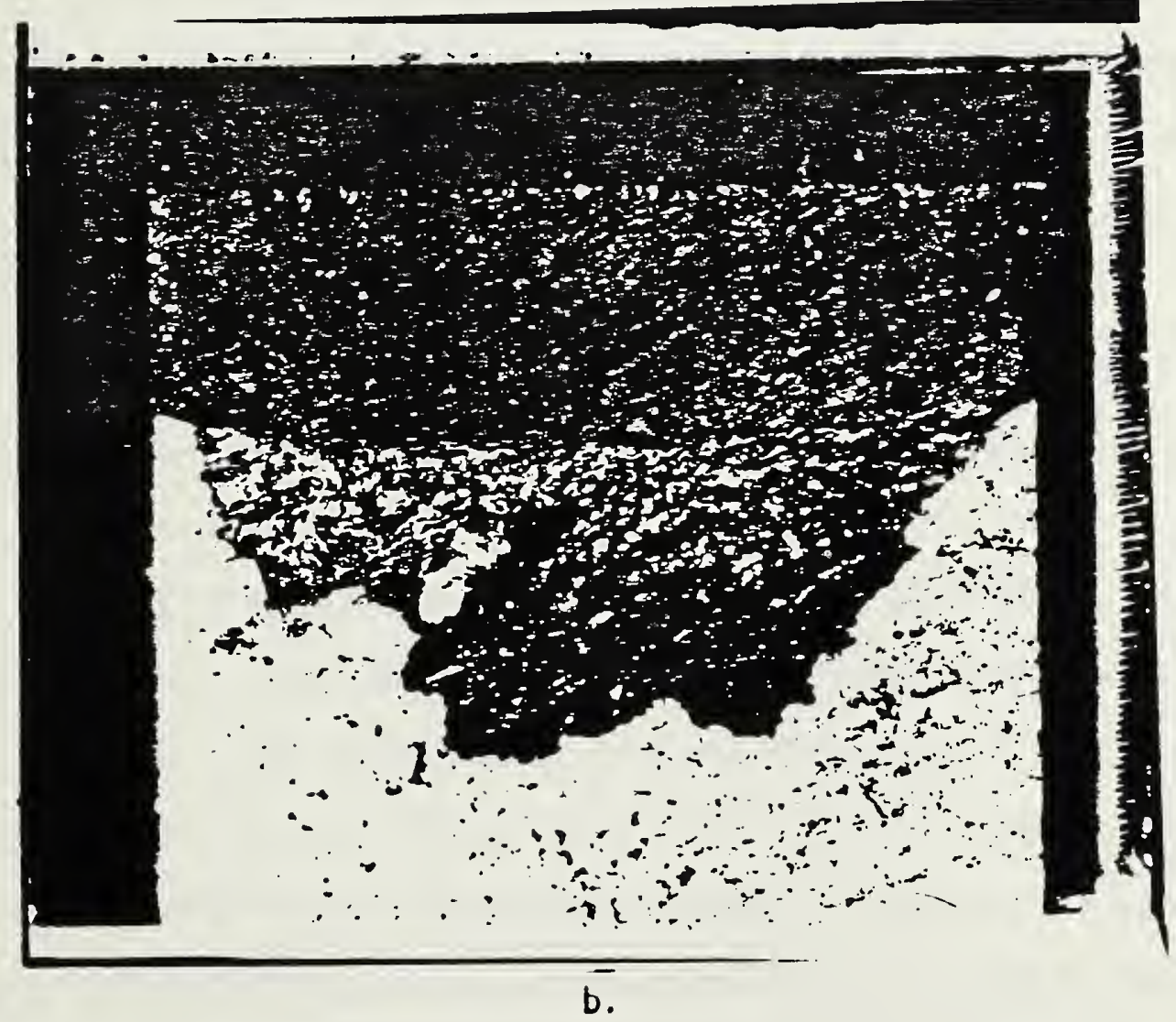

Fig. 7 - Fractographs of COD specimens illustrating the effect of compression pretreatment on fatigue crack growth. (a) Without compression pretreatment; type E8010 weld metal; test temperature $=+24^{\circ} \mathrm{C}\left(+75^{\circ} \mathrm{F}\right)$. (b) With compression pretreatment; type $E 8010$ weld metal; test temperature $=-45^{\circ} \mathrm{C}\left(-50^{\circ} \mathrm{F}\right)$. Magnification: $7 \mathrm{X}$. 

To reduce the effect of nonuniform fatigue cracks, the ligament region ahead of the notch in all CTOD specimens was compressed about $1 \%$ following the procedure of Dawes. ${ }^{5,6}$ The actual load necessary to cause a $1 \%$ plastic strain in the through-thickness direction was within $5 \%$ of the calculated load. Several studies with weld metal specimens have shown little effect of the compression treatment on the measured crack opening displacement. ${ }^{4,5}$

After the compression pretreatment, fatigue precracks were grown at $24^{\circ} \mathrm{C}$ by sinusoidal loading of each specimen at a frequency of $20 \mathrm{~Hz}$ with maximum and minimum loads of $6.7 \mathrm{kN}$ and $0.1 \mathrm{kN}$. The final ratio of crack length to specimen width was about 0.50 . The average number of cycles required was 25,500 for the automatic-weld specimens with a range of 22,300 cycles to 29,000 cycles. For the HAZ specimens, the average number of cycles was 24,660 with a range of 21,000 cycles to 27,000 cycles. For the manual weld and HAZ specimens, the averages were 21,130 cycles and 20,940 cycles, with ranges of 20,000 cycles to 22,500 cycles and 19,000 cycles to 22,000 cycles, respectively. The shapes of the resulting fatigue cracks were generally straight except near the surfaces, with little evidence of the bimodal behavior, as can be seen in Fig. $7 b$.

The crack-opening-displacement procedure followed was based on British Standard BS5762:1979. Each specimen was loaded in displacement control at a rate of $0.25 \mathrm{~mm} / \mathrm{min}$. The instantaneous load and displacement, as measured by a clip gage attached to the specimen surface, were continuously recorded. The temperature was monitored by a thermocouple attached to one of the screw-in knife edges on the specimen. At the conclusion of each test, the specimen was rapidly unloaded and heat-tinted to mark the extent of crack growth. Each specimen was then cooled in liquid nitrogen and broken, and the amount of crack extension was determined. 

The CTOD value for each test was calculated using the relations:

and

$$
C T O D=\frac{K^{2}\left(1-v^{2}\right)}{2 \sigma_{y} E}+\frac{0.4(W-a) v_{p}}{0.4 W+0.6\left(a+h_{c}\right)}
$$

$$
K=Y P /\left(t W^{\frac{1}{2}}\right)
$$

where: $Y=$ stress intensity coefficient at $a / W$

$P=1$ load

$t=$ specimen thickness

$W=$ specimen width

$V_{p}=$ plastic component of $c l i p$-gage opening displacement

$h_{c}^{p}=$ distance of clip gage from specimen surface

The interpretation of the calculated value of CTOD depends on observations of the load-displacement behavior and the type and extent of crack growth at the test temperature. The results of the analysis of the temperature dependence of the fracture toughness are summarized in Tables 3 and 4 and Fig. 8. The weld-metal data for both welding processes show a regular progression in crack growth behavior from low test temperatures to high test temperatures: for example, unstable or brittle fracture or pop-in without evidence of slow, stable crack growth at the lowest temperatures through unstable brittle fracture or pop-in after some slow, stable crack growth at intermediate temperatures, to only slow, stable crack growth through the maximum applied load at the highest temperatures. The higher-strength automatic weld did not exhibit slow, stable crack growth until the test temperature was increased to $-18^{\circ} \mathrm{C}$. The lower-strength manual weld exhibited slow, stable crack growth at $-46^{\circ} \mathrm{C}$.

The HAZ data for both welding processes showed a similar regularity in the crack growth behavior at the lowest and highest test temperatures: no stable crack growth at the lowest temperature and only slow, stable crack 

Table 3 - Temperature Dependence of Fracture Toughness for the Automatic Weld

\begin{tabular}{|c|c|c|c|c|}
\hline $\begin{array}{l}\text { Specimen } \\
\text { Number }\end{array}$ & Type* & $\begin{array}{c}\text { Temperature, } \\
{ }^{\circ} \mathrm{C}\end{array}$ & $a / w$ & $\begin{array}{c}\text { CTOD } \\
\text { Criteriont }\end{array}$ \\
\hline$W 133$ & $w$ & -73 & 0.5042 & $\delta_{c}=0.028$ \\
\hline W135 & W & -46 & 0.5287 & $\delta_{c}=0.076$ \\
\hline W128 & w & -18 & 0.5388 & $\delta_{u}=0.094$ \\
\hline W1214 & $w$ & -18 & 0.5215 & $\delta_{u}=0.089$ \\
\hline W129 & $w$ & -18 & 0.5189 & $\delta_{u}=0.079$ \\
\hline W139 & $w$ & 0 & 0.4971 & $\delta_{\max }=0.084$ \\
\hline W138 & $W$ & 21 & 0.5101 & $\delta_{\max }=0.122$ \\
\hline W134 & $W$ & 21 & 0.5093 & $\delta_{\max }=0.104$ \\
\hline$W 1216$ & $H A Z$ & -101 & 0.5018 & $\delta_{c}=0.018$ \\
\hline W123 & $H A Z$ & -101 & 0.4959 & $\delta_{c}=0.041$ \\
\hline W121 & $H A Z$ & -73 & 0.4854 & $\delta_{c}=0.061$ \\
\hline W1218 & $H A Z$ & -18 & 0.5064 & $\delta_{\max }=0.437$ \\
\hline$W 1212$ & $H A Z$ & 0 & 0.5133 & $\delta_{\max }=0.262$ \\
\hline W1213 & $H A Z$ & 0 & 0.5053 & $\delta_{\max }=0.508$ \\
\hline$W 1210$ & $H A Z$ & 24 & 0.5080 & $\delta_{\max }=0.442$ \\
\hline
\end{tabular}

* $W M=$ weld metal; $H A Z=$ heat-affected $z o n e$

t where: $\delta_{C}=$ CTOD at unstable fracture or onset of arrested brittle crack growth without evidence of slow crack growth.

$\delta_{u}=$ CTOD at unstable fracture on onset of arrested brittle crack growth with evidence of slow crack growth.

$\delta_{\max }=$ CTOD at maximum applied force. 

Table 4 - Temperature Dependence of Fracture Toughness for the Manual Weld

\begin{tabular}{|c|c|c|c|c|}
\hline $\begin{array}{l}\text { Specimen } \\
\text { Number }\end{array}$ & Type * & $\begin{array}{c}\text { Temperature, } \\
{ }^{\circ} \mathrm{C}\end{array}$ & $a / w$ & $\begin{array}{c}\text { CTOD } \\
\text { Criteriont }\end{array}$ \\
\hline W434 & WM & -101 & 0.5044 & $\delta_{c}=0.010$ \\
\hline W436 & WM & -101 & 0.5050 & $\delta_{c}=0.053$ \\
\hline W431 & WM & -73 & 0.5143 & $\delta_{c}=0.036$ \\
\hline W432 & WM & -46 & 0.5086 & $\delta_{u}=0.097$ \\
\hline W439 & WM & -18 & 0.5200 & $\delta_{u}=0.084$ \\
\hline W4117 & WM & -18 & 0.5033 & $\delta_{\max }=0.229$ \\
\hline W438 & WM & 0 & 0.5173 & $\delta_{\max }=0.226$ \\
\hline W433 & WM & +21 & 0.5054 & $\delta_{\max }=0.236$ \\
\hline W412 & $H A Z$ & -101 & 0.5026 & $\delta_{c}=0.053$ \\
\hline$W 4110$ & HAZ & -101 & 0.5132 & $\delta_{c}=0.018$ \\
\hline W415 & $H A Z$ & -73 & 0.5971 & $\delta_{C}=0.114$ \\
\hline W4114 & $\mathrm{HAZ}$ & -18 & 0.5074 & $\delta_{\max }=0.127$ \\
\hline W4113 & HAZ & -18 & 0.5142 & $\delta_{C}=0.076$ \\
\hline W417 & $H A Z$ & -18 & 0.5292 & $\delta_{u}=0.155$ \\
\hline W418 & $H A Z$ & 0 & 0.5117 & $\delta_{u}=0.188$ \\
\hline W4111 & $H A Z$ & 0 & 0.5121 & $\delta_{\max }=0.432$ \\
\hline W419 & $H A Z$ & 24 & 0.5159 & $\delta_{\max }=0.249$ \\
\hline W411 & $H A Z$ & 21 & 0.5057 & $\delta_{\max }=0.226$ \\
\hline
\end{tabular}

* $W M=$ weld metal ; HAZ = heat-affected zone

+ where: $\delta_{C}=$ CTOD at unstable fracture or onset of arrested brittle crack growth without evidence of slow crack growth.

$\delta_{u}=$ CTOD at unstable fracture on onset of arrested brittle crack growth with evidence of slow crack growth.

$\delta_{\max }=$ CTOD at maximum applied force. 



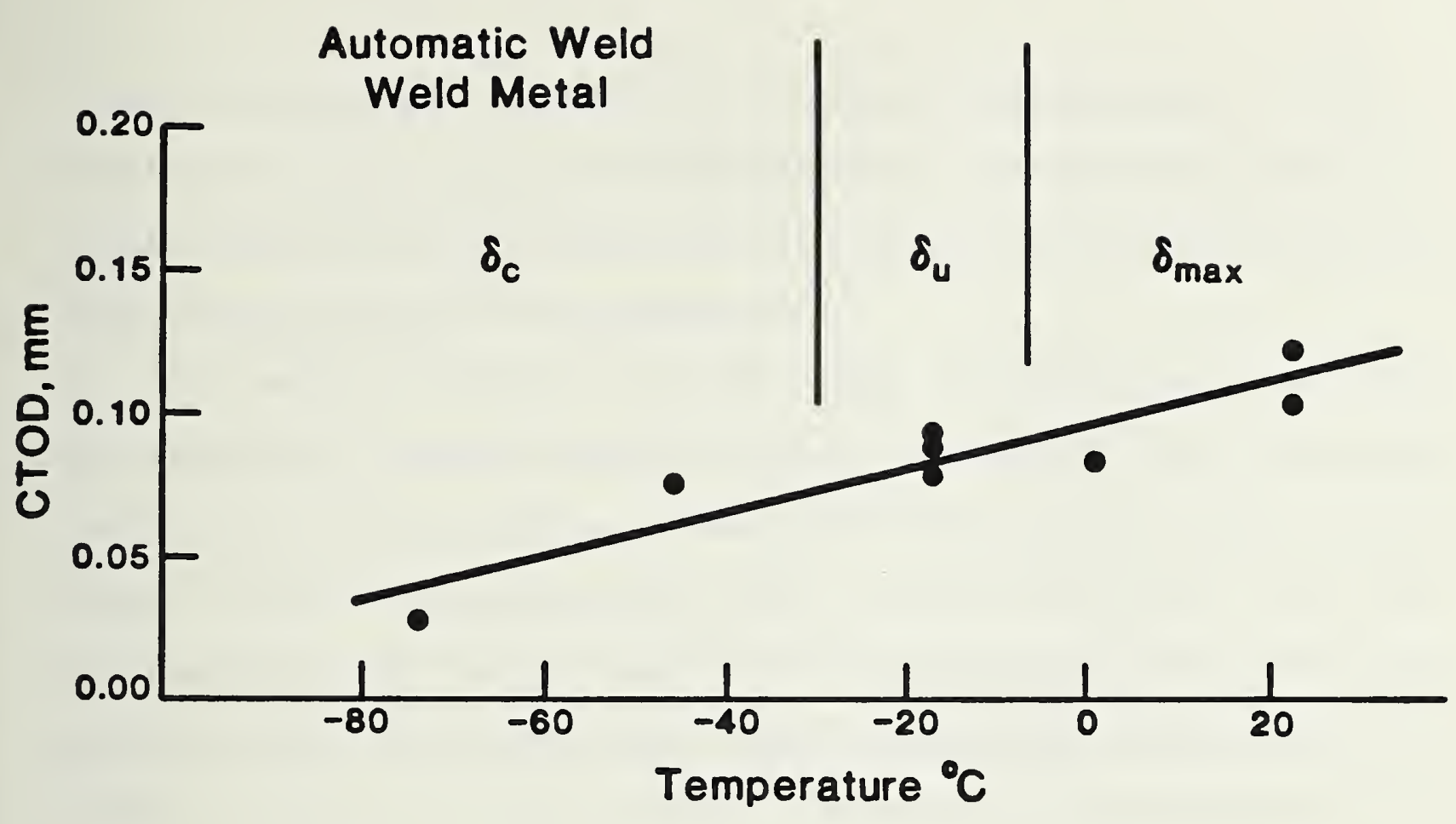

Fig. 8(a) - Temperature dependence of crack-opening displacement for the automatic GMA girth weld.

Automatic Weld

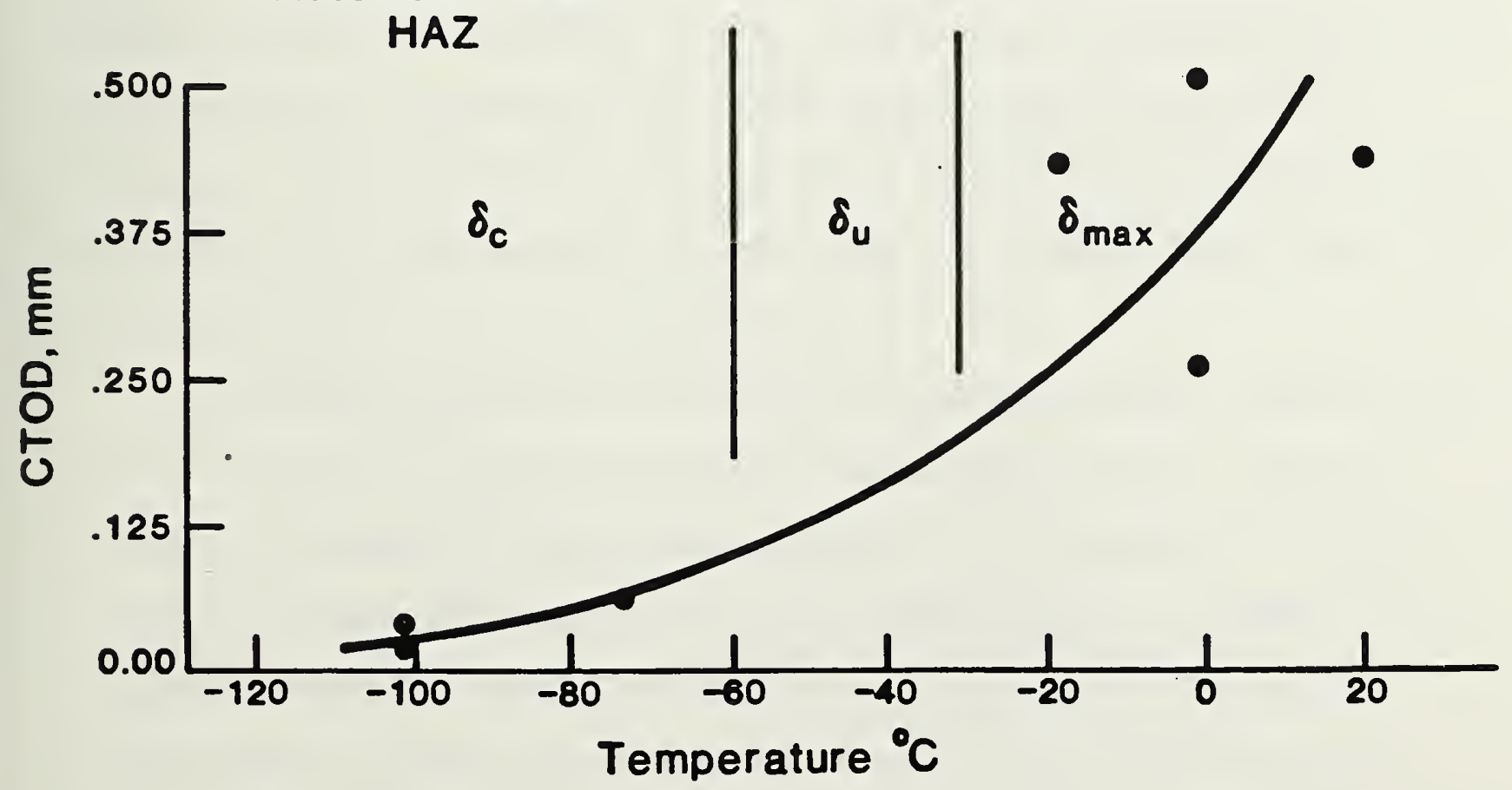

Fig. 8(b) - Temperature dependence of crack-opening displacement for the HAZ from the automatic GMA girth weld. 

growth through maximum applied load at the highest temperatures. However, considerable variability in crack growth behavior was observed at the intermediate temperatures, with less correlation between the presence or absence of slow, stable crack growth and temperatures.

The greater variability in the HAZ results is probably associated with the geometrical relationship between the weld and specimen notch orientation. The welds are single- $V$ type so that when the fatigue crack is grown, only the central portion of the crack (about 25\% of the crack front) lies in the HAZ. The remainder of the fatigue crack lies in the weld metal on one side and the plate base metal on the other side. Thus, the resulting or controlling fracture toughness is variable because it depends on the detailed shape of the fatigue crack, the resulting stress intensity due to the applied load, and the expected difference in toughness in each of these three regions.

Using the temperature dependence of the CTOD toughness parameter, as shown in Tables 3 and 4 , an estimate was made of the lowest temperature at which consistent and reproducible slow, stable crack growth would occur. At this temperature, arrested brittle crack growth or pop-in would not be expected. These temperatures were estimated to be $0^{\circ} \mathrm{C}$ for the automatic weld and $-18^{\circ} \mathrm{C}$ for the manual weld, and the temperatures for each $H A Z$ were estimated to be $-18^{\circ} \mathrm{C}$ and $0^{\circ} \mathrm{C}$, respectively.

A multiple-specimen resistance curve approach was used to determine the critical CTOD, $\delta_{i}$, at the initiation of stable crack growth. The crackopening-displacement procedure followed was based on Appendix A of British Standard BS5762:1979. ${ }^{1}$ Each fatigue precracked specimen was loaded in displacement control at a rate $0.25 \mathrm{~mm} / \mathrm{min}$ to various values of $\mathrm{clip}$-gage displacement to obtain various amounts of crack, $\delta$, for each increment of slow, stable crack growth. Then each specimen was rapidly unloaded and heat- 

tinted to mark the extent of stable crack growth. Each specimen was cooled in liquid nitrogen, fractured, and the amount of crack extension measured. The results of these tests are summarized in Tables 5 and 6 and Figs. 9a-d. The $\delta_{i}$ representative of the initiation of stable crack growth was taken as the value of crack-tip-opening displacement at zero crack extensions, $\Delta a=0$. The values of $\delta_{j}$ for the automatic and manual girth welds are $0.074 \mathrm{~mm}$ at $0^{\circ} \mathrm{C}$ and $0.079 \mathrm{~mm}$ at $-18^{\circ} \mathrm{C}$, respectively, and the $\mathrm{HAZ}$ values of $\delta_{j}$ are $0.14 \mathrm{~mm}$ at $-18^{\circ} \mathrm{C}$ and $0.17 \mathrm{~mm}$ at $0^{\circ} \mathrm{C}$, respectively.

The measured $\delta_{\max }$ of $0.083 \mathrm{~mm}$ at $0^{\circ} \mathrm{C}$ for the automatic weld is consistent with the calculated $\delta_{j}$ of $0.074 \mathrm{~mm}$ because of the lack of a strong temperature dependence of the CTOD Fig. $8 a$ and the very shallow slope of the CTOD-vs.-stable crack extension curve Fig. 9a. The $\delta_{\max }$ of $0.023 \mathrm{~mm}$ at $-18^{\circ} \mathrm{C}$ for the manual weld is consistent with the calculated $\delta_{i}$ of $0.079 \mathrm{~mm}$ because of the stronger temperature dependence of the CTOD (Fig. 8b) and the steeper slope of the CTOD-vs.-crack extension curve (Fig. 9b). Although considerably greater scatter was observed in the HAZ data, the same general relationship was observed when comparing the calculated $\delta_{\mathfrak{j}}$ to $\delta_{\max }$. 

Table 5 - Critical CTOD for Initiation of Slow, Stable Crack Growth for the Automatic Weld

\begin{tabular}{lllllll}
\hline $\begin{array}{l}\text { Specimen } \\
\text { Number }\end{array}$ & Type * & $\begin{array}{c}\text { Temp., } \\
{ }^{\circ} \mathrm{C}\end{array}$ & $\begin{array}{l}\mathrm{P}, \\
\mathrm{kN}\end{array}$ & $\mathrm{a} / \mathrm{W}$ & $\begin{array}{c}\Delta \mathrm{a}, \\
\mathrm{mm}\end{array}$ & $\begin{array}{l}\text { CTOD, } \\
\text { mm }\end{array}$ \\
\hline W122 & WM & 0 & 17.1 & 0.5018 & 0.431 & 0.076 \\
W126 & WM & 0 & 18.1 & 0.5006 & 0.356 & 0.097 \\
W124 & WM & 0 & 17.4 & 0.5060 & 0.660 & 0.099 \\
W1217 & WM & 0 & 17.5 & 0.5041 & 0.533 & 0.107 \\
W1215 & HAZ & -18 & 17.9 & 0.5081 & 0.152 & 0.109 \\
W1310 & HAZ & -18 & 18.9 & 0.5059 & 0.086 & 0.163 \\
W1311 & HAZ & -18 & 18.3 & 0.5113 & 0.401 & 0.190 \\
W1312 & HAZ & -18 & 19.4 & 0.5054 & 0.142 & 0.196 \\
\hline
\end{tabular}

* $W M=$ weld metal; HAZ = heat-affected zone 

Table 6 - Critical CTOD for Initiation of Slow, Stable Crack frowth for the Manual Weld

\begin{tabular}{|c|c|c|c|c|c|c|}
\hline $\begin{array}{l}\text { Specimen } \\
\text { Number }\end{array}$ & Type * & Temp. & P & $a / w$ & $\underset{\mathrm{mm}}{\Delta \mathrm{a}}$ & $\begin{array}{l}\text { CTOD, } \\
\text { mm }\end{array}$ \\
\hline W4119 & $W M$ & -18 & 13.92 & 0.5092 & 0.066 & 0.094 \\
\hline W4118 & WM! & -18 & 14.41 & 0.5042 & 0.122 & 0.119 \\
\hline W4116 & WM & -18 & 14.54 & 0.5137 & 0.277 & 0.142 \\
\hline W413 & WM & -18 & 14.52 & 0.5079 & 0.246 & 0.167 \\
\hline W414 & HAZ & -18 & 14.12 & 0.5140 & 0.361 & 0.185 \\
\hline W4120 & HAZ & 0 & 14.14 & 0.5117 & 0.129 & 0.150 \\
\hline W4112 & HAZ & 0 & 14.79 & 0.5169 & 1.85 & 0.178 \\
\hline W4115 & HAZ & 0 & 15.50 & 0.5131 & 0.081 & 0.231 \\
\hline W4121 & HAZ & 0 & 16.06 & 0.5087 & 0.193 & 0.300 \\
\hline
\end{tabular}

* WM = weld metal; HAZ = heat-affected zone 



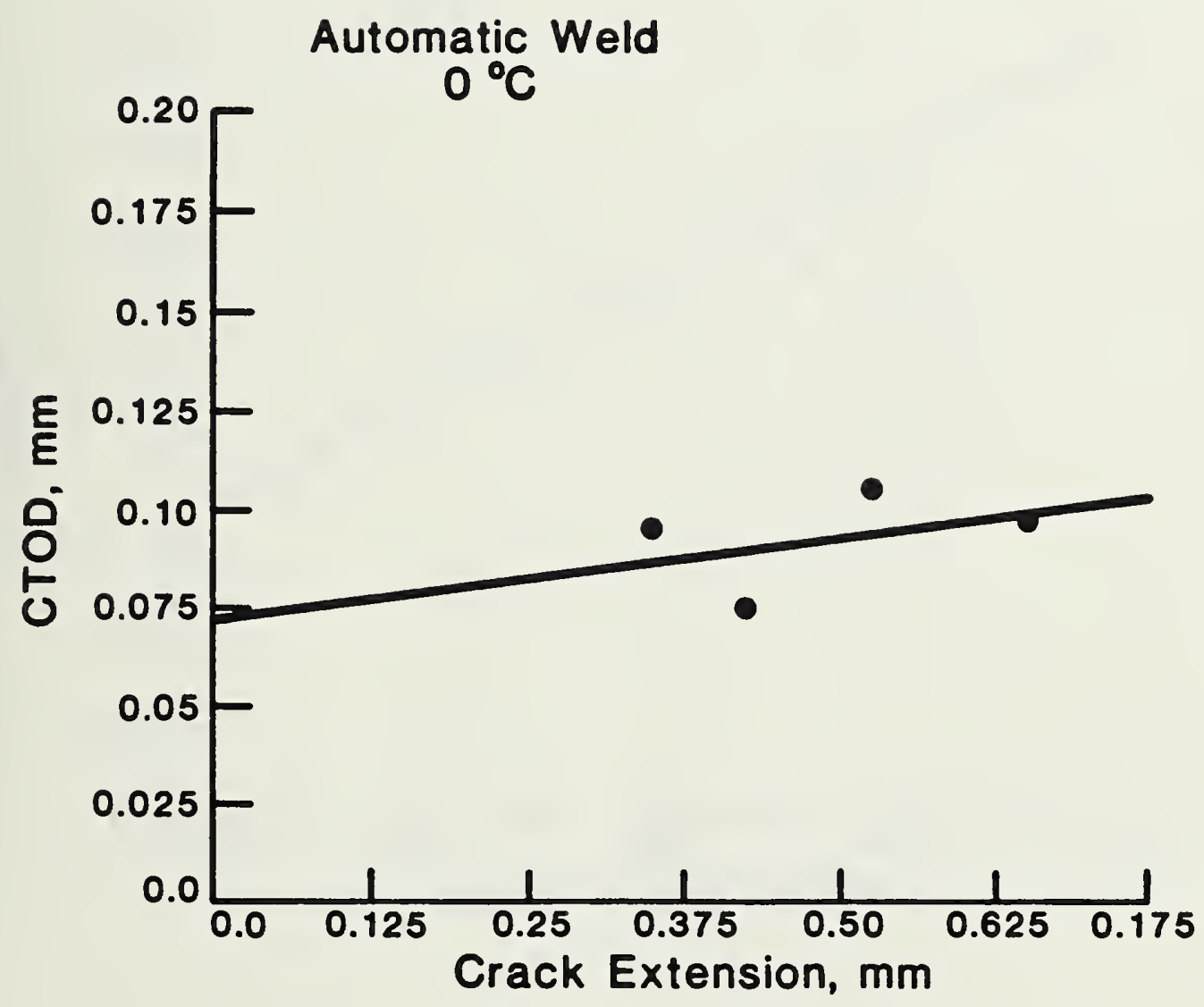

Fig. 9(a) - Crack opening displacement for the initiation of slow, stable crack growth for the automatic GMA girth weld at $0^{\circ} \mathrm{C}\left(32^{\circ} \mathrm{F}\right)$. 



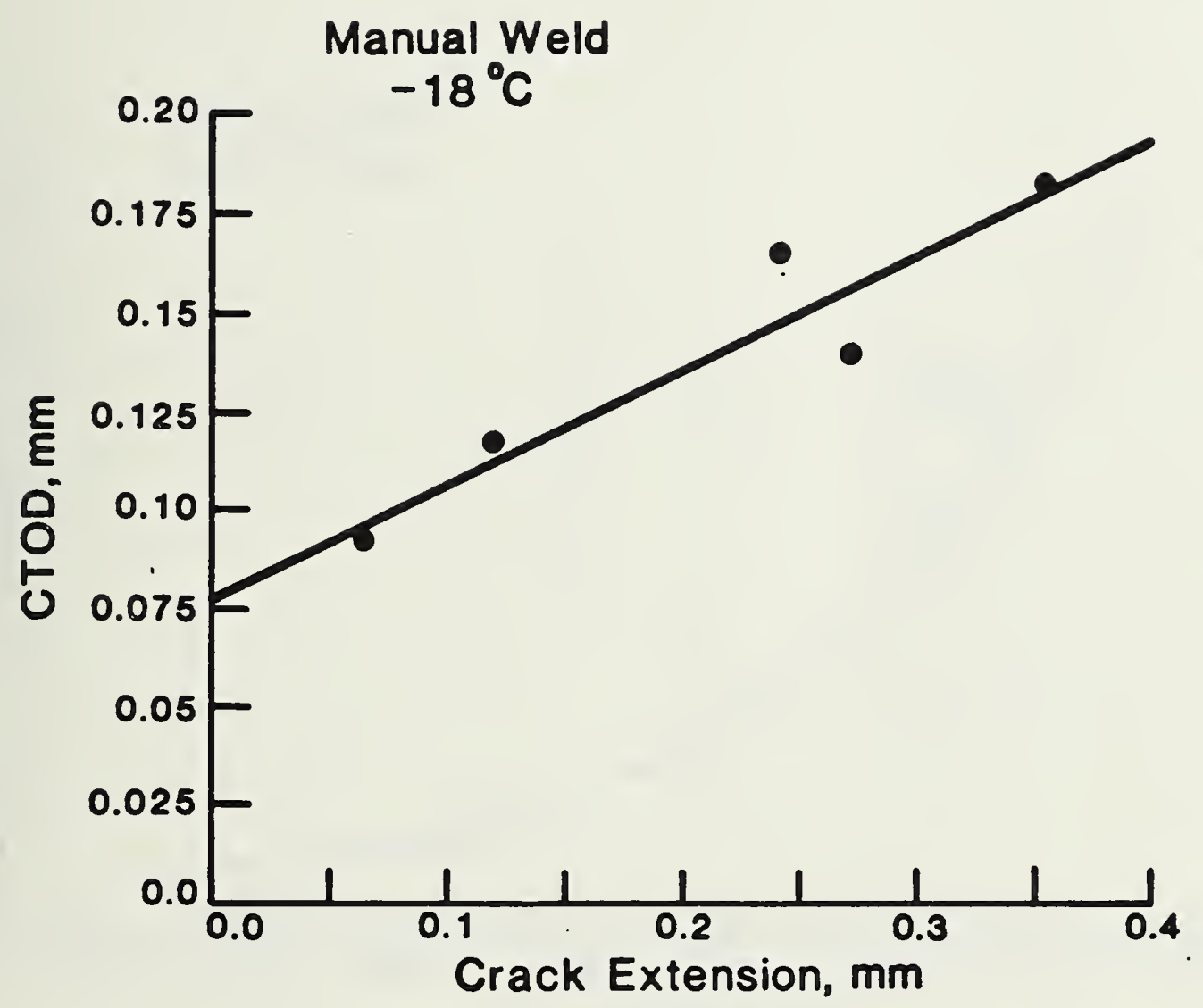

Fig. 9(b) - Crack-opening displacement for the initiation of slow, stable crack growth for the manual SMA girth weld. 



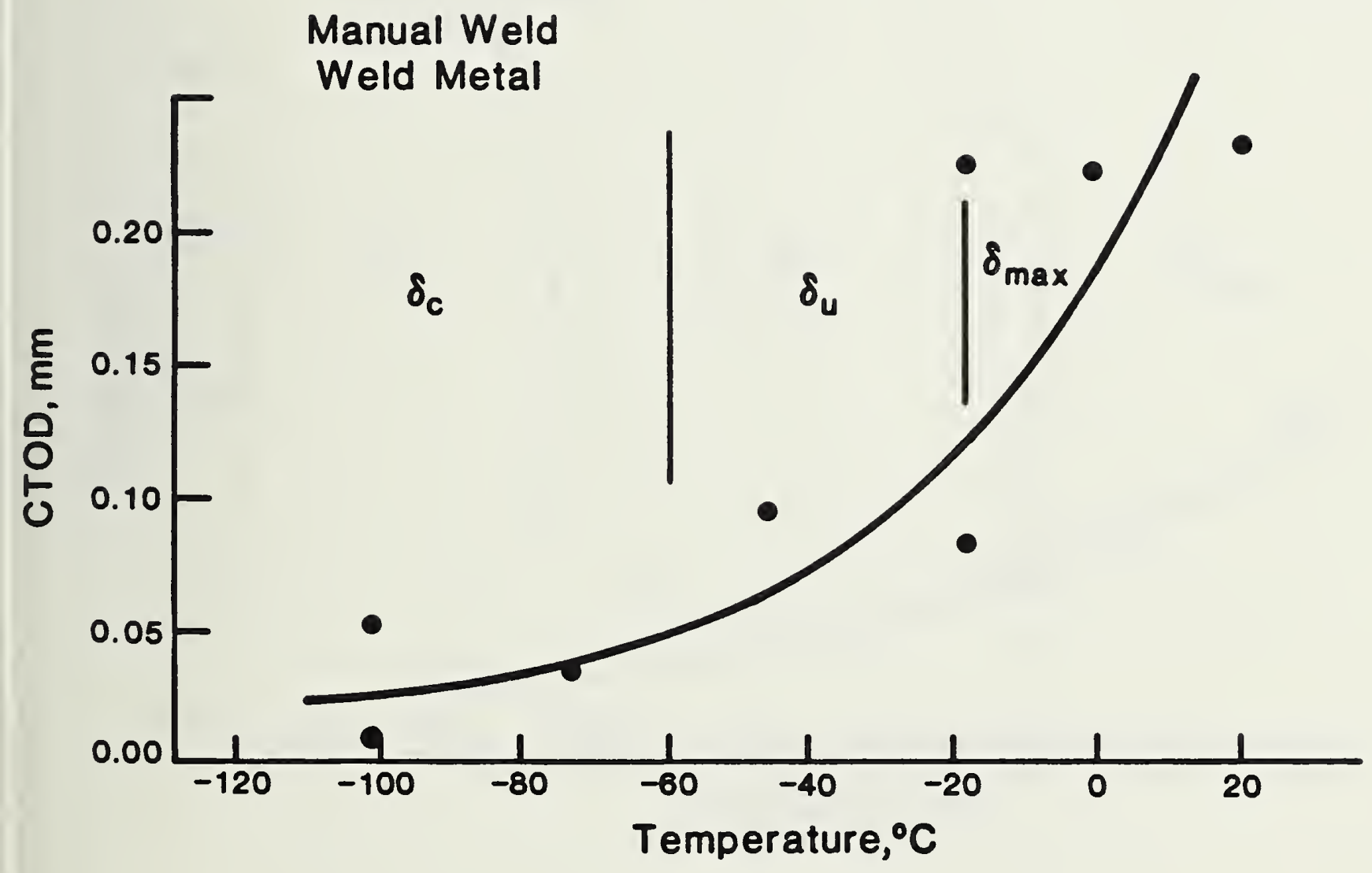

Fig. $9(c)$ - Temperature dependence of crack-opening displacement for the weld metal in the manual SMA girth weld. 



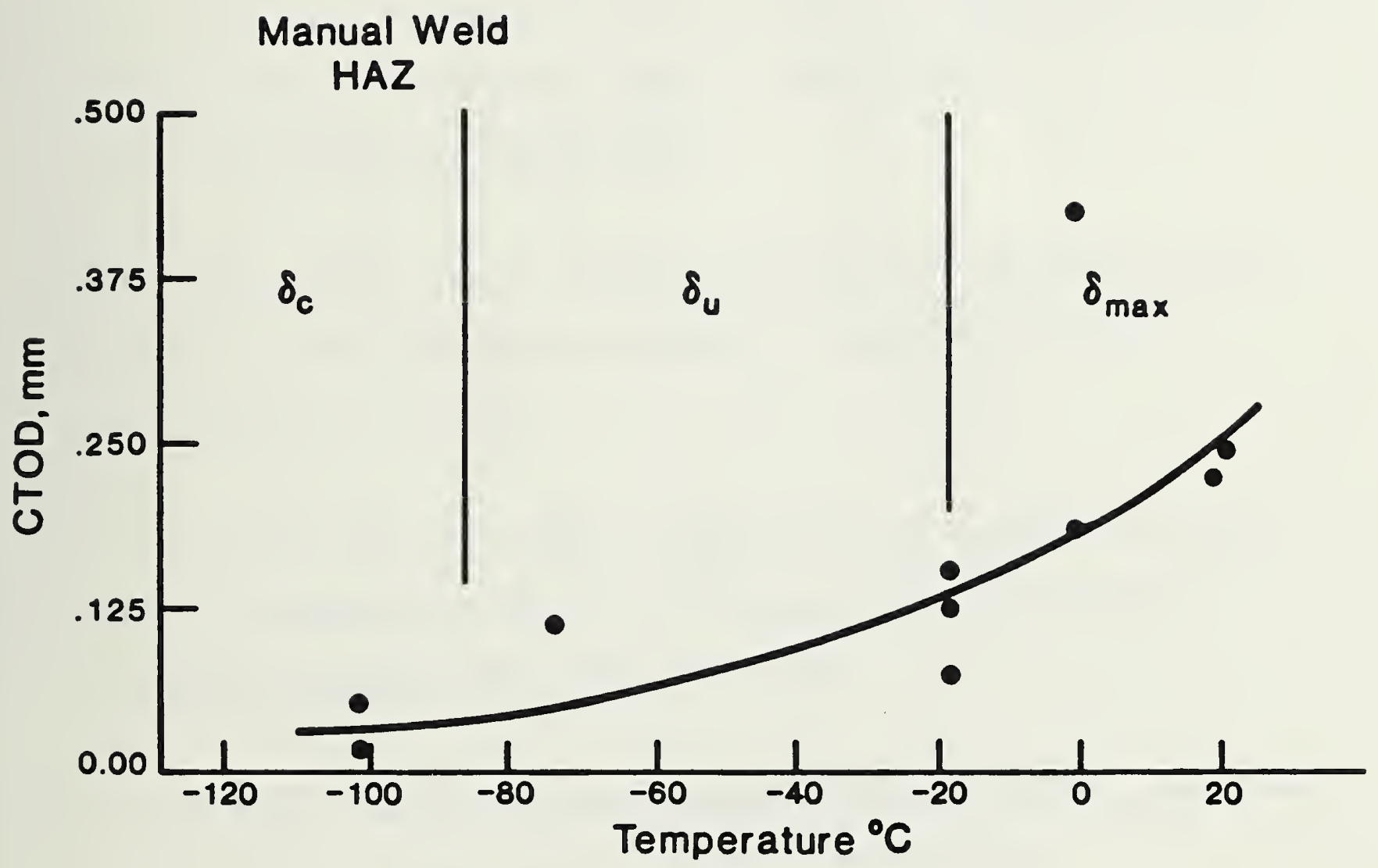

Fig. 9(d) - Temperature dependence of crack-opening displacement for the $H A Z$ from the manual SMA girth weld. 



\section{References}

1. "Methods for Crack Opening Displacement (COD) Testing," BS5762 British Standards Institution, London (1979).

2. J. D. Harrison, "The State-of-the-Art in Crack Tip Opening Displacement (CTOD) Testing and Analysis," Report No. 7302.10/80/210.2, The Welding Institute, Cambridge, England (1980).

3. G. Kaufman, "Developments in Fracture Mechanics Test Methods Standardization" ASTM STP 632, American Society for Testing and Materials, Philadelphia, 11- (1977).

4. H. G. Pisarski and M. G. Dawes, "Measurement of Crack Opening Displacement in Weldments with Particular Reference to Offshore Structures," Fracture Mechanics, Rome, Italy (June 1980).

5. M. G. Dawes, "Fatigue Precracking Weldment Fracture Mechanics Specimens," Met. Constr. and Brit. Weld. J., 3, No. 2, 61-65 (1971).

6. M. G. Dawes, "Contemporary Measurement of Weld Metal Fracture Toughness," Weld. ‥ 55, 1052-1057 (1976). 

Document describes a computer program; SF-185, FIPS Software Summary, is attached.

ABSTRACT (A 200-word or less foctual summary of most significant information. If document includes a significant bibliogrophy or literoture survey. mention it here)

Criteria have been developed for applying fitness-for-service analyses to flaws in e girth welds of the Alaska Natural Gas Transmission System pipeline. A critical ack-opening-displacement elastic-plastic fracture mechanics model was developed and perimentaliy verified. Procedures for constructing flaw acceptance criteria curves sed on this model are provided. A significantly improved ultrasonic method for tecting and dimensioning significant weld flaws was developed and demonstrated on peline sections. The probability of crack initiation from blunt flaws was shown to very low under severe low-cycie fatigue. Suggestions are offered for technical plementation of field inspection procedures and for practical implementation of the aw acceptance criteria.

KEY WORDS (Six to twelve entries: alphabetical order; capitalize only proper names; and separate key words by semicolons) Elastic-plastic fracture mechanics; fitness-for-service; nondestructive evaluation; pipeline; pipeline steel; weldment flaws

\section{AVAILABILITY}

Donlimited

For Official Distribution. Do Not Release to NTIS

Order From Superintendent of Documents, U.S. Government Printing Office, Washington, D.C. 20402.

Order From National Technical Information Service (NTIS), Springfield, VA. 22161
14. NO. OF

PRINTED PAGES

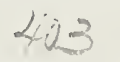

15. Price 


(16) 
